\title{
State Energy Price System: 1982 Update
}

K. L. Imhoff

J. M. Fang

October 1984

Prepared for the U.S. Department of Energy under Contract DE-AC06-76RLO 1830

Pacific Northwest Laboratory Operated for the U.S. Department of Energy by Battelle Memorial Institute 


\title{
DISCLAIMER
}

This report was prepared as an account of work sponsored by an agency of the United States Government. Neither the United States Government nor any agency thereof, nor any of their employees, makes any warranty, express or implied, or assumes any legal liability or responsibility for the accuracy, completeness, or usefulness of any information, apparatus, product, or process disclosed, or represents that its use would not infringe privately owned rights. Reference herein to any specific commercial product, process, or service by trade name, trademark, manufacturer, or otherwise, does not necessarily constitute or imply its endorsement, recommendation, or favoring by the United States Government or any agency thereof. The views and opinions of authors expressed herein do not necessarily state or reflect those of the United States Government or any agency thereof.

\author{
PACIFIC NORTHWEST LABORATORY \\ operated by \\ BATTELLE \\ for the \\ UNITED STATES DEPARTMENT OF ENERGY \\ under Contract DE-AC06-76RLO 1830
}

\begin{tabular}{|c|c|}
\hline \multirow{2}{*}{\multicolumn{2}{|c|}{ Printed in the United States of America }} \\
\hline & \\
\hline \multirow{2}{*}{\multicolumn{2}{|c|}{$\begin{array}{l}\text { National Technical Information Service } \\
\text { United States Department of Commerce }\end{array}$}} \\
\hline & \\
\hline \multirow{2}{*}{\multicolumn{2}{|c|}{$\begin{array}{c}5285 \text { Port Royal Road } \\
\text { Springfield, Virginia } 22161\end{array}$}} \\
\hline & \\
\hline \multirow{2}{*}{\multicolumn{2}{|c|}{$\begin{array}{l}\text { NTIS Price Codes } \\
\text { Microfiche A01 }\end{array}$}} \\
\hline & \\
\hline \multicolumn{2}{|c|}{ Printed Copy } \\
\hline & Price \\
\hline Pages & Codes \\
\hline $001-025$ & $\mathrm{~A} 02$ \\
\hline $026-050$ & $\mathrm{AO} 3$ \\
\hline $051-075$ & $\mathrm{AO4}$ \\
\hline $076-100$ & A05 \\
\hline $101-125$ & A06 \\
\hline $126-150$ & $\mathrm{~A} 07$ \\
\hline $151-175$ & A08 \\
\hline $176-200$ & A09 \\
\hline $201-225$ & $A 010$ \\
\hline $226-250$ & $A 011$ \\
\hline $251-275$ & $A 012$ \\
\hline $276-300$ & $A 013$ \\
\hline
\end{tabular}


STATE ENERGY PRICE SYSTEM:

1982 UPDATE

K. L. Imhoff

J. M. Fang

October 1984

Prepared for

the Office of Energy Markets and End Use

Energy Information Administration

U.S. Department of Energy

under Contract DE-AC06-76RL0 1830

Pacific Northwest Laboratory

Richland, Washington 99352 


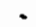

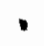

a

-

,

$\bullet$ 


\section{CONTENTS}

SUMMARY.................................................. ix

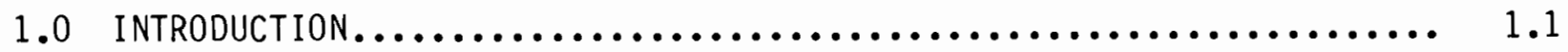

2.0 PHYSICAL UNIT PRICES $\ldots \ldots \ldots \ldots \ldots \ldots \ldots \ldots \ldots \ldots \ldots \ldots \ldots \ldots \ldots \ldots \ldots \ldots \ldots$

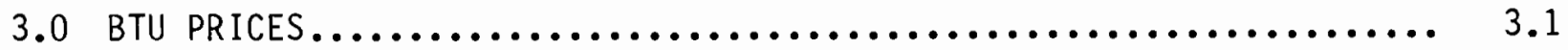

4.01982 STEPS UPDATE DOCUMENTATION....................... 4.1

4.1 ELECTRICITY $\ldots \ldots \ldots \ldots \ldots \ldots \ldots \ldots \ldots \ldots \ldots \ldots \ldots \ldots \ldots \ldots \ldots \ldots \ldots \ldots . \ldots \ldots$

4.2 NATURAL GAS $\ldots \ldots \ldots \ldots \ldots \ldots \ldots \ldots \ldots \ldots \ldots \ldots \ldots \ldots \ldots \ldots \ldots \ldots \ldots \ldots \ldots \ldots \ldots$

4.3 METALLURGICAL COAL........................... 4.2

4.4 STEAM COAL................................. 4.3

4.4 .1 Residential Sector.......................... 4.5

4.4 .2 Industrial Sector......................... 4.6

4.5 DISTILLATE FUEL. $\ldots \ldots \ldots \ldots \ldots \ldots \ldots \ldots \ldots \ldots \ldots \ldots \ldots \ldots \ldots \ldots \ldots \ldots$

4.5 .1 Residential Sector........................ 4.7

4.5 .2 Commercial Sector......................... 4.9

4.5 .3 Industrial Sector........................ 4.10

4.6 MOTOR GASOLINE.............................. 4.11

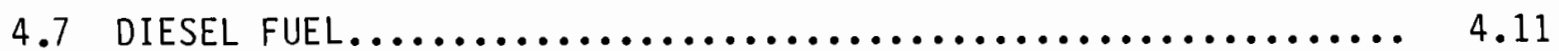

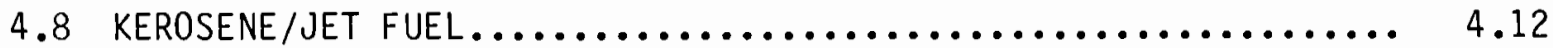

4.8.1 Residential Sector........................ 4.12

4.8 .2 Industrial Sector........................ 4.19

4.8.3 Transportation Sector....................... 4.19

4.8.4 Electric Utility Sector.................... 4.20

4.9 RESIDUAL FUEL............................... 4.20

4.9 .1 Industrial Sector.......................... 4.21 
4.9.2 Transportation Sector...................... 4.22

4.10 LIQUEF IEd PETROLEUM GAS........................ 4.23

4.10 .1 Residential Sector........................ 4.23

4.10 .2 Industrial Sector....................... 4.23

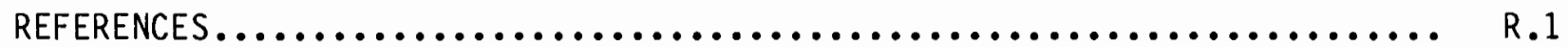

APPENDIX A: CONVERSION FACTORS ......................... A.1 


\section{TABLES}

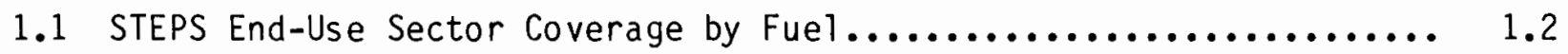

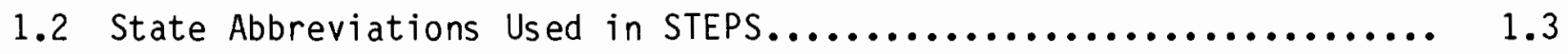

2.1 Electricity Prices: Residential Sector $(\$ / k W h) \ldots \ldots \ldots \ldots \ldots \ldots . . . .2$

2.2 Electricity Prices: Commercial Sector $(\$ / k w h) \ldots \ldots \ldots \ldots \ldots \ldots \ldots .6$

2.3 Electricity Prices: Industrial Sector $(\$ / k W h) \ldots \ldots \ldots \ldots \ldots \ldots . . . . . .2$

2.4 Electricity Prices: Transportation Sector $(\$ / k W h) \ldots \ldots \ldots \ldots \ldots . . .2 .12$

2.5 Natural Gas Prices: Residential Sector $(\$ / 1000 \mathrm{cu} f \mathrm{ft}) \ldots \ldots \ldots \ldots .2 .15$

2.6 Natural Gas Prices: Commercial Sector $(\$ / 1000 \mathrm{cu} f \mathrm{ft}) \ldots \ldots \ldots \ldots \ldots .2 .18$

2.7 Natural Gas Prices: Industrial Sector $(\$ / 1000 \mathrm{cu} f \mathrm{ft}) \ldots \ldots \ldots \ldots . .2 .21$

2.8 Natural Gas Prices: Transportation Sector $(\$ / 1000 \mathrm{cu} f \mathrm{ft}) \ldots \ldots \ldots .2 .24$

2.9 Natural Gas Prices: Electric Utility Sector $(\$ / 1000 \mathrm{cu} f \mathrm{t}) \ldots \ldots .2 .27$

2.10 Metallurgical Coal Prices: Industrial Sector $(\$ /$ ton $) .. . . . . . . .2 .30$

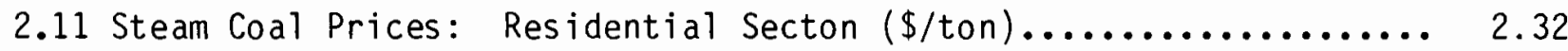

2.12 Steam Coal Prices: Industrial Sector (\$/ton)............... 2.34

2.13 Steam Coal Prices: Electric Utility Sector $(\$ /$ ton $) \ldots \ldots \ldots \ldots \ldots .2 .36$

2.14 Distillate Fuel Prices: Residential Sector (\$/gallon).......... 2.38

2.15 Distillate Fuel Prices: Commercial Sector (\$/gallon)........... 2.40

2.16 Distillate Fuel Prices: Industrial Sector (\$/gallon).......... 2.42

2.17 Distillate Fuel Prices: Electric Utility Sector (\$/gallon)....... 2.44

2.18 Motor Gasoline Prices: Transportation Sector (\$/gallon)......... 2.46

2.19 Diesel Fuel Prices: Transportation Sector ( $\$ /$ gallon)........... 2.48

2.20 Kerosene Prices: Residential Sector (\$/gallon)............... 2.50

2.21 Kerosene Prices: Industrial Sector (\$/gallon)............... 2.52

2.22 Jet Fuel Prices: Transportation Sector (\$/gallon)............. 2.54 
2.23 Kerosene Prices: Electric Utility Sector $(\$ /$ gallon)........... 2.56

2.24 Residual Fuel Prices: Commercial Sector $(\$ / b b 1) \ldots \ldots \ldots \ldots \ldots . . \ldots . . . .2 .58$

2.25 Residual Fuel Prices: Industrial Sector $(\$ / b b 1) \ldots . \ldots \ldots \ldots \ldots . . . . .2 .60$

2.26 Residual Fuel Prices: Transportation Sector $(\$ / b b 1) \ldots \ldots \ldots \ldots . . . .2 .62$

2.27 Residual Fuel Prices: Electric Utility Sector $(\$ / b b 1) \ldots . . \ldots \ldots . . .2 .64$

2.28 Liquefied Petroleum Gas Prices: Residential Sector (\$/gallon)..... 2.66

2.29 Liquefied Petroleum Gas Prices: Industrial Sector ( $\$ /$ gallon)..... 2.68

3.1 Electricity Prices: Residential Sector $(\$ / m i l l i o n$ Btu).......... 3.3

3.2 Electricity Prices: Commercial Sector $(\$ / m i l l i o n$ Btu).......... 3.6

3.3 Electricity Prices: Industrial Sector $(\$ / m i l l i o n$ Btu).......... 3.9

3.4 Electricity Prices: Transportation Sector $(\$ /$ million Btu) ....... 3.12

3.5 Natural Gas Prices: Residential Sector (\$/million Btu)......... 3.15

3.6 Natural Gas Prices: Commercial Sector $(\$ / m i l l i o n$ Btu)........... 3.18

3.7 Natural Gas Prices: Industrial Sector $(\$ / m i l l i o n$ Btu).......... 3.21

3.8 Natural Gas Prices: Transportation Sector (\$/million Btu)....... 3.24

3.9 Natural Gas Prices: Electric Utility Sector (\$/million Btu)...... 3.27

3.10 Metallurgical Coal Prices: Industrial Sector ( $\$ / m i l l i o n$ Btu)...... 3.30

3.11 Steam Coal Prices: Residential Sector ( $\$ /$ million Btu)........... 3.32

3.12 Steam Coal Prices: Industrial Sector $(\$ / m i l l i o n$ Btu)........... 3.34

3.13 Steam Coal Prices: Electric Utility Sector $(\$ / m i l l i o n$ Btu)....... 3.36

3.14 Distillate Fuel Prices: Residential Sector $(\$ / m i 11$ ion Btu)....... 3.38

3.15 Distillate Fuel Prices: Commercial Sector (\$/million Btu)........ 3.40

3.16 Distillate Fuel Prices: Industrial Sector $(\$ / m i l l i o n$ Btu) ........ 3.42

3.17 Distillate Fuel Prices: Electric Utility Sector
$(\$ /$ million Btu $) \ldots \ldots \ldots \ldots \ldots \ldots \ldots \ldots \ldots \ldots \ldots \ldots \ldots \ldots \ldots \ldots \ldots \ldots \ldots \ldots$

3.18 Motor Gasoline Prices: Transportation Sector ( $\$ /$ million Btu)...... 3.46 
3.19 Diese1 Fuel Prices: Transportation Sector ( $\$ /$ million Btu)........ 3.48

3.20 Kerosene Prices: Residential Sector (\$/million Btu)............ 3.50

3.21 Kerosene Prices: Industrial Sector (\$/million Btu)............. 3.52

3.22 Jet Fuel Prices: Transportation Sector ( $\$ /$ million Btu).......... 3.54

3.23 Kerosene Prices: Electric Utility Sector $(\$ / m i 11$ ion Btu)......... 3.56

3.24 Residual Fuel Prices: Commercial Sector ( $\$ /$ million Btu).......... 3.58

3.25 Residual Fuel Prices: Industrial Sector (\$/million Btu).......... 3.60

3.26 Residual Fuel Prices: Transportation Sector (\$/million Btu)...... 3.62

3.27 Residual Fuel Prices: Electricity Utility Sector

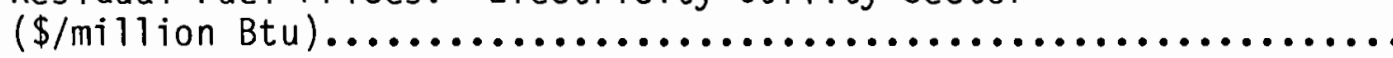

3.28 Liquefied Petroleum Gas Prices: Residential Sector

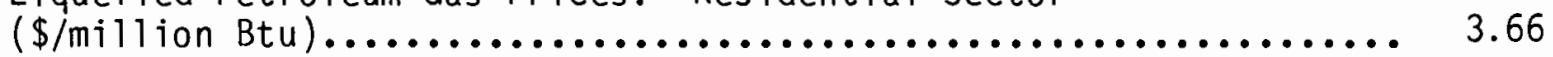

3.29 Liquefied Petroleum Gas Prices: Industrial Sector

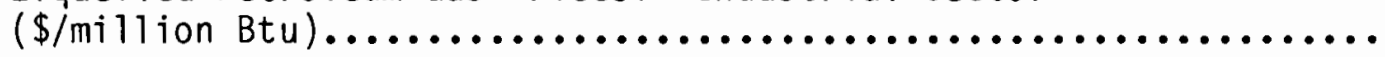

4.1 Revenues and Sales Data Table Numbers in the EEI Statistical Year

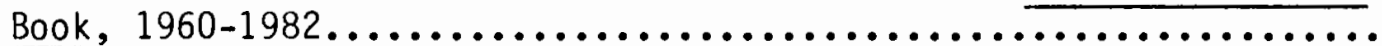

4.2 Metallurgical Coal State Price Assignments, 1970-1982........... 4.4

4.3 Estimated Values of $c_{X X}$ for Residential Sector Steam Coal Prices......................................... 4.5

4.4 Price Assignments for States Missing Cost and Quality Prices,

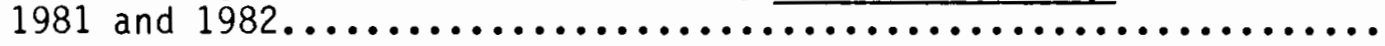
4.6

4.5 Residual Fuel Transportation Use List for 1982 Update........... 4.22 
. 
This report updates the State Energy Price Data System (STEPS) to include state-level energy prices by fuel and by end-use sectors through 1982. Both physical unit prices and prices per million Btu are presented. Basic documentation of the STEPS data base remains generally the same as in the original report: State Energy Price System, Volume 1: Overview and Technical Documentation (DOE/NBB-0029, Volume 1 of 2, November 1982). This report documents only the changes in procedures necessitated by the updates to 1981 and 1982, and corrections to the basic documentation. The most significant changes in procedures for the updates occur in the residential sector distillate series and the residential sector kerosene series. All physical unit and Btu prices are shown with three significant digits instead of with four significant digits as shown in the original documentation. Details of these and other changes are contained in this report, along with the updated data files. 


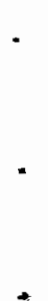




\subsection{INTRODUCTION}

The State Energy Price System (STEPS) is a data base containing annual energy prices by major end-use sectors by state. Published and maintained by the Energy Information Administration (EIA), STEPS serves three primary uses: 1) support for the EIA's regional energy analysis effort; 2) to update, with the consumption data from the EIA's State Energy Data System (SEDS), the State Price and Expenditure Report (SEPER); and 3) to provide for policy development and the general information needs of all levels of government and the public. The original STEPS series covered the period 1960 to 1980 for electricity and natural gas, and 1970 to 1980 for all other fuels.(a) Subsequently, the STEPS series were updated to include data for 1981 and an update report was issued to document changes in the original STEPS procedures needed for the update. (b) This report updates the price series to 1982, and contains documentation changes that occurred in both the 1981 and 1982 updates.

STEPS contains estimates of energy prices for ten major fuels (electricity, natural gas, metallurgical coal, steam coal, distillate, motor gasoline, diesel, kerosene/jet fuel, residual fuel, and 1 iquefied petroleum gas), by major end-use sectors (residential, commercial, industrial, transportation, and electric utility), and by state. Table 1.1 shows the sectors covered for each of the fuels. Both physical unit prices and prices per million Btu are included in STEPS.

The price estimates in STEPS are based on a variety of data sources and estimation procedures as needed to fill gaps in the data. Detailed documentation for STEPS can be found in State Energy Price System, Volume 1: Overview and Technical Documentation. The documentation in Chapter 4.0 of this report supplements and, where appropriate, updates the basic STEPS documentation, referring directly to the equation numbers contained in the original

(a) Energy Information Administration, U.S. Department of Energy. 1982. State Energy Price System, DOE/NBB-0029. Volumes 1 and 2. Washington, D.C.

(b) Fang, J. M., K. L. Imhoff, and L. J. Hood. 1983. State Energy Price System: 1981 Update, PNL-4802, Pacific Northwest Laboratory, Richland, Washington. 


\section{TABLE 1.1. STEPS End-Use Sector Coverage by Fuel}

\begin{tabular}{|c|c|c|c|c|c|}
\hline Fuel & Residential & Commercial & Industrial & Transportation & $\begin{array}{l}\text { Electric } \\
\text { Utility }\end{array}$ \\
\hline Electricity & * & * & * & * & -- \\
\hline Natural Gas & * & * & * & $\star$ & * \\
\hline $\begin{array}{l}\text { Metallurgical } \\
\text { Coal }\end{array}$ & -- & -- & * & -- & -- \\
\hline Steam Coal & * & -- & * & -- & $\star$ \\
\hline Distillate & * & * & * & -- & * \\
\hline Motor Gasoline & -- & -- & - & $\star$ & -- \\
\hline Diesel & -- & -- & -- & * & -- \\
\hline $\begin{array}{l}\text { Kerosene/Jet } \\
\text { Fuel }\end{array}$ & $\star$ & -- & * & * & * \\
\hline Residual $0 i 1$ & -- & * & * & * & * \\
\hline LPG & $\star$ & -- & * & -- & -- \\
\hline
\end{tabular}

Note: $" * "$ = contained in STEPS; "--" not contained in STEPS. Source: Pacific Northwest Laboratory.

documentation where necessary. This report should be regarded as a supplement to the STEPS documentation and not as a complete documentation, as it contains only the procedures that changed for the 1981 and 1982 updates from those given in State Energy Price System, Volume 1.

Chapter 2.0 of this report contains the updated physical unit price data base, while Chapter 3.0 contains the updated Btu price data base. All of the prices in these two chapters are shown with three significant digits rather than the four significant digits given in the original documentation. Table 1.2 gives the state code abbreviations used in the data base tables found in these two chapters. Chapter 4.0 first provides a brief summary of the major changes in the STEPS data base for 1981 and 1982, then specific changes are discussed on a fuel-by-fuel basis for all ten of the fuels contained in the data base. Appendix A presents the Btu conversion factors used for 1981 and 1982. 
TABLE 1.2. State Abbreviations Used in STEPS

\begin{tabular}{llll}
\multicolumn{1}{c}{ State } & Code & \multicolumn{1}{c}{ Code } \\
\cline { 1 - 1 } Alaska & AK & Montana & MT \\
Alabama & AL & North Carolina & NC \\
Arkansas & AR & North Dakota & ND \\
Arizona & AZ & Nebraska & NE \\
California & CA & New Hampshire & NH \\
Colorado & CO & New Jersey & NJ \\
Connecticut & CT & New Mexico & NM \\
District of Columbia & DC & Nevada & NV \\
Delaware & DE & New York & NY \\
Florida & FL & Ohio & OH \\
Georgia & GA & Oklahoma & OK \\
Hawaii & HI & Oregon & OR \\
Iowa & IA & Pennsylvania & PA \\
Idaho & ID & Rhode Island & RI \\
Illinois & IL & South Carolina & SC \\
Indiana & IN & South Dakota & SD \\
Kansas & KS & Tennessee & TN \\
Kentucky & KY & Texas & TX \\
Louisiana & LA & Utah & UT \\
Massachusetts & MA & Virginia & VA \\
Maryland & MD & Vermont & VT \\
Maine & ME & Washington & WA \\
Michigan & MI & Wisconsin & WI \\
Minnesota & MN & West Virginia & WV \\
Missouri & MO & Wyoming & WY \\
Mississippi & MS & United States & US \\
& & &
\end{tabular}

Source: Pacific Northwest Laboratory 

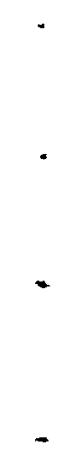

. 


\subsection{PHYSICAL UNIT PRICES}

This chapter presents the updated state-level physical unit energy prices in nominal dollars per common physical unit of the fuel concerned. The periods covered are 1960 through 1982 for electricity and natural gas, and 1970 through 1982 for metallurgical coal, steam coal, distillate, motor gasoline, diesel, kerosene/jet fuel, residual oil, and LPG. Please refer to Chapter 4.0 of State Energy Price System, Volume 1: Overview and Technical Documentation (DOE/NBB0029) for basic documentation of data sources and estimation procedures. Also refer to Chapter 4.0 of this report for all the procedural changes necessitated by the updates to include data for 1981 and 1982, and for the corrections made to the original STEPS documentation. Note that where Btu prices were converted into physical prices, the changes are made to the Btu data, then the physical prices are recalculated. Conversion factors used for the updates are found in Appendix A. 
.

,

. 
TABLE 2.1. Electricity Prices: Residential Sector ( $\$ / \mathrm{k} / \mathrm{lh})$

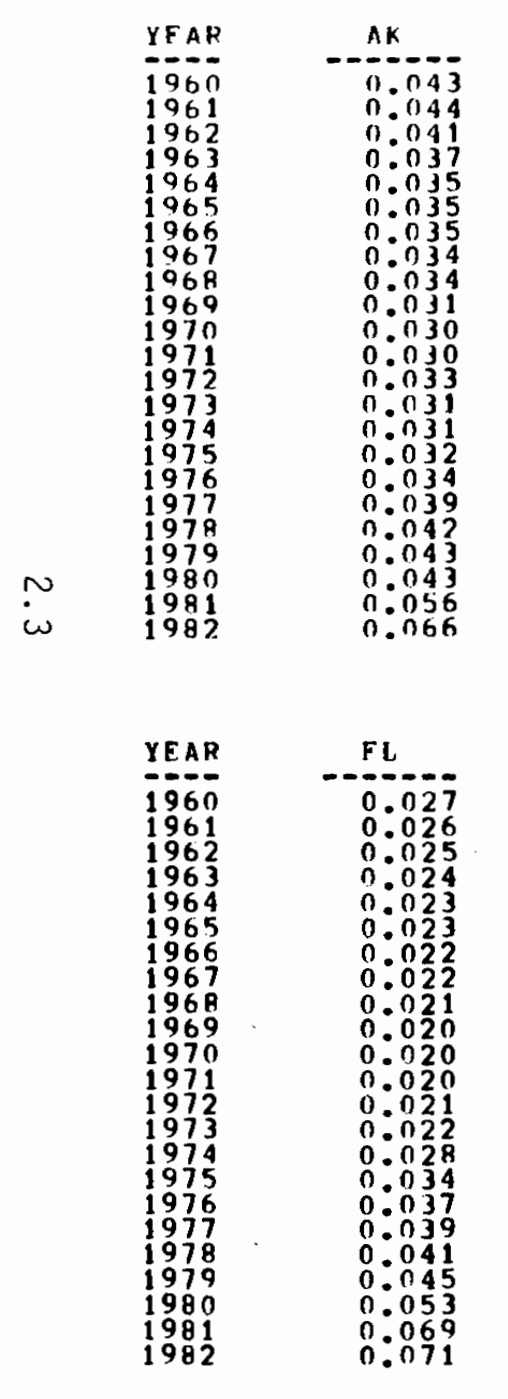

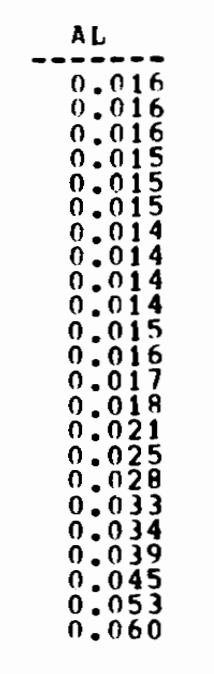
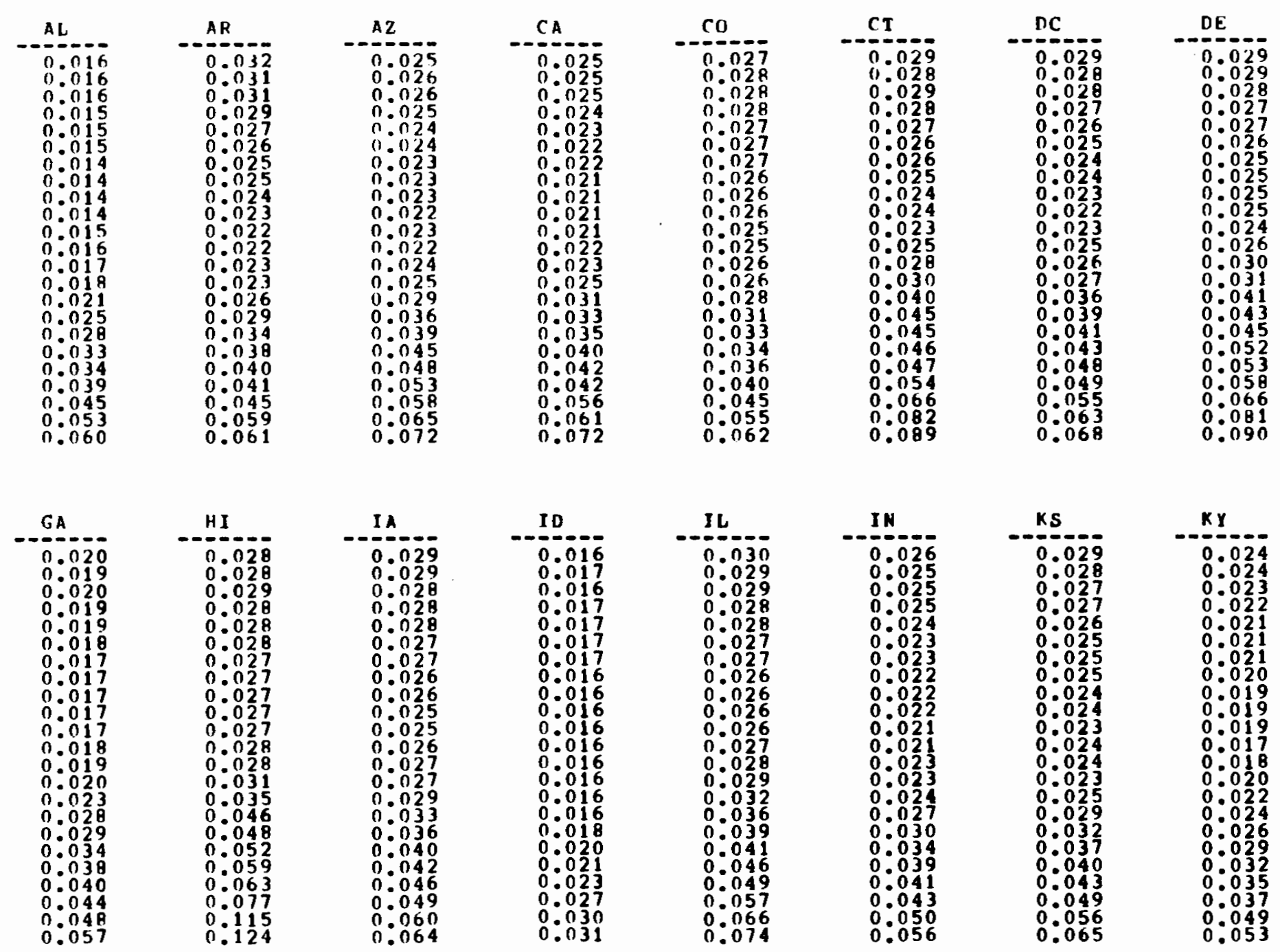

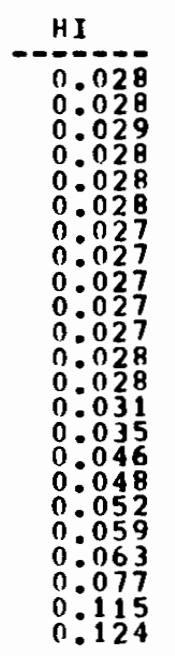

\begin{tabular}{|c|c|}
\hline I A & ID \\
\hline $\begin{array}{l}0.029 \\
0.029 \\
0.028 \\
0.028 \\
0.028 \\
00.027 \\
0.027 \\
00026 \\
0.026 \\
00025 \\
0.025 \\
0.026 \\
0.027 \\
0.027 \\
0.029 \\
0.033 \\
0.036 \\
0.040 \\
0.042 \\
0.046 \\
0.049 \\
0.060 \\
0.064\end{array}$ & $\begin{array}{l}0.016 \\
0: 017 \\
0: 016 \\
0: 017 \\
0: 017 \\
0: 017 \\
0: 017 \\
0: 016 \\
0: 016 \\
0: 016 \\
0: 016 \\
0: 016 \\
0: 016 \\
0: 016 \\
0: 016 \\
0: 016 \\
0: 018 \\
0: 020 \\
0: 021 \\
0: 023 \\
0.027 \\
0: 030 \\
0: 031\end{array}$ \\
\hline
\end{tabular}

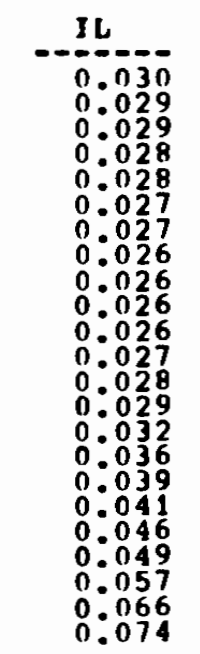
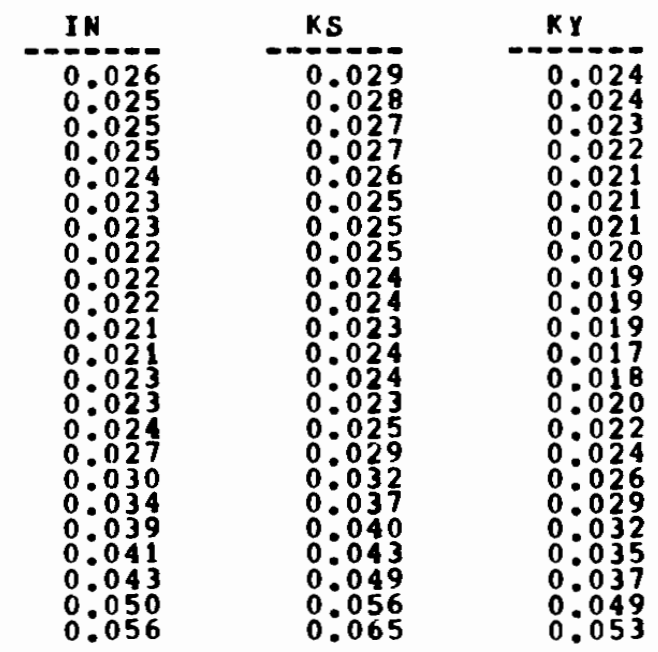
TABLE 2.1 (cont). Electricity Prices: Residential Sector ( $\$ / \mathrm{kWh})$

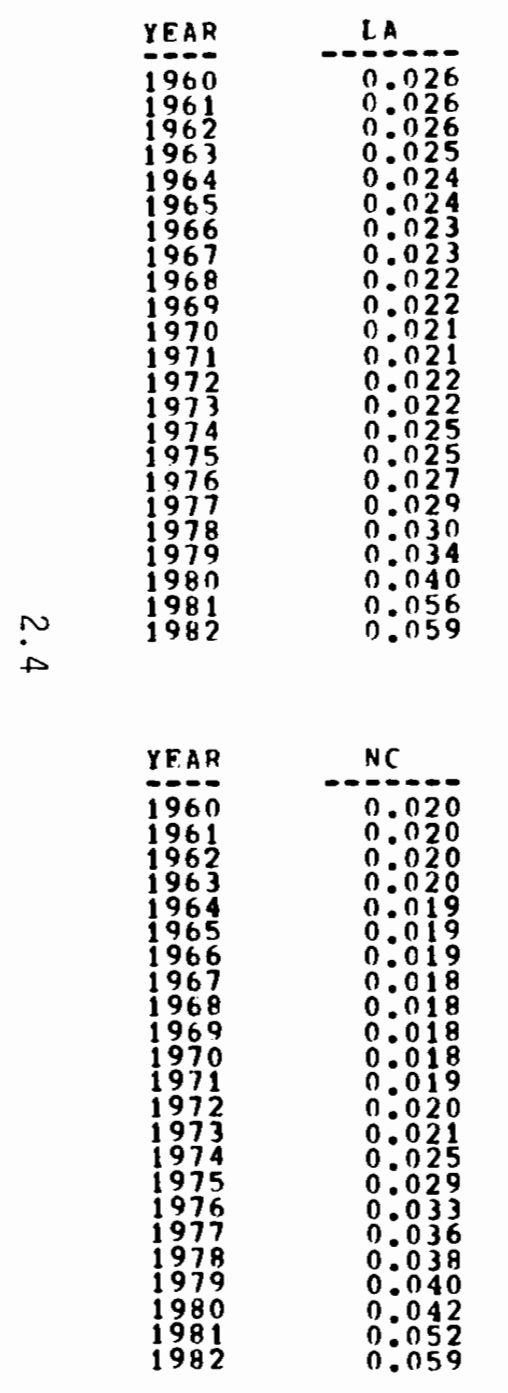

\begin{tabular}{ll}
$M A$ & $M D$ \\
\hline 0.035 & 0.029 \\
0.033 & 0.028 \\
0.033 & 0.028 \\
0.033 & 0.027 \\
0.033 & 0.026 \\
0.032 & 0.025 \\
0.031 & 0.024 \\
0.035 & 0.024 \\
0.029 & 0.023 \\
0.028 & 0.022 \\
0.028 & 0.023 \\
0.030 & 0.025 \\
0.031 & 0.026 \\
0.032 & 0.027 \\
0.043 & 0.036 \\
0.048 & 0.039 \\
0.050 & 0.041 \\
0.053 & 0.043 \\
0.053 & 0.048 \\
0.058 & 0.049 \\
0.069 & 0.055 \\
0.088 & 0.063 \\
0.084 & 0.068
\end{tabular}

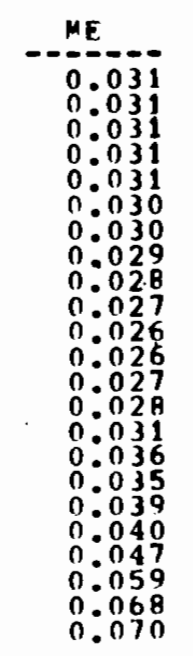
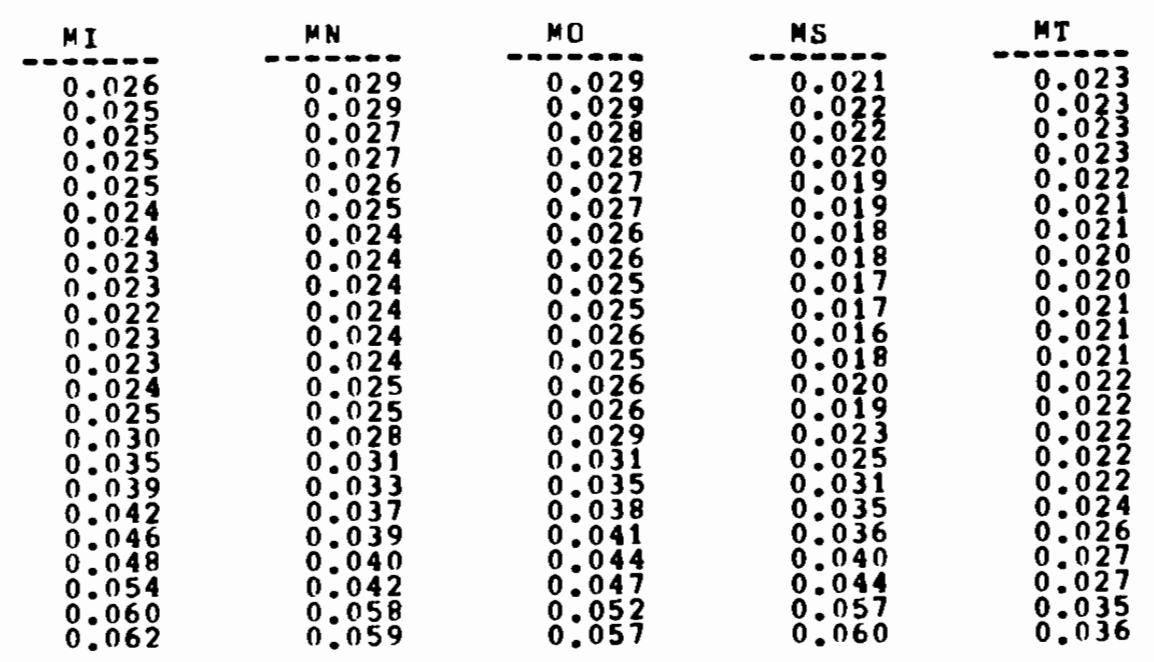

\begin{tabular}{ll} 
ND & NE \\
\hline 0.030 & 0.022 \\
0.029 & 0.023 \\
0.028 & 0.022 \\
0.028 & 0.023 \\
0.027 & 0.023 \\
0.027 & 0.022 \\
0.026 & 0.021 \\
0.025 & 0.021 \\
0.026 & 0.021 \\
0.026 & 0.020 \\
0.025 & 0.020 \\
0.025 & 0.021 \\
0.026 & 0.022 \\
0.026 & 0.021 \\
0.026 & 0.022 \\
0.029 & 0.025 \\
0.031 & 0.030 \\
0.033 & 0.033 \\
0.035 & 0.035 \\
0.037 & 0.037 \\
0.038 & 0.040 \\
0.051 & 0.047 \\
0.053 & 0.047 \\
\end{tabular}

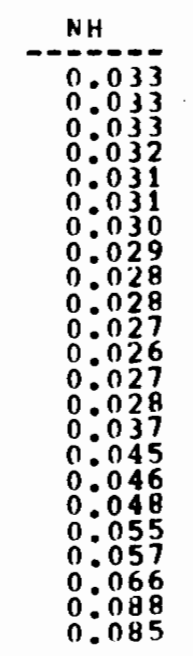

$N J$
$N=0.029$
0.029
0.029
0.029
0.028
0.028
0.027
0.026
0.026
0.026
0.025
0.025
0.029
0.031
0.032
0.044
0.049
0.053
0.059
0.060
0.065
0.078
0.090
0.098

$N M$
00.027
0.029
00029
0.028
0.028
0.027
00.027
0.027
0.027
0.027
0.026
0.026
0.027
0.027
0.029
0.033
0.036
0.040
0.047
0.054
0.058
0.068
0.076

\begin{tabular}{l}
$N V$ \\
\hdashline 0.016 \\
0.016 \\
0.015 \\
0.015 \\
0.015 \\
0.015 \\
0.014 \\
0.015 \\
0.015 \\
0.014 \\
0.014 \\
0.015 \\
0.016 \\
0.016 \\
0.019 \\
0.024 \\
0.028 \\
0.032 \\
0.034 \\
0.036 \\
0.047 \\
0.048 \\
0.056
\end{tabular}

$N Y$
00.032
0.032
0.031
0.031
0.030
0.030
0.030
0.030
0.029
0.028
0.029
0.031
0.033
0.035
0.047
0.051
0.055
0.059
0.061
0.067
0.076
0.093
0.098

\begin{tabular}{l} 
OH \\
\hdashline 0.026 \\
0.025 \\
0.026 \\
0.025 \\
0.025 \\
0.024 \\
0.024 \\
0.024 \\
0.023 \\
0.023 \\
0.023 \\
0.023 \\
0.024 \\
0.024 \\
0.029 \\
0.034 \\
0.0036 \\
0.040 \\
0.044 \\
0.047 \\
0.051 \\
0.060 \\
0.067
\end{tabular}


TABLE 2.1 (cont). Electricity Prices: Residential Sector ( $\$ / \mathrm{kWh})$
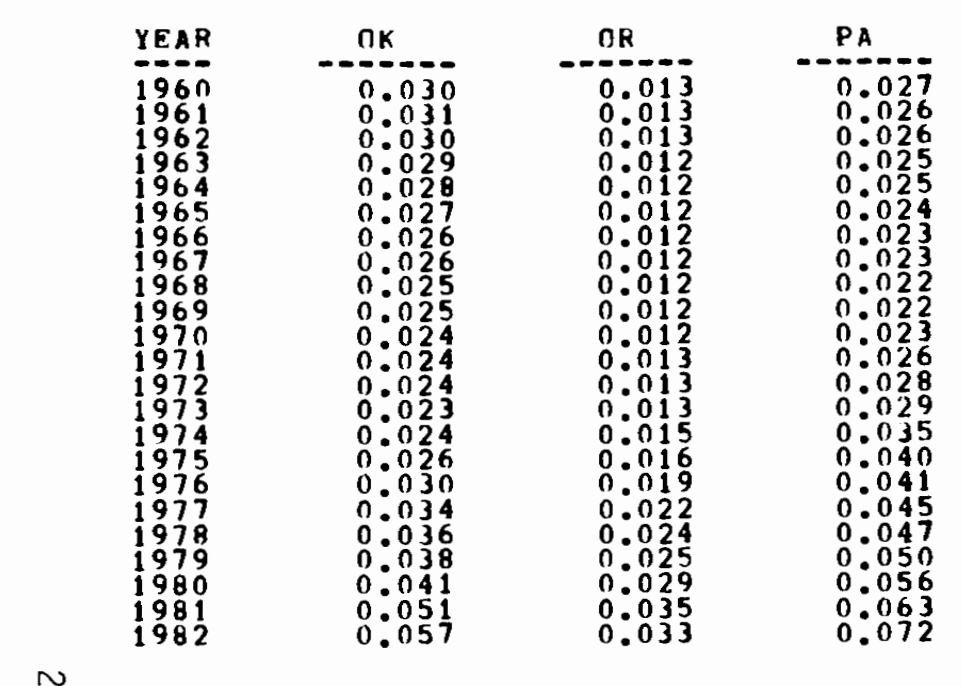

$\begin{array}{ll}R I & S C \\ 0.034 & 0.022 \\ 0.034 & 0.021 \\ 0.033 & 0.021 \\ 0.033 & 0.021 \\ 0.032 & 0.020 \\ 0.032 & 0.020 \\ 0.031 & 0.019 \\ 0.033 & 0.019 \\ 0.029 & 0.019 \\ 0.028 & 0.018 \\ 0.027 & 0.018 \\ 0.028 & 0.020 \\ 0.031 & 0.021 \\ 0.033 & 0.021 \\ 0.046 & 0.026 \\ 0.048 & 0.030 \\ 0.048 & 0.033 \\ 0.054 & 0.037 \\ 0.055 & 0.036 \\ 0.062 & 0.040 \\ 0.074 & 0.042 \\ 0.040 & 0.051 \\ 0.086 & 0.057\end{array}$
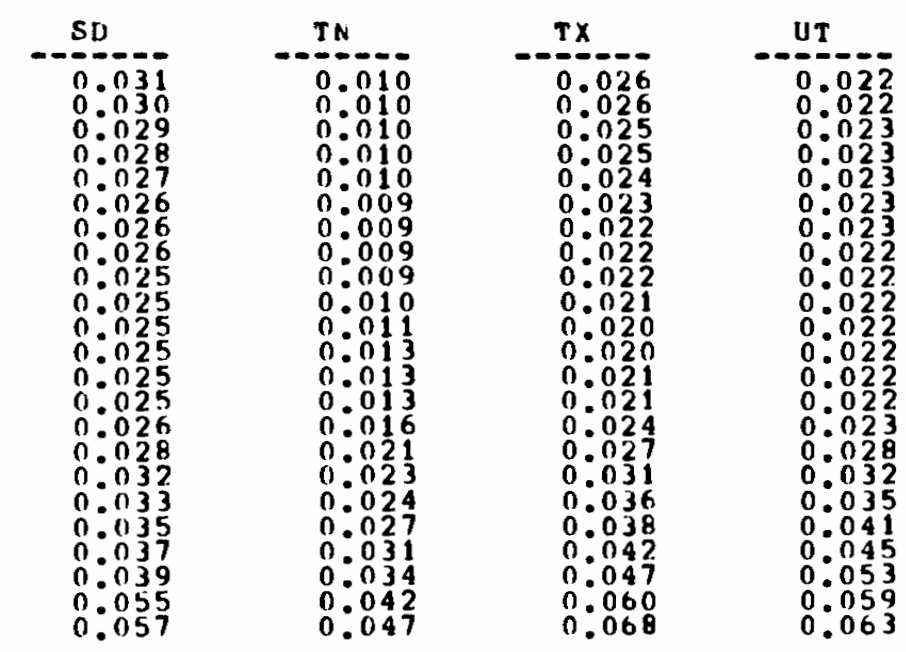

YEAR
1960
1960
1961
1962
1963
1964
1965
1966
1967
1968
1969
1970
1971
1972
1973
1974
1975
1976
1977
1978
1979
1980
1981
1982

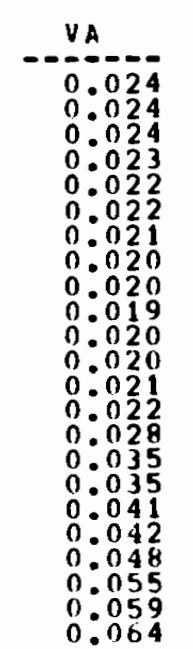
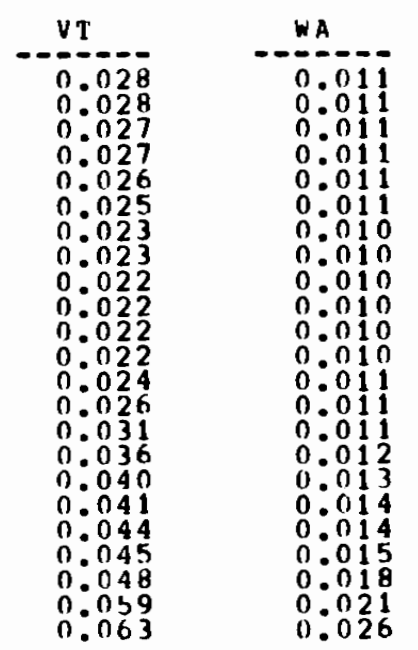

$\begin{array}{ll}w 1 & \\ -1 & 0.026 \\ 0.024 & 0.026 \\ 0.024 & 0.026 \\ 0.024 & 0.025 \\ 0.024 & 0.025 \\ 0.023 & 0.024 \\ 0.023 & 0.024 \\ 0.022 & 0.023 \\ 0.022 & 0.022 \\ 0.021 & 0.022 \\ 0.022 & 0.021 \\ 0.022 & 0.021 \\ 0.022 & 0.027 \\ 0.024 & 0.023 \\ 0.025 & 0.023 \\ 0.027 & 0.027 \\ 0.031 & 0.033 \\ 0.034 & 0.036 \\ 0.037 & 0.035 \\ 0.040 & 0.036 \\ 0.043 & 0.037 \\ 0.046 & 0.041 \\ 0.056 & 0.047 \\ 0.063 & 0.053\end{array}$

$W Y$
0.023
0.02
0.028
0.028
0.027
0.027
0.026
0.025
0.025
0.025
0.025
0.024
0.024
0.025
0.023
0.024
0.024
0.024
0.026
0.028
0.029
0.030
0.044
0.043

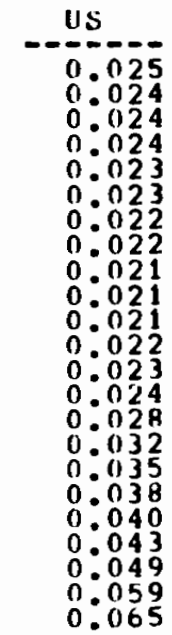

Source: Pacific Northwest Laboratory 
TABLE 2.2. Electricity Prices: Commercial Sector ( $\$ / k W h)$

\begin{tabular}{ll} 
YEAR & AK \\
\hline 1960 & 0.040 \\
1961 & 0.042 \\
1962 & 0.039 \\
1963 & 0.037 \\
1964 & 0.036 \\
1965 & 0.036 \\
1966 & 0.035 \\
1967 & 0.034 \\
1968 & 0.033 \\
1969 & 0.031 \\
1970 & 0.031 \\
1971 & 0.031 \\
1972 & 0.034 \\
1973 & 0.032 \\
1974 & 0.031 \\
1975 & 0.034 \\
1976 & 0.035 \\
1977 & 0.038 \\
1978 & 0.040 \\
1979 & 0.042 \\
1980 & 0.046 \\
1981 & 0.063 \\
1982 & 0.068
\end{tabular}

AL
00.019
0.019
0.019
0.019
0.018
0.018
0.018
0.018
0.017
0.017
0.017
0.018
0.019
0.021
0.024
0.028
0.030
0.038
0.038
0.044
0.050
0.057
0.064
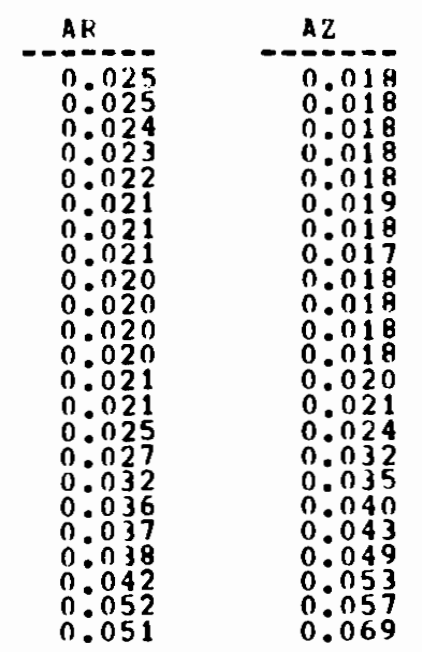

\begin{tabular}{ll}
$C A$ & $C 0$ \\
\hline 0.021 & -0.022 \\
0.021 & 0.022 \\
0.021 & 0.022 \\
0.020 & 0.021 \\
0.018 & 0.021 \\
0.018 & 0.021 \\
0.016 & 0.020 \\
0.016 & 0.020 \\
0.016 & 0.020 \\
0.016 & 0.020 \\
0.016 & 0.019 \\
0.017 & 0.019 \\
0.018 & 0.020 \\
0.020 & 0.020 \\
0.025 & 0.022 \\
0.028 & 0.025 \\
0.030 & 0.028 \\
0.038 & 0.030 \\
0.047 & 0.032 \\
0.041 & 0.036 \\
0.058 & 0.042 \\
0.062 & 0.052 \\
0.070 & 0.058
\end{tabular}
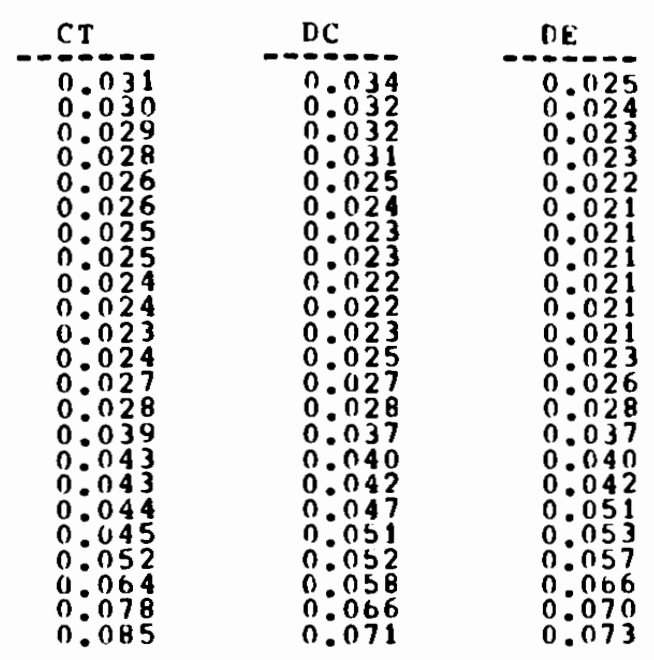

in

\begin{tabular}{ll} 
YEAR & FL \\
\hline 1960 & -0.030 \\
1961 & 0.026 \\
1962 & 0.026 \\
1963 & 0.025 \\
1964 & 0.024 \\
1965 & 0.024 \\
1966 & 0.022 \\
1967 & 0.021 \\
1968 & 0.021 \\
1969 & 0.020 \\
1970 & 0.020 \\
1971 & 0.021 \\
1972 & 0.022 \\
1973 & 0.023 \\
1974 & 0.030 \\
1975 & 0.036 \\
1976 & 0.038 \\
1977 & 0.040 \\
1978 & 0.042 \\
1979 & 0.047 \\
1980 & 0.055 \\
1981 & 0.069 \\
1982 & 0.065
\end{tabular}

$G A$
0.07
0.023
0.022
0.021
0.0221
0.021
0.020
0.020
0.020
0.019
0.019
0.019
0.020
0.021
0.022
0.027
0.034
0.036
0.038
0.041
0.044
0.048
0.050
0.058

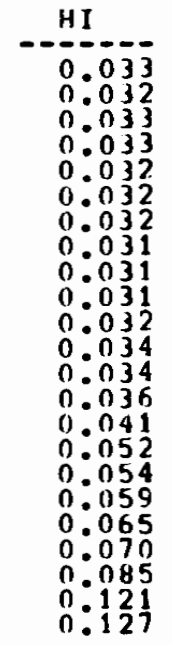

1.
00.031
0.029
0.028
0.027
0.027
0.026
0.026
0.026
0.025
0.025
0.025
0.025
0.026
0.027
0.029
0.033
0.036
0.039
0.041
0.046
0.050
0.055
0.063

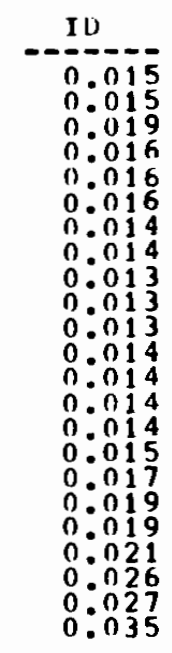

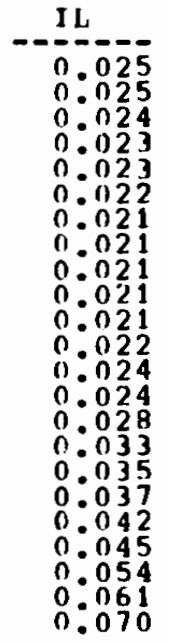

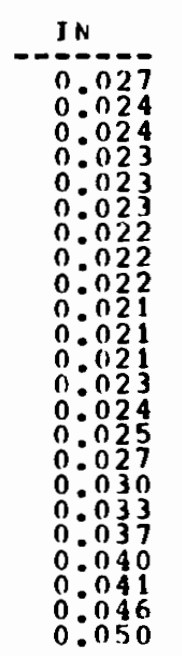

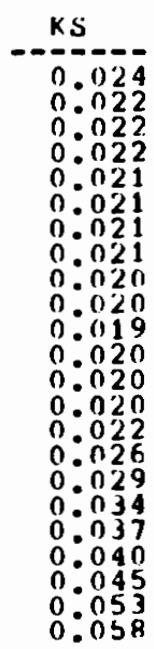

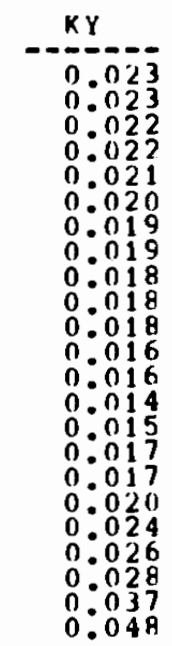


TABLE 2.2 (cont). Electricity Prices: Commercial Sector ( $\$ / \mathrm{kWh})$

\begin{tabular}{ll} 
YEAR & I.A \\
\hline 1960 & 0.024 \\
1961 & 0.022 \\
1962 & 0.022 \\
1963 & 0.020 \\
1964 & 0.020 \\
1965 & 0.019 \\
1966 & 0.018 \\
1967 & 0.017 \\
1968 & 0.017 \\
1969 & 0.016 \\
1970 & 0.016 \\
1971 & 0.016 \\
1972 & 0.017 \\
1973 & 0.018 \\
1974 & 0.021 \\
1975 & 0.022 \\
1976 & 0.025 \\
1977 & 0.027 \\
1978 & 0.028 \\
1979 & 0.032 \\
1980 & 0.037 \\
1981 & 0.047 \\
1982 & 0.049 \\
&
\end{tabular}

0.
0.038
0.035
0.032
0.031
0.031
0.030
0.029
0.028
0.026
0.026
0.026
0.028
0.029
0.029
0.042
0.045
0.048
0.051
0.052
0.057
0.069
0.085
0.082
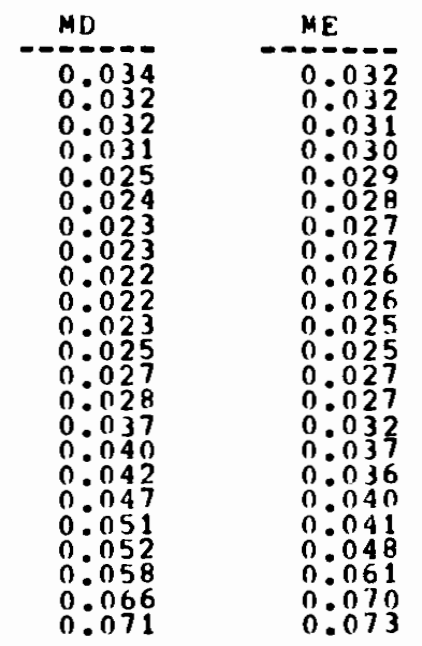

\begin{tabular}{|c|}
\hline 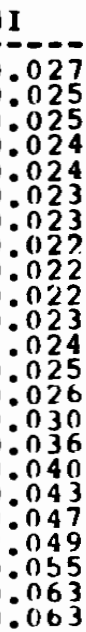 \\
\hline
\end{tabular}
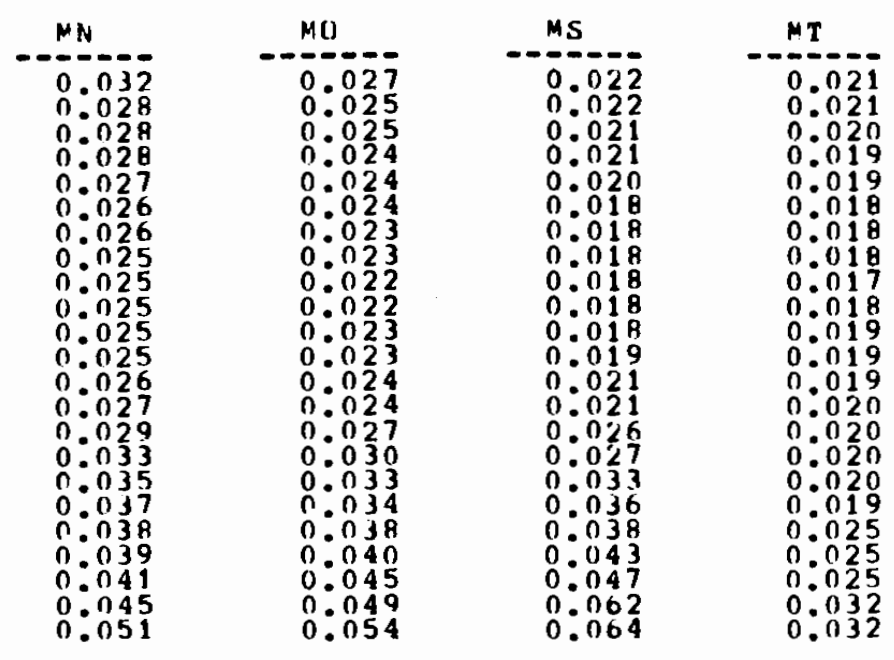

\begin{tabular}{ll} 
YEAR & NC \\
\hline 1960 & 0.020 \\
1961 & 0.018 \\
1962 & 0.018 \\
1963 & 0.018 \\
1964 & 0.017 \\
1965 & 0.016 \\
1966 & 0.016 \\
1967 & 0.016 \\
1968 & 0.015 \\
1969 & 0.015 \\
1970 & 0.015 \\
1971 & 0.016 \\
1972 & 0.017 \\
1973 & 0.018 \\
1974 & 0.022 \\
1975 & 0.027 \\
1976 & 0.029 \\
1977 & 0.032 \\
1978 & 0.034 \\
1979 & 0.035 \\
1980 & 0.038 \\
1981 & 0.045 \\
1982 & 0.052
\end{tabular}

$N D$
$0.0-0$
00.035
0.033
0.032
0.031
0.029
0.027
0.026
0.023
0.022
0.021
0.021
0.021
0.022
0.022
0.023
0.025
0.027
0.029
0.031
0.033
0.034
0.046
0.047

\begin{tabular}{l}
$N E$ \\
\hdashline 0.027 \\
0.0020 \\
0.0199 \\
0.0199 \\
0.018 \\
0.018 \\
0.018 \\
0.017 \\
0.016 \\
0.016 \\
0.016 \\
0.016 \\
0.017 \\
0.017 \\
0.019 \\
0.0222 \\
0.026 \\
0.028 \\
0.030 \\
0.0033 \\
0.0355 \\
0.045 \\
0.047
\end{tabular}

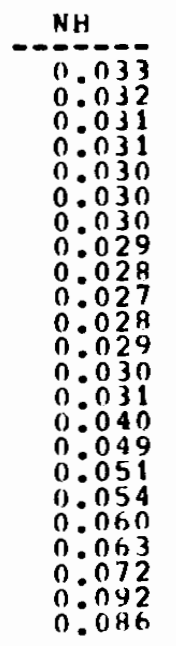

$\mathrm{N} J$
0.078
0.028
0.028
0.027
0.027
0.026
0.025
0.025
0.025
0.025
0.024
0.025
0.028
0.029
0.031
0.043
0.047
0.050
0.054
0.056
0.060
0.072
0.042
0.090

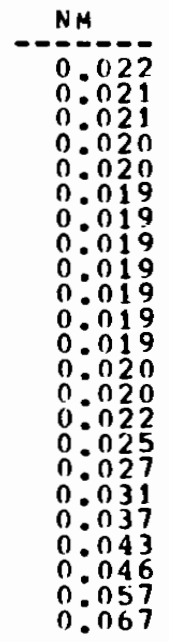

$N V$
00.017
00017
0.017
0.017
0.017
0.017
0.0116
0.016
0.015
0.015
0.015
0.0116
0.017
0.017
0.020
0.025
0.029
0.033
0.036
0.037
0.048
0.053
0.069

$N Y$
-0.029
00.028
0.029
0.025
0.025
0.025
0.024
0.025
0.025
0.024
0.025
0.029
0.031
0.033
0.048
0.052
0.055
0.059
0.057
0.0062
0.075
0.094
0.160

0.
$0.0-5$
0.0224
0.023
0.023
0.023
0.023
0.022
0.021
0.021
0.021
0.0220
0.0020
0.021
0.022
0.022
0.027
0.032
0.0033
0.038
0.042
0.045
0.050
0.057
0.061


TABLE 2.2 (cont). Electricity Prices: Commercial Sector ( $\$ / \mathrm{kWh})$
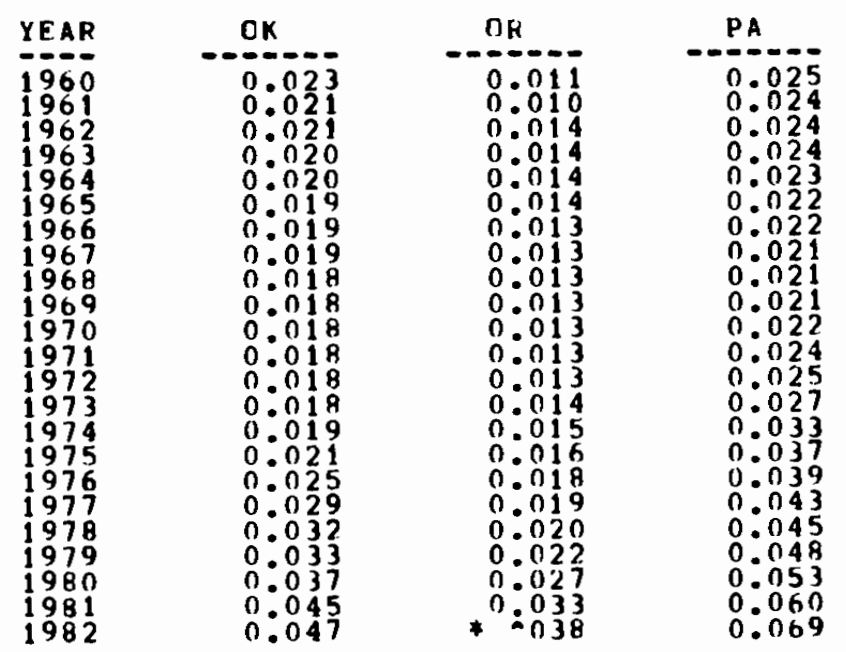

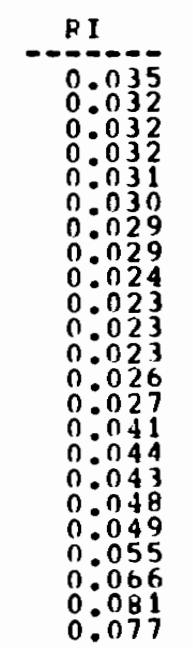
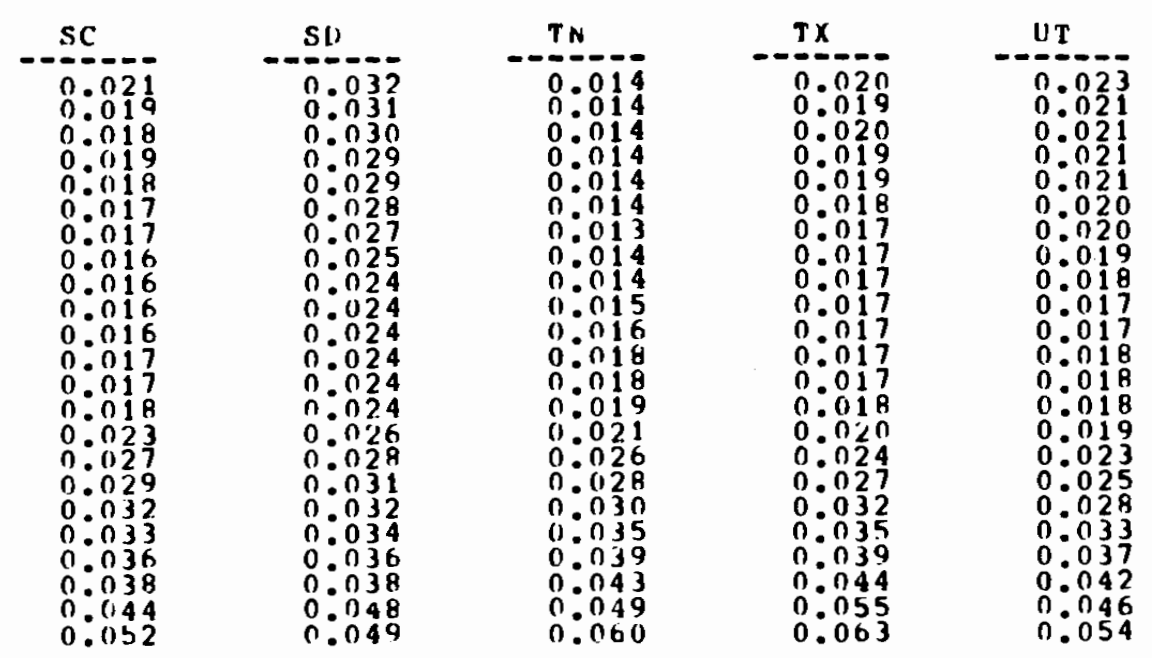

$i$

\begin{tabular}{ll} 
YEAR & VA \\
\hline 1960 & 0.020 \\
1961 & 0.019 \\
1962 & 0.019 \\
1963 & 0.018 \\
1964 & 0.017 \\
1965 & 0.017 \\
1966 & 0.016 \\
1967 & 0.016 \\
1968 & 0.016 \\
1969 & 0.015 \\
1970 & 0.016 \\
1971 & 0.016 \\
1972 & 0.017 \\
1973 & 0.017 \\
1974 & 0.023 \\
1975 & 0.030 \\
1976 & 0.031 \\
1977 & 0.037 \\
1978 & 0.038 \\
1979 & 0.042 \\
1980 & 0.049 \\
1981 & 0.052 \\
1982 & 0.055
\end{tabular}

$\begin{array}{ll}v T & \\ 00.031 & 0.014 \\ 0.026 & 0.013 \\ 0.025 & 0.013 \\ 0.026 & 0.013 \\ 0.025 & 0.013 \\ 0.025 & 0.013 \\ 0.024 & 0.011 \\ 0.023 & 0.010 \\ 0.023 & 0.010 \\ 0.023 & 0.010 \\ 0.022 & 0.010 \\ 0.022 & 0.010 \\ 0.024 & 0.011 \\ 0.026 & 0.011 \\ 0.032 & 0.012 \\ 0.036 & 0.013 \\ 0.039 & 0.013 \\ 0.039 & 0.015 \\ 0.042 & 0.014 \\ 0.042 & 0.015 \\ 0.046 & 0.018 \\ 0.063 & 0.022 \\ 0.066 & 0.027\end{array}$
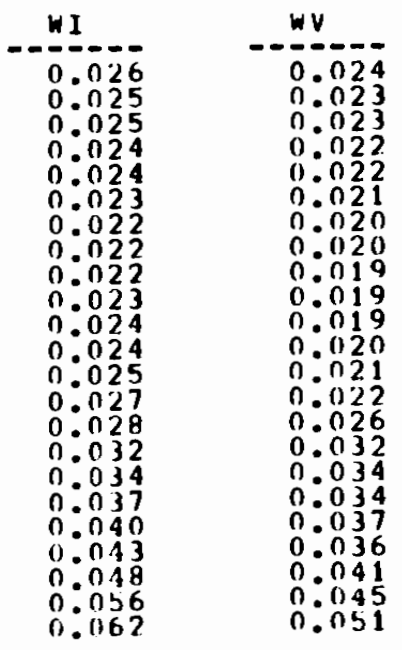

1
0.017
0.016
0.018
0.019
0.019
0.019
0.018
00018
0.017
0.017
0.017
0.017
0.018
0.017
0.017
0.017
0.018
0.020
0.022
0.023
0.024
0.042
0.040

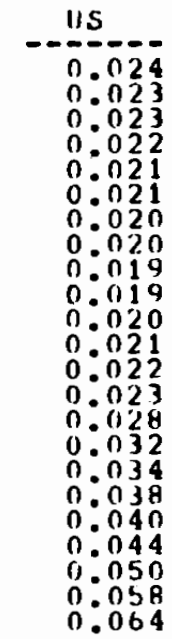


TABLE 2.3. Electricity Prices: Industrial Sector ( $\$ / k W h)$

\begin{tabular}{|c|c|c|c|c|c|c|c|c|c|}
\hline $\begin{array}{l}Y F A R \\
Y-1960 \\
1960 \\
1961 \\
1962 \\
1963 \\
1964 \\
1965 \\
1966 \\
1967 \\
1968 \\
1969 \\
1970 \\
1971 \\
1972 \\
1973 \\
1974 \\
1975 \\
1976 \\
1977 \\
1978 \\
1999 \\
1990 \\
1981 \\
1982\end{array}$ & 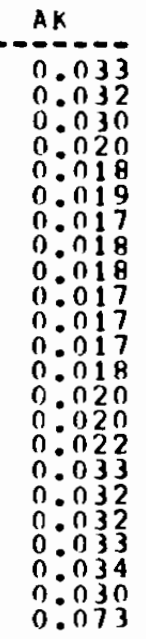 & 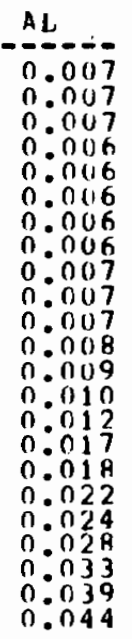 & 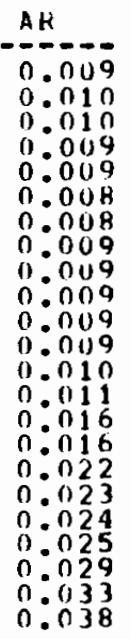 & 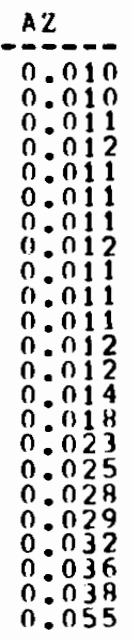 & 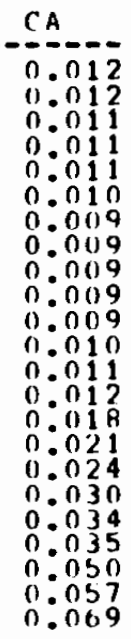 & 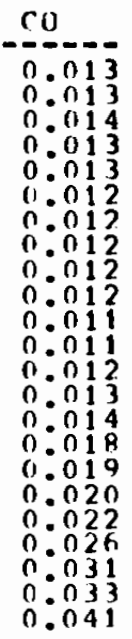 & 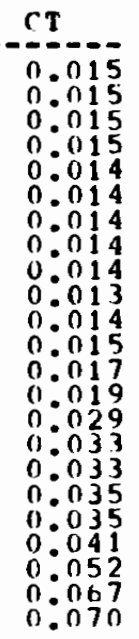 & 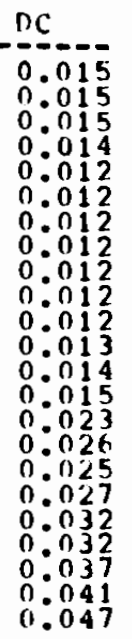 & 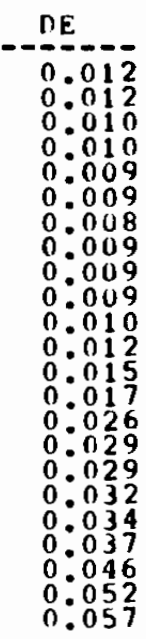 \\
\hline $\begin{array}{l}\text { YFAR } \\
1960 \\
1960 \\
1961 \\
1962 \\
1963 \\
1964 \\
1965 \\
1966 \\
1967 \\
1968 \\
1969 \\
1970 \\
1971 \\
1972 \\
1973 \\
1974 \\
1975 \\
1976 \\
1977 \\
1978 \\
1979 \\
1980 \\
1981 \\
1982\end{array}$ & 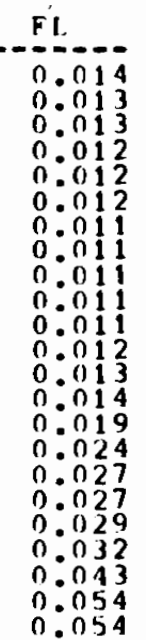 & 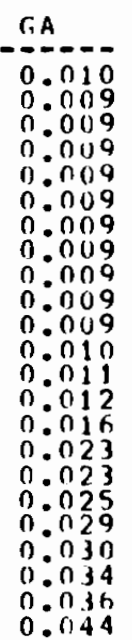 & 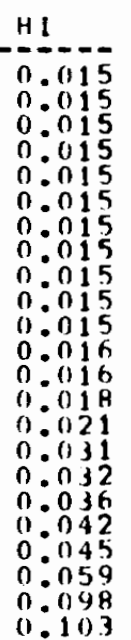 & 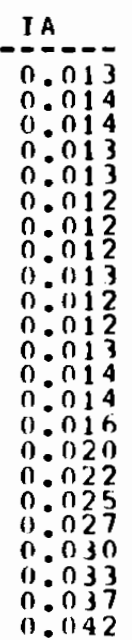 & 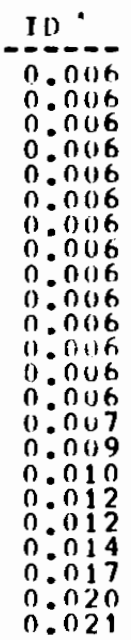 & 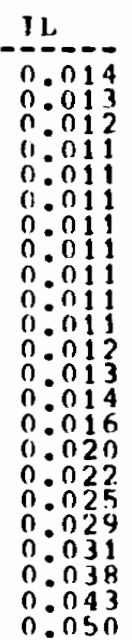 & 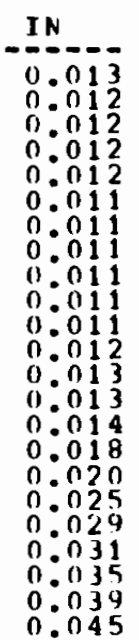 & 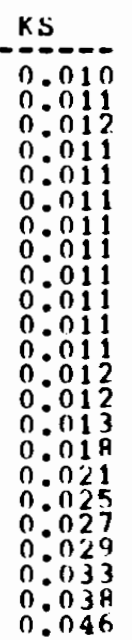 & \begin{tabular}{c}
$K Y$ \\
\hdashline $0: 005$ \\
$0: 005$ \\
$0: 005$ \\
$0: 005$ \\
$0: 005$ \\
$0: 005$ \\
$0: 006$ \\
$0: 006$ \\
$0: 006$ \\
$0: 006$ \\
$0: 007$ \\
0.008 \\
0.008 \\
$0: 009$ \\
$0: 010$ \\
$0: 014$ \\
0.016 \\
$0: 017$ \\
$0: 022$ \\
$0: 024$ \\
$0: 027$ \\
$0: 035$ \\
0.036
\end{tabular} \\
\hline
\end{tabular}


TABLE 2.3 (cont). Electricity Prices: Industrial Sector $(\$ / \mathrm{kWh})$

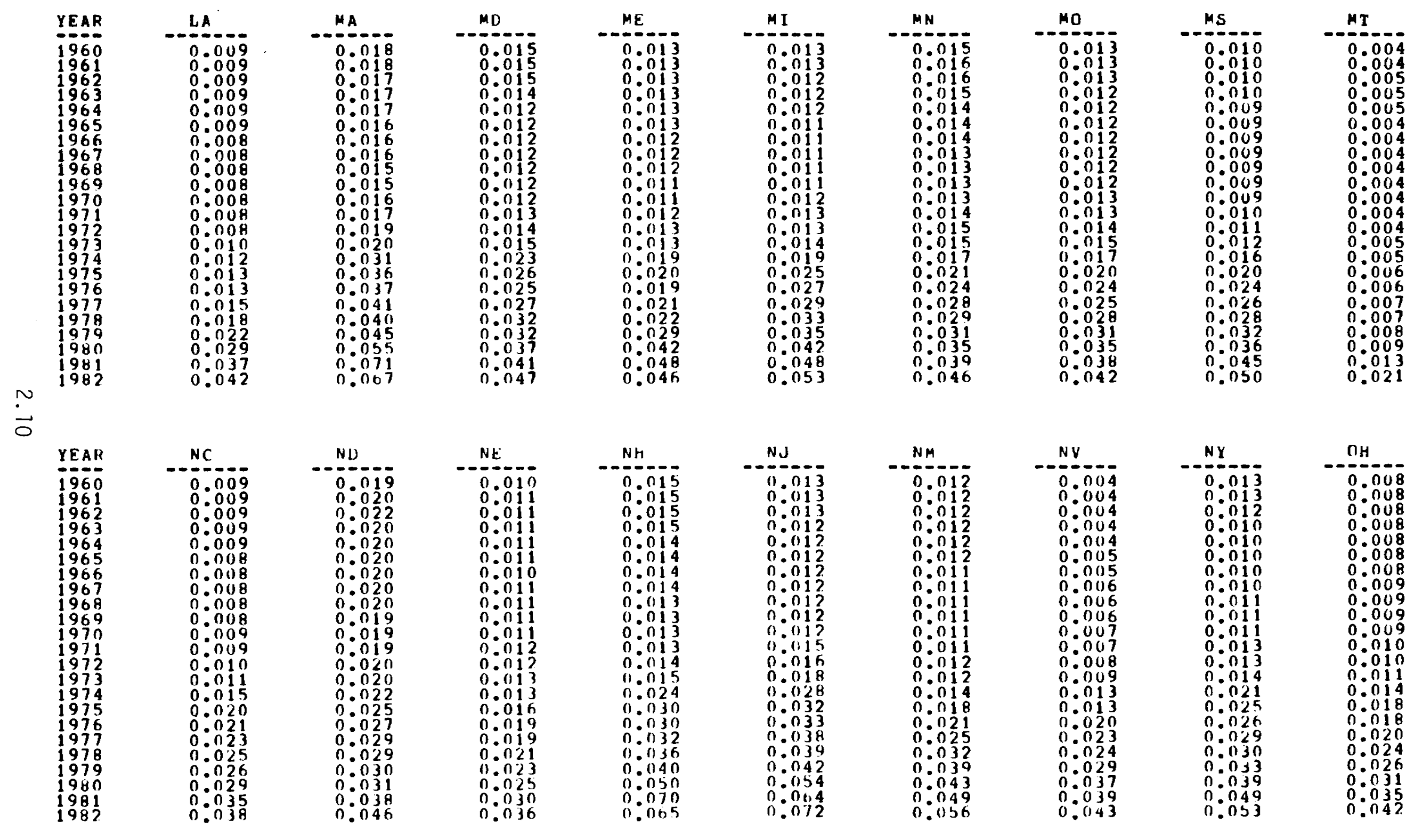


TABLE 2.3 (cont). Electricity Prices: Industrial Sector ( $\$ / \mathrm{kWh})$

\begin{tabular}{|c|c|c|c|c|c|c|c|c|c|}
\hline $\begin{array}{l}\text { YEAR } \\
1960 \\
1961 \\
1962 \\
1963 \\
1964 \\
1965 \\
1966 \\
1967 \\
1968 \\
1969 \\
1970 \\
1971 \\
1972 \\
1973 \\
1974 \\
1975 \\
1976 \\
1977 \\
1978 \\
1979 \\
1980 \\
1981 \\
1982\end{array}$ & 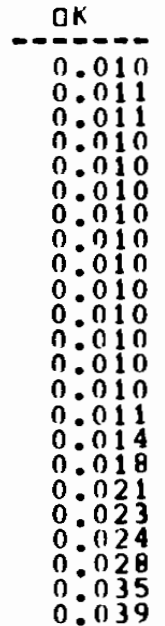 & 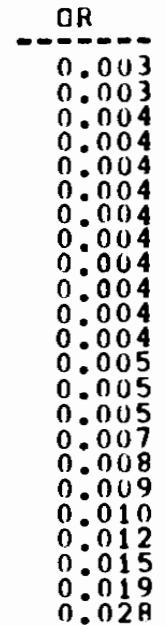 & 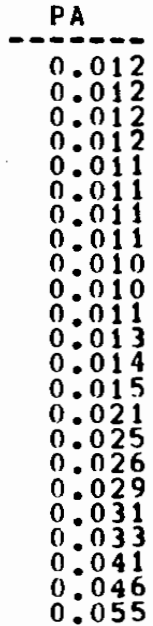 & 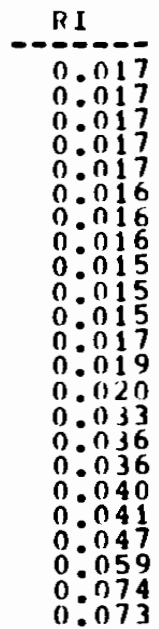 & 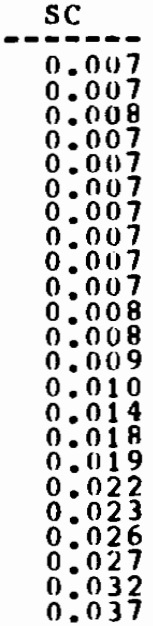 & 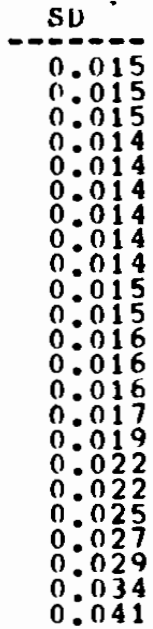 & 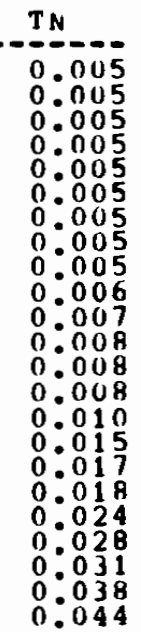 & $\begin{array}{l}T X \\
-50.010 \\
0.010 \\
0.010 \\
0.009 \\
0.009 \\
0.009 \\
0.008 \\
0.008 \\
0.008 \\
0.008 \\
0.008 \\
0.008 \\
0.008 \\
0.009 \\
0.012 \\
0.015 \\
0.018 \\
0.022 \\
0.024 \\
0.027 \\
0.032 \\
0.040 \\
0.048\end{array}$ & 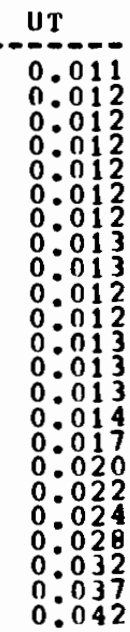 \\
\hline & & & & & & & & & \\
\hline $\begin{array}{l}\text { YFAR } \\
1960 \\
1961 \\
1962 \\
1963 \\
1964 \\
1965 \\
1966 \\
1967 \\
1968 \\
1969 \\
1970 \\
1971 \\
1972 \\
1973 \\
1974 \\
1975 \\
1976 \\
1977 \\
1978 \\
1979 \\
1980 \\
1981 \\
1982\end{array}$ & 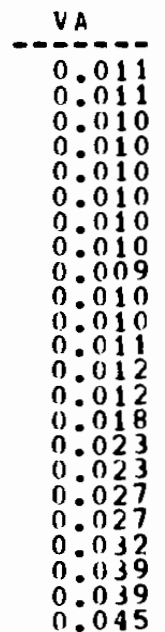 & $\begin{array}{l}V T \\
0.014 \\
0.015 \\
0.015 \\
0.015 \\
0.015 \\
0.014 \\
0.014 \\
0.014 \\
0.015 \\
0.014 \\
0.014 \\
0.015 \\
0.017 \\
0.018 \\
0.023 \\
0.024 \\
0.026 \\
0.026 \\
0.028 \\
0.029 \\
0.033 \\
0.045 \\
0.052\end{array}$ & \begin{tabular}{c}
$W A$ \\
\hdashline 0.003 \\
0.003 \\
0.003 \\
0.003 \\
0.003 \\
0.003 \\
0.003 \\
0.003 \\
0.003 \\
0.003 \\
0.003 \\
0.0003 \\
0.003 \\
0.003 \\
0.003 \\
0.004 \\
0.005 \\
0.004 \\
0.005 \\
0.005 \\
0.007 \\
0.01010 \\
0.018
\end{tabular} & 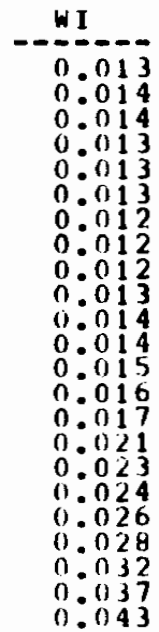 & $\begin{array}{l}W V \\
0.009 \\
0.009 \\
0.009 \\
0.008 \\
0.008 \\
0.008 \\
0.004 \\
0.008 \\
0.008 \\
0.008 \\
0.004 \\
0.010 \\
0.011 \\
0.011 \\
0.015 \\
0.021 \\
0.022 \\
0.022 \\
0.024 \\
0.024 \\
0.027 \\
0.031 \\
0.037\end{array}$ & 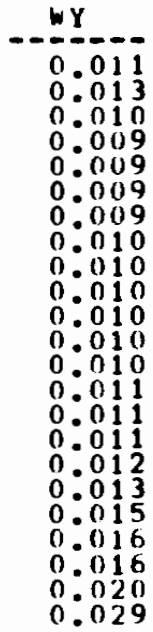 & 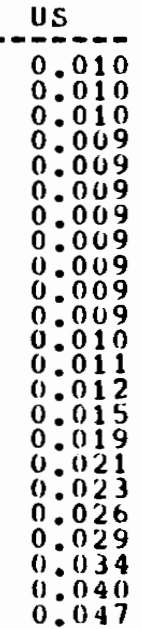 & & \\
\hline
\end{tabular}

Source: Pacific Northwest Laboratory 
TABLE 2.4. Electricity Prices: Transportation Sector ( $\$ / \mathrm{kWh})$

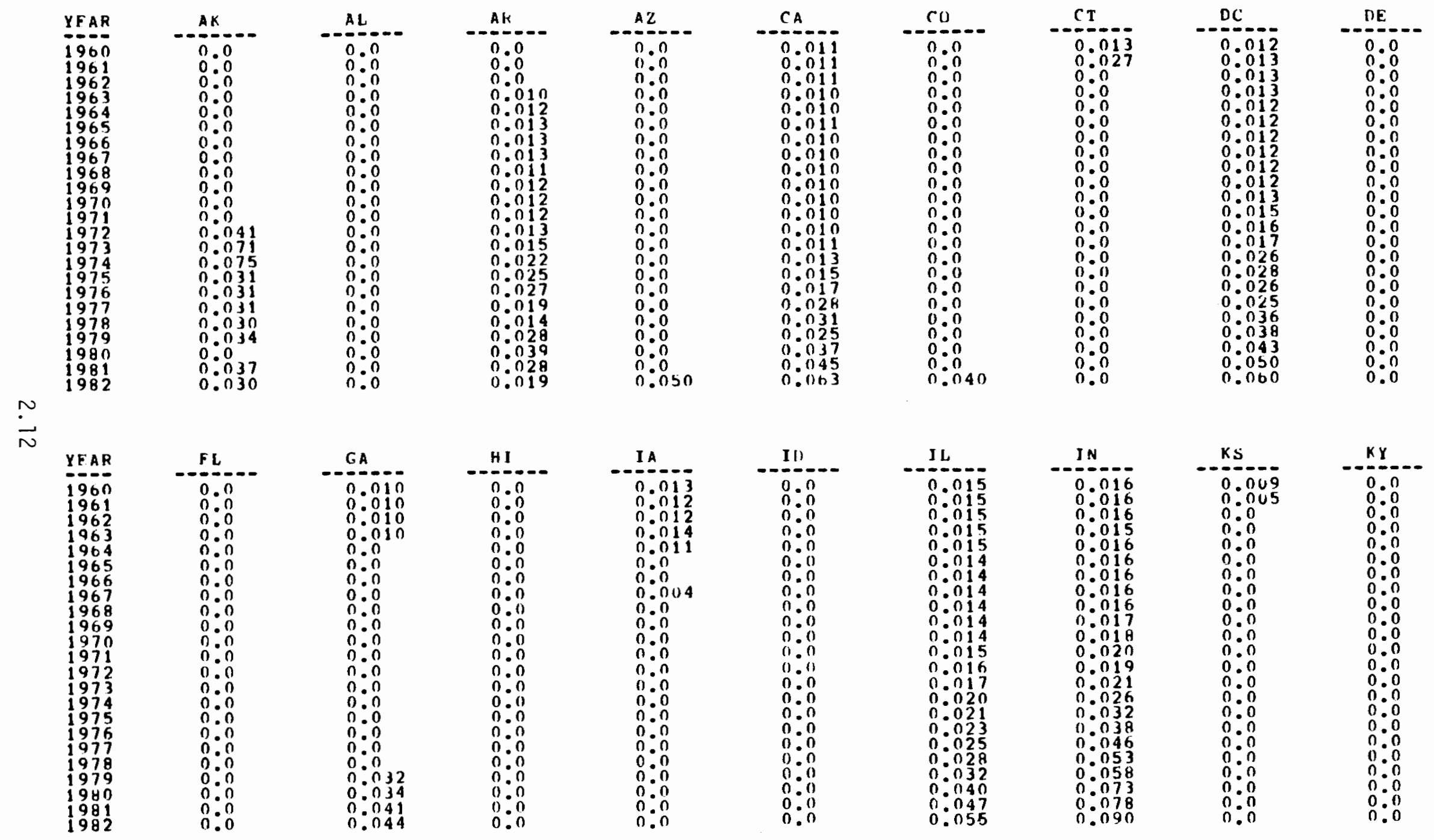


TABLE 2.4 (cont). Electricity Prices: Transportation Sector ( $\$ / k W h)$

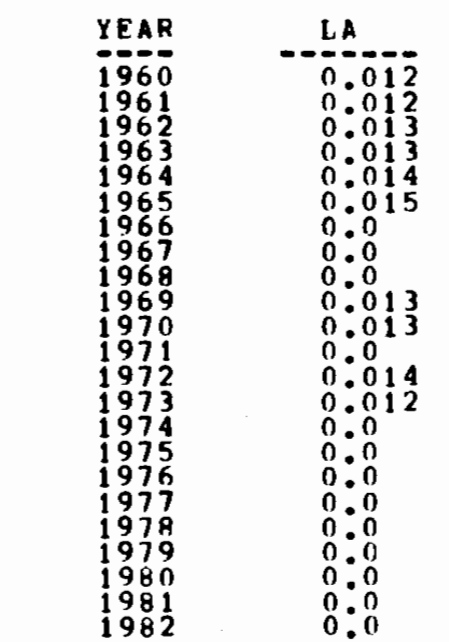

$\begin{array}{ll}M A & M 0 \\ 00.010 & 0.012 \\ 0.010 & 0.013 \\ 0.008 & 0.013 \\ 0.009 & 0.013 \\ 0.008 & 0.012 \\ 0.008 & 0.012 \\ 0.008 & 0.012 \\ 0.008 & 0.012 \\ 0.008 & 0.012 \\ 0.008 & 0.012 \\ 0.019 & 0.013 \\ 0.028 & 0.015 \\ 0.030 & 0.016 \\ 0.025 & 0.017 \\ 0.044 & 0.026 \\ 0.050 & 0.028 \\ 0.062 & 0.026 \\ 0.064 & 0.025 \\ 0.072 & 0.036 \\ 0.063 & 0.038 \\ 0.074 & 0.043 \\ 0.083 & 0.050 \\ 0.081 & 0.060\end{array}$
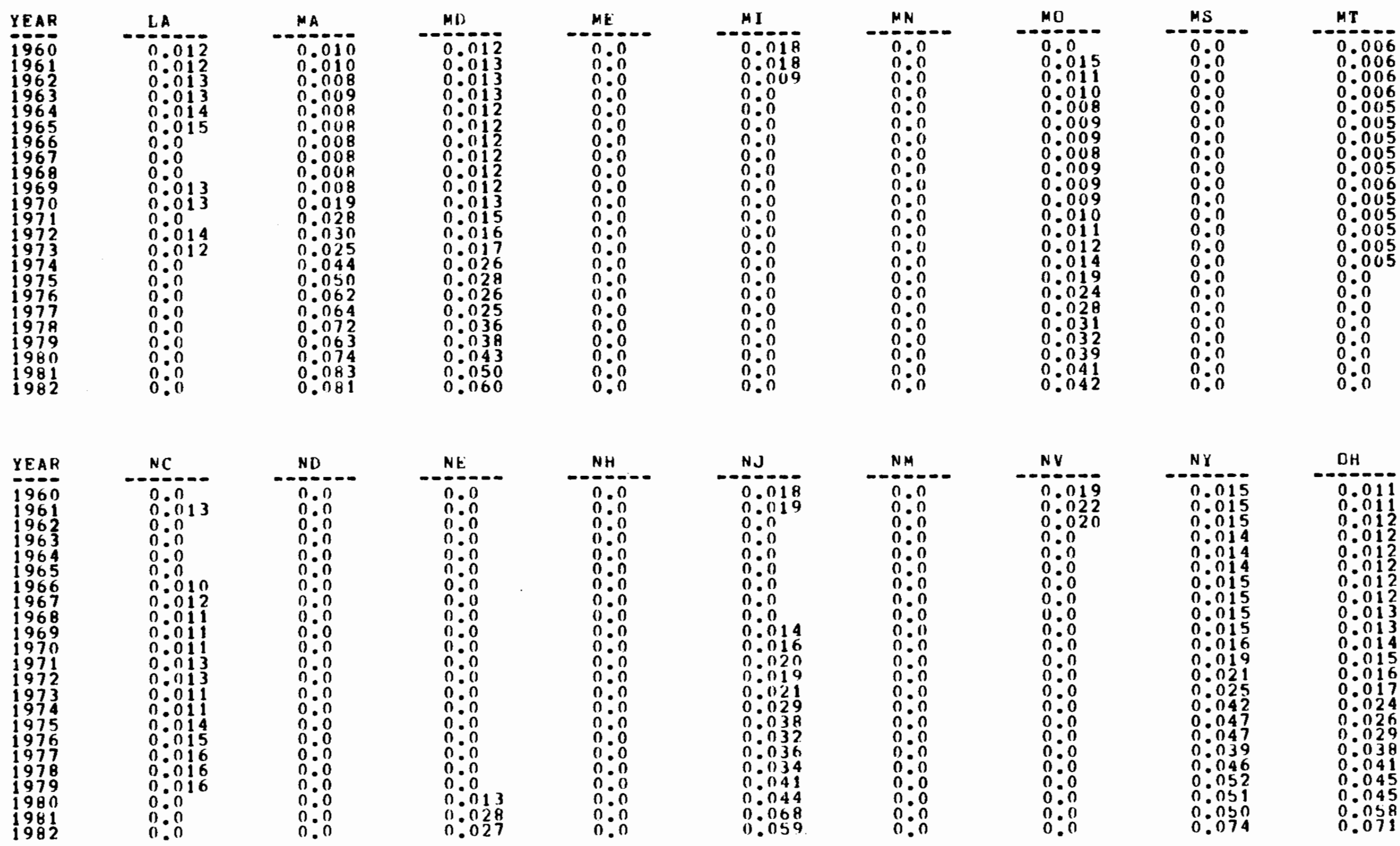

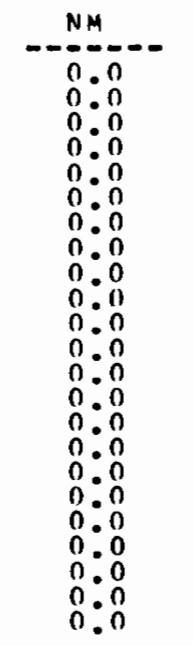
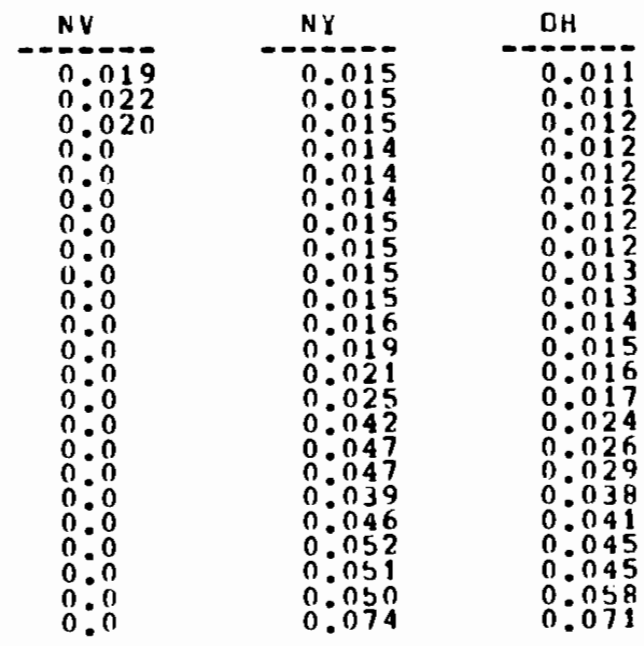
TABLE 2.4 (cont). Electricity Prices: Transportation Sector ( $\$ / \mathrm{kWh}$ )

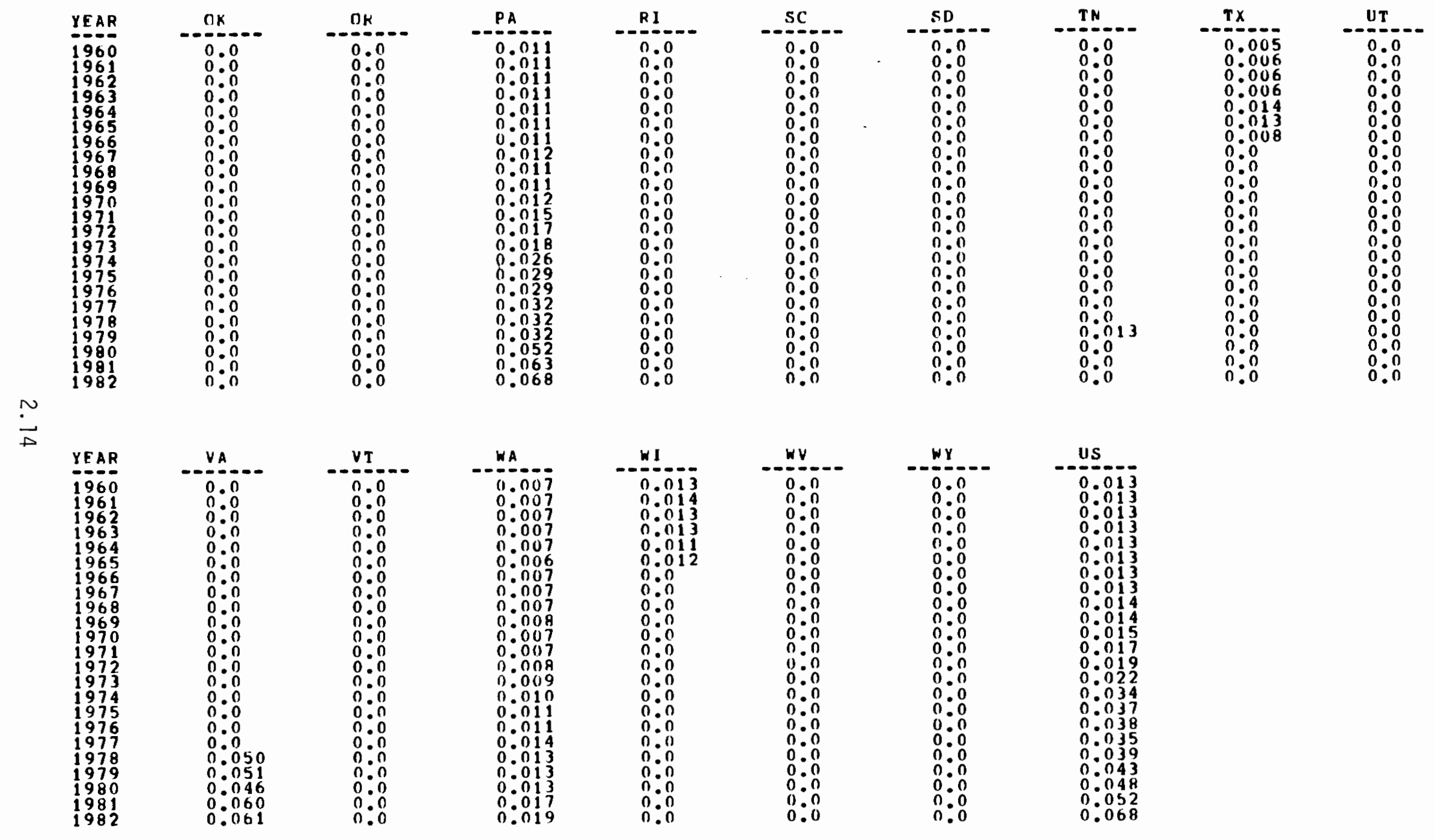

Source: Pacific Northwest Laboratory 
TABLE 2.5. Natural Gas Prices: Residential Sector (\$/1000 cu. ft.)

\begin{tabular}{|c|c|c|c|c|c|c|c|c|c|}
\hline $\begin{array}{l}Y E A R \\
1960 \\
1960 \\
1961 \\
1962 \\
1963 \\
1964 \\
1965 \\
1967 \\
1968 \\
1969 \\
1970 \\
1971 \\
1977 \\
1973 \\
1974 \\
1975 \\
1976 \\
1977 \\
1978 \\
1979 \\
1980 \\
1981 \\
1982\end{array}$ & \begin{tabular}{l}
$A K$ \\
\hdashline 0.135 \\
$0: 066$ \\
$0: 974$ \\
$1: 269$ \\
$1: 265$ \\
$1: 503$ \\
$1: 502$ \\
$1: 513$ \\
$1: 517$ \\
$1: 517$ \\
$1: 519$ \\
$1: 531$ \\
$1: 517$ \\
$1: 574$ \\
$1: 580$ \\
$1: 627$ \\
$1: 653$ \\
$1: 710$ \\
$1: 760$ \\
$1: 700$ \\
$1: 737$ \\
$1: 954$ \\
$1: 797$
\end{tabular} & 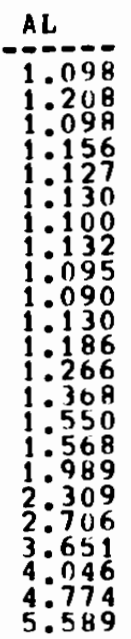 & 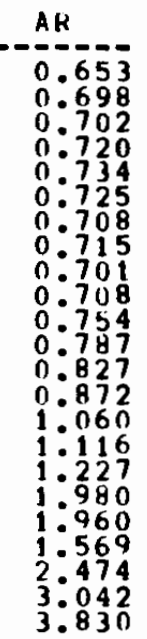 & 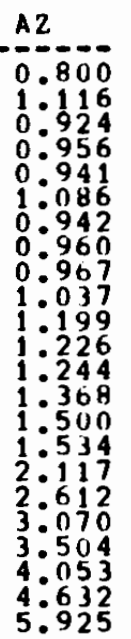 & 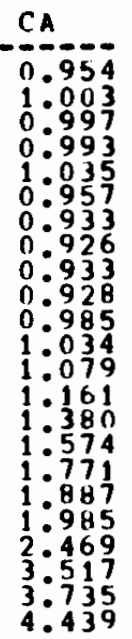 & 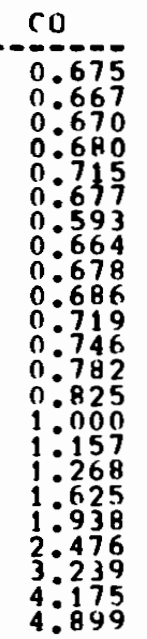 & 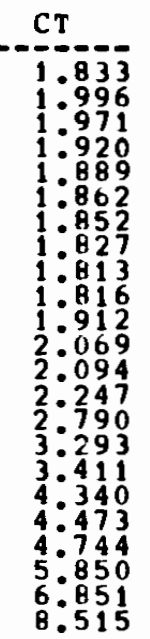 & 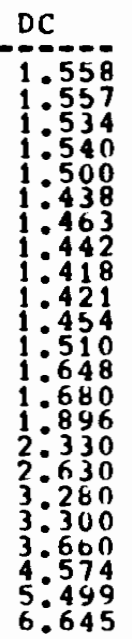 & $\begin{array}{l}D E \\
-1: 748 \\
1: 748 \\
1: 748 \\
1: 700 \\
1: 684 \\
1: 659 \\
1: 632 \\
1: 596 \\
1: 595 \\
1: 504 \\
1: 585 \\
1: 627 \\
1: 710 \\
1: 853 \\
2: 110 \\
2: 426 \\
2.609 \\
4: 249 \\
4: 060 \\
4: 025 \\
5: 500 \\
6: 042\end{array}$ \\
\hline & & & & & & & & & \\
\hline $\begin{array}{l}\text { YEAR } \\
1960 \\
19961 \\
1962 \\
1963 \\
1964 \\
1965 \\
1966 \\
1967 \\
1968 \\
1969 \\
1970 \\
1971 \\
1977 \\
1973 \\
1974 \\
1975 \\
1976 \\
1977 \\
1978 \\
1979 \\
1980 \\
1981 \\
1982\end{array}$ & 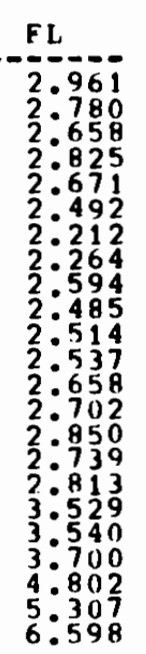 & 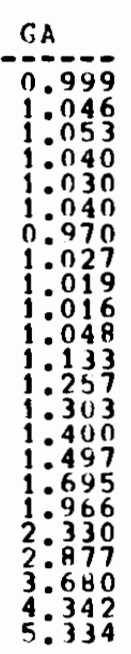 & 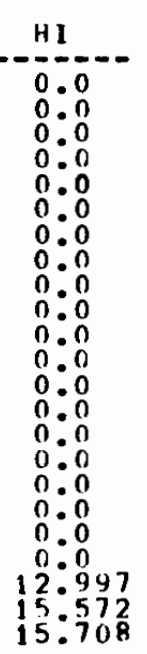 & 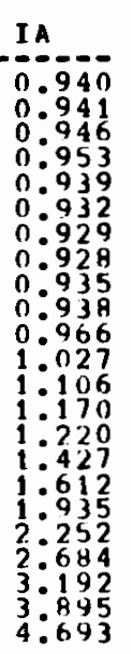 & 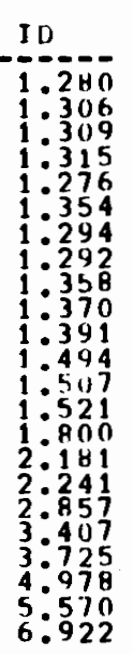 & 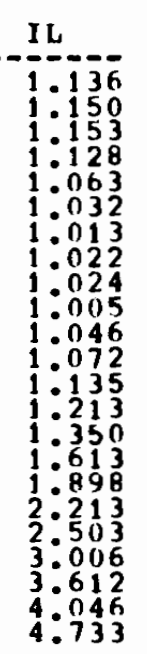 & 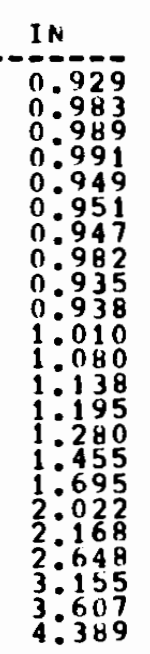 & 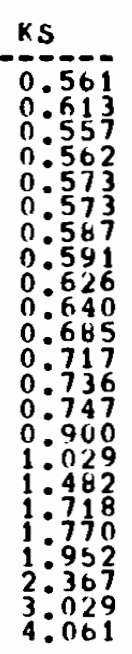 & $\begin{array}{l}K Y \\
-0.788 \\
0: 820 \\
0: 812 \\
0: 812 \\
0: 804 \\
0: 812 \\
0: 801 \\
0: 843 \\
00794 \\
0: 800 \\
0: 828 \\
0: 880 \\
0: 925 \\
0: 989 \\
1: 100 \\
1: 227 \\
1: 320 \\
1: 988 \\
2: 937 \\
3: 029 \\
3: 488 \\
4: 469\end{array}$ \\
\hline
\end{tabular}


TABLE 2.5 (cont). Natural Gas Prices: Residential Sector ( $\$ / 1000 \mathrm{cu}$. ft.)

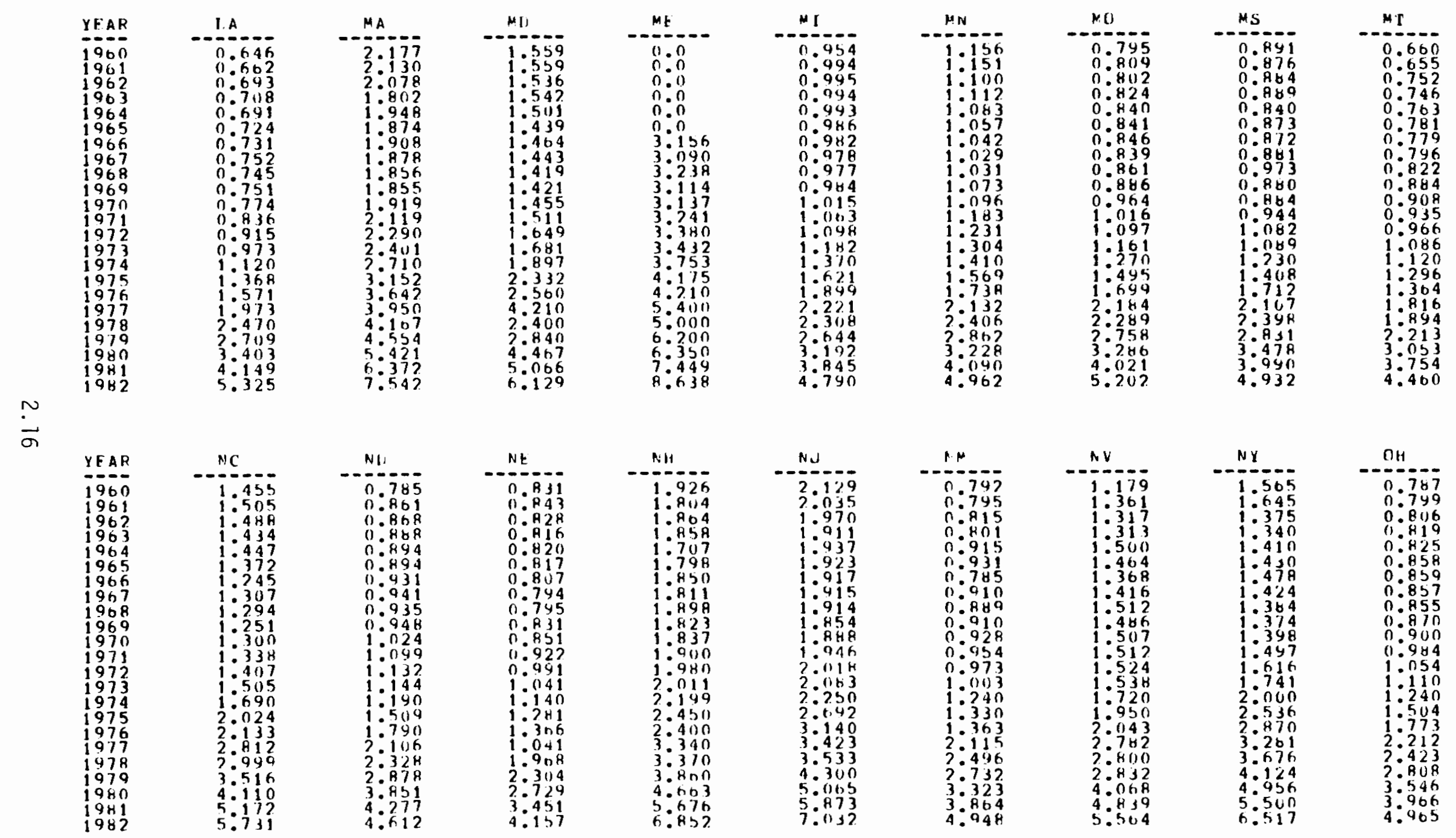


TABLE 2.5 (cont). Natural Gas Prices: Residential Sector ( $\$ / 1000 \mathrm{cu}$. ft.)

\begin{tabular}{|c|c|c|c|c|c|c|c|c|c|}
\hline $\begin{array}{l}\text { YEAR } \\
1960 \\
1960 \\
1961 \\
1962 \\
1963 \\
1964 \\
1965 \\
1966 \\
1967 \\
1969 \\
1969 \\
1970 \\
1971 \\
1972 \\
1973 \\
1994 \\
1975 \\
1976 \\
1997 \\
1978 \\
1979 \\
1990 \\
1981 \\
1998\end{array}$ & 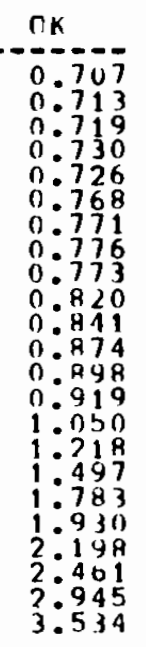 & 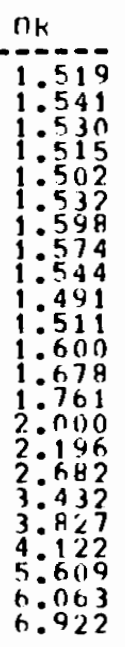 & 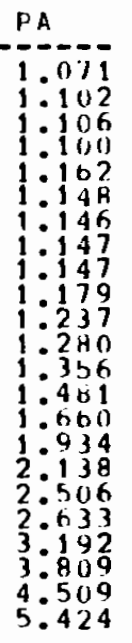 & 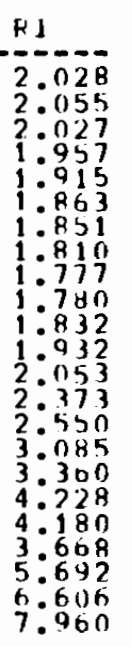 & 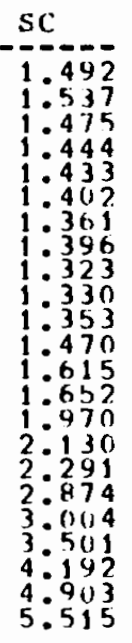 & 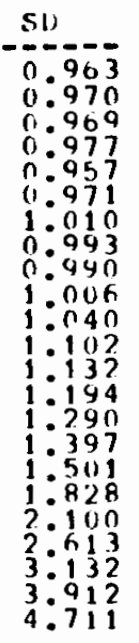 & 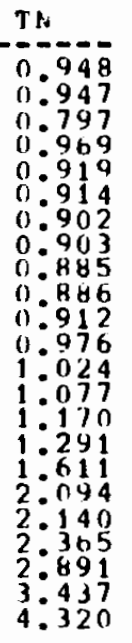 & 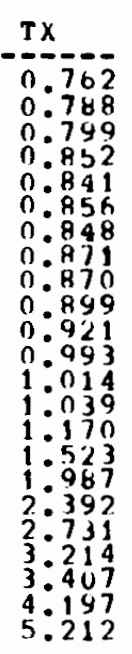 & 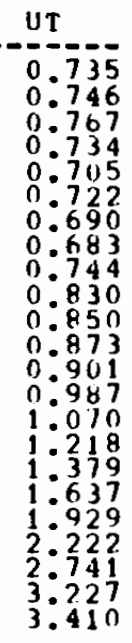 \\
\hline $\begin{array}{l}\text { YFAR } \\
1960 \\
1960 \\
1961 \\
1962 \\
1963 \\
1964 \\
1965 \\
1966 \\
1967 \\
1968 \\
1969 \\
1970 \\
1971 \\
1972 \\
1973 \\
1974 \\
1975 \\
1976 \\
1977 \\
1978 \\
1979 \\
1980 \\
19981 \\
1982\end{array}$ & 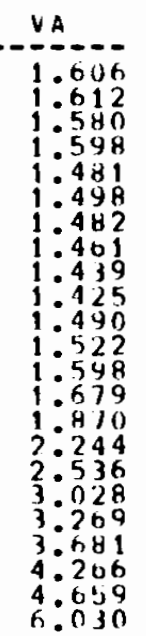 & 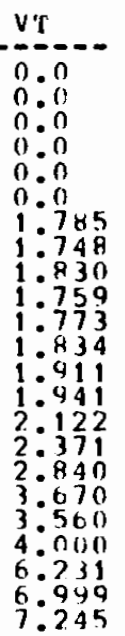 & 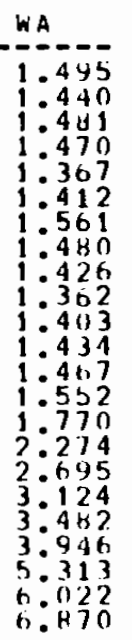 & 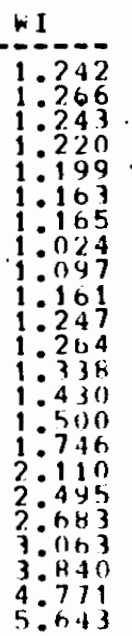 & 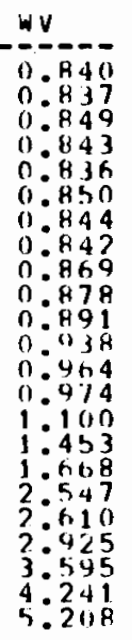 & 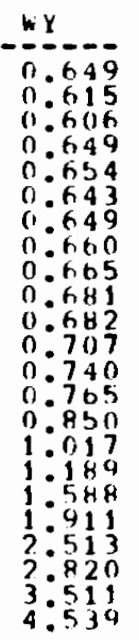 & 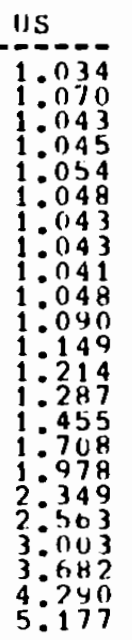 & & \\
\hline
\end{tabular}

Source: Pacific Northwest Laboratory 
TABLE 2.6. Natural Gas Prices: Commercial Sector ( $\$ / 1000 \mathrm{cu}$. ft.)

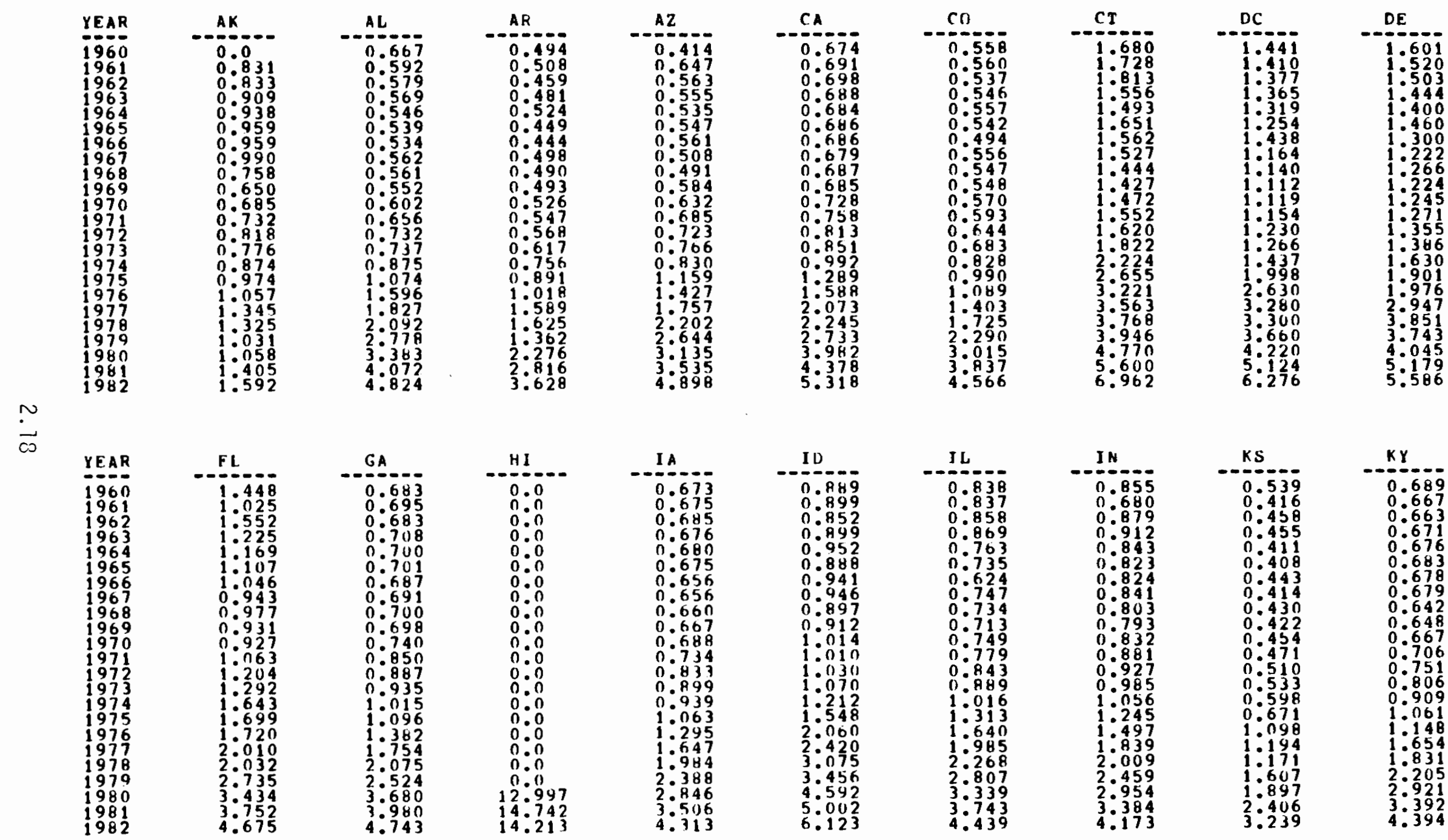


TABLE 2.6 (cont). Natural Gas Prices: Commercial Sector ( $\$ / 1000 \mathrm{cu}$. ft.)

\begin{tabular}{|c|c|c|c|c|c|c|c|c|c|}
\hline $\begin{array}{l}\text { YEAR } \\
1960 \\
1960 \\
1961 \\
1962 \\
1963 \\
1964 \\
1965 \\
1966 \\
1967 \\
1908 \\
1969 \\
1970 \\
1971 \\
1972 \\
1973 \\
1974 \\
1975 \\
1976 \\
1997 \\
1978 \\
19980 \\
1997 \\
1982\end{array}$ & 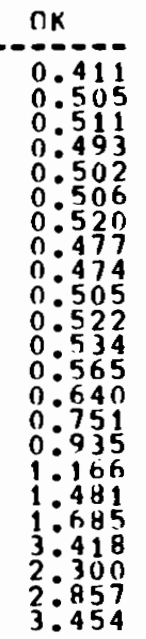 & $\begin{array}{l}D R \\
-1: 437 \\
1: 474 \\
1: 468 \\
1: 439 \\
1: 404 \\
1: 353 \\
1: 394 \\
1: 386 \\
1: 354 \\
1: 272 \\
1: 279 \\
: 312 \\
1: 432 \\
1: 452 \\
1: 710 \\
1: 963 \\
3: 373 \\
3: 077 \\
3: 387 \\
3: 718 \\
5: 110 \\
5: 430 \\
6.294\end{array}$ & 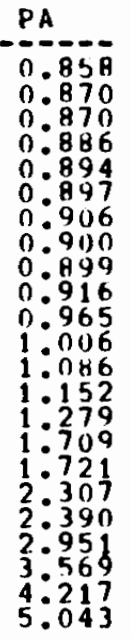 & 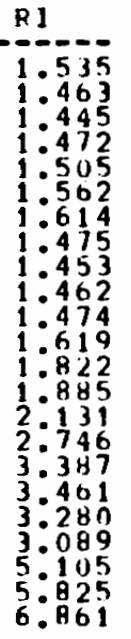 & 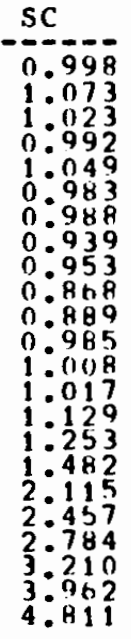 & 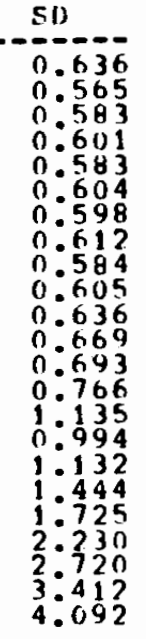 & 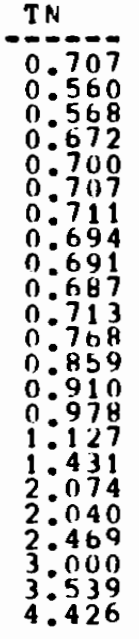 & $\begin{array}{l}I X \\
0.505 \\
0: 528 \\
0: 544 \\
0: 577 \\
0: 561 \\
0: 542 \\
0: 545 \\
0: 439 \\
0: 444 \\
0: 458 \\
0: 454 \\
0: 496 \\
0: 524 \\
0: 555 \\
0: 749 \\
1: 050 \\
1: 422 \\
10797 \\
2: 196 \\
2: 845 \\
2: 993 \\
3: 641 \\
4: 486\end{array}$ & $\begin{array}{l}\text { UT } \\
00.545 \\
0: 523 \\
0: 530 \\
0: 538 \\
0: 553 \\
0: 557 \\
0: 540 \\
0: 560 \\
0: 567 \\
0: 579 \\
0: 591 \\
0: 636 \\
0: 709 \\
0: 795 \\
0: 930 \\
1: 519 \\
10527 \\
10730 \\
1: 668 \\
2: 333 \\
55594 \\
5: 353 \\
3: 431\end{array}$ \\
\hline $\begin{array}{l}Y E A R \\
Y=A 6 \\
1960 \\
1961 \\
1962 \\
1963 \\
1964 \\
1965 \\
1966 \\
1967 \\
1968 \\
1969 \\
1970 \\
1971 \\
1972 \\
1973 \\
1974 \\
1995 \\
1976 \\
1977 \\
1978 \\
1979 \\
1990 \\
1981 \\
1982\end{array}$ & 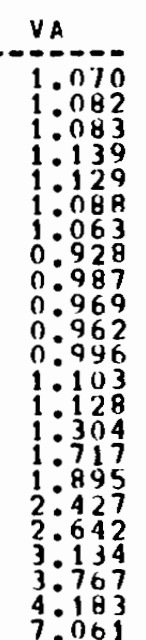 & 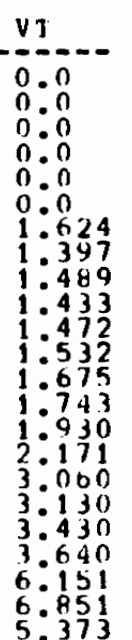 & 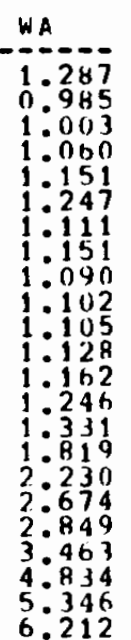 & 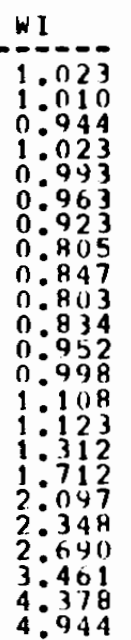 & 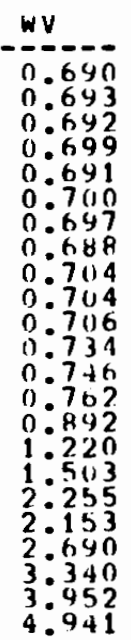 & 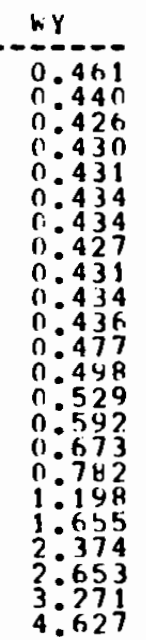 & 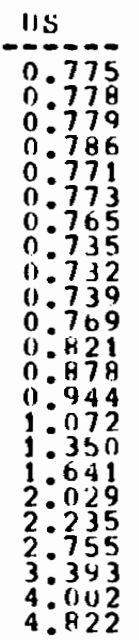 & & \\
\hline
\end{tabular}


TABLE 2.7. Natural Gas Prices: Industrial Sector ( $\$ / 1000 \mathrm{cu}$. ft.)

\begin{tabular}{|c|c|c|c|c|c|c|c|c|c|}
\hline $\begin{array}{l}\text { YEAR } \\
1919 \\
1960 \\
1961 \\
1962 \\
1963 \\
1964 \\
1965 \\
1966 \\
1967 \\
1968 \\
1969 \\
1970 \\
1971 \\
1972 \\
1973 \\
1974 \\
1975 \\
1976 \\
1977 \\
1978 \\
1979 \\
1990 \\
1981 \\
1982\end{array}$ & 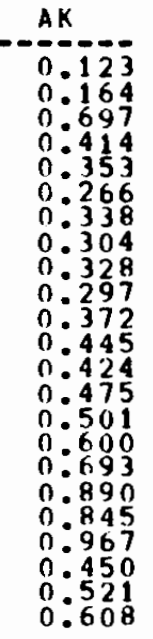 & 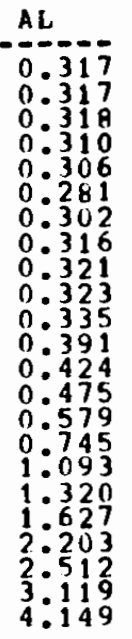 & 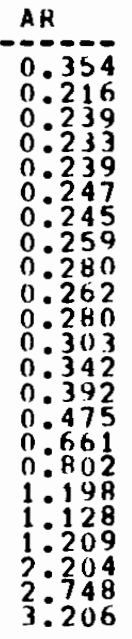 & 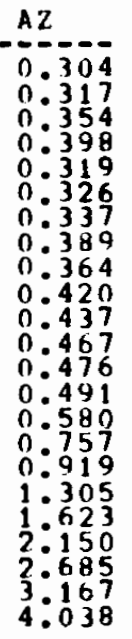 & 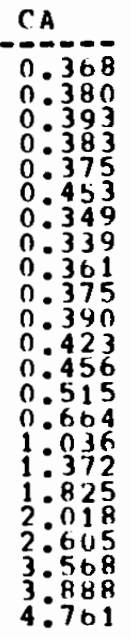 & \begin{tabular}{l}
$c 0$ \\
\hdashline 0.215 \\
$0: 228$ \\
$0: 226$ \\
$0: 221$ \\
$0: 231$ \\
$0: 249$ \\
$0: 236$ \\
$0: 237$ \\
$0: 263$ \\
$0: 264$ \\
$0: 273$ \\
$0: 297$ \\
$0: 322$ \\
$0: 363$ \\
$0: 474$ \\
$0: 638$ \\
$0: 779$ \\
$1: 075$ \\
$1: 282$ \\
$1: 687$ \\
20554 \\
$3: 282$ \\
$3: 716$
\end{tabular} & 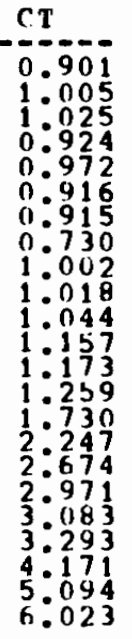 & 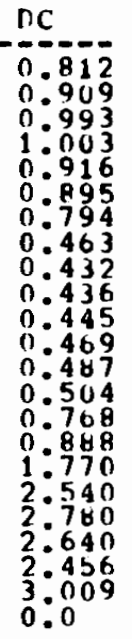 & 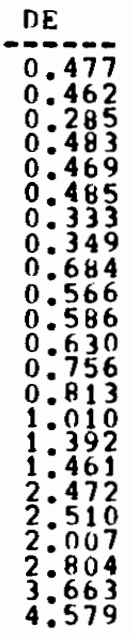 \\
\hline $\begin{array}{l}\text { YFAR } \\
1960 \\
1960 \\
1961 \\
1962 \\
1963 \\
1964 \\
1965 \\
1966 \\
1967 \\
1968 \\
1969 \\
1970 \\
1971 \\
1972 \\
1973 \\
1974 \\
1975 \\
1976 \\
1977 \\
1978 \\
1979 \\
19801 \\
1982\end{array}$ & 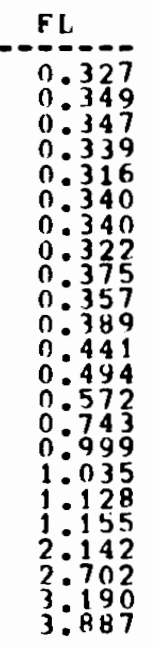 & 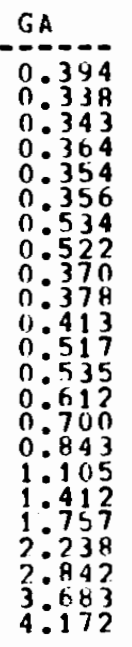 & 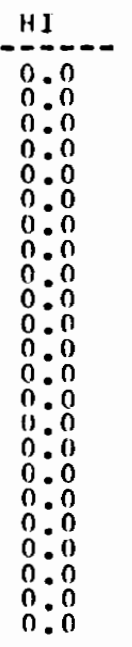 & 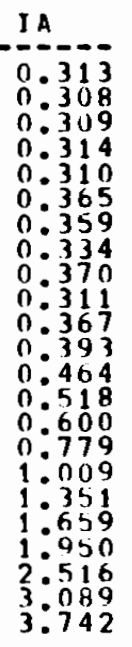 & 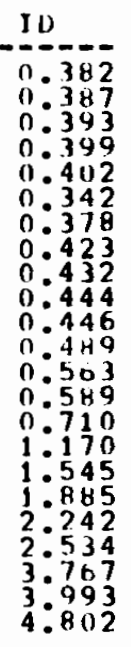 & 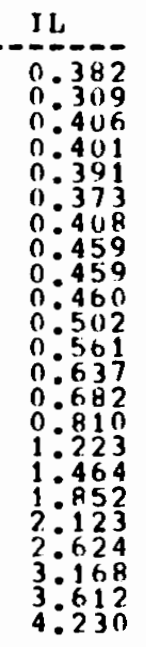 & 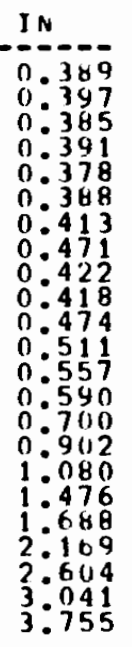 & 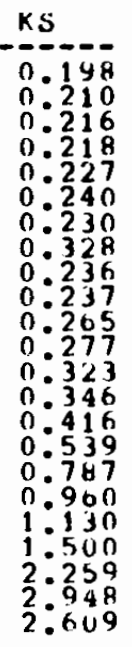 & 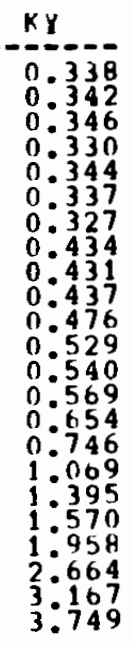 \\
\hline
\end{tabular}


TABLE 2.7 (cont). Natural Gas Prices: Industrial Sector $(\$ / 1000 \mathrm{cu} . \mathrm{ft}$.

\begin{tabular}{ll} 
YEAR & LA \\
\hline 1960 & 0.195 \\
1961 & 0.198 \\
1962 & 0.210 \\
1963 & 0.217 \\
1964 & 0.203 \\
1965 & 0.205 \\
1966 & 0.238 \\
1967 & 0.204 \\
1968 & 0.179 \\
1969 & 0.209 \\
1970 & 0.229 \\
1971 & 0.257 \\
1972 & 0.299 \\
1973 & 0.344 \\
1974 & 0.546 \\
1975 & 0.654 \\
1976 & 0.907 \\
1977 & 0.916 \\
1978 & 0.810 \\
1979 & 0.879 \\
1980 & 1.269 \\
1981 & 1.803 \\
1982 & 2.781
\end{tabular}

MA
-5.728
0.728
0.757
0.771
0.674
0.890
0.661
0.696
0.707
0.926
0.976
1.040
1.070
1.234
1.298
1.710
2.292
2.500
2.596
3.008
3.734
4.154
5.090
5.673
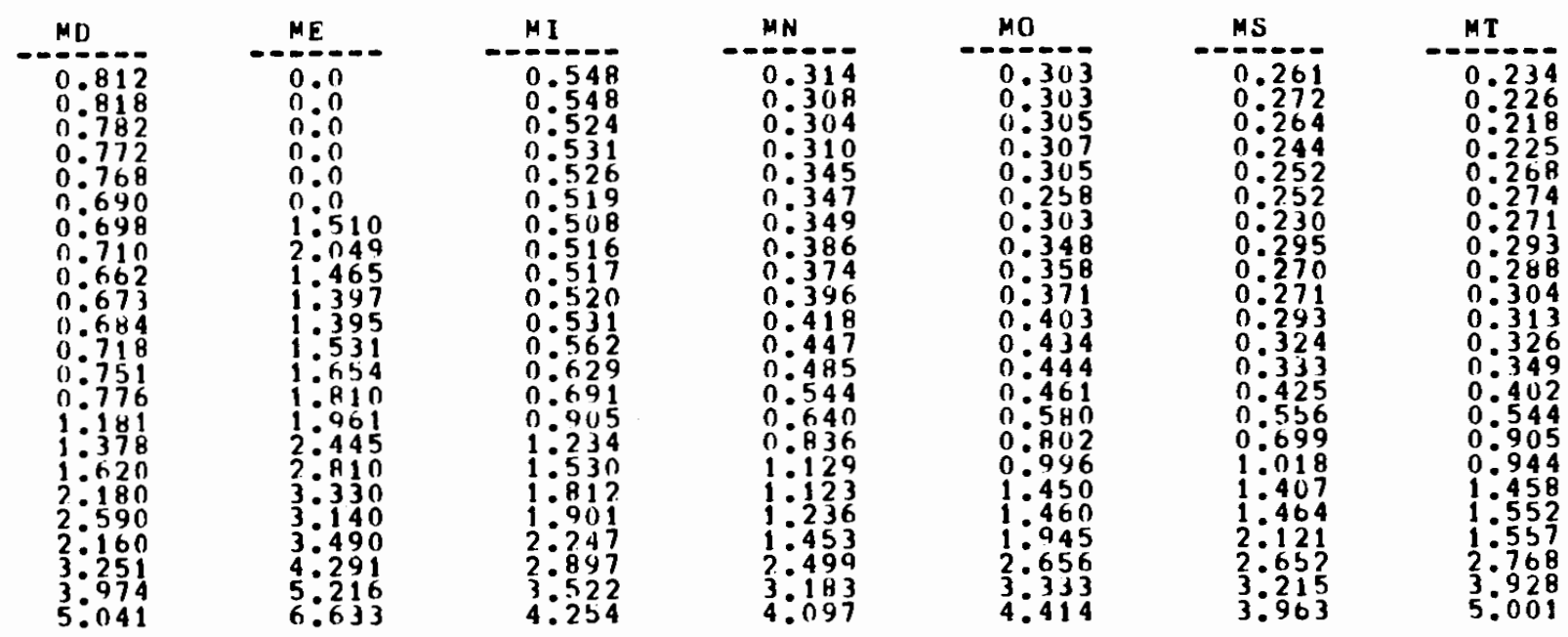

\begin{tabular}{ll} 
YEAR & NC \\
\hline 1960 & 0.267 \\
1961 & 0.266 \\
1962 & 0.274 \\
1963 & 0.265 \\
1964 & 0.274 \\
1965 & 0.278 \\
1966 & 0.282 \\
1967 & 0.491 \\
1968 & 0.496 \\
1969 & 0.506 \\
1970 & 0.512 \\
1971 & 0.535 \\
1972 & 0.597 \\
1973 & 0.682 \\
1974 & 0.870 \\
1975 & 1.363 \\
1976 & 1.684 \\
1977 & 2.368 \\
1978 & 1.961 \\
1979 & 2.676 \\
1980 & 3.360 \\
1981 & 4.416 \\
1982 & 4.642
\end{tabular}

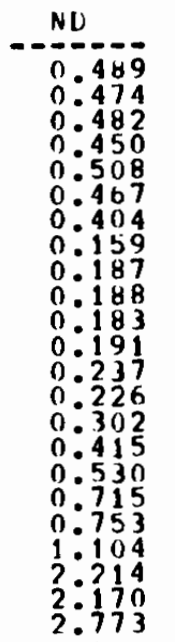

$N E$
0.212
0.147
0.154
0.186
0.198
0.210
0.168
0.244
0.300
0.312
0.316
$0: 362$
0.484
0.435
0.535
0.651
0.843
$1: 019$
1.098
1.223
2.044
20779
$3: 542$

$N H$
-9.781
0.78
0.860
0.906
0.857
0.852
0.930
0.986
1.341
0.958
0.914
0.912
1.000
1.082
1.185
1.282
1.600
2.610
3.180
2.600
3.210
3.931
4.631
5.564
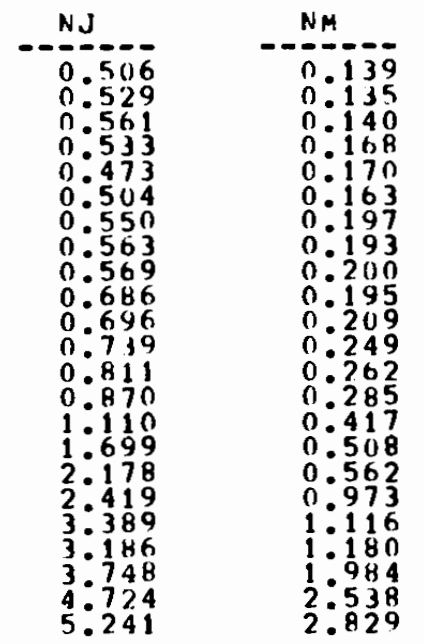

$N V$
0.610
0.620
0.620
0.625
0.445
0.457
0.443
0.472
0.457
0.493
0.562
0.574
0.715
0.793
0.880
1.134
1.427
1.968
1.780
2.331
2.990
$3: 589$
4.414
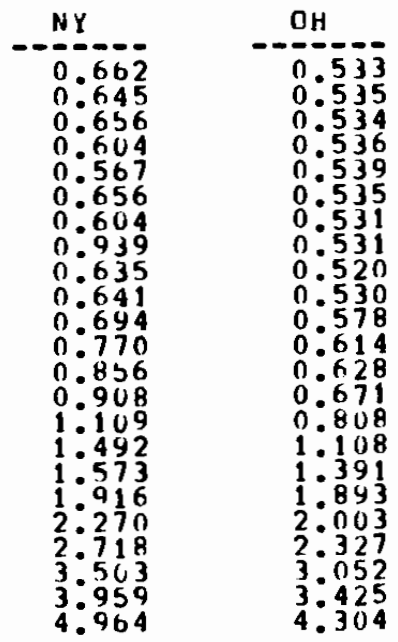
TABLE 2.7 (cont). Natural Gas Prices: Industrial Sector $(\$ / 1000 \mathrm{cu}$. ft.)

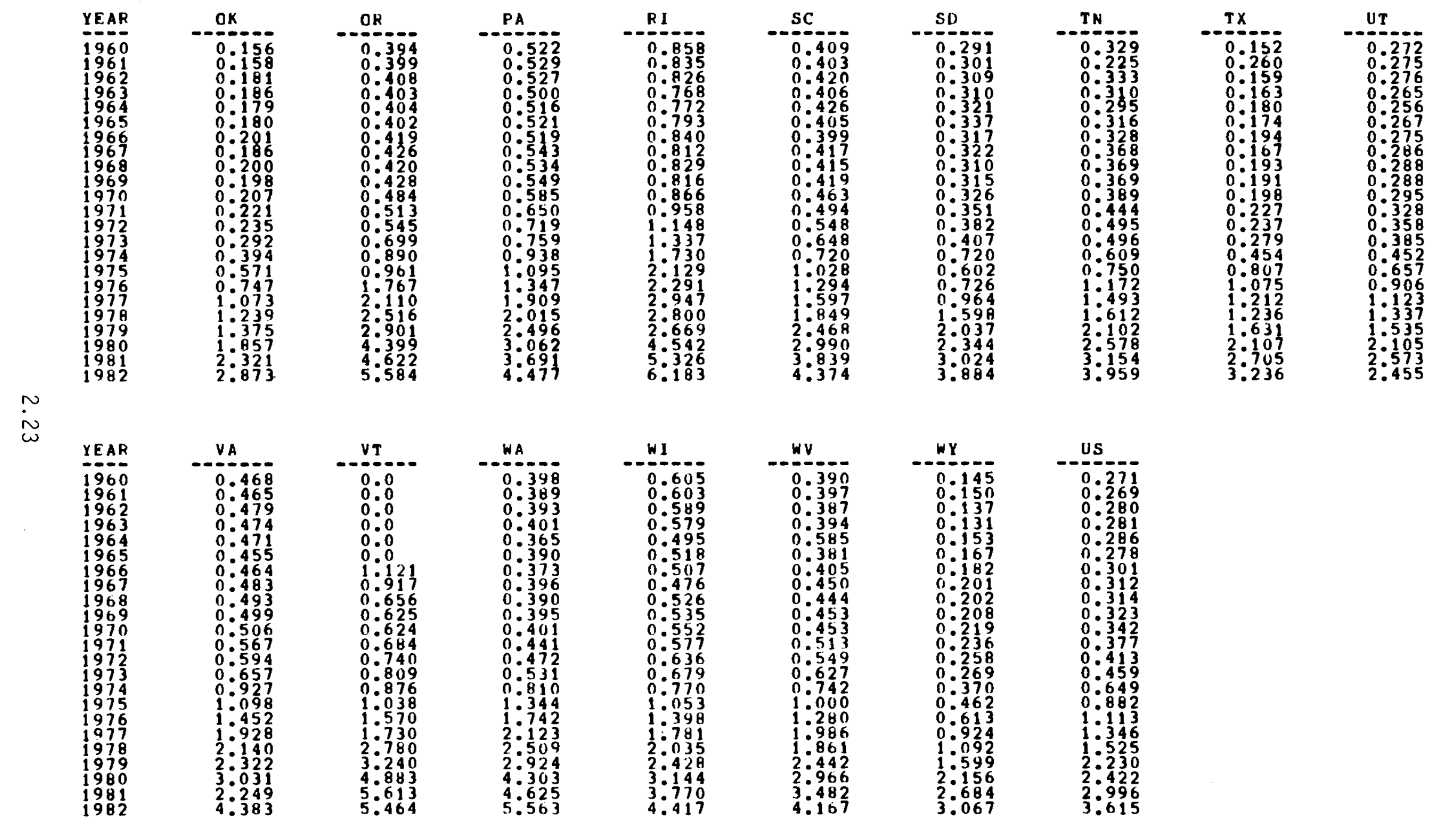

Source: Pacific Northwest Laboratory 
TABLE 2.8. Natural Gas Prices: Transportation Sector ( $\$ / 1000 \mathrm{cu} . \mathrm{ft}$.

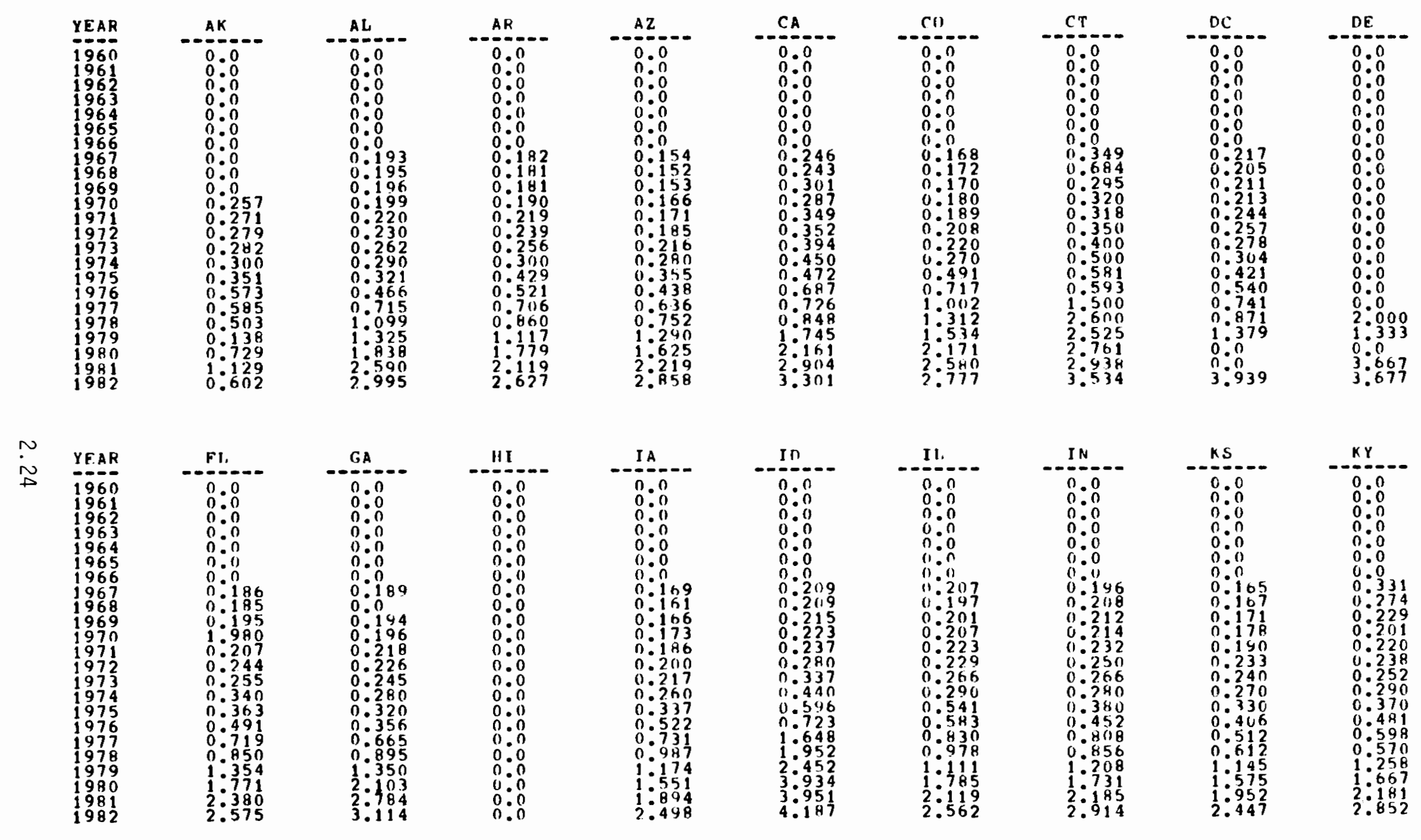


TABLE 2.8 (cont). Natural Gas Prices: Transportation Sector ( $\$ / 1000 \mathrm{cu}$. ft.)

\begin{tabular}{|c|c|c|c|c|c|c|c|c|c|}
\hline $\begin{array}{l}\text { YEAR } \\
1960 \\
1961 \\
1962 \\
1963 \\
1964 \\
1965 \\
1966 \\
1967 \\
1968 \\
1969 \\
1970 \\
1971 \\
1972 \\
1973 \\
1974 \\
1975 \\
1976 \\
1977 \\
1978 \\
1979 \\
19980 \\
19981 \\
1982\end{array}$ & 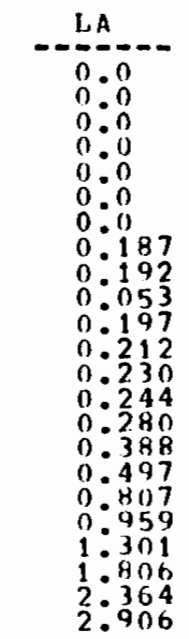 & \begin{tabular}{c} 
MA \\
\hdashline $0: 0$ \\
$0: 0$ \\
$0: 0$ \\
$0: 0$ \\
$0: 0$ \\
$0: 0$ \\
$0: 0$ \\
$0: 0$ \\
$0: 234$ \\
$0: 263$ \\
$0: 251$ \\
$0: 316$ \\
$0: 356$ \\
$0: 368$ \\
$0: 383$ \\
$0: 399$ \\
$0: 421$ \\
$0: 618$ \\
$0: 676$ \\
$0: 938$ \\
$1: 238$ \\
$1: 650$ \\
$2: 302$ \\
$4: 286$
\end{tabular} & 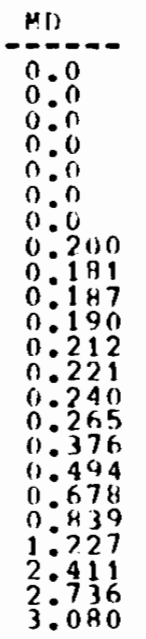 & \begin{tabular}{c}
$M F$ \\
\hdashline $0: 0$ \\
$0: 0$ \\
$0: 0$ \\
$0: 0$ \\
$0: 0$ \\
$0: 0$ \\
$0: 0$ \\
$0: 587$ \\
$0: 0$ \\
$0: 0$ \\
$0: 0$ \\
$0: 0$ \\
$0: 0$ \\
$0: 0$ \\
$0: 0$ \\
$0: 0$ \\
$0: 0$ \\
0 \\
$0: 0$ \\
$0: 0$ \\
$0: 628$ \\
$3: 200$ \\
$4: 919$
\end{tabular} & 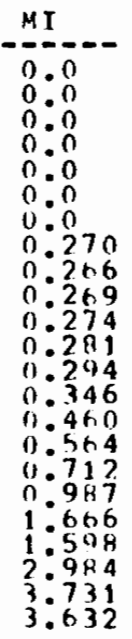 & 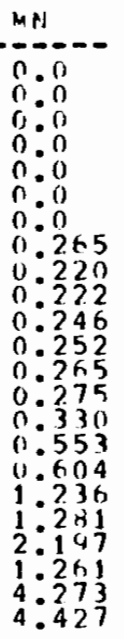 & 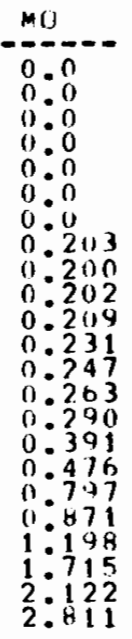 & 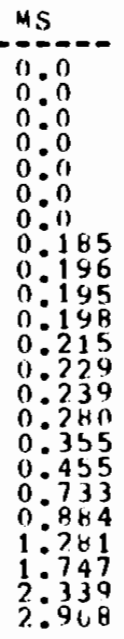 & 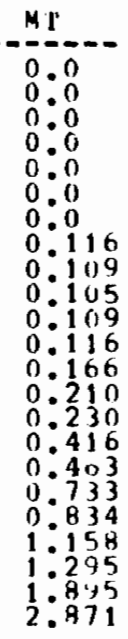 \\
\hline YFAR & $\mathrm{NC}$ & ND & $\mathrm{NF}$ & $\mathrm{NH}$ & N J J & 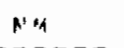 & NV & $\mathrm{HY}$ & nol \\
\hline $\begin{array}{l}1960 \\
1960 \\
1961 \\
1962 \\
1964 \\
1965 \\
1966 \\
1967 \\
1968 \\
1969 \\
1970 \\
1971 \\
1972 \\
1973 \\
1974 \\
1975 \\
1976 \\
1977 \\
1978 \\
1999 \\
1990 \\
1981 \\
1982\end{array}$ & 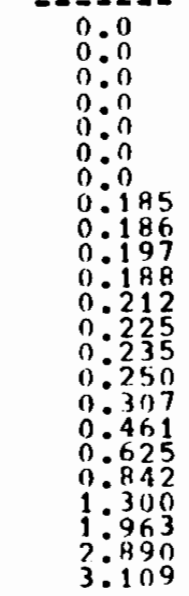 & $\begin{array}{l}0.0 \\
0: 0 \\
0: 0 \\
0: 0 \\
0: 0 \\
0: 0 \\
0: 0 \\
0: 273 \\
0: 167 \\
0: 167 \\
0: 200 \\
0: 2000 \\
0: 247 \\
0: 286 \\
0: 3113 \\
0: 568 \\
0: 570 \\
0: 747 \\
0: 953 \\
1: 549 \\
1: 813 \\
2: 345 \\
4: 112\end{array}$ & $\begin{array}{l}0: 0 \\
0: 0 \\
0: 0 \\
0: 0 \\
0: 0 \\
0: 0 \\
0: 0 \\
0: 143 \\
0: 145 \\
0: 150 \\
0: 155 \\
0: 164 \\
0: 178 \\
0: 187 \\
0: 240 \\
0: 324 \\
0: 418 \\
0: 575 \\
0: 726 \\
1: 193 \\
1: 358 \\
1: 813 \\
2: 353\end{array}$ & $\begin{array}{l}0: 0 \\
0: 0 \\
0: 0 \\
0: 0 \\
0: 0 \\
0: 0 \\
0: 0 \\
0: 367 \\
0: 0 \\
0: 0 \\
0: 0 \\
0: 0 \\
0: 0 \\
0: 0 \\
0: 0 \\
0: 0 \\
0: 0 \\
0: 0 \\
0: 0 \\
0: 0 \\
2: 7331 \\
2: 318 \\
4: 0824\end{array}$ & $\begin{array}{l}0: 0 \\
0: 0 \\
0: 0 \\
0: 0 \\
0: 0 \\
0: 0 \\
0: 0 \\
0: 249 \\
0: 211 \\
0: 212 \\
0: 223 \\
0: 234 \\
0: 241 \\
0: 249 \\
0: 310 \\
0: 333 \\
0: 408 \\
0: 626 \\
0: 849 \\
1: 247 \\
1: 902 \\
2: 750 \\
3: 070\end{array}$ & 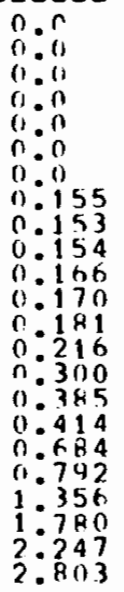 & $\begin{array}{l}0: 0 \\
0: 0 \\
0: 0 \\
0: 0 \\
0: 0 \\
0: 0 \\
0: 0 \\
0: 455 \\
0: 0 \\
0: 0 \\
0: 0 \\
0: 0 \\
0: 0 \\
0: 0 \\
0: 0 \\
0: 0 \\
0: 0 \\
0: 0 \\
0: 0 \\
0 \\
3: 0 \\
3: 265 \\
3: 726 \\
4: 318\end{array}$ & $\begin{array}{l}0.0 \\
0: 0 \\
0: 0 \\
0: 0 \\
0: 0 \\
0: 0 \\
0: 0 \\
0: 263 \\
0: 229 \\
0: 249 \\
0: 234 \\
0: 247 \\
0: 264 \\
0: 273 \\
0: 310 \\
0: 344 \\
0: 542 \\
0: 831 \\
1: 065 \\
1: 445 \\
1: 050 \\
2: 414 \\
3: 146\end{array}$ & $\begin{array}{l}0: 0 \\
0: 0 \\
0: 0 \\
0: 0 \\
0: 0 \\
0: 0 \\
0: 0 \\
0: 0223 \\
0: 028 \\
0: 227 \\
0: 229 \\
0: 267 \\
0: 077 \\
0: 303 \\
0: 320 \\
0: 0434 \\
0: 524 \\
0: 869 \\
1: 010 \\
1: 371 \\
1: 916 \\
2: 333 \\
3: 043\end{array}$ \\
\hline
\end{tabular}


TABLE 2.8 (cont). Natural Gas Prices: Transportation Sector $(\$ / 1000 \mathrm{cu}$. ft.)

\begin{tabular}{|c|c|c|c|c|c|c|c|c|c|}
\hline $\begin{array}{l}\text { YEAR } \\
19-1 \\
1960 \\
1961 \\
1962 \\
1963 \\
1964 \\
1965 \\
1966 \\
1967 \\
1968 \\
1969 \\
1970 \\
1971 \\
1972 \\
1973 \\
1974 \\
1975 \\
1976 \\
1977 \\
1978 \\
1979 \\
1980 \\
1981 \\
1992\end{array}$ & 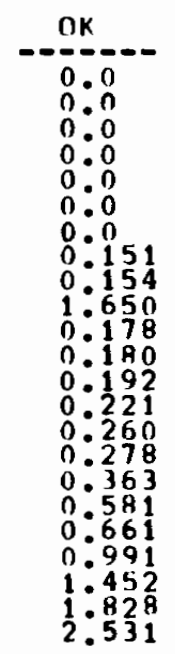 & 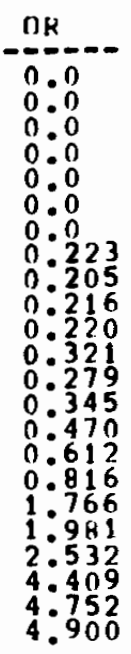 & 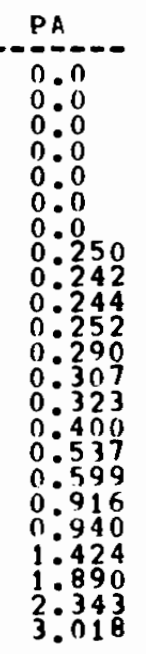 & 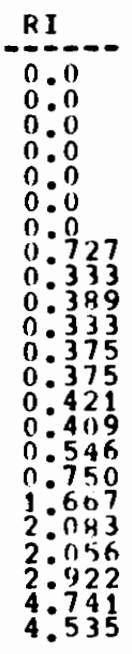 & 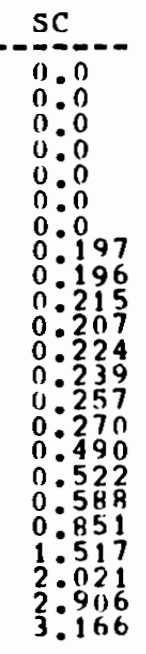 & 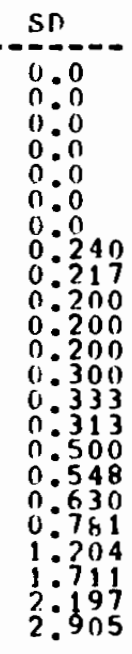 & 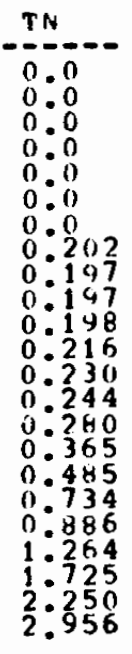 & 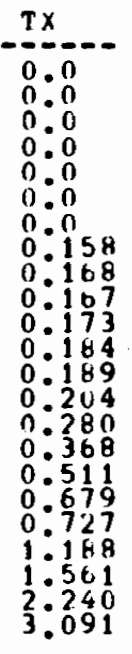 & 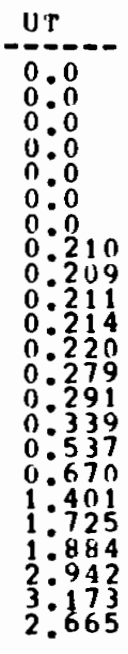 \\
\hline $\begin{array}{ll}\text { N } & \text { YFAR } \\
\sim & 1960 \\
\sigma & 1960 \\
1961 \\
1962 \\
1963 \\
1964 \\
11965 \\
1966 \\
1967 \\
1968 \\
1969 \\
1970 \\
1971 \\
1972 \\
1973 \\
1974 \\
1975 \\
1976 \\
1977 \\
1978 \\
1979 \\
1980 \\
1991 \\
1982\end{array}$ & 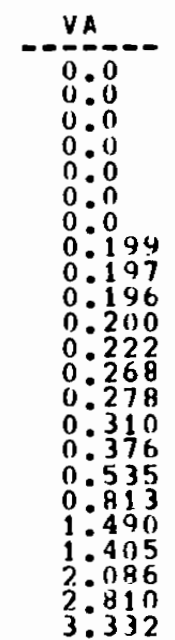 & 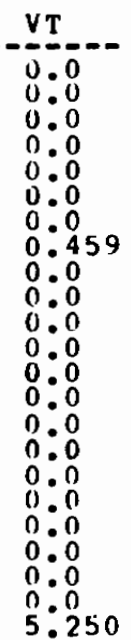 & 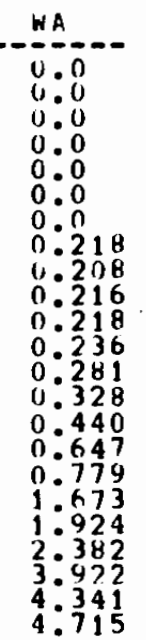 & 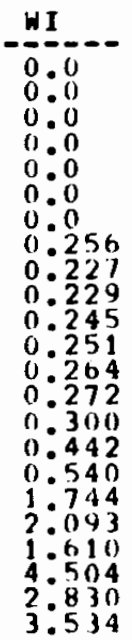 & 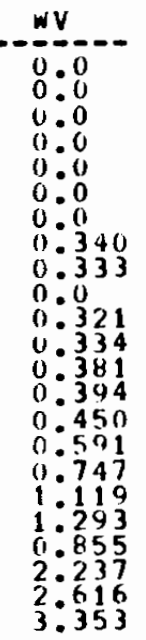 & 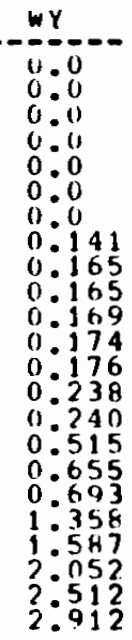 & 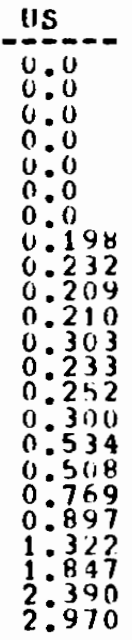 & & \\
\hline
\end{tabular}

Source: Pacific Northwest Laboratory 
TABLE 2.9. Natural Gas Prices: Electric Utility Sector $(\$ / 1000 \mathrm{cu} . \mathrm{ft}$.

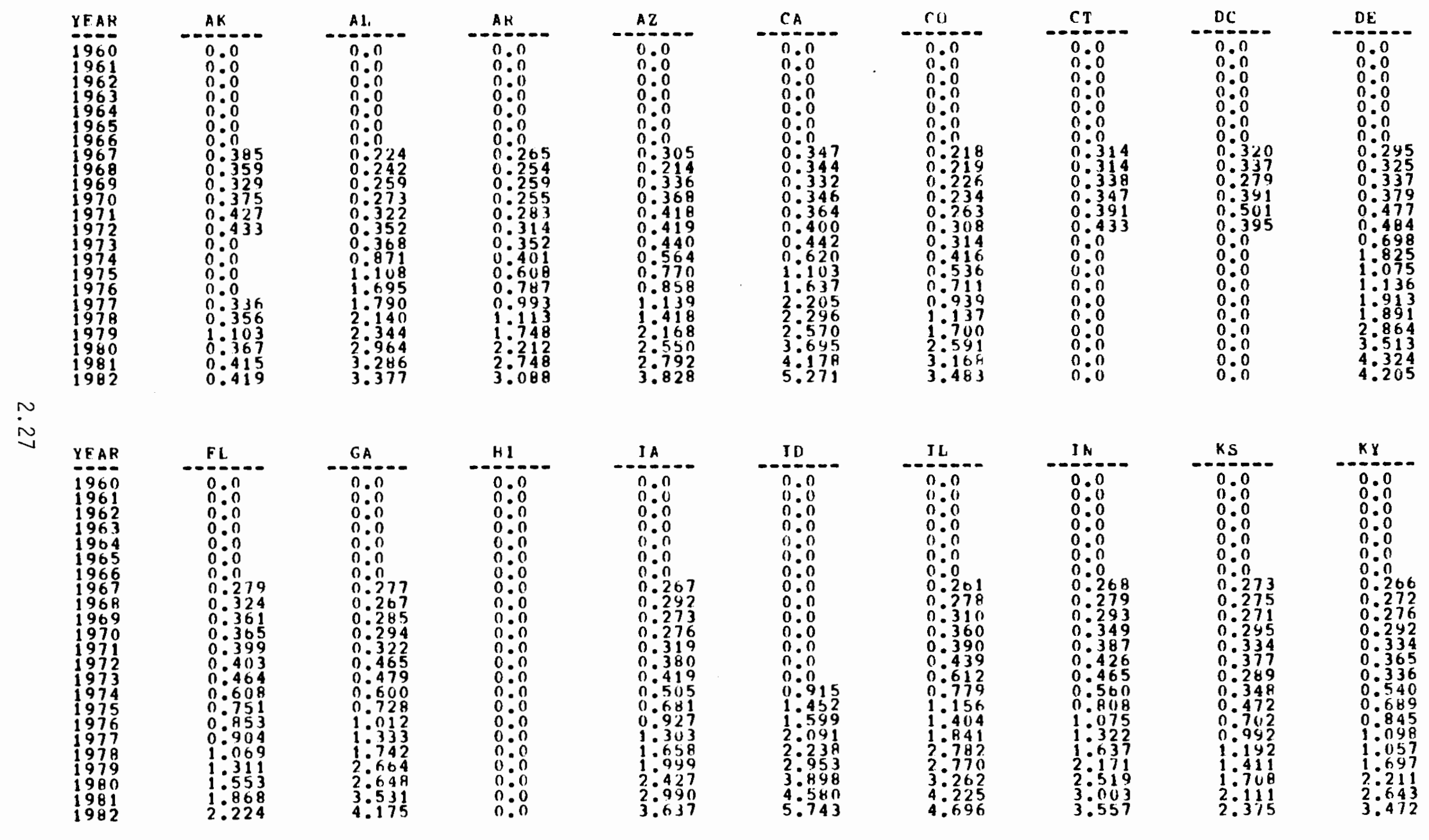


TABLE 2.9 (cont). Natural Gas Prices: Electric Utility Sector $(\$ / 1000 \mathrm{cu}$. ft.)

$\underset{\infty}{\sim}$
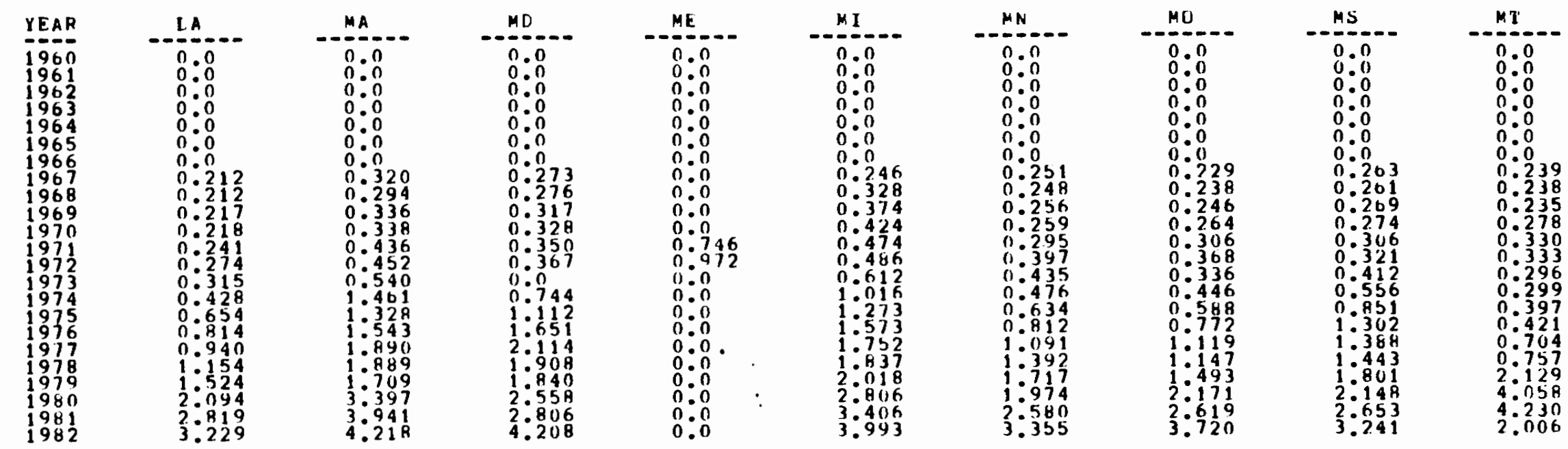

\begin{tabular}{ll} 
YFAR & NC \\
\hline 1960 & 0.0 \\
1961 & $0: 0$ \\
1962 & $0: 0$ \\
1963 & $0: 0$ \\
1964 & $0: 0$ \\
1965 & 0.0 \\
1966 & 000 \\
1967 & $0: 292$ \\
1968 & $0: 314$ \\
1969 & 0.344 \\
1970 & 0.381 \\
1971 & 0.480 \\
1972 & 0.502 \\
1973 & 0.520 \\
1974 & 0.940 \\
1975 & 1.429 \\
1976 & 3.444 \\
1977 & 4.233 \\
1978 & 3.074 \\
1979 & 3.018 \\
1980 & 3.260 \\
1981 & 4.630 \\
1982 & 4.625
\end{tabular}
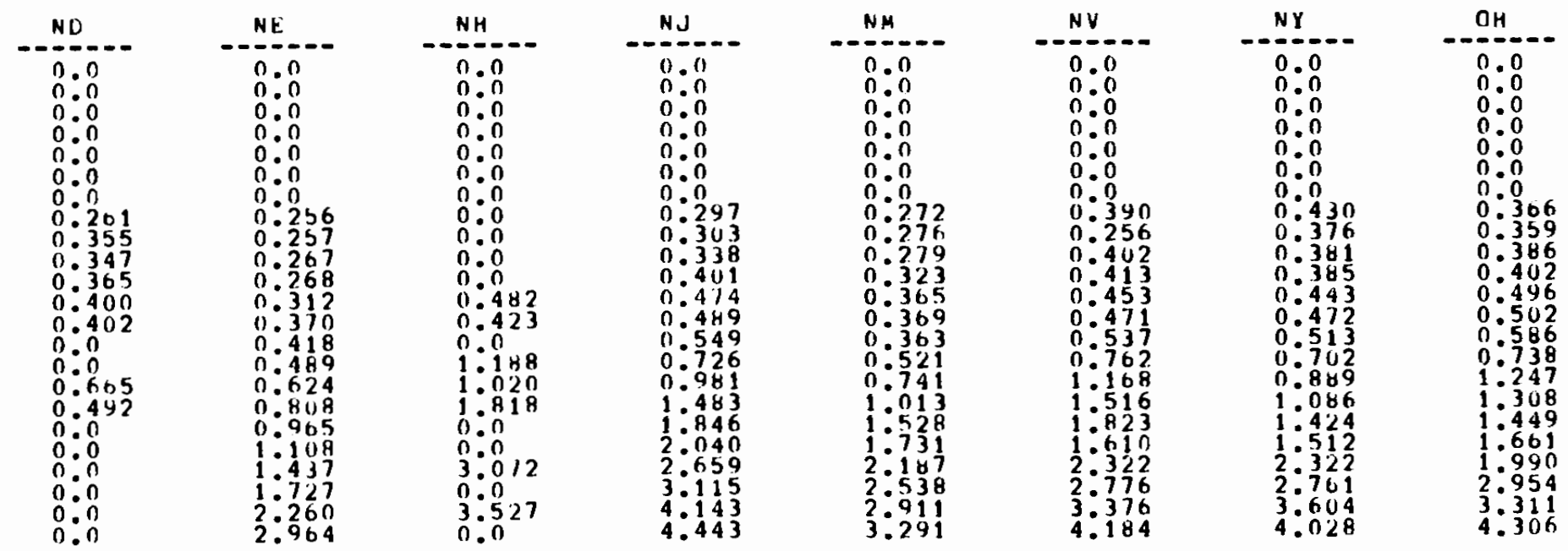
TABLE 2.9 (cont). Natural Gas Prices: Electric Utility Sector ( $\$ / 1000 \mathrm{cu}$. ft.)

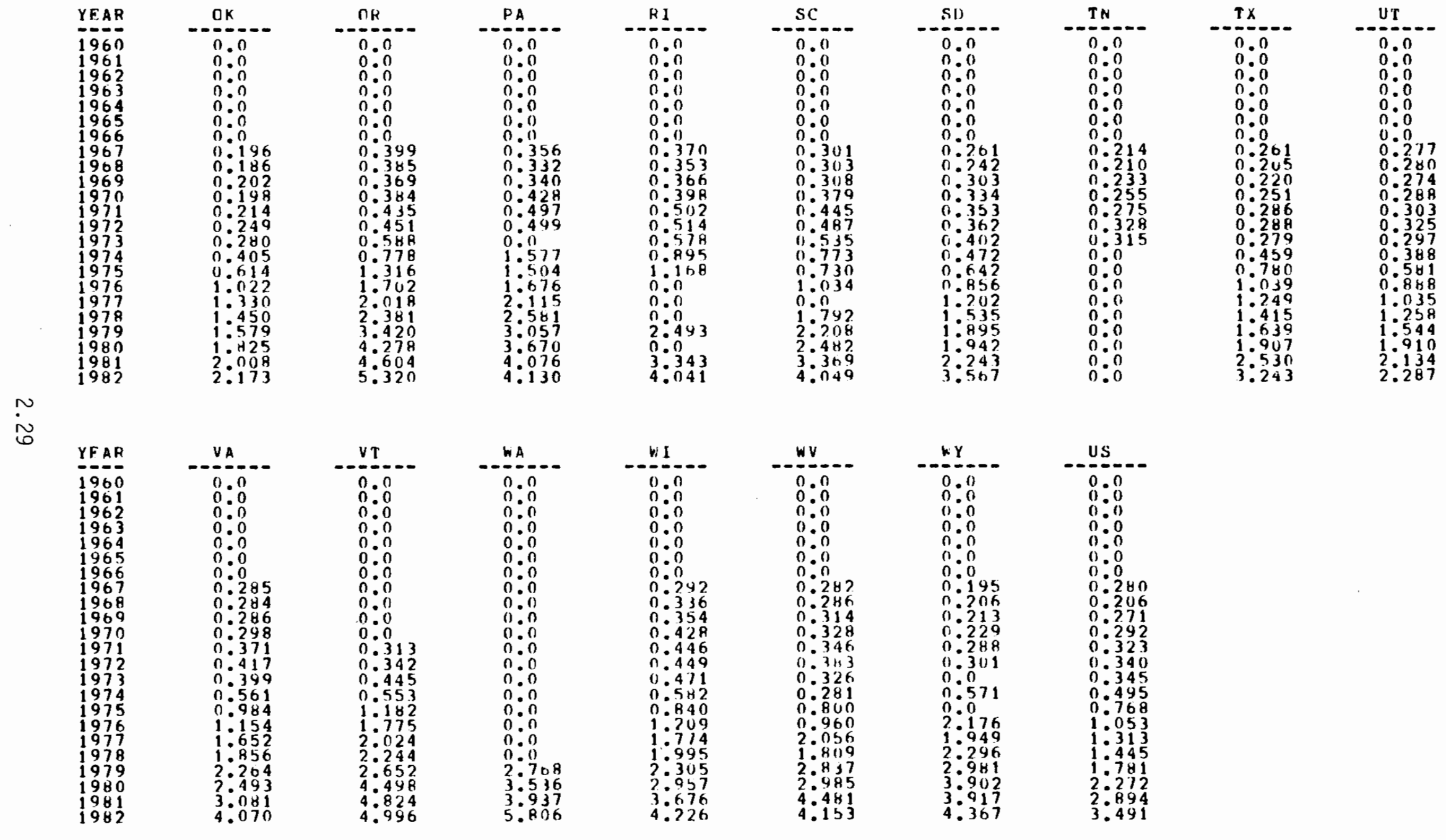

Source: Pacific Northwest Laboratory 
IABLE 2.10. Metallurgical Coal Prices: Industrial Sector (\$/ton)

\begin{tabular}{|c|c|c|c|c|c|c|c|c|c|}
\hline $\begin{array}{l}\text { YEAR } \\
1970 \\
1970 \\
1971 \\
1972 \\
1974 \\
1975 \\
1976 \\
1977 \\
1978 \\
1979 \\
1980 \\
1981 \\
1982\end{array}$ & \begin{tabular}{l}
$A K$ \\
\hdashline $0: 0$ \\
$00: 0$ \\
$0: 0$ \\
$0: 0$ \\
$0: 0$ \\
$0: 0$ \\
$0: 0$ \\
$0: 0$ \\
$0: 0$ \\
$0: 0$ \\
$0: 0$ \\
$0: 0$ \\
$0: 0$
\end{tabular} & $\begin{array}{r}\text { AL } \\
-11: 380 \\
12: 460 \\
14: 160 \\
17: 240 \\
33: 940 \\
40.270 \\
40: 050 \\
55: 230 \\
48: 690 \\
480280 \\
52: 560 \\
55: 370 \\
58: 440\end{array}$ & \begin{tabular}{c}
$A K$ \\
\hdashline $0: 0$ \\
$0:-0$ \\
$0: 0$ \\
$0: 0$ \\
$0: 0$ \\
$0: 0$ \\
$0: 0$ \\
$0: 0$ \\
$0: 0$ \\
$0: 0$ \\
$0: 0$ \\
$0: 0$ \\
$0: 0$
\end{tabular} & 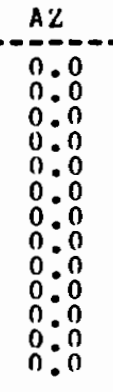 & 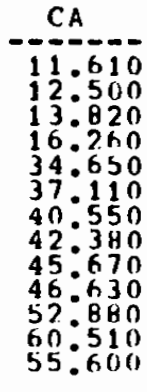 & $\begin{array}{r}C 0 \\
-11.610 \\
13: 500 \\
13: 820 \\
16.260 \\
34.650 \\
37.110 \\
40.550 \\
42: 300 \\
45.670 \\
46.630 \\
52.880 \\
60.510 \\
55.600\end{array}$ & \begin{tabular}{ccc}
$C T$ \\
\hdashline $0: 0$ \\
$0: 0$ \\
$0: 0$ \\
$0: 0$ \\
$0: 0$ \\
$0: 0$ \\
$0: 00$ \\
$0: 0$ \\
$0: 0$ \\
$0: 0$ \\
$0: 0$ \\
0 & 0 \\
0 & 0 \\
0 & 0
\end{tabular} & \begin{tabular}{ll}
$D C$ \\
\hdashline 0 \\
0 & 0 \\
0 & 0 \\
0 & 0 \\
0 & 0 \\
0 & 0 \\
0 & 0 \\
0 & 0 \\
0 & 0 \\
0 & 0 \\
0 & 0 \\
0 & 0 \\
0 & 0 \\
0 & 0 \\
0 & 0
\end{tabular} & \begin{tabular}{rl}
$D E$ \\
\hdashline $0: 0$ \\
0 & 0 \\
0 & 0 \\
0 & 0 \\
0 & 0 \\
0 & 0 \\
0 & 0 \\
0 & 0 \\
0 & 0 \\
0 & 0 \\
0 & 0 \\
0 & 0 \\
0 & 0
\end{tabular} \\
\hline $\begin{array}{l}\text { YEAR } \\
\text { YEA7 } \\
1970 \\
1971 \\
1972 \\
1973 \\
1974 \\
1997 \\
1977 \\
1978 \\
1979 \\
1980 \\
1991 \\
1982\end{array}$ & \begin{tabular}{l}
$\mathbf{F} \mathbf{L}$ \\
\hdashline $0: 0$ \\
$0: 0$ \\
$0: 0$ \\
$0: 0$ \\
$0: 0$ \\
$0: 0$ \\
$0: 0$ \\
$0: 0$ \\
$0: 0$ \\
$0: 0$ \\
$0: 0$ \\
$0: 0$ \\
$0: 0$ \\
$0: 0$
\end{tabular} & \begin{tabular}{c}
$G$ A \\
\hdashline $0: 0$ \\
00 \\
$0: 0$ \\
$0: 0$ \\
$0: 0$ \\
$0: 0$ \\
$0: 0$ \\
$0: 0$ \\
$0: 0$ \\
$0: 0$ \\
$0: 0$ \\
$0: 0$ \\
$0: 0$ \\
0.0
\end{tabular} & \begin{tabular}{cc}
$H I$ \\
\hdashline \\
\hdashline $0: 0$ \\
$0: 0$ \\
$0: 0$ \\
$0: 0$ \\
$0: 0$ \\
$0: 0$ \\
$0: 0$ \\
$0: 0$ \\
$0: 0$ \\
$0: 0$ \\
$0: 0$ \\
$0: 0$ \\
$0: 0$
\end{tabular} & \begin{tabular}{l}
$1 A$ \\
\hdashline 0 \\
$0: 0$ \\
$0: 0$ \\
$0: 0$ \\
$0: 0$ \\
$0: 0$ \\
0 \\
0 \\
0 \\
0 \\
0 \\
0 \\
0 \\
0 \\
0 \\
0 \\
0 \\
0 \\
0 \\
0 \\
0
\end{tabular} & \begin{tabular}{c}
$I 0$ \\
\hdashline $0: 0$ \\
$0: 0$ \\
$0: 0$ \\
$0: 0$ \\
$0: 0$ \\
$0: 0$ \\
$0: 0$ \\
$0: 0$ \\
$0: 0$ \\
$0: 0$ \\
$0: 0$ \\
$0: 0$ \\
$0: 0$
\end{tabular} & \begin{tabular}{r}
$I 4$ \\
\hdashline 11.160 \\
$13: 410$ \\
$14: 940$ \\
$16: 140$ \\
32.630 \\
39.800 \\
38.480 \\
$47: 290$ \\
49.710 \\
48.960 \\
51.750 \\
$56: 100$ \\
59.790
\end{tabular} & $\begin{array}{r}I N \\
-11.50 \\
11.440 \\
15: 730 \\
18: 390 \\
370030 \\
47: 250 \\
40.640 \\
45: 180 \\
54: 150 \\
50: 250 \\
57: 050 \\
67: 060 \\
69.400\end{array}$ & 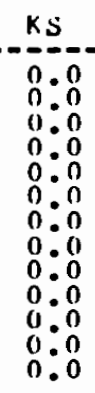 & \begin{tabular}{r}
$x y$ \\
\hdashline 11.840 \\
$11: 730$ \\
$15: 280$ \\
17.710 \\
$32: 830$ \\
42.870 \\
$44: 670$ \\
470040 \\
$50: 620$ \\
47.020 \\
48.520 \\
$54: 510$ \\
54.770
\end{tabular} \\
\hline $\begin{array}{l}\text { YFAR } \\
1970 \\
1970 \\
1972 \\
1973 \\
1974 \\
1975 \\
1996 \\
1977 \\
1979 \\
1998 \\
1981 \\
1982\end{array}$ & \begin{tabular}{l}
$1 . A$ \\
\hdashline $0: 0$ \\
0 \\
$0: 0$ \\
$0: 0$ \\
$0: 0$ \\
$0: 0$ \\
$0: 0$ \\
$0: 0$ \\
$0: 0$ \\
$0: 0$ \\
$0: 0$ \\
$0: 0$ \\
$0: 0$ \\
0.0
\end{tabular} & $\begin{array}{l}M A \\
00.0 \\
0: 0 \\
0: 0 \\
0: 0 \\
0: 0 \\
0: 0 \\
0: 0 \\
0: 0 \\
0: 0 \\
0: 0 \\
0: 0 \\
0: 0 \\
0: 0 \\
0.0\end{array}$ & \begin{tabular}{c}
$M D$ \\
\hdashline 15.630 \\
$18: 940$ \\
200520 \\
$22: 590$ \\
$42: 700$ \\
$57: 270$ \\
$56: 650$ \\
$57: 210$ \\
$62: 670$ \\
$62: 200$ \\
630900 \\
$68: 480$ \\
$73: 150$
\end{tabular} & \begin{tabular}{l}
$M E$ \\
\hdashline $0:-0$ \\
$0: 0$ \\
$0: 0$ \\
$0: 0$ \\
$0: 0$ \\
$0: 0$ \\
$0: 0$ \\
$0: 0$ \\
$0: 0$ \\
$0: 0$ \\
$0: 0$ \\
$0: 0$ \\
$0: 0$ \\
$0: 0$
\end{tabular} & \begin{tabular}{l}
$M 1$ \\
\hdashline 10.220 \\
$17: 710$ \\
$19: 380$ \\
$21: 300$ \\
$44: 240$ \\
$55: 430$ \\
53.460 \\
$53: 760$ \\
$56: 220$ \\
$59: 020$ \\
00770 \\
$64: 700$ \\
66.210
\end{tabular} & \begin{tabular}{c}
$M N$ \\
\hdashline 14.150 \\
$18: 990$ \\
181600 \\
$21: 290$ \\
38.640 \\
$48: 370$ \\
$48: 700$ \\
$48: 4100$ \\
$65: 290$ \\
$59: 020$ \\
0.0 \\
$0: 0$ \\
0.0
\end{tabular} & $\begin{array}{r}M 0 \\
-11: 840 \\
11: 730 \\
15: 280 \\
17: 710 \\
32: 830 \\
42: 870 \\
44: 670 \\
47: 040 \\
50.620 \\
47: 020 \\
48.520 \\
54: 510 \\
54: 770\end{array}$ & 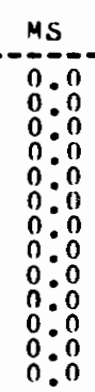 & $\begin{array}{l}0: 0 \\
0: 0 \\
0: 0 \\
0: 0 \\
0: 0 \\
0: 0 \\
0: 0 \\
0: 0 \\
0: 0 \\
0: 0 \\
0: 0 \\
0: 0\end{array}$ \\
\hline
\end{tabular}


TABLE 2.10 (cont). Metallurgical Coal Prices: Industrial Sector (\$/ton)

\begin{tabular}{|c|c|c|c|c|c|c|c|c|c|}
\hline $\begin{array}{l}\text { YFAR } \\
1970 \\
1970 \\
1971 \\
1972 \\
1973 \\
1974 \\
1975 \\
1976 \\
1977 \\
1978 \\
1979 \\
1980 \\
1981 \\
1982\end{array}$ & $\begin{array}{l}N C \\
-:-.- \\
00: 0 \\
0: 0 \\
0: 0 \\
0: 0 \\
0: 0 \\
0: 0 \\
0: 0 \\
0: 0 \\
0: 0 \\
0: 0 \\
0: 0 \\
0: 0 \\
0: 0\end{array}$ & \begin{tabular}{c} 
ND \\
\hdashline $0: 0$ \\
$0: 0$ \\
$0: 0$ \\
$0: 0$ \\
$0: 0$ \\
$0: 0$ \\
$0: 0$ \\
$0: 0$ \\
$0: 0$ \\
$0: 0$ \\
$0: 0$ \\
$0: 0$ \\
$0: 0$
\end{tabular} & \begin{tabular}{l}
$N E$ \\
\hdashline $0:-0$ \\
$0: 0$ \\
$0: 0$ \\
$0: 0$ \\
$0: 0$ \\
$0: 0$ \\
$0: 0$ \\
$0: 0$ \\
$0: 0$ \\
$0: 0$ \\
$0: 0$ \\
$0: 0$ \\
$0: 0$
\end{tabular} & \begin{tabular}{ll}
$N H$ \\
\hdashline $0: 0$ \\
$0: 0$ \\
$0: 0$ \\
$0: 0$ \\
$0: 0$ \\
$0: 0$ \\
$0: 0$ \\
$0: 0$ \\
$0: 0$ \\
$0: 0$ \\
$0: 0$ \\
$0: 0$ \\
$0: 0$ \\
0 & 0
\end{tabular} & $\begin{array}{c}N J \\
-15: 630 \\
150: 940 \\
00: 0 \\
00 \\
0: 0 \\
0: 0 \\
0: 0 \\
0: 0 \\
0: 0 \\
0: 0 \\
0: 0 \\
0: 0 \\
0: 0\end{array}$ & 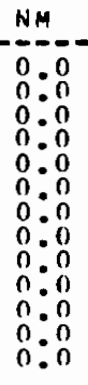 & \begin{tabular}{c}
$N \mathrm{~N}$ \\
\hdashline $0: 0$ \\
$0: 0$ \\
$0: 0$ \\
$0: 0$ \\
$0: 0$ \\
$0: 0$ \\
$0: 0$ \\
$0: 0$ \\
$0: 0$ \\
$0: 0$ \\
$0: 0$ \\
$0: 0$ \\
$0: 0$
\end{tabular} & $\begin{array}{l}\text { NY } \\
-15.630 \\
18: 940 \\
20: 520 \\
22: 590 \\
42.700 \\
57.270 \\
56: 650 \\
57: 210 \\
62: 670 \\
62.200 \\
63: 900 \\
68.480 \\
73.150\end{array}$ & 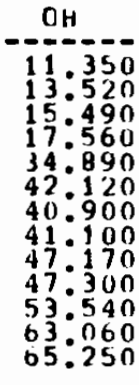 \\
\hline $\begin{array}{l}\text { YEAR } \\
1970 \\
1970 \\
1971 \\
1972 \\
1973 \\
1974 \\
1975 \\
1976 \\
1977 \\
1978 \\
1979 \\
1980 \\
1981 \\
1992\end{array}$ & \begin{tabular}{c}
$n k$ \\
\hdashline $0: 0$ \\
$0: 0$ \\
$0: 0$ \\
$0: 0$ \\
$0: 0$ \\
$0: 0$ \\
$0: 0$ \\
$0: 0$ \\
$0: 0$ \\
$0: 0$ \\
$0: 0$ \\
$0: 0$ \\
$0: 0$
\end{tabular} & \begin{tabular}{c}
$0 R$ \\
$0 R$ \\
\hdashline $0: 0$ \\
$0: 0$ \\
$0: 0$ \\
$0: 0$ \\
$0: 0$ \\
$0: 0$ \\
$0: 0$ \\
$0: 0$ \\
$0: 0$ \\
$0: 0$ \\
$0: 0$ \\
$0: 0$ \\
$0: 0$
\end{tabular} & \begin{tabular}{r} 
PA \\
\hdashline $11: 760$ \\
$12: 370$ \\
$14: 880$ \\
$17: 950$ \\
$36: 440$ \\
$40: 690$ \\
430450 \\
$43: 430$ \\
$52: 960$ \\
$50: 370$ \\
580930 \\
$63: 590$ \\
67.570
\end{tabular} & \begin{tabular}{c} 
RI \\
\hdashline $0: 0$ \\
$0: 0$ \\
$0: 0$ \\
$0: 0$ \\
$0: 0$ \\
$0: 0$ \\
$0: 0$ \\
$0: 0$ \\
$0: 0$ \\
$0: 0$ \\
$0: 0$ \\
$0: 0$ \\
$0: 0$
\end{tabular} & $\begin{array}{c}S C \\
-0 .-- \\
00: 0 \\
0: 0 \\
0: 0 \\
0: 0 \\
0: 0 \\
0: 0 \\
0: 0 \\
0: 0 \\
0: 0 \\
0: 0 \\
0: 0 \\
0.0\end{array}$ & \begin{tabular}{ll}
50 \\
\hdashline 0 \\
0 & 0 \\
0 & 0 \\
0 & 0 \\
0 & 0 \\
0 & 0 \\
0 & 0 \\
0 & 0 \\
0 & 0 \\
0 & 0 \\
0 & 0 \\
0 & 0 \\
0 & 0 \\
0 & 0 \\
0 & 0
\end{tabular} & 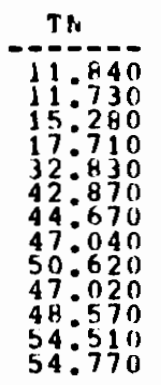 & \begin{tabular}{r}
$T X$ \\
\hdashline 11.840 \\
$11: 730$ \\
$15: 280$ \\
$17: 710$ \\
$32: 830$ \\
$42: 870$ \\
$44: 670$ \\
47040 \\
50.620 \\
47020 \\
48.520 \\
$54: 510$ \\
54.770
\end{tabular} & 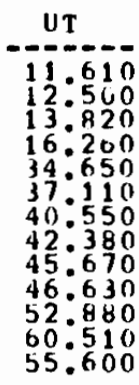 \\
\hline $\begin{array}{l}\text { YEAR } \\
\text { 1977 } \\
1971 \\
1971 \\
1973 \\
1973 \\
1974 \\
1975 \\
1976 \\
1977 \\
1978 \\
1979 \\
1980 \\
1981 \\
1982\end{array}$ & \begin{tabular}{c}
$V A$ \\
\hdashline 0.0 \\
$0: 0$ \\
$0: 0$ \\
$0: 0$ \\
$0: 0$ \\
$0: 0$ \\
$0: 0$ \\
$0: 0$ \\
$46: 460$ \\
47.690 \\
$49: 760$ \\
$52: 460$ \\
$54: 990$
\end{tabular} & \begin{tabular}{ll}
$v$ & 1 \\
\hdashline & - \\
0 & 0 \\
0 & 0 \\
0 & 0 \\
0 & 0 \\
0 & 0 \\
0 & 0 \\
0 & 0 \\
0 & 0 \\
0 & 0 \\
0 & 0 \\
0 & 0 \\
0 & 0 \\
0 & 0 \\
0 & 0 \\
0 & 0
\end{tabular} & \begin{tabular}{ll}
$W$ & $=$ \\
\hdashline 0 & 0 \\
0 & 0 \\
0 & 0 \\
0 & 0 \\
0 & 0 \\
0 & 0 \\
0 & 0 \\
0 & 0 \\
0 & 0 \\
0 & 0 \\
0 & 0 \\
0 & 0 \\
0 & 0 \\
0 & 0
\end{tabular} & $\begin{array}{r}W I \\
14.150 \\
18: 940 \\
180160 \\
21.290 \\
38.640 \\
48.370 \\
48.700 \\
48.410 \\
65.290 \\
59.020 \\
60.740 \\
64.790 \\
66.210\end{array}$ & $\begin{array}{c}W v \\
10.760 \\
12: 520 \\
13: 500 \\
15: 100 \\
30: 450 \\
40: 540 \\
43: 930 \\
45: 250 \\
46: 460 \\
47.640 \\
49.760 \\
52: 460 \\
54.890\end{array}$ & $\begin{array}{ll} & y \\
0 & 0 \\
0 & 0 \\
0 & 0 \\
0 & 0 \\
0 & 0 \\
0 & 0 \\
0 & 0 \\
0 & 0 \\
0 & 0 \\
0 & 0 \\
0 & 0 \\
0 & 0 \\
0 & 0 \\
0 & 0 \\
0 & 0\end{array}$ & $\begin{array}{l}11 S \\
-12.210 \\
14: 000 \\
15: 730 \\
18: 3200 \\
36: 490 \\
44.210 \\
44: 160 \\
46: 240 \\
52: 050 \\
50.690 \\
56.330 \\
62.860 \\
64.970\end{array}$ & & \\
\hline
\end{tabular}

Source: Pacific Northwest Laboratory 
TABLE 2.11. Steam Coal Prices: Residential Sector ( $\$ /$ ton)

\begin{tabular}{|c|c|c|c|c|c|c|c|c|c|}
\hline $\begin{array}{l}\text { YEAR } \\
1970 \\
1971 \\
1971 \\
1977 \\
1974 \\
1975 \\
1976 \\
1977 \\
1978 \\
1979 \\
1980 \\
1981 \\
1982\end{array}$ & 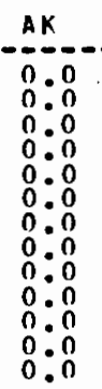 & \begin{tabular}{r}
$A 1$ \\
\hdashline 7.470 \\
99823 \\
23.850 \\
$31: 414$ \\
48.635 \\
$47: 077$ \\
$49: 153$ \\
$50: 511$ \\
$72: 828$ \\
59.985 \\
64.176 \\
$80: 563$ \\
71.273
\end{tabular} & $\begin{array}{l}A H \\
00 \\
0: 0 \\
0: 0 \\
0: 0 \\
0: 0 \\
0: 0 \\
0: 0 \\
0: 0 \\
0: 0 \\
0: 0 \\
0: 0 \\
0: 0 \\
0.0\end{array}$ & \begin{tabular}{l}
$A:$ \\
\hdashline$-10-$ \\
$0: 0$ \\
$0: 0$ \\
$0: 0$ \\
$0: 0$ \\
$0: 0$ \\
$0: 0$ \\
$0: 0$ \\
$0: 0$ \\
$0: 0$ \\
$0: 0$ \\
$0: 0$ \\
$0: 0$ \\
$0: 0$
\end{tabular} & 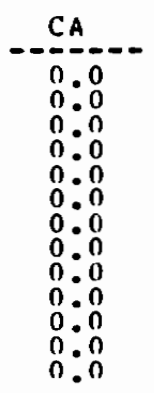 & 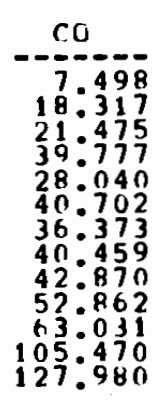 & \begin{tabular}{r}
$C T$ \\
\hdashline 12.511 \\
$22: 400$ \\
$14: 900$ \\
$28: 286$ \\
$20: 513$ \\
27.658 \\
27.329 \\
$44: 882$ \\
5502029 \\
$35: 570$ \\
38.114 \\
95.247 \\
107.957
\end{tabular} & \begin{tabular}{c}
$D C$ \\
\hdashline 11.024 \\
$30: 000$ \\
$35: 000$ \\
$37: 278$ \\
$59: 289$ \\
$45: 116$ \\
$45: 221$ \\
$67: 097$ \\
$71: 449$ \\
$64: 053$ \\
$69: 948$ \\
$82: 971$ \\
$84: 008$
\end{tabular} & 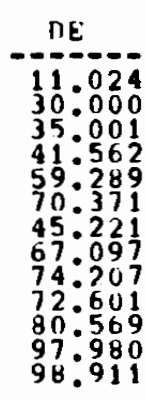 \\
\hline $\begin{array}{l}\text { YEAR } \\
1 E A 7 \\
1970 \\
1977 \\
1972 \\
1977 \\
1974 \\
1975 \\
1977 \\
1978 \\
1979 \\
1980 \\
1981 \\
1982\end{array}$ & \begin{tabular}{c}
$F L$ \\
\hdashline$-:-$ \\
00 \\
$0: 0$ \\
$0: 0$ \\
$0: 0$ \\
$0: 0$ \\
$0: 0$ \\
$0: 0$ \\
$0: 0$ \\
$0: 0$ \\
$0: 0$ \\
$0: 0$ \\
$0: 0$ \\
0 \\
0
\end{tabular} & \begin{tabular}{r} 
GA \\
\hdashline 10.733 \\
19.399 \\
$22: 906$ \\
26.008 \\
$34: 740$ \\
83.366 \\
$39: 3222$ \\
$57: 296$ \\
$73: 079$ \\
62.417 \\
67.534 \\
$78: 123$ \\
80.656
\end{tabular} & \begin{tabular}{l}
$H \mathrm{I}$ \\
\hdashline-0. \\
\hdashline $0: 0$ \\
$0: 0$ \\
$0: 0$ \\
$0: 0$ \\
$0: 0$ \\
$0: 0$ \\
$0: 0$ \\
$0: 0$ \\
$0: 0$ \\
$0: 0$ \\
$0: 0$ \\
$0: 0$ \\
$0: 0$
\end{tabular} & $\begin{array}{l}\text { IA } \\
-99.149 \\
24: 150 \\
30: 000 \\
31: 108 \\
690479 \\
95: 136 \\
69: 142 \\
56: 894 \\
80.975 \\
65: 096 \\
73065 \\
95: 223 \\
92.152\end{array}$ & 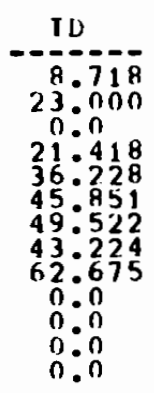 & $\begin{aligned} \text { Il } \\
-8.776 \\
30: 900 \\
31: 495 \\
29: 947 \\
44: 356 \\
54368 \\
57: 263 \\
57.497 \\
59.541 \\
77.278 \\
87.181 \\
67.454 \\
74.623\end{aligned}$ & $\begin{aligned} \text { IN } \\
-77.171 \\
22: 750 \\
26: 890 \\
31: 476 \\
59: 726 \\
64: 928 \\
65: 210 \\
61: 600 \\
64: 346 \\
66: 935 \\
70.614 \\
70: 700 \\
71.463\end{aligned}$ & 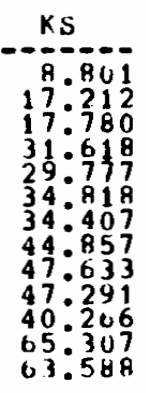 & $\begin{array}{r}K Y \\
-6.257 \\
199: 000 \\
22: 001 \\
32: 319 \\
49.132 \\
54: 511 \\
55: 420 \\
600480 \\
62.257 \\
57.267 \\
59.021 \\
67.137 \\
65.997\end{array}$ \\
\hline $\begin{array}{l}\text { YEAR } \\
\text { YEA } \\
1970 \\
1971 \\
1972 \\
1973 \\
1974 \\
1975 \\
1975 \\
1977 \\
1979 \\
1979 \\
1980 \\
1982\end{array}$ & \begin{tabular}{c} 
LA \\
\hdashline $0:-$ \\
$0: 0$ \\
$0: 0$ \\
$0: 0$ \\
$0: 0$ \\
$0: 0$ \\
$0: 0$ \\
$0: 0$ \\
$0: 0$ \\
$0: 0$ \\
$0: 0$ \\
$0: 0$ \\
$0: 0$
\end{tabular} & \begin{tabular}{c}
$M A$ \\
\hdashline 12.511 \\
22.400 \\
14.900 \\
28.286 \\
20.515 \\
27.658 \\
27.329 \\
44.882 \\
$55: 029$ \\
35.570 \\
38.114 \\
95.247 \\
107.957
\end{tabular} & \begin{tabular}{c}
$M D$ \\
\hdashline 11.024 \\
$30: 000$ \\
$35: 000$ \\
$37: 278$ \\
$59: 249$ \\
$45: 116$ \\
$45: 221$ \\
$67: 097$ \\
$71: 449$ \\
$64: 053$ \\
$69: 998$ \\
$82: 971$ \\
$84: 001$
\end{tabular} & \begin{tabular}{l} 
ME \\
\hdashline $0: 0$ \\
$0: n$ \\
$0: 0$ \\
$0: 0$ \\
$0: n$ \\
$0: 0$ \\
$0: 0$ \\
$0: 0$ \\
$0: 0$ \\
$0: 0$ \\
$0: 0$ \\
$0: 0$ \\
$0: 0$ \\
$0: 0$
\end{tabular} & 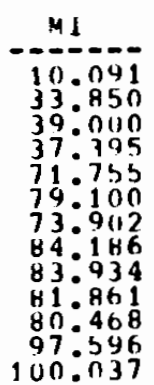 & \begin{tabular}{r}
$M N$ \\
\hdashline 9.742 \\
$0: 0$ \\
010 \\
$30: 343$ \\
$\forall 4: 368$ \\
$69: 967$ \\
770137 \\
750641 \\
970272 \\
860262 \\
87.419 \\
128.431 \\
130.234
\end{tabular} & $\begin{array}{r}M O 1 \\
-77.251 \\
22: 650 \\
22: 800 \\
20: 653 \\
36: 640 \\
49: 380 \\
42: 216 \\
50: 260 \\
51: 059 \\
57: 177 \\
51: 157 \\
58: 890 \\
57.228\end{array}$ & \begin{tabular}{l}
$M S$ \\
\hdashline $0: 0$ \\
$0: 0$ \\
$0: 0$ \\
$0: 0$ \\
$0: 0$ \\
$0: 0$ \\
$0: 0$ \\
$0: 0$ \\
$0: 0$ \\
$0: 0$ \\
$0: 0$ \\
$0: 0$ \\
$0: 0$
\end{tabular} & 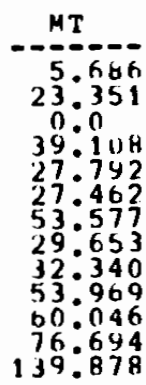 \\
\hline
\end{tabular}


TABLE 2.12. Steam Coal Prices: Industrial Sector ( $\$ /$ ton)

\begin{tabular}{|c|c|c|c|c|c|c|c|c|c|}
\hline $\begin{array}{l}\text { YEAR } \\
19770 \\
1970 \\
1971 \\
1972 \\
1973 \\
1975 \\
1975 \\
1977 \\
1978 \\
1979 \\
1980 \\
1981 \\
1982\end{array}$ & 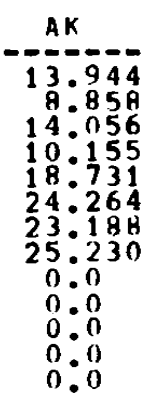 & 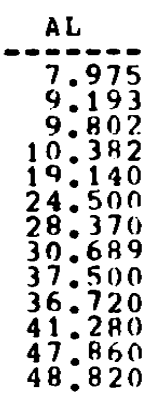 & 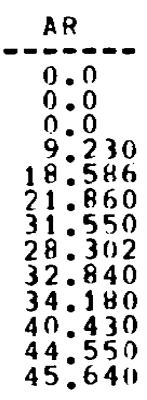 & 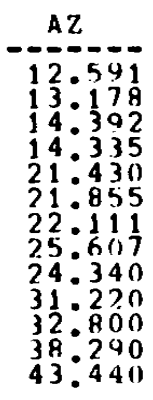 & $\begin{array}{r}C A \\
-8.072 \\
12.65 \% \\
13.356 \\
13.187 \\
24.210 \\
21.170 \\
25.620 \\
28.296 \\
26.930 \\
35.070 \\
38.860 \\
45.800 \\
51.010\end{array}$ & 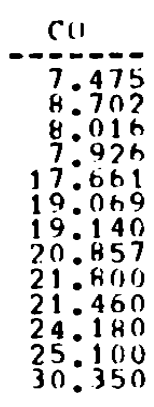 & \begin{tabular}{c} 
Cr \\
\hdashline 17.377 \\
18.868 \\
19.660 \\
$23: 537$ \\
36.803 \\
47.350 \\
38.1100 \\
46.938 \\
39.540 \\
44.130 \\
40.850 \\
550000 \\
51.550
\end{tabular} & 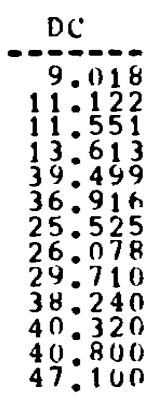 & 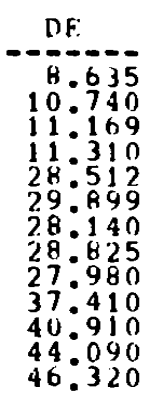 \\
\hline $\begin{array}{l}\text { YEAR } \\
1970 \\
1970 \\
1971 \\
1972 \\
1973 \\
1974 \\
1975 \\
1976 \\
1977 \\
1978 \\
1979 \\
1980 \\
1981 \\
1992\end{array}$ & 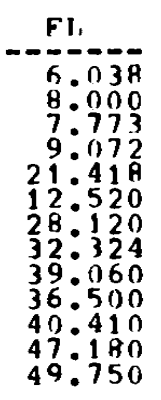 & 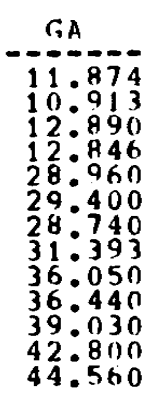 & \begin{tabular}{c}
$H I$ \\
\hdashline 0.0 \\
$0: 0$ \\
$0: 0$ \\
$0: 0$ \\
$0: 0$ \\
$0: 0$ \\
$0: 0$ \\
$0: 0$ \\
$0: 0$ \\
$0: 0$ \\
$0: 0$ \\
$0: 0$ \\
0.0
\end{tabular} & 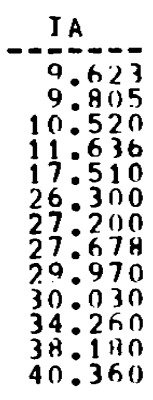 & 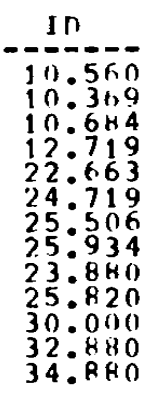 & 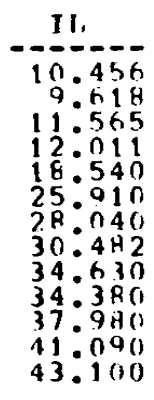 & 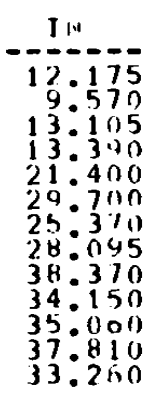 & 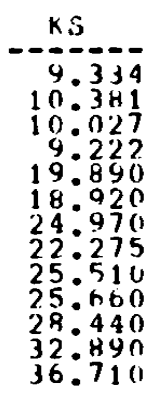 & 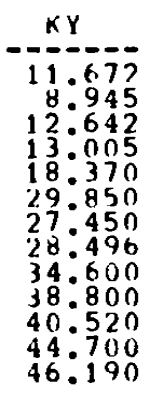 \\
\hline $\begin{array}{l}\text { YEAR } \\
\text { YEA } \\
1971 \\
1971 \\
1972 \\
1973 \\
1974 \\
1975 \\
1976 \\
1977 \\
1978 \\
1979 \\
1980 \\
1981 \\
1982\end{array}$ & $\begin{array}{c}\text { LA } \\
-0.0 \\
0: 0 \\
0: 0 \\
0: 0 \\
0: 0 \\
0: 0 \\
0: 0 \\
0: 0 \\
30.303 \\
33: 330 \\
26: 050 \\
31: 840 \\
350 \\
48.360 \\
48\end{array}$ & $\begin{array}{r}M A \\
-18.357 \\
14.970 \\
21.260 \\
26.157 \\
41.240 \\
62.500 \\
43.800 \\
490000 \\
51.800 \\
49.340 \\
54.960 \\
53.640 \\
53.670\end{array}$ & 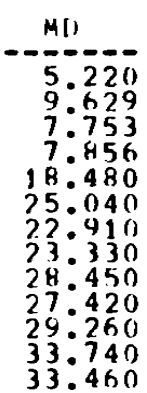 & \begin{tabular}{l}
$M F:$ \\
\hdashline 21.973 \\
$20: 000$ \\
$24: 044$ \\
$24: 336$ \\
$36: 440$ \\
450644 \\
$46: 674$ \\
470358 \\
$17: 220$ \\
$44: 740$ \\
$55: 470$ \\
55.930 \\
60.500
\end{tabular} & 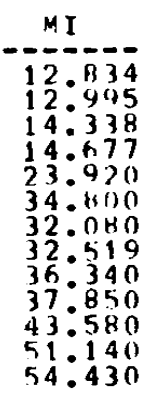 & 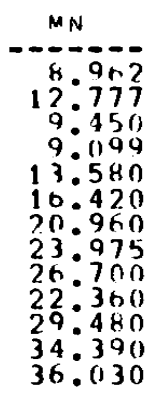 & 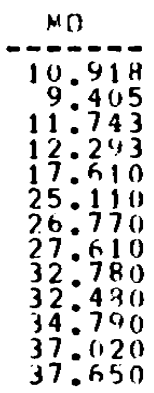 & \begin{tabular}{r}
$M_{D}$ \\
\hdashline 3.940 \\
$4: 428$ \\
$5: 615$ \\
$6: 175$ \\
$18: 259$ \\
$21: 549$ \\
$24: 574$ \\
$25: 240$ \\
370260 \\
$30: 200$ \\
$36: 150$ \\
$44: 850$ \\
47.610
\end{tabular} & $\begin{array}{l}M r \\
-16.604 \\
13: 369 \\
16: 855 \\
16: 900 \\
26.384 \\
26: 735 \\
26.954 \\
26.764 \\
22: 610 \\
25: 300 \\
38: 890 \\
36.660 \\
35.570\end{array}$ \\
\hline
\end{tabular}


TABLE 2.12 (cont). Steam Coal Prices: Industrial Sector ( $\$ /$ ton)
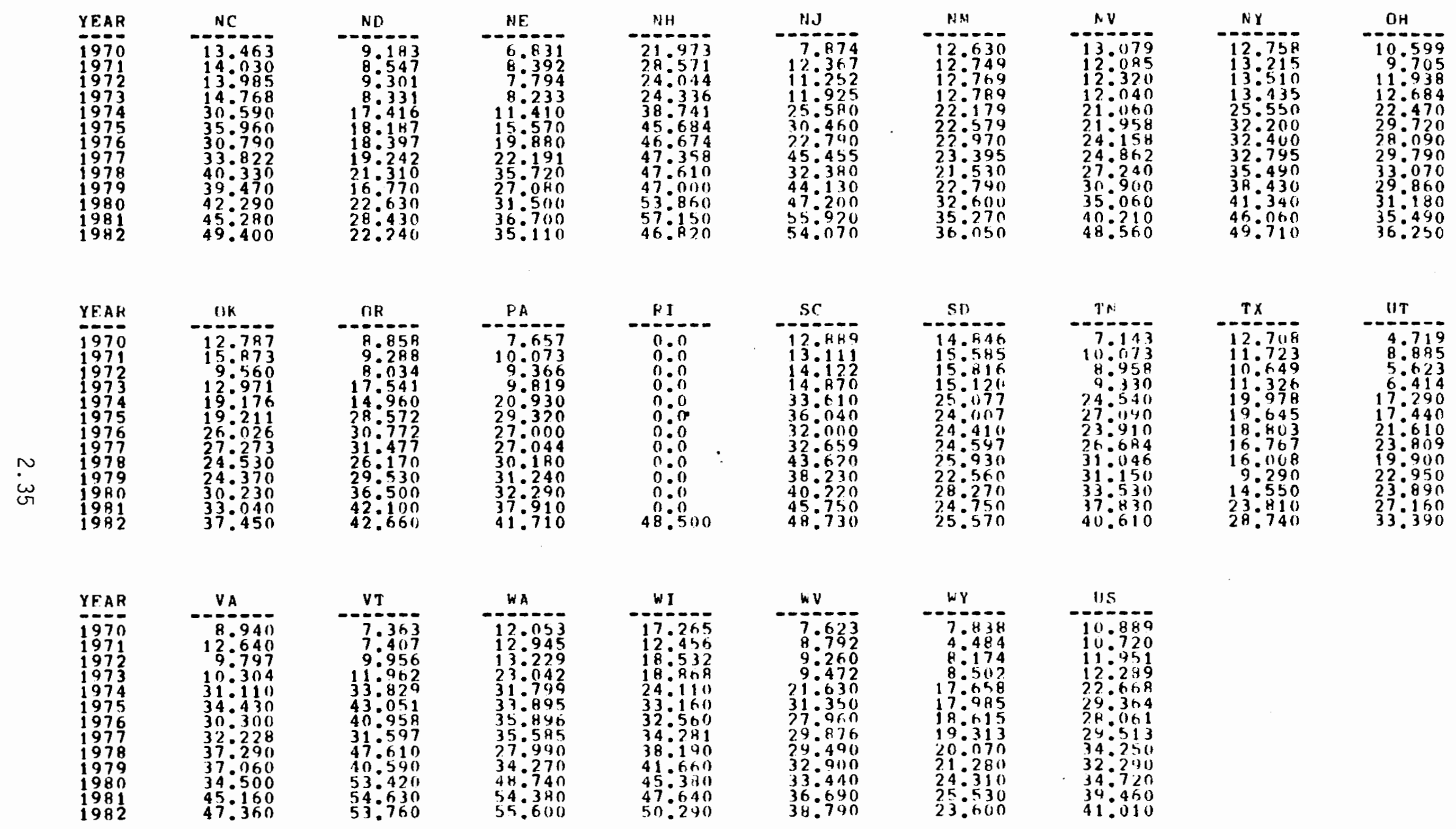

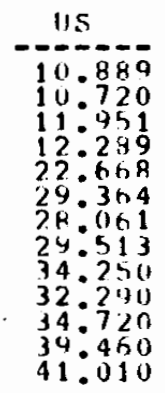

Source: Pacific Northwest Laboratory 
TABLE 2.13. Steam Coal Prices: Electric Utility Sector ( $\$ /$ ton)
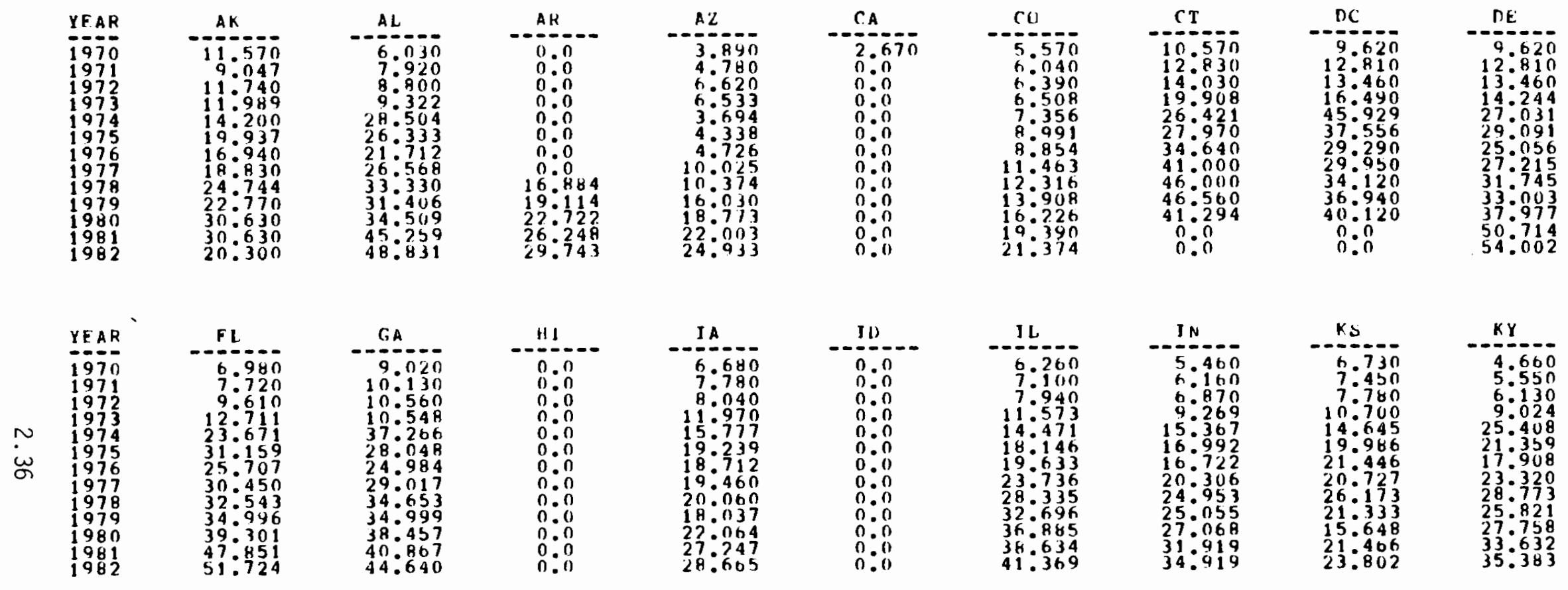

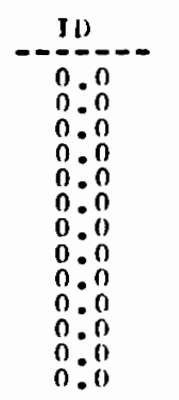
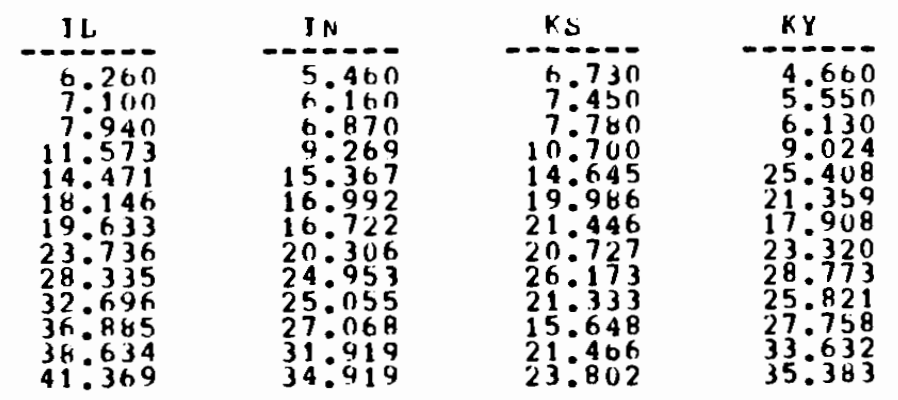

\begin{tabular}{ll} 
YEAR & LA \\
\hline 1970 & 0.0 \\
1971 & 0.0 \\
1972 & 000 \\
1973 & 0.0 \\
1974 & 0.0 \\
1975 & 000 \\
1976 & 000 \\
1977 & 000 \\
1978 & 0.0 \\
1979 & 0.0 \\
1980 & $31: 357$ \\
1981 & 34.400 \\
1982 & 37.982
\end{tabular}

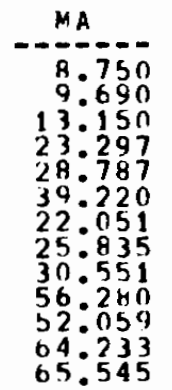

$M D$
-9.070
12.810
13.460
13.076
28.554
28.891
25.741
27.088
32.610
33.519
37.279
44.347
47.663

ME
0.0
$0: 0$
$0: 0$
0.0
$0: 0$
0.0
0.0
0.0
0.0
$0: 0$
$0: 0$
0.0
0.0

$M 1$
-9.600
10.110
10.880
12.640
31.705
26.842
26.475
25.065
28.619
33.311
32.461
41.007
46.344
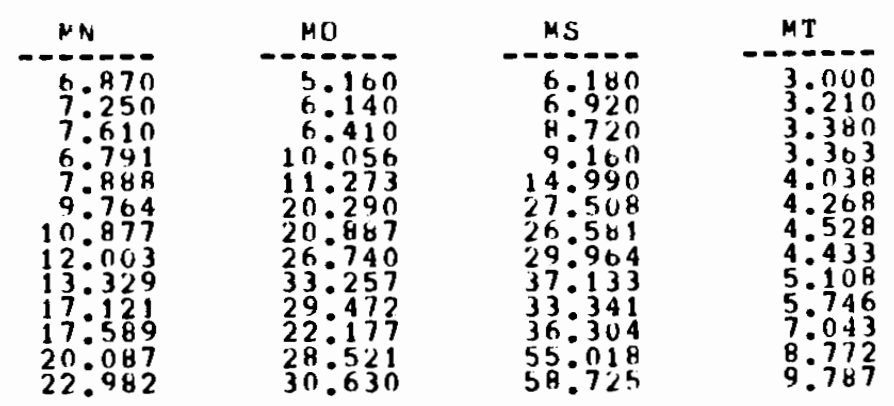
TABLE 2.13 (cont). Steam Coal Prices: Electric Utility Sector ( $\$ /$ ton)
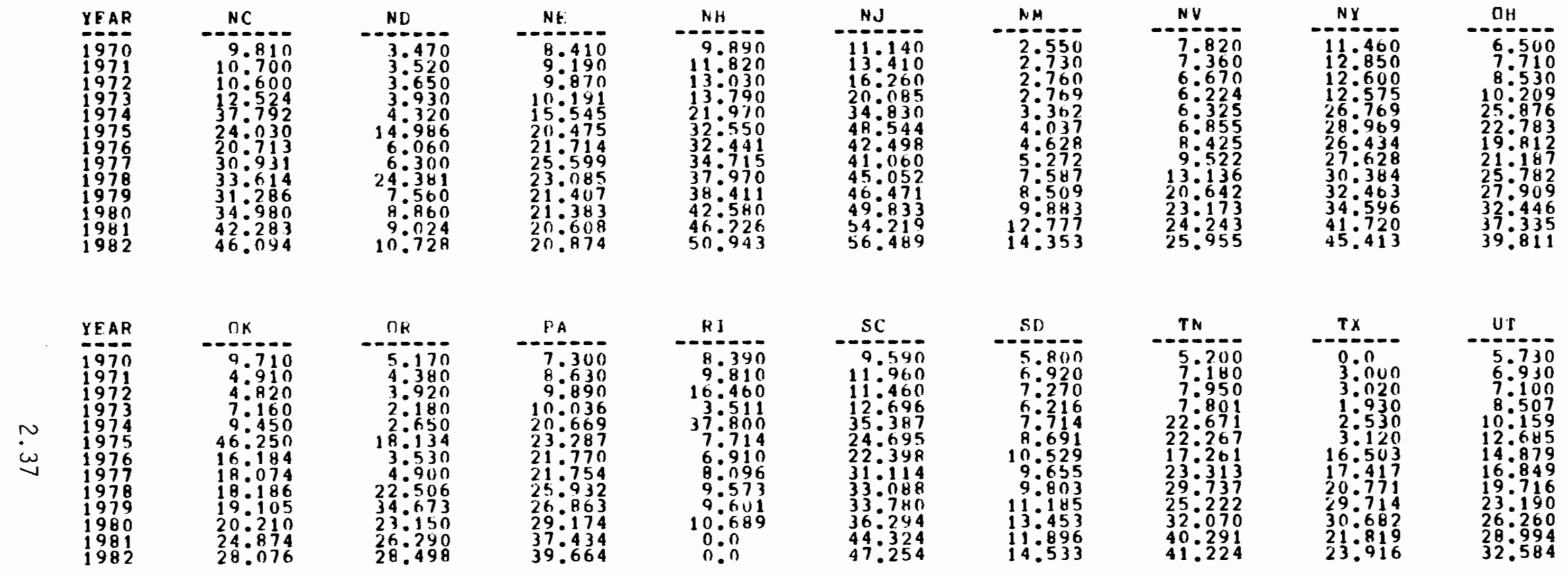

\begin{tabular}{ll} 
YFAR & VA \\
\hline 1970 & -99.560 \\
1971 & 11.140 \\
1972 & 10.860 \\
1973 & 13.523 \\
1974 & 36.345 \\
1975 & 29.954 \\
1976 & 26.800 \\
1977 & 36.392 \\
1978 & 41.390 \\
1979 & 41.573 \\
1980 & 44.057 \\
1981 & 47.851 \\
1982 & 48.164
\end{tabular}
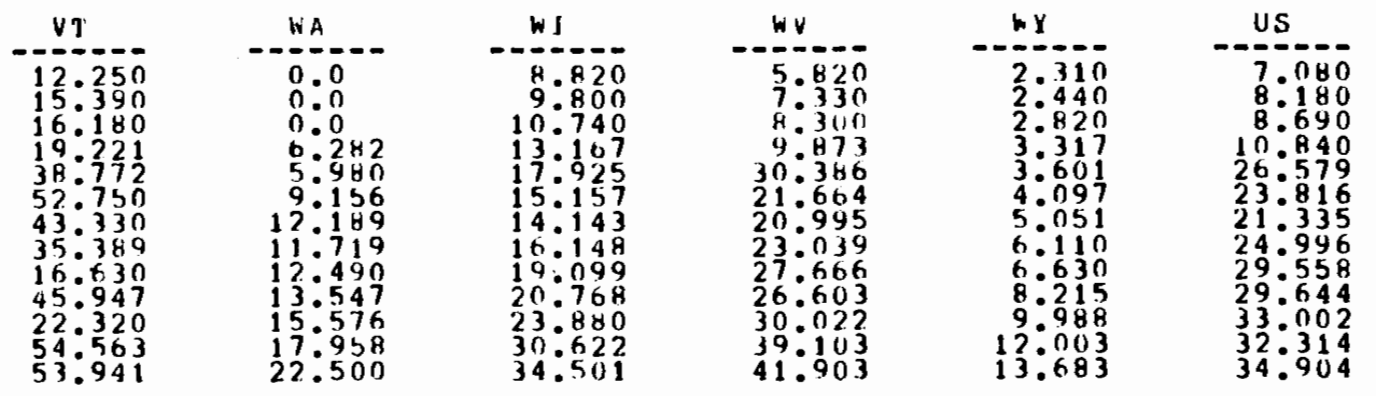

Source: Pacific Northwest Laboratory 
TABLE 2.14. Distillate Fuel Prices: Residential Sector (\$/gallon)

\begin{tabular}{|c|c|c|c|c|c|c|c|c|c|}
\hline $\begin{array}{l}\text { YFAR } \\
1970 \\
1971 \\
1972 \\
1973 \\
1974 \\
1975 \\
1976 \\
1977 \\
1978 \\
1979 \\
1980 \\
1981 \\
1982\end{array}$ & \begin{tabular}{l}
$A K$ \\
\hdashline$-:-154$ \\
$0: 184$ \\
$0: 196$ \\
$0: 197$ \\
$0: 228$ \\
$0: 3303$ \\
$0: 388$ \\
$0: 431$ \\
$0: 450$ \\
$0: 473$ \\
$0: 655$ \\
$0: 942$ \\
$1: 170$ \\
$1: 179$
\end{tabular} & 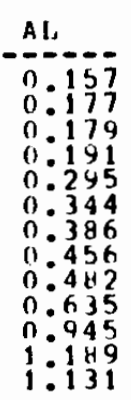 & \begin{tabular}{l}
$A K$ \\
\hdashline $0: 122$ \\
$0: 133$ \\
$0: 134$ \\
$0: 114$ \\
$0: 312$ \\
$0: 330$ \\
$0: 352$ \\
$0: 393$ \\
$0: 409$ \\
$0: 630$ \\
$0: 902$ \\
$1: 112$ \\
10050
\end{tabular} & \begin{tabular}{l}
$A Z$ \\
\hdashline 0.149 \\
$0: 102$ \\
$0: 166$ \\
$0: 207$ \\
$0: 357$ \\
$0: 394$ \\
$0: 420$ \\
$0: 432$ \\
$0: 450$ \\
$0: 617$ \\
$0: 975$ \\
$1: 007$ \\
$1: 076$
\end{tabular} & \begin{tabular}{l}
$C A$ \\
\hdashline 0.149 \\
$0: 162$ \\
$0: 106$ \\
$0: 207$ \\
$0: 357$ \\
$0: 388$ \\
$0: 431$ \\
$0: 450$ \\
$0: 432$ \\
$0: 625$ \\
$0: 927$ \\
$0: 996$ \\
$1: 041$
\end{tabular} & 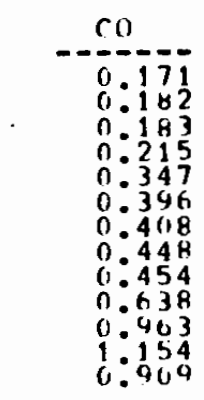 & \begin{tabular}{l}
$C T$ \\
\hdashline 0.195 \\
$0: 206$ \\
0.207 \\
$0: 240$ \\
00.397 \\
0.394 \\
0.421 \\
$0: 473$ \\
$0: 5106$ \\
0.695 \\
0.995 \\
1.239 \\
1.217
\end{tabular} & $\begin{array}{l}n c \\
-00.190 \\
0: 195 \\
0: 195 \\
0: 230 \\
0: 359 \\
0: 374 \\
0.403 \\
0: 400 \\
0: 496 \\
0: 657 \\
1: 025 \\
1: 312 \\
10302\end{array}$ & \begin{tabular}{l} 
De \\
\hdashline 0.190 \\
$0: 195$ \\
0.195 \\
$0: 230$ \\
$0: 359$ \\
$0: 374$ \\
$0: 403$ \\
00460 \\
$0: 496$ \\
$0: 657$ \\
0.973 \\
$1: 191$ \\
$1: 158$
\end{tabular} \\
\hline $\begin{array}{l}\text { YFAR } \\
1970 \\
1970 \\
1971 \\
1972 \\
1973 \\
1974 \\
1975 \\
1977 \\
1978 \\
1979 \\
1980 \\
1981 \\
1982\end{array}$ & \begin{tabular}{l}
$F L$ \\
\hdashline $0.17-$ \\
$0: 2804$ \\
$0: 206$ \\
$0: 205$ \\
$0: 345$ \\
$0: 364$ \\
$0: 398$ \\
$0: 447$ \\
$0: 186$ \\
$0: 633$ \\
$0: 957$ \\
$1: 208$ \\
1.161
\end{tabular} & $\begin{array}{l}C A \\
-3 A \\
0.172 \\
0: 193 \\
0: 195 \\
0: 224 \\
0: 348 \\
0: 354 \\
0: 401 \\
0: 455 \\
0: 148 \\
0.641 \\
0: 957 \\
1: 199 \\
1: 158\end{array}$ & $\begin{array}{l}H 1 \\
-10 \\
00149 \\
0: 102 \\
0: 106 \\
0: 207 \\
0.357 \\
0: 388 \\
0: 431 \\
0: 450 \\
0.432 \\
0.625 \\
0.927 \\
0: 996 \\
1.041\end{array}$ & 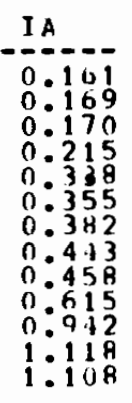 & \begin{tabular}{l}
11 \\
\hdashline 0.184 \\
$0: 196$ \\
$0: 197$ \\
$0: 228$ \\
$0: 363$ \\
$0: 344$ \\
$0: 420$ \\
$0: 449$ \\
$0: 445$ \\
$0: 645$ \\
$0: 4.41$ \\
$1: 130$ \\
1.147
\end{tabular} & \begin{tabular}{l}
$\mathrm{IL}$ \\
\hdashline 0.163 \\
$0: 175$ \\
$0: 177$ \\
$0: 723$ \\
$0: 356$ \\
$0: 356$ \\
$0: 340$ \\
0.444 \\
0.471 \\
0.644 \\
$0: 904$ \\
10189 \\
1.153
\end{tabular} & $\begin{array}{l}\text { IN } \\
0.103 \\
0: 175 \\
0: 177 \\
0: 223 \\
1: 356 \\
0: 356 \\
0: 390 \\
0: 444 \\
0: 471 \\
0: 648 \\
0: 940 \\
1: 179 \\
1: 144\end{array}$ & \begin{tabular}{l}
$k s$ \\
\hdashline 0.137 \\
$0: 171$ \\
$0: 172$ \\
$0: 142$ \\
$0: 337$ \\
$0: 363$ \\
$0: 396$ \\
$0: 446$ \\
$0: 458$ \\
$0: 651$ \\
$0: 951$ \\
$1: 126$ \\
10100
\end{tabular} & $\begin{array}{l}K Y \\
K Y \\
0: 152 \\
0: 170 \\
01772 \\
0: 199 \\
0: 316 \\
0: 344 \\
0: 3444 \\
0: 444 \\
0: 470 \\
0: 642 \\
0: 981 \\
1: 188 \\
1.125\end{array}$ \\
\hline $\begin{array}{l}\text { YFAR } \\
1977 \\
1970 \\
1971 \\
1972 \\
1977 \\
1974 \\
1975 \\
1977 \\
1978 \\
1979 \\
1990 \\
1991 \\
1982\end{array}$ & \begin{tabular}{l}
$1 . A$ \\
\hdashline 0.127 \\
$0: 139$ \\
$0: 140$ \\
$0: 164$ \\
$0: 2991$ \\
$0: 309$ \\
$0: 352$ \\
$0: 382$ \\
$0: 409$ \\
0.636 \\
$0: 945$ \\
1.136 \\
1.050
\end{tabular} & $\begin{array}{l}M A \\
-0.197 \\
0: 107 \\
0: 208 \\
0: 209 \\
0: 245 \\
0.367 \\
0: 395 \\
0.021 \\
0.475 \\
0.504 \\
0.693 \\
0.945 \\
1.234 \\
1.222\end{array}$ & \begin{tabular}{l}
$M 1$ \\
\hdashline 0.190 \\
$0: 195$ \\
$0: 195$ \\
$0: 230$ \\
$0: 359$ \\
$0: 374$ \\
$0: 403$ \\
0.4601 \\
$0: 446$ \\
0.657 \\
0.973 \\
1.211 \\
1.144
\end{tabular} & \begin{tabular}{l}
$M E$ \\
\hdashline $0: 198$ \\
$0: 210$ \\
$0: 211$ \\
$0: 242$ \\
$0: 375$ \\
$0: 398$ \\
$0: 424$ \\
$0: 176$ \\
$0: 512$ \\
$0: 6790$ \\
$0: 976$ \\
$1: 223$ \\
1.185
\end{tabular} & 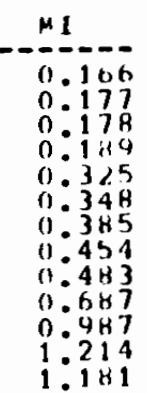 & \begin{tabular}{l}
$N \mathbb{N}$ \\
\hdashline 0.970 \\
$0: 177$ \\
$0: 176$ \\
$0: 2116$ \\
$0: 348$ \\
$0: 353$ \\
$0: 388$ \\
$0: 443$ \\
$0: 477$ \\
$0: 654$ \\
$0: 962$ \\
$1: 133$ \\
1.115
\end{tabular} & 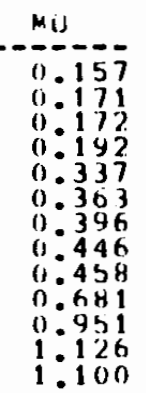 & $\begin{array}{l}M S \\
00.152 \\
0: 170 \\
0: 172 \\
0: 199 \\
0: 316 \\
0: 344 \\
0: 364 \\
0: 443 \\
0: 470 \\
10: 642 \\
0: 981 \\
1: 140 \\
1: 125\end{array}$ & \begin{tabular}{l}
$M 1$ \\
\hdashline 0.171 \\
$0: 1182$ \\
$0: 183$ \\
$0: 215$ \\
$0: 347$ \\
$0: 396$ \\
$0: 408$ \\
$0: 406$ \\
$0: 407$ \\
$0: 665$ \\
$0: 958$ \\
$1: 147$ \\
0.963
\end{tabular} \\
\hline
\end{tabular}


TABLE 2.14 (cont). Distillate Fuel Prices: Residential Sector (\$/gallon)

\begin{tabular}{|c|c|c|c|c|c|c|c|c|c|}
\hline $\begin{array}{l}\text { YFAR } \\
1970 \\
1971 \\
1971 \\
1973 \\
1974 \\
1975 \\
1976 \\
1977 \\
1978 \\
1979 \\
1980 \\
1981 \\
1982\end{array}$ & 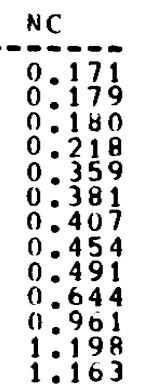 & 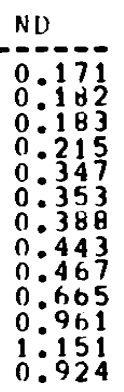 & \begin{tabular}{l}
$N E$ \\
\hdashline 0.157 \\
$0: 1171$ \\
$0: 172$ \\
$0: 192$ \\
$0: 337$ \\
$0: 363$ \\
$0: 396$ \\
$0: 4446$ \\
$0: 458$ \\
$0: 481$ \\
$0: 951$ \\
$1: 126$ \\
1.100
\end{tabular} & $\begin{array}{l}\mathrm{NH} \\
-0.158 \\
0: 2190 \\
0: 210 \\
0: 242 \\
0: 375 \\
0: 398 \\
0: 424 \\
0: 476 \\
0: 512 \\
0: 679 \\
0: 946 \\
1: 214 \\
1: 177\end{array}$ & $\begin{array}{l}\text { NJ } \\
0.192 \\
0: 203 \\
0: 204 \\
0: 222 \\
0: 447 \\
0: 387 \\
0: 410 \\
0: 464 \\
0: 500 \\
0: 674 \\
0: 982 \\
1: 2117 \\
1: 186\end{array}$ & $\begin{array}{l}N M \\
-0.150 \\
0: 1127 \\
0: 130 \\
0: 170 \\
0: 335 \\
0: 394 \\
0: 420 \\
0: 446 \\
0: 4110 \\
0: 428 \\
0: 937 \\
1: 1113 \\
1: 070\end{array}$ & $\begin{array}{l}N V \\
-19 \\
0: 149 \\
0: 162 \\
0: 166 \\
0: 207 \\
0: 357 \\
0: 394 \\
0: 420 \\
0: 432 \\
0: 432 \\
0: 625 \\
0: 927 \\
0: 996 \\
1: 041\end{array}$ & $\begin{array}{l}N Y \\
-10.18 B \\
0: 200 \\
0: 201 \\
0: 244 \\
0: 327 \\
0: 391 \\
0: 412 \\
0: 468 \\
0: 506 \\
0.676 \\
0.965 \\
1: 251 \\
1: 237\end{array}$ & 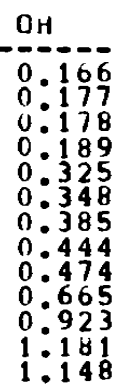 \\
\hline $\begin{array}{l}\text { YEAR } \\
1970 \\
1971 \\
1972 \\
1973 \\
1974 \\
1975 \\
1976 \\
1977 \\
1997 \\
1990 \\
1981 \\
1982\end{array}$ & \begin{tabular}{l}
$n k$ \\
\hdashline 0.117 \\
$0: 119$ \\
$0: 119$ \\
$0: 149$ \\
$0: 300$ \\
$0: 330$ \\
$0: 358$ \\
$0: 399$ \\
$0: 420$ \\
$0: 650$ \\
$0: 910$ \\
$1: 103$ \\
1.040
\end{tabular} & 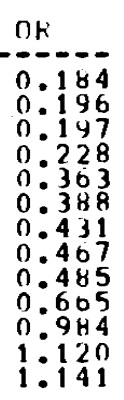 & $\begin{array}{l}P A \\
00181 \\
0: 194 \\
0: 145 \\
0: 228 \\
0: 365 \\
0: 390 \\
0: 407 \\
0: 461 \\
0: 495 \\
0: 666 \\
0: 961 \\
1: 184 \\
1: 145\end{array}$ & 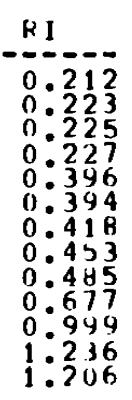 & $\begin{array}{l}S C \\
00: 172 \\
0: 187 \\
0: 188 \\
0: 213 \\
0: 336 \\
0: 3800 \\
0: 407 \\
0: 456 \\
0: 441 \\
0: 616 \\
0: 950 \\
1: 111 \\
1: 160\end{array}$ & \begin{tabular}{l}
50 \\
\hdashline 0.171 \\
$0: 1182$ \\
$0: 183$ \\
$0: 2115$ \\
$0: 347$ \\
$0: 353$ \\
$0: 344$ \\
$0: 4443$ \\
$0: 467$ \\
$0: 665$ \\
$0: 961$ \\
$1: 151$ \\
0.924
\end{tabular} & 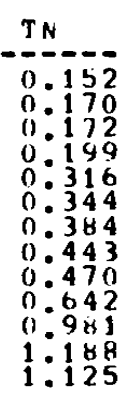 & \begin{tabular}{l}
$T x$ \\
\hdashline 0.127 \\
$0: 139$ \\
$0: 140$ \\
$0: 164$ \\
$0: 291$ \\
$0: 309$ \\
$0: 352$ \\
$0: 342$ \\
$0: 409$ \\
$0: 636$ \\
0.898 \\
$1: 108$ \\
$1: 038$
\end{tabular} & $\begin{array}{l}11 \\
-17 \\
0: 152 \\
0: 167 \\
0: 172 \\
0: 204 \\
0: 353 \\
0: 346 \\
0: 408 \\
0: 448 \\
0: 457 \\
0: 621 \\
0: 953 \\
1: 1940 \\
0.943\end{array}$ \\
\hline $\begin{array}{l}Y E A R \\
1970 \\
1971 \\
1972 \\
1973 \\
1974 \\
1975 \\
1976 \\
1977 \\
1978 \\
1979 \\
1980 \\
1981 \\
1982\end{array}$ & \begin{tabular}{l}
$V A$ \\
$V A$ \\
\hdashline 0.183 \\
$0: 196$ \\
$0: 197$ \\
0.222 \\
$0: 353$ \\
$0: 371$ \\
$0: 403$ \\
10.459 \\
$0: 493$ \\
0.667 \\
$0: 993$ \\
$1: 233$ \\
1.199
\end{tabular} & $\begin{array}{l}V 1 \\
-1.198 \\
0: 290 \\
0: 211 \\
0: 242 \\
0: 375 \\
0: 348 \\
0: 424 \\
0: 474 \\
0: 512 \\
0: 679 \\
1: 024 \\
1: 260 \\
1: 237\end{array}$ & \begin{tabular}{l}
$W A$ \\
\hdashline 0.184 \\
$0: 196$ \\
$0: 197$ \\
$0: 228$ \\
$0: 363$ \\
$0: 3884$ \\
$0: 431$ \\
$0: 467$ \\
$0: 485$ \\
$0: 655$ \\
$1: 001$ \\
101848 \\
$1: 222$
\end{tabular} & 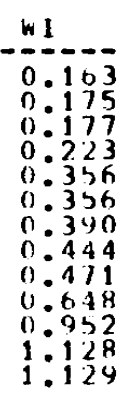 & 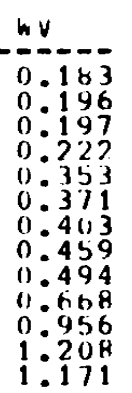 & $\begin{array}{l}W Y \\
-10 \\
0: 171 \\
0: 182 \\
0: 183 \\
0: 2115 \\
0: 347 \\
0: 396 \\
0: 408 \\
0: 448 \\
0: 452 \\
0: 639 \\
0: 960 \\
1: 1445 \\
0.931\end{array}$ & \begin{tabular}{l}
115 \\
\hdashline 0.185 \\
$0: 196$ \\
$0: 197$ \\
$0: 228$ \\
$0: 360$ \\
$0: 377$ \\
0.406 \\
$0: 460$ \\
0.494 \\
0.656 \\
0.979 \\
$1: 205$ \\
1.185
\end{tabular} & & \\
\hline
\end{tabular}

Source: Pacific Northwest Laboratory 
TABLE 2.15. Distillate Fuel Prices: Commercial Sector ( $\$ / g a l 1$ on)

\begin{tabular}{|c|c|c|c|c|c|c|c|c|c|}
\hline $\begin{array}{l}\text { YFAR } \\
19710 \\
1971 \\
19712 \\
1973 \\
19794 \\
1975 \\
1976 \\
19777 \\
19798 \\
1990 \\
1981 \\
1982\end{array}$ & 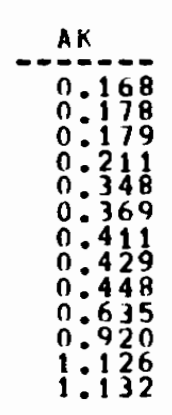 & 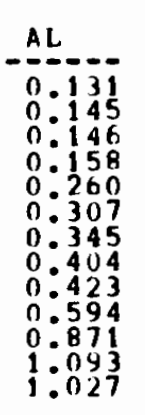 & 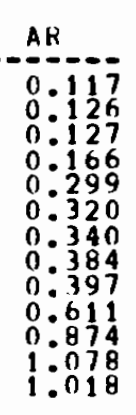 & 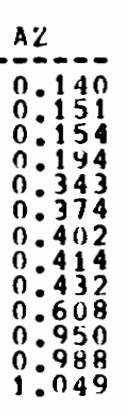 & 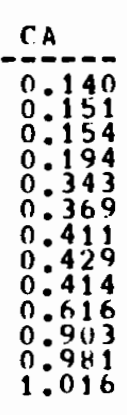 & 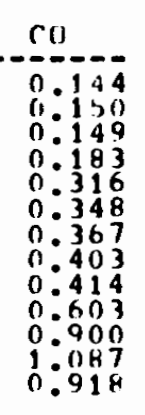 & 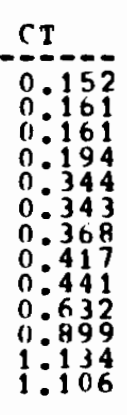 & 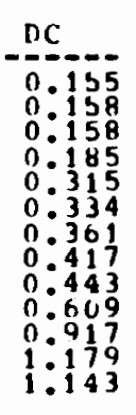 & \begin{tabular}{l} 
DE \\
\hdashline $0: 155$ \\
$0: 158$ \\
$0: 158$ \\
$0: 158$ \\
$0: 145$ \\
$0: 315$ \\
$0: 334$ \\
$0: 361$ \\
$0: 417$ \\
$0: 443$ \\
$0: 609$ \\
$0: 890$ \\
$1: 116$ \\
$1: 068$
\end{tabular} \\
\hline $\begin{array}{l}\text { YEAR } \\
1977 \\
1971 \\
19727 \\
1973 \\
1977 \\
1975 \\
1976 \\
19777 \\
1979 \\
9980 \\
9981 \\
1982\end{array}$ & 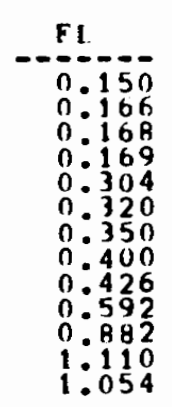 & 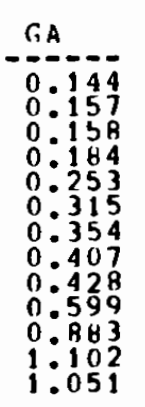 & 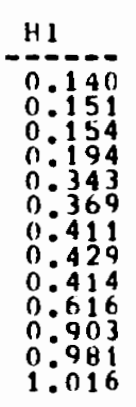 & 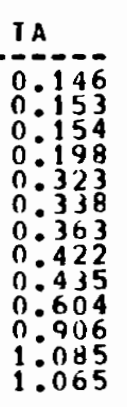 & 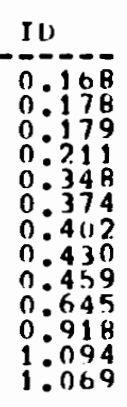 & 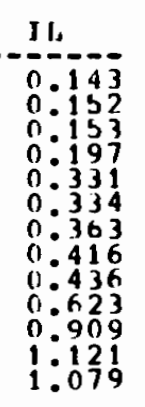 & 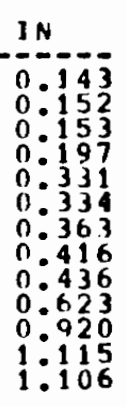 & 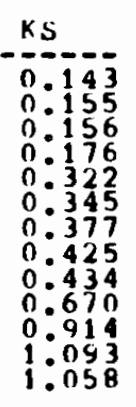 & \begin{tabular}{l}
$\mathrm{kY}$ \\
\hdashline $0: 135$ \\
$0: 149$ \\
$0: 151$ \\
$0: 171$ \\
$0: 179$ \\
$0: 319$ \\
$0: 319$ \\
$0: 357$ \\
$0: 410$ \\
$0: 432$ \\
$0: 615$ \\
$0: 931$ \\
$1: 125$ \\
$1: 057$
\end{tabular} \\
\hline $\begin{array}{l}\text { YEAR } \\
19 A 70 \\
1971 \\
1971 \\
1973 \\
1974 \\
1975 \\
19767 \\
1978 \\
1979 \\
1990 \\
1981 \\
1982\end{array}$ & 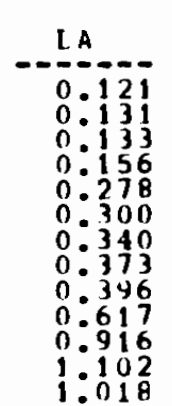 & \begin{tabular}{l}
$M A$ \\
$M A$ \\
\hdashline $0: 153$ \\
$0: 162$ \\
00162 \\
$0: 198$ \\
$0: 318$ \\
$0: 344$ \\
$0: 369$ \\
$0: 419$ \\
$0: 442$ \\
$0: 641$ \\
$0: 891$ \\
$1: 134$ \\
$1: 108$
\end{tabular} & 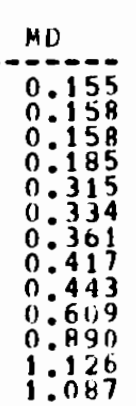 & 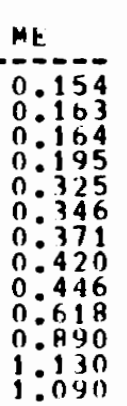 & 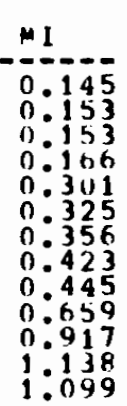 & 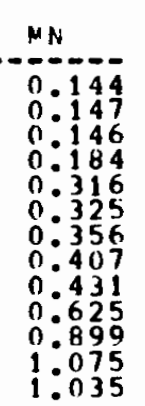 & 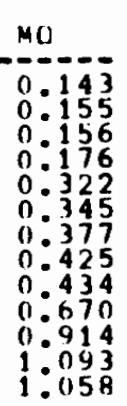 & 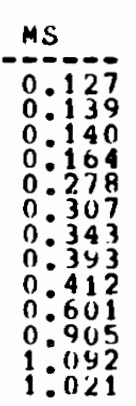 & \begin{tabular}{l}
$\mu \mathrm{T}$ \\
\hdashline $0: 144$ \\
$0: 150$ \\
$0: 149$ \\
$0: 183$ \\
$0: 316$ \\
$0: 348$ \\
$0: 367$ \\
$0: 420$ \\
$0: 426$ \\
$0: 628$ \\
$0: 828$ \\
$0: 095$ \\
0.981 \\
0.972
\end{tabular} \\
\hline
\end{tabular}


TABLE 2.15 (cont). Distillate Fuel Prices: Commercial Sector ( $\$ /$ gallon)

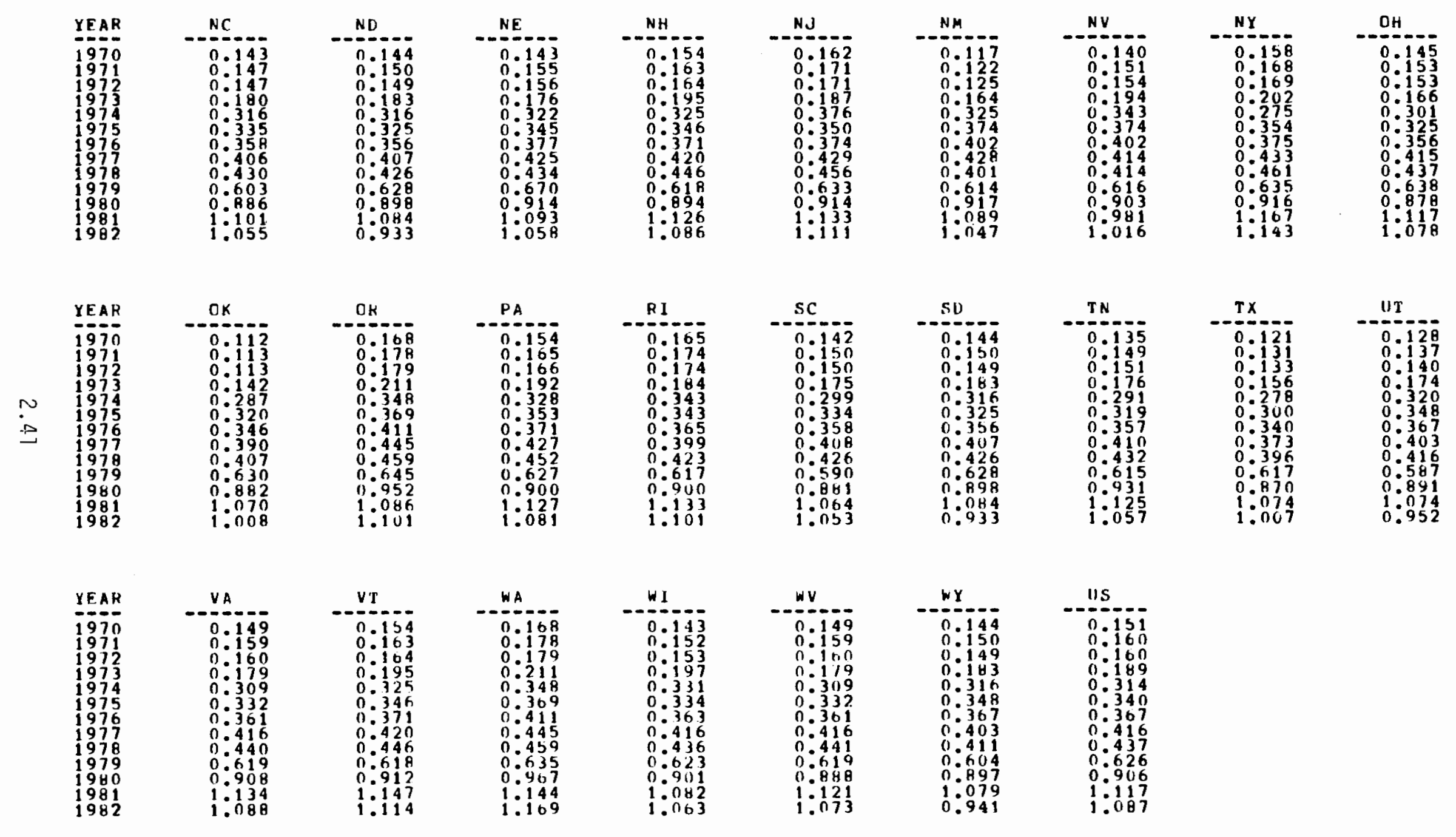


TABLE 2.16. Distillate Fuel Prices: Industrial Sector $(\$ / \text { gallon })^{(a)}$
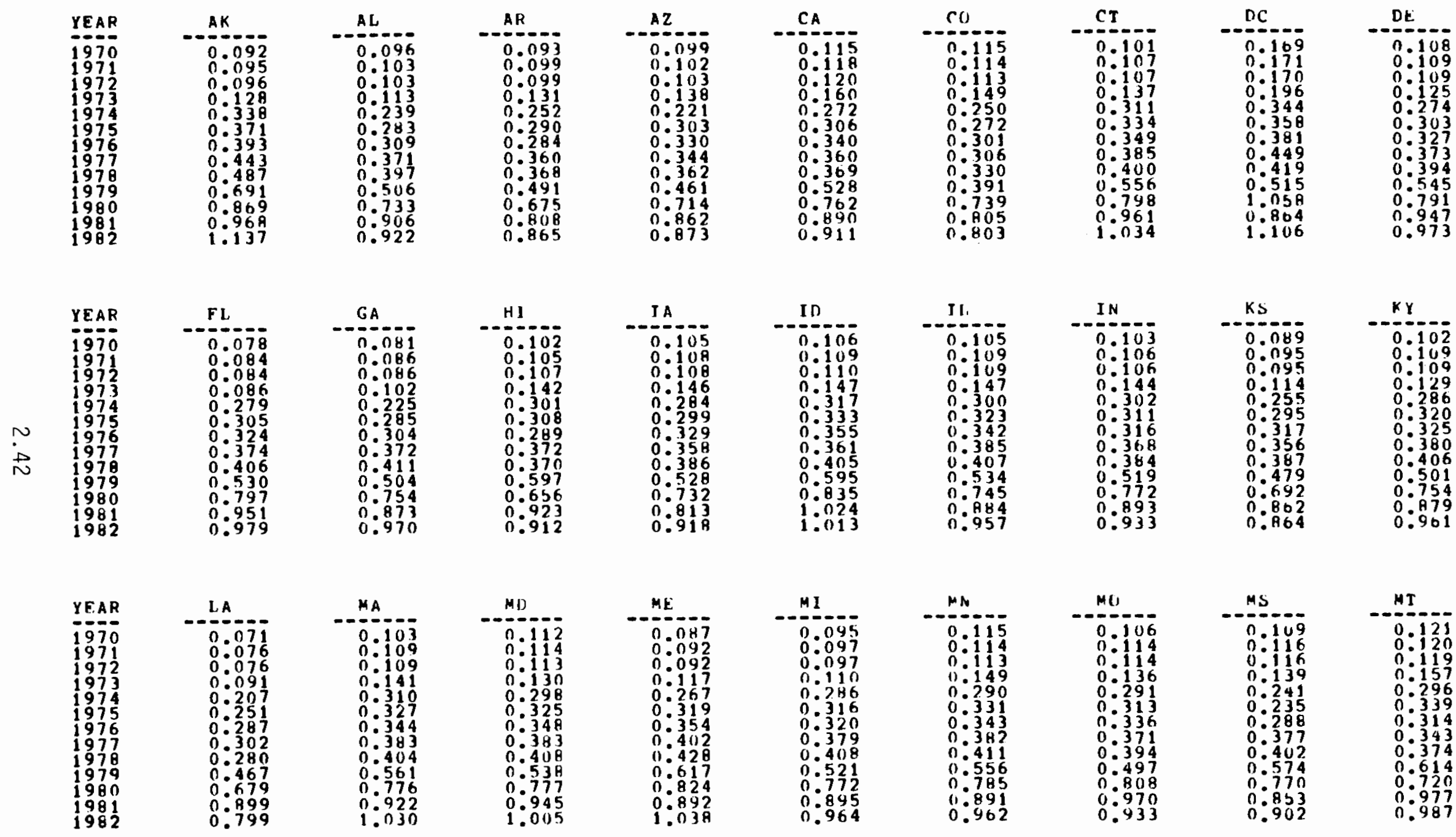

(a) Data for 1982 are provisional estimates, and will be updated when data from Annual Survey of Manufactures become available. 
TABLE 2.16 (cont). Distillate Fuel Prices: Industrial Sector (\$/gallon)(a)

\begin{tabular}{|c|c|c|c|c|c|c|c|c|c|}
\hline $\begin{array}{l}\text { YEAR } \\
\text { Y. } \\
1970 \\
1971 \\
1972 \\
1973 \\
1974 \\
1975 \\
1976 \\
19777 \\
1979 \\
1980 \\
1981 \\
1982\end{array}$ & 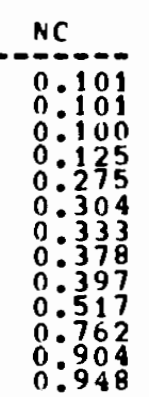 & 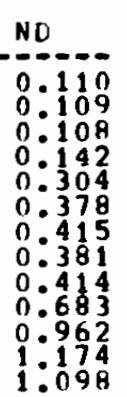 & $\begin{array}{l}\text { Nk } \\
-00 \\
0: 102 \\
0: 109 \\
0: 109 \\
0: 130 \\
0: 257 \\
0: 311 \\
0: 332 \\
0: 351 \\
0: 382 \\
0: 464 \\
0: 685 \\
0: 888 \\
0: 873\end{array}$ & \begin{tabular}{l} 
NH \\
\hdashline $0: 095$ \\
$0: 09101$ \\
$0: 101$ \\
$0: 128$ \\
$0: 304$ \\
$0: 318$ \\
$0: 325$ \\
$0: 376$ \\
$0: 301$ \\
$0: 401$ \\
$0: 791$ \\
$0: 795$ \\
$0: 878$ \\
0.971
\end{tabular} & \begin{tabular}{l} 
NJ \\
\hdashline $0:-105$ \\
$0: 112$ \\
$0: 112$ \\
$0: 123$ \\
$0: 301$ \\
$0: 327$ \\
$0: 333$ \\
$0: 382$ \\
$0: 397$ \\
$0: 544$ \\
$0: 759$ \\
$0: 915$ \\
$1: 015$
\end{tabular} & 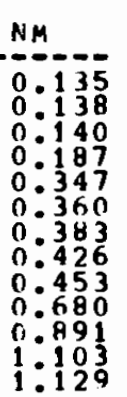 & $\begin{array}{l}N V \\
-0.130 \\
0: 137 \\
0: 139 \\
0: 185 \\
0: 311 \\
0: 324 \\
0: 347 \\
0: 390 \\
0: 417 \\
0: 650 \\
0: 906 \\
1: 036 \\
1: 024\end{array}$ & 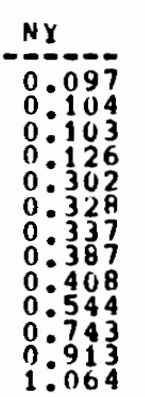 & 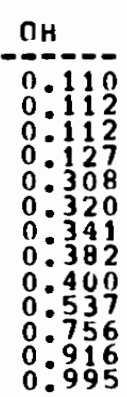 \\
\hline $\begin{array}{l}\text { YEAR } \\
19770 \\
1971 \\
1972 \\
1973 \\
19774 \\
1975 \\
1976 \\
1977 \\
1978 \\
19790 \\
1981 \\
1982\end{array}$ & 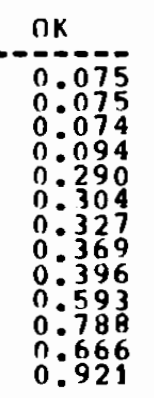 & 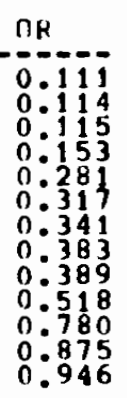 & $\begin{array}{l}\text { PA } \\
-00 \\
0: 097 \\
0: 104 \\
0: 103 \\
0: 118 \\
0: 301 \\
0: 330 \\
0: 335 \\
0: 387 \\
0: 396 \\
0: 530 \\
0: 746 \\
0: 917 \\
1: 003\end{array}$ & \begin{tabular}{l} 
RI \\
\hdashline $0: 099$ \\
$0: 105$ \\
$0: 105$ \\
$0: 117$ \\
$0: 298$ \\
$0: 324$ \\
$0: 309$ \\
$0: 391$ \\
$0: 415$ \\
$0: 576$ \\
$0: 786$ \\
$1: 006$ \\
$1: 040$
\end{tabular} & 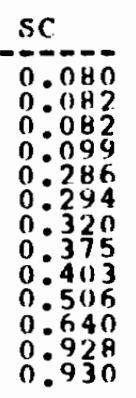 & 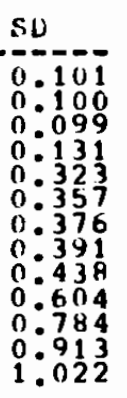 & $\begin{array}{l}\text { TN } \\
-0: 101 \\
0: 108 \\
0: 108 \\
0: 108 \\
0: 277 \\
0: 293 \\
0: 313 \\
0: 313 \\
0: 401 \\
0: 500 \\
0: 760 \\
0: 964 \\
0: 946\end{array}$ & 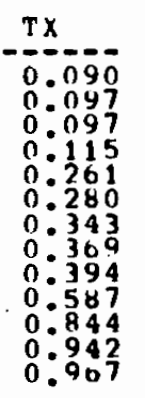 & 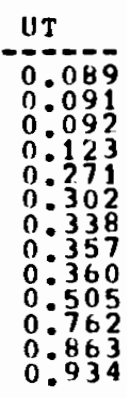 \\
\hline $\begin{array}{l}\text { YEAR } \\
19-770 \\
1971 \\
1972 \\
1973 \\
1974 \\
1975 \\
1976 \\
1977 \\
1979 \\
1980 \\
1981 \\
1982\end{array}$ & 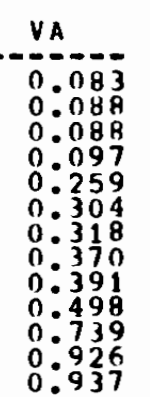 & 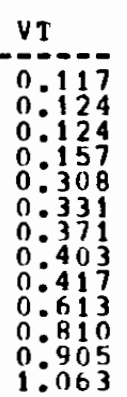 & 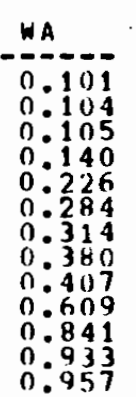 & $\begin{array}{l}\text { W1 } \\
-10-106 \\
0: 109 \\
0: 109 \\
0: 148 \\
0: 1995 \\
0: 310 \\
0: 337 \\
0: 381 \\
0: 399 \\
0: 590 \\
0: 718 \\
0: 956 \\
0: 960\end{array}$ & 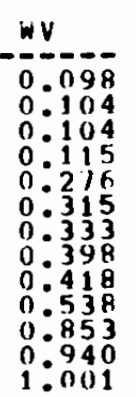 & 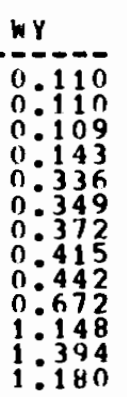 & \begin{tabular}{l} 
us \\
\hdashline $0: 100$ \\
$0: 100$ \\
$0: 104$ \\
$0: 128$ \\
$0: 277$ \\
$0: 306$ \\
$0: 306$ \\
$0: 371$ \\
$0: 371$ \\
$0: 349$ \\
$0: 542$ \\
$0: 981$ \\
$0: 921$ \\
0.954
\end{tabular} & & \\
\hline
\end{tabular}

Source: Pacific Northwest Laboratory 
TABLE 2.17. Distillate Fuel Prices: Electric Utility Sector (\$/gallon)
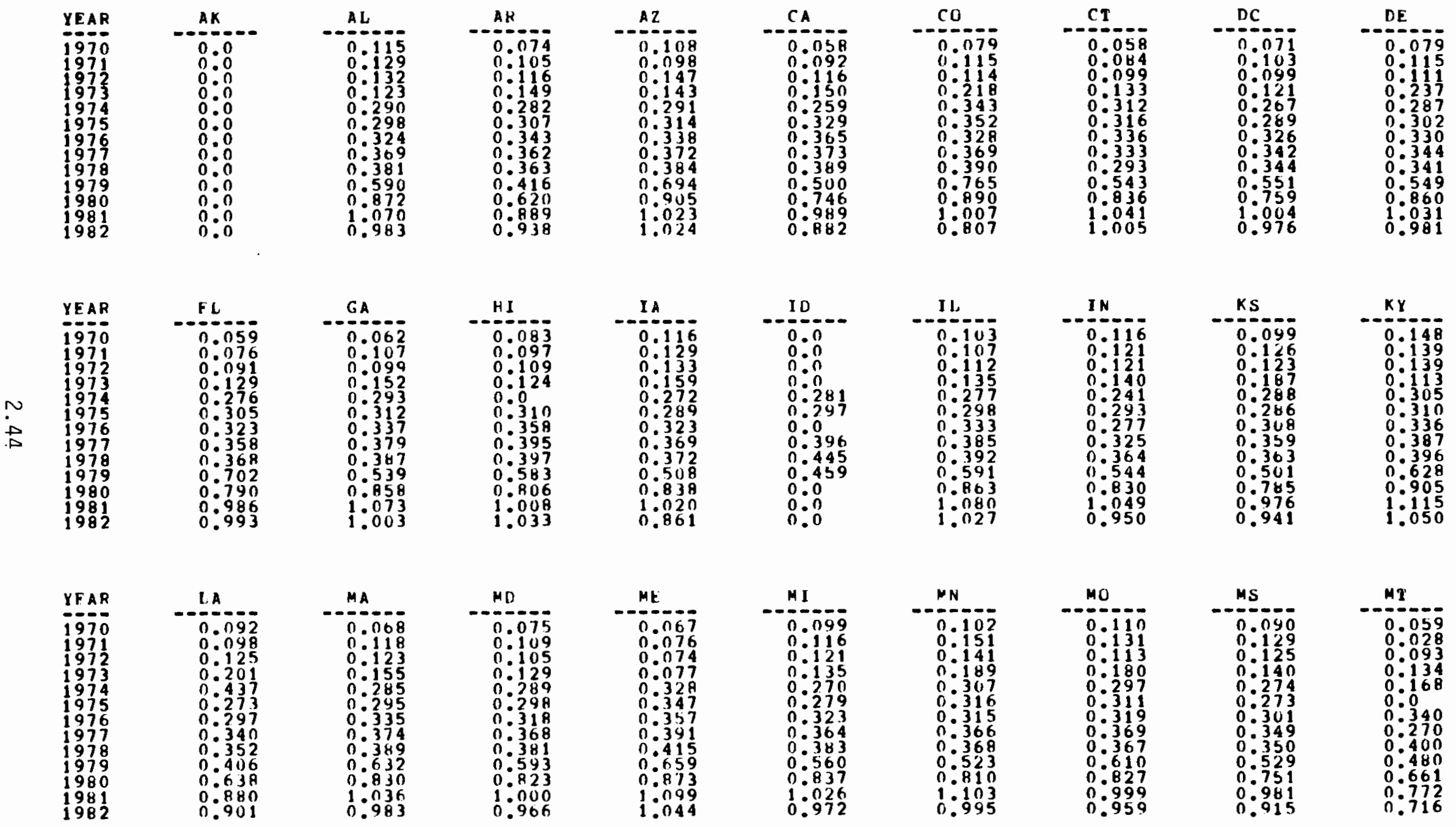

$M 0$
-0.075
0.109
0.105
0.129
0.789
0.298
0.318
0.368
0.381
0.593
0.823
1.000
0.966
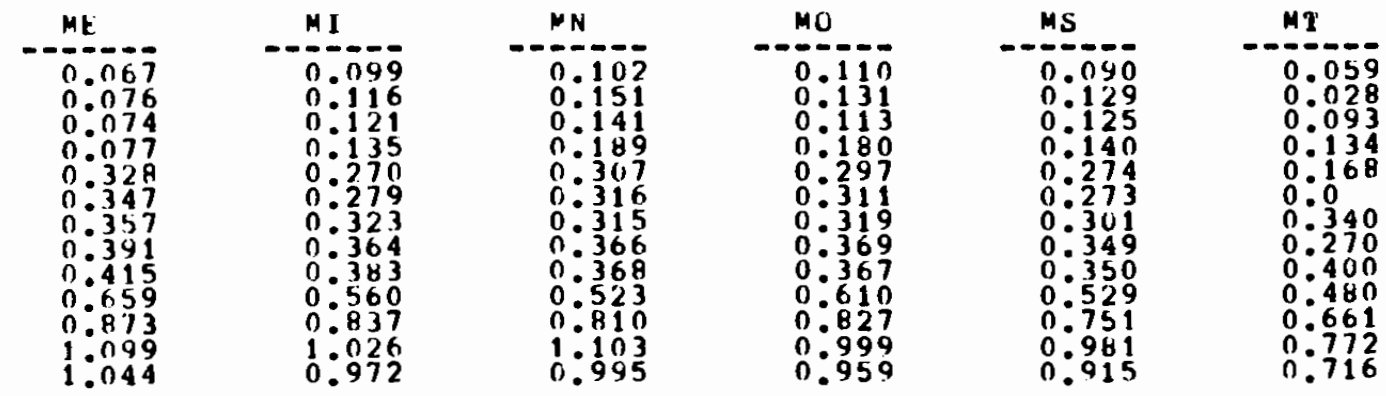
TABLE 2.17 (cont). Distillate Fuel Prices: Electric Utility Sector (\$/gallon)
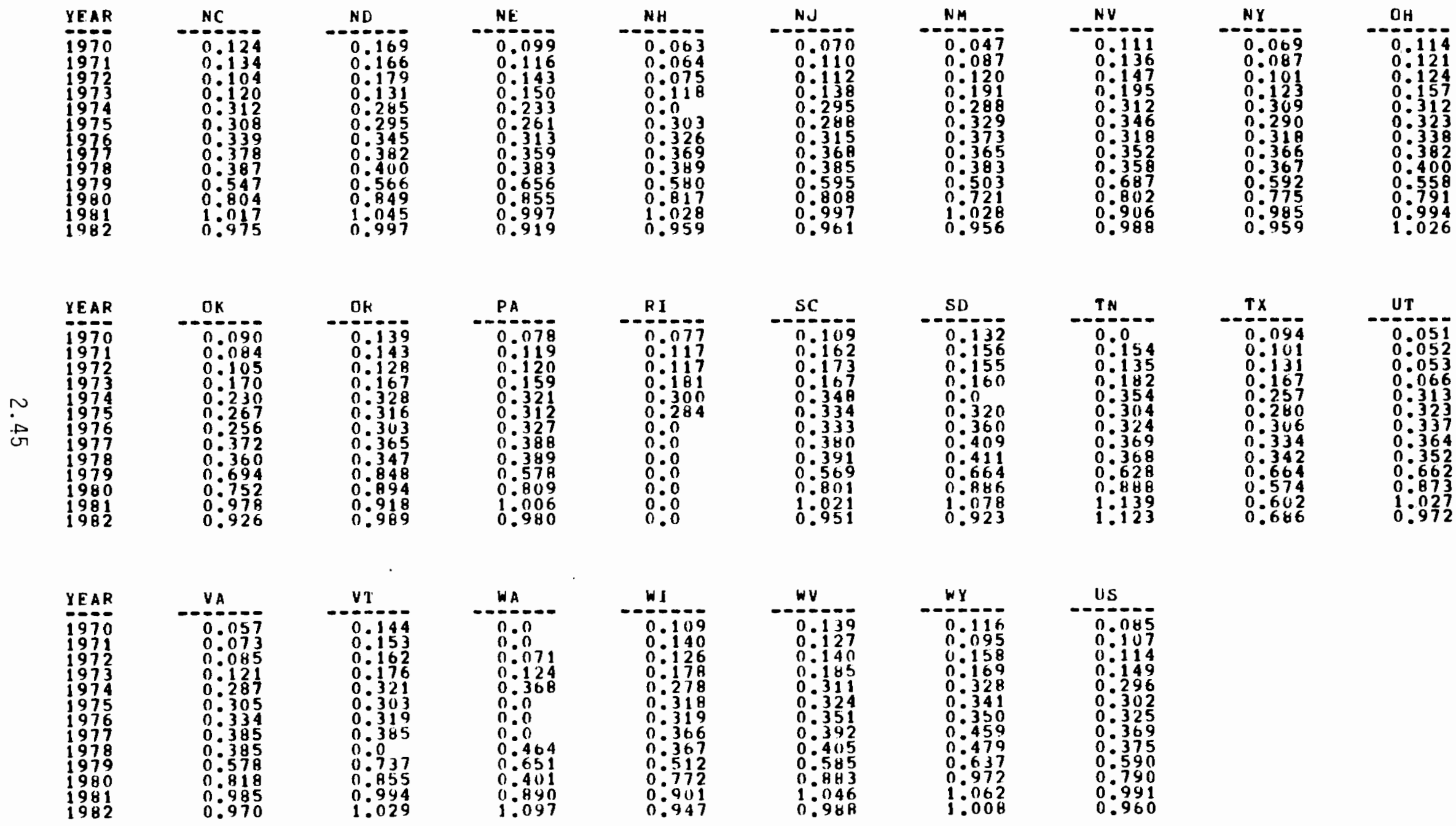

Source: Pacific Northwest Laboratory 
TABLE 2.18. Motor Gasoline Prices: Transportation Sector (\$/gallon)
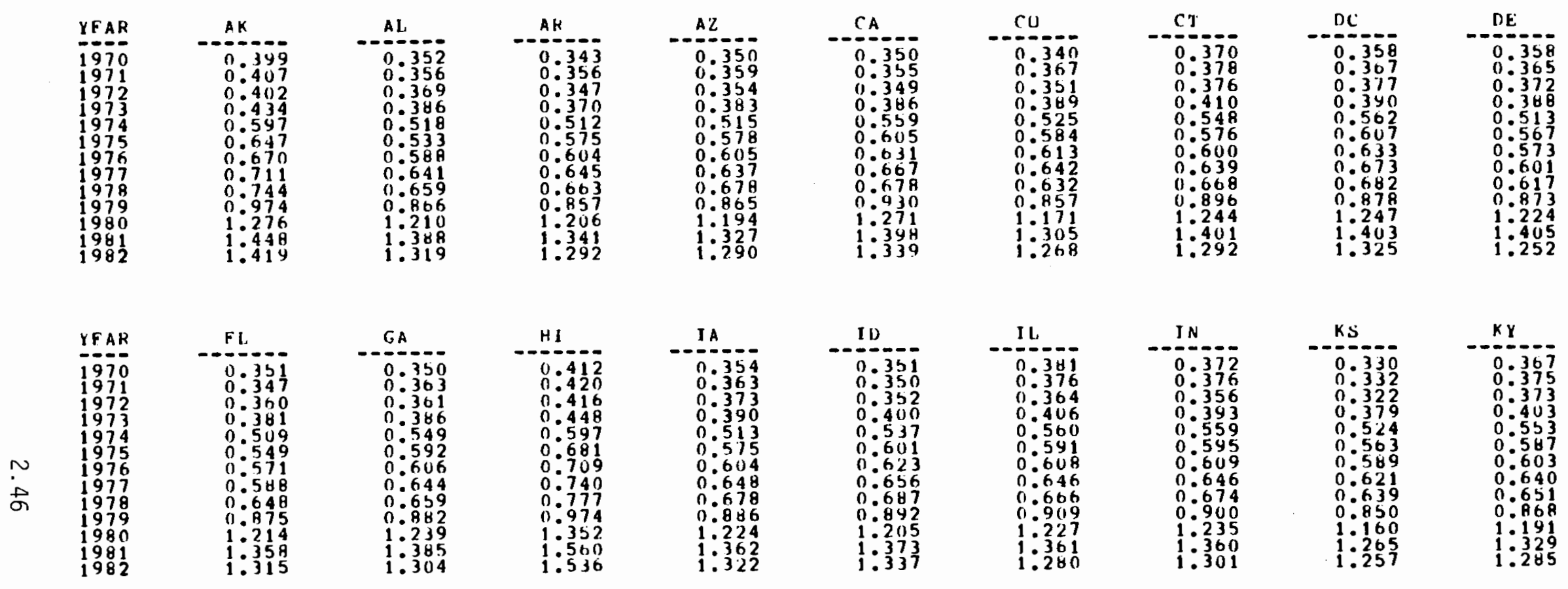

\begin{tabular}{ll} 
YEAR & $1 . A$ \\
\hline 1970 & 0.358 \\
1971 & 0.356 \\
1972 & 0.341 \\
1973 & 0.365 \\
1974 & 0.500 \\
1975 & 0.562 \\
1976 & 0.604 \\
1977 & 0.630 \\
1978 & 0.665 \\
1979 & 0.875 \\
1980 & 1.219 \\
1981 & 1.364 \\
1982 & 1.282
\end{tabular}

$M A$
0.358
0.366
0.359
0.391
0.548
0.592
0.602
0.645
0.644
0.674
1.212
1.354
1.261

$M 1$
0.357
0.361
0.377
0.392
0.565
0.604
0.620
0.654
0.666
0.878
1.242
1.376
1.310

$M E$
-0.378
0.391
0.341
0.402
0.544
0.571
0.548
0.629
0.652
0.982
1.222
1.356
1.259
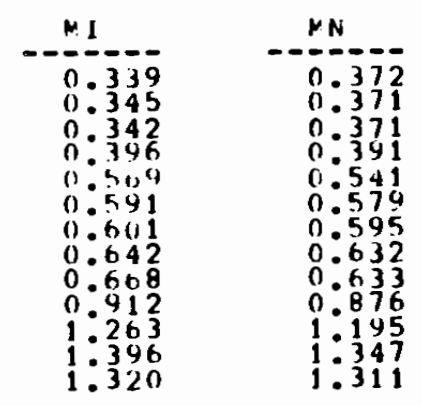

$m 0$
-0.54
0.341
0.362
0.358
0.392
0.541
0.569
0.582
0.618
0.626
0.866
1.166
1.281
1.227
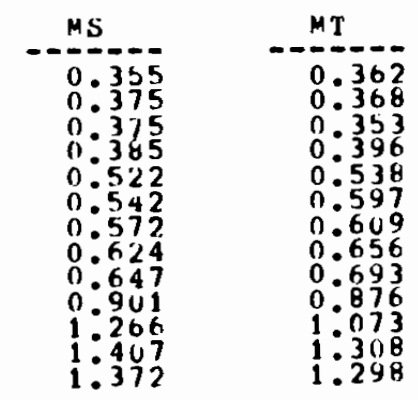
TABLE 2.18 (cont). Motor Gasoline Prices: Transportation Sector (\$/gallon)

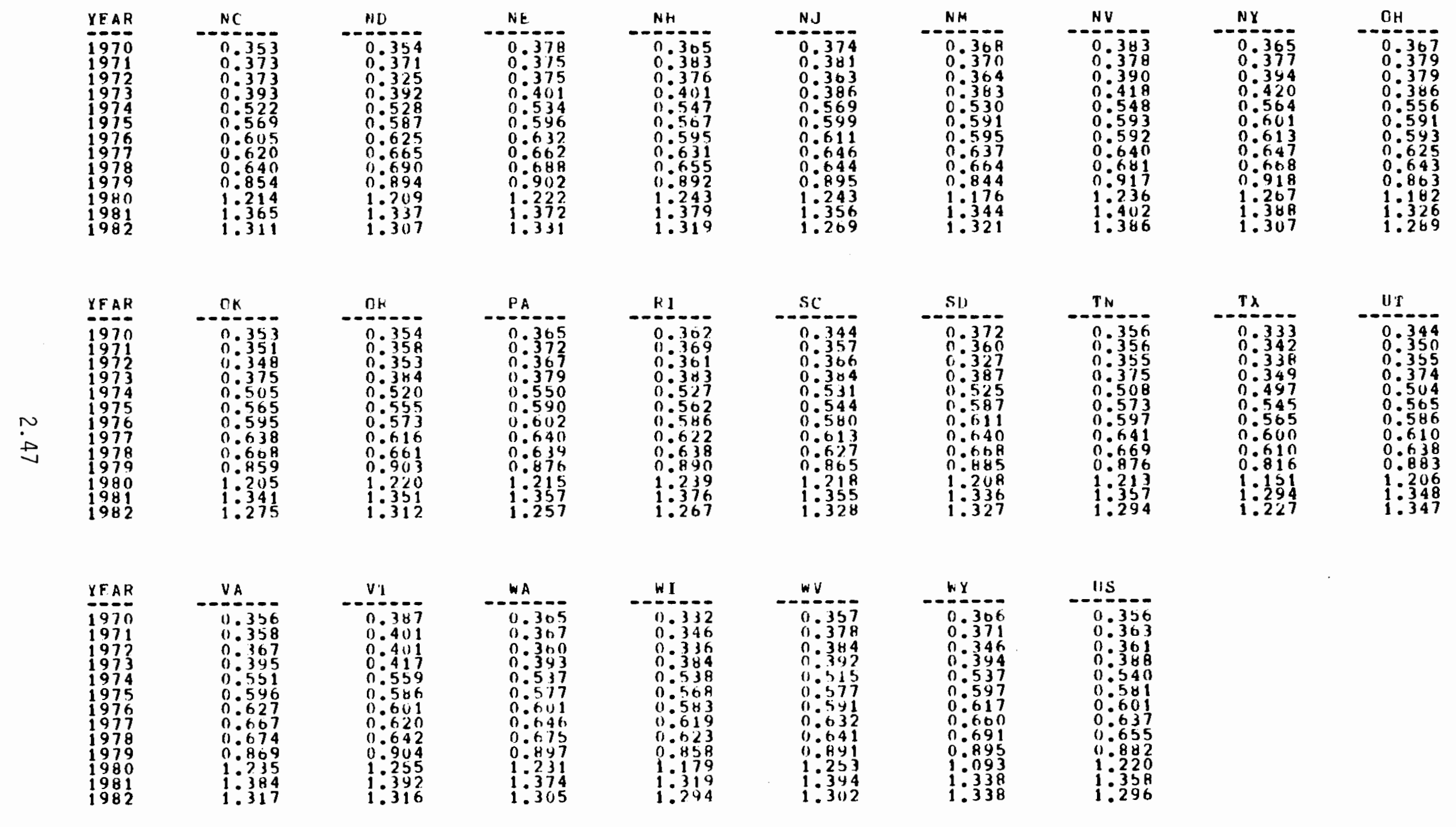

Source: Pacific Northwest Laboratory 
TABLE 2.19. Diesel Fuel Prices: Transportation Sector (\$/gallon)

\begin{tabular}{|c|c|c|c|c|c|c|c|c|c|}
\hline $\begin{array}{l}\text { YEAR } \\
19770 \\
19711 \\
1972 \\
1973 \\
1974 \\
19756 \\
1977 \\
1978 \\
1979 \\
1980 \\
1981 \\
1982\end{array}$ & \begin{tabular}{l}
$A K$ \\
\hdashline $0:-.$. \\
$0: 0$ \\
$0: 0$ \\
$0: 0$ \\
$0: 0$ \\
$0: 0$ \\
$0: 0$ \\
$0: 0$ \\
$0: 0$ \\
$0: 0$ \\
$0: 0$ \\
$0: 0$ \\
$0: 0$ \\
$0: 0$
\end{tabular} & \begin{tabular}{c} 
AL \\
\hdashline 0.184 \\
$0: 187$ \\
$0: 197$ \\
$0: 197$ \\
$0: 373$ \\
$0: 405$ \\
$0: 428$ \\
$0: 463$ \\
$0: 457$ \\
$0: 660$ \\
$0: 969$ \\
$1: 187$ \\
$1: 124$
\end{tabular} & 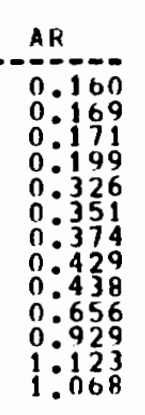 & 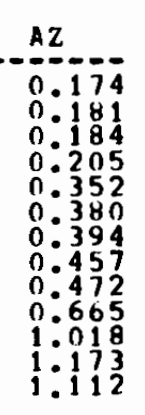 & 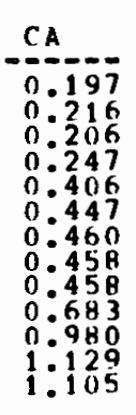 & 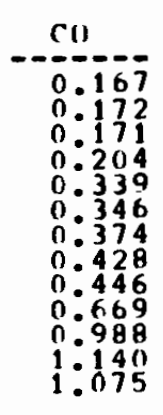 & \begin{tabular}{l}
$C T$ \\
\hdashline 0.193 \\
$0: 0207$ \\
$0: 208$ \\
$0: 250$ \\
$0: 377$ \\
$0: 403$ \\
$0: 430$ \\
$0: 501$ \\
$0: 526$ \\
$0: 738$ \\
$1: 027$ \\
10250 \\
1.208
\end{tabular} & 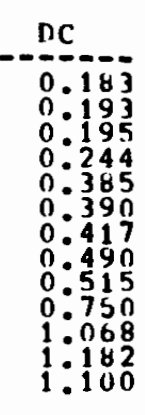 & 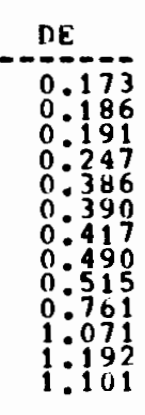 \\
\hline $\begin{array}{l}\text { YEAR } \\
19970 \\
1971 \\
1972 \\
1973 \\
1974 \\
1975 \\
1976 \\
19777 \\
1979 \\
1990 \\
1991 \\
1982\end{array}$ & \begin{tabular}{c}
$\mathrm{FL}$ \\
\hdashline 0.200 \\
$0: 200$ \\
$0: 216$ \\
$0: 256$ \\
$0: 359$ \\
$0: 401$ \\
$0: 440$ \\
$0: 491$ \\
$0: 522$ \\
$0: 769$ \\
$0: 070$ \\
$1: 271$ \\
$1: 139$
\end{tabular} & \begin{tabular}{l}
$G A$ \\
\hdashline $0: 159$ \\
$0: 208$ \\
$0: 209$ \\
$0: 243$ \\
$0: 371$ \\
$0: 419$ \\
$0: 434$ \\
$0: 488$ \\
$0: 500$ \\
$0: 722$ \\
$1: 038$ \\
$1: 213$ \\
$1: 098$
\end{tabular} & 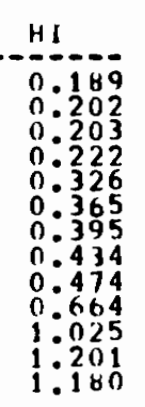 & 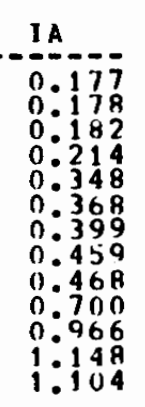 & 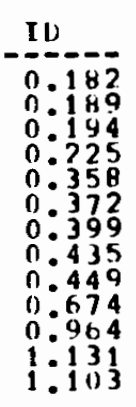 & 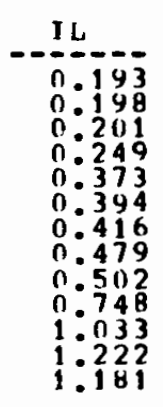 & 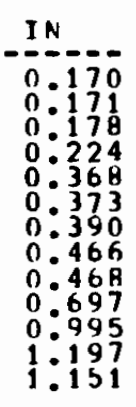 & 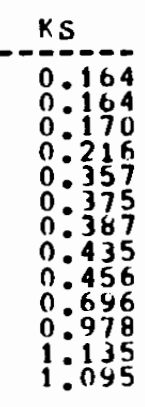 & 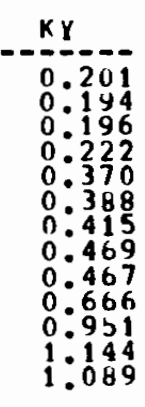 \\
\hline 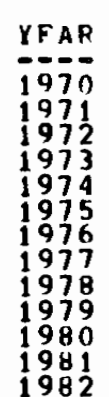 & 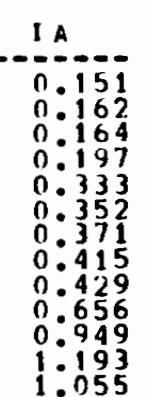 & \begin{tabular}{c}
$M A$ \\
\hdashline $0: 189$ \\
$0: 206$ \\
$0: 209$ \\
$0: 250$ \\
$0: 379$ \\
$0: 403$ \\
$0: 430$ \\
$0: 501$ \\
$0: 526$ \\
$0: 741$ \\
$1: 026$ \\
$1: 256$ \\
$1: 213$
\end{tabular} & 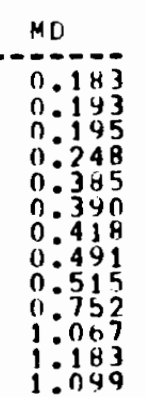 & 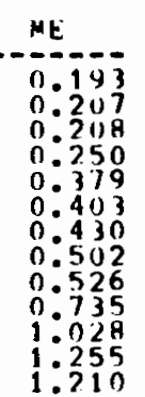 & 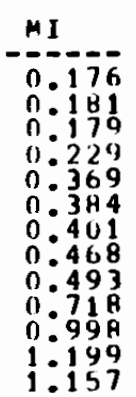 & 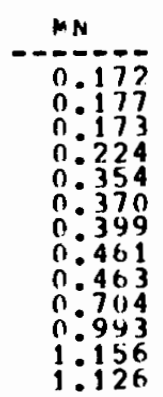 & 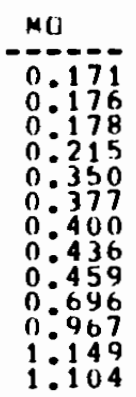 & 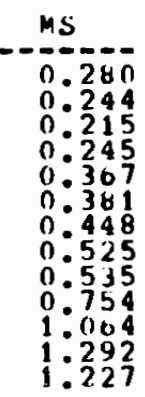 & 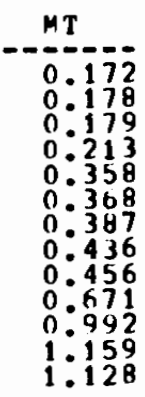 \\
\hline
\end{tabular}


IABLE 2.19 (cont). Diesel Fuel Prices: Transportation Sector (\$/gallon)

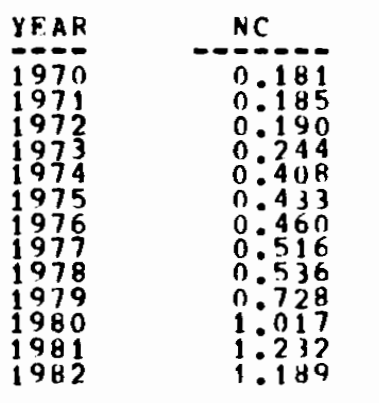

$N D$
00.184
0.183
0.185
0.222
$0: 354$
0.310
0.405
0.453
0.471
0.766
1.003
1.189
1.143
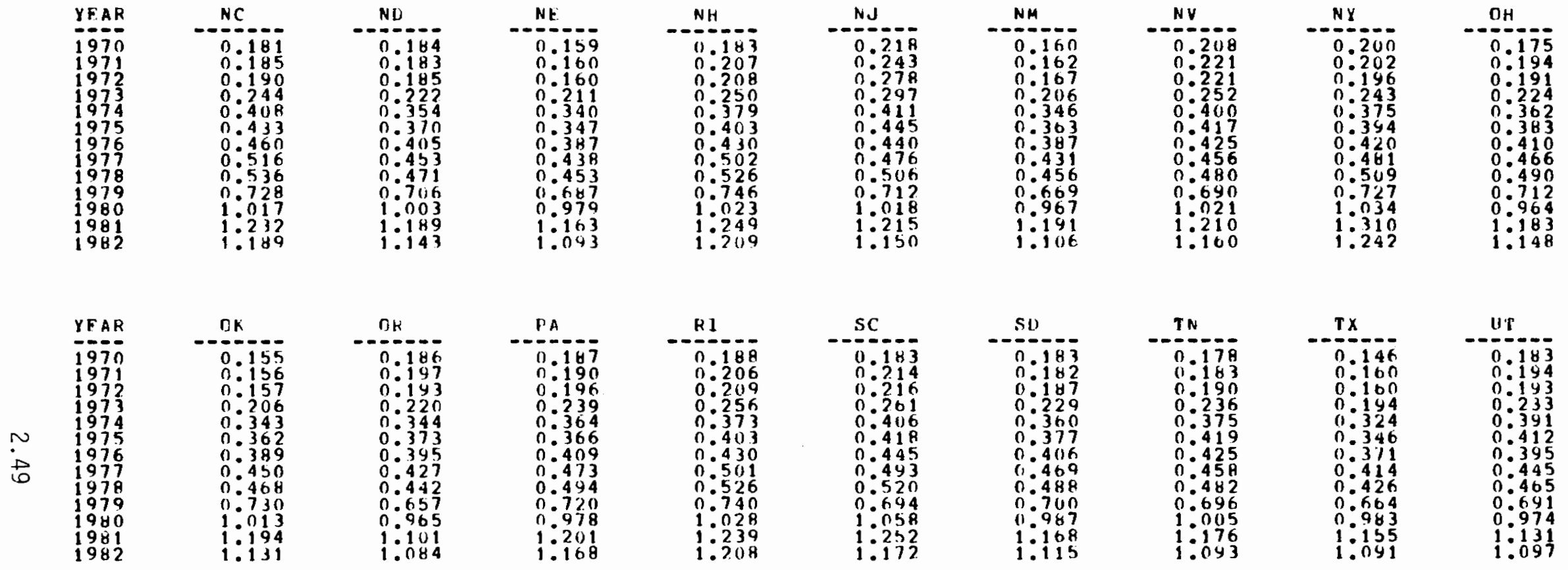

\begin{tabular}{ll} 
YFAR & VA \\
\hline 1970 & 0.173 \\
1971 & 0.183 \\
1972 & 0.184 \\
1973 & 0.220 \\
1974 & 0.358 \\
1975 & 0.378 \\
1976 & 0.403 \\
1977 & 0.462 \\
1978 & 0.487 \\
1979 & 0.690 \\
1980 & 1.009 \\
1981 & 1.225 \\
1982 & 1.176
\end{tabular}

$v 1$
-0.798
0.1907
0.207
0.208
0.251
0.378
0.403
0.430
0.502
0.526
0.745
0.945
1.251
1.210

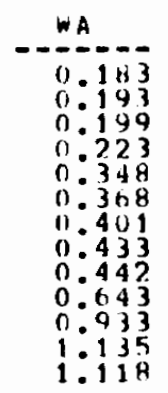

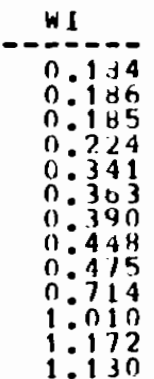

$W V$
0.238
0.265
0.269
0.346
0.5116
0.551
0.507
0.560
0.600
0.826
1.160
1.412
1.365

$W Y$
-0.181
0.1144
0.1184
0.181
0.209
0.355
0.375
0.401
0.449
0.466
0.693
1.025
1.165
1.117

115
00.181
0.190
0.190
0.221
$0: 366$
00391
0.413
0.449
0.463
0.647
0.948
1.160
1.1114


TABLE 2.20. Kerosene Prices: Residential Sector (\$/gal1on)

\begin{tabular}{|c|c|c|c|c|c|c|c|c|c|}
\hline $\begin{array}{l}\text { YEAR } \\
1970 \\
19971 \\
19972 \\
1973 \\
1974 \\
1975 \\
1976 \\
1977 \\
1978 \\
1979 \\
1980 \\
1989 \\
1982\end{array}$ & \begin{tabular}{c}
$A K$ \\
\hdashline $0: 0$ \\
$0: 0$ \\
$0: 0$ \\
$0: 0$ \\
$0: 0$ \\
$0: 0$ \\
$0: 0$ \\
$0: 0$ \\
$0: 0$ \\
$0: 0$ \\
$0: 0$ \\
$0: 0$ \\
0.0
\end{tabular} & \begin{tabular}{l}
$A L$ \\
\hdashline 0.219 \\
$0: 225$ \\
$0: 228$ \\
$0: 262$ \\
$0: 399$ \\
$0: 447$ \\
0.480 \\
$0: 521$ \\
$0: 576$ \\
$0: 943$ \\
$0: 811$ \\
$1: 507$ \\
1.458
\end{tabular} & $\begin{array}{l}\text { AR } \\
-0.189 \\
0: 1191 \\
0: 200 \\
0: 227 \\
0: 371 \\
0: 378 \\
0: 391 \\
0: 463 \\
0.487 \\
0.699 \\
1: 044 \\
1: 270 \\
1.251\end{array}$ & \begin{tabular}{r}
$A Z$ \\
\hdashline $0: 391$ \\
$0: 363$ \\
$0: 330$ \\
$0: 346$ \\
$0: 535$ \\
$0: 6258$ \\
$0: 619$ \\
$0: 704$ \\
$0: 749$ \\
$0: 853$ \\
$1: 576$ \\
$1: 927$ \\
1.934
\end{tabular} & $\begin{array}{c}C A \\
-0.347 \\
0: 371 \\
0: 409 \\
0: 479 \\
0.721 \\
0: 685 \\
0: 701 \\
0: 773 \\
0: 814 \\
10163 \\
1: 756 \\
20065 \\
2.099\end{array}$ & \begin{tabular}{c}
$C 0$ \\
\hdashline $0: 204$ \\
$0: 200$ \\
$0: 210$ \\
$0: 246$ \\
$0: 387$ \\
$0: 399$ \\
$0: 431$ \\
$0: 483$ \\
$0: 5114$ \\
$0: 571$ \\
$1: 077$ \\
$1: 320$ \\
$1: 326$
\end{tabular} & \begin{tabular}{l} 
CT \\
\hdashline 0.229 \\
$0: 225$ \\
$0: 228$ \\
$0: 262$ \\
$0: 407$ \\
$00: 427$ \\
$0: 452$ \\
$0: 506$ \\
$0: 541$ \\
$0: 719$ \\
$1: 100$ \\
$1: 422$ \\
1.456
\end{tabular} & $\begin{array}{l}D C \\
-0.203 \\
0: 248 \\
0: 227 \\
0: 257 \\
0: 397 \\
0: 454 \\
0: 443 \\
0: 520 \\
0: 552 \\
0: 746 \\
1: 154 \\
1: 458 \\
1.426\end{array}$ & \begin{tabular}{l}
$D E$ \\
\hdashline 0.181 \\
$00: 194$ \\
$0: 295$ \\
$0: 257$ \\
$0: 396$ \\
$0: 454$ \\
$0: 443$ \\
$0: 520$ \\
$0: 552$ \\
$0: 746$ \\
$1: 154$ \\
$1: 458$ \\
$1: 426$
\end{tabular} \\
\hline $\begin{array}{l}\text { YEAR } \\
1970 \\
1971 \\
1972 \\
1973 \\
1974 \\
1975 \\
1976 \\
1977 \\
1978 \\
1979 \\
1980 \\
1981 \\
1982\end{array}$ & \begin{tabular}{l}
$F L$ \\
\hdashline 0.220 \\
$0: 216$ \\
0.216 \\
$0: 248$ \\
0.400 \\
0.442 \\
00.480 \\
0.546 \\
0.584 \\
0.791 \\
1.203 \\
1.515 \\
1.502
\end{tabular} & \begin{tabular}{l}
$G A$ \\
\hdashline 0.799 \\
$0: 207$ \\
$0: 222$ \\
$0: 254$ \\
$0: 401$ \\
$0: 452$ \\
$0: 459$ \\
0.537 \\
$0: 574$ \\
$0: 779$ \\
$1: 184$ \\
$1: 491$ \\
1.478
\end{tabular} & \begin{tabular}{c}
$H I$ \\
$-I=-$ \\
\hdashline 0.396 \\
$0: 427$ \\
$0: 468$ \\
$0: 487$ \\
$0: 619$ \\
$0: 700$ \\
$0: 760$ \\
$0: 849$ \\
$0: 894$ \\
$1: 277$ \\
$1: 928$ \\
$2: 267$ \\
$2: 304$
\end{tabular} & \begin{tabular}{c} 
IA \\
\hdashline 0.212 \\
$0: 219$ \\
$0: 219$ \\
$0: 251$ \\
$0: 375$ \\
$0: 403$ \\
$0: 440$ \\
$0: 503$ \\
$0: 535$ \\
$0: 737$ \\
$1: 093$ \\
$1: 337$ \\
$1: 298$
\end{tabular} & \begin{tabular}{l} 
ID \\
\hdashline 0.337 \\
$0: 336$ \\
$0: 417$ \\
$0: 434$ \\
$0: 536$ \\
$0: 550$ \\
$0: 588$ \\
0.645 \\
$0: 647$ \\
$0: 763$ \\
$1: 439$ \\
$1: 764$ \\
$1: 772$
\end{tabular} & $\begin{aligned} I L \\
-10-52 \\
0.222 \\
0: 227 \\
0: 225 \\
0: 259 \\
0: 396 \\
0: 429 \\
0: 452 \\
0: 526 \\
0: 551 \\
0: 786 \\
1: 175 \\
1: 443 \\
1: 402\end{aligned}$ & \begin{tabular}{l} 
IN \\
\hdashline $0: 215$ \\
$0: 205$ \\
$0: 187$ \\
$0: 238$ \\
$0: 382$ \\
$0: 420$ \\
$0: 439$ \\
$0: 516$ \\
$0: 541$ \\
$0: 772$ \\
$1: 154$ \\
$1: 417$ \\
$1: 377$
\end{tabular} & $\begin{array}{l}K S \\
0.190 \\
0: 193 \\
0: 199 \\
0: 235 \\
0: 365 \\
0: 384 \\
0: 407 \\
0: 477 \\
0: 507 \\
0.699 \\
1: 036 \\
1: 268 \\
1: 231\end{array}$ & $\begin{array}{l}K Y \\
-1 \\
0: 234 \\
0: 215 \\
0: 217 \\
0: 255 \\
0: 399 \\
0: 422 \\
0: 446 \\
0: 485 \\
0: 536 \\
0: 836 \\
0: 825 \\
1: 405 \\
1: 355\end{array}$ \\
\hline $\begin{array}{l}\text { YEAR } \\
1970 \\
1979 \\
1971 \\
1973 \\
1974 \\
1975 \\
1976 \\
1977 \\
1978 \\
1979 \\
1980 \\
1981 \\
1982\end{array}$ & \begin{tabular}{l}
$\mathrm{L} A$ \\
\hdashline 0.216 \\
$0: 228$ \\
$0: 237$ \\
$0: 274$ \\
$0: 437$ \\
$0: 461$ \\
$0: 511$ \\
$0: 597$ \\
$0: 627$ \\
$0: 900$ \\
$1: 345$ \\
$1: 636$ \\
$1: 611$
\end{tabular} & $\begin{array}{l}M A \\
-9.719 \\
0: 219 \\
0: 225 \\
0: 228 \\
0: 262 \\
0: 407 \\
0: 427 \\
0: 452 \\
0: 506 \\
0: 541 \\
0: 719 \\
1: 100 \\
1: 422 \\
1.456\end{array}$ & \begin{tabular}{l}
$M D$ \\
\hdashline 0.203 \\
0.244 \\
0.227 \\
$0: 257$ \\
0.397 \\
0.454 \\
0.443 \\
0.520 \\
0.552 \\
0.746 \\
$1: 154$ \\
$1: 458$ \\
1.426
\end{tabular} & \begin{tabular}{l}
$M E$ \\
\hdashline 0.216 \\
$0: 225$ \\
$0: 228$ \\
$0: 262$ \\
0.407 \\
0.427 \\
0.452 \\
$0: 506$ \\
$0: 541$ \\
$0: 719$ \\
$1: 100$ \\
$1: 422$ \\
1.456
\end{tabular} & $\begin{array}{l}M 1 \\
-1 \\
0.211 \\
0: 200 \\
0: 212 \\
0: 256 \\
0: 405 \\
0: 421 \\
0: 436 \\
0: 509 \\
0: 533 \\
0: 761 \\
1: 1138 \\
1: 397 \\
1: 357\end{array}$ & $\begin{array}{l}M N \\
-0 .-105 \\
0: 205 \\
0: 200 \\
0: 212 \\
0: 242 \\
0: 374 \\
0: 393 \\
0: 426 \\
0.498 \\
0: 530 \\
0: 730 \\
1: 082 \\
1: 325 \\
1.286\end{array}$ & \begin{tabular}{l}
$M 0$ \\
\hdashline 0.193 \\
$0: 211$ \\
$0: 213$ \\
$0: 247$ \\
$0: 375$ \\
$0: 389$ \\
$0: 425$ \\
$0: 494$ \\
$0: 525$ \\
$0: 723$ \\
$1: 073$ \\
$1: 313$ \\
$1: 275$
\end{tabular} & $\begin{array}{l}M S \\
0.278 \\
0: 288 \\
0: 245 \\
0: 306 \\
0.427 \\
0: 513 \\
0: 553 \\
0: 598 \\
0.661 \\
1: 083 \\
0: 932 \\
1: 730 \\
1.674\end{array}$ & $\begin{array}{l}M T \\
0.232 \\
0: 241 \\
0: 244 \\
0: 291 \\
0: 416 \\
0: 427 \\
0: 439 \\
0: 501 \\
0: 533 \\
0: 592 \\
1: 117 \\
1: 369 \\
1.375\end{array}$ \\
\hline
\end{tabular}


TABLE 2.20 (cont). Kerosene Prices: Residential Sector (\$/gallon)
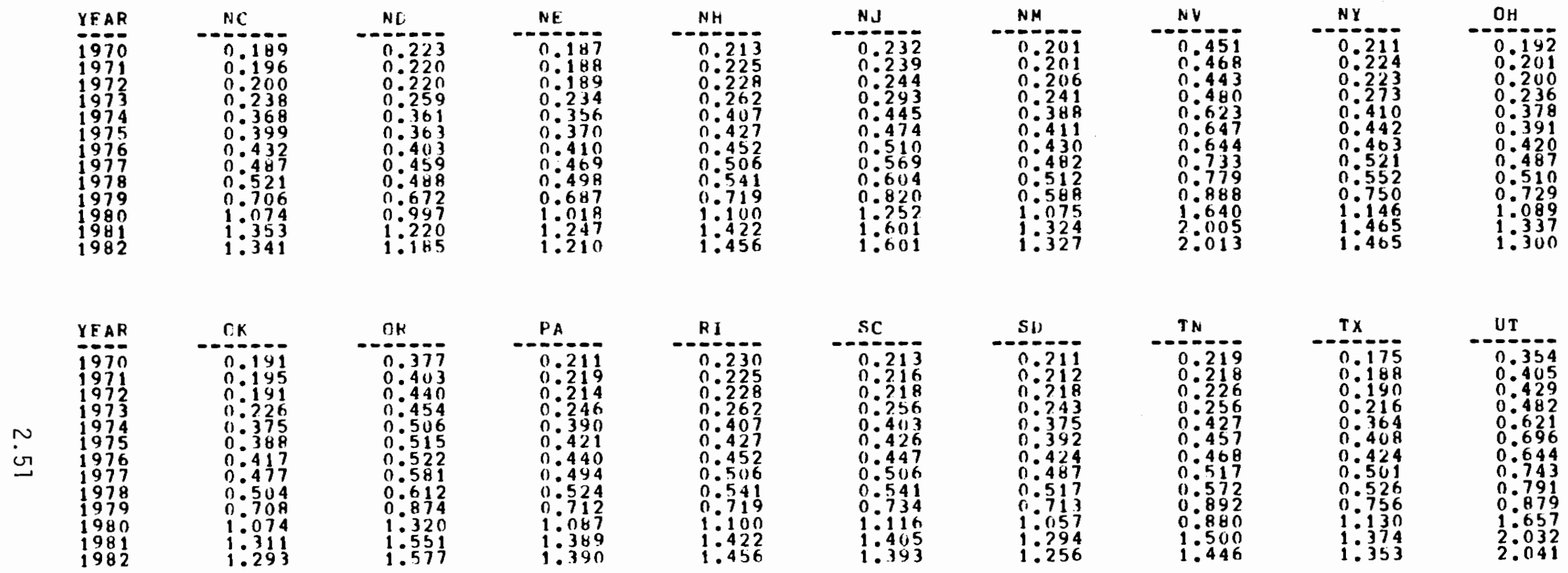

0.
0.377
0.343
0.403
0.440
0.454
0.506
0.515
0.522
0.581
0.612
0.874
1.320
1.551
1.577
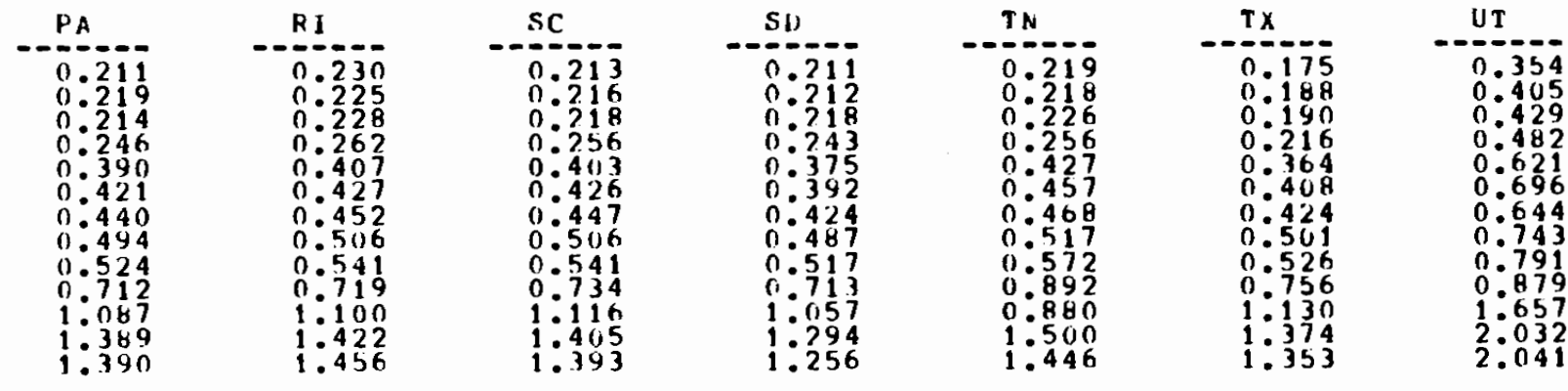

\begin{tabular}{lr} 
YEAR & VA \\
\hline 1970 & 0.194 \\
1971 & 0.203 \\
1972 & 0.294 \\
1973 & 0.241 \\
1974 & 0.372 \\
1975 & 0.404 \\
1976 & 0.430 \\
1977 & 0.487 \\
1978 & 0.521 \\
1979 & 0.707 \\
1980 & 1.075 \\
1981 & 1.354 \\
1982 & 1.342
\end{tabular}

$V T$
-0.220
0.225
0.228
0.262
0.407
0.427
0.452
0.506
0.541
0.719
1.100
1.422
1.456

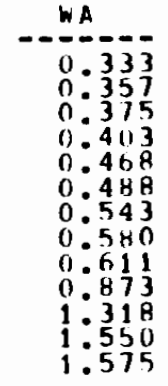

$W 1$
-0.198
0.205
0.214
0.749
0.379
0.400
0.419
0.490
0.513
0.732
1.095
1.344
1.307
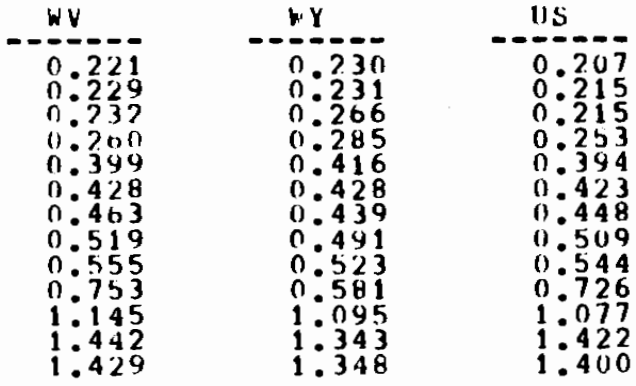

Source: Pacific Northwest Laboratory 
TABLE 2.21. Kerosene Prices: Industrial Sector (\$/gallon)
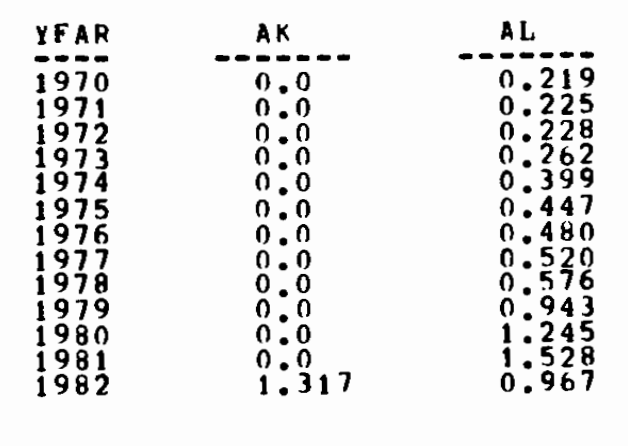

$\begin{array}{ll}A R & A Z \\ -0.189 & 0.391 \\ 0.191 & 0.363 \\ 0.200 & 0.330 \\ 0.227 & 0.346 \\ 0.371 & 0.515 \\ 0.378 & 0.628 \\ 0.391 & 0.619 \\ 0.463 & 0.705 \\ 0.486 & 0.749 \\ 0.698 & 1.006 \\ 1.043 & 1.578 \\ 1.181 & 1.927 \\ 0.947 & 0.949\end{array}$

$C A$
$0.7-58$
0.371
0.409
0.479
0.721
0.685
0.701
0.773
0.814
1.163
1.756
2.064
1.055
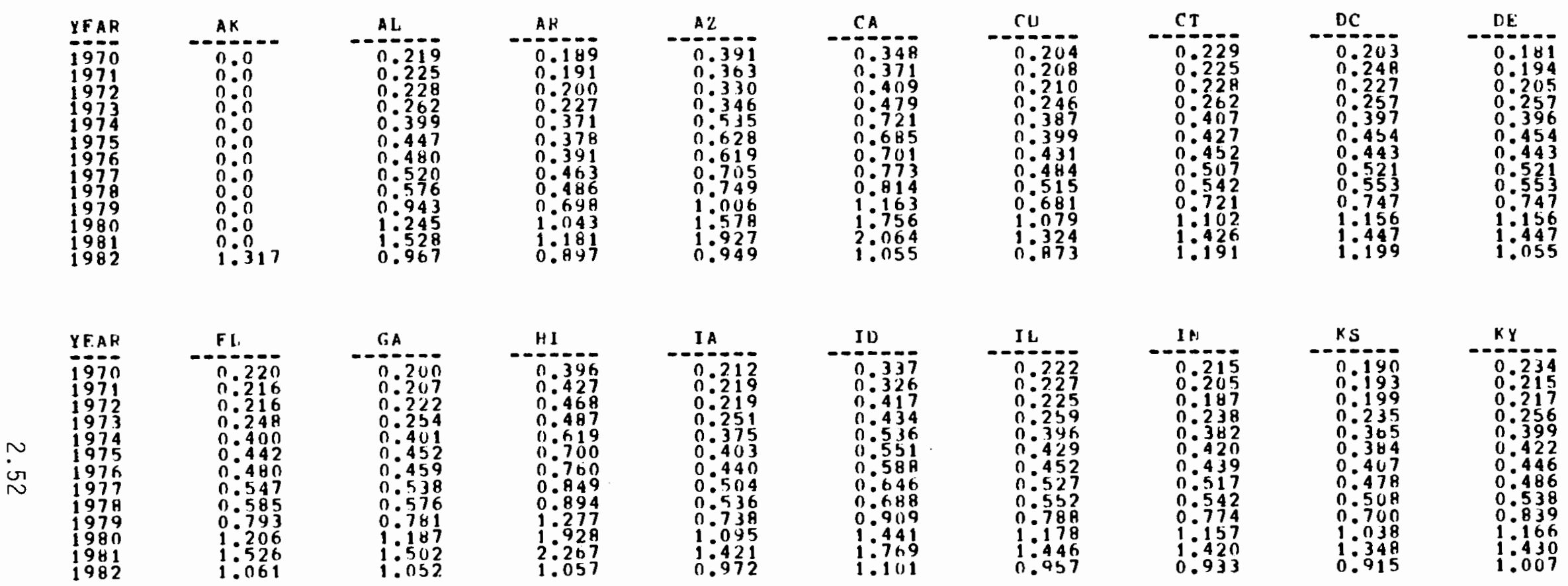

$H I$
0.396
0.427
0.468
0.467
0.619
0.700
0.760
0.849
0.894
1.277
1.928
2.267
1.057
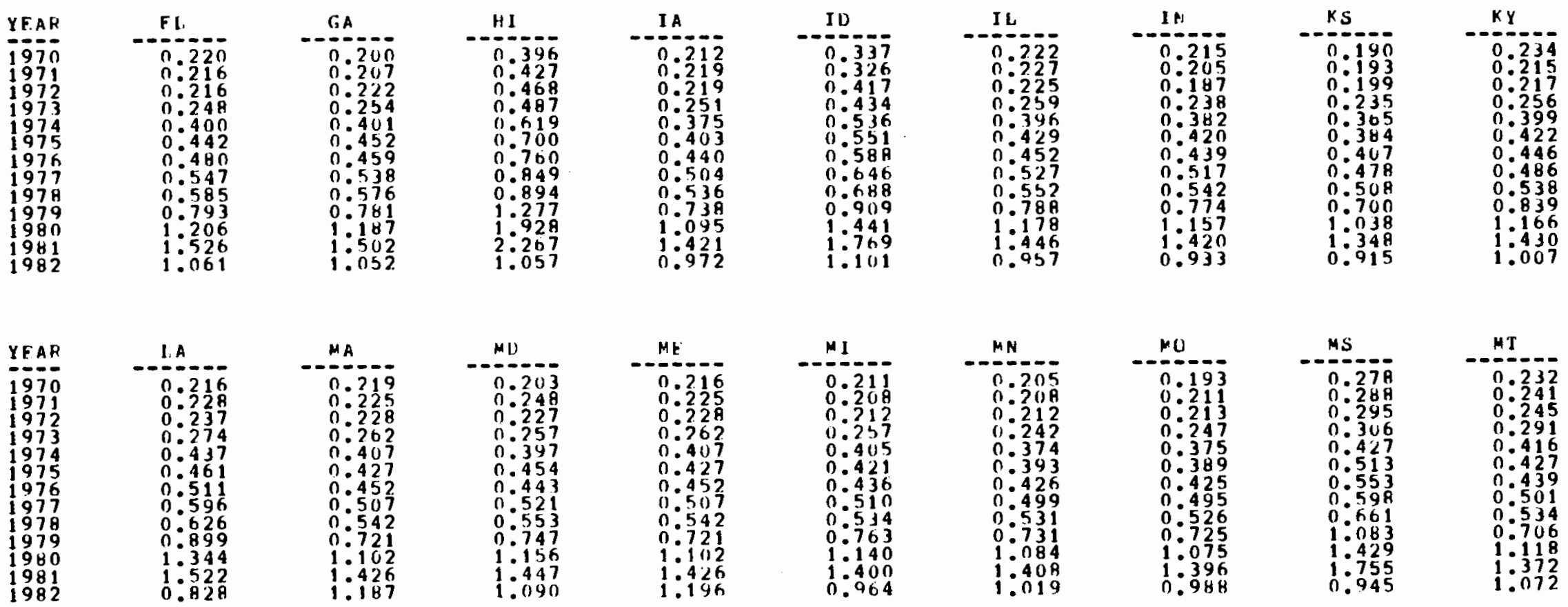

\begin{tabular}{ll}
$M D$ & $M E$ \\
\hline 0.203 & 0.216 \\
0.248 & 0.225 \\
0.227 & 0.228 \\
0.257 & 0.262 \\
0.397 & 0.407 \\
0.454 & 0.427 \\
0.443 & 0.452 \\
0.521 & 0.507 \\
0.553 & 0.542 \\
0.747 & 0.721 \\
1.156 & 1.102 \\
1.447 & 1.426 \\
1.090 & 1.196
\end{tabular}

01
0.011
0.208
0.2112
0.257
0.405
0.421
0.436
0.510
0.514
$0: 763$
1.1440
1.400
0.964
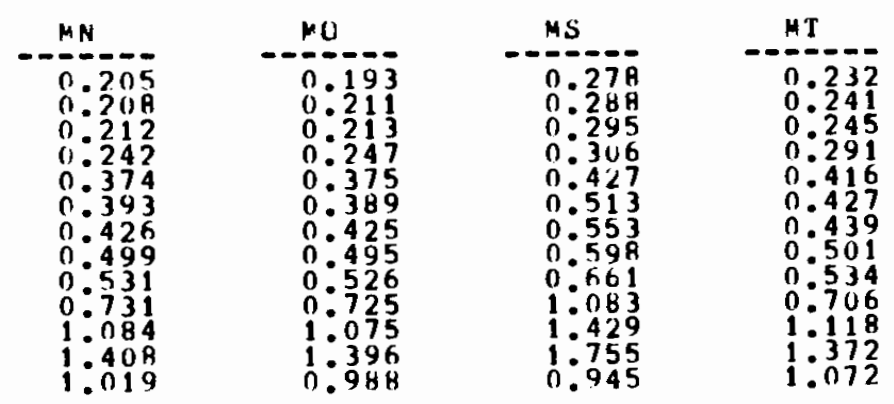
TABLE 2.27 (cont). Kerosene Prices: Industrial Sector (\$/gallon)

\begin{tabular}{|c|c|c|c|c|c|c|c|c|c|}
\hline \begin{tabular}{l} 
YEAR \\
\hdashline 1970 \\
1971 \\
1972 \\
1973 \\
1974 \\
1975 \\
1976 \\
1977 \\
1978 \\
1979 \\
1980 \\
1981 \\
1982
\end{tabular} & $\begin{array}{r}N C \\
-0.189 \\
0: 196 \\
0: 200 \\
0: 239 \\
0: 369 \\
0: 399 \\
0: 432 \\
0: 498 \\
0: 522 \\
0.708 \\
1: 077 \\
1: 362 \\
10028\end{array}$ & \begin{aligned}$N D \\
$\hdashline 0.223 \\
$0: 220 \\
0: 220 \\
0: 259 \\
0: 361 \\
0: 363 \\
0: 403 \\
0: 460 \\
0: 489 \\
0.673 \\
0: 999 \\
1: 297 \\
1: 163\end{aligned}$ & \begin{tabular}{l}
$N E$ \\
\hdashline $0: 187$ \\
$0: 188$ \\
$0: 189$ \\
$0: 234$ \\
$0: 356$ \\
$0: 370$ \\
$0: 410$ \\
$0: 470$ \\
$0: 499$ \\
$0: 688$ \\
$1: 020$ \\
$1: 325$ \\
0.924
\end{tabular} & \begin{tabular}{l}
$\mathrm{NH}$ \\
\hdashline 0.213 \\
$0: 225$ \\
$0: 22 \mathrm{~B}$ \\
$0: 262$ \\
$0: 407$ \\
$0: 422$ \\
$0: 452$ \\
$0: 507$ \\
$0: 542$ \\
$0: 721$ \\
$1: 102$ \\
$1: 426$ \\
$1: 1198$
\end{tabular} & \begin{tabular}{l} 
NJ \\
\hdashline 0.232 \\
$0: 239$ \\
$0: 245$ \\
$0: 299$ \\
$0: 445$ \\
$0: 474$ \\
$0: 5110$ \\
$0: 571$ \\
$0: 605$ \\
$0: 422$ \\
1.254 \\
$1: 604$ \\
1.1111
\end{tabular} & \begin{tabular}{l} 
NM \\
\hdashline 0.201 \\
$0: 201$ \\
$0: 206$ \\
$0: 241$ \\
$0: 388$ \\
$0: 411$ \\
$0: 430$ \\
$0: 482$ \\
$0: 513$ \\
$0: 684$ \\
$1: 076$ \\
$1: 320$ \\
$1: 227$
\end{tabular} & \begin{tabular}{l}
$N V$ \\
\hdashline 0.451 \\
0.460 \\
0.443 \\
$0: 480$ \\
0.623 \\
0.647 \\
0.644 \\
$0: 734$ \\
0.780 \\
$1: 047$ \\
1.642 \\
2.006 \\
$1: 113$
\end{tabular} & \begin{tabular}{l}
$N Y$ \\
\hdashline 0.211 \\
$0: 225$ \\
$0: 223$ \\
$0: 273$ \\
$0: 410$ \\
$0: 442$ \\
$0: 463$ \\
$0: 522$ \\
$0: 554$ \\
$0: 752$ \\
$1: 148$ \\
$1: 468$ \\
$1: 164$
\end{tabular} & $\begin{array}{l}\mathrm{OH} \\
-00192 \\
0: 201 \\
0.200 \\
0: 236 \\
0: 378 \\
0: 391 \\
0: 420 \\
0: 488 \\
0: 512 \\
0: 730 \\
1: 092 \\
1: 340 \\
0.995\end{array}$ \\
\hline $\begin{array}{l}\text { YFAR } \\
1970 \\
1971 \\
1972 \\
1973 \\
1974 \\
1975 \\
1976 \\
1977 \\
1979 \\
1979 \\
1980 \\
1981 \\
1982\end{array}$ & \begin{tabular}{l}
$0 K$ \\
\hdashline 0.791 \\
$0: 195$ \\
01191 \\
$0: 226$ \\
$0: 376$ \\
$0: 388$ \\
$0: 417$ \\
$0: 179$ \\
$0: 506$ \\
0.710 \\
$1: 077$ \\
$1: 227$ \\
0.955
\end{tabular} & $\begin{array}{l}0 R \\
-0.575 \\
0.377 \\
0: 403 \\
0: 440 \\
0: 454 \\
0: 506 \\
0: 516 \\
0: 522 \\
0: 581 \\
0: 612 \\
0: 874 \\
1: 319 \\
1: 551 \\
1.096\end{array}$ & \begin{tabular}{l}
$P A$ \\
\hdashline 0.212 \\
$0: 219$ \\
$0: 2114$ \\
$0: 246$ \\
$0: 3990$ \\
$0: 421$ \\
0.440 \\
0.495 \\
0.525 \\
0.7113 \\
$1: 089$ \\
$1: 392$ \\
1.097
\end{tabular} & \begin{tabular}{l}
$\mathrm{RI}$ \\
\hdashline 0.230 \\
$0: 225$ \\
$0: 228$ \\
$0: 262$ \\
$0: 407$ \\
$0: 427$ \\
0.452 \\
$0: 507$ \\
$0: 542$ \\
$0: 721$ \\
$1: 102$ \\
$1: 426$ \\
1.198
\end{tabular} & \begin{tabular}{l}
$s c$ \\
\hdashline 0.213 \\
$0: 2116$ \\
$0: 211$ \\
$0: 256$ \\
$0: 403$ \\
$0: 426$ \\
0.447 \\
$0: 507$ \\
$0: 542$ \\
$0: 736$ \\
$1: 119$ \\
$1: 415$ \\
$1: 008$
\end{tabular} & \begin{tabular}{l}
$S D$ \\
\hdashline 0.211 \\
$0: 013$ \\
$0: 218$ \\
$0: 243$ \\
$0: 375$ \\
$0: 393$ \\
0.424 \\
0.488 \\
0.518 \\
0.714 \\
$1: 059$ \\
$1: 375$ \\
$1: 083$
\end{tabular} & \begin{tabular}{l}
$T N$ \\
\hdashline 0.219 \\
$0: 2118$ \\
$0: 226$ \\
$0: 256$ \\
$0: 427$ \\
$0: 457$ \\
$0: 468$ \\
$0: 5119$ \\
$0: 574$ \\
$0: 895$ \\
$1: 244$ \\
$1: 526$ \\
0.992
\end{tabular} & \begin{tabular}{c}
$T X$ \\
\hdashline $0: 175$ \\
$0: 188$ \\
$0: 190$ \\
$0: 216$ \\
$0: 364$ \\
$0: 408$ \\
$0: 424$ \\
$0: 501$ \\
$0: 526$ \\
$0: 755$ \\
$1: 129$ \\
$1: 278$ \\
1003
\end{tabular} & \begin{tabular}{l} 
UT \\
\hdashline 0.354 \\
0.4405 \\
0.429 \\
0.482 \\
0.621 \\
0.696 \\
0.644 \\
0.744 \\
0.792 \\
10047 \\
10659 \\
20036 \\
1.015
\end{tabular} \\
\hline $\begin{array}{l}\text { YEAR } \\
1970 \\
1970 \\
1971 \\
1972 \\
1973 \\
1974 \\
1975 \\
1976 \\
1977 \\
1978 \\
1979 \\
1980 \\
1981 \\
1982\end{array}$ & \begin{tabular}{l}
$V A$ \\
\hdashline 0.195 \\
$0: 203$ \\
$0: 204$ \\
$0: 241$ \\
$0: 372$ \\
$0: 404$ \\
$0: 430$ \\
$0: 489$ \\
$0: 522$ \\
$0: 709$ \\
$1: 074$ \\
$1: 363$ \\
1.016
\end{tabular} & \begin{tabular}{l}
$v T$ \\
\hdashline 0.220 \\
$0: 225$ \\
$0: 220$ \\
$0: 262$ \\
0.4407 \\
0.427 \\
$0: 452$ \\
0.507 \\
$0: 542$ \\
$0: 721$ \\
$1: 102$ \\
$1: 426$ \\
1.225
\end{tabular} & \begin{tabular}{l}
$W A$ \\
\hdashline 0.333 \\
$0: 357$ \\
$0: 375$ \\
0.403 \\
0.468 \\
0.488 \\
$0: 544$ \\
$0: 580$ \\
$0: 611$ \\
$0: 877$ \\
$1: 3118$ \\
$1: 549$ \\
1.108
\end{tabular} & \begin{tabular}{l}
$w 1$ \\
\hdashline 0.198 \\
$0: 205$ \\
$0: 214$ \\
$0: 249$ \\
$0: 379$ \\
$0: 400$ \\
$0: 419$ \\
$0: 491$ \\
0.5114 \\
$0: 734$ \\
$1: 097$ \\
$1: 347$ \\
0.960
\end{tabular} & \begin{tabular}{l}
$w V$ \\
\hdashline 0.221 \\
$0: 229$ \\
$0: 232$ \\
$0: 260$ \\
$0: 399$ \\
$0: 428$ \\
$0: 463$ \\
0.521 \\
$0: 557$ \\
$0: 755$ \\
$1: 148$ \\
1.452 \\
1.085
\end{tabular} & 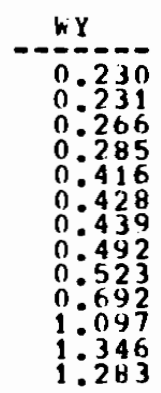 & \begin{tabular}{l} 
us \\
\hdashline 0.209 \\
$0: 216$ \\
$0: 218$ \\
$0: 254$ \\
$0: 400$ \\
$0: 429$ \\
$0: 454$ \\
$0: 521$ \\
$0: 557$ \\
$0: 751$ \\
$1: 127$ \\
$1: 430$ \\
0.994
\end{tabular} & & \\
\hline
\end{tabular}

Source: Pacific Northwest Laboratory 
TABLE 2.22. Jet Fuel Prices: Transportation Sector (\$/gallon)(a)

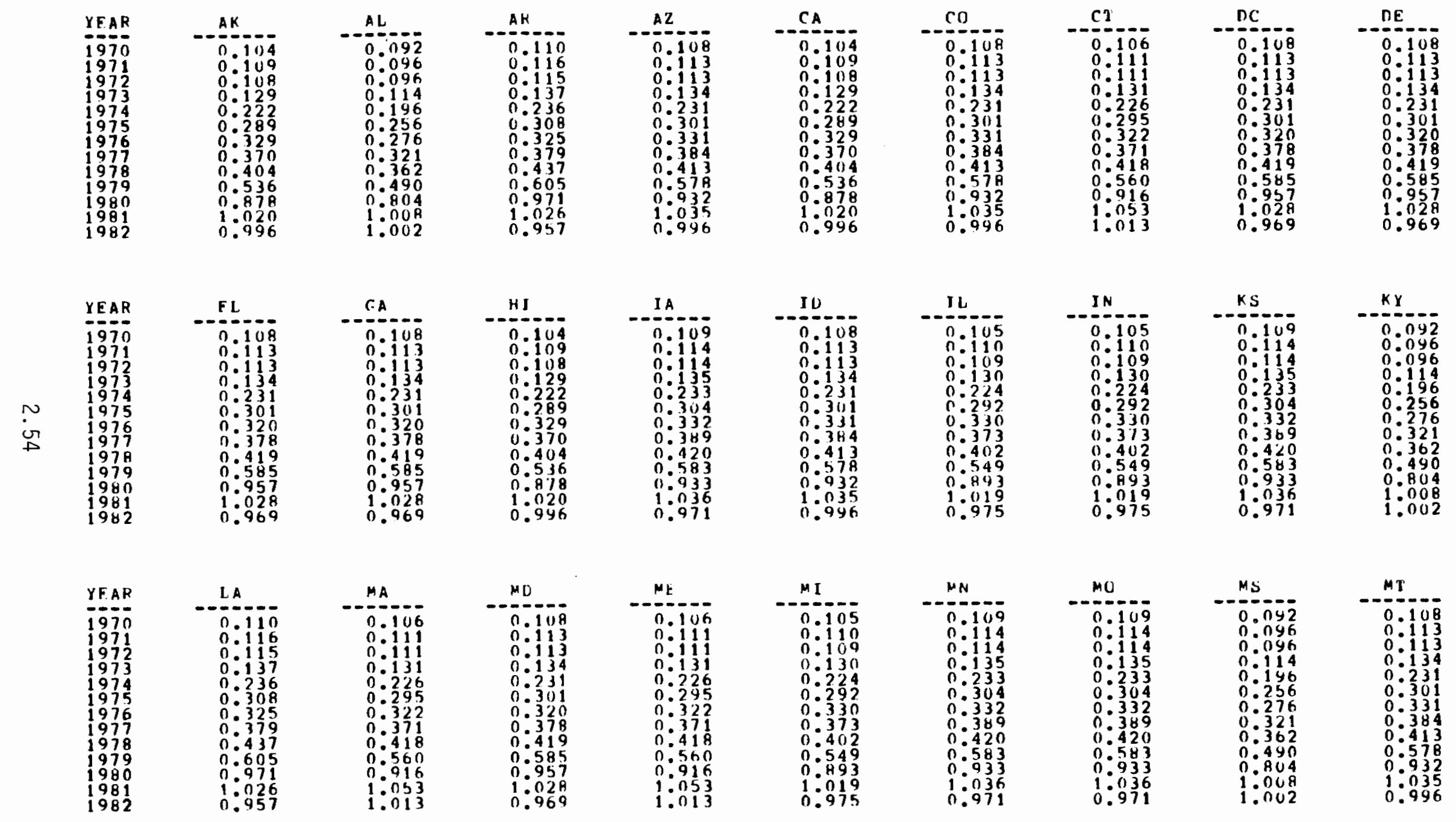

(a) Data are wholesale regional prices assigned to states. 
TABLE 2.22 (cont). Jet Fuel Prices: Transportation Sector (\$/gallon) ${ }^{(a)}$

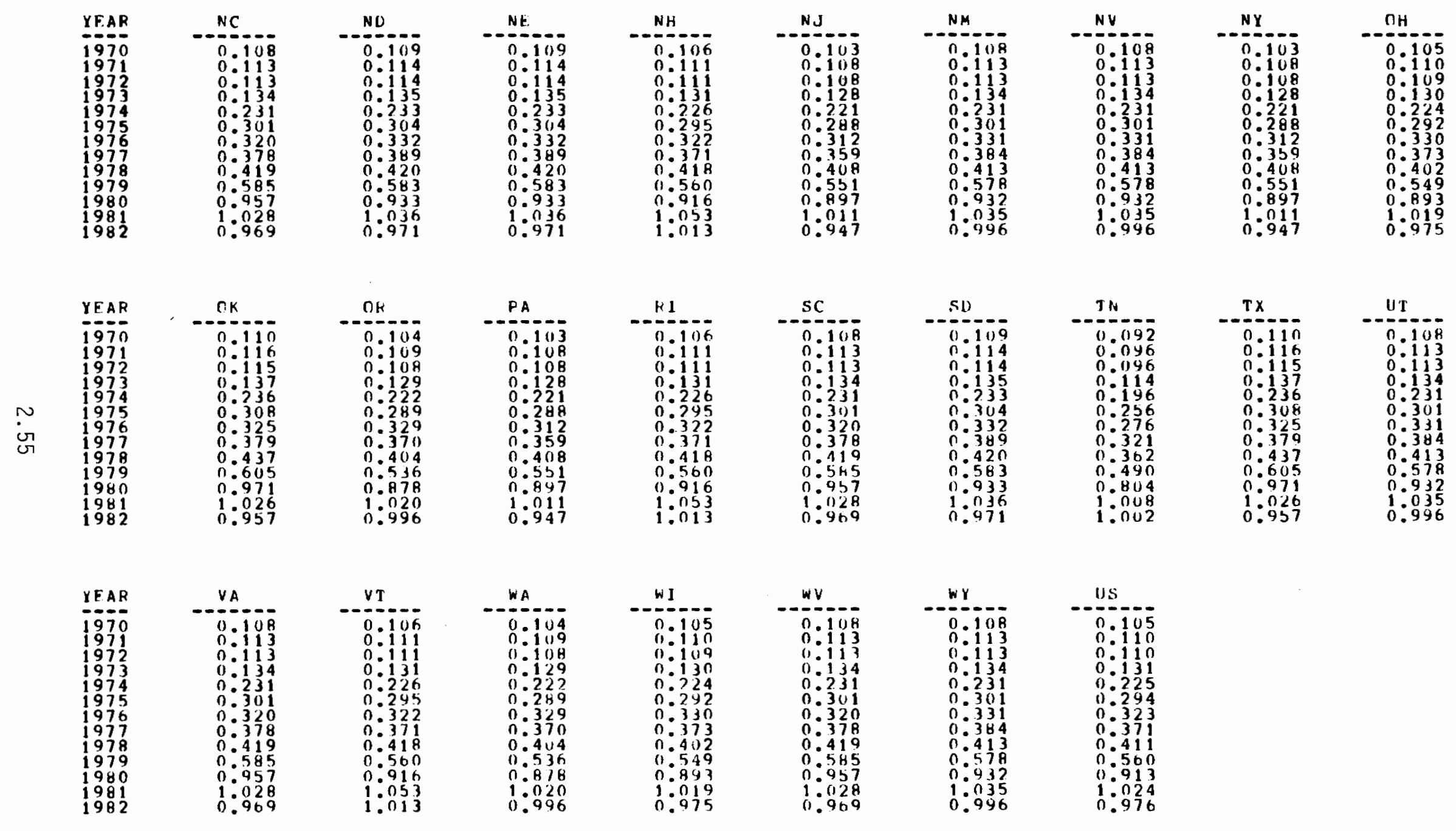

Source: Pacific Northwest Laboratory 
TABLE 2.23. Kerosene Prices: Electric Utility Sector ( $\$ /$ gallon)

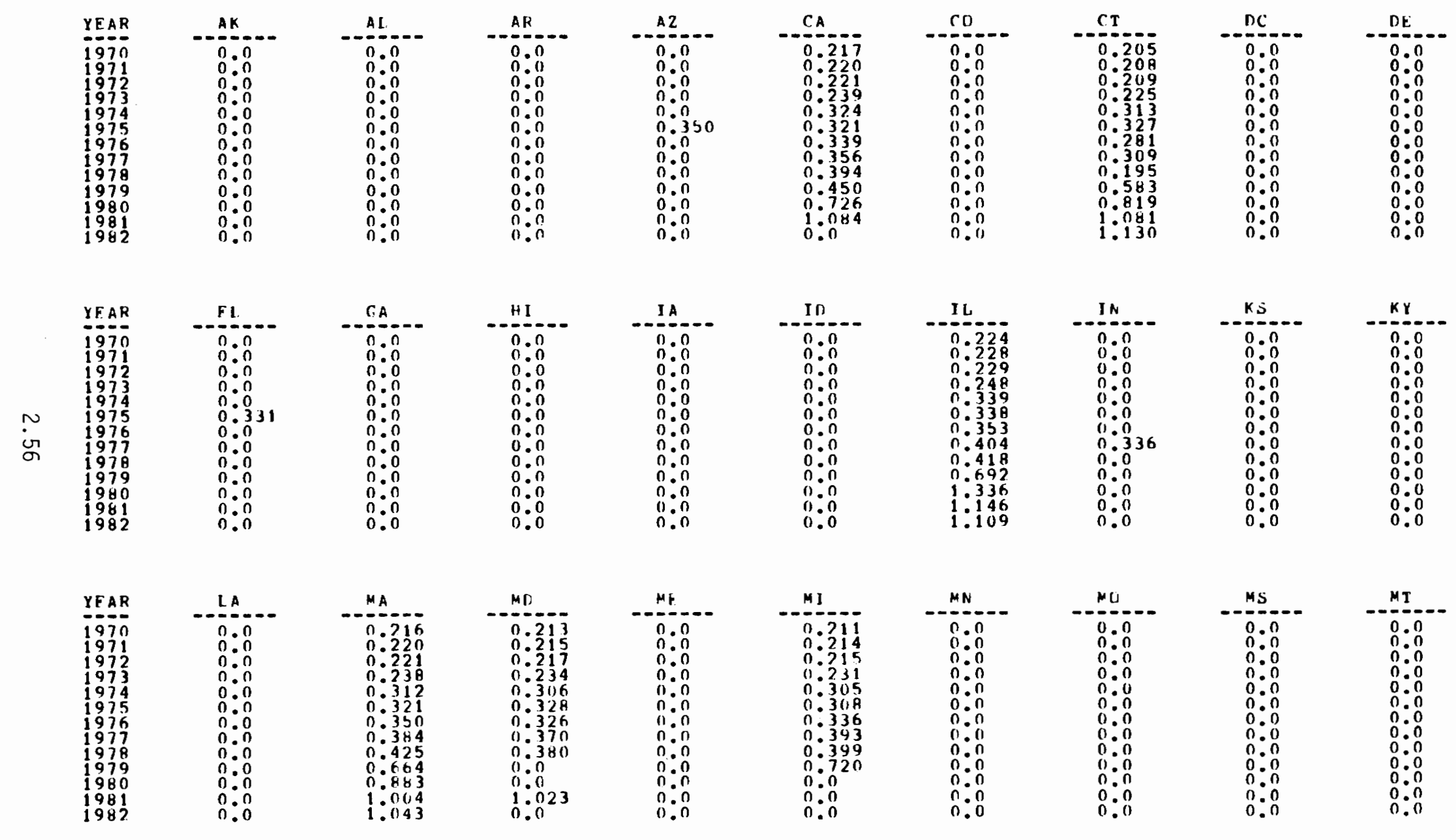


TABLE 2.23 (cont). Kerosene Prices: Electric Utility Sector (\$/gallon)
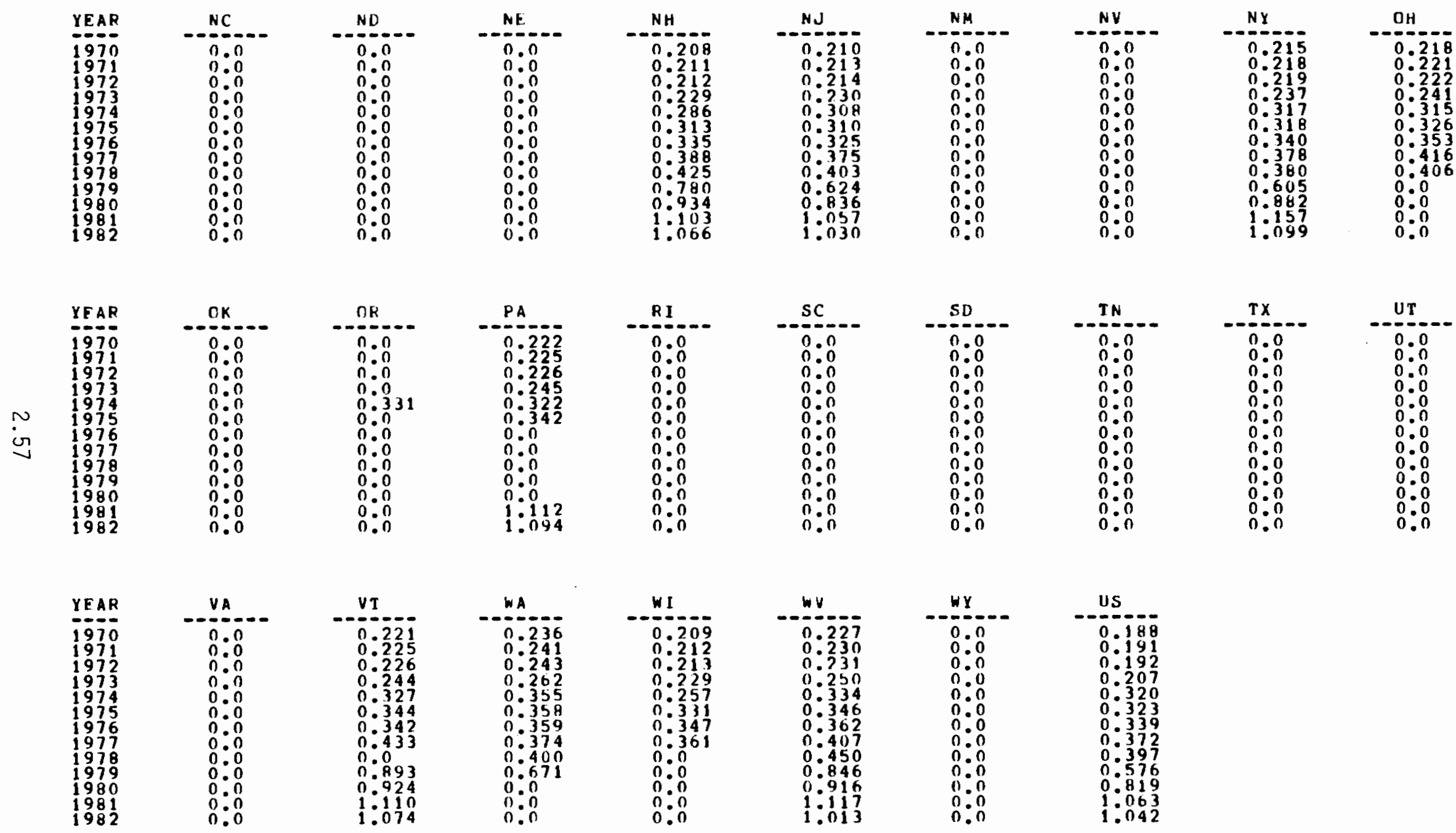

Source: Pacific Northwest Laboratory 
TABLE 2.24. Residual Fuel Prices: Commercial Sector (\$/bbl)
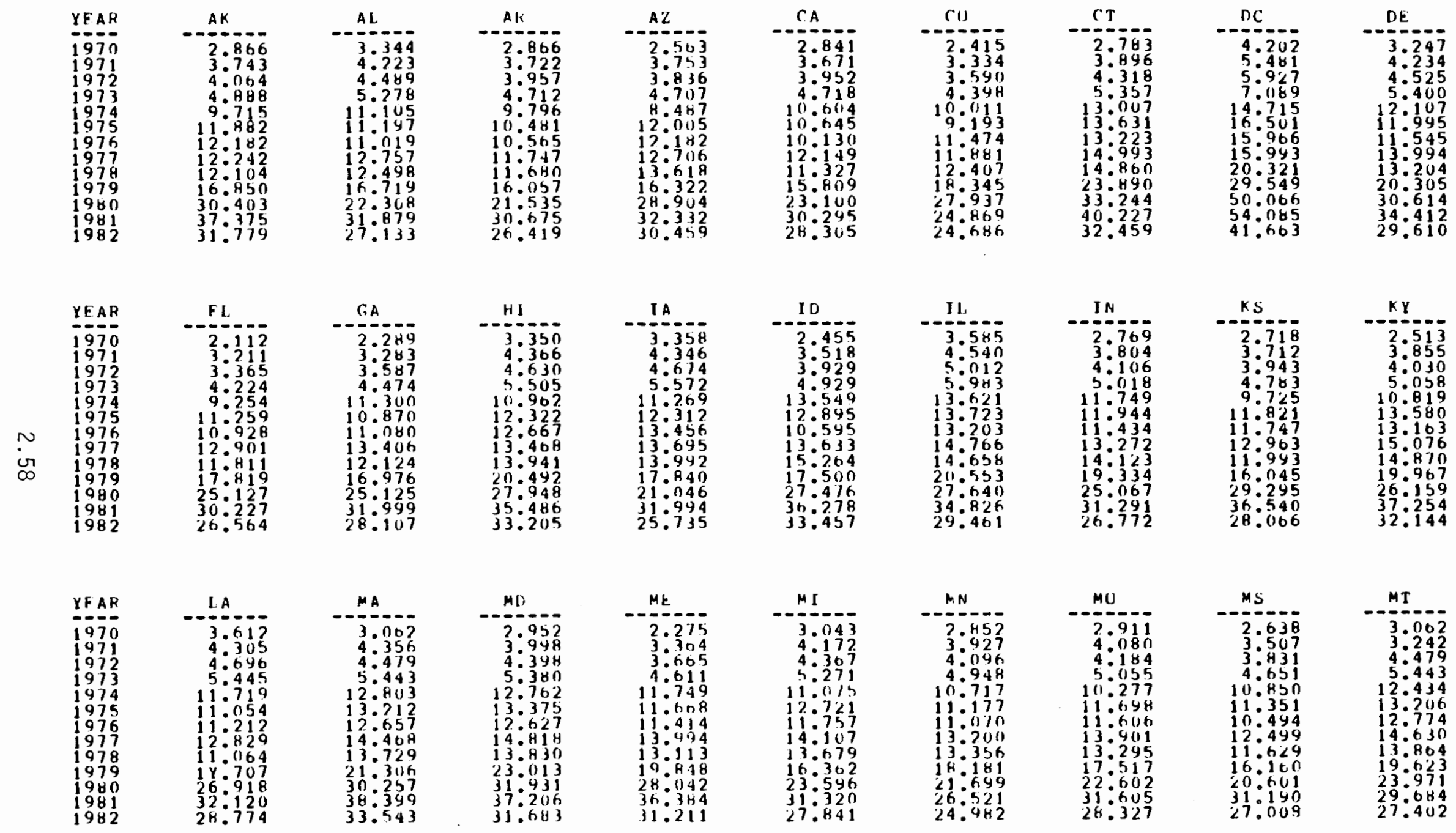

(a) Data for 1982 are provisional estimates and will be updated when data from Annual Survey of Manufactures become available. 
TABLE 2.24 (cont). Residual Fuel Prices: Commercial Sector $(\$ / b b 1)^{(a)}$
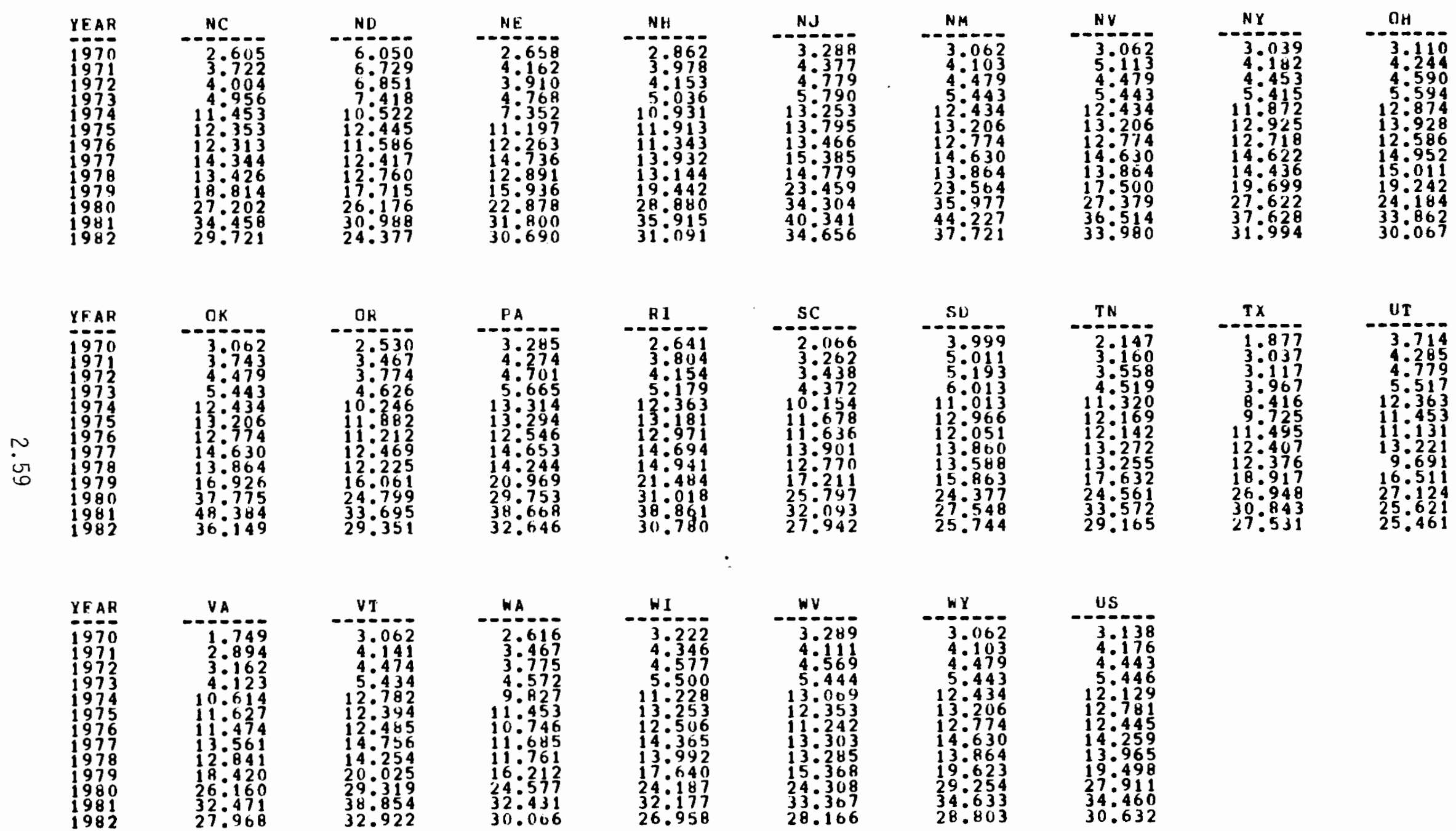

Source: Pacific Northwest Laboratory 
TABLE 2.25. Residual Fuel Prices: Industrial Sector $(\$ / b b 7)^{(a)}$

\begin{tabular}{|c|}
\hline 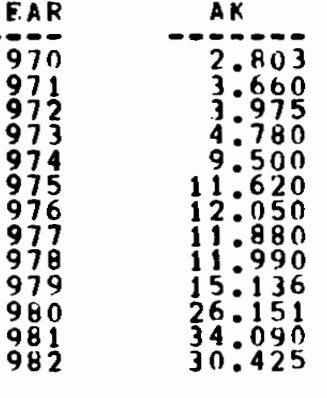 \\
\hline
\end{tabular}

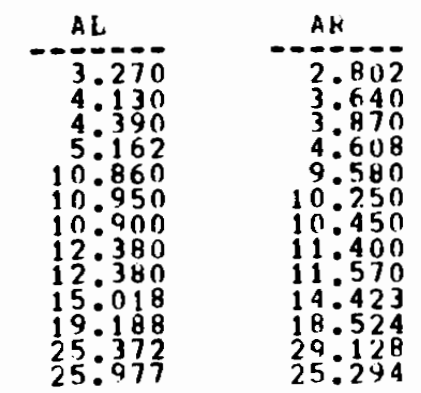
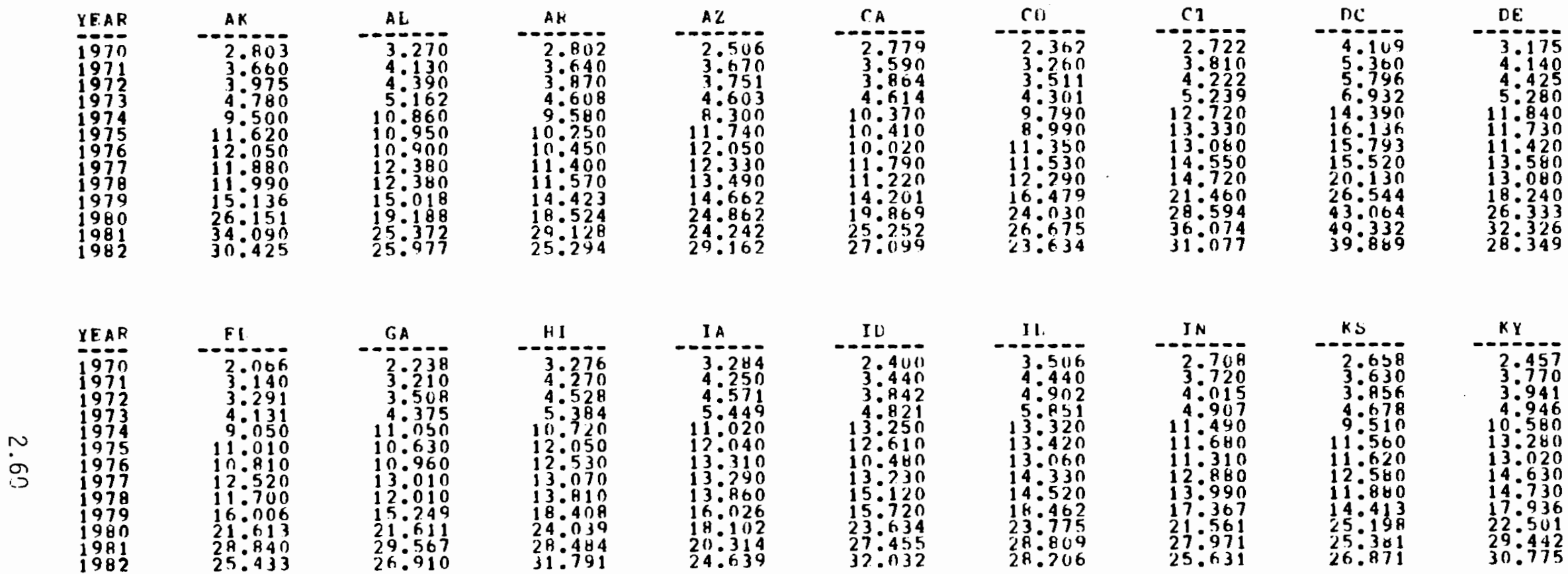

\begin{tabular}{lr} 
YFAR & \multicolumn{1}{l}{ IA } \\
\hline 1970 & -1.532 \\
1971 & 4.210 \\
1972 & 4.592 \\
1973 & 5.325 \\
1974 & 11.460 \\
1975 & 10.810 \\
1976 & 11.090 \\
1977 & 17.450 \\
1978 & 10.960 \\
1979 & 15.906 \\
1980 & 23.154 \\
1961 & 29.824 \\
1982 & 27.549
\end{tabular}

$M A$
-92.995
2.260
4.360
5.323
12.520
12.920
12.520
14.040
13.600
190139
26.025
31.181
32.115
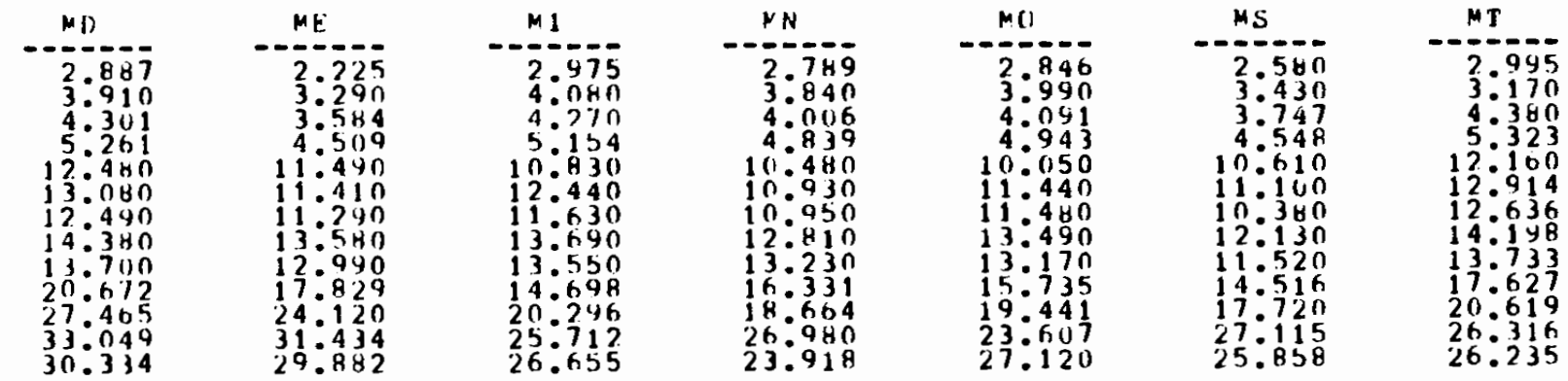

(a) Data for 1982 are provisional estimates and will be updated when data from Annual Survey of Manufactures become available. 
TABLE 2.25 (cont). Residual Fuel Prices: Industrial Sector $(\$ / b b l)(a)$
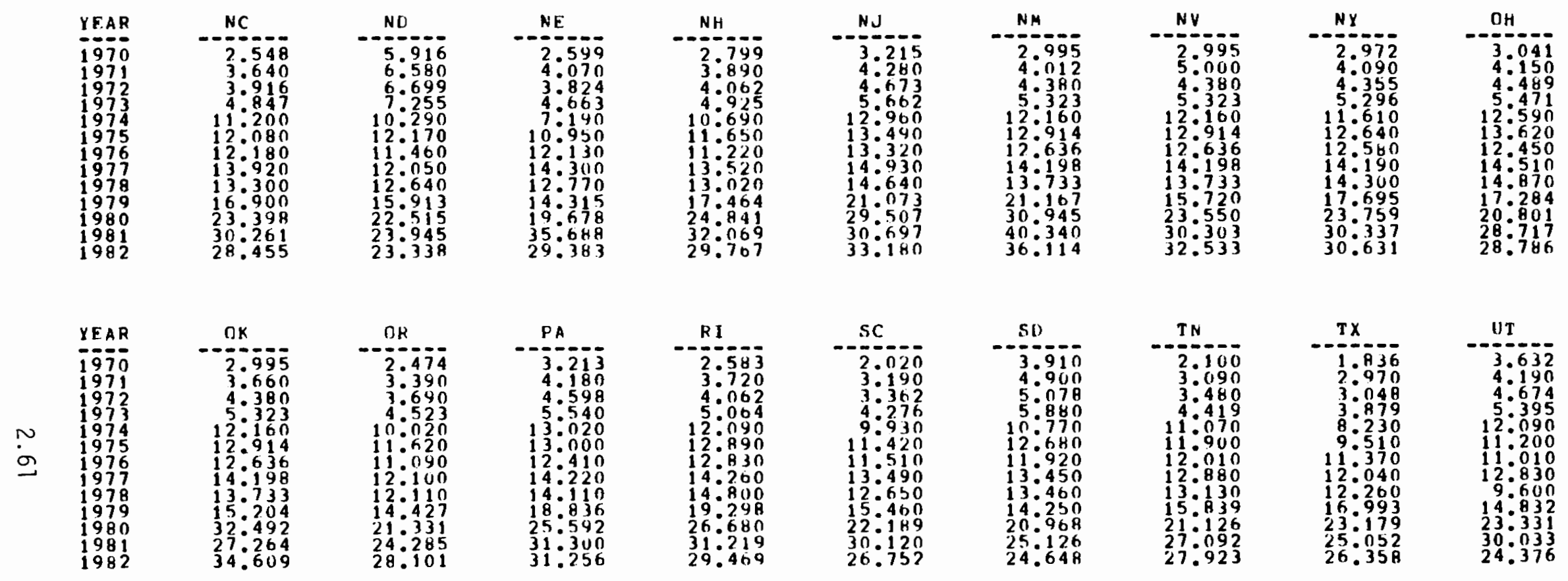

\begin{tabular}{lr} 
YEAR & VA \\
\hline 1970 & -1.710 \\
1971 & 2.830 \\
1972 & 3.092 \\
1973 & 4.032 \\
1974 & 10.380 \\
1975 & 11.370 \\
1976 & 11.350 \\
1977 & 13.160 \\
1978 & 12.720 \\
1979 & 16.547 \\
1980 & 22.501 \\
1981 & 29.820 \\
1982 & 26.777
\end{tabular}
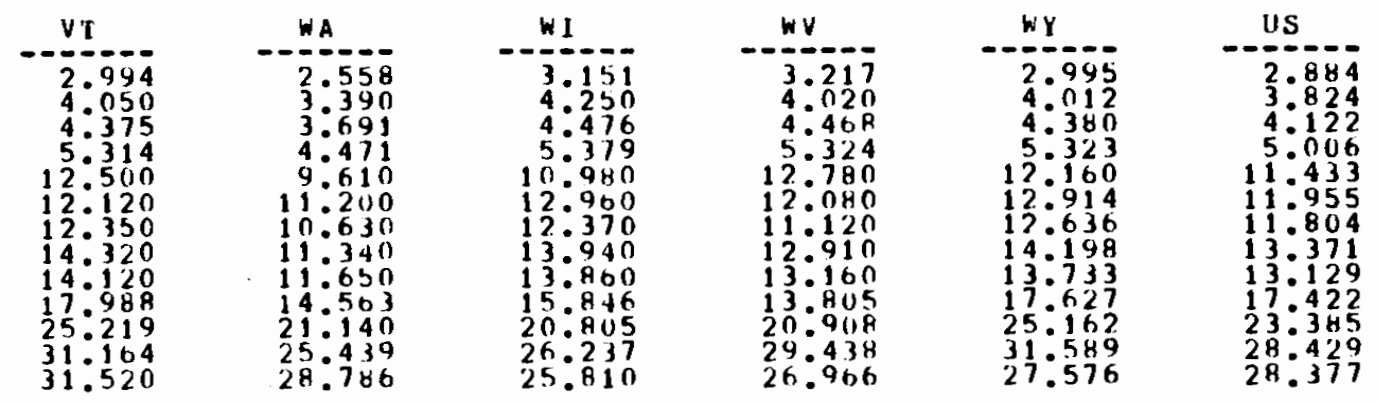
TABLE 2.26. Residual Fuel Prices: Transportation Sector $(\$ / b b 1)^{(a)}$

\begin{tabular}{|c|c|c|c|c|c|c|c|c|c|}
\hline $\begin{array}{l}\text { YEAR } \\
1970 \\
1970 \\
1971 \\
1973 \\
1974 \\
1974 \\
1975 \\
1976 \\
1979 \\
1979 \\
1980 \\
1981 \\
1982\end{array}$ & 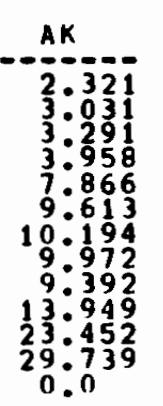 & 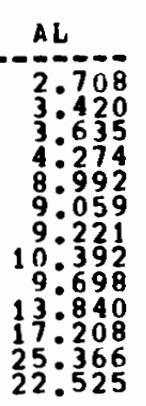 & \begin{tabular}{cc}
$A R$ \\
\hdashline 0 \\
\hdashline 0 & 0 \\
0 & 0 \\
0 & 0 \\
0 & 0 \\
0 & 0 \\
0 & 0 \\
0 & 0 \\
0 & 0 \\
0 & 0 \\
0 & 0 \\
0 & 0 \\
0 & 0 \\
0 & 0 \\
0 & 0
\end{tabular} & \begin{tabular}{cc}
$A Z$ & -1 \\
\hdashline 0 & 0 \\
0 & 0 \\
0 & 0 \\
0 & 0 \\
0 & 0 \\
0 & 0 \\
0 & 0 \\
0 & 0 \\
0 & 0 \\
0 & 0 \\
0 & 0 \\
0 & 0 \\
0 & 0 \\
0 & 0 \\
0 & 0 \\
0 & 0
\end{tabular} & 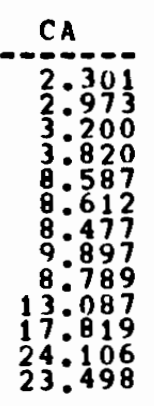 & 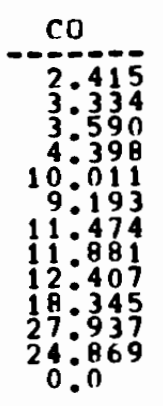 & 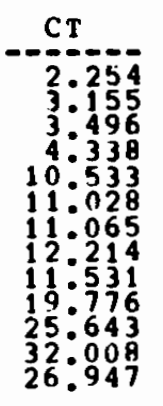 & \begin{tabular}{cc}
$D C$ \\
\hdashline $0 .-1$ \\
0 & 0 \\
0 & 0 \\
0 & 0 \\
0 & 0 \\
0 & 0 \\
0 & 0 \\
0 & 0 \\
0 & 0 \\
0 & 0 \\
0 & 0 \\
0 & 0 \\
0 & 0 \\
0 & 0 \\
0 & 0 \\
0 & 0
\end{tabular} & 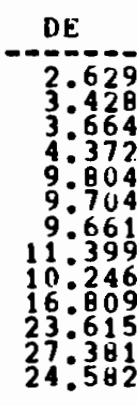 \\
\hline $\begin{array}{l}\text { YEAR } \\
1970 \\
1970 \\
1971 \\
1972 \\
1973 \\
1974 \\
1975 \\
1976 \\
1977 \\
1978 \\
1979 \\
1980 \\
1981 \\
1982\end{array}$ & 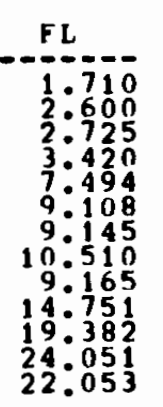 & 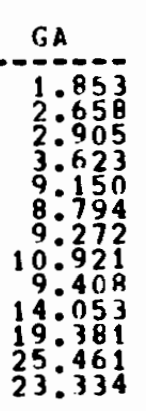 & 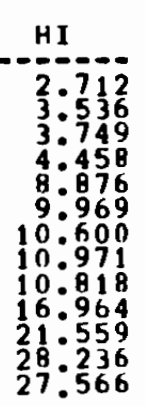 & 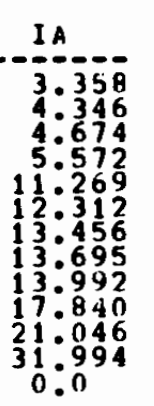 & 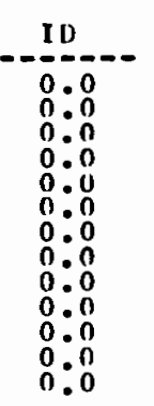 & 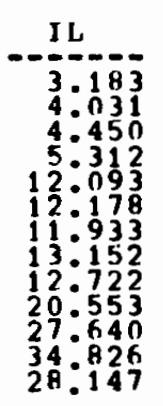 & \begin{tabular}{r}
$1 N$ \\
$-1 N-5$ \\
\hdashline $2: 242$ \\
$3: 080$ \\
$3: 325$ \\
$4: 064$ \\
$9: 514$ \\
90663 \\
$9: 560$ \\
$10: 812$ \\
$10: 959$ \\
$16: 005$ \\
$19: 336$ \\
$24: 898$ \\
$22: 225$
\end{tabular} & 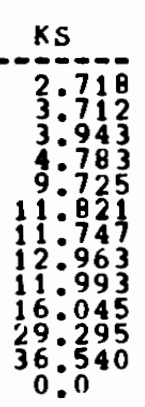 & \begin{aligned}$K Y \\
$\hdashline $0:- \\
0: 0 \\
0: 0 \\
0: 0 \\
0: 0 \\
0: 0 \\
0: 0 \\
0: 0 \\
0: 0 \\
0: 0 \\
0: 0 \\
0 \\
0: 0 \\
0\end{aligned}$ \\
\hline $\begin{array}{l}\text { YEAR } \\
1971 \\
1971 \\
1971 \\
1973 \\
1973 \\
1974 \\
1975 \\
1976 \\
1977 \\
1978 \\
1980 \\
1981 \\
1982\end{array}$ & 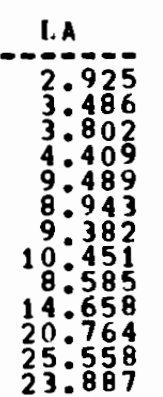 & 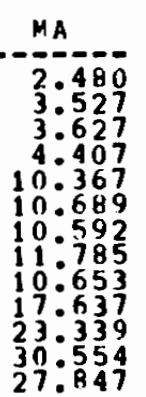 & 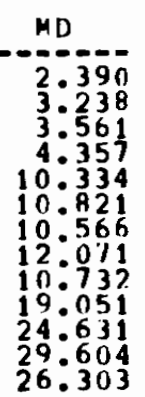 & 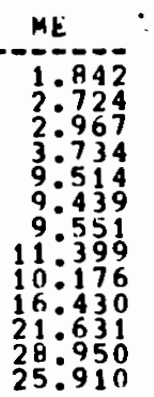 & \begin{tabular}{r}
$M I$ \\
\hdashline 2.464 \\
$3: 378$ \\
$3: 536$ \\
$4: 568$ \\
$8: 968$ \\
$10: 291$ \\
$9: 839$ \\
$11: 492$ \\
$10: 614$ \\
$13: 545$ \\
$18: 202$ \\
$24: 921$ \\
$23: 996$
\end{tabular} & 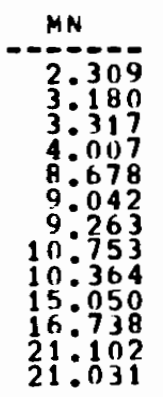 & \begin{tabular}{r}
$M L$ \\
\hdashline $2: 911$ \\
$4: 080$ \\
$4: 184$ \\
$5: 055$ \\
$10: 277$ \\
$11: 698$ \\
$11: 606$ \\
$13: 901$ \\
130295 \\
$17: 517$ \\
$22: 602$ \\
$31: 605$ \\
$23: 516$
\end{tabular} & 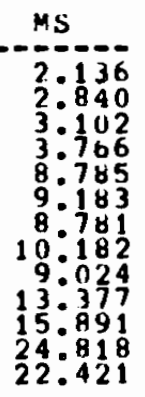 & $\begin{array}{cc}M T \\
-0 .- \\
0 & 0 \\
0 & 0 \\
0 & 0 \\
0 & 0 \\
0 & 0 \\
0 & 0 \\
0 & 0 \\
0 & 0 \\
0 & 0 \\
0 & 0 \\
0 & 0 \\
0 & 0 \\
0 & 0 \\
0 & 0\end{array}$ \\
\hline
\end{tabular}

(a) Data for 1982 are provisional estimates and will be updated when data from Annual Survey of Manufactures become available. 
TABLE 2.26 (cont). Residual Fuel Prices: Transportation Sector $(\$ / b b 1)^{(a)}$
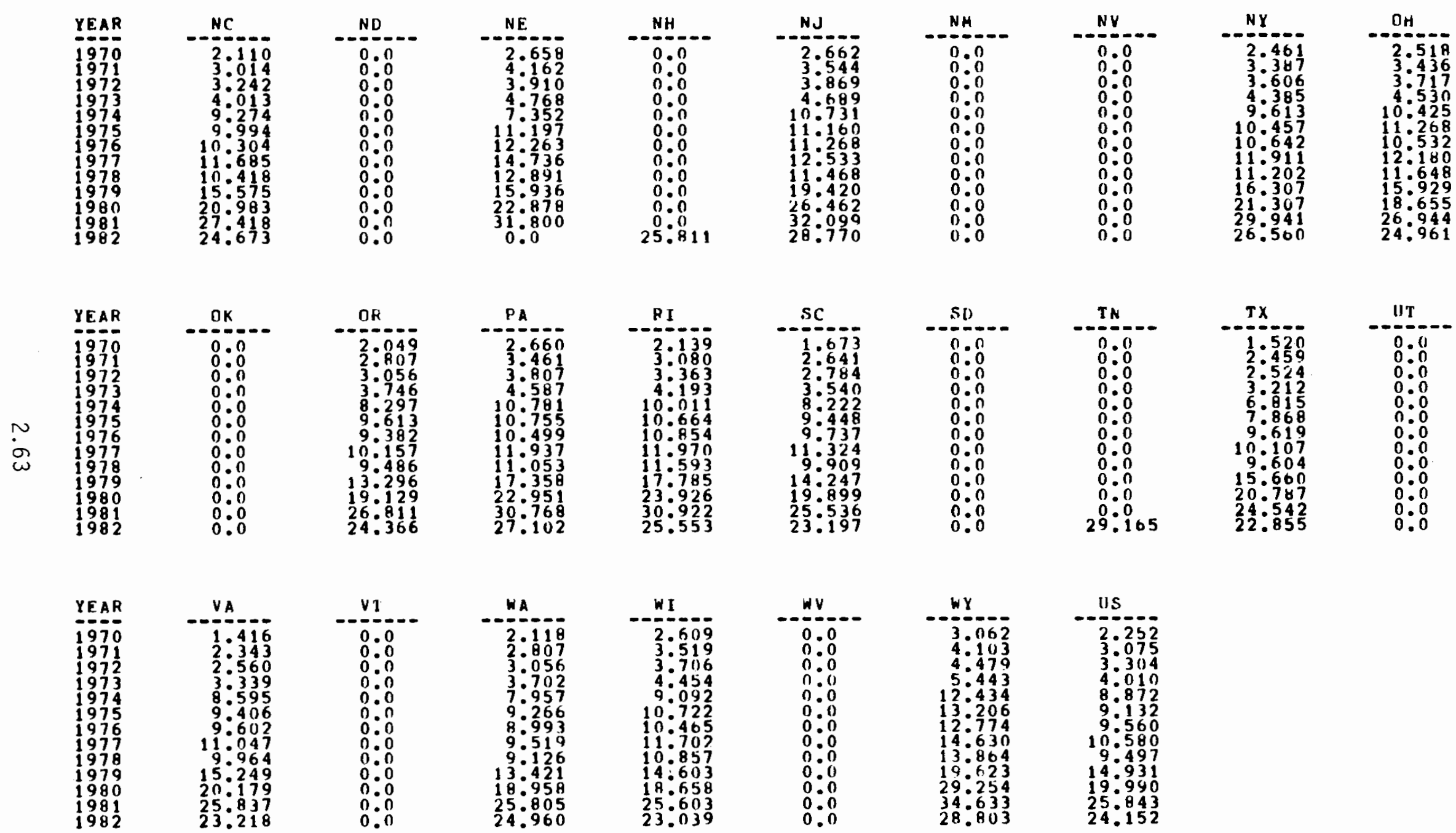

\begin{tabular}{l}
$v 1$ \\
\hdashline $0: 0$ \\
$0: 0$ \\
$0: 0$ \\
$0: 0$ \\
$0: 0$ \\
$0: 0$ \\
$0: 0$ \\
$0: 0$ \\
$0: 0$ \\
$0: 0$ \\
$0: 0$ \\
$0: 0$ \\
0.0
\end{tabular}
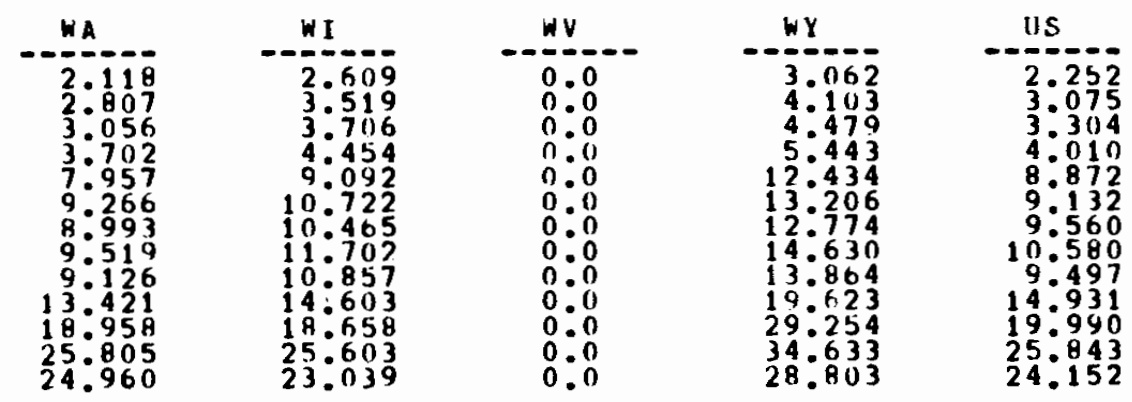

Source: Pacific Horthwest Laboratory 
TABLE 2.27. Residual Fuel Prices: Electric Utility Sector $(\$ / b b 1)$
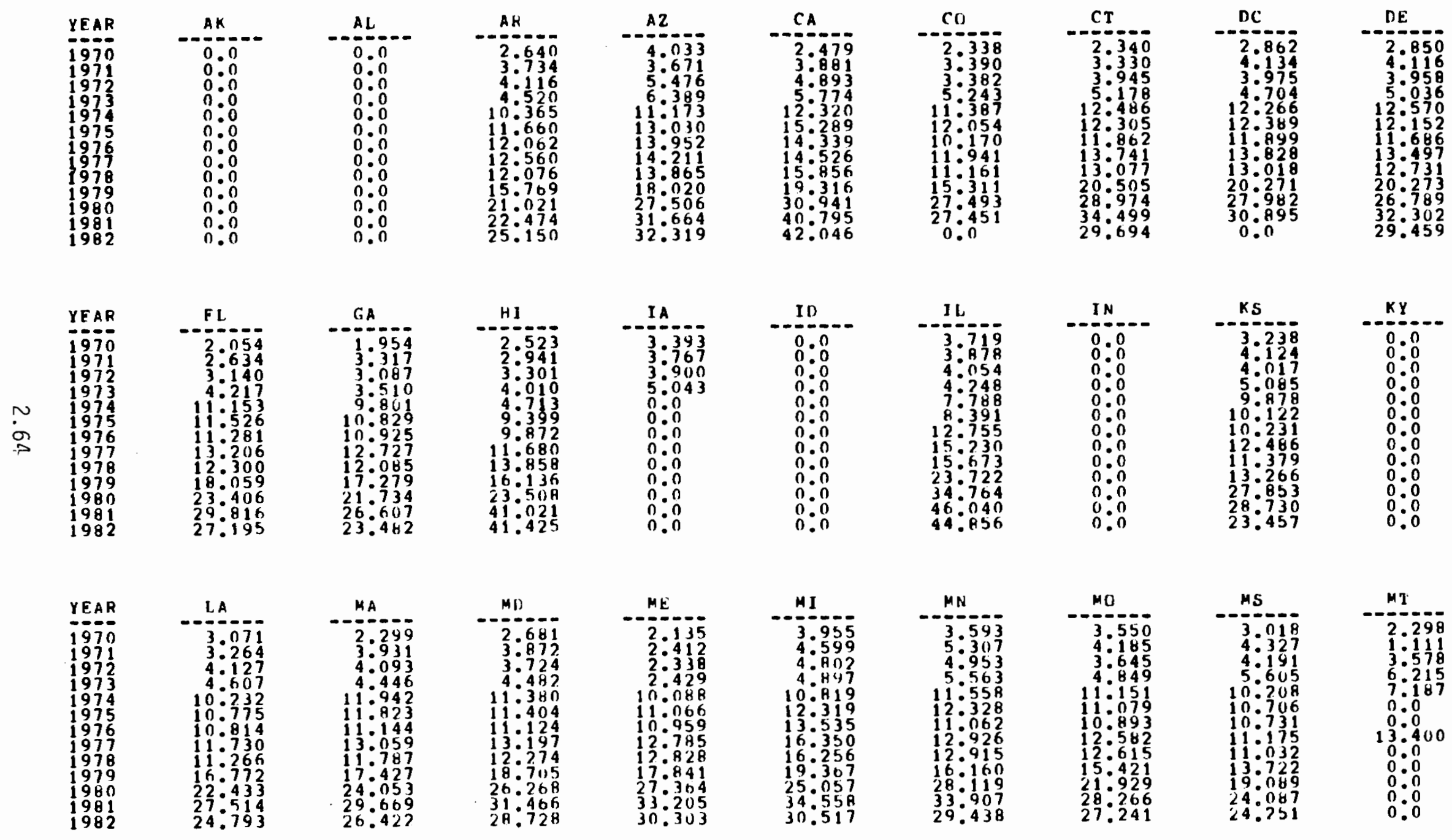
TABLE 2.27 (cont). Residual Fuel Prices: Electric Utility Sector ( $\$ / b b 1)$
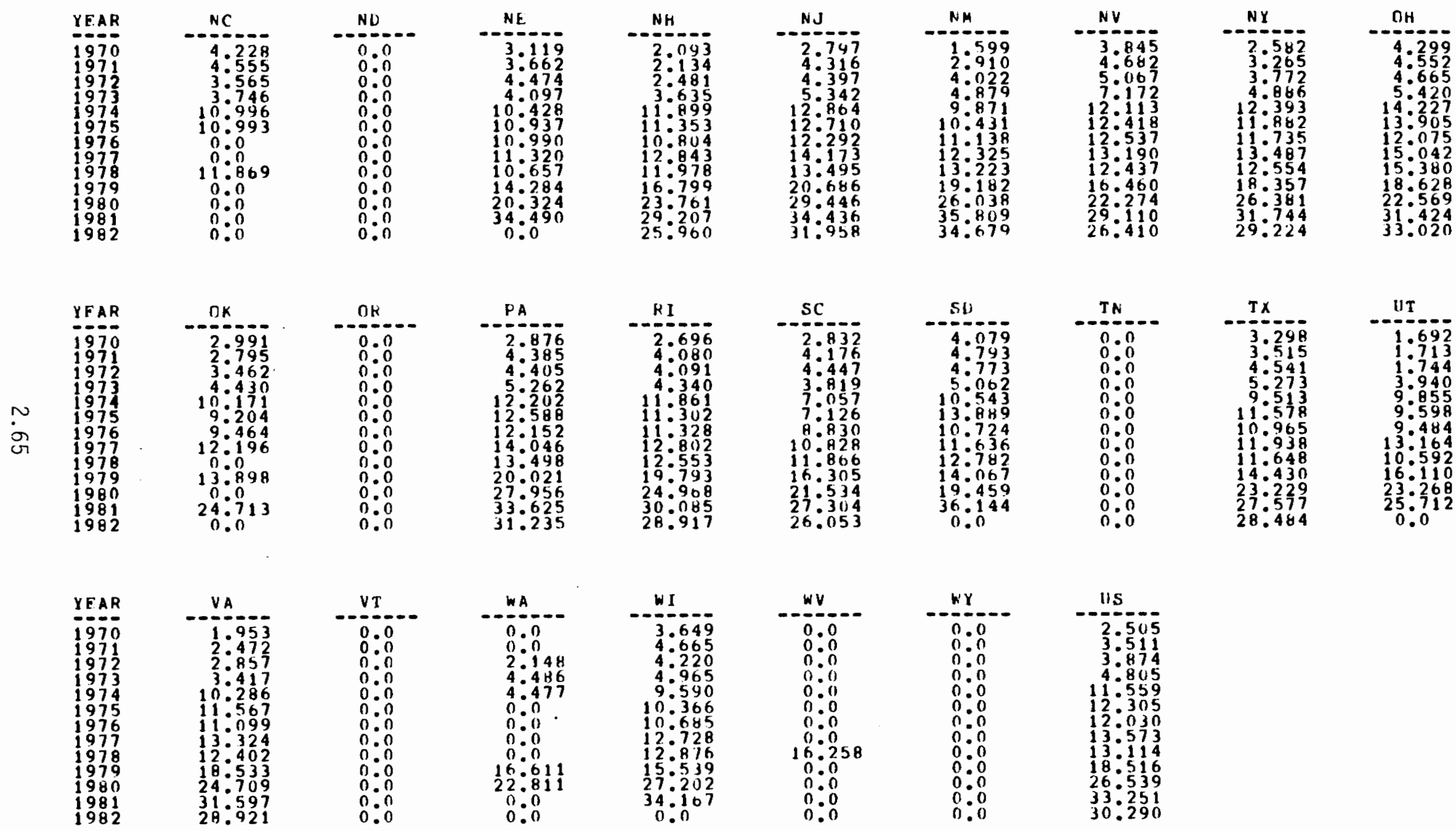
TABLE 2.28. Liquefied Petroleum Gas Prices: Residential Sector (\$/gallon)

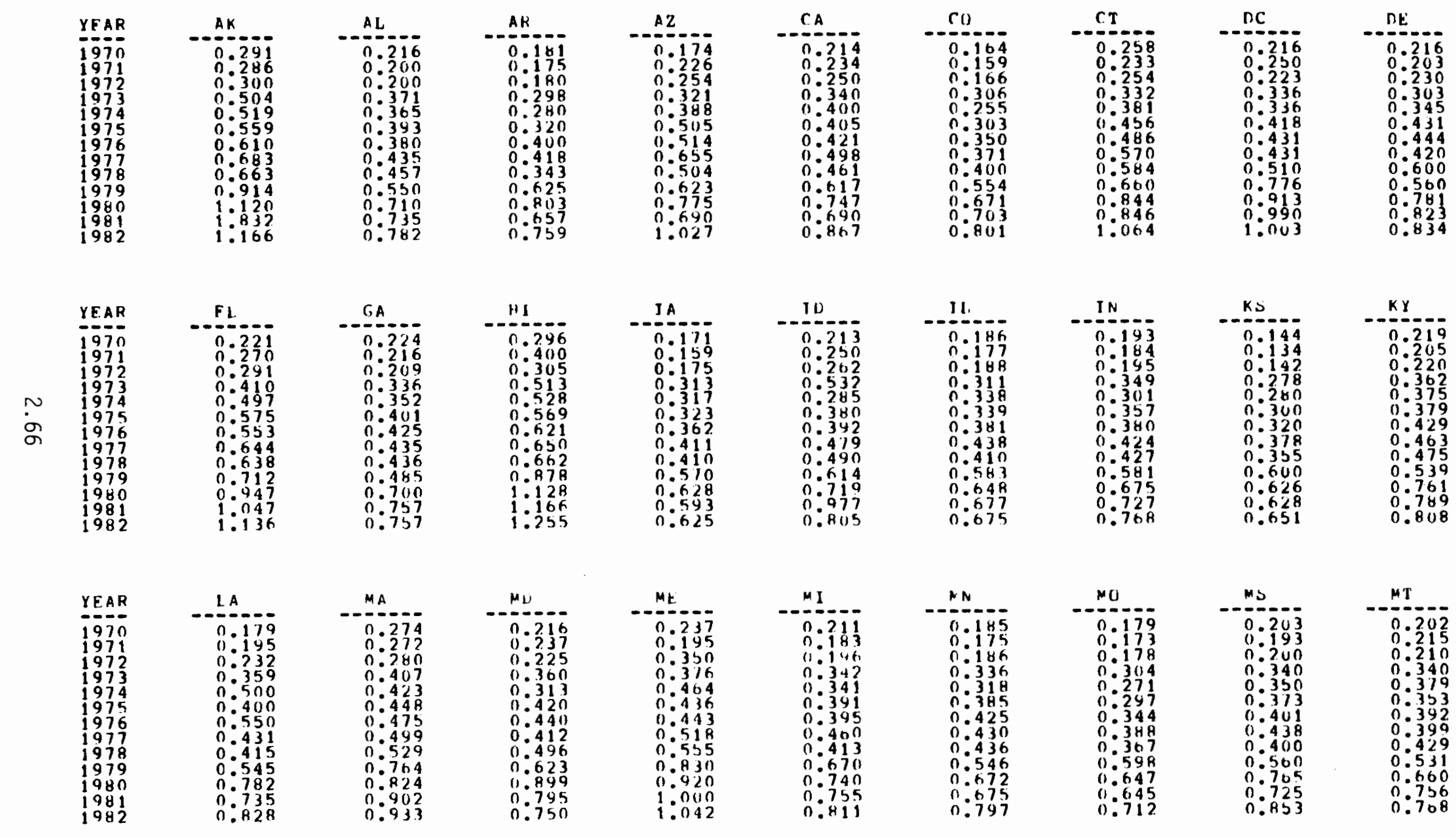


TABLE 2.28 (cont). Liquefied Petroleum Gas Prices: Residential Sector (\$/gallon)
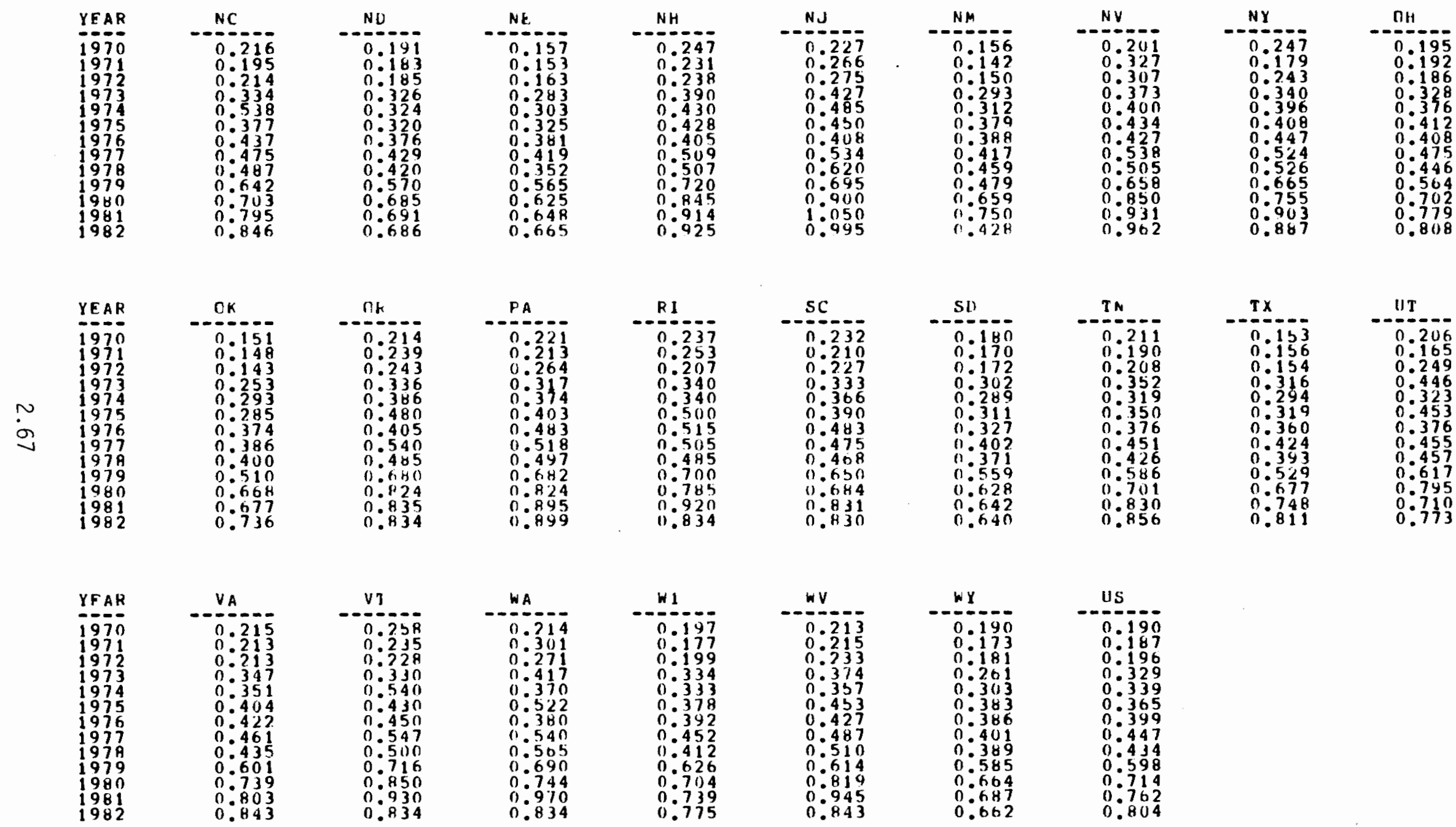
TABLE 2.29. Liquefied Petroleum Gas Prices: Industrial Sector (\$/gallon) (a)
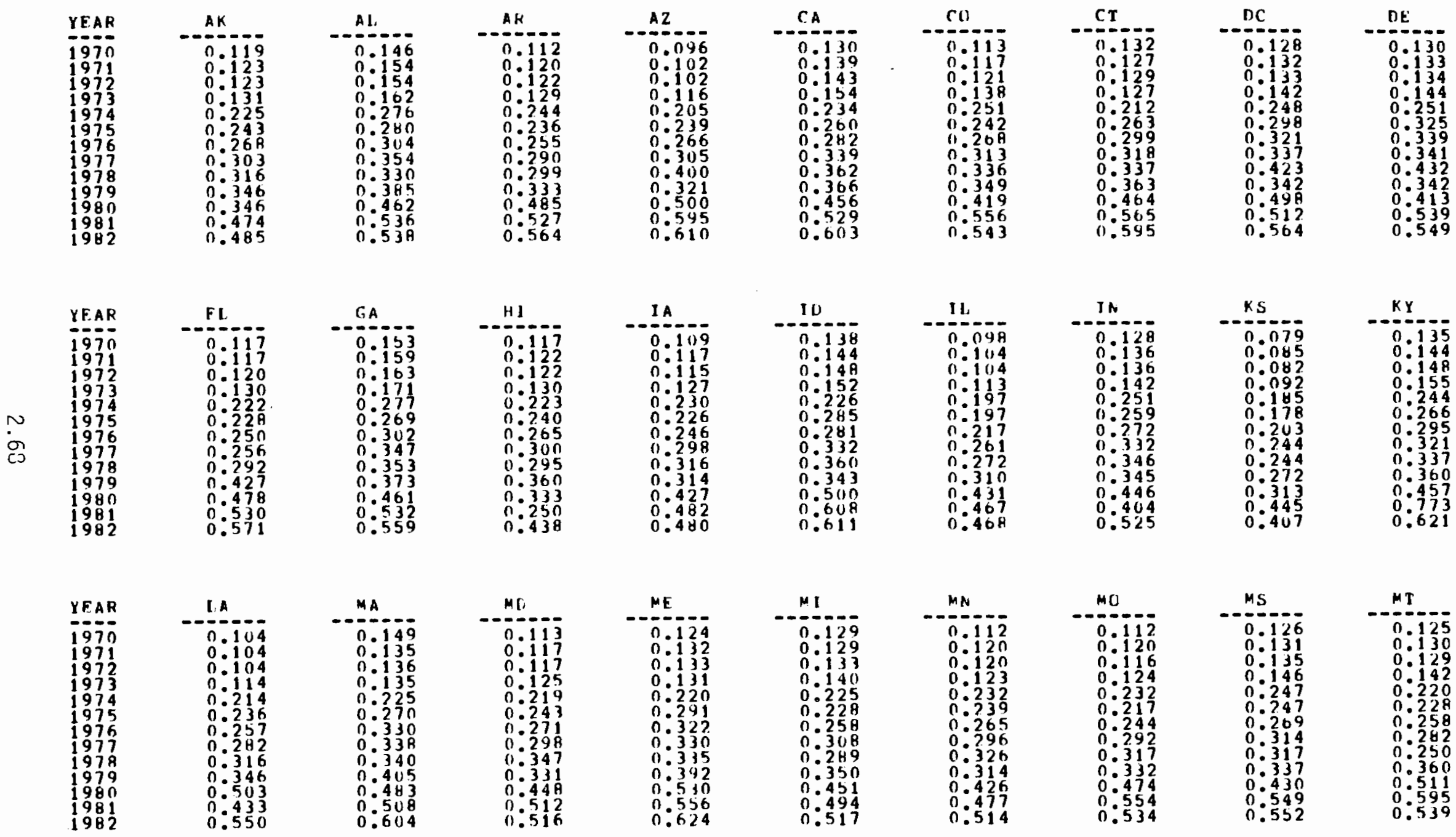

(a) Data for 1982 are provisional estimates and will be updated when data from Annual Survey of Manufactures become available. 
TABLE 2.29 (cont). Liquefied Petroleum Gas Prices: Industrial Sector (\$/gallon) ${ }^{(a)}$

\begin{tabular}{|c|c|c|c|c|c|c|c|c|c|}
\hline $\begin{array}{l}Y E A R \\
1970 \\
1970 \\
1971 \\
1973 \\
1974 \\
1975 \\
1976 \\
1977 \\
1978 \\
1979 \\
1980 \\
1981 \\
1982\end{array}$ & $\begin{array}{l}\text { NC } \\
-0.5 \\
0: 126 \\
0: 126 \\
0: 126 \\
0: 137 \\
0: 217 \\
0: 235 \\
0: 235 \\
0: 285 \\
0: 320 \\
0: 311 \\
0: 459 \\
0: 557 \\
0.540\end{array}$ & 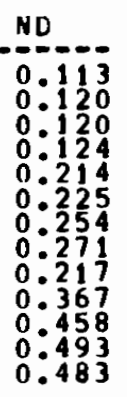 & 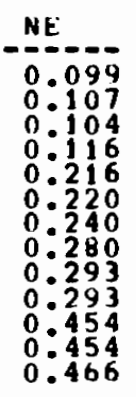 & 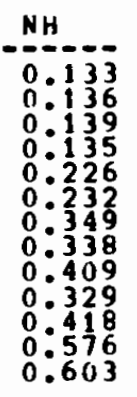 & 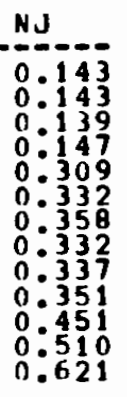 & 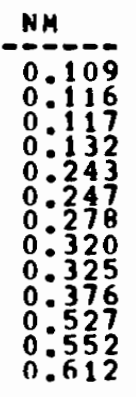 & 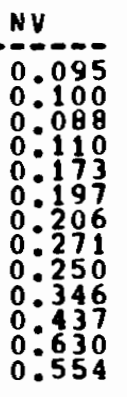 & 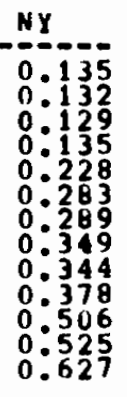 & \begin{tabular}{l} 
OH \\
\hdashline $0:-134$ \\
$0: 0137$ \\
$0: 137$ \\
$0: 147$ \\
$0: 252$ \\
$0: 269$ \\
$0: 266$ \\
$0: 333$ \\
$0: 329$ \\
$0: 381$ \\
$0: 467$ \\
$0: 512$ \\
$0: 586$
\end{tabular} \\
\hline $\begin{array}{l}\text { YEAR } \\
1970 \\
1970 \\
1971 \\
1972 \\
1973 \\
1974 \\
1975 \\
1976 \\
1977 \\
1978 \\
1979 \\
1980 \\
1981 \\
1982\end{array}$ & \begin{tabular}{l} 
ok \\
\hdashline $0: 103$ \\
$0: 113$ \\
$0: 109$ \\
$0: 122$ \\
$0: 243$ \\
$0: 230$ \\
$0: 257$ \\
$0: 324$ \\
$0: 346$ \\
$0: 355$ \\
$0: 300$ \\
$0: 545$ \\
0.575
\end{tabular} & 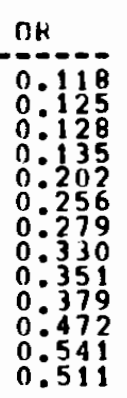 & 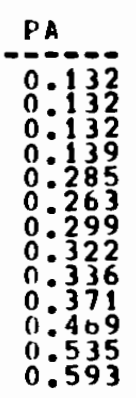 & 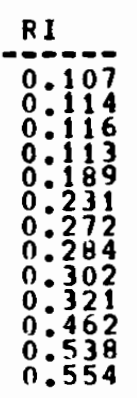 & 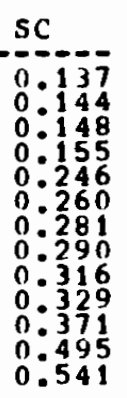 & 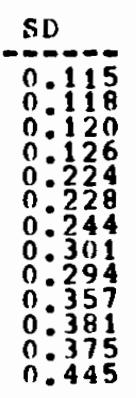 & 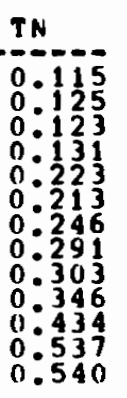 & 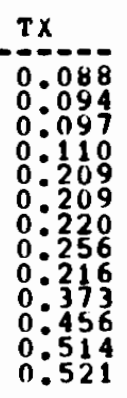 & 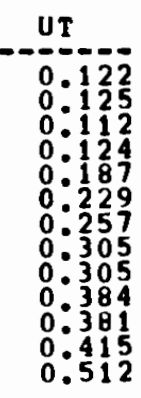 \\
\hline $\begin{array}{l}\text { YEAR } \\
1970 \\
1971 \\
1972 \\
1973 \\
1974 \\
1975 \\
1976 \\
1977 \\
1978 \\
1979 \\
1980 \\
1981 \\
1982\end{array}$ & \begin{tabular}{l}
$V A$ \\
\hdashline $0: 134$ \\
$0: 140$ \\
$0: 141$ \\
$0: 150$ \\
$0: 243$ \\
$0: 269$ \\
$0: 276$ \\
$0: 325$ \\
$0: 313$ \\
$0: 372$ \\
$0: 402$ \\
$0: 506$ \\
0.545
\end{tabular} & 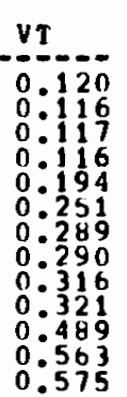 & 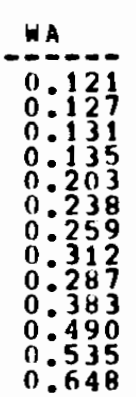 & 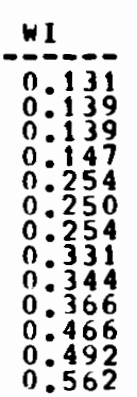 & 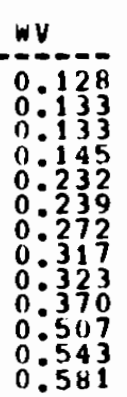 & 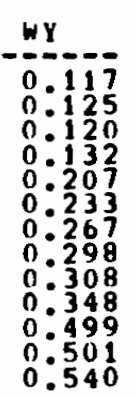 & 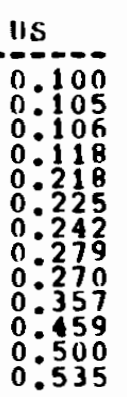 & & \\
\hline
\end{tabular}

Source: Pacific Northwest Laboratory 


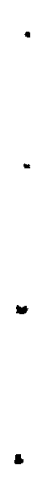

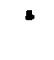$$
\text { . }
$$ 


\subsection{BTU PRICES}

This chapter presents the updated state-level Btu prices, in nominal dollars per million Btu. The periods covered are 1960 through 1982 for electricity and natural gas, and 1970 through 1982 for metallurgical coal, steam coal, distillate, motor gasoline, diesel, kerosene/jet fuel, residual oil, and LPG. Please refer to Chapter 4.0 of State Energy Price System, Volume 1: Overview and Technical Documentation for basic documentation of data sources and estimation procedures. Also refer to Chapter 4.0 of this report for all the procedural changes necessitated by the updates to include price data for 1981 and 1982, and for the corrections made to the original STEPS documentation. Note that where changes are made in the physical price data, Btu prices are recalculated so the two data bases accurately translate into each other. Conversion factors used for the updates are found in Appendix $A$. 
IABLE 3.1. Electricity Prices: Residential Sector (\$/million Btu)

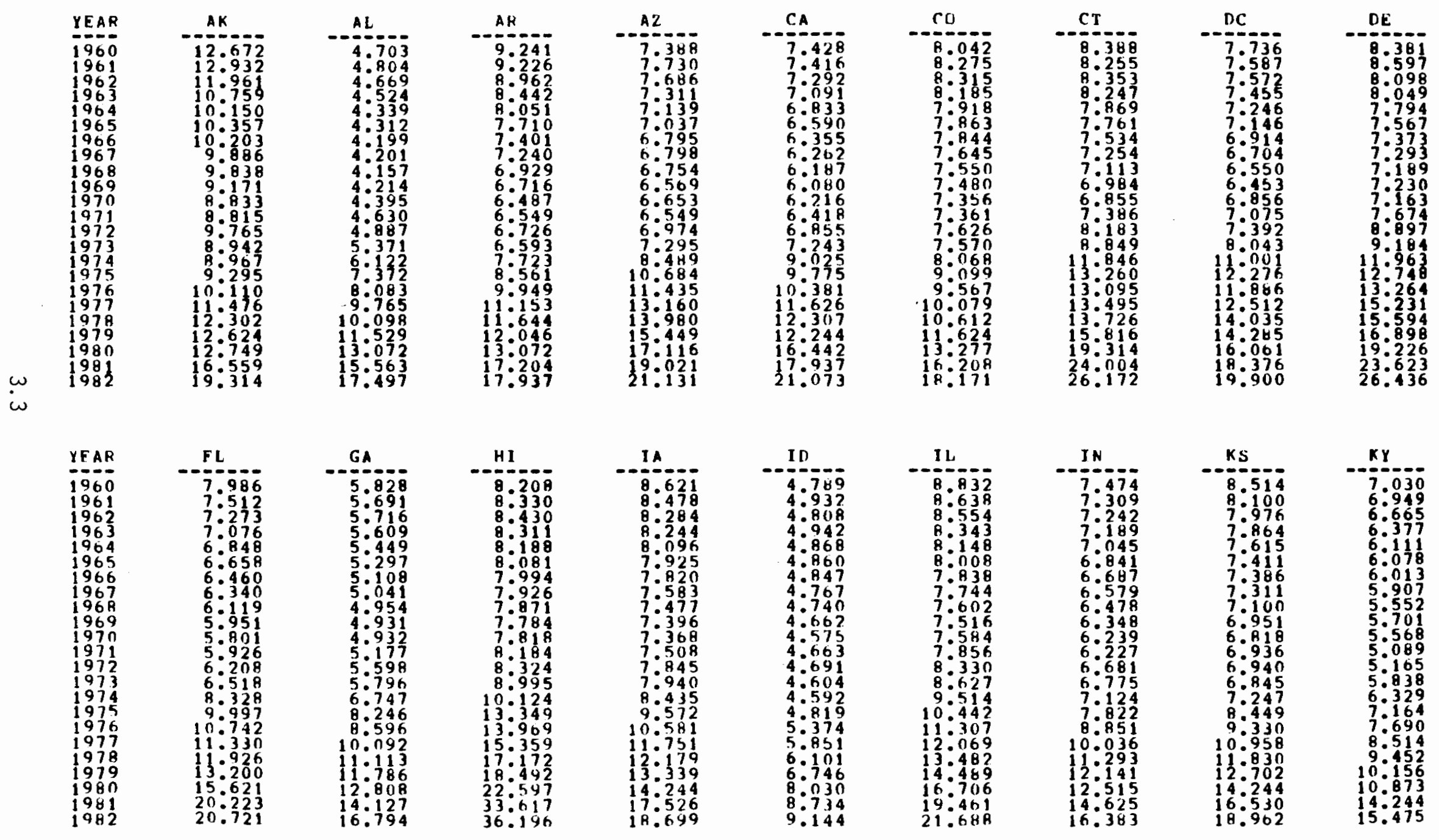


TABLE 3.1 (cont). Electricity Prices: Residential Sector (\$/millian Btu)

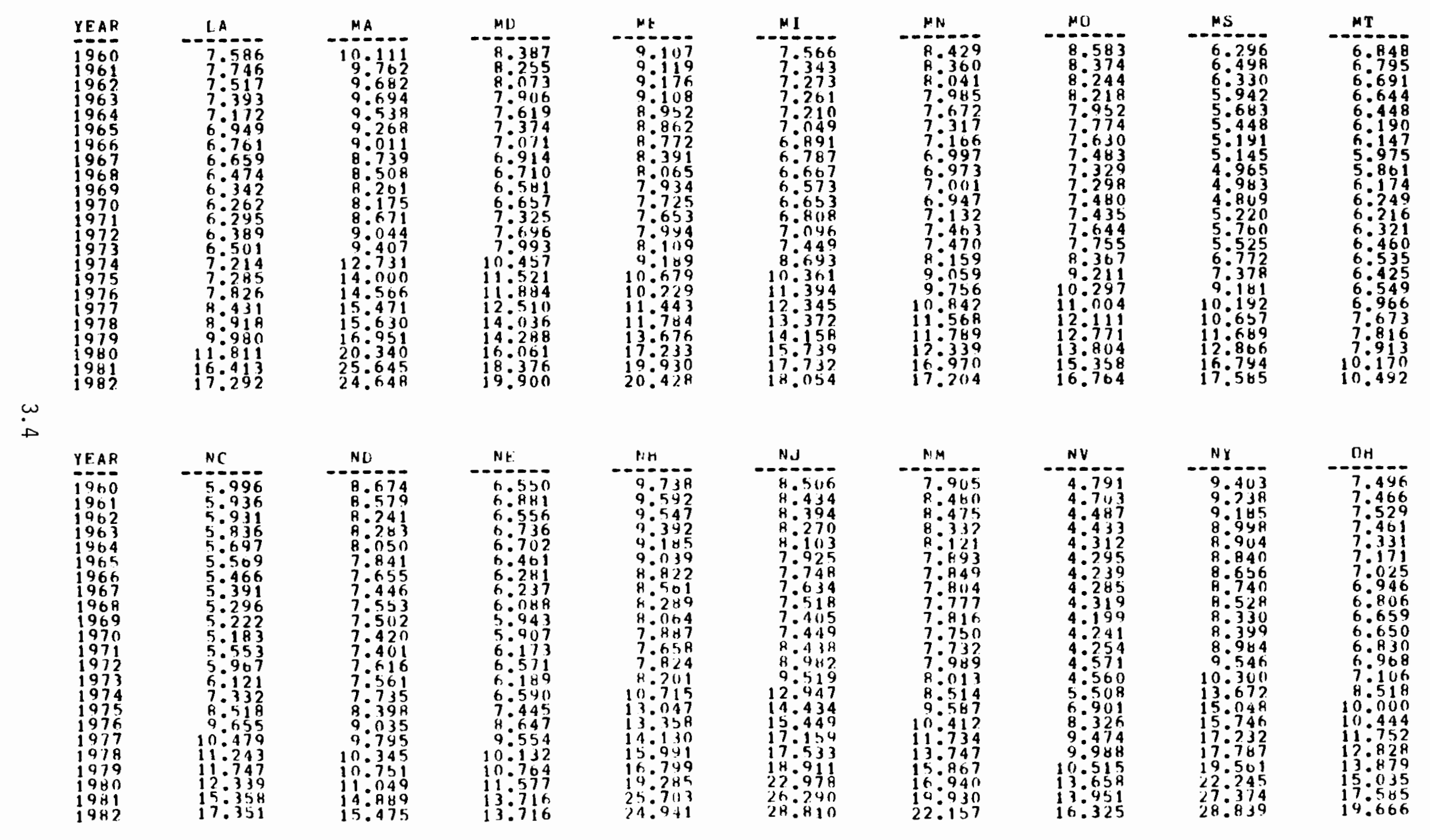


TABLE 3.1 (cont). Electricity Prices: Residential Sector ( $\$ / m i 11$ ion Btu)

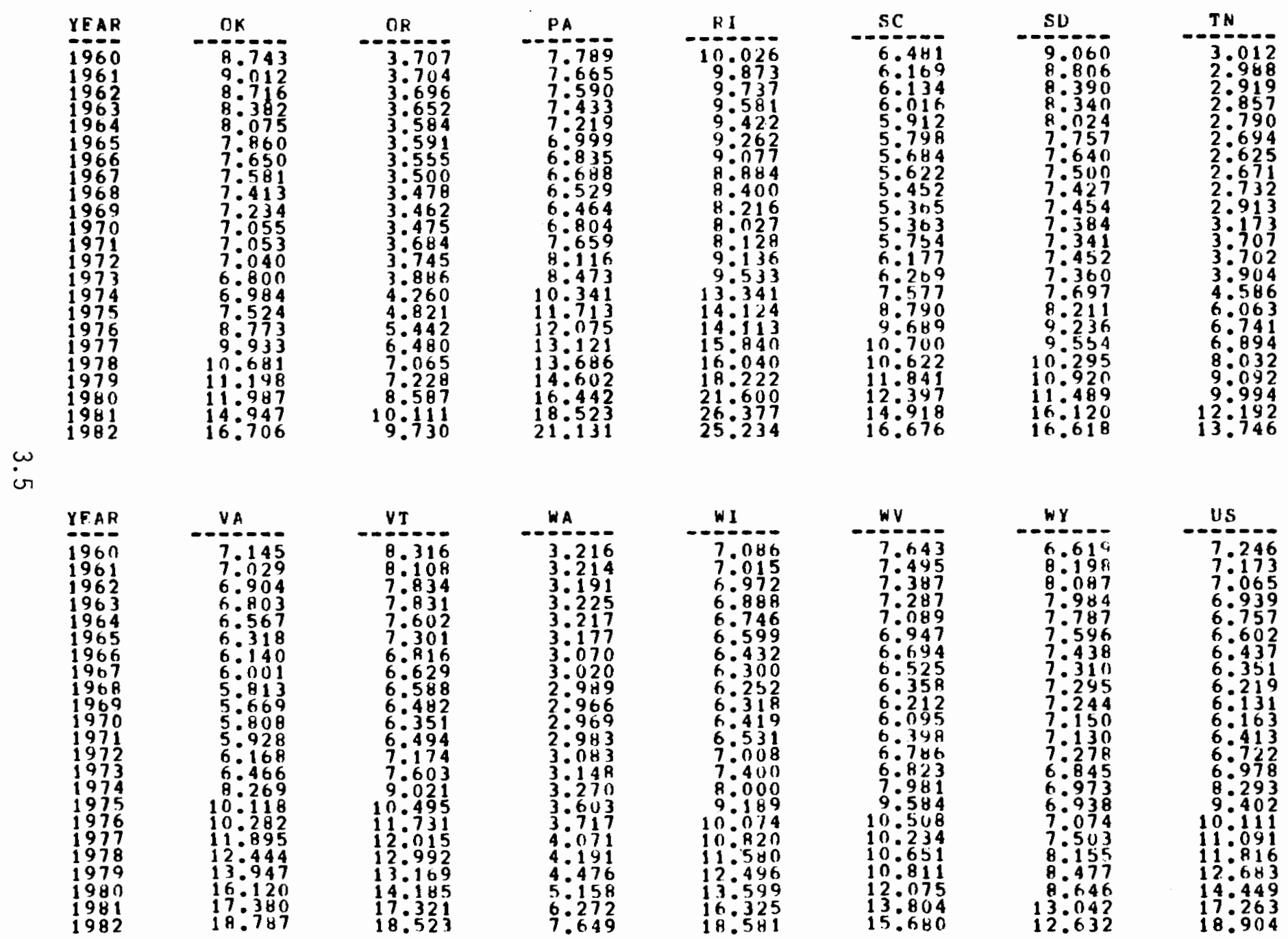


TABLE 3.2. Electricity Prices: Commercial Sector $(\$ / m i l l i o n$ Btu)

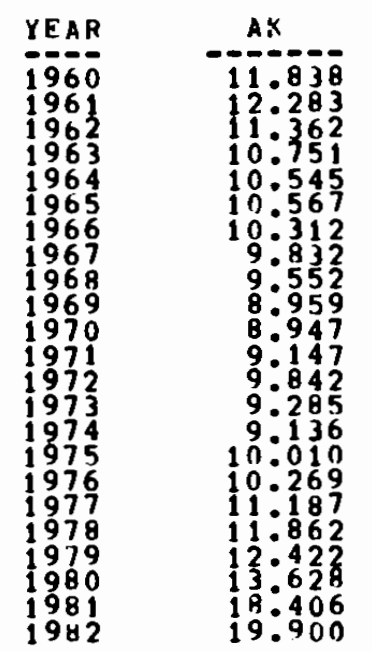

AL
-5.641
5.501
5.508
$5: 450$
$5: 365$
$5: 214$
$5: 177$
5.164
$5: 072$
50.050
5.097
5.349
$5: 588$
$6: 265$
7.086
$8: 306$
0.861
11.185
11.238
12.980
14.654
$16: 735$
18.728
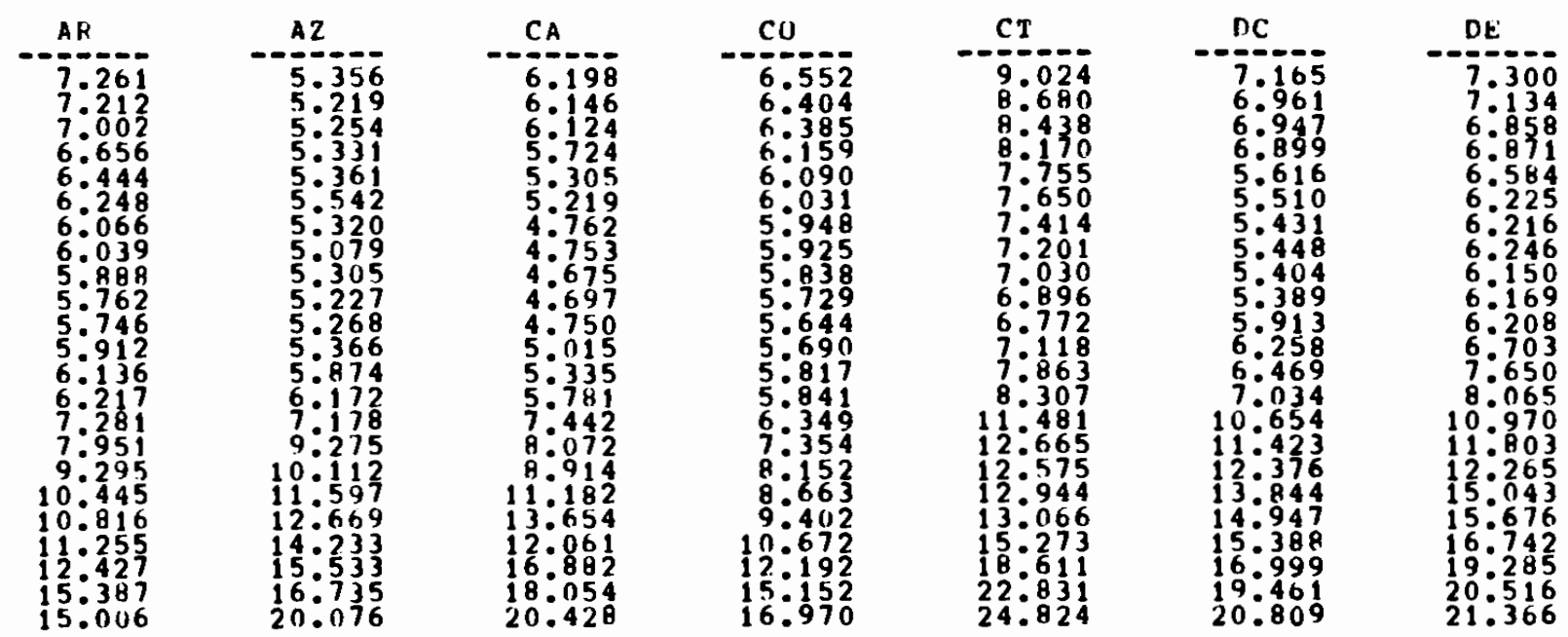

o

\begin{tabular}{lr} 
YEAR & FL \\
\hline 1960 & --1.75 \\
1961 & 7.756 \\
1962 & 7.546 \\
1962 & 7.317 \\
1963 & 7.161 \\
1964 & 6.917 \\
1965 & 6.591 \\
1966 & 6.296 \\
1967 & 6.141 \\
1968 & 5981 \\
1969 & 6.904 \\
1970 & 6.315 \\
1971 & 6.790 \\
1972 & 8.876 \\
1973 & 10.580 \\
1974 & 11.041 \\
1975 & 11.856 \\
1976 & 12.451 \\
1977 & 13.801 \\
1978 & 16.090 \\
1979 & 20.076 \\
1980 & 18.992 \\
1981 &
\end{tabular}
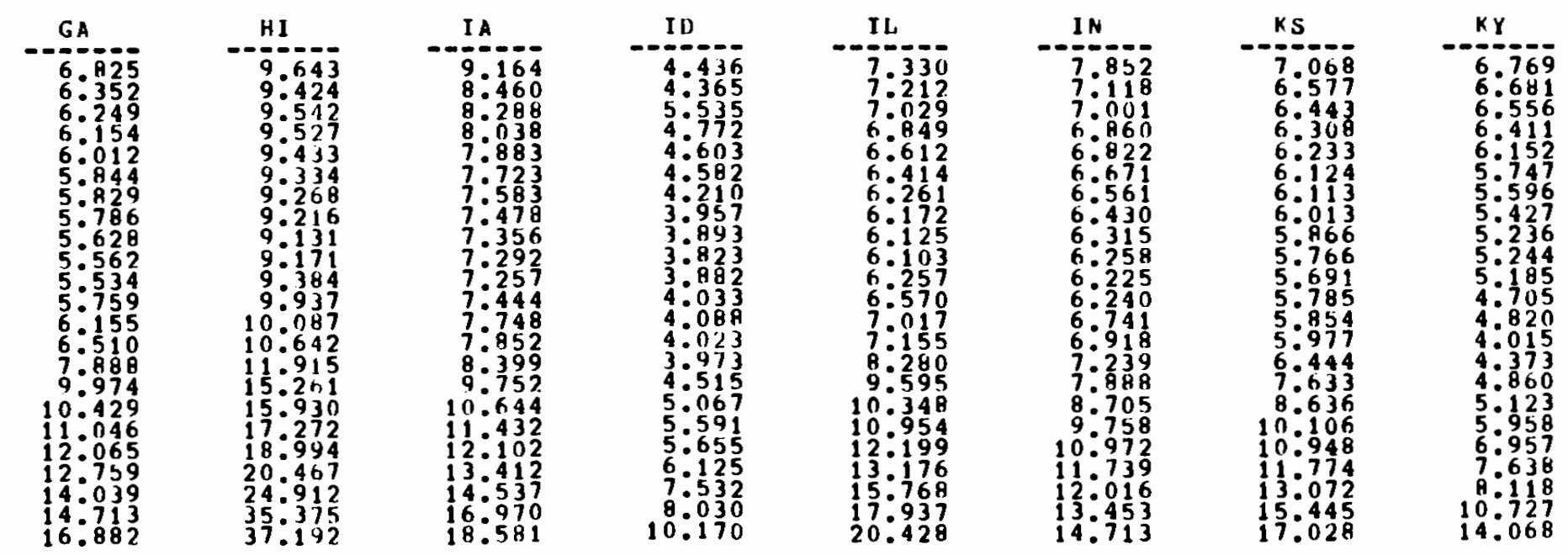
TABLE 3.2 (cont). Electricity Prices: Commercial Sector ( $\$ /$ million Btu)

\begin{tabular}{|c|c|c|c|c|c|c|c|c|c|}
\hline $\begin{array}{l}\text { YEAR } \\
1960 \\
1960 \\
1961 \\
1962 \\
1963 \\
1964 \\
1965 \\
1966 \\
1967 \\
1968 \\
1969 \\
1970 \\
1971 \\
1972 \\
1973 \\
1974 \\
1975 \\
1976 \\
1977 \\
1978 \\
1979 \\
1980 \\
1981 \\
1982\end{array}$ & 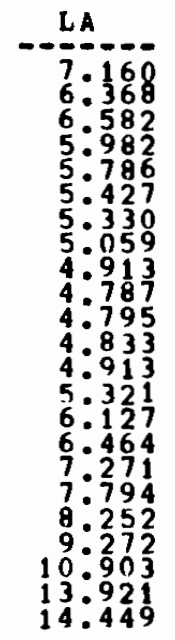 & \begin{tabular}{r}
$M A$ \\
\hdashline $11: 276$ \\
$10: 291$ \\
$9: 336$ \\
$9: 176$ \\
$9: 105$ \\
$8: 850$ \\
$8: 569$ \\
$8: 301$ \\
$7: 750$ \\
$7: 655$ \\
$7: 611$ \\
$8: 126$ \\
$8: 372$ \\
$8: 618$ \\
$12: 236$ \\
$13: 309$ \\
$14: 015$ \\
$14: 971$ \\
$15: 222$ \\
$16: 569$ \\
20193 \\
$24: 971$ \\
$24: 091$
\end{tabular} & 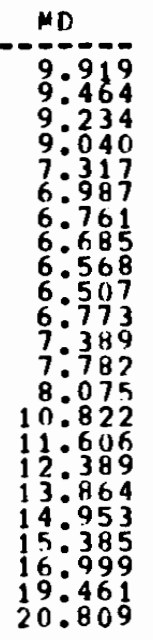 & 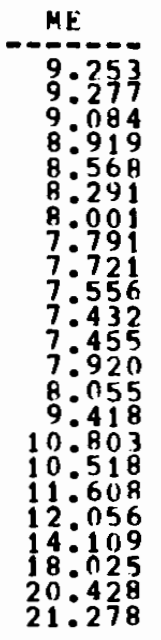 & 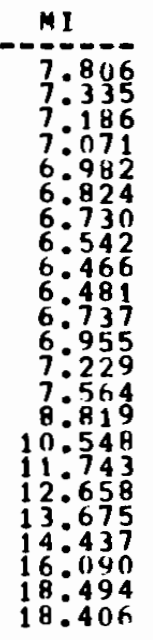 & 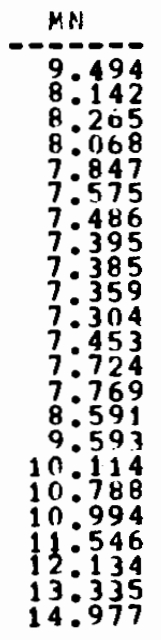 & 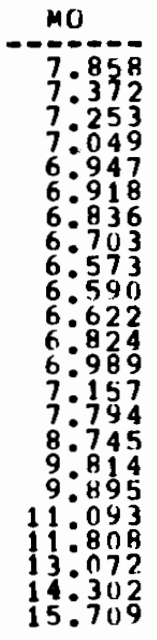 & \begin{tabular}{r}
$M S$ \\
\hdashline 6.483 \\
$6: 347$ \\
$6: 247$ \\
$6: 043$ \\
$5: 715$ \\
$5: 280$ \\
$5: 151$ \\
$5: 160$ \\
$5: 214$ \\
$5: 201$ \\
$5: 234$ \\
$5: 566$ \\
$6: 014$ \\
6027 \\
$7: 473$ \\
$7: 946$ \\
90710 \\
$10: 580$ \\
$10: 994$ \\
$12: 518$ \\
130834 \\
$18: 200$ \\
$18: 640$
\end{tabular} & $\begin{array}{c}M T \\
-6.228 \\
6: 033 \\
5: 840 \\
5: 687 \\
5: 544 \\
5: 350 \\
5: 302 \\
5: 130 \\
5: 033 \\
5: 355 \\
5: 432 \\
5: 457 \\
5: 590 \\
5: 784 \\
5: 802 \\
5: 908 \\
5: 910 \\
5: 535 \\
7: 199 \\
7: 242 \\
7: 386 \\
9: 379 \\
9: 437\end{array}$ \\
\hline & & & & & & & & & \\
\hline $\begin{array}{l}\text { YEAR } \\
1960 \\
1960 \\
1961 \\
1962 \\
1963 \\
1964 \\
1965 \\
1966 \\
1967 \\
1968 \\
1969 \\
1970 \\
1971 \\
1972 \\
1973 \\
1974 \\
1975 \\
1976 \\
1997 \\
1978 \\
1997 \\
1990 \\
1981 \\
1982\end{array}$ & 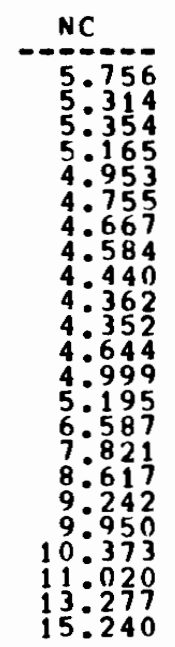 & \begin{tabular}{r} 
ND \\
\hdashline $10: 399$ \\
$9: 761$ \\
$9: 314$ \\
$9: 014$ \\
$8: 572$ \\
$7: 863$ \\
$7: 583$ \\
$6: 612$ \\
$6: 324$ \\
$6: 252$ \\
$6: 258$ \\
$6: 206$ \\
$6: 433$ \\
$6: 427$ \\
$6: 712$ \\
7.246 \\
$7: 902$ \\
$8: 515$ \\
$9: 077$ \\
$9: 532$ \\
$9: 818$ \\
$13: 482$ \\
13.892
\end{tabular} & 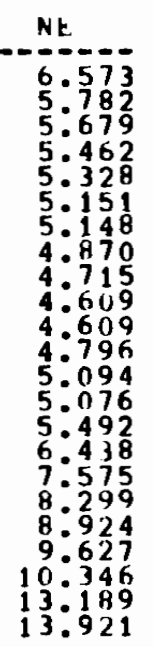 & 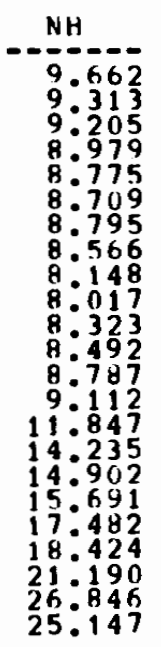 & 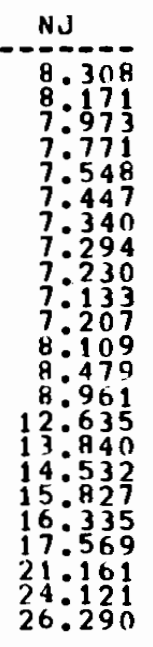 & 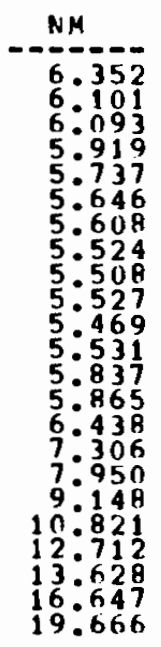 & $\begin{array}{r}N V \\
-4.996 \\
4: 996 \\
4: 112 \\
4: 893 \\
4: 947 \\
4: 949 \\
4: 846 \\
4: 693 \\
4: 564 \\
4: 486 \\
4: 440 \\
4: 479 \\
4: 602 \\
4: 931 \\
5: 028 \\
5: 935 \\
7: 404 \\
8: 576 \\
9: 668 \\
10: 501 \\
10.769 \\
14: 097 \\
15: 542 \\
20: 281\end{array}$ & 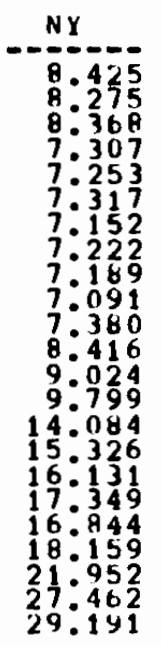 & $\begin{array}{r}6.996 \\
6: 840 \\
6: 855 \\
6: 690 \\
6: 519 \\
6: 304 \\
6: 197 \\
6: 145 \\
6: 049 \\
6: 003 \\
5: 991 \\
6: 274 \\
6: 411 \\
6: 564 \\
8: 013 \\
9033 \\
9: 714 \\
11: 205 \\
12: 239 \\
130280 \\
14.566 \\
16: 647 \\
17.701\end{array}$ \\
\hline
\end{tabular}


TABLE 3.2 (cont). Electricity Prices: Commercial Sector ( $\$ /$ million Btu)
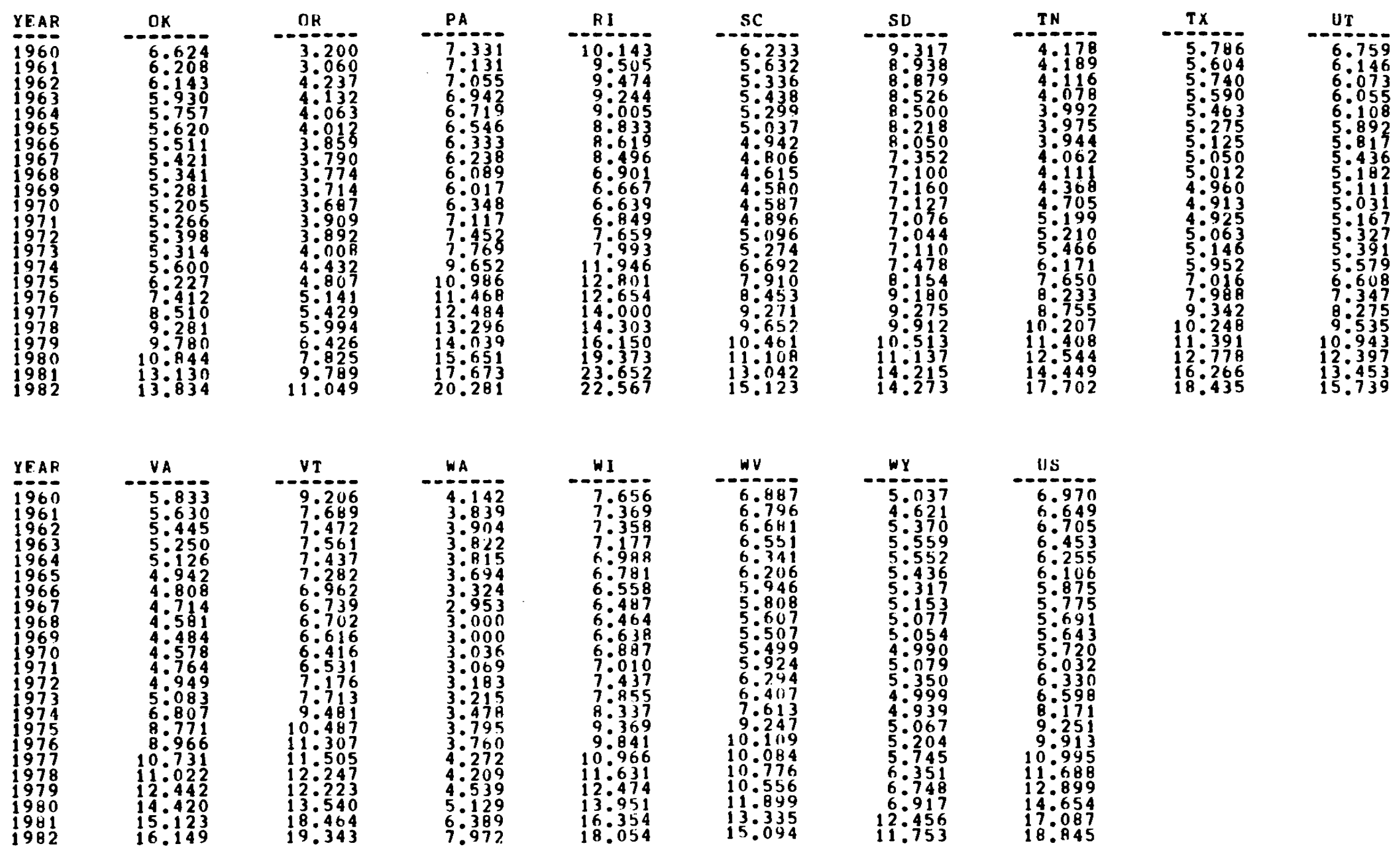

$W V$
-6.887
6.796
6.796
6.641
6.551
6.341
6.206
5.946
5.808
5.607
5.507
5.499
5.924
6.294
6.407
7.613
90.247
10.1199
10.084
10.776
10.556
11.899
13.335
15.094
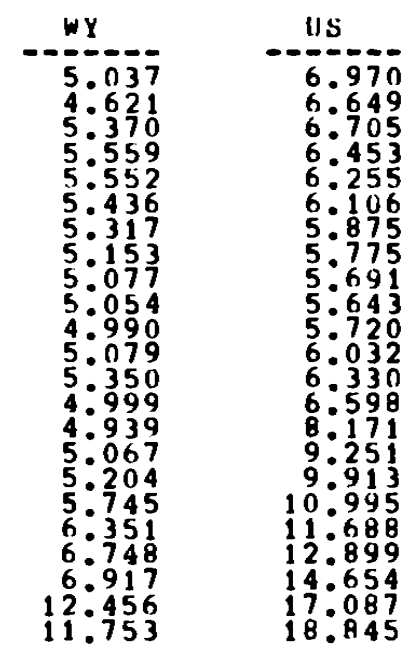
TABLE 3.3. Electricity Prices: Industrial Sector (\$/million Btu)
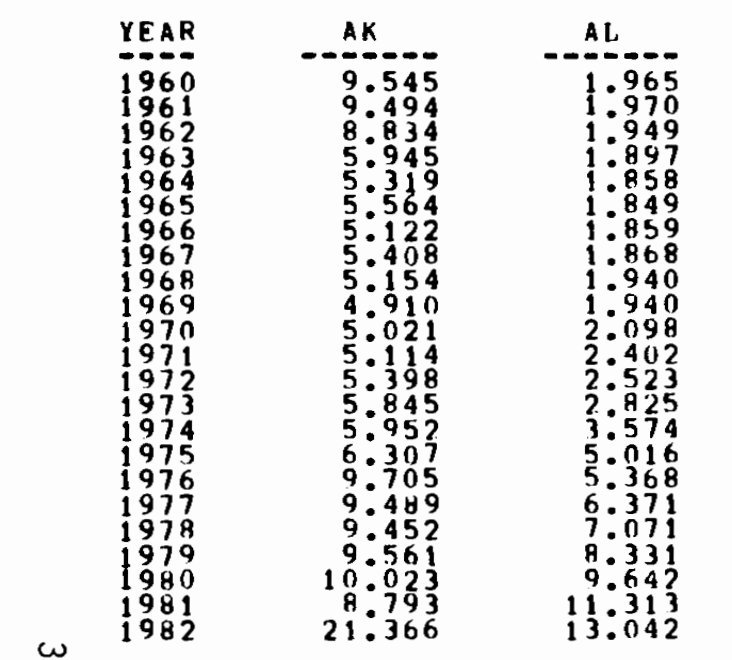

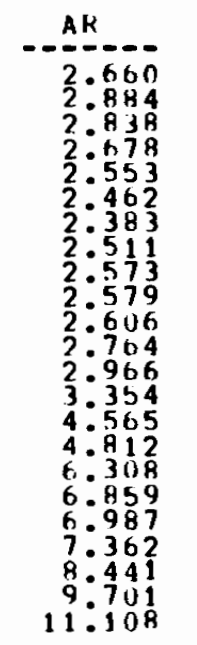
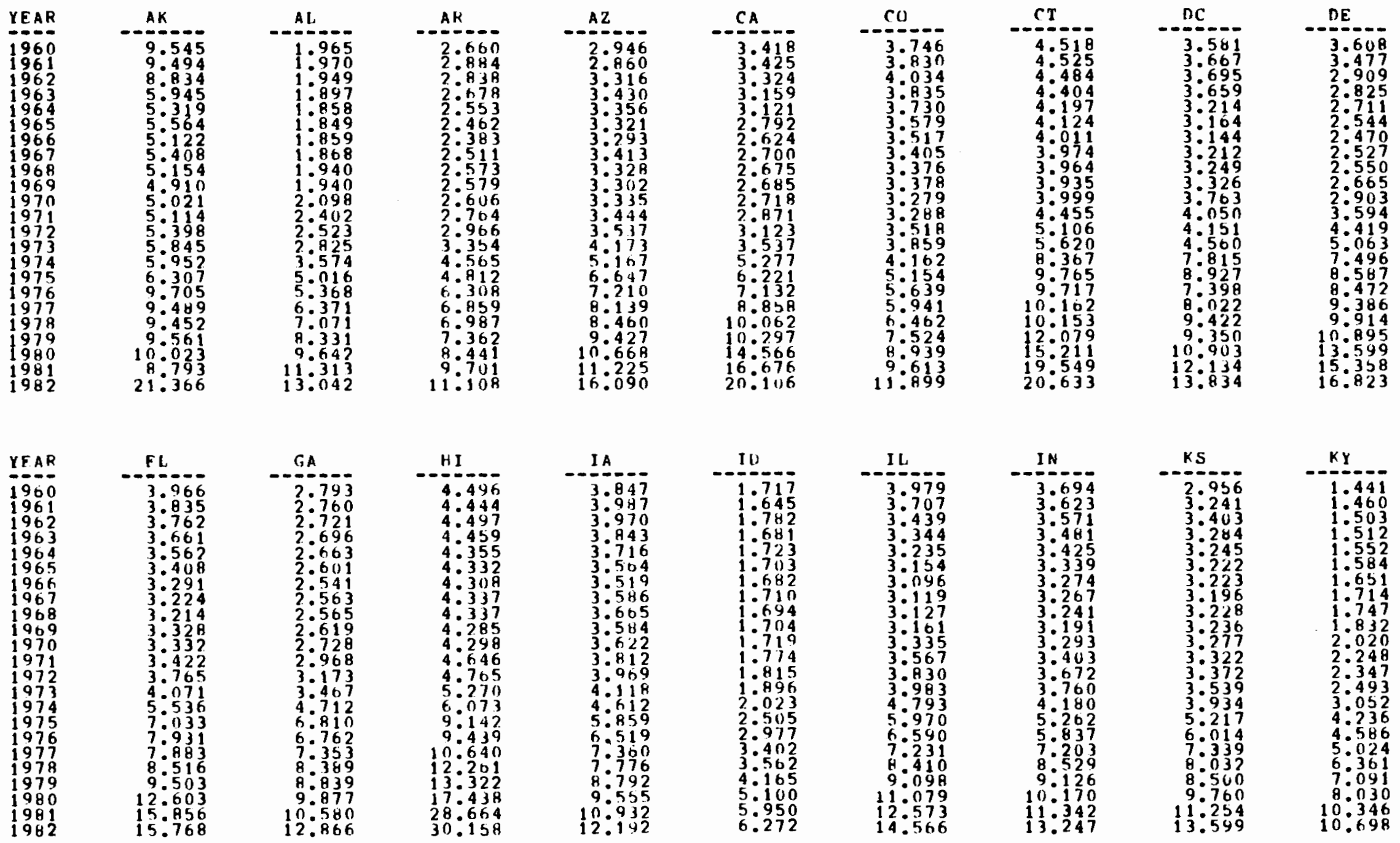
TABLE 3.3 (cont). Electricity Prices: Industrial Sector (\$/million Btu)

\begin{tabular}{|c|c|c|c|c|c|c|c|c|c|}
\hline $\begin{array}{l}\text { YEAR } \\
1960 \\
1960 \\
1961 \\
1962 \\
1963 \\
1964 \\
1965 \\
1966 \\
1967 \\
1968 \\
1969 \\
1970 \\
1971 \\
1972 \\
1973 \\
1974 \\
1975 \\
1976 \\
1977 \\
1978 \\
1999 \\
1980 \\
1981\end{array}$ & 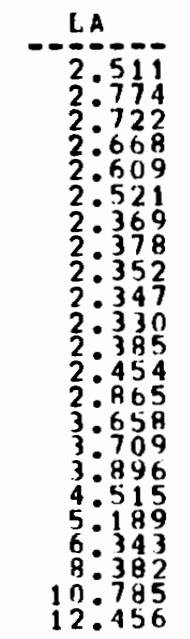 & 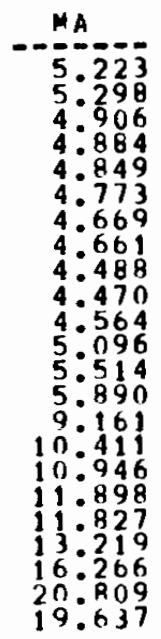 & 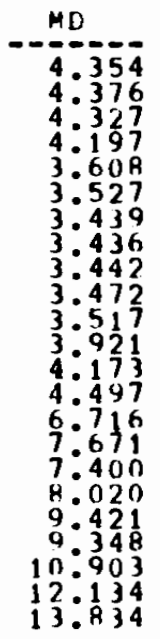 & 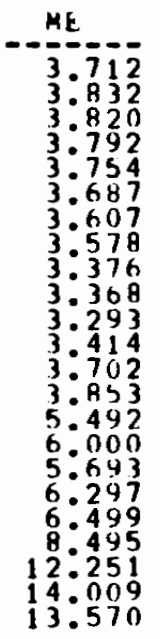 & 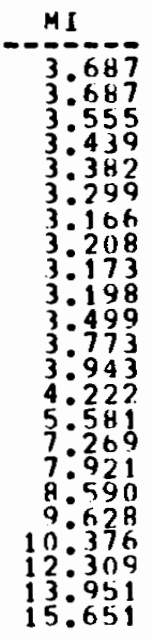 & $\begin{array}{r}M N \\
-4.480 \\
4: 595 \\
4: 601 \\
4: 368 \\
4: 227 \\
4: 142 \\
4: 048 \\
3: 938 \\
3: 829 \\
3: 845 \\
3: 905 \\
4: 110 \\
4: 313 \\
4: 357 \\
5097 \\
6: 248 \\
6: 930 \\
8: 188 \\
80591 \\
8.951 \\
102029 \\
11: 489 \\
13.453\end{array}$ & 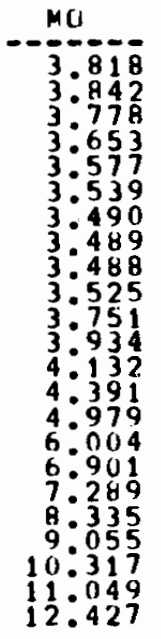 & 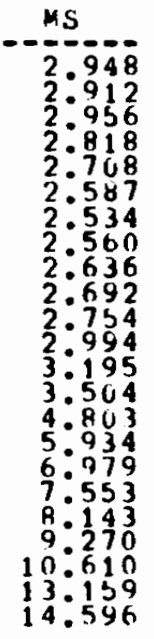 & $\begin{array}{l}M T \\
-1:-258 \\
1: 308 \\
1: 342 \\
1: 328 \\
1: 321 \\
1: 290 \\
1: 246 \\
1: 241 \\
1: 184 \\
1: 189 \\
1: 244 \\
1: 252 \\
1: 553 \\
1: 473 \\
1: 821 \\
1: 786 \\
1: 968 \\
2: 116 \\
2: 336 \\
2: 784 \\
3: 693 \\
6: 096\end{array}$ \\
\hline & & & & & & & & & \\
\hline 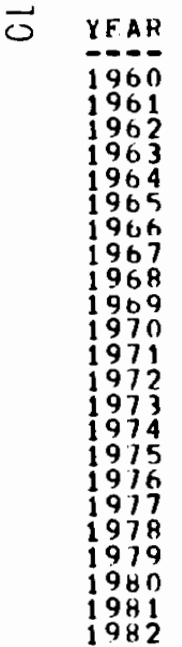 & 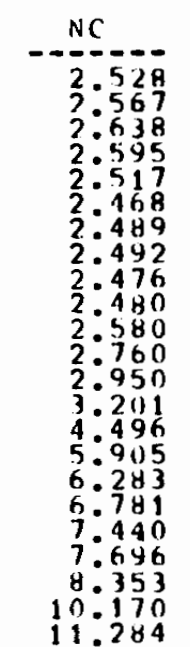 & 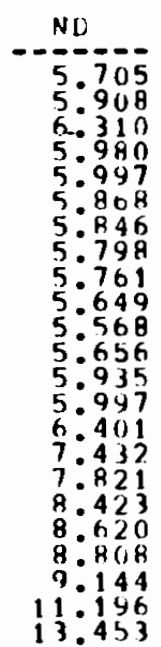 & 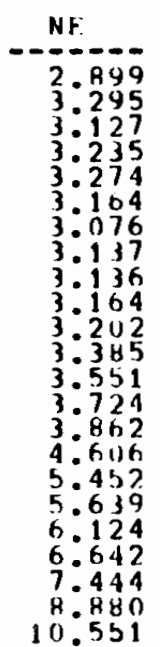 & $\begin{array}{r}\text { NH } \\
-44: 285 \\
4: 275 \\
4: 379 \\
4: 261 \\
4: 171 \\
4: 115 \\
4: 109 \\
4: 077 \\
3: 914 \\
3: 912 \\
3: 914 \\
3: 873 \\
4: 017 \\
4: 445 \\
6: 913 \\
8: 715 \\
8: 677 \\
9: 241 \\
10: 681 \\
11: 790 \\
14: 771 \\
20.428 \\
19.1199\end{array}$ & 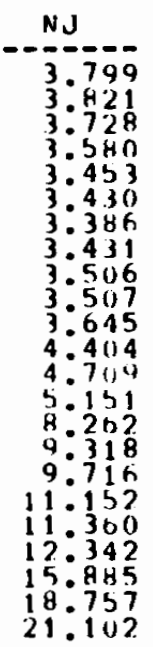 & 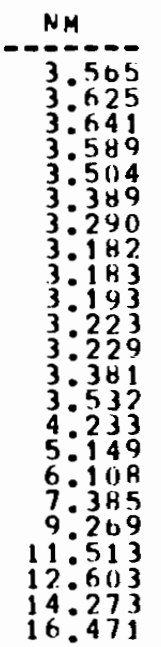 & 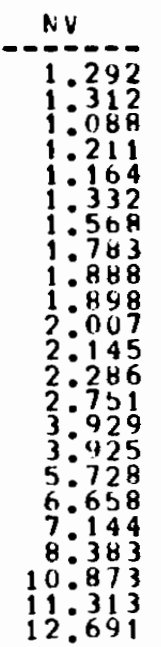 & 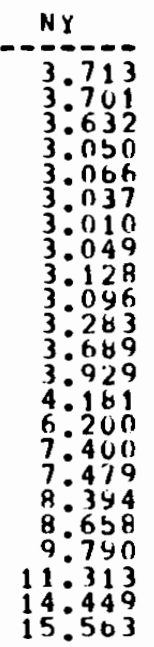 & 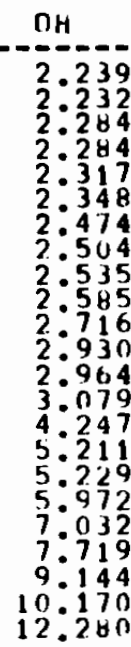 \\
\hline
\end{tabular}


TABLE 3.3 (cont). Electricity Prices: Industrial Sector (\$/million Btu)

\begin{tabular}{|c|c|c|c|c|c|c|c|}
\hline \begin{tabular}{l} 
YFAR \\
\hdashline 1960 \\
1960 \\
1961 \\
1962 \\
1963 \\
1964 \\
1965 \\
1966 \\
1967 \\
1968 \\
1969 \\
1970 \\
1971 \\
1972 \\
1973 \\
1974 \\
1975 \\
1976 \\
1977 \\
1978 \\
1979 \\
1980 \\
1981 \\
1982
\end{tabular} & 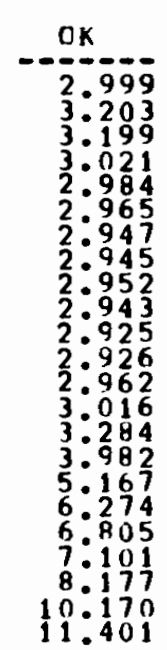 & 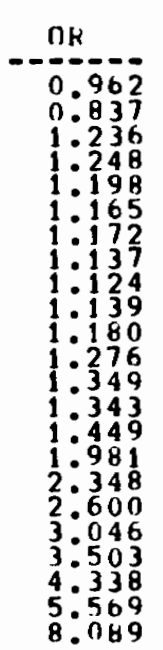 & 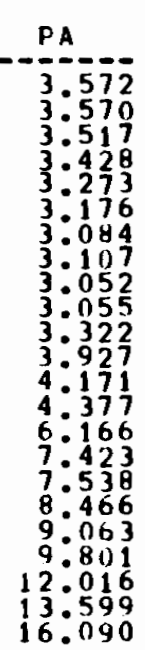 & 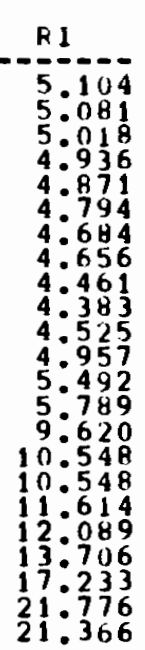 & \begin{tabular}{r} 
sc \\
\hdashline $2:-18$ \\
$2: 182$ \\
$2: 157$ \\
$2: 212$ \\
$2: 167$ \\
$2: 168$ \\
$2: 157$ \\
$2: 161$ \\
$2: 171$ \\
$2: 159$ \\
$2: 153$ \\
$2: 260$ \\
$2: 478$ \\
$2: 569$ \\
$2: 890$ \\
$4: 128$ \\
$5: 387$ \\
$5: 667$ \\
$6: 328$ \\
$6: 735$ \\
$7: 476$ \\
$7: 972$ \\
$9: 320$ \\
$10: 815$
\end{tabular} & \begin{tabular}{r}
$S I$ \\
\hdashline $4:-5$ \\
$4: 468$ \\
$4: 299$ \\
$4: 368$ \\
$4: 218$ \\
$4: 123$ \\
$4: 112$ \\
$4: 125$ \\
$4: 203$ \\
$4: 229$ \\
$4: 251$ \\
$4: 416$ \\
$4: 663$ \\
$4: 828$ \\
$4: 819$ \\
$5: 124$ \\
$5: 577$ \\
$6: 325$ \\
$6: 570$ \\
$7: 466$ \\
$7: 779$ \\
$8: 587$ \\
$10: 053$ \\
$11: 967$
\end{tabular} & 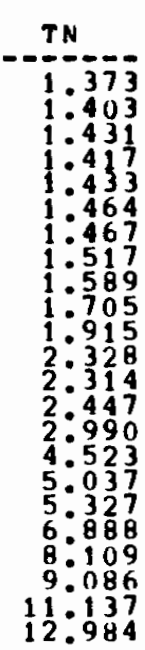 \\
\hline \\
\hline YEAR & $\checkmark A$ & VT & $w A$ & WI & wv & $W Y$ & ."1s \\
\hline $\begin{array}{l}1960 \\
19601 \\
1961\end{array}$ & $\begin{array}{l}3 \\
3: 121\end{array}$ & 4.232 & 0.947 & $\begin{array}{l}3.900 \\
4055\end{array}$ & $\begin{array}{r}2 \\
2: 560 \\
551\end{array}$ & $\begin{array}{r}3 \\
3: 219 \\
3\end{array}$ & $\frac{2}{2}:$ \\
\hline $\begin{array}{l}1961 \\
1962 \\
1963 \\
1964\end{array}$ & $\begin{array}{l}3: 013 \\
33006 \\
3\end{array}$ & $\begin{array}{l}43393 \\
4: 3932 \\
48263\end{array}$ & $\begin{array}{l}0.969 \\
0.956 \\
0945\end{array}$ & $\begin{array}{l}4.012 \\
43912 \\
3: 9812\end{array}$ & $\begin{array}{l}2: 503 \\
22447 \\
2: 387\end{array}$ & $\begin{array}{l}3: 065 \\
2: 769 \\
27 \\
732\end{array}$ & 206 \\
\hline $\begin{array}{l}1964 \\
1965 \\
1966 \\
1967\end{array}$ & $\begin{array}{l}2: 81 \\
2: 791 \\
2.791\end{array}$ & $\begin{array}{r}4: 203 \\
4: 156 \\
4: 052\end{array}$ & $\begin{array}{l}0.943 \\
0 \\
0 \\
0112\end{array}$ & $\begin{array}{l}3: 775 \\
3: 661 \\
3 \\
3\end{array}$ & $\begin{array}{l}2: 371 \\
2: 356 \\
2.356\end{array}$ & $\begin{array}{l}2067 \\
2067 \\
2077 \\
207\end{array}$ & \\
\hline $\begin{array}{l}1967 \\
1968 \\
1969\end{array}$ & $\begin{array}{l}2: 817 \\
2: 775 \\
2: 786\end{array}$ & $\begin{array}{l}4: 212 \\
4: 281 \\
4: 241\end{array}$ & $\begin{array}{l}0: 072 \\
0 \\
0\end{array}$ & $\begin{array}{l}3.653 \\
3: 630 \\
3.750\end{array}$ & $\begin{array}{r}2: 3800 \\
2: 346 \\
2: 359\end{array}$ & 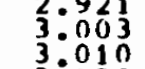 & $\begin{array}{l}639 \\
639\end{array}$ \\
\hline $\begin{array}{l}1970 \\
1971 \\
1972\end{array}$ & $\begin{array}{l}2: 805 \\
3 \\
3 \\
3\end{array}$ & $\begin{array}{l}4: 231 \\
4: 371 \\
4: 376\end{array}$ & $\begin{array}{l}0: 912 \\
0 \\
0 \\
0937\end{array}$ & 3958 & $\begin{array}{l}2.458 \\
2: 802 \\
3.087\end{array}$ & $\begin{array}{l}3: 026 \\
3: 026 \\
3 \\
3\end{array}$ & $\begin{array}{l}2: 74 \\
3.03 \\
3.03\end{array}$ \\
\hline $\begin{array}{l}1972 \\
1973 \\
1974\end{array}$ & $\begin{array}{l}3 \\
3 \\
5: 548 \\
5: 347 \\
3497\end{array}$ & 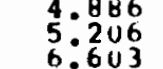 & $\begin{array}{l}0.941 \\
0993 \\
0: 975\end{array}$ & $\begin{array}{l}4.5302 \\
4: 613 \\
5: 025\end{array}$ & $\begin{array}{l}3: 196 \\
4: 467 \\
4\end{array}$ & 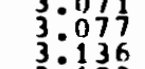 & $\begin{array}{l}3: 54 \\
4: 53 \\
4: 53\end{array}$ \\
\hline $\begin{array}{l}1975 \\
1976\end{array}$ & $\begin{array}{l}642 \\
6: 908\end{array}$ & 77:066 & $\begin{array}{l}1.274 \\
1: 32\end{array}$ & $\begin{array}{l}6: 154 \\
6: 871\end{array}$ & $\begin{array}{l}6092 \\
60392\end{array}$ & $\begin{array}{l}3: 193 \\
3: 1932 \\
3 \\
3\end{array}$ & $\begin{array}{l}5: 63 \\
6.00\end{array}$ \\
\hline $\begin{array}{l}1977 \\
1978 \\
1979\end{array}$ & $\begin{array}{l}7.924 \\
7894 \\
9.843\end{array}$ & $\begin{array}{l}7.611 \\
8: 254 \\
8.411\end{array}$ & $\begin{array}{l}1: 264 \\
1: 345 \\
1: 65\end{array}$ & $\begin{array}{l}7.064 \\
7.566 \\
8340\end{array}$ & $\begin{array}{l}6.405 \\
70102 \\
7: 131 \\
131\end{array}$ & $\begin{array}{l}3.839 \\
4.518 \\
4.558\end{array}$ & $\begin{array}{r}7.6 \\
8.3\end{array}$ \\
\hline $\begin{array}{l}1979 \\
1980 \\
1981\end{array}$ & $\begin{array}{r}9: 430 \\
11: 313 \\
11: 489 \\
11\end{array}$ & $\begin{array}{r}8041 \\
9730 \\
135306\end{array}$ & $\begin{array}{rl}2 & : 40 \\
2 & 081 \\
2.843 & 843\end{array}$ & $\begin{array}{r}8.340 \\
10.291 \\
10998\end{array}$ & $\begin{array}{r}8030 \\
90056\end{array}$ & $\begin{array}{l}4: 530 \\
4: 907 \\
5.950\end{array}$ & $\begin{array}{r}9: 90 \\
11: 09 \\
11\end{array}$ \\
\hline & & & & & & & \\
\hline
\end{tabular}


TABLE 3.4. Electricity Prices: Transportation Sector ( $\$ / m i 11$ ion Btu)

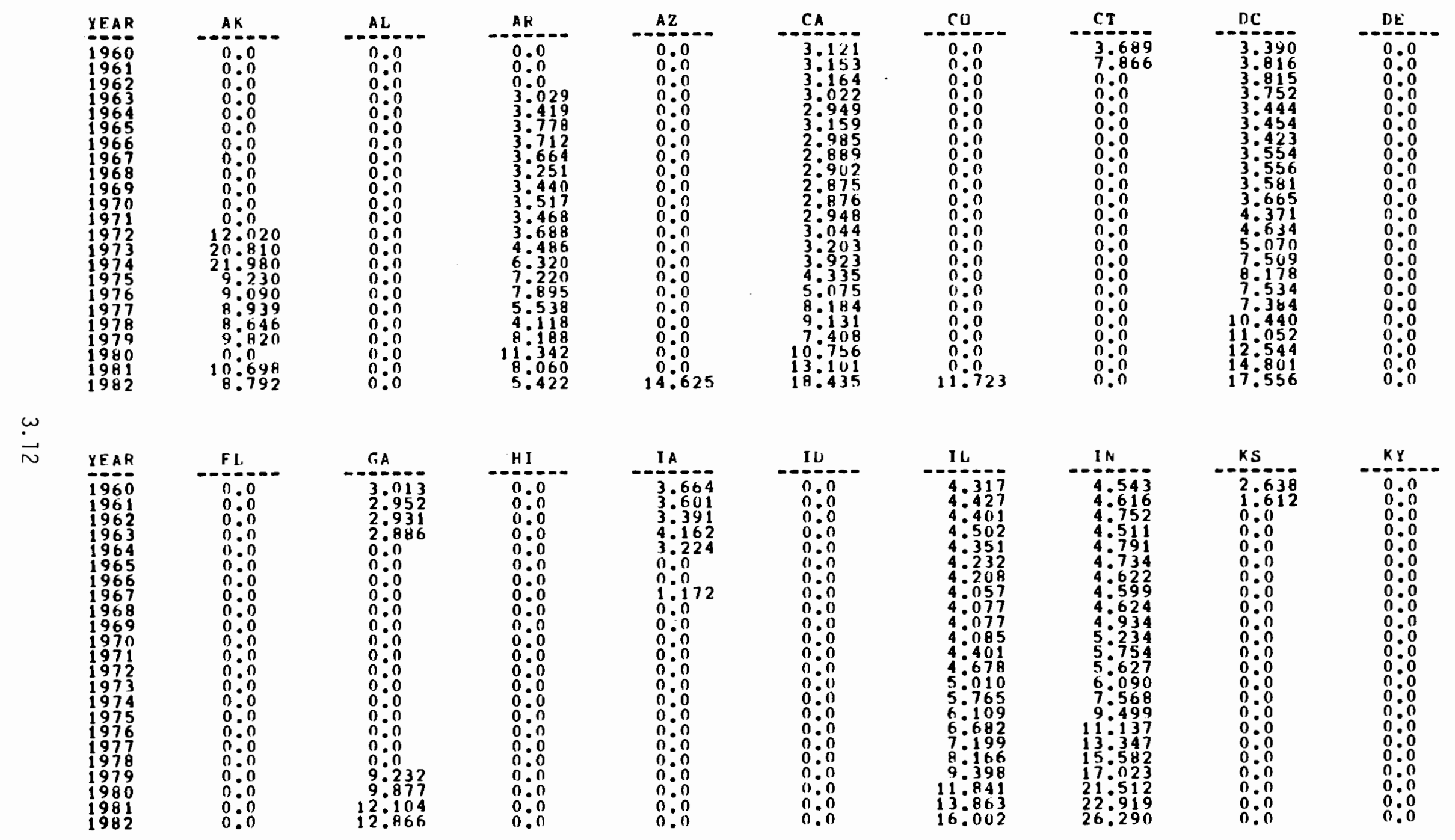


TABLE 3.4 (cont). Electricity Prices: Transportation Sector (\$/million Btu)

\begin{tabular}{|c|c|c|c|c|c|c|c|c|c|}
\hline $\begin{array}{l}\text { YEAR } \\
\text { YEA } \\
1960 \\
1961 \\
1962 \\
1963 \\
1964 \\
1965 \\
1966 \\
1967 \\
1968 \\
1969 \\
1970 \\
1971 \\
1972 \\
1973 \\
1974 \\
1975 \\
1976 \\
1977 \\
1978 \\
1979 \\
1980 \\
1981 \\
1942\end{array}$ & 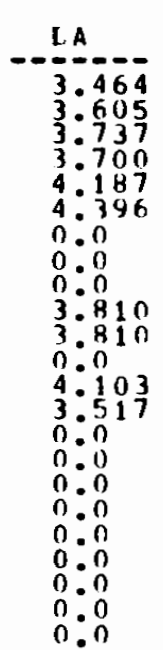 & 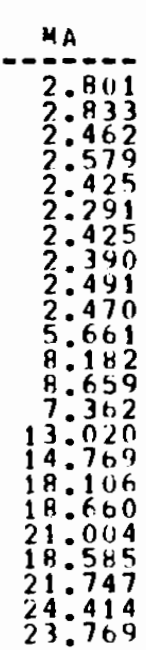 & 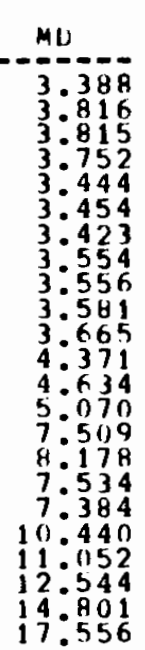 & 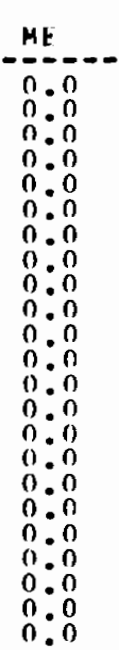 & 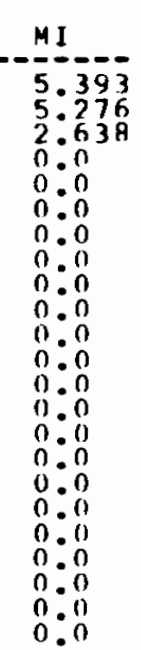 & 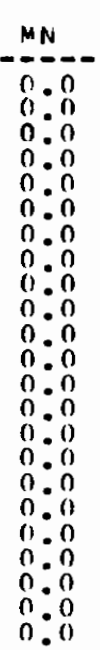 & 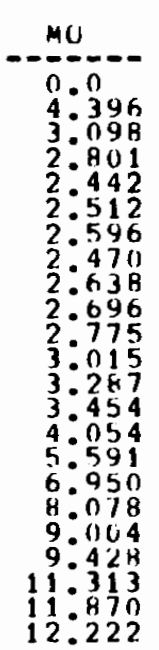 & 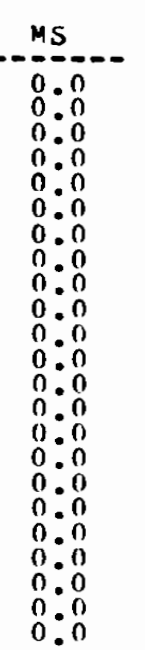 & 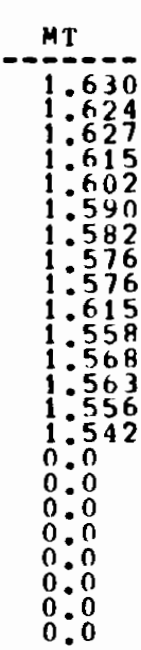 \\
\hline & & & & & & & & & \\
\hline YEAR & $N C$ & No & NE & NH & N & $\sim M$ & NV & $\mathrm{NY}$ & $\mathrm{OH}$ \\
\hline $\begin{array}{l}7960 \\
1961 \\
1962 \\
1963 \\
1964 \\
1965 \\
1966 \\
1967 \\
1968 \\
1969 \\
1970 \\
1971 \\
1977 \\
1973 \\
1974 \\
1975 \\
1976 \\
1977 \\
1978 \\
1979 \\
1980 \\
1981 \\
1982\end{array}$ & 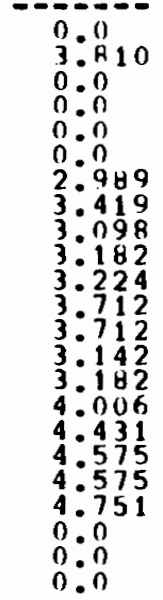 & 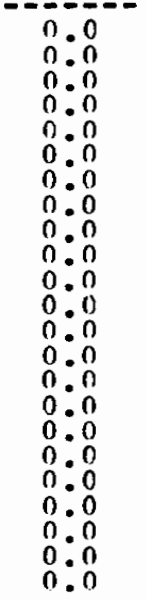 & 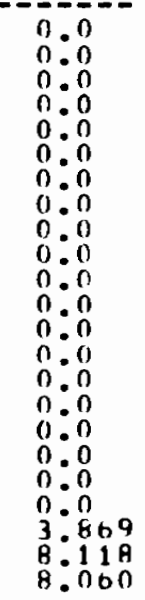 & 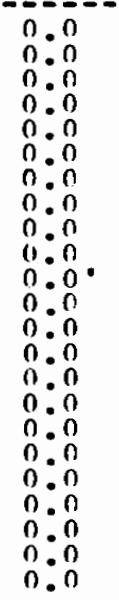 & 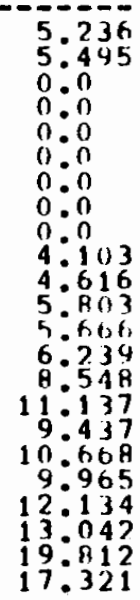 & 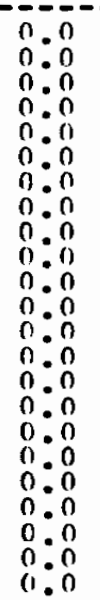 & 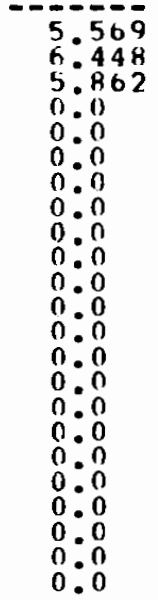 & 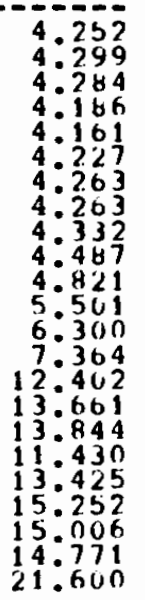 & 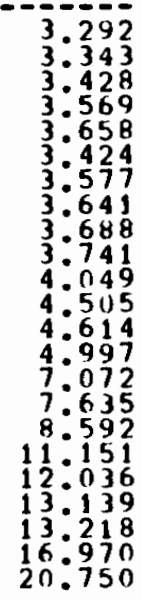 \\
\hline
\end{tabular}


TABLE 3.4 (cont). Electricity Prices: Transportation Sector ( $\$ /$ million Btu)

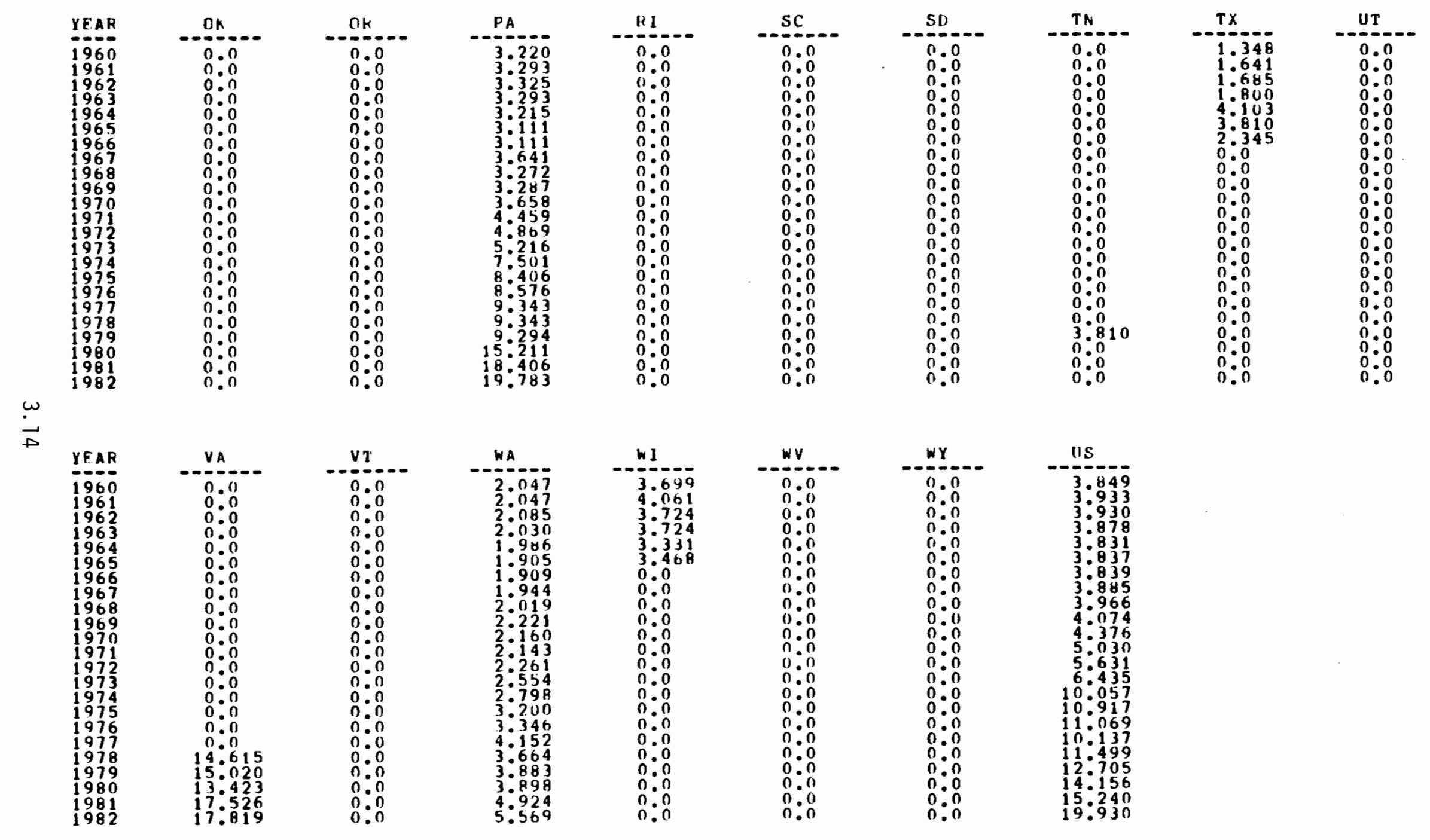


IABLE 3.5. Natural Gas Prices: Residential Sector (\$/million Btu)

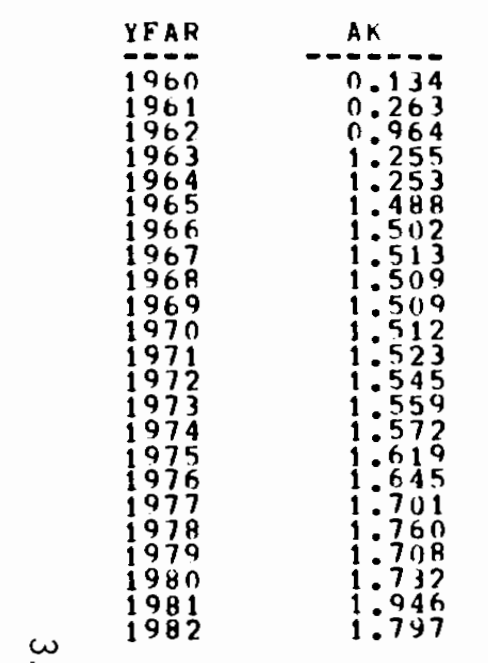

$4 l$
1.064
1.171
1.064
1.126
1.087
1.092
1.065
1.098
1.060
1.055
1.095
1.149
1.228
1.329
1.508
1.524
1.931
2.240
2.521
3.550
3.913
4.608
5.312

\begin{tabular}{|c|}
\hline 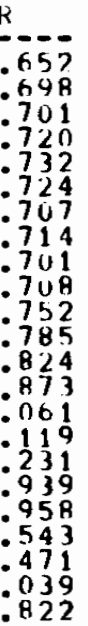 \\
\hline
\end{tabular}
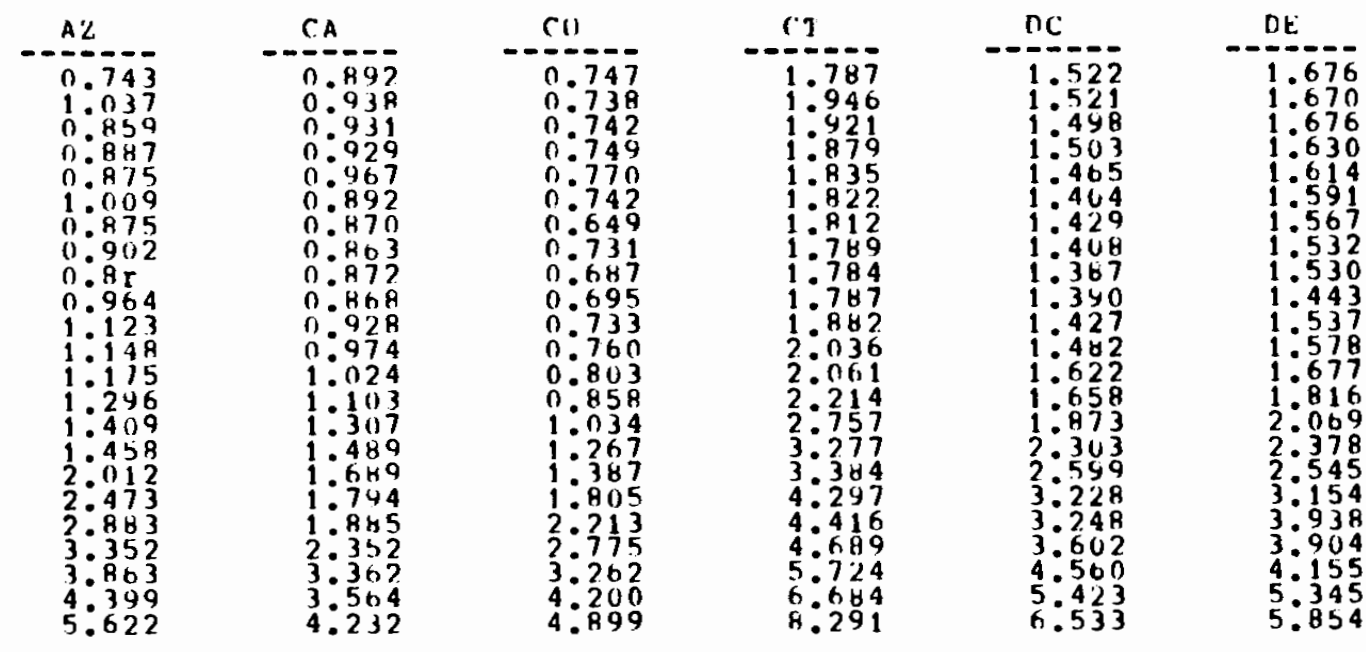

\begin{tabular}{lr} 
YFAR & FL \\
\hline 1960 & $-1 .-1$ \\
1961 & 2.863 \\
1962 & 2.581 \\
1962 & 2.570 \\
1963 & 2.753 \\
1964 & 2.566 \\
1965 & 2.403 \\
1966 & 2.129 \\
1967 & 2.179 \\
1968 & 2.475 \\
1969 & 2.371 \\
1970 & 2.406 \\
1971 & 2.428 \\
1972 & 2.553 \\
1973 & 2.591 \\
1974 & 2.733 \\
1975 & 2.626 \\
1976 & 2.702 \\
1977 & 3.777 \\
1978 & 3.381 \\
1979 & 7.567 \\
1980 & 1.613 \\
1981 & 5.011 \\
1982 & 6.320
\end{tabular}

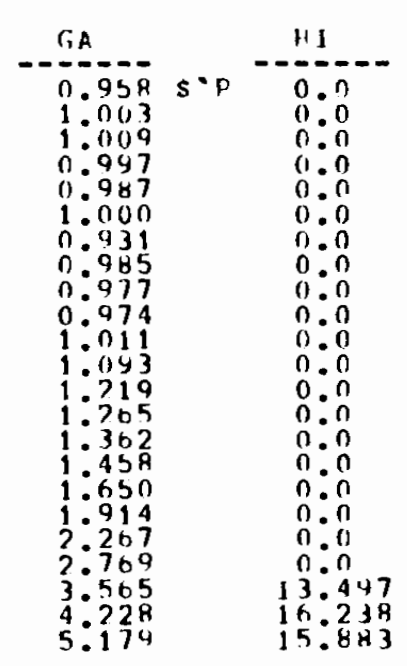

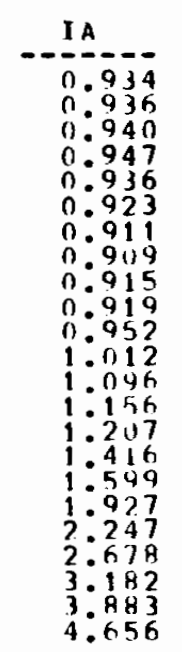

$I U$
-1.198
1.222
$1: 225$
1.233
1.192
1.272
1.198
1.233
1.327
1.339
1.334
1.434
1.420
1.438
1.728
2.067
2.120
2.695
3.234
3.557
4.727
5.205
6.457

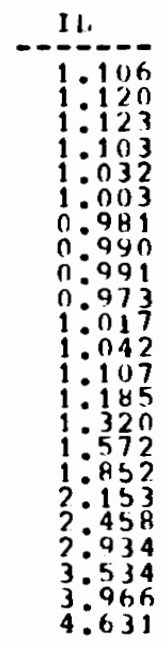

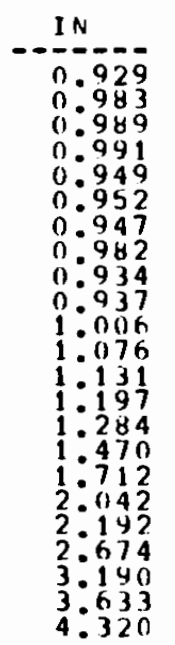

$K S$
-0.564
0.616
0.559
0.565
0.576
0.576
0.591
0.597
0.634
0.648
0.690
0.722
0.737
0.756
0.913
1.046
1.511
1.752
1.810
1.996
2.396
3.068
4.065

$K Y$
-0.761
0.791
0.784
0.777
0.783
0.790
0.782
0.823
0.777
0.783
0.812
0.863
0.910
0.971
1.084
1.217
$1: 306$
1.708
$1: 918$
2.331
3.002
3.440
4.407


TABLE 3.5 (cont). Natural Gas Prices: Residential Sector ( $\$ /$ mil1ion Btu)
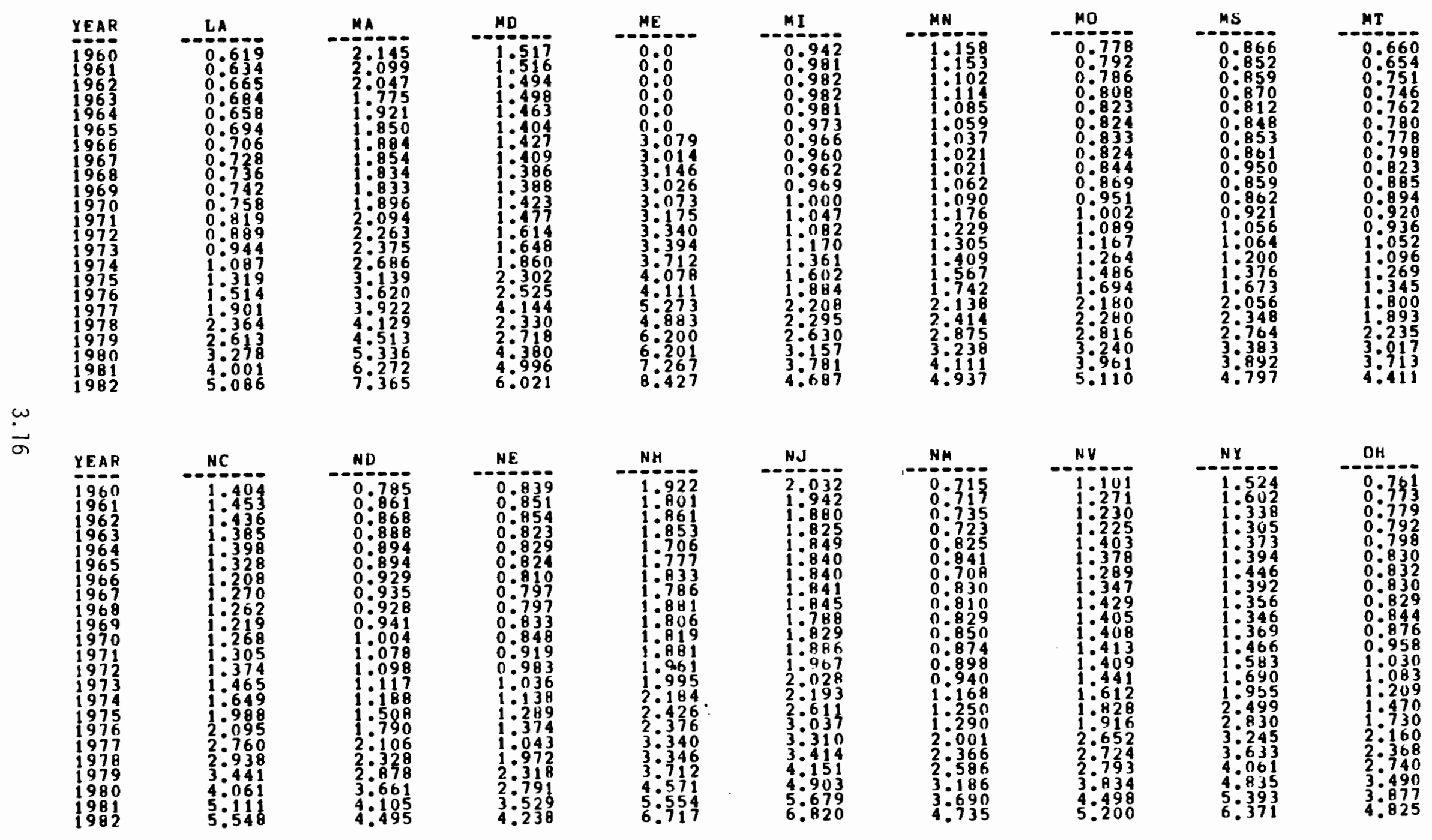
TABLE 3.5 (cont). Natural Gas Prices: Residential Sector ( $\$ /$ million Btu)

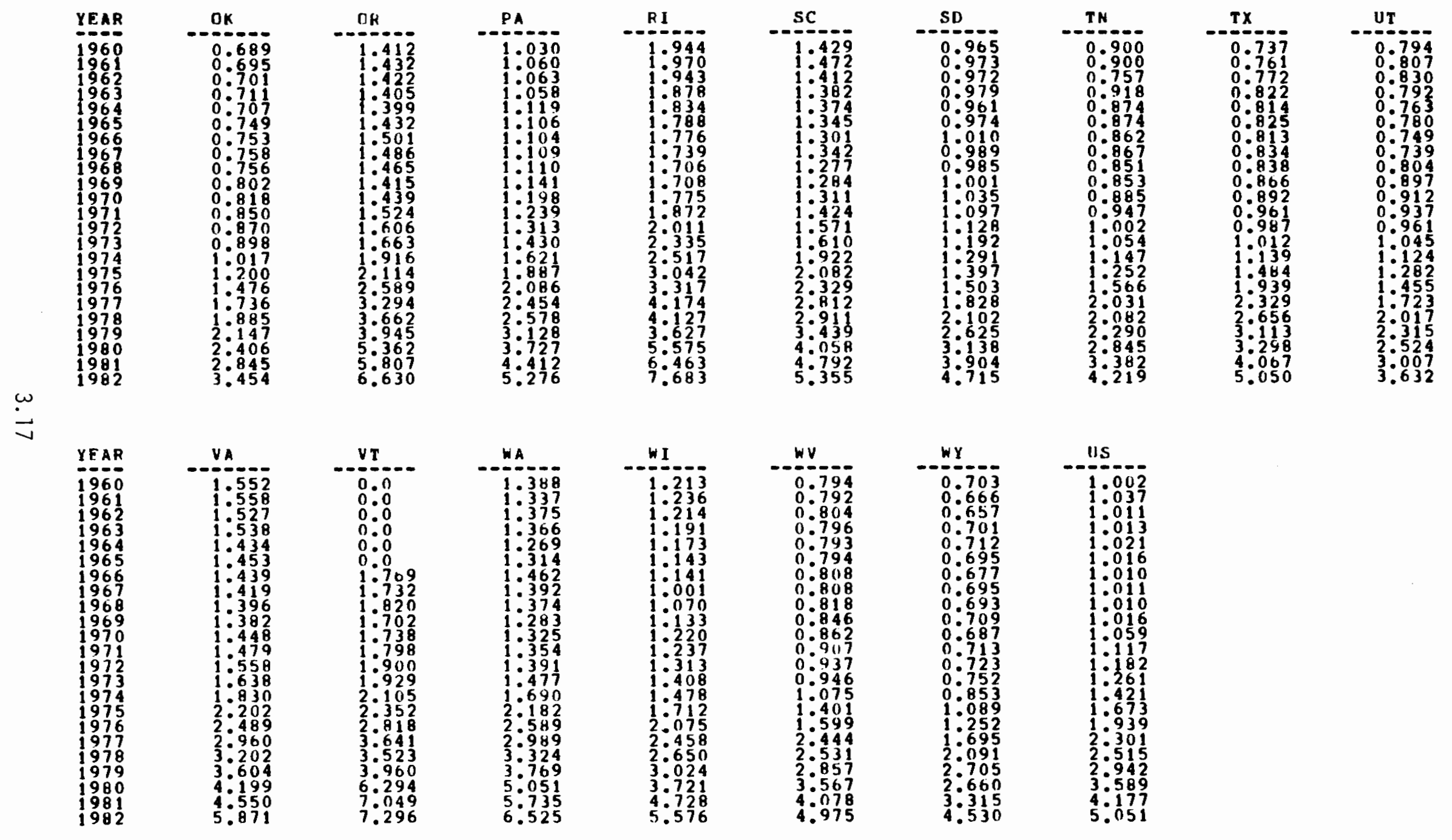


TABLE 3.6. Natural Gas Prices: Commercial Sector ( $\$ / m i l l i o n$ Btu)
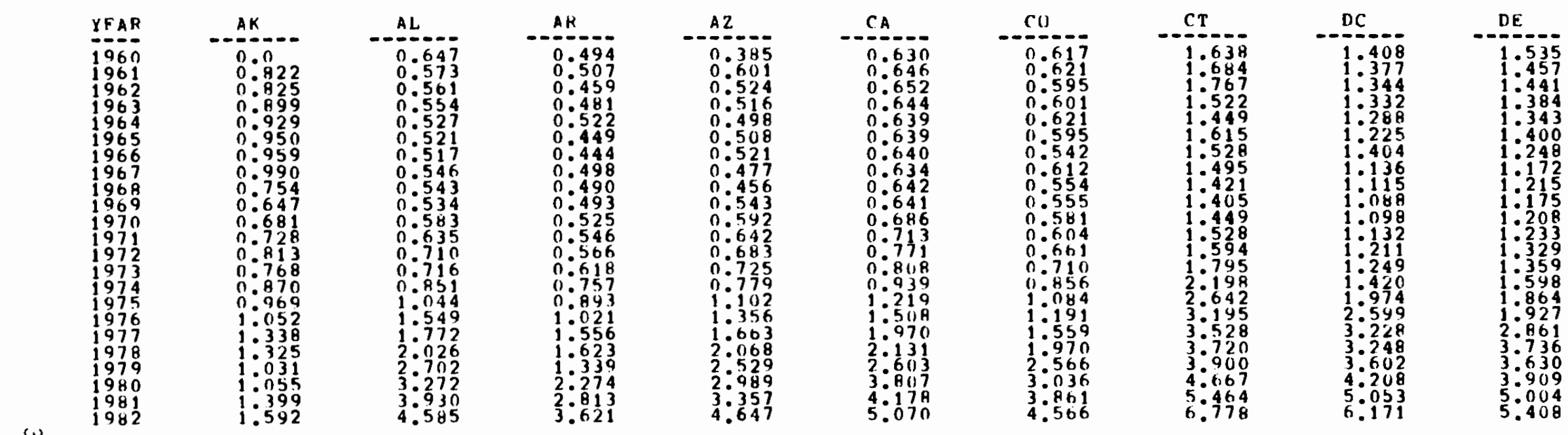

$\vec{\infty}$

\begin{tabular}{ll} 
YFAR & $F L$ \\
\hline 1960 & -1.400 \\
1961 & 0.991 \\
1962 & 1.501 \\
1963 & 1.194 \\
1964 & 1.123 \\
1965 & 1.067 \\
1966 & 1.007 \\
1967 & 0.908 \\
1968 & 0.932 \\
1969 & 0.889 \\
1970 & 0.887 \\
1971 & 1.017 \\
1972 & 1.157 \\
1973 & 1.239 \\
1974 & 1.575 \\
1975 & 1.629 \\
1976 & 1.652 \\
1977 & 1.923 \\
1978 & 1.941 \\
1979 & 2.637 \\
1980 & 3.298 \\
1981 & 3.543 \\
1982 & 4.478
\end{tabular}

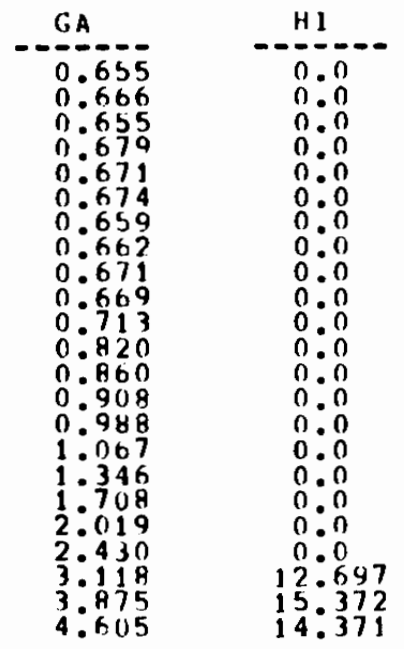

JA
-0.669
0.671
0.681
0.671
0.678
0.668
0.644
0.642
0.646
0.653
0.678
0.723
0.125
0.848
0.929
1.054
1.284
1.641
1.979
2.383
2.837
3.496
4.279

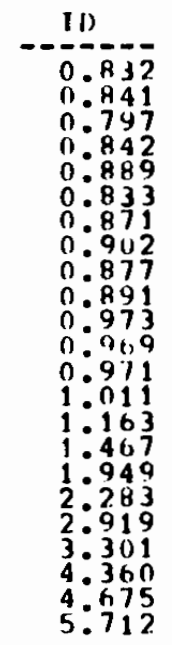

1
0.016
0.015
0.836
0.649
0.741
0.715
0.604
0.723
0.710
0.690
0.728
0.757
0.822
0.869
0.943
1.279
1.600
1.931
2.227
2.740
3.267
3.669
4.344

IN
-0.855
0.85
0.680
0.879
0.912
0.843
0.824
0.824
0.841
0.803
0.793
0.829
0.879
0.921
0.987
1.059
1.257
1.513
1.854
2.031
2.482
2.987
3.408
4.107
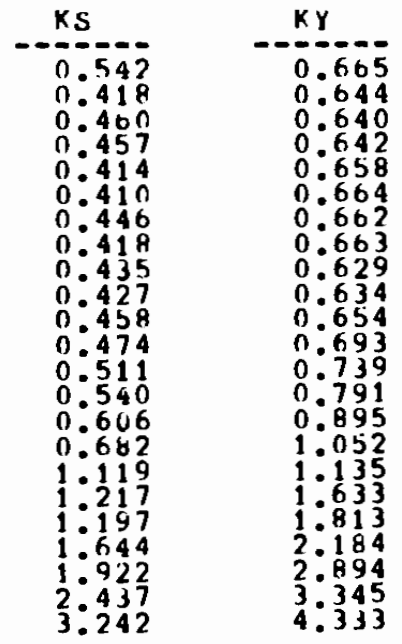
TABLE 3.6 (cont). Natural Gas Prices: Commercial Sector ( $\$ / m i 11$ ion Btu)

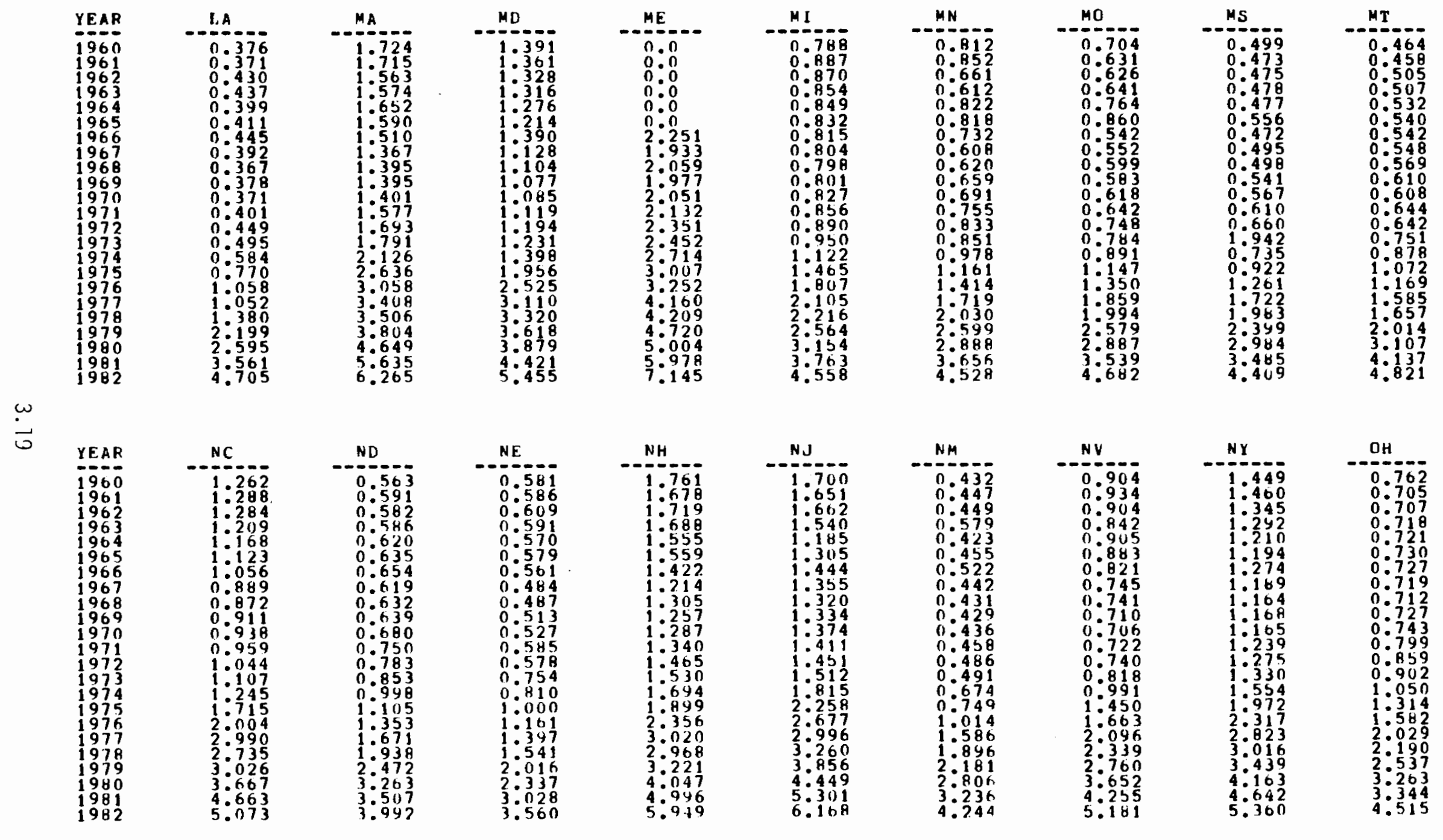


TABLE 3.6 (cont). Natural Gas Prices: Commercial Sector ( $\$ /$ million Btu)

\begin{tabular}{|c|c|c|c|c|c|c|c|}
\hline $\begin{array}{l}\text { YEAR } \\
Y 965 \\
1960 \\
1961 \\
1962 \\
1963 \\
1964 \\
1965 \\
1966 \\
1967 \\
1968 \\
1969 \\
1970 \\
1971 \\
1972 \\
1973 \\
1974 \\
1975 \\
1996 \\
1977 \\
1978 \\
1979 \\
1980 \\
1981 \\
1982\end{array}$ & 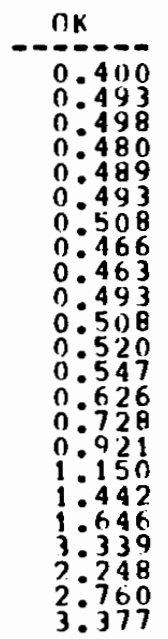 & 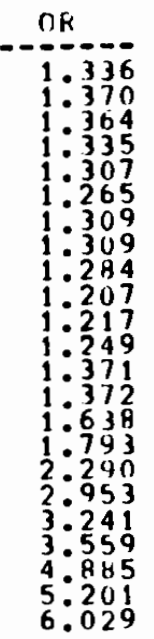 & 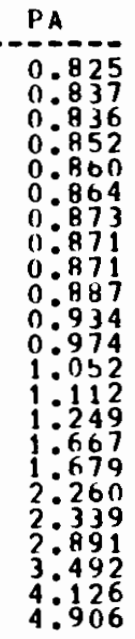 & 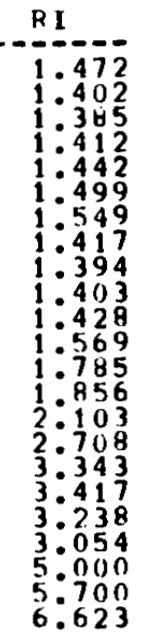 & 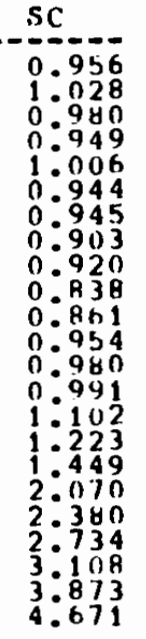 & 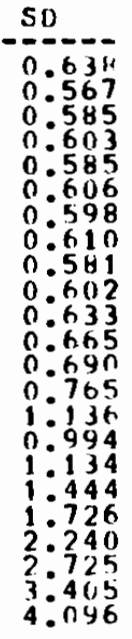 & 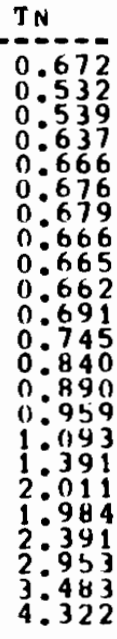 \\
\hline $\begin{array}{l}\text { YFAR } \\
\text { YFG } \\
1960 \\
1961 \\
1962 \\
1963 \\
1964 \\
1965 \\
1965 \\
1967 \\
1967 \\
1968 \\
1969 \\
1970 \\
1971 \\
1972 \\
1973 \\
1974 \\
1975 \\
1976 \\
1977 \\
1978 \\
1997 \\
1990 \\
19981 \\
1982\end{array}$ & 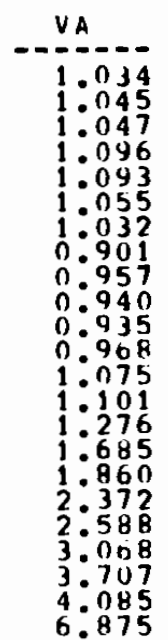 & \begin{tabular}{c}
$v \mathrm{~V}$ \\
\hdashline $0: 0--$ \\
$0: 0$ \\
$0: 0$ \\
$0: 0$ \\
$0: 0$ \\
$0: 0$ \\
$1: 609$ \\
$1: 385$ \\
$1: 440$ \\
$1: 387$ \\
$1: 443$ \\
$1: 502$ \\
$1: 665$ \\
$1: 732$ \\
$1: 915$ \\
$2: 153$ \\
30036 \\
$3: 105$ \\
$3: 394$ \\
$3: 504$ \\
$6: 213$ \\
$6: 849$ \\
$5: 411$
\end{tabular} & 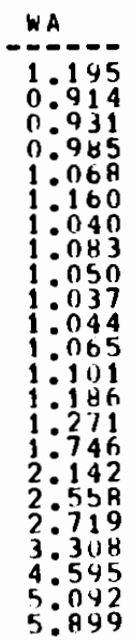 & 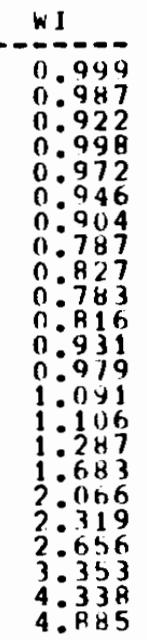 & 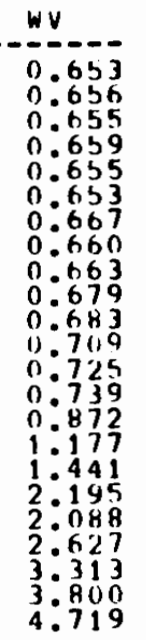 & 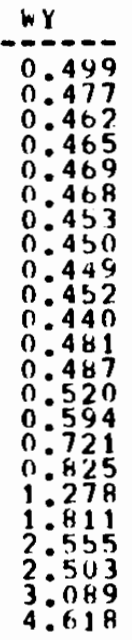 & 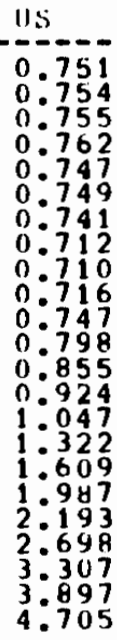 \\
\hline
\end{tabular}

Source: Pacific Northwest Laboratory 
TABLE 3.7. Natural Gas Prices: Industrial Sector ( $\$ / m i 11$ ion Btu)

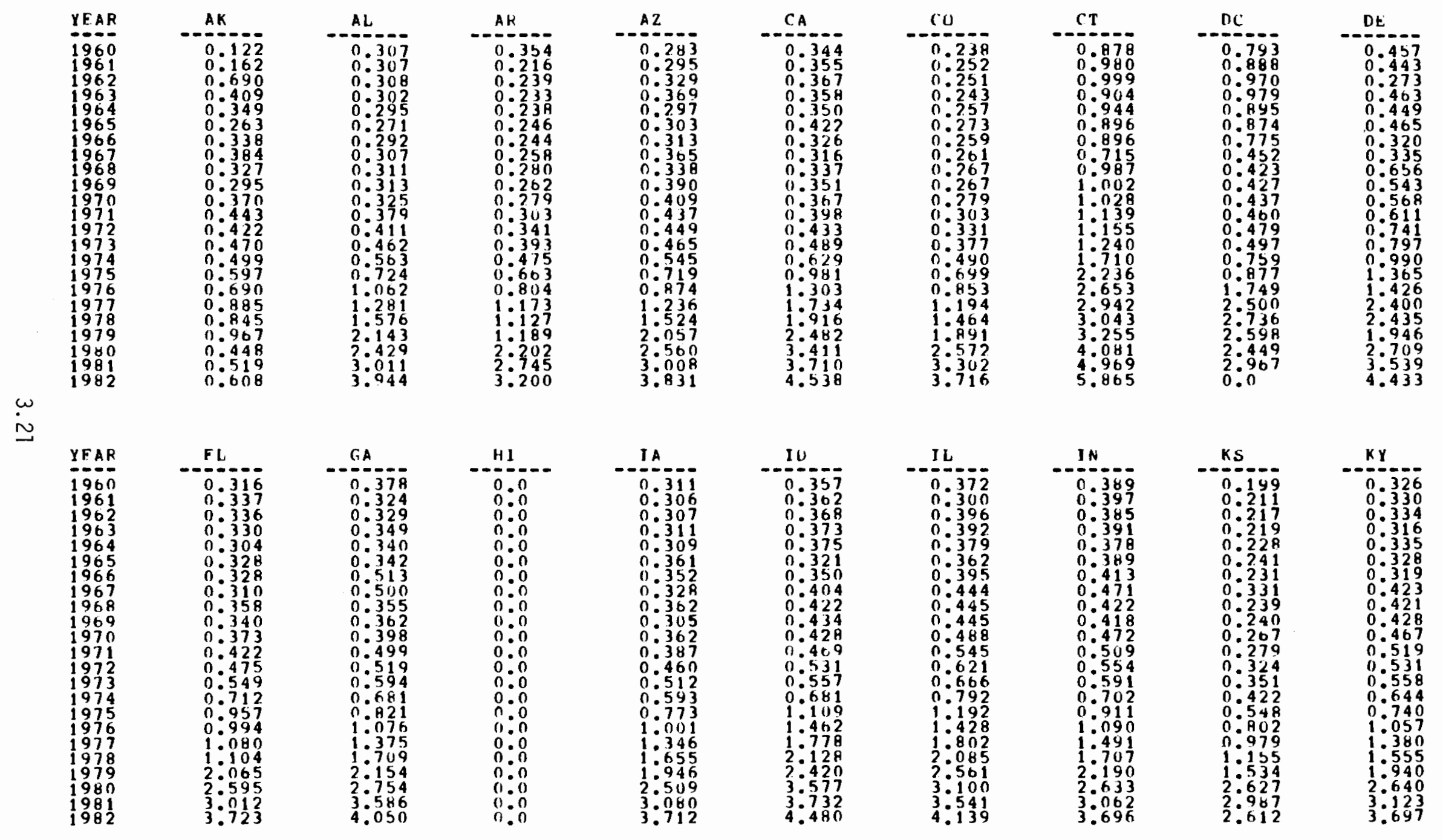


TABLE 3.7 (cont). Natural Gas Prices: Industrial Sector (\$/million Btu)

\begin{tabular}{|c|c|c|c|c|c|c|c|c|c|}
\hline $\begin{array}{l}\text { YEAR } \\
1960 \\
1961 \\
1961 \\
1963 \\
1964 \\
1965 \\
1966 \\
1967 \\
1968 \\
1969 \\
1970 \\
1971 \\
1972 \\
1973 \\
1974 \\
1975 \\
1976 \\
1977 \\
1978 \\
1979 \\
1980 \\
1981\end{array}$ & 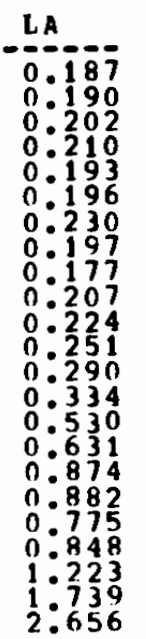 & 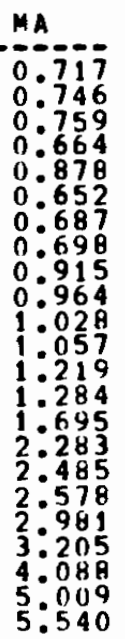 & 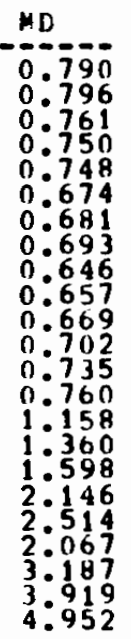 & 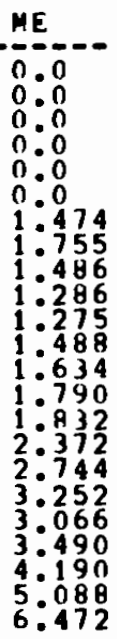 & \begin{tabular}{l}
$M 1$ \\
\hdashline $0: 541$ \\
$0: 540$ \\
$0: 517$ \\
$0: 525$ \\
$0: 519$ \\
$0: 512$ \\
$0: 499$ \\
$0: 507$ \\
$0: 509$ \\
$0: 513$ \\
$0: 523$ \\
$0: 554$ \\
$0: 620$ \\
$0: 644$ \\
$0: 899$ \\
$1: 220$ \\
$1: 518$ \\
$1: 901$ \\
$1: 891$ \\
$2: 235$ \\
$2: 965$ \\
$3: 463$ \\
$4: 162$
\end{tabular} & \begin{tabular}{l}
$M N$ \\
\hdashline $0-315$ \\
$0: 315$ \\
$0: 309$ \\
$0: 305$ \\
$0: 310$ \\
$0: 346$ \\
$0: 347$ \\
$0: 347$ \\
$0: 383$ \\
$0: 370$ \\
$0: 392$ \\
$0: 416$ \\
$0: 444$ \\
$0: 484$ \\
$0: 545$ \\
$0: 639$ \\
$0: 835$ \\
$1: 131$ \\
$1: 126$ \\
$1: 240$ \\
$1: 460$ \\
$2: 506$ \\
$3: 199$ \\
$4: 076$
\end{tabular} & 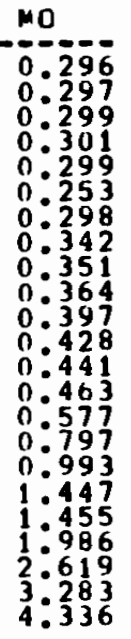 & 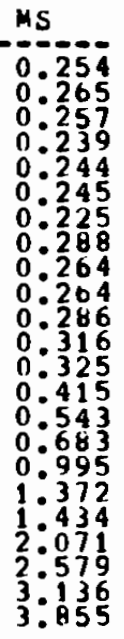 & \begin{tabular}{l}
$M T$ \\
\hdashline $0: 234$ \\
$0: 226$ \\
$0: 218$ \\
$0: 225$ \\
$0: 267$ \\
$0: 274$ \\
$0: 271$ \\
$0: 293$ \\
$0: 288$ \\
$0: 304$ \\
$0: 308$ \\
$0: 321$ \\
$0: 338$ \\
$0: 390$ \\
$0: 530$ \\
$0: 387$ \\
$0: 931$ \\
$1: 545$ \\
$1: 551$ \\
$1: 572$ \\
$2: 520$ \\
$3: 885$ \\
$4: 946$
\end{tabular} \\
\hline & & & & & & & & & \\
\hline YEAR & $N C$ & ND & NF. & NH & NS & NM & NV & $\begin{array}{l}\text { NY } \\
.\end{array}$ & OH \\
\hline $\begin{array}{l}19-1 \\
1960 \\
1961 \\
1962 \\
1963 \\
1964 \\
1965 \\
1966 \\
1967 \\
1968 \\
1969 \\
1970 \\
1971 \\
1972 \\
1973 \\
1974 \\
1975 \\
1976 \\
1977 \\
1978 \\
1979 \\
1980 \\
1981 \\
1982\end{array}$ & $\begin{array}{l}0: 257 \\
0: 256 \\
0: 264 \\
0: 256 \\
0: 264 \\
0: 269 \\
0: 273 \\
0: 477 \\
0: 443 \\
0: 493 \\
0: 500 \\
0: 522 \\
0: 583 \\
0: 664 \\
0: 829 \\
1: 339 \\
1: 654 \\
2: 324 \\
1: 921 \\
2: 619 \\
2: 735 \\
4: 363 \\
4: 494\end{array}$ & $\begin{array}{l}0.489 \\
0: 474 \\
0: 482 \\
0: 450 \\
0: 508 \\
0: 467 \\
0: 404 \\
0: 158 \\
0: 186 \\
0: 186 \\
0: 179 \\
0: 187 \\
0: 230 \\
0: 220 \\
0: 302 \\
0: 414 \\
0: 530 \\
0: 715 \\
0: 753 \\
1: 104 \\
3: 194 \\
2: 083 \\
2: 702\end{array}$ & 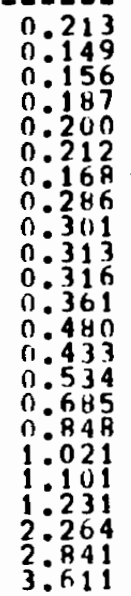 & $\begin{array}{l}0.779 \\
0: 858 \\
0: 904 \\
0: 855 \\
0: 851 \\
0: 919 \\
0: 977 \\
1: 102 \\
0: 959 \\
0: 977 \\
0: 954 \\
0: 971 \\
1: 071 \\
1: 176 \\
10380 \\
1: 975 \\
20584 \\
3: 180 \\
2: 581 \\
3: 087 \\
2: 004 \\
4: 531 \\
5.455\end{array}$ & $\begin{array}{l}0.443 \\
0: 505 \\
0: 535 \\
0: 509 \\
0: 451 \\
0: 482 \\
0: 528 \\
0: 541 \\
0: 549 \\
0: 662 \\
0.674 \\
0: 716 \\
0: 790 \\
0: 847 \\
10042 \\
10648 \\
20106 \\
20339 \\
30274 \\
3: 076 \\
30006 \\
4: 569 \\
5.083\end{array}$ & $\begin{array}{l}0: 126 \\
0: 122 \\
0: 126 \\
0: 152 \\
0: 154 \\
0: 147 \\
0: 178 \\
0: 176 \\
0: 183 \\
0: 178 \\
0: 191 \\
0: 228 \\
0: 242 \\
0: 267 \\
0: 393 \\
0: 478 \\
0: 532 \\
0: 920 \\
1: 058 \\
1: 117 \\
1: 902 \\
2: 424 \\
2: 707\end{array}$ & $\begin{array}{l}0.570 \\
0: 579 \\
0: 579 \\
0: 583 \\
0: 416 \\
0: 430 \\
0: 4118 \\
0: 449 \\
0: 432 \\
0: 466 \\
0: 525 \\
0: 536 \\
0: 661 \\
0: 743 \\
0: 025 \\
1: 063 \\
1: 339 \\
1: 873 \\
1: 732 \\
2: 299 \\
2: 809 \\
3: 335 \\
4: 125\end{array}$ & 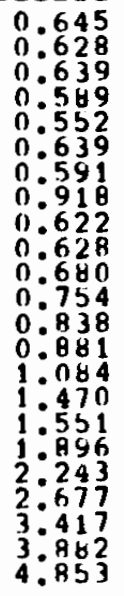 & $\begin{array}{l}0: 516 \\
0: 519 \\
0: 517 \\
0: 518 \\
0: 522 \\
0: 518 \\
0: 514 \\
0: 514 \\
0: 505 \\
0: 514 \\
0: 563 \\
0: 598 \\
0: 614 \\
0: 655 \\
0: 788 \\
1: 083 \\
1: 357 \\
1: 849 \\
1: 958 \\
2: 271 \\
3: 004 \\
3: 348 \\
4: 182\end{array}$ \\
\hline
\end{tabular}


TABLE 3.7 (cont). Natural Gas Prices: Industrial Sector ( $\$ /$ million Btu)
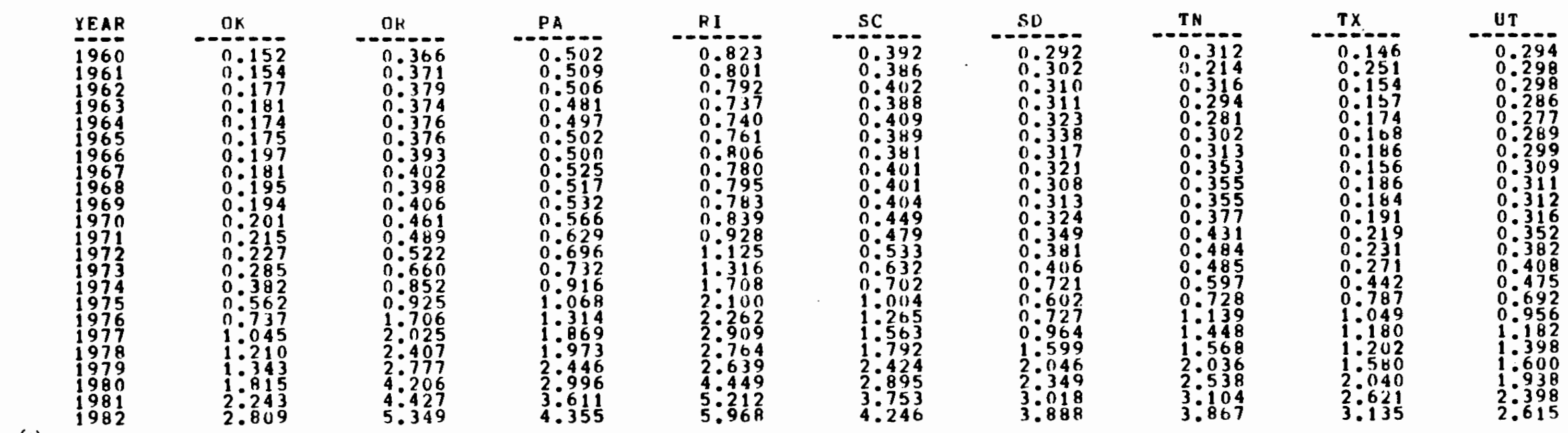

$\omega$

\begin{tabular}{ll} 
YEAR & VA \\
\hline 1960 & -1.55 \\
1961 & 0.452 \\
1962 & 0.449 \\
1963 & 0.463 \\
1964 & 0.456 \\
1965 & 0.456 \\
1966 & 0.441 \\
1967 & 0.450 \\
1968 & 0.469 \\
1969 & 0.478 \\
1970 & 0.484 \\
1971 & 0.592 \\
1972 & 0.579 \\
1973 & 0.641 \\
1974 & 0.907 \\
1975 & 1.077 \\
1976 & 1.425 \\
1977 & 1.885 \\
1978 & 2.097 \\
1979 & 2.273 \\
1980 & 2.984 \\
1981 & 2.197 \\
1982 & 4.268
\end{tabular}
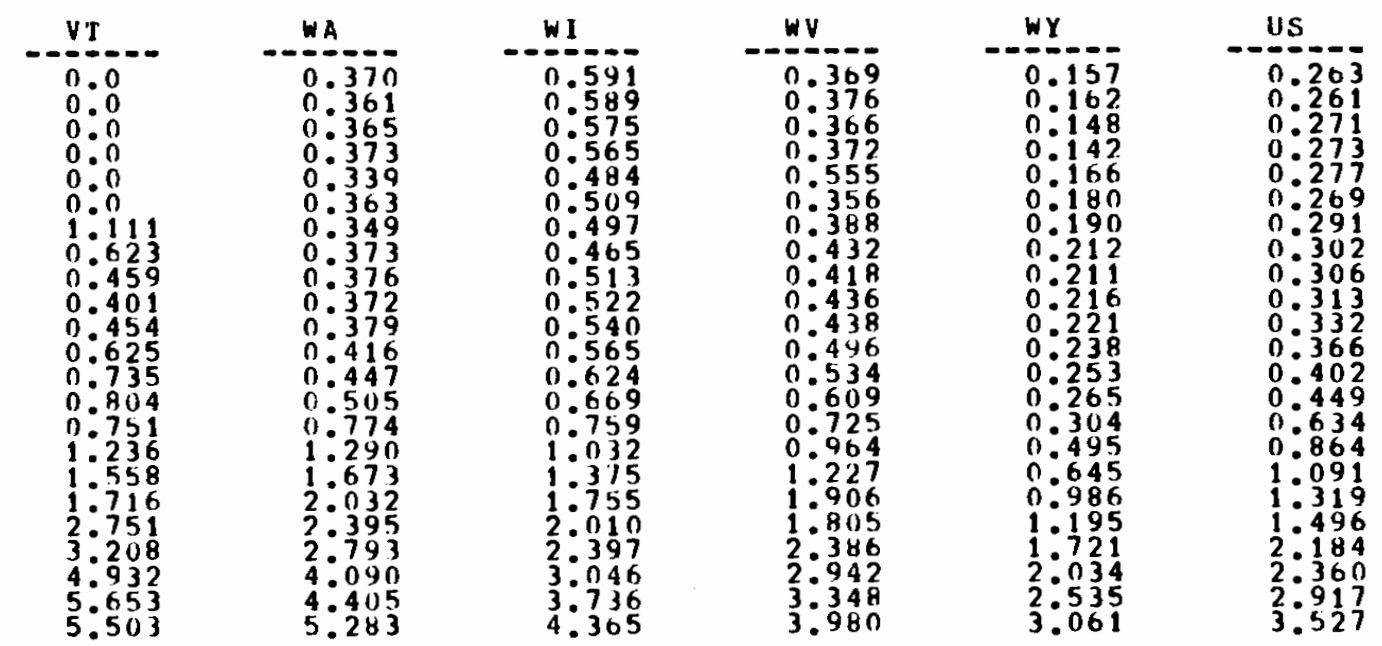

Source: Pacific Northwest Laboratory 
TABLE 3.8. Natural Gas Prices: Transportation Sector ( $\$ /$ million Btu)

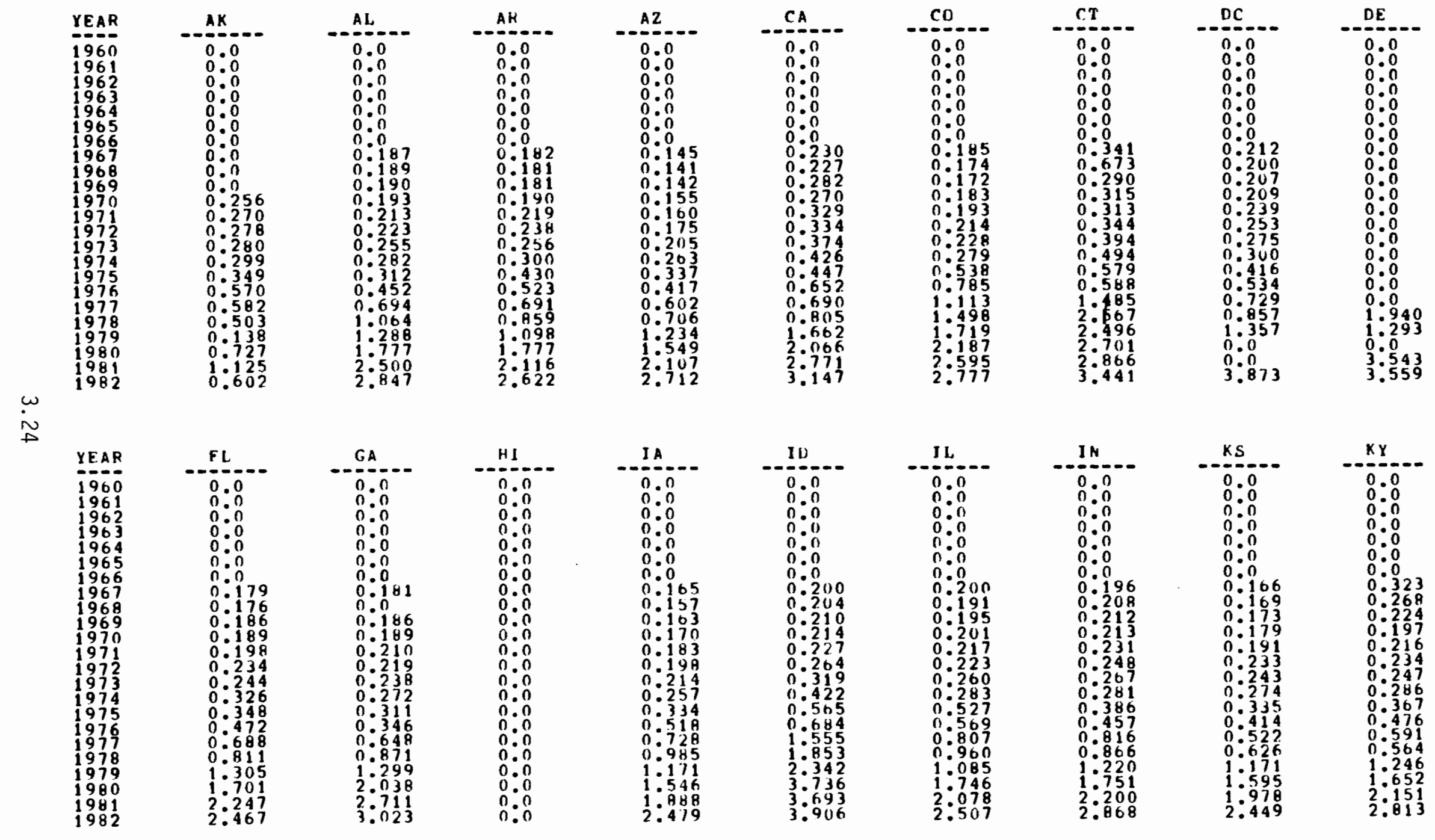


TABLE 3.8 (cont). Natural Gas Prices: Transportation Sector ( $\$ /$ million Btu)

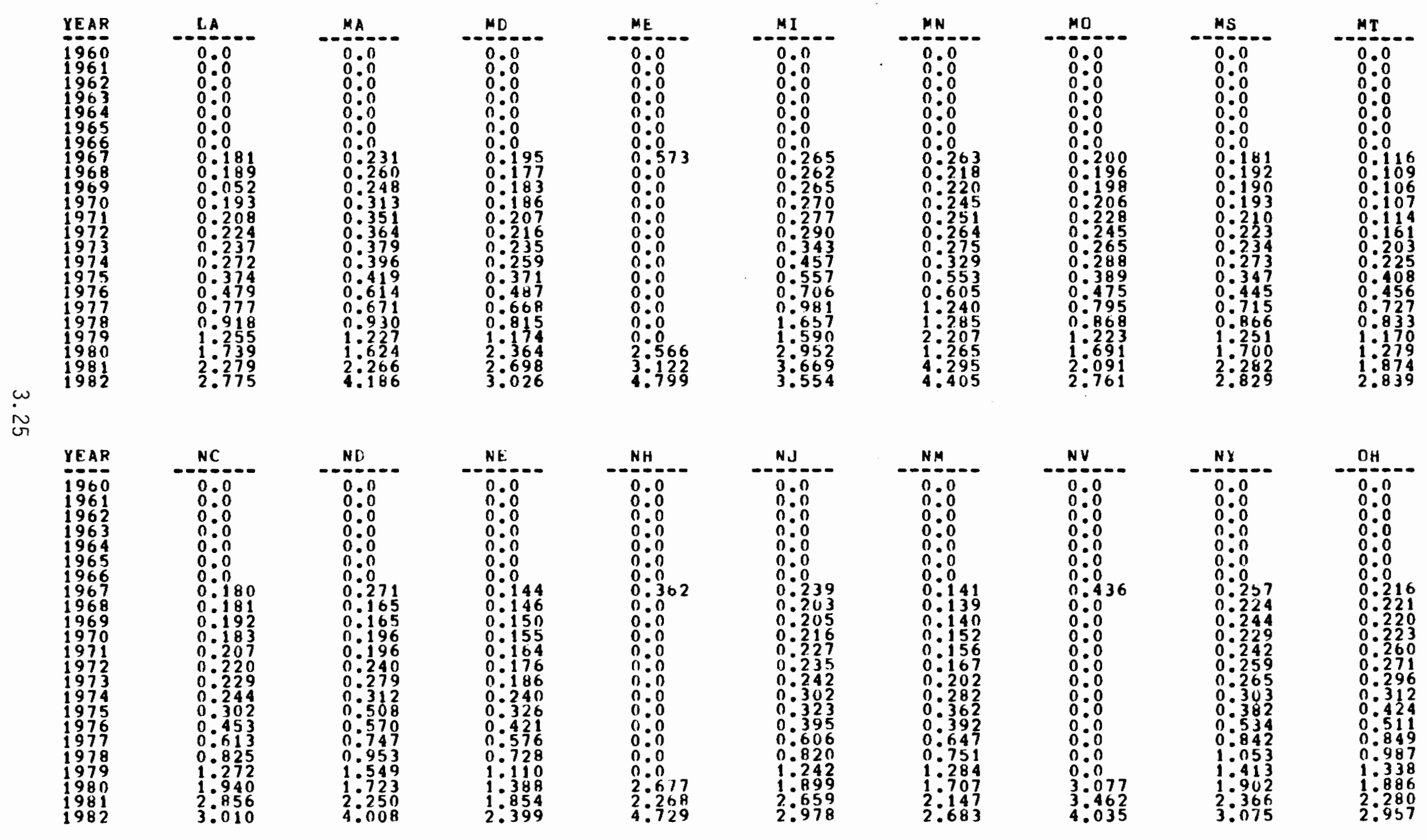


TABLE 3.8 (cont). Natural Gas Prices: Transportation Sector (\$/miliion Btu)

\begin{tabular}{|c|c|c|c|c|c|c|c|c|}
\hline $\begin{array}{l}Y E A R \\
Y E A R \\
1960 \\
1961 \\
1962 \\
1963 \\
1964 \\
1965 \\
1966 \\
1967 \\
1968 \\
1969 \\
1970 \\
1971 \\
1977 \\
1973 \\
1974 \\
1975 \\
1977 \\
1978 \\
1979 \\
1980 \\
1981 \\
1982\end{array}$ & 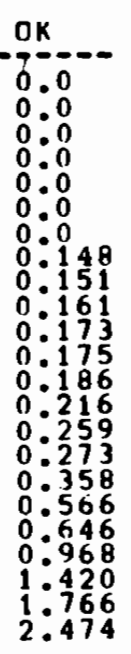 & 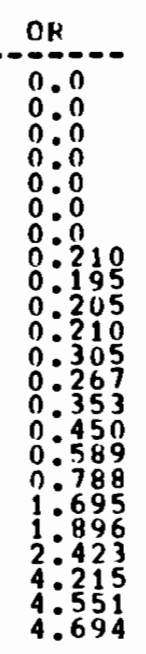 & 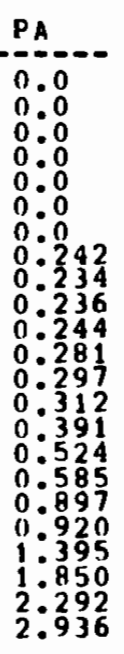 & \begin{tabular}{l}
$R 1$ \\
\hdashline $1 .--$ \\
0.0 \\
$0: 0$ \\
$0: 0$ \\
$0: 0$ \\
$0: 0$ \\
$0: 0$ \\
$0: 0$ \\
$0: 699$ \\
$0: 320$ \\
$0: 373$ \\
$0: 323$ \\
$0: 363$ \\
$0: 367$ \\
$0: 414$ \\
$0: 404$ \\
$0: 538$ \\
$0: 740$ \\
$1: 645$ \\
$2: 057$ \\
$2: 033$ \\
$2: 862$ \\
$4: 639$ \\
$4: 377$
\end{tabular} & \begin{tabular}{l}
$s c$ \\
\hdashline $0 .--$ \\
$0: 0$ \\
$0: 0$ \\
$0: 0$ \\
$0: 0$ \\
$0: 0$ \\
$0: 0$ \\
$0: 190$ \\
$0: 189$ \\
$0: 207$ \\
$0: 201$ \\
$0: 217$ \\
$0: 232$ \\
$0: 251$ \\
$0: 263$ \\
$0: 478$ \\
$0: 510$ \\
$0: 575$ \\
$0: 425$ \\
$1: 490$ \\
$1: 957$ \\
$2: 841$ \\
$3: 074$
\end{tabular} & 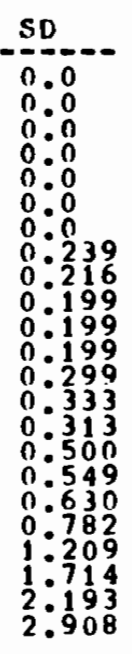 & 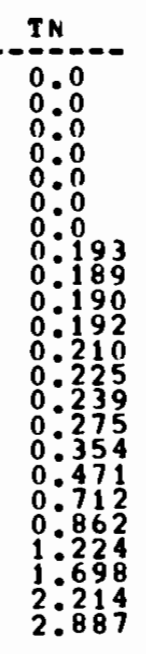 & 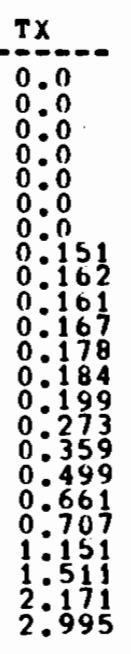 \\
\hline $\begin{array}{l}\text { YEAR } \\
1960 \\
1960 \\
1961 \\
1962 \\
1963 \\
1964 \\
1965 \\
1966 \\
1967 \\
1968 \\
1969 \\
1970 \\
1971 \\
1972 \\
1973 \\
1975 \\
1976 \\
1977 \\
1978 \\
1979 \\
1980 \\
1981 \\
1982\end{array}$ & 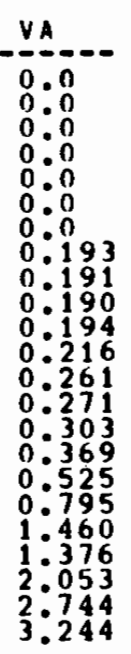 & \begin{tabular}{ll}
$V T$ \\
\hdashline 0 \\
0 & 0 \\
0 & 0 \\
0 & 0 \\
0 & 0 \\
0 & 0 \\
0 & 0 \\
0 & 0 \\
0 & 0 \\
0 & 0 \\
0 & 0 \\
0 & 0 \\
0 & 0 \\
0 & 0 \\
0 & 0 \\
0 & 0 \\
0 & 0 \\
0 & 0 \\
0 & 0 \\
0 & 0 \\
0 & 0 \\
0 & 0 \\
0 & 0 \\
5 & 287
\end{tabular} & $\begin{array}{l}W A \\
-A \\
0: 0 \\
0: 0 \\
0: 0 \\
0: 0 \\
0: 0 \\
0: 0 \\
0: 00 \\
0: 205 \\
0: 201 \\
0: 203 \\
0: 206 \\
0: 223 \\
0: 266 \\
0: 312 \\
0: 420 \\
0: 621 \\
0: 748 \\
1: 601 \\
1: 837 \\
2: 275 \\
3: 728 \\
4: 134 \\
4: 478\end{array}$ & $\begin{array}{l}w 1 \\
0: 0-- \\
0: 0 \\
0: 0 \\
0: 0 \\
0: 0 \\
0: 0 \\
0: 0 \\
0: 0 \\
0: 250 \\
0: 221 \\
0: 223 \\
0: 240 \\
0: 246 \\
0: 259 \\
0: 268 \\
0: 296 \\
0: 433 \\
0: 531 \\
1: 718 \\
2: 068 \\
1: 589 \\
4: 304 \\
2: 804 \\
3: 492\end{array}$ & $\begin{array}{l}\forall y \\
0:-.- \\
0: 0 \\
0: 0 \\
0: 0 \\
0: 0 \\
0: 0 \\
0: 0 \\
0: 0 \\
0: 326 \\
0: 313 \\
0: 0 \\
0: 310 \\
0: 328 \\
0: 370 \\
0: 382 \\
0: 440 \\
0: 570 \\
0: 716 \\
1: 074 \\
1: 254 \\
0: 835 \\
2: 219 \\
2: 515 \\
3: 203\end{array}$ & 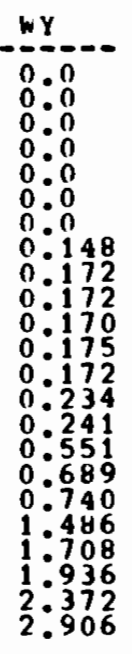 & \begin{tabular}{l} 
US \\
\hdashline $0:-.-$ \\
$0: 0$ \\
$0: 0$ \\
$0: 0$ \\
$0: 0$ \\
$0: 0$ \\
$0: 0$ \\
$0: 192$ \\
$0: 225$ \\
$0: 203$ \\
$0: 204$ \\
$0: 295$ \\
$0: 226$ \\
$0: 247$ \\
$0: 293$ \\
$0: 523$ \\
$0: 498$ \\
$0: 754$ \\
0.881 \\
$1: 295$ \\
$1: 800$ \\
$2: 328$ \\
$2: 898$
\end{tabular} & \\
\hline
\end{tabular}


TABLE 3.9. Natural Gas Prices: Electric Utility Sector ( $\$ /$ million Btu)

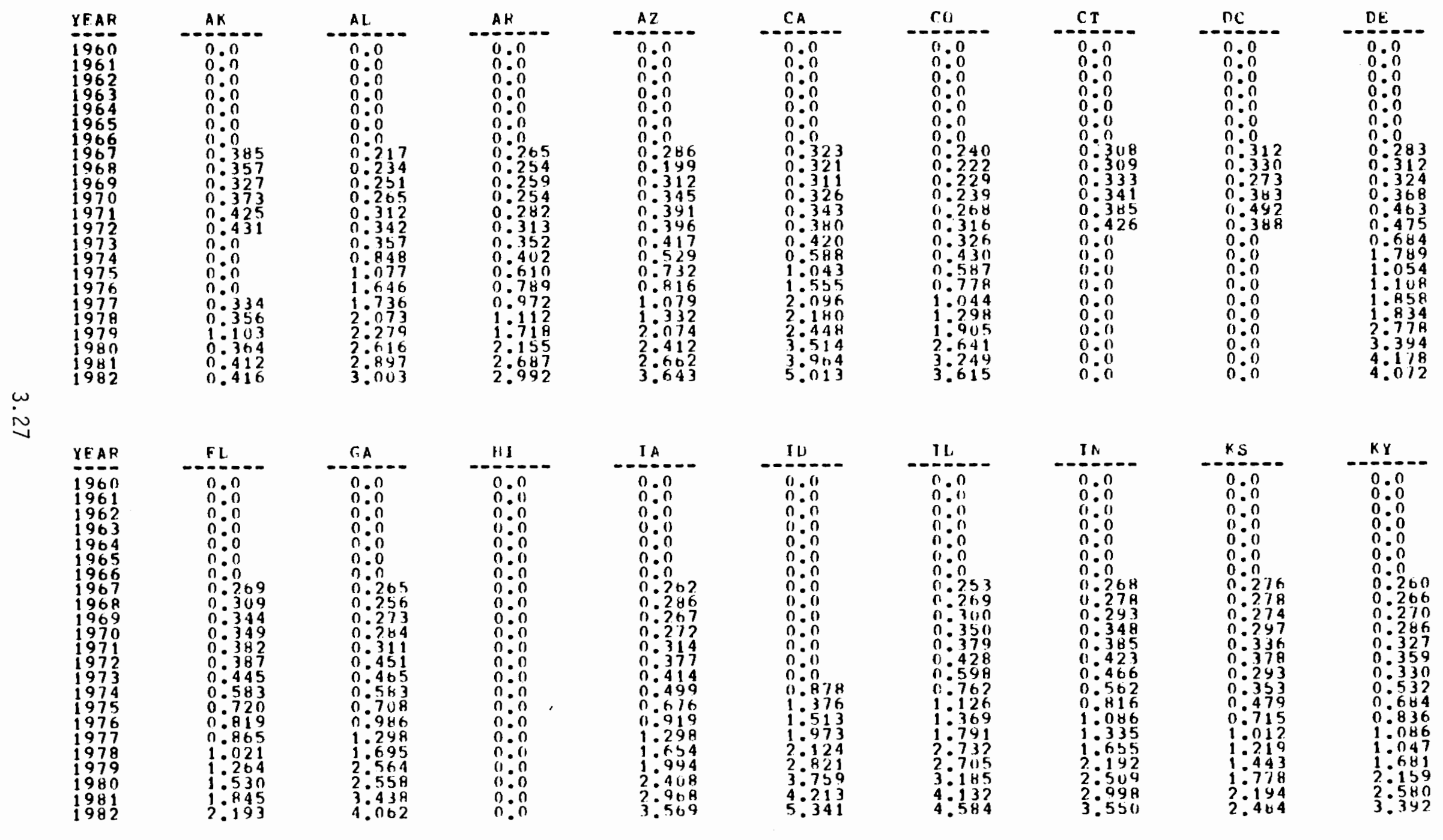


TABLE 3.9 (cont). Natural Gas Prices: Electric Utility Sector (\$/million Btu)

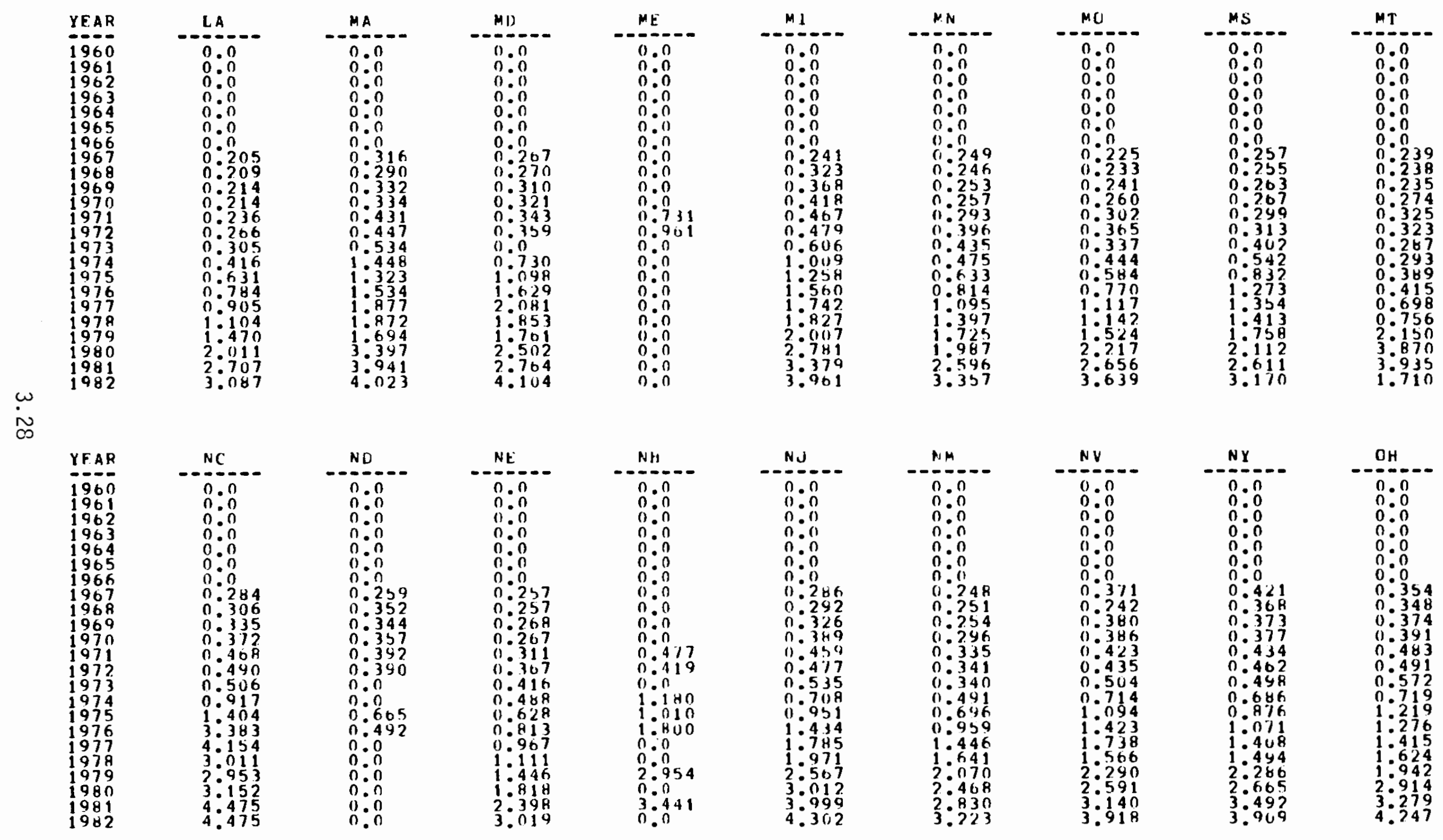


TABLE 3.9 (cont). Natural Gas Prices: Electric Utility Sector (\$/million Btu)

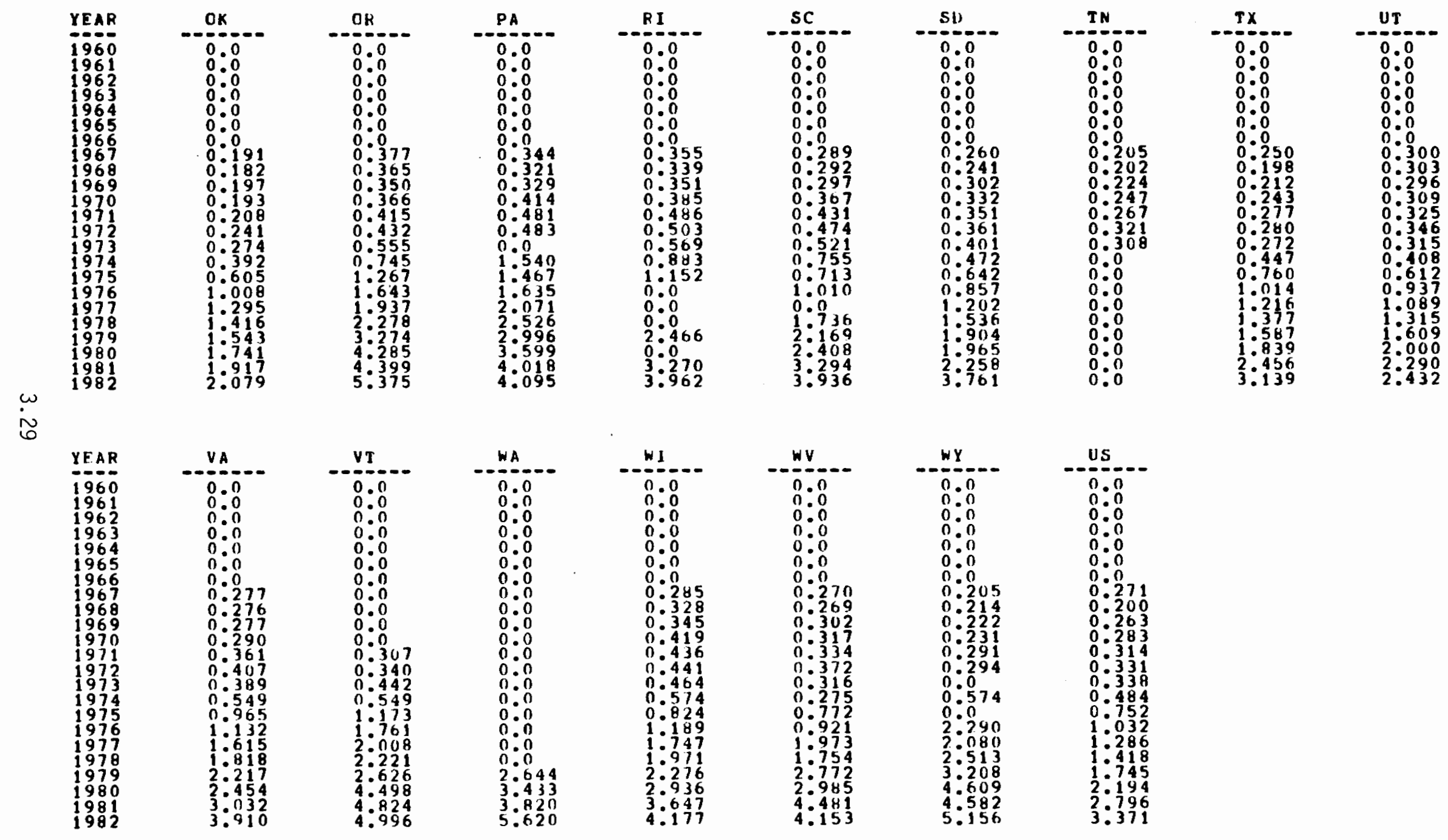

Source: Pacific Northwest Laboratory 
TABLE 3.10. Metallurgical Coal Prices: Industrial Sector ( $\$ /$ million Btu)
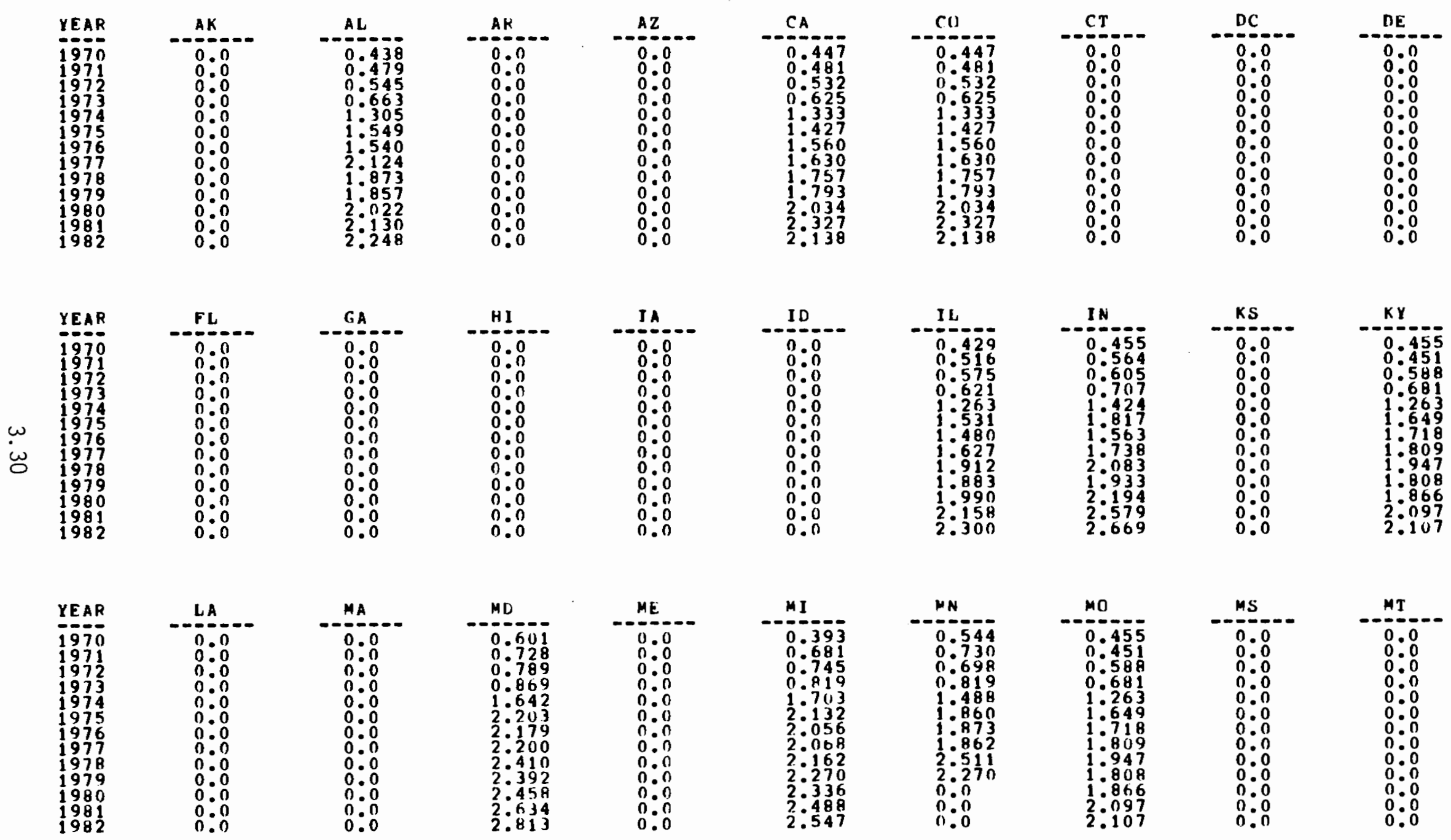

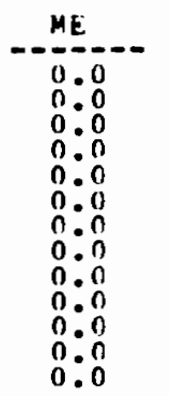
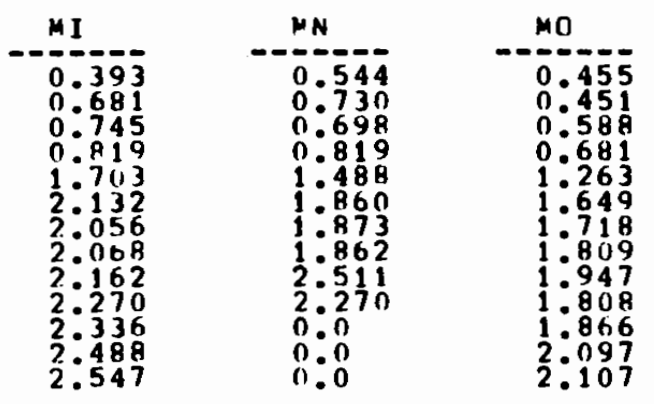

$m S$
00.0
000
0.0
0.0
0.0
0.0
0.0
$0: 0$
0.0
0.0
0.0
0.0
0.0

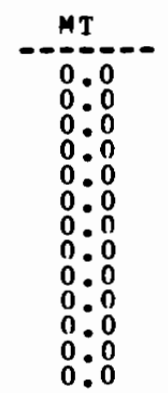


TABLE 3.10 (cont). Metallurgical Coal Prices: Industrial Sector $(\$ / m i l l i o n$ Btu)

\begin{tabular}{|c|c|c|c|c|c|c|c|c|c|}
\hline $\begin{array}{l}\text { YEAR } \\
1970 \\
1970 \\
1971 \\
1972 \\
1973 \\
1974 \\
1975 \\
1976 \\
1977 \\
1978 \\
1979 \\
1980 \\
1981 \\
1982\end{array}$ & \begin{tabular}{c}
$N C$ \\
\hdashline $0: 0$ \\
$0: 0$ \\
$0: 0$ \\
$0: 0$ \\
$0: 0$ \\
$0: 0$ \\
$0: 0$ \\
$0: 0$ \\
$0: 0$ \\
$0: 0$ \\
$0: 0$ \\
$0: 0$ \\
0.0
\end{tabular} & $\begin{array}{ll}N D \\
0 & 0 \\
0 & 0 \\
0 & 0 \\
0 & 0 \\
0 & 0 \\
0 & 0 \\
0 & 0 \\
0 & 0 \\
0 & 0 \\
0 & 0 \\
0 & 0 \\
0 & 0 \\
0 & 0 \\
0 & 0\end{array}$ & \begin{tabular}{c}
$N E$ \\
\hdashline $0: 0$ \\
$0: 0$ \\
$0: 0$ \\
$0: 0$ \\
$0: 0$ \\
$0: 0$ \\
$0: 0$ \\
$0: 0$ \\
$0: 0$ \\
$0: 00$ \\
$0: 0$ \\
$0: 0$ \\
0.0
\end{tabular} & \begin{tabular}{c}
$N H$ \\
\hdashline $0: 0$ \\
$0: 0$ \\
$0: 0$ \\
$0: 0$ \\
$0: 0$ \\
$0: 0$ \\
$0: 0$ \\
$0: 0$ \\
$0: 0$ \\
$0: 0$ \\
$0: 0$ \\
$0: 0$ \\
$0: 0$
\end{tabular} & \begin{tabular}{l}
$N \mathrm{~J}$ \\
\hdashline 0.601 \\
0.728 \\
0.0 \\
0.0 \\
$0: 0$ \\
$0: 0$ \\
$0: 0$ \\
$0: 0$ \\
$0: 0$ \\
$0: 0$ \\
$0: 0$ \\
0.00 \\
0.0
\end{tabular} & $\begin{array}{c}N M \\
0 \\
0\end{array}$ & $\begin{array}{cc}N V \\
0 & 0 \\
0 & 0 \\
0 & 0 \\
0 & 0 \\
0 & 0 \\
0 & 0 \\
0 & 0 \\
0 & 0 \\
0 & 0 \\
0 & 0 \\
0 & 0 \\
0 & 0 \\
0 & 0 \\
0 & 0 \\
0 & 0\end{array}$ & \begin{tabular}{l}
$N Y$ \\
\hdashline 0.601 \\
$0: 728$ \\
0.789 \\
0.869 \\
$1: 642$ \\
$2: 203$ \\
2.179 \\
$2: 200$ \\
$2: 410$ \\
$2: 392$ \\
$2: 458$ \\
2.634 \\
2.813
\end{tabular} & $\begin{array}{l}\text { OH } \\
-0.537 \\
0.520 \\
0.596 \\
0.675 \\
1: 342 \\
1.620 \\
1.573 \\
1.581 \\
1.814 \\
1.819 \\
2.059 \\
2.425 \\
2.510\end{array}$ \\
\hline $\begin{array}{ll} & \text { YEAR } \\
& -1970 \\
& 1970 \\
1971 \\
1972 \\
1973 \\
1974 \\
\omega \\
1975 \\
\omega \\
\omega & 1976 \\
1977 \\
1978 \\
1979 \\
1980 \\
1981 \\
1982\end{array}$ & \begin{tabular}{ll}
$0 K$ \\
\hdashline 0 & 0 \\
0 & 0 \\
0 & 0 \\
0 & 0 \\
0 & 0 \\
0 & 0 \\
0 & 0 \\
0 & 0 \\
0 & 0 \\
0 & 0 \\
0 & 0 \\
0 & 0 \\
0 & 0 \\
0 & 0
\end{tabular} & 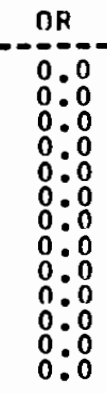 & $\begin{array}{l}P A \\
0.452 \\
0.476 \\
0.572 \\
0.690 \\
1.402 \\
1.565 \\
1.671 \\
1.670 \\
2.037 \\
1.937 \\
2.267 \\
2.446 \\
2.603\end{array}$ & \begin{tabular}{c}
$R I$ \\
\hdashline $0: 0$ \\
$0: 0$ \\
$0: 0$ \\
$0: 0$ \\
$0: 0$ \\
$0: 0$ \\
$0: 0$ \\
$0: 0$ \\
$0: 0$ \\
$0: 0$ \\
$0: 00$ \\
$0: 0$ \\
$0: 0$
\end{tabular} & \begin{tabular}{c}
$s c$ \\
\hdashline $0: 0$ \\
$00: 0$ \\
$0: 0$ \\
$0: 0$ \\
$0: 0$ \\
$0: 0$ \\
$0: 0$ \\
$0: 0$ \\
00 \\
$0: 0$ \\
$0: 0$ \\
$0: 0$ \\
$0: 0$
\end{tabular} & \begin{tabular}{ll}
$S 0$ \\
\hdashline 0 & 0 \\
0 & 0 \\
0 & 0 \\
0 & 0 \\
0 & 0 \\
0 & 0 \\
0 & 0 \\
0 & 0 \\
0 & 0 \\
0 & 0 \\
0 & 0 \\
0 & 0 \\
0 & 0 \\
0 & 0 \\
0 & 0
\end{tabular} & $\begin{array}{r}T N \\
0.455 \\
0.451 \\
0.588 \\
0.681 \\
1.263 \\
1: 649 \\
1.718 \\
1.809 \\
1.947 \\
1.808 \\
1.868 \\
2.097 \\
2.107\end{array}$ & $\begin{array}{l}T X \\
0.455 \\
0.451 \\
0.588 \\
0.681 \\
1: 263 \\
1.649 \\
1.718 \\
1.809 \\
1.947 \\
1.808 \\
1.866 \\
2.097 \\
2.107\end{array}$ & $\begin{array}{l}\text { UT } \\
0.447 \\
0.481 \\
0.532 \\
0.625 \\
1: 333 \\
1: 427 \\
1: 560 \\
1.630 \\
1: 757 \\
1: 793 \\
2.034 \\
2: 327 \\
2.138\end{array}$ \\
\hline $\begin{array}{l}\text { YEAR } \\
1970 \\
1970 \\
1971 \\
1972 \\
1973 \\
1974 \\
1975 \\
1976 \\
1977 \\
1978 \\
1979 \\
1980 \\
1981 \\
1982\end{array}$ & $\begin{array}{l}\text { VA } \\
0.0 \\
0: 0 \\
0: 0 \\
0: 0 \\
0: 0 \\
0.0 \\
0: 0 \\
0: 0 \\
1.787 \\
1.834 \\
1: 914 \\
2.0118 \\
2.111\end{array}$ & \begin{tabular}{ll}
$V T$ \\
\hdashline 0 & 0 \\
0 & 0 \\
0 & 0 \\
0 & 0 \\
0 & 0 \\
0 & 0 \\
0 & 0 \\
0 & 0 \\
0 & 0 \\
0 & 0 \\
0 & 0 \\
0 & 0 \\
0 & 0 \\
0 & 0
\end{tabular} & \begin{tabular}{ll}
$W A$ \\
\hdashline $0: 0$ \\
$0: 0$ \\
$0: 0$ \\
$0: 0$ \\
$0: 0$ \\
$0: 0$ \\
$0: 0$ \\
$0: 0$ \\
$0: 0$ \\
$0: 0$ \\
$0: 0$ \\
$0: 0$ \\
0.00
\end{tabular} & \begin{tabular}{l}
$W I$ \\
\hdashline 0.544 \\
0.730 \\
0.698 \\
0.819 \\
1.488 \\
1.860 \\
1.873 \\
1.862 \\
2.511 \\
2.270 \\
2.336 \\
2.488 \\
2.547
\end{tabular} & $\begin{array}{l}w V \\
0.414 \\
0.482 \\
0.519 \\
0.581 \\
1.171 \\
1.561 \\
1.690 \\
1.740 \\
1.787 \\
1.834 \\
1.914 \\
2.018 \\
2.111\end{array}$ & 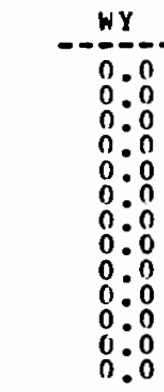 & $\begin{array}{l}\text { us } \\
0.470 \\
0.538 \\
0.605 \\
0.705 \\
1.403 \\
1.700 \\
1.698 \\
1.778 \\
2.002 \\
1.950 \\
2.167 \\
2.418 \\
2.499\end{array}$ & & \\
\hline
\end{tabular}


TABLE 3.11. Steam Coal Prices: Residential Sector ( $\$ /$ million Btu)

\begin{tabular}{|c|c|c|c|c|c|c|c|c|c|}
\hline $\begin{array}{l}\text { YEAR } \\
1970 \\
1970 \\
1971 \\
1977 \\
1974 \\
1975 \\
1976 \\
1977 \\
1978 \\
1998 \\
1980 \\
1981 \\
1982\end{array}$ & $\begin{array}{l}A K \\
0:-0 \\
0: 0 \\
0: 0 \\
0: 00 \\
0: 0 \\
0: 00 \\
0: 0 \\
0: 00 \\
0: 0 \\
0: 0 \\
0: 0 \\
0: 0 \\
0: 0 \\
0: 0\end{array}$ & $\begin{array}{l}A L \\
0.287 \\
0: 378 \\
0: 917 \\
1: 170 \\
1: 862 \\
1: 824 \\
1: 900 \\
2: 010 \\
2: 905 \\
2: 374 \\
2: 561 \\
3: 215 \\
2: 856\end{array}$ & \begin{tabular}{l} 
AR \\
\hdashline $0:---$ \\
$0: 0$ \\
$0: 0$ \\
$0: 0$ \\
$0: 0$ \\
$0: 00$ \\
$0: 0$ \\
$0: 0$ \\
$0: 0$ \\
$0: 0$ \\
$0: 0$ \\
$0: 0$ \\
$0: 0$ \\
$0: 0$
\end{tabular} & \begin{tabular}{l}
$A Z$ \\
\hdashline$:-0$ \\
$0: 0$ \\
$0: 0$ \\
$0: 0$ \\
$0: 0$ \\
0 \\
$0: 0$ \\
$0: 0$ \\
$0: 0$ \\
$0: 0$ \\
$0: 0$ \\
$0: 0$ \\
0 \\
0 \\
0 \\
0
\end{tabular} & \begin{tabular}{l}
$C A$ \\
\hdashline $0---$ \\
$00: 0$ \\
$0: 0$ \\
$0: 0$ \\
$0: 0$ \\
$0: 0$ \\
$0: 0$ \\
$0: 0$ \\
$0: 0$ \\
$0: 0$ \\
$0: 0$ \\
$0: 0$ \\
$0: 0$ \\
$0: 0$
\end{tabular} & \begin{tabular}{r}
$C D$ \\
\hdashline $0.28 B$ \\
0.704 \\
0.826 \\
$1: 482$ \\
$1: 074$ \\
$1: 577$ \\
$1: 406$ \\
10610 \\
$1: 710$ \\
$2: 109$ \\
2.515 \\
$4: 209$ \\
5.127
\end{tabular} & 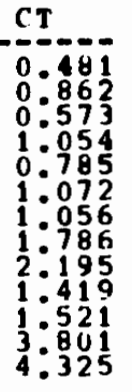 & \begin{tabular}{l} 
DC \\
\hdashline 0.424 \\
$1: 154$ \\
$1: 346$ \\
$1: 389$ \\
$2: 270$ \\
$1: 748$ \\
$1: 748$ \\
$2: 670$ \\
$2: 850$ \\
$2: 556$ \\
$2: 793$ \\
$3: 311$ \\
$3: 366$
\end{tabular} & $\begin{array}{l}D E \\
-0.424 \\
1: 154 \\
1: 346 \\
1: 549 \\
2: 270 \\
2: 727 \\
1: 748 \\
2: 670 \\
2: 960 \\
2: 897 \\
33: 215 \\
3: 910 \\
3.963\end{array}$ \\
\hline $\begin{array}{l}\text { YEAR } \\
1970 \\
1971 \\
1972 \\
1973 \\
1974 \\
1975 \\
1976 \\
1977 \\
1978 \\
1990 \\
1980 \\
1982\end{array}$ & 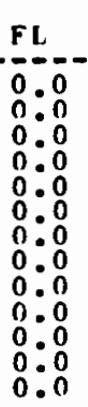 & $\begin{array}{l}G A \\
0.713 \\
0: 413 \\
0: 746 \\
0: 881 \\
0: 969 \\
1: 330 \\
3: 230 \\
1: 5200 \\
2: 280 \\
2: 915 \\
2.491 \\
2.695 \\
3: 117 \\
3.231\end{array}$ & \begin{tabular}{l}
$H I$ \\
\hdashline $0:---$ \\
$0: 0$ \\
$0: 0$ \\
$0: 0$ \\
$0: 0$ \\
$0: 0$ \\
$0: 0$ \\
$0: 0$ \\
$0: 0$ \\
$0: 0$ \\
$0: 0$ \\
$0: 0$ \\
0.0
\end{tabular} & 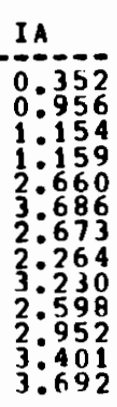 & \begin{tabular}{l}
10 \\
-10 \\
\hdashline $0: 335$ \\
$0: 885$ \\
$0: 0$ \\
$0: 798$ \\
$1: 387$ \\
$1: 777$ \\
19914 \\
$1: 720$ \\
$2: 500$ \\
$0: 0$ \\
000 \\
$0: 0$ \\
0.0
\end{tabular} & \begin{tabular}{l}
$I L$ \\
\hdashline 0.326 \\
$1: 1188$ \\
$1: 211$ \\
$1: 116$ \\
$1: 698$ \\
$2: 106$ \\
$2: 214$ \\
$2: 288$ \\
$2: 375$ \\
3.084 \\
3.479 \\
$2: 692$ \\
2.990
\end{tabular} & $\begin{array}{l}I N \\
-0.276 \\
0: 875 \\
1: 034 \\
1: 173 \\
2: 287 \\
2: 516 \\
2: 521 \\
2: 451 \\
2: 567 \\
2: 671 \\
2: 8118 \\
2: 821 \\
2: 863\end{array}$ & \begin{tabular}{l}
$k s$ \\
$-S$ \\
\hdashline 039 \\
$0: 662$ \\
0.684 \\
$1: 178$ \\
$1: 140$ \\
$1: 349$ \\
$1: 330$ \\
$1: 785$ \\
$1: 900$ \\
$1: 987$ \\
$1: 607$ \\
$2: 606$ \\
$2: 548$
\end{tabular} & 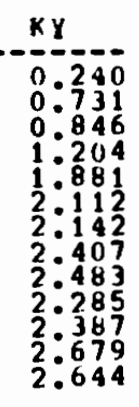 \\
\hline $\begin{array}{l}\text { YEAR } \\
1970 \\
1970 \\
1972 \\
1973 \\
1974 \\
1975 \\
1976 \\
1977 \\
1978 \\
1979 \\
1980 \\
1981 \\
1982\end{array}$ & \begin{tabular}{l}
$L A$ \\
\hdashline $0: 0$ \\
$0: 0$ \\
$0: 0$ \\
$0: 0$ \\
$0: 0$ \\
$0: 0$ \\
$0: 0$ \\
$0: 0$ \\
$0: 0$ \\
$0: 0$ \\
$0: 0$ \\
$0: 0$ \\
$0: 0$
\end{tabular} & 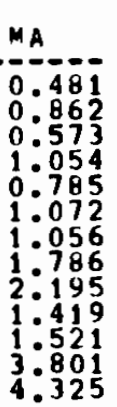 & $\begin{array}{l}M D \\
-1042- \\
1: 154 \\
1: 154 \\
1: 349 \\
2: 389 \\
1: 270 \\
1: 748 \\
1: 748 \\
2: 670 \\
2: 850 \\
2: 556 \\
2: 793 \\
3: 311 \\
3.366\end{array}$ & $\begin{array}{l}M E \\
0.0- \\
0: 0 \\
0: 0 \\
0: 0 \\
0: 0 \\
0: 0 \\
0: 0 \\
0: 0 \\
0: 0 \\
0: 0 \\
0: 0 \\
0: 0 \\
0: 0 \\
0: 0\end{array}$ & \begin{tabular}{l}
$M 1$ \\
\hdashline 0.380 \\
$1: 302$ \\
$1: 500$ \\
$1: 393$ \\
$2: 747$ \\
$3: 065$ \\
$2: 057$ \\
$3: 350$ \\
$33: 348$ \\
$3: 267$ \\
$33: 211$ \\
$3: 895$ \\
4.008
\end{tabular} & \begin{tabular}{l}
$M N$ \\
\hdashline $0: 375$ \\
$0: 0$ \\
$0: 0$ \\
$1: 131$ \\
$3: 230$ \\
$2: 711$ \\
$2: 982$ \\
$3: 010$ \\
$3: 880$ \\
$3: 442$ \\
$3: 488$ \\
$5: 125$ \\
$5: 218$
\end{tabular} & $\begin{array}{l}M L \\
0.279 \\
0: 871 \\
0: 877 \\
0: 770 \\
1: 403 \\
1: 720 \\
10632 \\
2: 000 \\
2: 037 \\
2: 282 \\
2: 041 \\
2: 350 \\
2.293\end{array}$ & 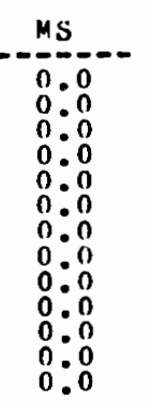 & \begin{tabular}{l}
$M T$ \\
\hdashline 0.219 \\
$0: 898$ \\
$0: 0$ \\
$1: 457$ \\
$1: 064$ \\
$1: 064$ \\
$2: 071$ \\
$1: 180$ \\
$1: 290$ \\
20154 \\
$2: 1996$ \\
$3: 060$ \\
5.604
\end{tabular} \\
\hline
\end{tabular}


TABLE 3.11 (cont). Steam Coal Prices: Residential Sector ( $\$ /$ million Btu)

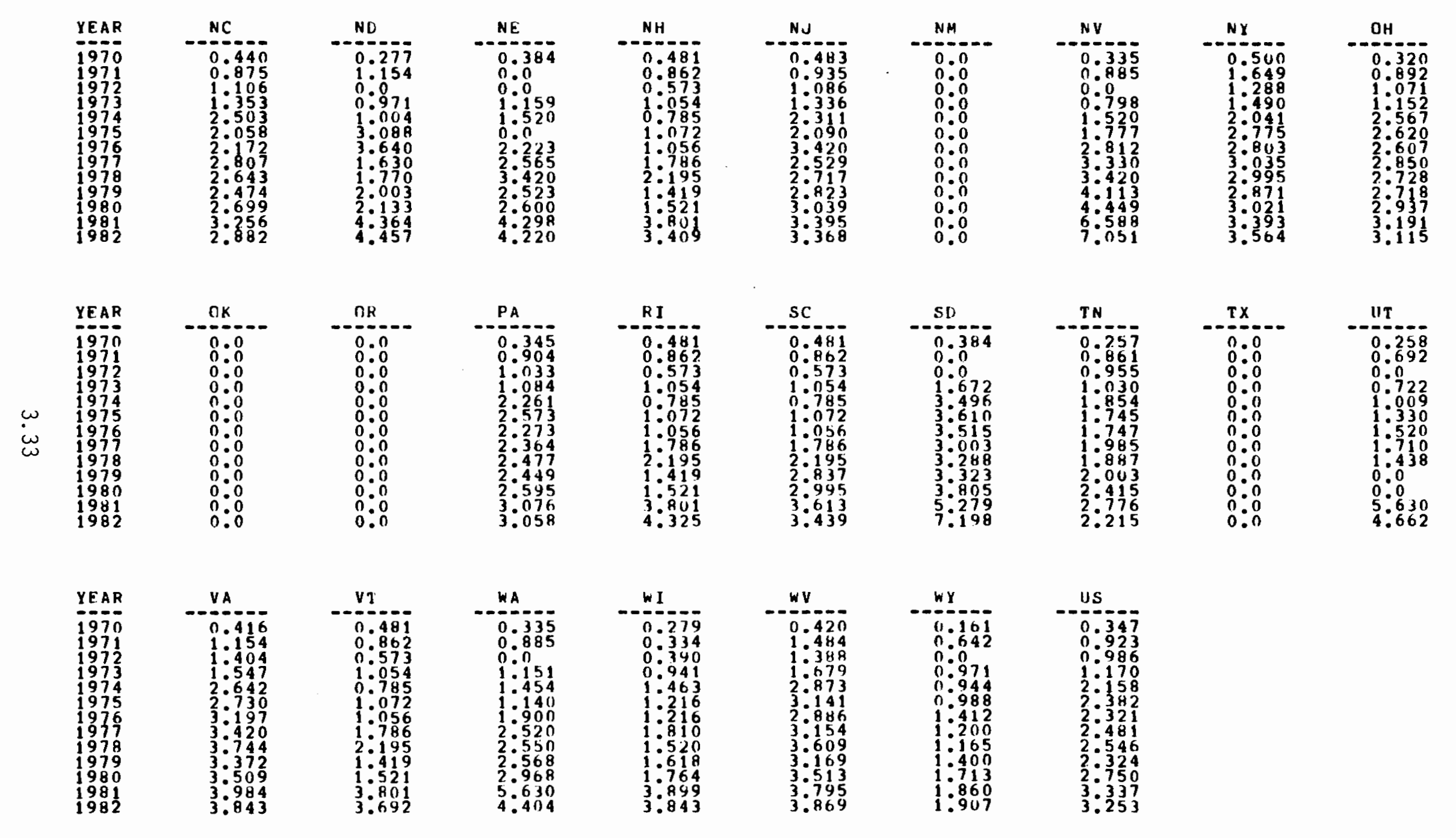


TABLE 3.12. Steam Coal Prices: Industrial Sector ( $\$ /$ million Btu)
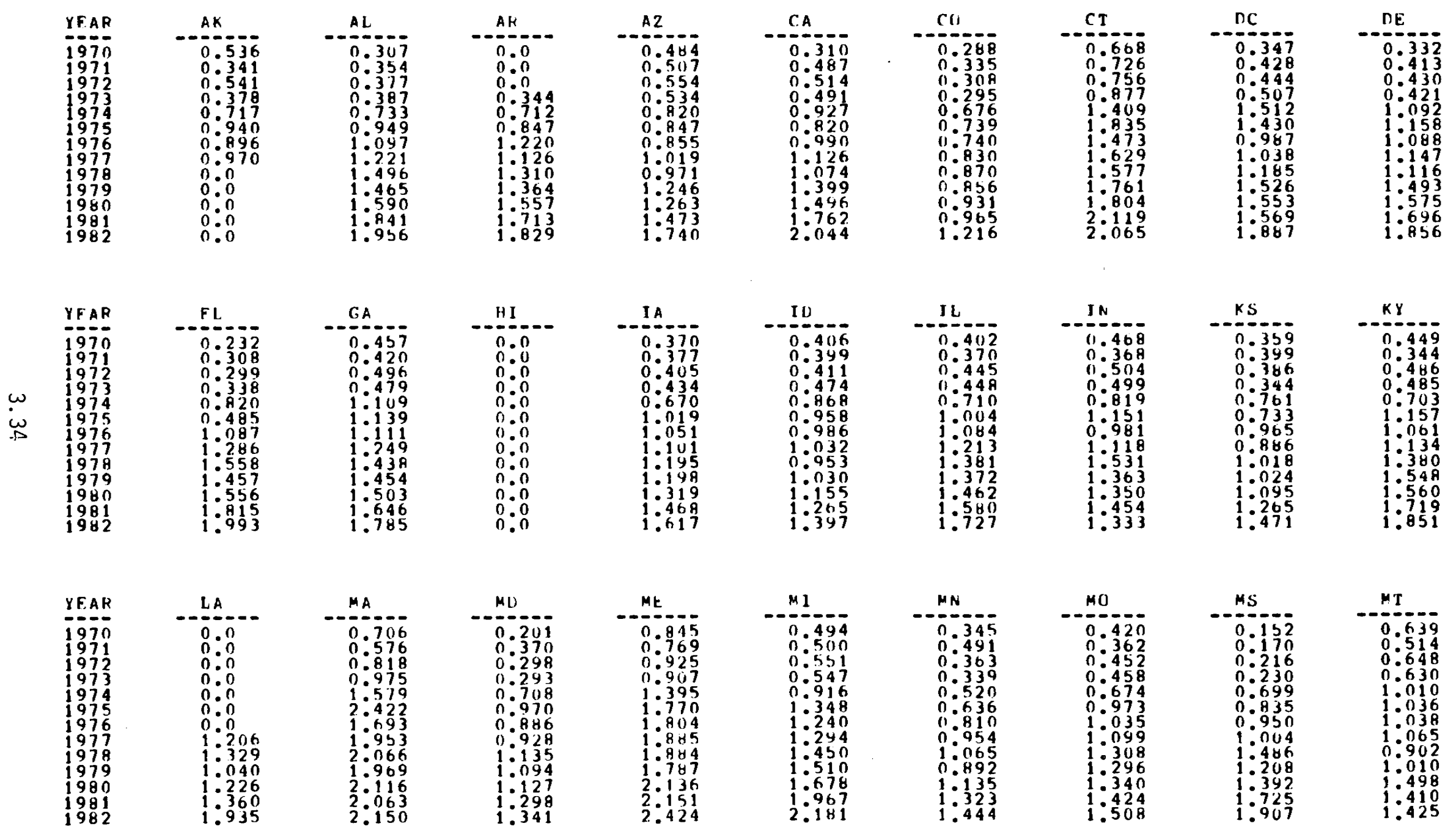
TABLE 3.12 (cont). Steam Coal Prices: Industrial Sector ( $\$ /$ million Btu)

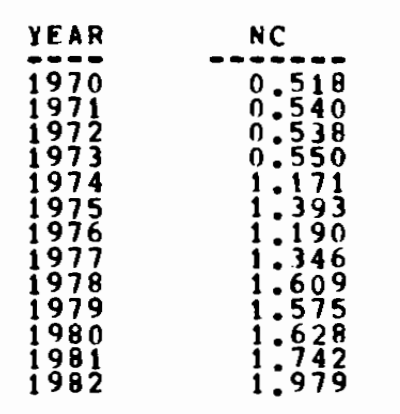

\begin{tabular}{ll}
$N D$ & $N E$ \\
\hdashline 0.353 & 0.263 \\
0.329 & 0.323 \\
0.358 & 0.300 \\
0.310 & 0.307 \\
0.667 & 0.437 \\
0.705 & 0.603 \\
0.711 & 0.768 \\
0.766 & 0.883 \\
0.850 & 1.425 \\
0.669 & 1.081 \\
0.871 & 1.213 \\
1.093 & 1.412 \\
0.891 & 1.407
\end{tabular}
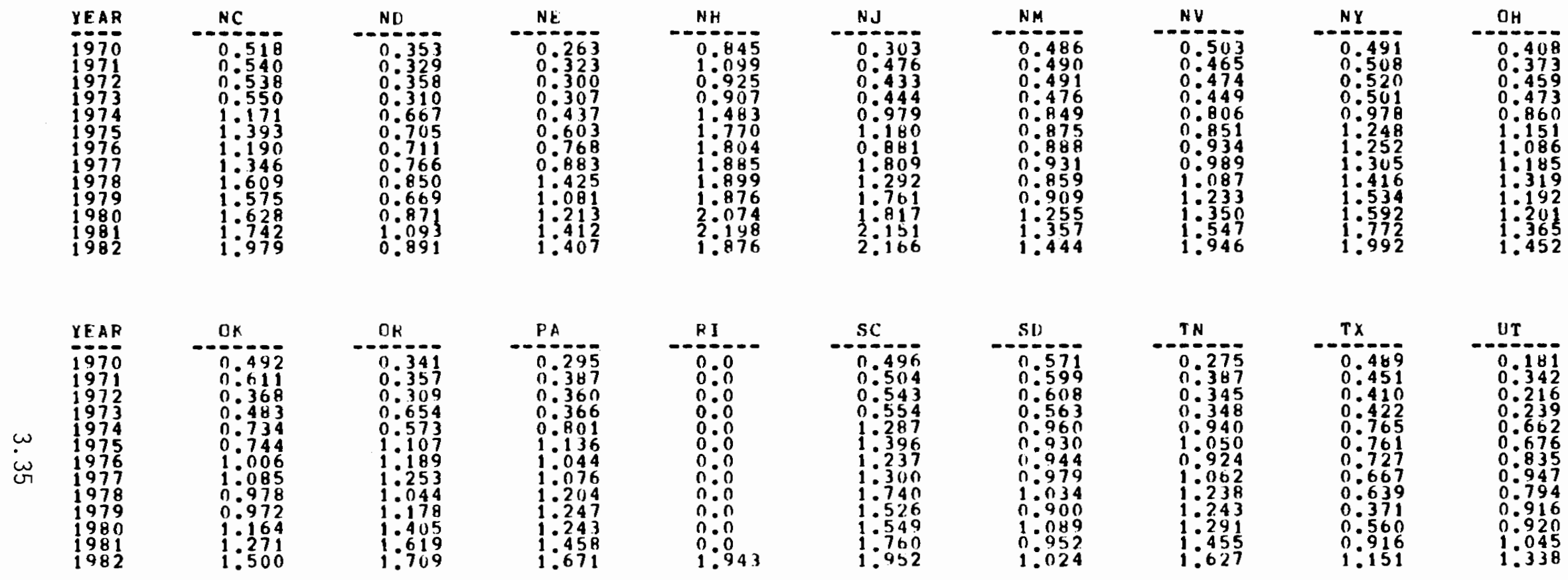

\begin{tabular}{ll} 
YFAR & VA \\
\hline 1970 & 0.344 \\
1971 & 0.486 \\
1972 & 0.377 \\
1973 & 0.384 \\
1974 & $1: 191$ \\
1975 & $1: 334$ \\
1976 & 1.171 \\
1977 & 1.242 \\
1978 & 1.487 \\
1979 & 1.479 \\
1980 & 1.328 \\
1981 & 1.737 \\
1982 & 1.897
\end{tabular}
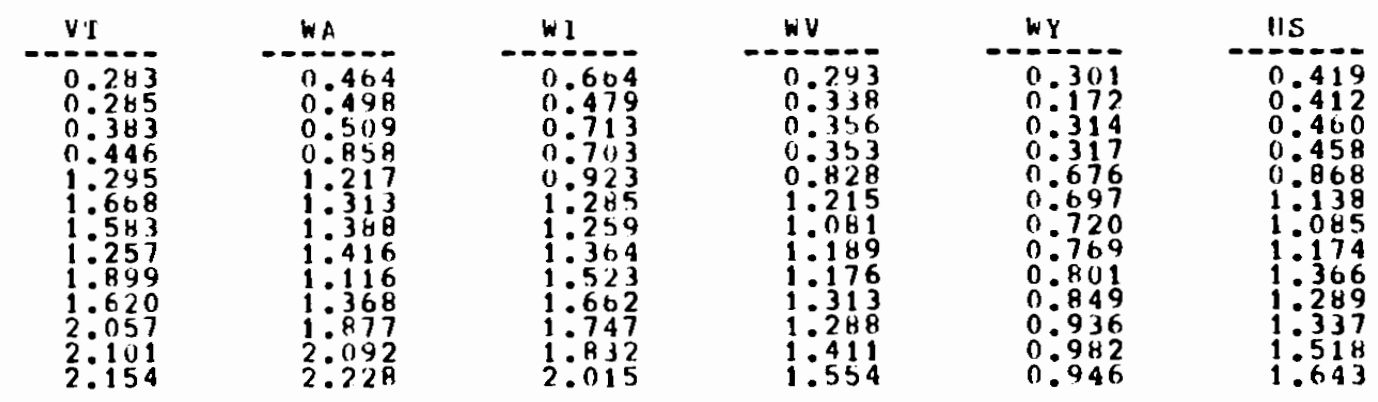
TABLE 3.13. Steam Coal Prices: Electric Utility Sector ( $\$ / m i 11$ ion Btu)

\begin{tabular}{|c|c|c|c|c|c|c|c|c|c|}
\hline 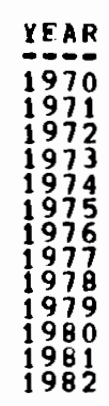 & 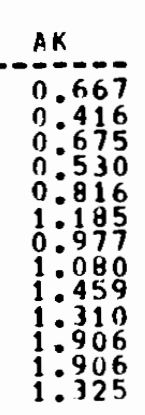 & \begin{tabular}{l} 
AL \\
\hdashline $0: 259$ \\
$0: 346$ \\
$0: 380$ \\
$0: 390$ \\
$1: 232$ \\
$1: 118$ \\
$0: 915$ \\
$1: 128$ \\
$1: 387$ \\
$1: 304$ \\
$1: 424$ \\
$1: 886$ \\
$2: 031$
\end{tabular} & 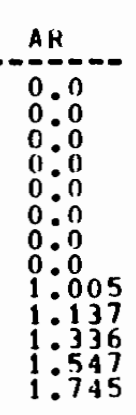 & 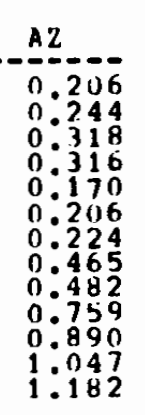 & 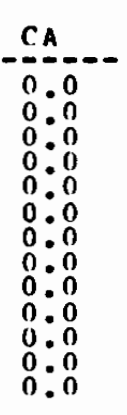 & 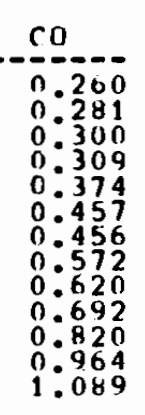 & 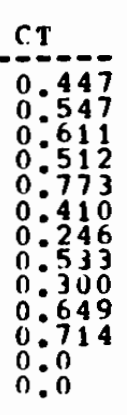 & 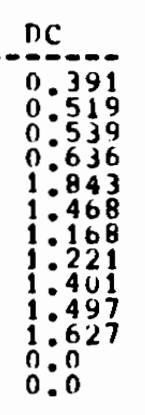 & 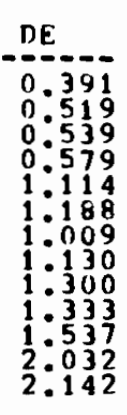 \\
\hline 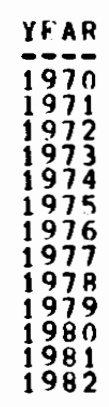 & 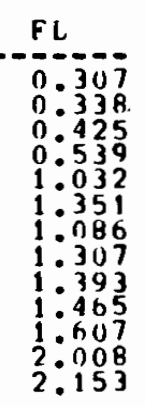 & \begin{tabular}{l}
$6 A$ \\
\hdashline-380 \\
$0: 385$ \\
$0: 435$ \\
$0: 447$ \\
$1: 597$ \\
$1: 175$ \\
$1: 034$ \\
$1: 218$ \\
$1: 455$ \\
$1: 440$ \\
$1: 571$ \\
$1: 709$ \\
$1: 861$
\end{tabular} & 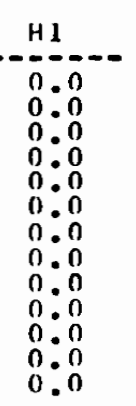 & 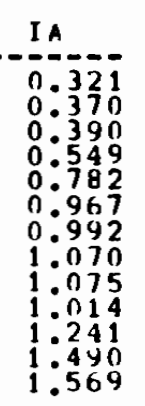 & \begin{tabular}{c}
10 \\
\hdashline $0:-0$ \\
$0: 0$ \\
$0: 0$ \\
$0: 0$ \\
$0: 0$ \\
$0: 0$ \\
$0: 0$ \\
$0: 0$ \\
$0: 0$ \\
$0: 0$ \\
$0: 0$ \\
$0: 0$ \\
0.0 \\
0.0
\end{tabular} & 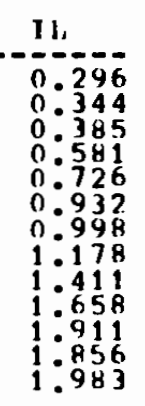 & 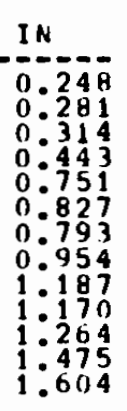 & 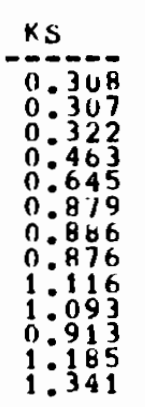 & 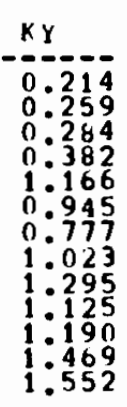 \\
\hline 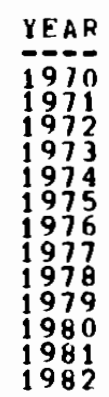 & 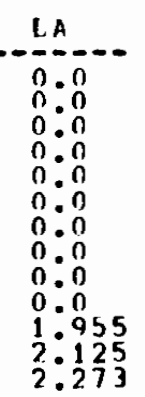 & \begin{tabular}{c}
$M A$ \\
\hdashline 0.311 \\
$0: 336$ \\
$0: 454$ \\
$0: 943$ \\
$1: 255$ \\
$1: 694$ \\
$1: 512$ \\
$1: 782$ \\
$2: 117$ \\
$2: 435$ \\
$1: 946$ \\
$2: 460$ \\
$2: 491$
\end{tabular} & 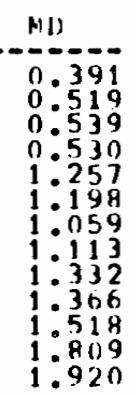 & 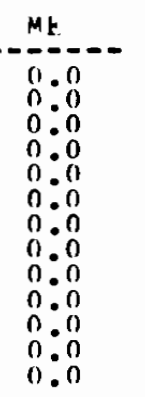 & 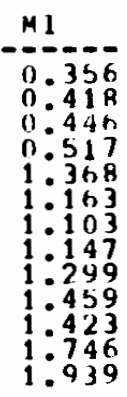 & 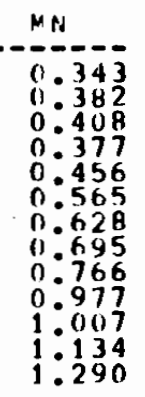 & 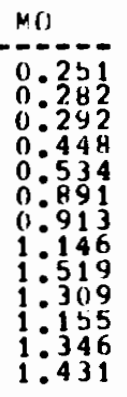 & 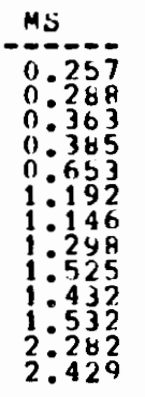 & 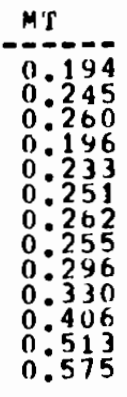 \\
\hline
\end{tabular}


JABLE 3.33 (cont). Steam Coal Prices: Electric Utility Sector $(\$ /$ million Btu)

\begin{tabular}{|c|c|c|c|c|c|c|c|c|c|}
\hline $\begin{array}{l}\text { YEAR } \\
-1970 \\
1970 \\
1971 \\
1972 \\
1973 \\
1974 \\
1975 \\
1976 \\
1977 \\
1978 \\
1979 \\
1980 \\
1981 \\
1982\end{array}$ & \begin{tabular}{l}
$N C$ \\
\hdashline 0.407 \\
0.454 \\
0.443 \\
0.513 \\
1.616 \\
1.016 \\
0.871 \\
1.303 \\
1.409 \\
1.295 \\
1.439 \\
1.730 \\
1.878
\end{tabular} & \begin{tabular}{l}
$N U$ \\
\hdashline 0.249 \\
0.254 \\
0.266 \\
0.285 \\
0.314 \\
0.869 \\
0.431 \\
0.452 \\
1.520 \\
0.543 \\
0.626 \\
0.681 \\
0.807
\end{tabular} & $\begin{array}{l}N E \\
-0.352 \\
0.403 \\
0.430 \\
0.465 \\
0.727 \\
0.962 \\
10026 \\
10156 \\
1.101 \\
10116 \\
10166 \\
1.144 \\
1.169\end{array}$ & $\begin{array}{l}N H \\
-0.351 \\
0.439 \\
0.479 \\
0.510 \\
0.819 \\
1.236 \\
1: 232 \\
1.288 \\
1.502 \\
1.475 \\
1.626 \\
1.715 \\
1.984\end{array}$ & $\begin{array}{l}N J \\
0.449 \\
0.547 \\
0.644 \\
0.804 \\
1.428 \\
1.858 \\
1.594 \\
1.568 \\
1.684 \\
1.756 \\
1.894 \\
2.067 \\
2.140\end{array}$ & \begin{tabular}{l}
$N M$ \\
\hdashline 0.143 \\
$0: 151$ \\
$0: 153$ \\
$0: 155$ \\
$0: 189$ \\
$0: 226$ \\
$0: 259$ \\
$0: 294$ \\
$0: 421$ \\
$0: 479$ \\
$0: 559$ \\
$0: 699$ \\
0.785
\end{tabular} & $\begin{array}{l}N V \\
00.305 \\
0: 310 \\
0: 297 \\
0: 278 \\
0: 282 \\
0: 308 \\
0: 385 \\
0: 437 \\
0.604 \\
0.944 \\
1: 050 \\
1.099 \\
1.175\end{array}$ & $\begin{array}{l}N Y \\
-0.465 \\
0.528 \\
0.515 \\
0.521 \\
1.157 \\
1.208 \\
1.063 \\
1.133 \\
1.266 \\
1.318 \\
1.414 \\
1.709 \\
1.828\end{array}$ & \begin{tabular}{l}
$O H$ \\
\hdashline 0.290 \\
$0: 349$ \\
$0: 378$ \\
$0: 447$ \\
$1: 199$ \\
$1: 042$ \\
$0: 915$ \\
$0: 988$ \\
$1: 193$ \\
$1: 235$ \\
$1: 375$ \\
1.644 \\
1.723
\end{tabular} \\
\hline $\begin{array}{l}\text { YEAR } \\
1970 \\
1971 \\
1972 \\
1973 \\
1974 \\
1975 \\
1975 \\
1976 \\
1977 \\
1978 \\
1979 \\
1980 \\
1981 \\
1982\end{array}$ & \begin{tabular}{l}
$0 K$ \\
\hdashline 0.349 \\
0.212 \\
0.194 \\
0.303 \\
0.409 \\
$1: 793$ \\
0.979 \\
1.076 \\
1.065 \\
1.096 \\
1.154 \\
1.453 \\
1.646
\end{tabular} & $\begin{array}{l}0 R \\
-0.228 \\
0: 194 \\
0.174 \\
0.097 \\
0.121 \\
0: 587 \\
0: 165 \\
0.228 \\
0: 978 \\
1.729 \\
1.412 \\
1.586 \\
1.715\end{array}$ & \begin{tabular}{l}
$P A$ \\
\hdashline 0.314 \\
0.362 \\
0.400 \\
0.431 \\
0.904 \\
0.996 \\
0.912 \\
0.924 \\
1.093 \\
1.123 \\
1.216 \\
1.555 \\
1.634
\end{tabular} & \begin{tabular}{l}
$R I$ \\
\hdashline 0.289 \\
$0: 338$ \\
$0: 567$ \\
0.567 \\
$1: 562$ \\
$0: 0$ \\
$0: 0$ \\
$0: 0$ \\
$0: 0$ \\
$0: 0$ \\
$0: 0$ \\
$0: 0$ \\
0.0
\end{tabular} & \begin{tabular}{l}
$s c$ \\
\hdashline 0.433 \\
$0: 443$ \\
$0: 470$ \\
0.521 \\
1.504 \\
$1: 032$ \\
$0: 916$ \\
1.312 \\
$1: 392$ \\
$1: 376$ \\
$1: 477$ \\
$1: 801$ \\
1.908
\end{tabular} & \begin{tabular}{l}
51 \\
\hdashline 0.352 \\
0.405 \\
0.429 \\
0.341 \\
0.473 \\
0.531 \\
0.620 \\
0.592 \\
0.607 \\
0.696 \\
0.848 \\
0.942 \\
1.146
\end{tabular} & \begin{tabular}{l}
$T N$ \\
\hdashline 0.230 \\
$0: 325$ \\
$0: 360$ \\
$0: 341$ \\
$1: 044$ \\
$0: 990$ \\
0.737 \\
1.028 \\
$1: 296$ \\
$1: 042$ \\
$1: 346$ \\
$1: 735$ \\
1.745
\end{tabular} & \begin{tabular}{l}
$T x$ \\
\hdashline 0.0 \\
$0: 214$ \\
$0: 216$ \\
$0: 138$ \\
$0: 181$ \\
$0: 238$ \\
$1: 004$ \\
1.050 \\
$1: 217$ \\
1.594 \\
$1: 760$ \\
1.455 \\
1.596
\end{tabular} & $\begin{array}{l}11 T \\
0.251 \\
0.281 \\
0: 291 \\
0.353 \\
0.427 \\
0: 558 \\
0: 653 \\
0.757 \\
0: 844 \\
0: 991 \\
1.152 \\
1: 265 \\
1.412\end{array}$ \\
\hline $\begin{array}{l}\text { YFAR } \\
-1970 \\
1971 \\
1971 \\
1972 \\
1973 \\
1974 \\
1975 \\
1976 \\
1977 \\
1978 \\
1979 \\
1980 \\
1981 \\
1982\end{array}$ & $\begin{array}{l}\text { VA } \\
0.383 \\
0.446 \\
0.434 \\
0.551 \\
1.543 \\
1.725 \\
1.083 \\
1.465 \\
1.645 \\
1.620 \\
1.798 \\
1.930 \\
1.929\end{array}$ & $\begin{array}{l}V T \\
-0.486 \\
0.611 \\
0.619 \\
0.726 \\
1.504 \\
2.049 \\
1.759 \\
1.377 \\
1.268 \\
1.772 \\
2.126 \\
2.174 \\
2.145\end{array}$ & $\begin{array}{l}1 A \\
00.0 \\
0: 0 \\
0: 0 \\
00388 \\
0.369 \\
0.565 \\
0.757 \\
0.723 \\
0.771 \\
0.836 \\
0.962 \\
1.109 \\
1.389\end{array}$ & $\begin{array}{l}W J \\
0.387 \\
0.431 \\
0.465 \\
0.541 \\
0.887 \\
0.805 \\
0.777 \\
0.875 \\
1.036 \\
10115 \\
1.365 \\
1.548 \\
1.717\end{array}$ & $\begin{array}{l}w V \\
0.251 \\
0: 319 \\
0: 354 \\
0.423 \\
1: 344 \\
0: 958 \\
0.891 \\
1.045 \\
1: 194 \\
1: 1400 \\
1: 234 \\
1.615 \\
1.714\end{array}$ & \begin{tabular}{l}
$W Y$ \\
\hdashline 0.140 \\
$0: 150$ \\
$0: 171$ \\
$0: 198$ \\
0.212 \\
$0: 746$ \\
$0: 288$ \\
$0: 347$ \\
$0: 378$ \\
$0: 470$ \\
0.568 \\
0.693 \\
0.769
\end{tabular} & \begin{tabular}{l} 
US \\
\hdashline 0.312 \\
$0: 363$ \\
$0: 384$ \\
0.463 \\
$1: 189$ \\
$1: 046$ \\
$0: 920$ \\
1.084 \\
$1: 284$ \\
$1: 263$ \\
$1: 388$ \\
1.532 \\
1.646
\end{tabular} & & \\
\hline
\end{tabular}

Source: Pacific Northwest Laboratory 
TABLE 3.14. Distillate Fuel Prices: Residential Sector ( $\$ / \mathrm{million}$ Btu)

\begin{tabular}{|c|c|c|c|c|c|c|c|c|c|}
\hline $\begin{array}{l}\text { YEAR } \\
1970 \\
1970 \\
1971 \\
1972 \\
1973 \\
1974 \\
1975 \\
1976 \\
1977 \\
1979 \\
1980 \\
1981 \\
1982\end{array}$ & 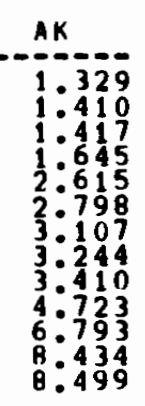 & 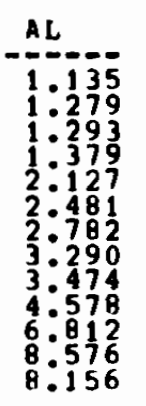 & 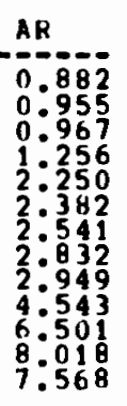 & 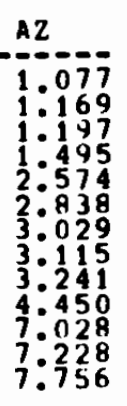 & 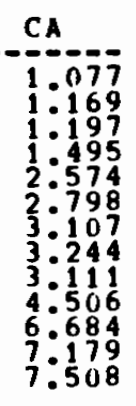 & 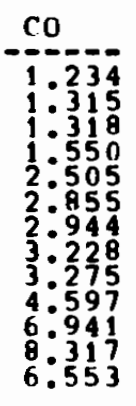 & 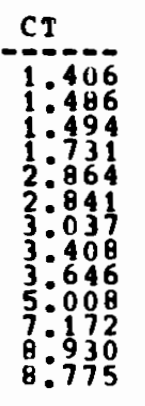 & 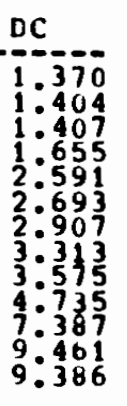 & 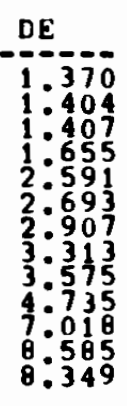 \\
\hline $\begin{array}{l}\text { YEAR } \\
7970 \\
1971 \\
1972 \\
1973 \\
1974 \\
1975 \\
1976 \\
1977 \\
1978 \\
1979 \\
1980 \\
1981 \\
1982\end{array}$ & 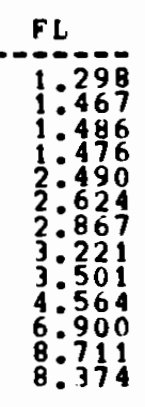 & 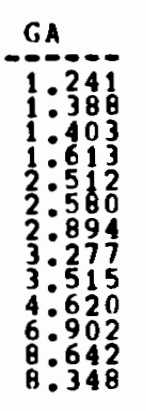 & 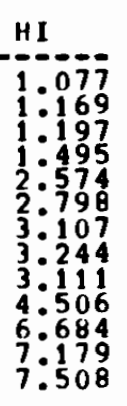 & 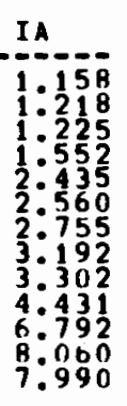 & 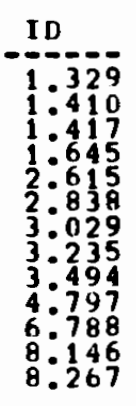 & 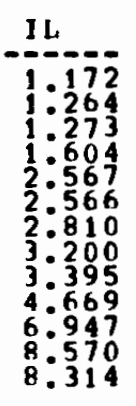 & 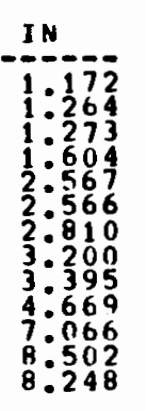 & 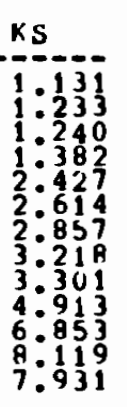 & 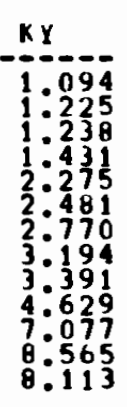 \\
\hline $\begin{array}{l}Y E A R \\
Y 1970 \\
1971 \\
1972 \\
1973 \\
1974 \\
1975 \\
1976 \\
1977 \\
1978 \\
1979 \\
1980 \\
1981 \\
1982\end{array}$ & 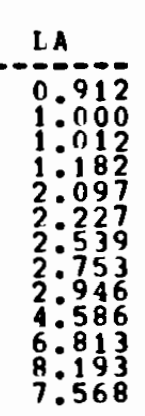 & $\begin{array}{l}\text { MA } \\
-1: 419 \\
1: 499 \\
1: 506 \\
1: 763 \\
2: 647 \\
2: 849 \\
3: 038 \\
3: 422 \\
3: 659 \\
5: 000 \\
7: 170 \\
8: 927 \\
8: 913\end{array}$ & 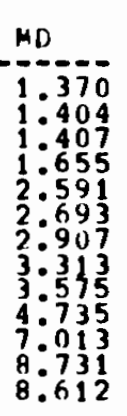 & 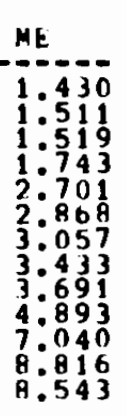 & 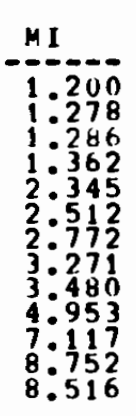 & 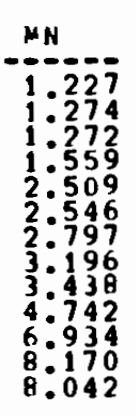 & 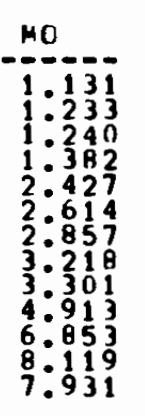 & 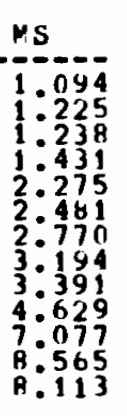 & 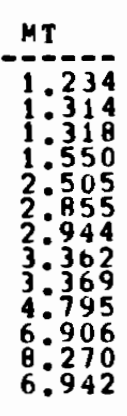 \\
\hline
\end{tabular}


IABLE 3.14 (cont). Distillate Fuel Prices: Residential Sector (\$/million Btu)

\begin{tabular}{|c|c|c|c|c|c|c|c|c|c|}
\hline $\begin{array}{l}\text { YEAR } \\
1970 \\
1970 \\
1971 \\
1973 \\
1973 \\
1975 \\
1976 \\
1977 \\
1978 \\
1979 \\
1980 \\
1981 \\
1982\end{array}$ & \begin{tabular}{r}
$N C$ \\
\hdashline 1.236 \\
$1: 292$ \\
$1: 298$ \\
$1: 571$ \\
$2: 586$ \\
$2: 744$ \\
$2: 933$ \\
$3: 276$ \\
$3: 537$ \\
$4: 644$ \\
6.930 \\
$8: 636$ \\
8.383
\end{tabular} & $\begin{array}{l}\text { ND } \\
-1.234 \\
1: 314 \\
1: 318 \\
1: 550 \\
2: 505 \\
2: 546 \\
2: 797 \\
3: 196 \\
3.369 \\
4: 795 \\
6.927 \\
8: 298 \\
6.659\end{array}$ & \begin{tabular}{l} 
NE \\
\hdashline 1.131 \\
$1: 233$ \\
$1: 240$ \\
$1: 382$ \\
$2: 427$ \\
$2: 614$ \\
$2: 857$ \\
$3: 219$ \\
$3: 301$ \\
$4: 913$ \\
6.853 \\
$8: 119$ \\
7.931
\end{tabular} & \begin{tabular}{l} 
NH \\
\hdashline 1.430 \\
1.511 \\
1.519 \\
10743 \\
2.701 \\
2.868 \\
33.057 \\
3.433 \\
3.691 \\
4.893 \\
7.107 \\
8.750 \\
8.487
\end{tabular} & \begin{tabular}{l} 
NJ \\
\hdashline 1.387 \\
1.466 \\
1.472 \\
1.601 \\
3.221 \\
2.787 \\
2.959 \\
3.347 \\
3.608 \\
4.861 \\
7.078 \\
9.773 \\
8.555
\end{tabular} & 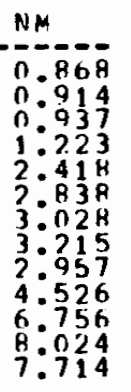 & 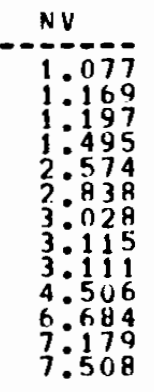 & 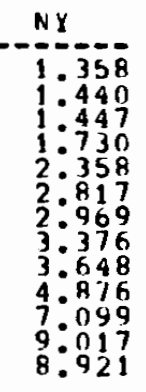 & \begin{tabular}{l}
$0 H$ \\
\hdashline 1.200 \\
$1: 278$ \\
$1: 286$ \\
$1: 362$ \\
$2: 345$ \\
$2: 512$ \\
$2: 772$ \\
$3: 202$ \\
$3: 421$ \\
$4: 796$ \\
$6: 656$ \\
8.513 \\
8.277
\end{tabular} \\
\hline $\begin{array}{l}\text { YFAR } \\
1970 \\
1971 \\
1972 \\
1973 \\
1974 \\
1975 \\
1976 \\
1977 \\
1978 \\
1979 \\
1980 \\
1981 \\
1982\end{array}$ & \begin{tabular}{r}
$n k$ \\
\hdashline 0.844 \\
$0: 858$ \\
0.858 \\
$1: 075$ \\
$2: 162$ \\
$2: 380$ \\
$2: 584$ \\
$2: 879$ \\
$3: 026$ \\
$4: 684$ \\
6.502 \\
79956 \\
7.495
\end{tabular} & 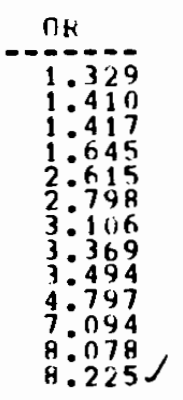 & $\begin{array}{l}P A \\
-1.505 \\
1: 399 \\
1: 407 \\
1: 641 \\
2: 630 \\
2: 808 \\
2: 934 \\
3: 326 \\
3: 566 \\
4: 798 \\
6.931 \\
8: 534 \\
8.254\end{array}$ & \begin{tabular}{r} 
RI \\
\hdashline 1.530 \\
$1: 611$ \\
1.619 \\
10640 \\
20853 \\
2.841 \\
30011 \\
3.264 \\
3.497 \\
4.883 \\
7.200 \\
8.913 \\
8.695
\end{tabular} & 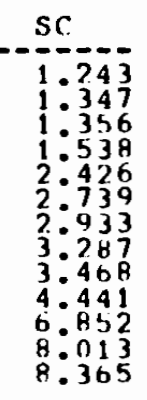 & $\begin{array}{l}50 \\
1: 234 \\
1: 314 \\
1: 318 \\
1: 550 \\
2: 505 \\
3: 546 \\
2: 797 \\
30196 \\
3: 369 \\
4: 795 \\
6: 927 \\
80298 \\
6.659\end{array}$ & 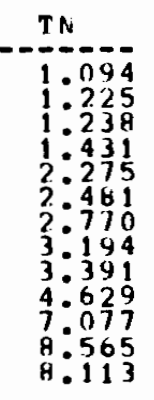 & 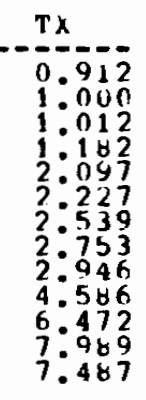 & $\begin{array}{l}11 T \\
-1.095 \\
1: 206 \\
1: 238 \\
1: 409 \\
2: 543 \\
2: 855 \\
2: 944 \\
33: 228 \\
3: 293 \\
4.480 \\
6.871 \\
8.217 \\
6.800\end{array}$ \\
\hline $\begin{array}{l}\text { YFAR } \\
1970 \\
1970 \\
1971 \\
1972 \\
1973 \\
1974 \\
1975 \\
1976 \\
1977 \\
1978 \\
1979 \\
1980 \\
1981 \\
1982\end{array}$ & \begin{tabular}{l}
$V A$ \\
\hdashline $1: 316$ \\
$1: 411$ \\
$1: 419$ \\
$1: 603$ \\
2.547 \\
2.676 \\
$2: 907$ \\
3.910 \\
30556 \\
$4: 913$ \\
7.161 \\
8.890 \\
9.644
\end{tabular} & \begin{tabular}{r}
$V T$ \\
\hdashline 1.430 \\
1.511 \\
1.519 \\
$1: 743$ \\
$2: 701$ \\
2.868 \\
30057 \\
3.433 \\
30691 \\
40893 \\
70340 \\
901042 \\
8.917
\end{tabular} & \begin{tabular}{l} 
WA \\
\hdashline $1: 329$ \\
$1: 410$ \\
$1: 417$ \\
$1: 645$ \\
$2: 615$ \\
$2: 798$ \\
$3: 1116$ \\
$3: 309$ \\
$3: 494$ \\
$4: 723$ \\
$7: 216$ \\
8.563 \\
8.808
\end{tabular} & 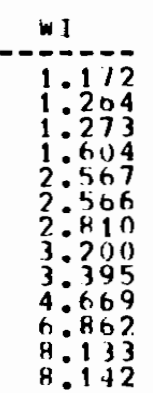 & \begin{tabular}{l}
$W v$ \\
\hdashline $1: 316$ \\
$1: 411$ \\
$1: 419$ \\
10613 \\
2.547 \\
2.676 \\
$2: 907$ \\
$3: 310$ \\
$3: 561$ \\
$4: 813$ \\
6.892 \\
8.710 \\
8.443
\end{tabular} & $\begin{array}{l}6 Y \\
1: 234 \\
1: 314 \\
1: 318 \\
1: 550 \\
2: 565 \\
2: 855 \\
2: 944 \\
3: 228 \\
3: 256 \\
4: 610 \\
6: 921 \\
8: 256 \\
6.716\end{array}$ & \begin{tabular}{l}
$11 s$ \\
\hdashline 1.334 \\
$1: 413$ \\
$1: 420$ \\
$1: 644$ \\
2.596 \\
$2: 718$ \\
$2: 927$ \\
$3: 317$ \\
30562 \\
$4: 730$ \\
7.052 \\
8.648 \\
9.544
\end{tabular} & & \\
\hline
\end{tabular}

Source: Pacific Northwest Laboratory 
TABLE 3.15. Distillate Fuel Prices: Commercial Sector ( $\$ / m i 11$ ion Btu)

\begin{tabular}{|c|c|c|c|c|c|c|c|c|c|}
\hline $\begin{array}{l}\text { YEAR } \\
1970 \\
1970 \\
1971 \\
1972 \\
1973 \\
1974 \\
1975 \\
1976 \\
1977 \\
1978 \\
1979 \\
1989 \\
1981 \\
1982\end{array}$ & \begin{tabular}{r}
$A K$ \\
\hdashline $1: 213$ \\
$1: 282$ \\
$1: 290$ \\
$1: 522$ \\
$2: 506$ \\
$2: 664$ \\
$2: 965$ \\
$3: 092$ \\
$3: 228$ \\
$4: 578$ \\
$6: 632$ \\
$8: 118$ \\
$8: 161$
\end{tabular} & $\begin{array}{r}A L \\
-0.947 \\
1: 045 \\
1: 052 \\
1: 137 \\
1: 872 \\
20210 \\
2: 485 \\
20916 \\
3: 046 \\
4: 282 \\
6: 281 \\
7: 881 \\
7: 403\end{array}$ & \begin{tabular}{l}
$A R$ \\
\hdashline $0: 842$ \\
$0: 906$ \\
$0: 914$ \\
$1: 193$ \\
$2: 154$ \\
$2: 310$ \\
$2: 454$ \\
$2: 767$ \\
$2: 859$ \\
$4: 404$ \\
$6: 302$ \\
$7: 773$ \\
$7: 337$
\end{tabular} & \begin{tabular}{r}
$A Z$ \\
\hdashline $1: 009$ \\
$1: 087$ \\
$1: 112$ \\
$1: 401$ \\
$2: 472$ \\
2.696 \\
$2: 902$ \\
$2: 987$ \\
$3: 111$ \\
$4: 382$ \\
$6: 848$ \\
$7: 120$ \\
$7: 565$
\end{tabular} & 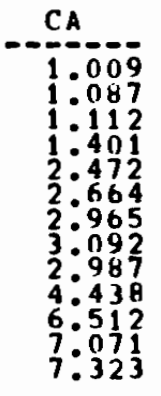 & \begin{tabular}{r}
$C 0$ \\
\hdashline $1: 038$ \\
$1: 079$ \\
$1: 077$ \\
$1: 320$ \\
$2: 275$ \\
$2: 506$ \\
$2: 643$ \\
$2: 906$ \\
$2: 982$ \\
$4: 344$ \\
$6: 489$ \\
$7: 836$ \\
$6: 617$
\end{tabular} & \begin{tabular}{l} 
CT \\
\hdashline $1: 093$ \\
$1: 158$ \\
10160 \\
$1: 398$ \\
$2: 484$ \\
$2: 471$ \\
$2: 656$ \\
3007 \\
30178 \\
$4: 559$ \\
$6: 479$ \\
80177 \\
$7: 975$
\end{tabular} & $\begin{array}{l}\text { DC } \\
1: 118 \\
1: 141 \\
1: 141 \\
1: 331 \\
2: 268 \\
2: 409 \\
2: 602 \\
30005 \\
3: 192 \\
4: 393 \\
6: 612 \\
8: 502 \\
8: 244\end{array}$ & \begin{tabular}{l}
$D E$ \\
\hdashline $1: 118$ \\
$1: 141$ \\
$1: 141$ \\
$1: 331$ \\
$2: 268$ \\
$2: 409$ \\
$2: 602$ \\
$3: 005$ \\
$3: 1992$ \\
$4: 393$ \\
$6: 519$ \\
$8: 043$ \\
$7: 701$
\end{tabular} \\
\hline $\begin{array}{l}\text { YEAR } \\
\text { YEAR } \\
1970 \\
1971 \\
1972 \\
1973 \\
1974 \\
1975 \\
1976 \\
1977 \\
1978 \\
1979 \\
1980 \\
1981 \\
1982\end{array}$ & \begin{aligned}$F L \\
$\hdashline $1: 083 \\
1: 199 \\
1: 209 \\
1: 217 \\
2: 192 \\
2: 309 \\
2: 525 \\
2: 800 \\
3070 \\
4: 269 \\
6: 361 \\
80005 \\
7: 600\end{aligned}$ & \begin{tabular}{l} 
GA \\
\hdashline $1: 036$ \\
$1: 134$ \\
$1: 141$ \\
$1: 330$ \\
$1: 822$ \\
$2: 271$ \\
$2: 549$ \\
$2: 931$ \\
$3: 082$ \\
$4: 321$ \\
$6: 364$ \\
$7: 942$ \\
$7: 577$
\end{tabular} & 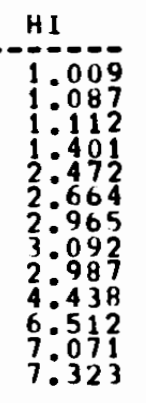 & 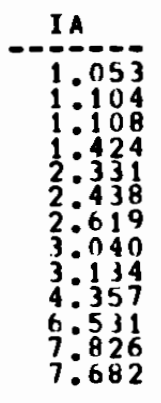 & \begin{aligned} ID \\
\hdashline 1.2113 \\
$1: 282 \\
1: 290 \\
1: 522 \\
2: 506 \\
2.696 \\
2: 902 \\
3.103 \\
3.3018 \\
4: 650 \\
6.619 \\
7.984 \\
7: 711\end{aligned}$ & 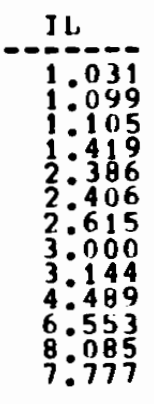 & $\begin{array}{l}1 N \\
1.031 \\
1: 099 \\
1: 105 \\
10419 \\
2: 386 \\
2: 406 \\
2: 615 \\
33000 \\
30144 \\
4: 489 \\
6: 630 \\
8.041 \\
7.973\end{array}$ & 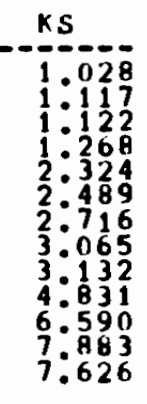 & $\begin{array}{l}k Y \\
-10-974 \\
1: 077 \\
1: 086 \\
1: 266 \\
2: 096 \\
2: 302 \\
2: 576 \\
2: 955 \\
3: 116 \\
4: 433 \\
6: 7113 \\
8108 \\
7: 619\end{array}$ \\
\hline $\begin{array}{l}\text { YEAR } \\
19-7 \\
1970 \\
1971 \\
1972 \\
1973 \\
1974 \\
1975 \\
1976 \\
1977 \\
1978 \\
1979 \\
1980 \\
1981 \\
1982\end{array}$ & \begin{tabular}{r}
1. \\
\hdashline 0.870 \\
00948 \\
$0: 957$ \\
$1: 123$ \\
20007 \\
$2: 159$ \\
$2: 452$ \\
2.690 \\
$2: 856$ \\
$4: 446$ \\
$6: 605$ \\
77942 \\
7.337
\end{tabular} & \begin{tabular}{l}
$M A$ \\
\hdashline $1: 103$ \\
$1: 167$ \\
$1: 169$ \\
$1: 425$ \\
$2: 296$ \\
$2: 478$ \\
20658 \\
30020 \\
$3: 189$ \\
$4: 551$ \\
6.473 \\
8.175 \\
7.985
\end{tabular} & 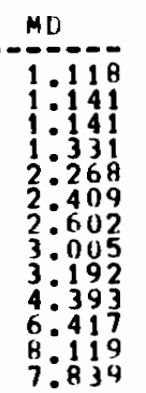 & \begin{aligned}$M E \\
$\hdashline $1: 111 \\
1: 177 \\
1: 179 \\
2: 408 \\
2: 343 \\
2.494 \\
2.674 \\
3: 029 \\
3: 217 \\
4: 454 \\
6.418 \\
8.146 \\
7: 854\end{aligned}$ & $\begin{array}{r}M 1 \\
-1: 046 \\
1: 100 \\
1: 1145 \\
1: 195 \\
2: 169 \\
2: 345 \\
2: 567 \\
3: 053 \\
30205 \\
4: 749 \\
6.613 \\
9: 202 \\
7: 922\end{array}$ & $\begin{array}{r}M N \\
-1: 035 \\
1: 057 \\
1052 \\
1: 325 \\
2: 277 \\
2: 340 \\
2: 564 \\
2: 938 \\
30105 \\
4: 503 \\
6.479 \\
7: 749 \\
7.464\end{array}$ & $\begin{array}{l}M 0 \\
1: 028 \\
1: 117 \\
1: 122 \\
1: 268 \\
2: 324 \\
2: 489 \\
2: 716 \\
30065 \\
3: 132 \\
4: 831 \\
6: 590 \\
70.843 \\
7.626\end{array}$ & $\begin{array}{l}M S \\
-0.912 \\
1: 001 \\
1: 007 \\
1: 100 \\
2: 003 \\
2: 210 \\
2: 475 \\
2: 031 \\
2: 973 \\
4: 331 \\
6: 525 \\
7.871 \\
7.363\end{array}$ & $\begin{array}{l}M T \\
1: 038 \\
1: 078 \\
1: 077 \\
1: 320 \\
2: 275 \\
2: 506 \\
2: 643 \\
30026 \\
3: 069 \\
4: 531 \\
6: 456 \\
7: 791 \\
7: 010\end{array}$ \\
\hline
\end{tabular}


TABLE 3.15 (cont). Distillate Fuel Prices: Commercial Sector ( $\$ /$ million Btu)

\begin{tabular}{|c|c|c|c|c|c|c|c|c|c|}
\hline $\begin{array}{l}\text { YFAR } \\
-1970 \\
1970 \\
1971 \\
1972 \\
1973 \\
1974 \\
1975 \\
1976 \\
1977 \\
1978 \\
1979 \\
1980 \\
1981 \\
1982\end{array}$ & \begin{tabular}{r}
$N C$ \\
\hdashline 1.031 \\
$1: 056$ \\
1.056 \\
1.295 \\
2.276 \\
2.415 \\
2.584 \\
2.930 \\
3.102 \\
4.344 \\
6.389 \\
7.937 \\
7.608
\end{tabular} & $\begin{array}{l}N D \\
1.038 \\
1.078 \\
1.077 \\
1.320 \\
2.275 \\
2.340 \\
2.564 \\
2.938 \\
3.069 \\
4.531 \\
6.475 \\
7.917 \\
6.724\end{array}$ & $\begin{array}{l}N E \\
1.028 \\
1: 117 \\
1: 122 \\
1.268 \\
2.324 \\
2.489 \\
2.716 \\
3.065 \\
3: 132 \\
4.831 \\
6.590 \\
7.883 \\
7.626\end{array}$ & $\begin{array}{l}\text { NH } \\
1.119 \\
1.177 \\
1: 179 \\
1.4198 \\
2.343 \\
2.494 \\
2.674 \\
3.029 \\
3.217 \\
4.454 \\
6.449 \\
8.115 \\
7.832\end{array}$ & $\begin{array}{r}\text { NJ } \\
1.165 \\
1.233 \\
1: 236 \\
1: 350 \\
2.708 \\
2: 527 \\
2.695 \\
3.095 \\
3: 284 \\
4.567 \\
6: 587 \\
8: 172 \\
8.010\end{array}$ & $\begin{array}{l}N M \\
0.840 \\
0.891 \\
0.9182 \\
1.181 \\
2.346 \\
2.696 \\
2.901 \\
3.083 \\
2.894 \\
4.430 \\
6.613 \\
7.854 \\
7.551\end{array}$ & $\begin{array}{l}N V \\
1.009 \\
1: 087 \\
1: 112 \\
1.401 \\
2.472 \\
2.696 \\
2.901 \\
2: 987 \\
2: 987 \\
4: 438 \\
6: 512 \\
7: 071 \\
7.323\end{array}$ & \begin{tabular}{l}
$N Y$ \\
\hdashline $1: 141$ \\
$1: 211$ \\
$1: 215$ \\
$1: 459$ \\
$1: 982$ \\
$2: 554$ \\
$2: 704$ \\
$3: 1222$ \\
$3: 321$ \\
$4: 582$ \\
$6: 606$ \\
$8: 413$ \\
8.239
\end{tabular} & \begin{tabular}{l}
$0 H$ \\
\hdashline 1.046 \\
$1: 100$ \\
$1: 105$ \\
$1: 195$ \\
$2: 169$ \\
$2: 345$ \\
$2: 567$ \\
2.989 \\
$3: 151$ \\
$4: 599$ \\
$6: 327$ \\
$8: 054$ \\
7.774
\end{tabular} \\
\hline $\begin{array}{l}\text { YEAR } \\
-1970 \\
1970 \\
1971 \\
1972 \\
1973 \\
1974 \\
1975 \\
1976 \\
1977 \\
1978 \\
1979 \\
1980 \\
1981 \\
1982\end{array}$ & $\begin{array}{l}C K \\
0.805 \\
0.814 \\
0.811 \\
1.021 \\
2.069 \\
2.307 \\
2.495 \\
2.813 \\
2.933 \\
4.541 \\
6: 362 \\
7.713 \\
7.266\end{array}$ & $\begin{array}{r}r H \\
1.213 \\
1.282 \\
1.290 \\
1.522 \\
2.506 \\
2.664 \\
2.964 \\
3.211 \\
3.301 \\
4.650 \\
6.867 \\
7.829 \\
7.935\end{array}$ & $\begin{array}{r}P A \\
1.111 \\
1.189 \\
1.193 \\
1.383 \\
2.366 \\
2.546 \\
2.673 \\
3.076 \\
3.259 \\
4.520 \\
6.492 \\
8.125 \\
7.797\end{array}$ & \begin{tabular}{l}
$R I$ \\
\hdashline 1.189 \\
1.254 \\
1.257 \\
$1: 325$ \\
2.474 \\
2.471 \\
20634 \\
2.840 \\
3.048 \\
4.445 \\
6.492 \\
8.169 \\
7.937
\end{tabular} & $\begin{array}{l}\text { sc } \\
1.021 \\
1.081 \\
10083 \\
1.263 \\
2.155 \\
2.4110 \\
2.583 \\
2.940 \\
3.074 \\
4.251 \\
6.354 \\
7.673 \\
7.592\end{array}$ & $\begin{array}{r}s 0 \\
1.038 \\
1: 078 \\
1: 077 \\
1: 320 \\
2.275 \\
2.340 \\
2.594 \\
2.938 \\
3.069 \\
4.531 \\
6.475 \\
7.817 \\
6.724\end{array}$ & 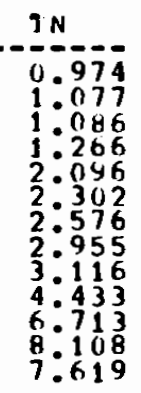 & $\begin{array}{l}T x \\
0.870 \\
0.948 \\
0.957 \\
1.123 \\
2.007 \\
2.159 \\
2.452 \\
2.690 \\
2.956 \\
4.446 \\
6.274 \\
7.745 \\
7.258\end{array}$ & \begin{tabular}{l}
$U T$ \\
\hdashline 0.921 \\
$0: 990$ \\
$1: 011$ \\
$1: 251$ \\
$2: 310$ \\
2.506 \\
20643 \\
2.906 \\
2.999 \\
4.234 \\
6.424 \\
7.741 \\
6.867
\end{tabular} \\
\hline $\begin{array}{l}\text { YEAR } \\
-1970 \\
1970 \\
1971 \\
1972 \\
1973 \\
1974 \\
1975 \\
1976 \\
1977 \\
1978 \\
1979 \\
1980 \\
1981 \\
1982\end{array}$ & $\begin{array}{l}\text { VA } \\
1.074 \\
1.147 \\
1.150 \\
1.290 \\
2.229 \\
2.393 \\
2.602 \\
3.002 \\
3.174 \\
4.465 \\
6.544 \\
8.174 \\
7.841\end{array}$ & $\begin{array}{r}V I \\
-1.111 \\
1.177 \\
1.179 \\
1.408 \\
2.343 \\
2.494 \\
2.674 \\
3.029 \\
3.217 \\
4.454 \\
6.578 \\
8.272 \\
8.034\end{array}$ & $\begin{array}{l}W A \\
-1.213 \\
1: 282 \\
1: 290 \\
1: 522 \\
2.506 \\
2.664 \\
2.964 \\
3: 211 \\
3: 308 \\
4.578 \\
6.975 \\
8.247 \\
8.431\end{array}$ & $\begin{array}{l}W \\
1.031 \\
1.099 \\
1.105 \\
1.419 \\
2.386 \\
2.406 \\
2.615 \\
30000 \\
3.144 \\
4.489 \\
6.498 \\
7.804 \\
7.666\end{array}$ & \begin{tabular}{c}
$w v$ \\
\hdashline 1.074 \\
1.147 \\
1.100 \\
1.290 \\
2.229 \\
2.393 \\
2.602 \\
30002 \\
3.179 \\
4.465 \\
6.403 \\
8.080 \\
7.736
\end{tabular} & $\begin{array}{r}W y \\
1.038 \\
1.078 \\
1.077 \\
1.320 \\
2.275 \\
2.506 \\
2.643 \\
2.906 \\
2.965 \\
4.357 \\
6.470 \\
7.777 \\
6.781\end{array}$ & $\begin{array}{l}\text { IIS } \\
1.090 \\
1: 155 \\
1: 154 \\
1.360 \\
2: 265 \\
2.453 \\
2.643 \\
2.999 \\
3.150 \\
4.511 \\
6.532 \\
8.056 \\
7.836\end{array}$ & & . \\
\hline
\end{tabular}

Source: Pacific Northwest Laboratory 
TABLE 3.16. Distillate Fuel Prices: Industrial Sector $(\$ /$ million Btu) $(a)$

\begin{tabular}{|c|c|c|c|c|c|c|c|c|c|}
\hline $\begin{array}{l}Y \text { YEAR } \\
1970 \\
1971 \\
1971 \\
1973 \\
1974 \\
1975 \\
1976 \\
1977 \\
1978 \\
1979 \\
1981 \\
1982\end{array}$ & 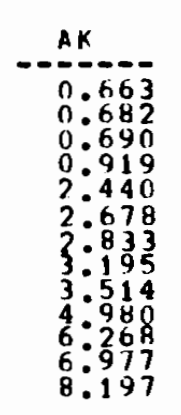 & 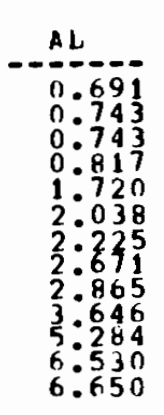 & 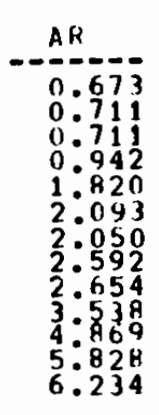 & 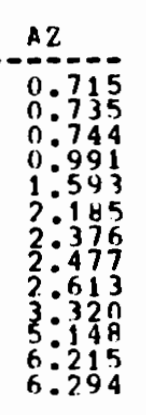 & 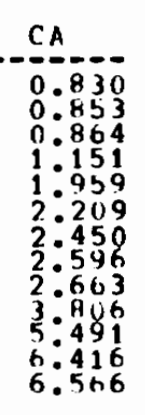 & 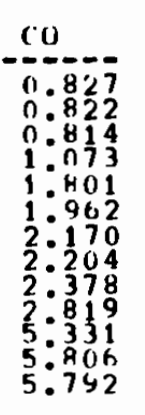 & 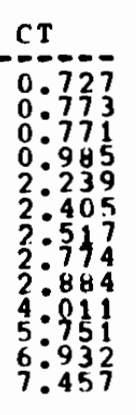 & 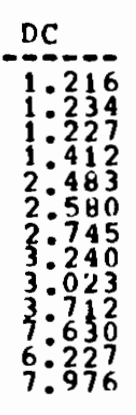 & 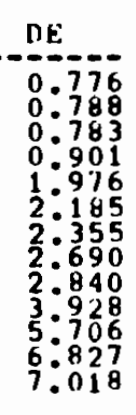 \\
\hline $\begin{array}{l}\text { YEAR } \\
1970 \\
1970 \\
1971 \\
1972 \\
1973 \\
1974 \\
1975 \\
1976 \\
1977 \\
1978 \\
1979 \\
1980 \\
1981 \\
1982\end{array}$ & 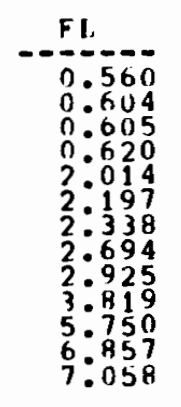 & 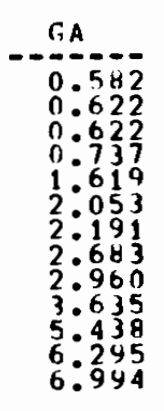 & 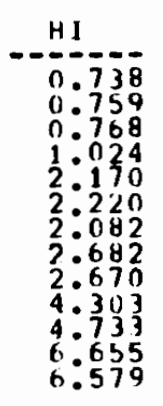 & 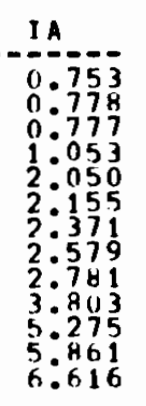 & 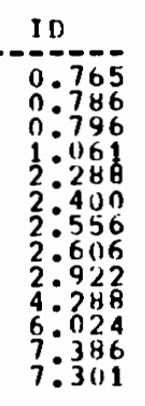 & 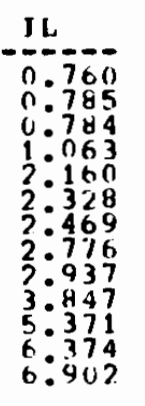 & $\begin{array}{l}I N \\
-1 N \\
0: 742 \\
0: 766 \\
0: 765 \\
1: 037 \\
2: 180 \\
2: 240 \\
2: 280 \\
2: 280 \\
2: 656 \\
2: 769 \\
3: 739 \\
5: 566 \\
6: 438 \\
6: 726\end{array}$ & 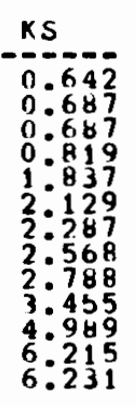 & 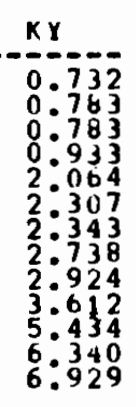 \\
\hline $\begin{array}{l}\text { YFAR } \\
19970 \\
1971 \\
19712 \\
1973 \\
1974 \\
1975 \\
1976 \\
19777 \\
1979 \\
1980 \\
1981 \\
1982\end{array}$ & 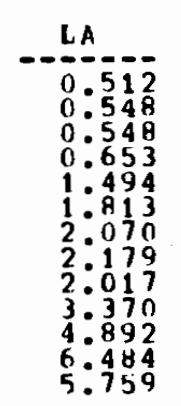 & 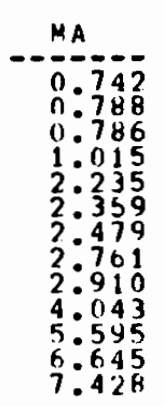 & 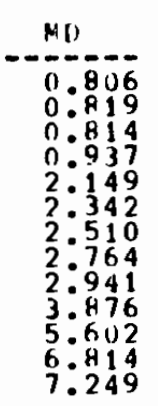 & 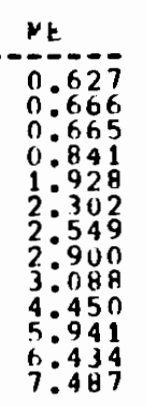 & 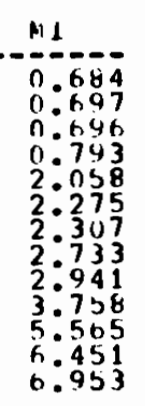 & 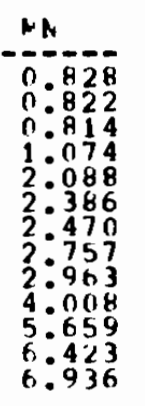 & 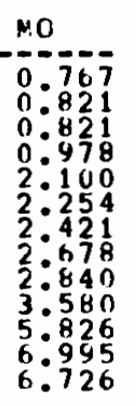 & 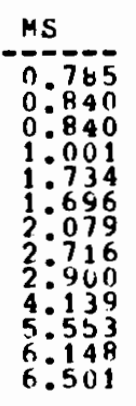 & 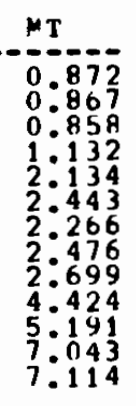 \\
\hline
\end{tabular}

(a) Data for 1982 are provisional estimates and will be updated when data from Annual Survey of Manufactures become available. 
TABLE 3.16 (cont). Distillate Fuel Prices: Industrial Sector $(\$ / m i 11$ ion Btu) $(a)$

\begin{tabular}{|c|c|c|c|c|c|c|c|c|c|}
\hline \begin{tabular}{l} 
YEAR \\
\hdashline 1970 \\
1970 \\
1972 \\
1973 \\
1974 \\
1975 \\
1976 \\
1977 \\
1978 \\
1979 \\
1980 \\
1981 \\
1982
\end{tabular} & \begin{tabular}{l}
$N C$ \\
\hdashline 0.727 \\
$0: 726$ \\
$0: 722$ \\
$0: 901$ \\
$1: 983$ \\
$2: 1992$ \\
20402 \\
$2: 728$ \\
$2: 864$ \\
$3: 726$ \\
$5: 491$ \\
$6: 517$ \\
6.837
\end{tabular} & \begin{tabular}{l} 
ND \\
\hdashline 0.790 \\
0.785 \\
0.777 \\
1.025 \\
2.191 \\
2.725 \\
2.994 \\
29749 \\
2.984 \\
4.994 \\
6.938 \\
8.461 \\
7.918
\end{tabular} & \begin{tabular}{l} 
NE \\
\hdashline 0.735 \\
$0: 786$ \\
$0: 786$ \\
$0: 937$ \\
$1: 851$ \\
$2: 246$ \\
$2: 393$ \\
$2: 527$ \\
$2: 755$ \\
$3: 344$ \\
$4: 941$ \\
$6: 401$ \\
6.292
\end{tabular} & $\begin{array}{c}N H \\
-0.587 \\
00: 730 \\
0: 728 \\
0: 921 \\
2: 189 \\
2: 295 \\
2: 340 \\
2: 711 \\
2: 894 \\
2: 894 \\
5: 732 \\
6: 329 \\
7.000\end{array}$ & 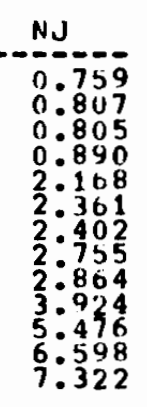 & \begin{aligned}$N M \\
$\hdashline 0.970 \\
$0: 997 \\
1: 010 \\
1: 345 \\
2: 500 \\
2: 596 \\
2: 366 \\
3: 070 \\
3: 264 \\
4: 900 \\
6: 422 \\
7: 954 \\
8: 137\end{aligned}$ & \begin{tabular}{l} 
NV \\
\hdashline 0.962 \\
$0: 989$ \\
$1: 001$ \\
$1: 334$ \\
$2: 240$ \\
$2: 337$ \\
$2: 503$ \\
$2: 810$ \\
$3: 004$ \\
$4: 683$ \\
$6: 531$ \\
$7: 468$ \\
$7: 382$
\end{tabular} & \begin{tabular}{l}
$N Y$ \\
\hdashline 0.701 \\
$0: 747$ \\
$0: 746$ \\
$0: 906$ \\
$2: 175$ \\
$2: 362$ \\
$2: 428$ \\
$2: 790$ \\
$2: 941$ \\
$3: 920$ \\
$5: 355$ \\
$6: 562$ \\
$7: 674$
\end{tabular} & \begin{tabular}{c} 
OH \\
\hdashline 0.792 \\
$0: 807$ \\
$0: 806$ \\
$0: 918$ \\
$2: 223$ \\
$2: 307$ \\
$2: 460$ \\
$2: 755$ \\
$2: 886$ \\
$3: 871$ \\
$5: 450$ \\
$6: 603$ \\
7.174
\end{tabular} \\
\hline 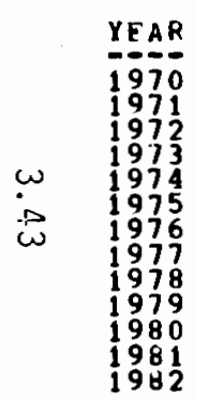 & \begin{tabular}{l}
$0 K$ \\
\hdashline 0.542 \\
0.537 \\
0.531 \\
0.679 \\
2.094 \\
20190 \\
$2: 356$ \\
20664 \\
20858 \\
4.279 \\
5.679 \\
4.8001 \\
6.641
\end{tabular} & $\begin{array}{r}0 K \\
-0.797 \\
0.719 \\
0.819 \\
0.829 \\
1.105 \\
2.026 \\
2.288 \\
2.460 \\
2.759 \\
2.803 \\
3.738 \\
5.622 \\
6.310 \\
6.821\end{array}$ & \begin{tabular}{r}
$P A$ \\
\hdashline 0.702 \\
$0: 747$ \\
$0: 745$ \\
$0: 850$ \\
$2: 170$ \\
$2: 379$ \\
$2: 416$ \\
$2: 788$ \\
20853 \\
$3: 822$ \\
5.666 \\
6.609 \\
7.229
\end{tabular} & $\begin{array}{r}R I \\
-0.716 \\
0: 757 \\
0: 755 \\
0: 844 \\
2: 149 \\
2: 338 \\
2: 230 \\
2: 816 \\
2: 991 \\
4: 154 \\
5: 646 \\
7: 254 \\
7: 500\end{array}$ & \begin{tabular}{l}
$S C$ \\
\hdashline 0.579 \\
$0: 594$ \\
$0: 591$ \\
$0: 715$ \\
20064 \\
$2: 122$ \\
$2: 304$ \\
$2: 706$ \\
$2: 905$ \\
3.647 \\
4.618 \\
$6: 649$ \\
6.706
\end{tabular} & \begin{tabular}{l} 
sv \\
\hdashline 0.726 \\
$0: 721$ \\
$0: 7114$ \\
$0: 942$ \\
$2: 328$ \\
$2: 5772$ \\
$2: 709$ \\
$2: 821$ \\
$3: 159$ \\
$4: 354$ \\
$5: 652$ \\
$6: 580$ \\
7.369
\end{tabular} & \begin{tabular}{c}
$\mathrm{TN}$ \\
\hdashline 0.726 \\
$0: 776$ \\
$0: 776$ \\
$0: 925$ \\
$1: 997$ \\
$2: 115$ \\
$2: 259$ \\
$2: 743$ \\
$2: 882$ \\
$3: 650$ \\
$5: 505$ \\
$6: 619$ \\
6.822
\end{tabular} & \begin{tabular}{l}
$T X$ \\
\hdashline 0.652 \\
$0: 697$ \\
$0: 697$ \\
$0: 831$ \\
$1: 882$ \\
$2: 016$ \\
$2: 472$ \\
$2: 663$ \\
$2: 838$ \\
$4: 235$ \\
$6: 088$ \\
$6: 7878$ \\
6.975
\end{tabular} & \begin{tabular}{l}
$U T$ \\
\hdashline 0.640 \\
$0: 658$ \\
$0: 666$ \\
$0: 887$ \\
$1: 957$ \\
$2: 180$ \\
2.438 \\
$2: 573$ \\
$2: 592$ \\
$3: 643$ \\
$5: 495$ \\
$6: 225$ \\
$6: 736$
\end{tabular} \\
\hline $\begin{array}{l}\text { YEAR } \\
1970 \\
1970 \\
1971 \\
1972 \\
1973 \\
1974 \\
1975 \\
1976 \\
1977 \\
1978 \\
1979 \\
1980 \\
1981 \\
1982\end{array}$ & \begin{tabular}{l} 
VA \\
\hdashline 0.596 \\
0.6634 \\
$0: 632$ \\
0.699 \\
$1: 868$ \\
2.189 \\
2.290 \\
$2: 664$ \\
2.819 \\
$3: 590$ \\
$5: 331$ \\
$6: 677$ \\
6.759
\end{tabular} & 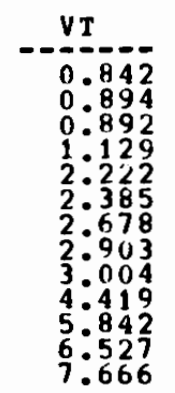 & \begin{tabular}{l}
$W A$ \\
\hdashline 0.728 \\
$0: 749$ \\
$0: 758$ \\
$1: 010$ \\
1.631 \\
2.050 \\
$2: 264$ \\
$2: 737$ \\
$2: 937$ \\
$4: 394$ \\
$6: 060$ \\
$6: 730$ \\
6.898
\end{tabular} & \begin{tabular}{c}
$W 1$ \\
\hdashline 0.762 \\
$0: 786$ \\
$0: 786$ \\
1065 \\
$2: 065$ \\
2.232 \\
2.428 \\
$2: 749$ \\
2.879 \\
$4: 254$ \\
$5: 180$ \\
$6: 891$ \\
6.923
\end{tabular} & $\begin{array}{r}W V \\
-10-708 \\
0: 752 \\
0: 750 \\
0: 829 \\
1: 991 \\
2: 268 \\
2: 403 \\
2: 869 \\
30015 \\
3: 879 \\
6: 148 \\
6: 780 \\
7.215\end{array}$ & \begin{tabular}{r}
$W Y$ \\
\hdashline 0.796 \\
$0: 791$ \\
$0: 783$ \\
$1: 033$ \\
$2: 421$ \\
$2: 518$ \\
2.684 \\
$2: 992$ \\
30185 \\
$4: 844$ \\
8.275 \\
105050 \\
$8: 512$
\end{tabular} & \begin{tabular}{l} 
US \\
\hdashline 0.721 \\
$0: 751$ \\
$0: 747$ \\
$0: 921$ \\
$1: 994$ \\
$2: 210$ \\
2.378 \\
$2: 677$ \\
2.808 \\
30907 \\
50631 \\
$6: 637$ \\
6.881
\end{tabular} & & \\
\hline
\end{tabular}

Source: Pacific Northwest Laboratory 
TABLE 3.17. Distillate Fuel Prices: Electric Utility Sector ( $\$ / \mathrm{mi}$ ]1ion Btu)

\begin{tabular}{|c|c|c|c|c|c|c|c|c|c|}
\hline $\begin{array}{l}\text { YEAR } \\
1970 \\
1971 \\
19773 \\
1973 \\
1975 \\
1976 \\
1977 \\
1978 \\
1979 \\
1990 \\
1981 \\
1982\end{array}$ & \begin{tabular}{c}
$A K$ \\
\hdashline $0: 0$ \\
$0: 0$ \\
$0: 0$ \\
$0: 0$ \\
$0: 0$ \\
$0: 0$ \\
$0: 0$ \\
$0: 0$ \\
$0: 0$ \\
$0: 0$ \\
$0: 0$ \\
$0: 0$ \\
$0: 0$
\end{tabular} & $\begin{array}{l}A L \\
-0.843 \\
0: 946 \\
0: 959 \\
0: 893 \\
2: 095 \\
2: 165 \\
2: 358 \\
2: 665 \\
2: 732 \\
4: 310 \\
6.351 \\
7: 713 \\
7.115\end{array}$ & $\begin{array}{l}A H \\
-10.527 \\
0: 749 \\
0.809 \\
1.065 \\
2.031 \\
2.221 \\
2.471 \\
2.622 \\
2.587 \\
20933 \\
4.343 \\
6.408 \\
6.741\end{array}$ & 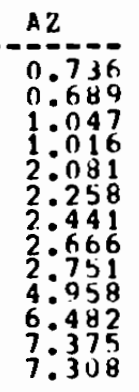 & $\begin{array}{l}C A \\
00.420 \\
0: 663 \\
0.838 \\
1: 078 \\
1: 867 \\
2: 372 \\
2.643 \\
2.691 \\
2.804 \\
3: 804 \\
5.790 \\
7.130 \\
6.712\end{array}$ & 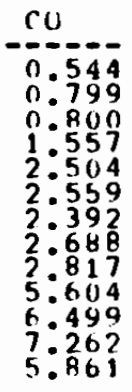 & \begin{tabular}{l}
$C T$ \\
\hdashline 0.415 \\
$0: 597$ \\
$0: 728$ \\
$0: 971$ \\
$2: 228$ \\
$2: 288$ \\
2.438 \\
2.422 \\
$2: 124$ \\
$3: 944$ \\
$6: 112$ \\
$7: 508$ \\
7.295
\end{tabular} & 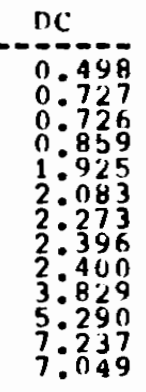 & $\begin{array}{l}D E \\
-0.570 \\
0: 832 \\
0: 830 \\
1: 658 \\
2: 069 \\
2: 181 \\
2: 374 \\
2: 083 \\
2: 459 \\
3: 958 \\
6: 214 \\
7: 435 \\
7.013\end{array}$ \\
\hline $\begin{array}{l}\text { YEAR } \\
1970 \\
1971 \\
1972 \\
1973 \\
1974 \\
1975 \\
1976 \\
1977 \\
1978 \\
1979 \\
1980 \\
1981 \\
1982\end{array}$ & 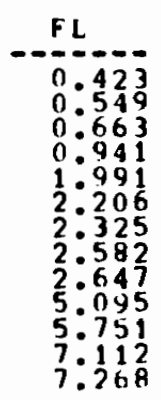 & 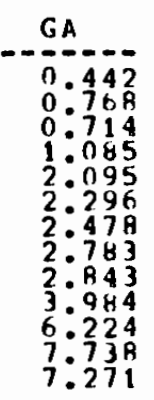 & \begin{tabular}{c}
$H I$ \\
\hdashline 0.581 \\
$0: 700$ \\
$0: 792$ \\
$0: 916$ \\
$0: 0$ \\
$2: 266$ \\
$2: 6003$ \\
$2: 975$ \\
$2: 900$ \\
$4: 194$ \\
$5: 813$ \\
$7: 267$ \\
7.451
\end{tabular} & $\begin{array}{l}1 A \\
0 .-18 \\
0.821 \\
0: 918 \\
0: 917 \\
1: 158 \\
1: 985 \\
20108 \\
2: 351 \\
2: 668 \\
20692 \\
30697 \\
60056 \\
7: 357 \\
6: 224\end{array}$ & 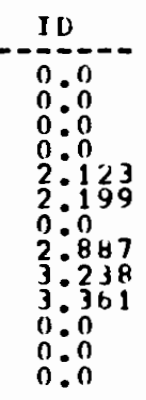 & 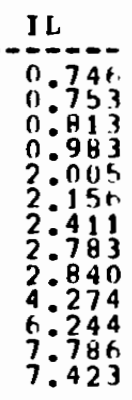 & \begin{tabular}{l} 
IN \\
\hdashline 0.831 \\
$0: 871$ \\
$0: 804$ \\
$1: 005$ \\
10745 \\
20119 \\
$2: 0113$ \\
$2: 361$ \\
2.657 \\
$3: 942$ \\
$5: 985$ \\
$7: 566$ \\
$6: 078$
\end{tabular} & 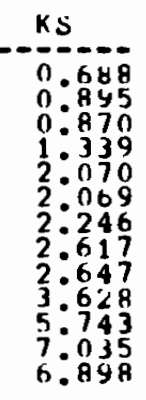 & $\begin{array}{l}K Y \\
-1:-0 \\
1: 148 \\
1: 028 \\
1: 020 \\
0: 810 \\
2: 207 \\
2: 250 \\
2: 453 \\
2: 806 \\
2: 874 \\
4: 545 \\
6: 542 \\
8: 039 \\
7.545\end{array}$ \\
\hline $\begin{array}{l}\text { YEAR } \\
1 E 97 \\
1970 \\
1971 \\
1972 \\
1973 \\
1974 \\
1975 \\
1977 \\
1977 \\
1978 \\
1979 \\
1980 \\
1981 \\
1982\end{array}$ & \begin{tabular}{r} 
LA \\
\hdashline 0.657 \\
$0: 685$ \\
$0: 926$ \\
$1: 456$ \\
$3: 156$ \\
$1: 913$ \\
$2: 066$ \\
$2: 421$ \\
$2: 526$ \\
$2: 904$ \\
$4: 372$ \\
$6: 347$ \\
6.307
\end{tabular} & $\begin{array}{l}M A \\
-90.997 \\
0: 847 \\
0: 897 \\
1: 113 \\
2: 066 \\
2: 146 \\
20422 \\
2: 7118 \\
2: 926 \\
4: 578 \\
5: 992 \\
7: 467 \\
7.072\end{array}$ & 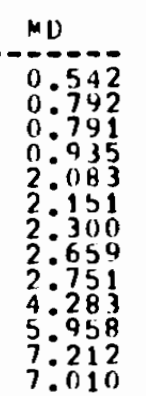 & 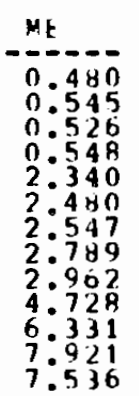 & $\begin{array}{l}M 1 \\
-0.711 \\
0: 7931 \\
0: 873 \\
0: 942 \\
1: 972 \\
2: 034 \\
2: 349 \\
2: 645 \\
2: 7880 \\
4: 040 \\
60065 \\
7: 0398 \\
7.0338\end{array}$ & 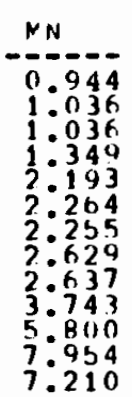 & $\begin{array}{l}m 0 \\
0.777 \\
0: 927 \\
0: 789 \\
1: 306 \\
2: 161 \\
2: 256 \\
2: 311 \\
2: 679 \\
2: 666 \\
4: 421 \\
6: 015 \\
7: 202 \\
6: 960\end{array}$ & $\begin{array}{l}M S \\
-0.633 \\
0: 943 \\
0: 927 \\
0: 992 \\
1: 959 \\
1: 949 \\
2: 107 \\
2: 515 \\
20505 \\
3: 729 \\
5: 409 \\
70073 \\
6: 729\end{array}$ & \begin{tabular}{l}
$M I$ \\
\hdashline 0.339 \\
$0: 1168$ \\
$0: 564$ \\
$0: 930$ \\
$1: 147$ \\
$0: 00$ \\
$2: 528$ \\
$1: 814$ \\
$2: 974$ \\
$3: 369$ \\
5010 \\
$5: 563$ \\
5.421
\end{tabular} \\
\hline
\end{tabular}


TABLE 3.17 (cont). Distillate Fuel Prices: Electric Utility Sector (\$/million Btu)

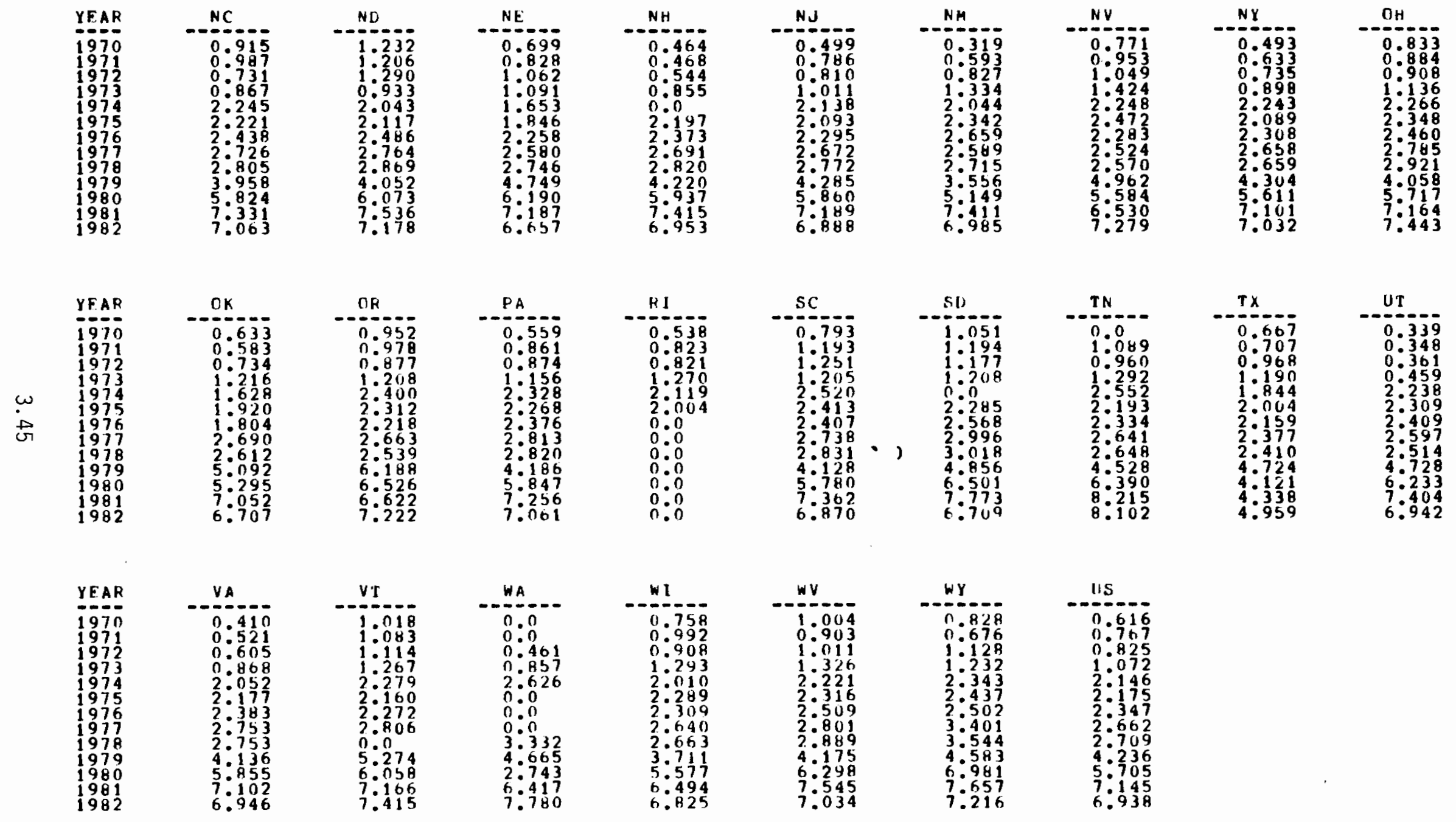

Source: Pacific Northwest Laboratory 
IABLE 3.18. Motor Gasoline Prices: Transportation Sector ( $\$ / m i l 1$ ion Btu)
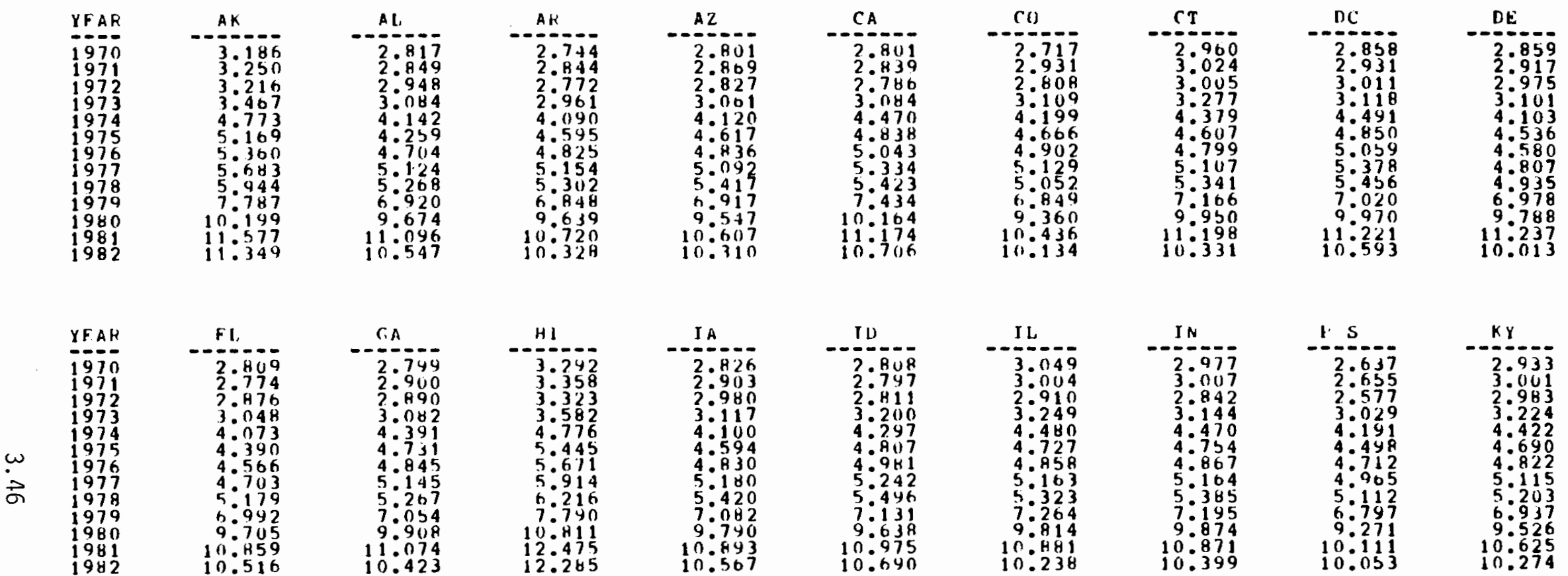

\begin{tabular}{|c|c|c|}
\hline $\begin{array}{l}\text { YFAR } \\
1970 \\
1971 \\
1972 \\
1973 \\
1974 \\
1975 \\
1976 \\
1977 \\
1978 \\
1979 \\
1980 \\
1981 \\
1982\end{array}$ & $\begin{array}{r}1 . A \\
-2.859 \\
2.845 \\
2.727 \\
2.920 \\
3.994 \\
4.441 \\
4.832 \\
5.035 \\
5.321 \\
6.944 \\
9.742 \\
10.903 \\
10.249\end{array}$ & $\begin{array}{r}M A \\
-2.939 \\
2.926 \\
2.873 \\
3.124 \\
4.384 \\
4.730 \\
4.114 \\
5.155 \\
5.146 \\
5.984 \\
9.692 \\
10.822 \\
10.084\end{array}$ \\
\hline
\end{tabular}
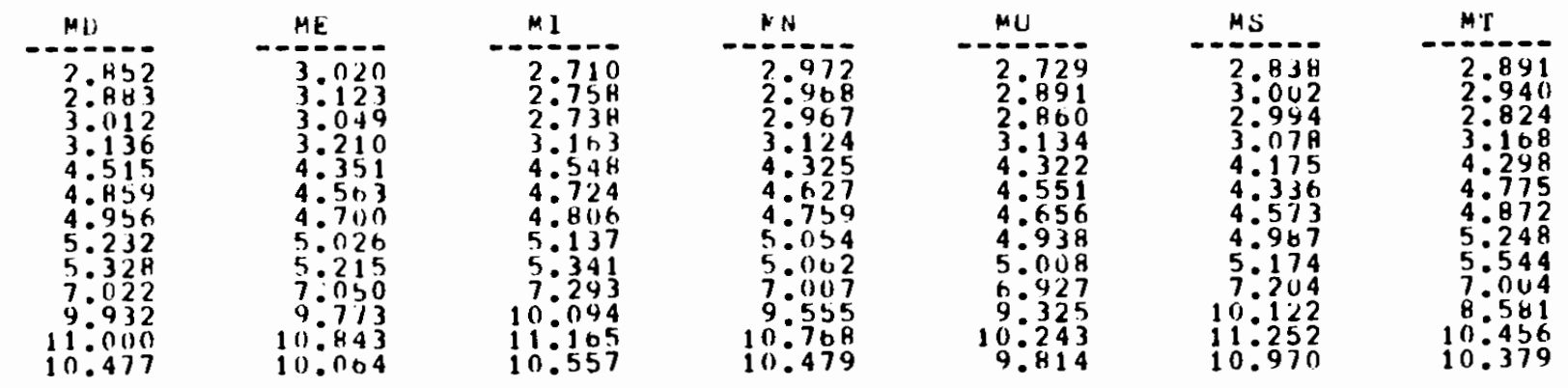
TABLE 3.18 (cont). Motor Gasoline Prices: Transportation Sector ( $\$ /$ million Btu)

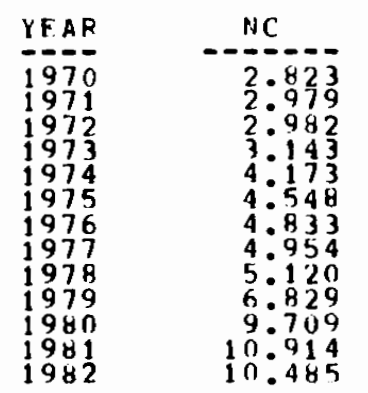

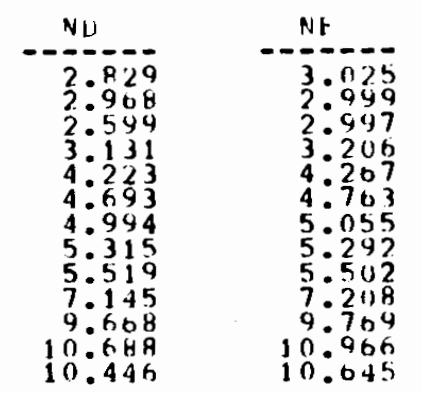
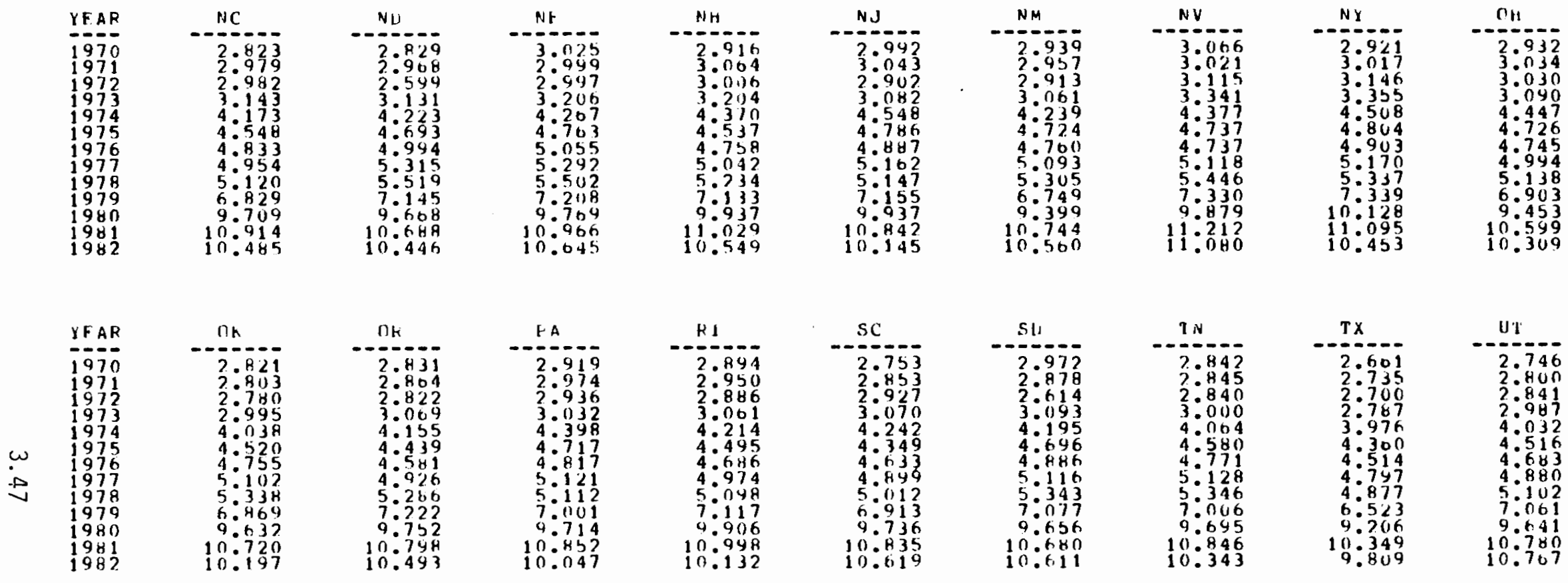

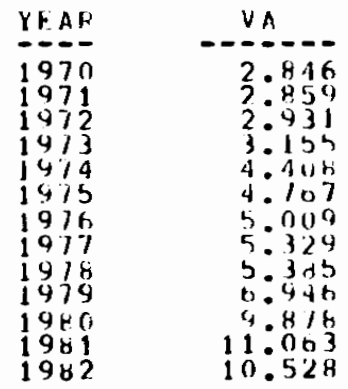

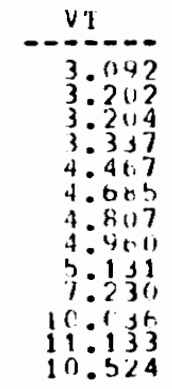
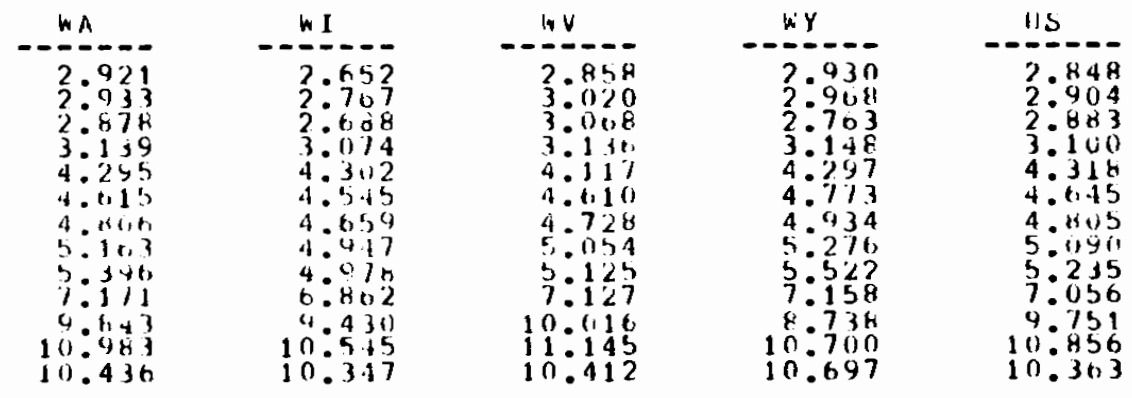

Source: Pacific Northwest Laboratory 
TABLE 3.19. Diesel Fuel Prices: Transportation Sector ( $\$ / \mathrm{million}$ Btu)

\begin{tabular}{|c|c|c|c|c|c|c|c|c|c|}
\hline $\begin{array}{l}\text { YEAR } \\
19710 \\
1971 \\
1972 \\
1973 \\
1974 \\
1975 \\
1977 \\
1977 \\
1978 \\
1979 \\
1980 \\
1981 \\
1982\end{array}$ & \begin{tabular}{rl}
$A K$ & \\
\hdashline-1 & 0 \\
0 & 0 \\
0 & 0 \\
0 & 0 \\
0 & 0 \\
0 & 0 \\
0 & 0 \\
0 & 0 \\
0 & 0 \\
0 & 0 \\
0 & 0 \\
0 & 0 \\
0 & 0 \\
0 & 0 \\
0 & 0 \\
0 & 0
\end{tabular} & 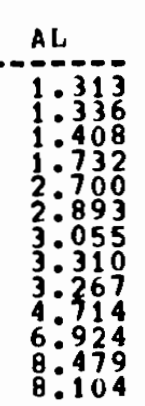 & 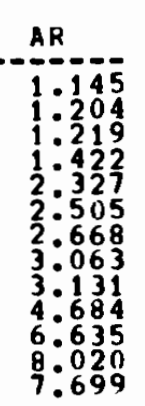 & 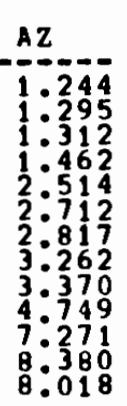 & 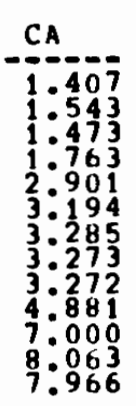 & 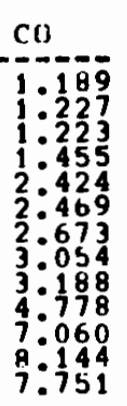 & 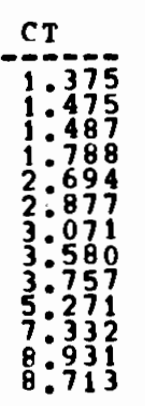 & 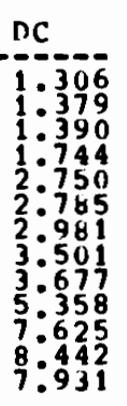 & $\begin{array}{l}\text { DE } \\
-1: 23 \\
1: 235 \\
1: 325 \\
1: 364 \\
1: 764 \\
2: 755 \\
2: 785 \\
2: 981 \\
3: 500 \\
3: 678 \\
5: 438 \\
7: 647 \\
8: 510 \\
7: 936\end{array}$ \\
\hline $\begin{array}{l}\text { YEAR } \\
\text { YEA70 } \\
1970 \\
1971 \\
1972 \\
1973 \\
1974 \\
1975 \\
1976 \\
1977 \\
1979 \\
1979 \\
19801 \\
1982\end{array}$ & 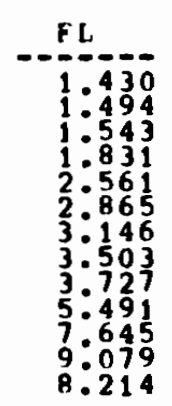 & \begin{tabular}{l}
$G A$ \\
\hdashline $1: 138$ \\
$11: 484$ \\
$1: 491$ \\
$1: 733$ \\
$2: 651$ \\
$2: 992$ \\
3096 \\
$33: 485$ \\
$3: 574$ \\
$5: 156$ \\
$77: 414$ \\
$8: 664$ \\
$7: 917$
\end{tabular} & 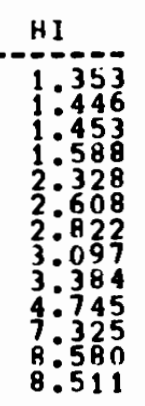 & 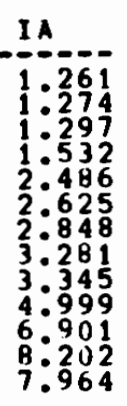 & 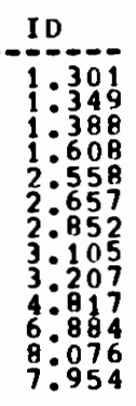 & 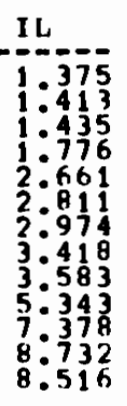 & 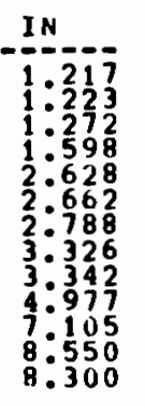 & 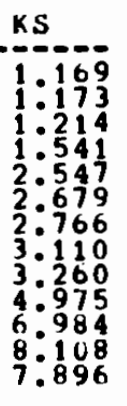 & \begin{tabular}{r}
$K Y$ \\
\hdashline $1: 438$ \\
$1: 384$ \\
$1: 398$ \\
$1: 583$ \\
$2: 646$ \\
$2: 769$ \\
$2: 964$ \\
$2: 964$ \\
$3: 350$ \\
$3: 337$ \\
$4: 756$ \\
$6: 192$ \\
$8: 169$ \\
$7: 855$
\end{tabular} \\
\hline $\begin{array}{l}\text { YEAR } \\
1970 \\
1970 \\
1971 \\
1973 \\
1974 \\
1975 \\
1976 \\
1977 \\
1978 \\
1979 \\
1980 \\
1981 \\
1982\end{array}$ & 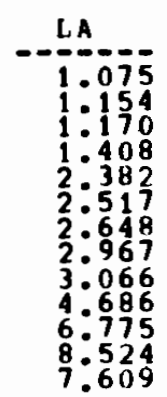 & 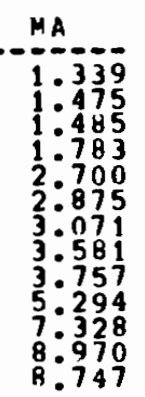 & 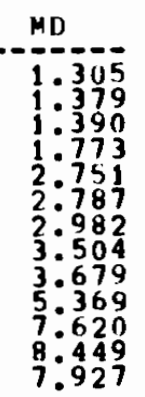 & 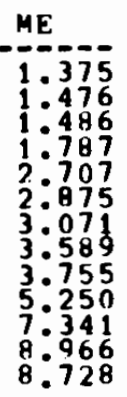 & 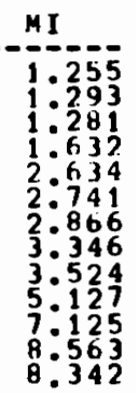 & 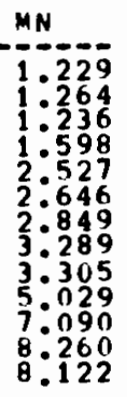 & 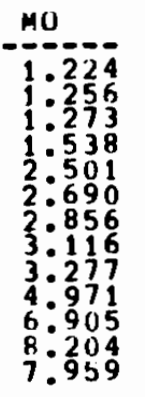 & 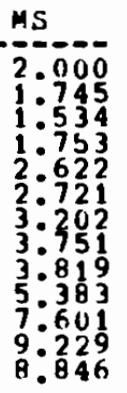 & 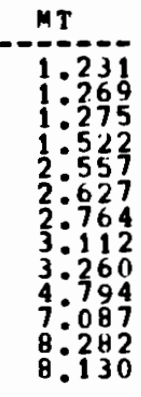 \\
\hline
\end{tabular}


TABLE 3.19 (cont). Diesel Fuel Prices: Transportation Sector (\$/million Btu)
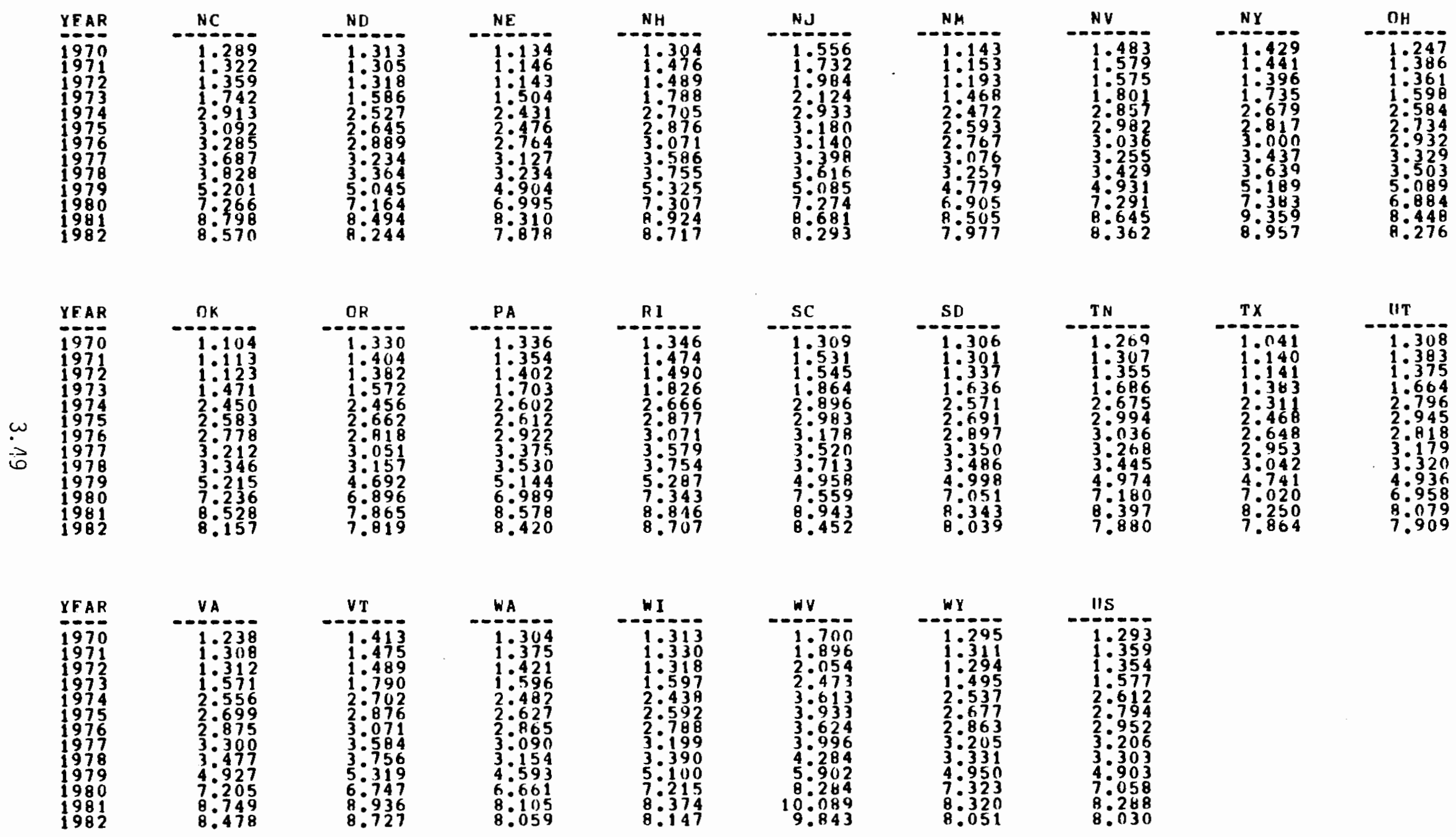
TABLE 3.20. Kerosene Prices: Residential Sector ( $\$ /$ million Btu)
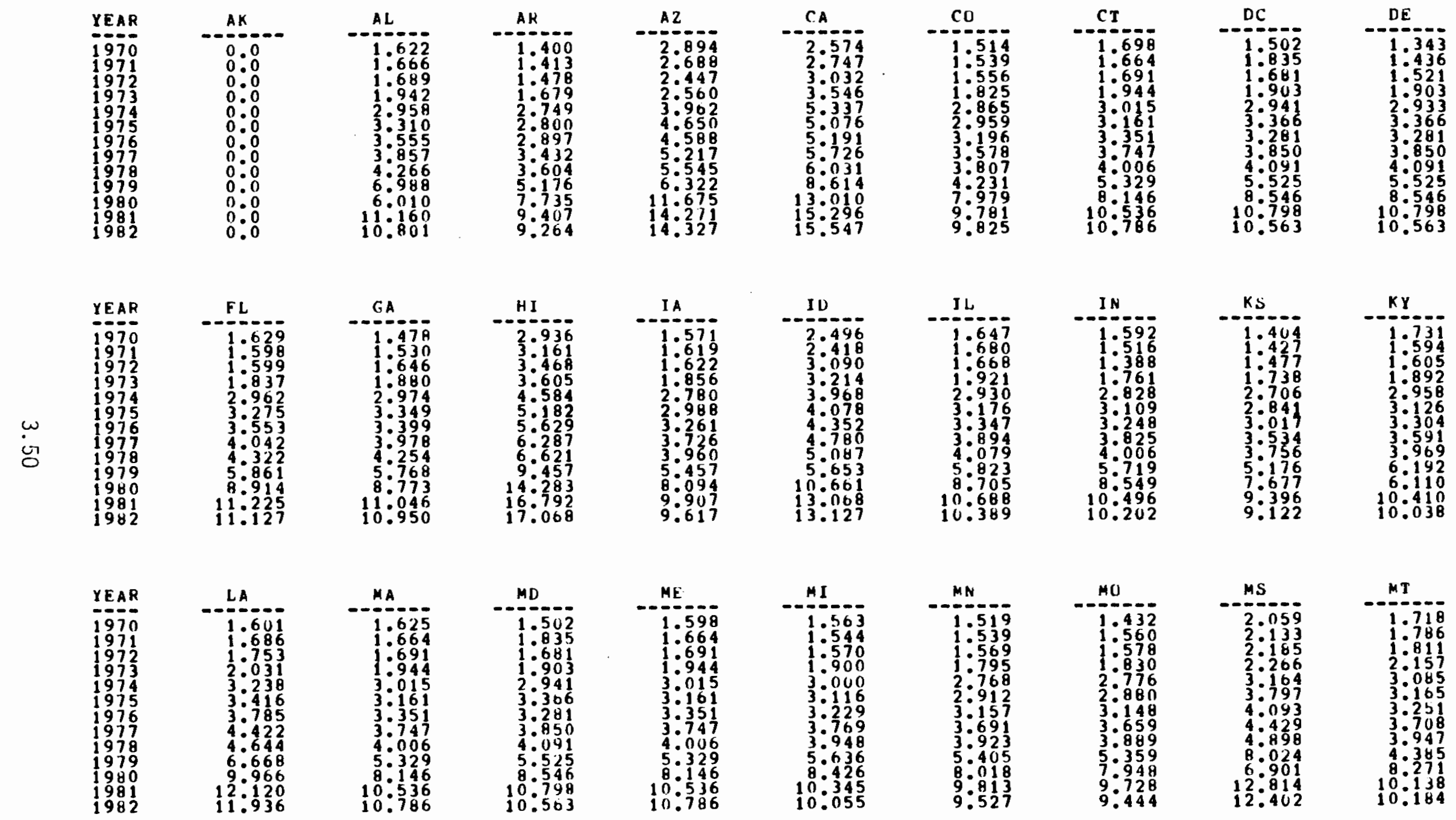
TABLE 3.20 (cont). Kerosene Prices: Residential Sector ( $\$ /$ million Btu)

\begin{tabular}{|c|c|c|c|c|c|c|c|c|c|}
\hline $\begin{array}{l}\text { YEAR } \\
1970 \\
1970 \\
1971 \\
1972 \\
1973 \\
1974 \\
1975 \\
1976 \\
1977 \\
1978 \\
1979 \\
1980 \\
1981 \\
1982\end{array}$ & \begin{tabular}{r}
$N C$ \\
\hdashline 1.401 \\
$1: 453$ \\
$1: 480$ \\
10767 \\
20730 \\
20957 \\
330199 \\
30609 \\
30959 \\
50233 \\
70958 \\
$10: 021$ \\
9.933
\end{tabular} & \begin{tabular}{c}
$N D$ \\
\hdashline 1.649 \\
$1: 633$ \\
$1: 627$ \\
$1: 927$ \\
2.672 \\
2.688 \\
2.987 \\
$3: 400$ \\
3.613 \\
$4: 978$ \\
7.385 \\
9.038 \\
8.774
\end{tabular} & \begin{tabular}{l}
$N E$ \\
\hdashline 1.388 \\
$1: 391$ \\
$1: 398$ \\
$1: 735$ \\
$2: 634$ \\
$2: 742$ \\
30.036 \\
30473 \\
30691 \\
$5: 086$ \\
7.544 \\
$9: 234$ \\
8.964
\end{tabular} & $\begin{array}{r}N H \\
1: 581 \\
1: 664 \\
1: 691 \\
1094 \\
3.015 \\
3.161 \\
3.351 \\
3: 747 \\
4006 \\
5: 329 \\
8: 146 \\
10: 536 \\
10.786\end{array}$ & \begin{tabular}{r} 
NJ \\
\hdashline 1.715 \\
$1: 767$ \\
10811 \\
$2: 167$ \\
$3: 295$ \\
$3: 512$ \\
$3: 779$ \\
$4: 218$ \\
4.470 \\
$6: 073$ \\
$9: 273$ \\
$11: 857$ \\
11.861
\end{tabular} & \begin{tabular}{l} 
NM \\
\hdashline $1: 491$ \\
$1: 489$ \\
$1: 526$ \\
$1: 786$ \\
$2: 874$ \\
$3: 048$ \\
$3: 182$ \\
$3: 568$ \\
$3: 794$ \\
$4: 354$ \\
79963 \\
$9: 910$ \\
9.828
\end{tabular} & $\begin{array}{r}N V \\
-N 3.340 \\
3: 468 \\
3: 282 \\
3: 555 \\
4: 617 \\
4: 791 \\
4: 767 \\
5: 430 \\
5: 7770 \\
6: 579 \\
12: 151 \\
14: 852 \\
14: 911\end{array}$ & \begin{tabular}{r}
$N Y$ \\
\hdashline 1.562 \\
1.663 \\
$1: 649$ \\
2.023 \\
$3: 036$ \\
3.276 \\
3.432 \\
3.860 \\
4.091 \\
5.556 \\
8.487 \\
10.852 \\
10.855
\end{tabular} & \begin{tabular}{r}
$\mathrm{OH}$ \\
\hdashline 1.424 \\
$1: 491$ \\
$1: 480$ \\
$1: 748$ \\
2.798 \\
2.897 \\
3.109 \\
30610 \\
$3: 781$ \\
50398 \\
8069 \\
90907 \\
9.630
\end{tabular} \\
\hline $\begin{array}{l}\text { YEAR } \\
1970 \\
1970 \\
1971 \\
1972 \\
1973 \\
1974 \\
1975 \\
1976 \\
1977 \\
1978 \\
1979 \\
1980 \\
1981 \\
1982\end{array}$ & 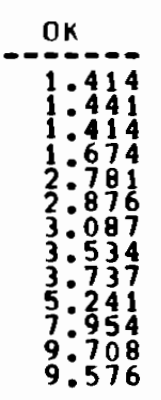 & \begin{tabular}{r}
$0 K$ \\
\hdashline 2.793 \\
$2: 986$ \\
$3: 260$ \\
$3: 361$ \\
$3: 748$ \\
$3: 811$ \\
3.868 \\
$4: 302$ \\
4.531 \\
6.477 \\
9.775 \\
11.492 \\
11.681
\end{tabular} & 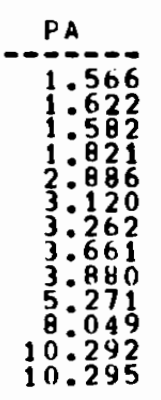 & 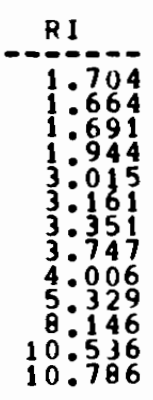 & 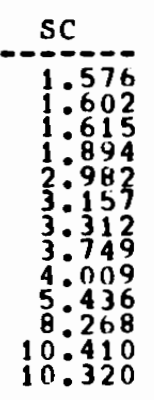 & \begin{tabular}{l}
$S D$ \\
\hdashline $1: 566$ \\
$1: 574$ \\
$1: 612$ \\
$1: 800$ \\
$2: 776$ \\
$2: 907$ \\
30139 \\
30606 \\
$3: 832$ \\
$5: 280$ \\
70832 \\
$9: 586$ \\
$9: 306$
\end{tabular} & 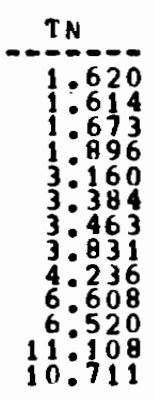 & \begin{tabular}{r}
$T X$ \\
\hdashline $1: 296$ \\
$1: 390$ \\
$1: 408$ \\
10600 \\
20694 \\
$3: 020$ \\
30144 \\
30714 \\
30900 \\
50600 \\
$8: 369$ \\
$10: 178$ \\
10.023
\end{tabular} & \begin{tabular}{r} 
UT \\
\hdashline 2.619 \\
$2: 998$ \\
$3: 175$ \\
$3: 579$ \\
$4: 599$ \\
$5: 156$ \\
$4: 767$ \\
$5: 504$ \\
$5: 858$ \\
$6: 509$ \\
$12: 276$ \\
$15: 048$ \\
15.115
\end{tabular} \\
\hline $\begin{array}{l}\text { YEAR } \\
197- \\
1970 \\
1971 \\
1972 \\
1973 \\
1974 \\
1975 \\
1976 \\
1977 \\
1978 \\
1979 \\
1980 \\
1981 \\
1982\end{array}$ & \begin{tabular}{r} 
VA \\
\hdashline 1.441 \\
$1: 505$ \\
$1: 509$ \\
1.785 \\
$2: 758$ \\
2.993 \\
$3: 184$ \\
3.611 \\
$3: 861$ \\
50236 \\
$7: 963$ \\
10.027 \\
9.939
\end{tabular} & \begin{tabular}{r}
$V T$ \\
\hdashline 1.628 \\
1.664 \\
$1: 6991$ \\
$1: 944$ \\
$3: 015$ \\
3.161 \\
$3: 351$ \\
$3: 747$ \\
$4: 006$ \\
50.329 \\
$8: 146$ \\
10.536 \\
10.786
\end{tabular} & $\begin{array}{r}\text { WA } \\
2.465 \\
2.641 \\
2: 779 \\
2.986 \\
3.468 \\
3.614 \\
4: 026 \\
4.297 \\
4.526 \\
6.404 \\
9.763 \\
11.478 \\
11.067\end{array}$ & \begin{tabular}{r}
$W I$ \\
\hdashline $1: 460$ \\
$1: 518$ \\
$1: 584$ \\
$1: 842$ \\
$2: 806$ \\
2.965 \\
$3: 107$ \\
$3: 629$ \\
$3: 800$ \\
5.426 \\
8.111 \\
9.958 \\
9.640
\end{tabular} & \begin{tabular}{r}
$W v$ \\
\hdashline 1.637 \\
$1: 695$ \\
$1: 719$ \\
1.925 \\
$2: 953$ \\
$3: 169$ \\
3.427 \\
3.846 \\
$4: 113$ \\
5.578 \\
80443 \\
10.641 \\
10.588
\end{tabular} & \begin{tabular}{l}
$W y$ \\
\hdashline $1: 702$ \\
$1: 713$ \\
$1: 973$ \\
$2: 108$ \\
$3: 085$ \\
$3: 167$ \\
$3: 252$ \\
$3: 637$ \\
$3: 071$ \\
$4: 302$ \\
$8: 113$ \\
$9: 945$ \\
9.969
\end{tabular} & \begin{tabular}{r} 
US \\
\hdashline 1.536 \\
$1: 589$ \\
$1: 592$ \\
$1: 874$ \\
$2: 919$ \\
$3: 130$ \\
$3: 330$ \\
$3: 7774$ \\
$4: 032$ \\
$5: 379$ \\
70974 \\
10.533 \\
10.373
\end{tabular} & & \\
\hline
\end{tabular}


TABLE 3.21. Kerosene Prices: Industrial Sector ( $\$ /$ million Btu)

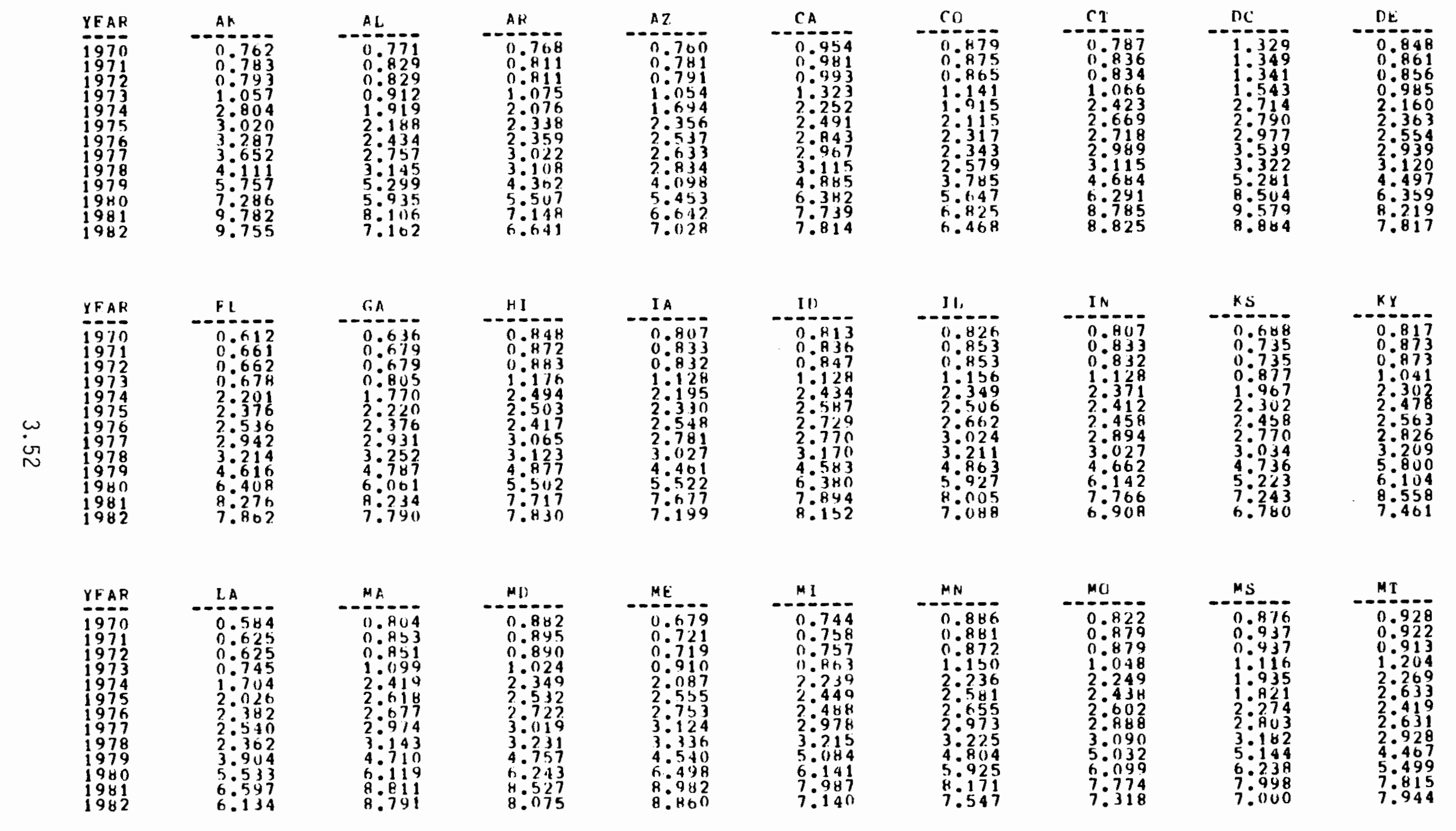


TABLE 3.21 (cont). Kerosene Prices: Industrial Sector ( $\$ /$ million Btu)

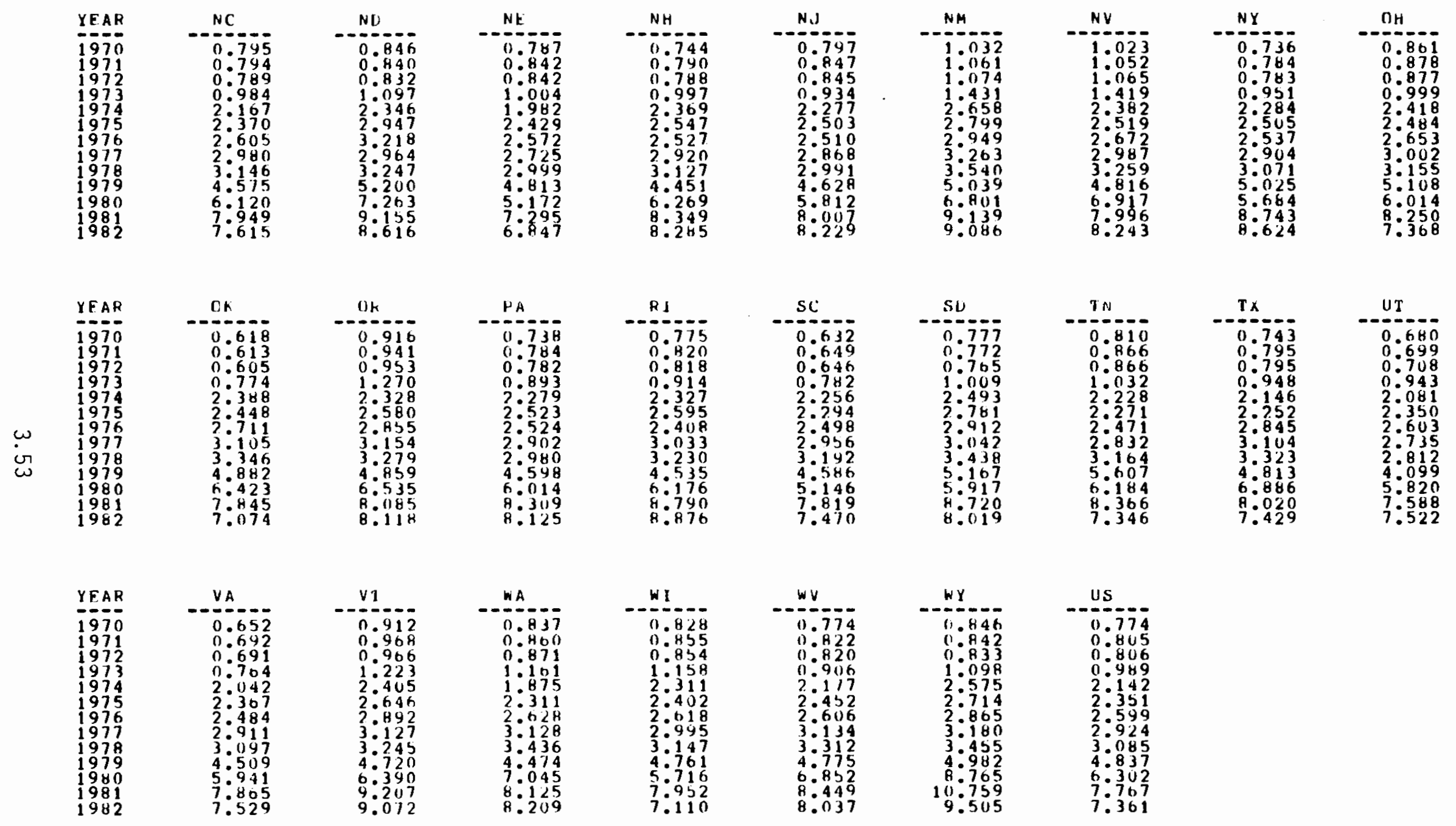

Source: Pacific Northwest Laboratory 
TABLE 3.22. Jet Fuel Prices: Transportation Sector $(\$ / \mathrm{mi} 11 \text { ion Btu })^{(a)}$

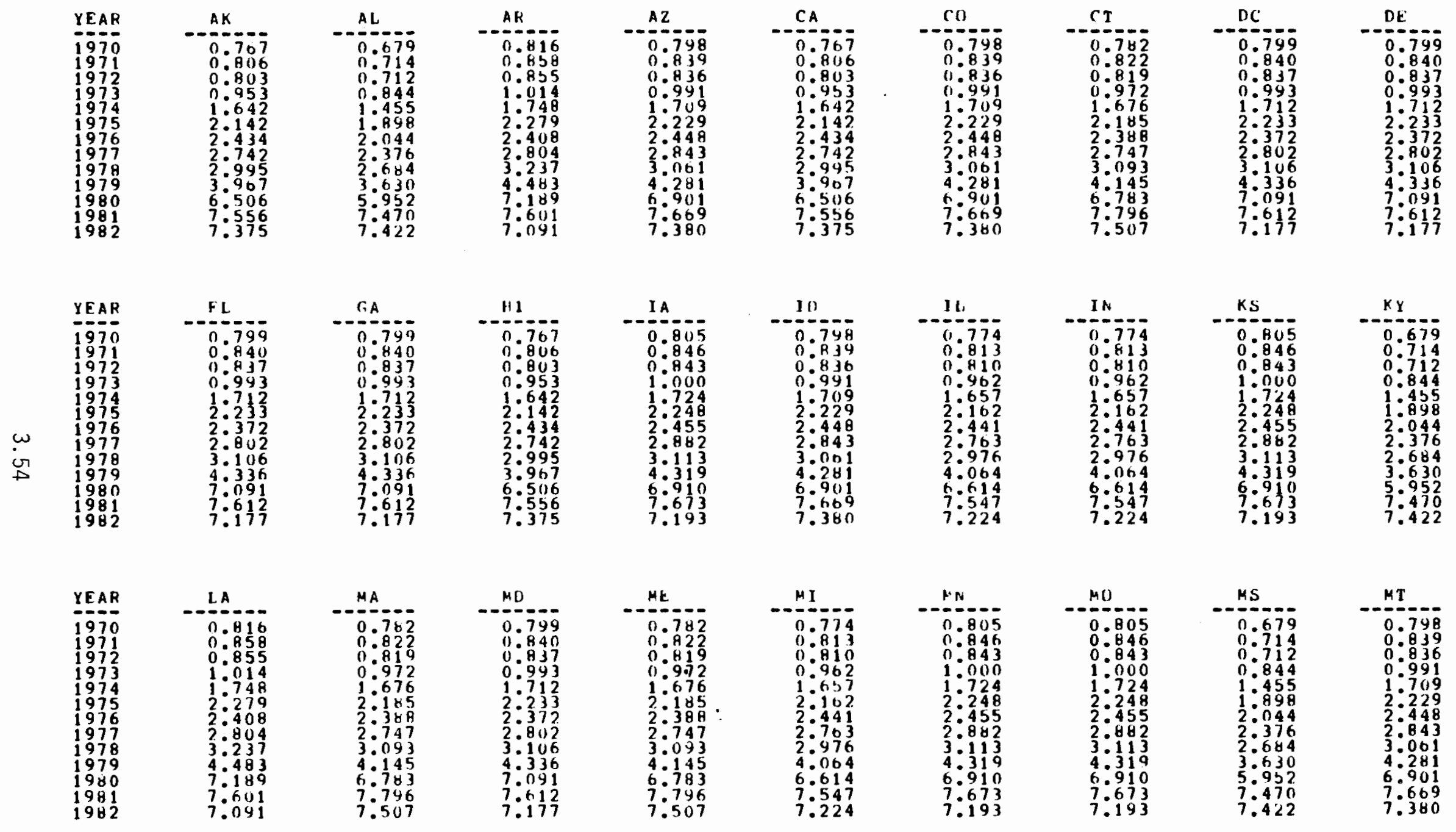

Ta) Data are wholesale regional prices assigned to states. 
TABLE 3.22 (cont). Jet Fuel Prices: Transportation Sector $\left(\$ /\right.$ million Btu) ${ }^{(a)}$

\begin{tabular}{|c|c|c|c|c|c|c|c|c|c|}
\hline $\begin{array}{l}\text { YEAR } \\
Y E A- \\
1970 \\
1971 \\
1972 \\
1973 \\
1974 \\
1975 \\
1976 \\
1977 \\
1978 \\
1979 \\
1981 \\
1981\end{array}$ & 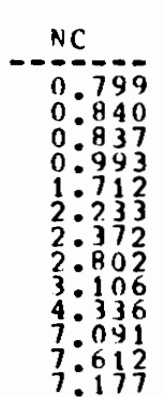 & 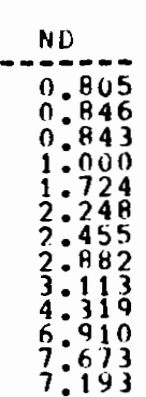 & 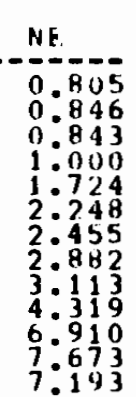 & 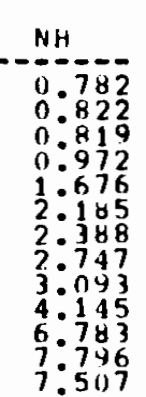 & 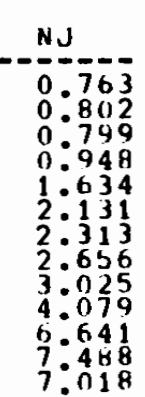 & 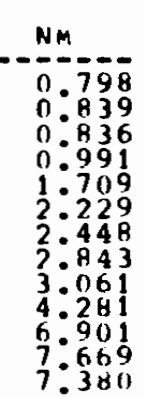 & 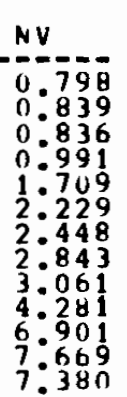 & 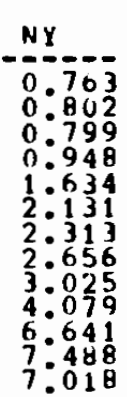 & \begin{tabular}{l}
$0 H$ \\
\hdashline $0: 774$ \\
$0: 813$ \\
$0: 810$ \\
$0: 962$ \\
$1: 657$ \\
$2: 162$ \\
$2: 441$ \\
$2: 763$ \\
$2: 976$ \\
$4: 064$ \\
$6: 614$ \\
$7: 547$ \\
$7: 224$
\end{tabular} \\
\hline $\begin{array}{l}\text { YEAR } \\
-1970 \\
1971 \\
1972 \\
1973 \\
1974 \\
1975 \\
1976 \\
1977 \\
1978 \\
1979 \\
1980 \\
1981 \\
1982\end{array}$ & 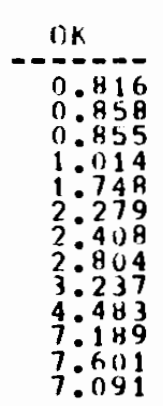 & \begin{tabular}{c} 
OK \\
\hdashline $0: 707$ \\
$0: 806$ \\
$0: 803$ \\
$0: 953$ \\
$1: 642$ \\
$1: 642$ \\
$2: 142$ \\
$2: 434$ \\
$2: 742$ \\
$2: 995$ \\
$2: 995$ \\
30967 \\
$6: 506$ \\
$7: 556$ \\
$7: 375$
\end{tabular} & 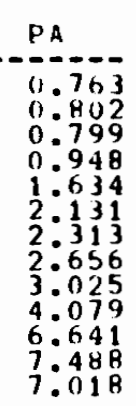 & 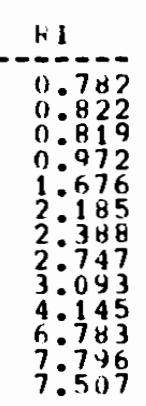 & 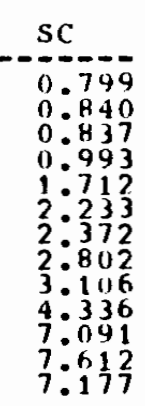 & \begin{tabular}{l} 
s1 \\
\hdashline 0.805 \\
$0: 846$ \\
$0: 843$ \\
$1: 000$ \\
$1: 724$ \\
$2: 248$ \\
$7: 455$ \\
$2: 887$ \\
$3: 113$ \\
$4: 319$ \\
$6: 910$ \\
$7: 673$ \\
$7: 193$
\end{tabular} & 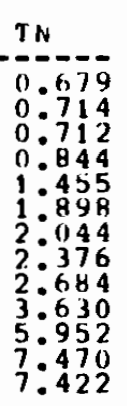 & 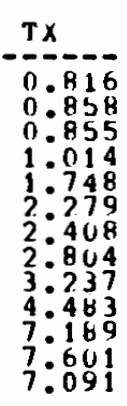 & 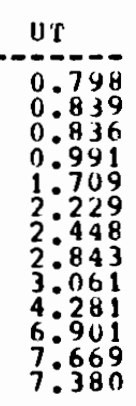 \\
\hline $\begin{array}{l}\text { YEAR } \\
19 \text { AR } \\
1971 \\
1971 \\
1972 \\
1973 \\
1974 \\
1975 \\
1976 \\
1977 \\
1978 \\
1979 \\
1981 \\
1981 \\
1982\end{array}$ & 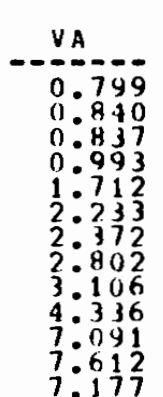 & 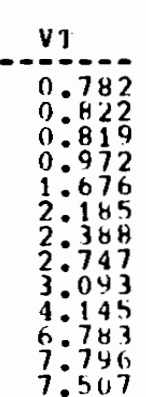 & 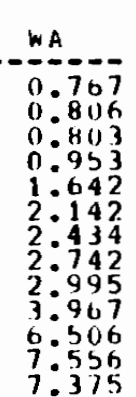 & 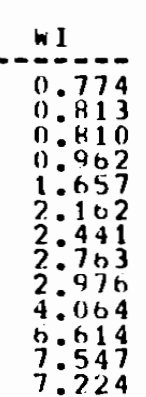 & \begin{tabular}{l}
$w v$ \\
\hdashline $0: 799$ \\
$0: 840$ \\
$0: 837$ \\
$0: 993$ \\
$1: 712$ \\
$2: 233$ \\
$2: 372$ \\
$2: 802$ \\
$3: 106$ \\
$4: 336$ \\
$7: 091$ \\
$7: 012$ \\
$7: 177$
\end{tabular} & 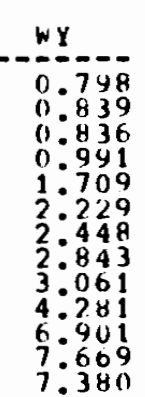 & 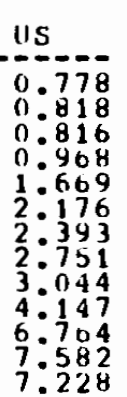 & & \\
\hline
\end{tabular}

Source: Pacific Northwest Laboratory 
TABLE 3.23. Kerosene Prices: Electric Utility Sector ( $\$ / \mathrm{milli}$ ion Btu)

\begin{tabular}{|c|c|c|c|c|c|c|c|c|c|}
\hline $\begin{array}{l}\text { YEAR } \\
1970 \\
1991 \\
1972 \\
1973 \\
1974 \\
1975 \\
1976 \\
1977 \\
1978 \\
1979 \\
1980 \\
1981 \\
1982\end{array}$ & \begin{tabular}{ll}
$A K$ \\
\hdashline $0: 0$ \\
$0: 0$ \\
$0: 0$ \\
000 \\
$0: 0$ \\
$0: 0$ \\
$0: 0$ \\
000 \\
$0: 0$ \\
$0: 0$ \\
$0: 0$ \\
$0: 0$ \\
0.0
\end{tabular} & \begin{tabular}{l} 
AL \\
\hdashline $0: 0$ \\
$0: 0$ \\
$0: 0$ \\
$0: 0$ \\
$0: 0$ \\
$0: 0$ \\
$0: 0$ \\
000 \\
$0: 0$ \\
$0: 0$ \\
$0: 0$ \\
$0: 0$ \\
$0: 0$ \\
0.0
\end{tabular} & \begin{tabular}{ll}
$A H$ \\
\hdashline $0: 0$ \\
$0: 0$ \\
$0: 0$ \\
$0: 0$ \\
$0: 0$ \\
$0: 0$ \\
$0: 0$ \\
$0: 0$ \\
$0: 0$ \\
$0: 0$ \\
$0: 0$ \\
$0: 0$ \\
0.0
\end{tabular} & \begin{tabular}{l}
$A 2$ \\
\hdashline $0: 0$ \\
000 \\
$0: 0$ \\
$0: 0$ \\
$0: 0$ \\
$2: 549$ \\
$0: 0$ \\
$0: 0$ \\
$0: 0$ \\
$0: 0$ \\
$0: 0$ \\
$0: 0$ \\
0.0
\end{tabular} & \begin{tabular}{l} 
CA \\
\hdashline 1.604 \\
$1: 627$ \\
$1: 636$ \\
$1: 768$ \\
$2: 402$ \\
$2: 374$ \\
$2: 512$ \\
2.639 \\
$2: 915$ \\
$3: 333$ \\
$5: 376$ \\
8.026 \\
0.0
\end{tabular} & \begin{tabular}{l}
$C 0$ \\
\hdashline 0.00 \\
00 \\
$0: 0$ \\
$0: 0$ \\
$0: 0$ \\
$0: 0$ \\
$0: 0$ \\
$0: 0$ \\
$0: 0$ \\
$0: 0$ \\
$0: 0$ \\
$0: 0$ \\
0.00 \\
0.0
\end{tabular} & \begin{tabular}{l} 
CT \\
\hdashline 1.517 \\
$1: 542$ \\
1.548 \\
1.667 \\
2.321 \\
2.419 \\
2.080 \\
2.285 \\
1.448 \\
$4: 315$ \\
6.070 \\
8.010 \\
8.458
\end{tabular} & \begin{tabular}{ll}
$D C$ \\
\hdashline 0 & 0 \\
0 & 0 \\
0 & 0 \\
0 & 0 \\
0 & 0 \\
0 & 0 \\
0 & 0 \\
0 & 0 \\
0 & 0 \\
0 & 0 \\
0 & 0 \\
0 & 0 \\
0 & 0 \\
0 & 0
\end{tabular} & \begin{tabular}{ll}
$D E$ \\
\hdashline 0 & 0 \\
0 & 0 \\
0 & 0 \\
0 & 0 \\
0 & 0 \\
0 & 0 \\
0 & 0 \\
0 & 0 \\
0 & 0 \\
0 & 0 \\
0 & 0 \\
0 & 0 \\
0 & 0 \\
0 & 0
\end{tabular} \\
\hline $\begin{array}{l}\text { YEAR } \\
-1970 \\
1970 \\
1971 \\
1972 \\
1973 \\
1974 \\
1975 \\
1976 \\
1977 \\
1978 \\
1979 \\
1980 \\
1981 \\
1982\end{array}$ & \begin{tabular}{l}
$\mathrm{FL}$ \\
\hdashline $0: 0$ \\
$0: 0$ \\
000 \\
$0: 0$ \\
$0: 0$ \\
$2: 454$ \\
000 \\
000 \\
000 \\
$0: 0$ \\
$0: 0$ \\
000 \\
0.0
\end{tabular} & \begin{tabular}{l}
$0 . A$ \\
\hdashline $0: 0$ \\
$0: 0$ \\
$0: 0$ \\
$0: 0$ \\
$0: 0$ \\
$0: 0$ \\
$0: 0$ \\
$00: 0$ \\
$0: 0$ \\
$0: 0$ \\
$0: 0$ \\
$0: 0$ \\
0.0
\end{tabular} & \begin{tabular}{c}
$H \mathbf{I}$ \\
\hdashline $0: 0$ \\
$0: 0$ \\
$0: 0$ \\
$0: 0$ \\
$0: 0$ \\
$0: 0$ \\
$0: 0$ \\
$0: 0$ \\
$0: 0$ \\
$0: 0$ \\
$0: 0$ \\
0.0 \\
0.0
\end{tabular} & \begin{tabular}{lll}
1 & 1 \\
\hdashline 0 & 0 \\
0 & 0 \\
0 & 0 \\
0 & 0 \\
0 & 0 \\
0 & 0 \\
0 & 0 \\
0 & 0 \\
0 & 0 \\
0 & 0 \\
0 & 0 \\
0 & 0 \\
0 & 0 \\
0 & 0 \\
0 & 0
\end{tabular} & $\begin{array}{ll}1 & 0 \\
0 & 0 \\
0 & 0 \\
0 & 0 \\
0 & 0 \\
0 & 0 \\
0 & 0 \\
0 & 0 \\
0 & 0 \\
0 & 0 \\
0 & 0 \\
0 & 0 \\
0 & 0 \\
0 & 0 \\
0.0 & 0 \\
0 & 0\end{array}$ & \begin{tabular}{l} 
IL \\
\hdashline 1.661 \\
1.685 \\
1.693 \\
1.833 \\
20509 \\
$2: 503$ \\
2.611 \\
2.693 \\
30.993 \\
50126 \\
9.895 \\
8.490 \\
8.252
\end{tabular} & \begin{tabular}{ll}
1 & $N$ \\
\hdashline 0 & 0 \\
0 & 0 \\
0 & 0 \\
0 & 0 \\
0 & 0 \\
0 & 0 \\
0 & 0 \\
0 & 490 \\
0 & 49 \\
0 & 0 \\
0 & 0 \\
0 & 0 \\
0 & 0 \\
0 & 0 \\
0 & 0
\end{tabular} & \begin{tabular}{ll}
$K S$ \\
\hdashline 0 & 0 \\
0 & 0 \\
0 & 0 \\
0 & 0 \\
0 & 0 \\
0 & 0 \\
0 & 0 \\
0 & 0 \\
0 & 0 \\
0 & 0 \\
0 & 0 \\
0 & 0 \\
0 & 0 \\
0 & 0 \\
0 & 0
\end{tabular} & \begin{tabular}{ll}
$K Y$ \\
\hdashline $0: 0$ \\
$0: 0$ \\
$0: 0$ \\
$0: 0$ \\
$0: 0$ \\
$0: 0$ \\
$0: 0$ \\
$0: 0$ \\
$0: 0$ \\
$0: 0$ \\
0 & 0 \\
0 & 0 \\
0 & 0 \\
0 & 0
\end{tabular} \\
\hline $\begin{array}{l}\text { YEAR } \\
1970 \\
1971 \\
1972 \\
1973 \\
1974 \\
1975 \\
1976 \\
1977 \\
1978 \\
1979 \\
1980 \\
1981 \\
1982\end{array}$ & $\begin{array}{lll}L & A \\
0 & 0 & 0 \\
0 & 0 \\
0 & 0 \\
0 & 0 \\
0 & 0 \\
0 & 0 \\
0 & 0 \\
0 & 0 \\
0 & 0 \\
0 & 0 \\
0 & 0 \\
0 & 0 \\
0 & 0 \\
0 & 0 \\
0 & 0 \\
0 & 0 \\
0 & 0\end{array}$ & \begin{tabular}{l}
$M A$ \\
\hdashline 1.603 \\
1.626 \\
$1: 634$ \\
10766 \\
$2: 312$ \\
$2: 376$ \\
$2: 594$ \\
2.843 \\
3.145 \\
4.919 \\
6.538 \\
7.434 \\
7.760
\end{tabular} & $\begin{array}{l}M D \\
1.576 \\
1.595 \\
1.604 \\
1.732 \\
2.268 \\
2.426 \\
2.413 \\
2.740 \\
2.811 \\
0.0 \\
0.0 \\
7.574 \\
0.0\end{array}$ & \begin{tabular}{ll} 
ME \\
\hdashline $0: 0$ \\
$0: 0$ \\
$0: 0$ \\
$0: 0$ \\
$0: 0$ \\
$0: 0$ \\
$0: 0$ \\
$0: 0$ \\
$0: 0$ \\
$0: 0$ \\
$0: 0$ \\
$0: 0$ \\
$0: 0$
\end{tabular} & \begin{tabular}{l}
$M I$ \\
\hdashline 1.561 \\
$1: 584$ \\
$1: 591$ \\
$1: 714$ \\
2.259 \\
$2: 279$ \\
$2: 488$ \\
2.908 \\
$2: 958$ \\
$5: 334$ \\
$0: 0$ \\
$0: 0$ \\
0.0
\end{tabular} & \begin{tabular}{ll}
$M N$ \\
\hdashline $0: 0$ \\
$0: 0$ \\
$0: 0$ \\
$0: 0$ \\
$0: 0$ \\
$0: 0$ \\
$0: 0$ \\
$0: 0$ \\
$0: 0$ \\
$0: 0$ \\
$0: 0$ \\
$0: 0$ \\
0.00 \\
0.0
\end{tabular} & \begin{tabular}{l}
$M 0$ \\
\hdashline $0: 0$ \\
$0: 0$ \\
$0: 0$ \\
$0: 0$ \\
$0: 0$ \\
$0: 0$ \\
$0: 0$ \\
000 \\
$0: 0$ \\
$0: 0$ \\
$0: 0$ \\
$0: 0$ \\
0.0
\end{tabular} & $\begin{array}{ll}M S \\
00 & 0 \\
0 & 0 \\
0 & 0 \\
0 & 0 \\
0 & 0 \\
0 & 0 \\
0 & 0 \\
0 & 0 \\
0 & 0 \\
0 & 0 \\
0 & 0 \\
0 & 0 \\
0 & 0 \\
0 & 0 \\
0 & 0\end{array}$ & $\begin{array}{ll}M T \\
00 \\
0: 0 \\
0: 0 \\
0: 0 \\
0: 0 \\
0: 0 \\
0: 0 \\
0: 0 \\
0: 0 \\
0: 0 \\
0: 0 \\
0.00 \\
0.0\end{array}$ \\
\hline
\end{tabular}


TABLE 3.23 (cont). Kerosene Prices: Electric Utility Sector (\$/million Btu)

\begin{tabular}{|c|c|c|c|c|c|c|c|c|c|}
\hline $\begin{array}{l}\text { YEAR } \\
1970 \\
1970 \\
1971 \\
1972 \\
1973 \\
1974 \\
1975 \\
1976 \\
1977 \\
1978 \\
1979 \\
1980 \\
1981 \\
1982\end{array}$ & 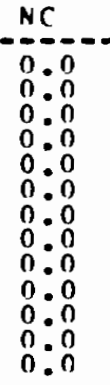 & \begin{tabular}{l} 
NU \\
\hdashline $0: 0$ \\
$0: 0$ \\
$0: 0$ \\
$0: 0$ \\
$0: 0$ \\
$0: 0$ \\
$0: 0$ \\
$0: 0$ \\
$0: 0$ \\
$0: 0$ \\
$0: 0$ \\
$0: 0$ \\
$0: 0$
\end{tabular} & $\begin{array}{l}\text { NE } \\
00.0 \\
00: 0 \\
0: 0 \\
0: 0 \\
0: 0 \\
0: 0 \\
0: 0 \\
0: 0 \\
0: 0 \\
0: 0 \\
0: 0 \\
0: 0 \\
0: 0\end{array}$ & $\begin{array}{l}\mathrm{NH} \\
-1.544 \\
1: 564 \\
1: 570 \\
1: 693 \\
2: 115 \\
2: 320 \\
2: 479 \\
2: 876 \\
3: 144 \\
5: 776 \\
69920 \\
89171 \\
7.925\end{array}$ & $\begin{array}{l}\text { NJ } \\
-1.554 \\
1: 576 \\
1: 582 \\
1: 705 \\
2: 283 \\
2: 293 \\
2: 403 \\
2: 779 \\
2: 982 \\
4: 620 \\
6.195 \\
7: 826 \\
7: 656\end{array}$ & 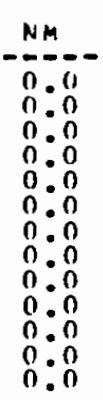 & 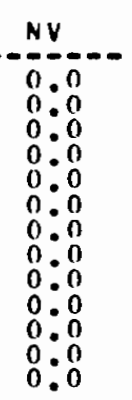 & $\begin{array}{l}\text { NY } \\
-1: 593 \\
1: 616 \\
1: 624 \\
1: 753 \\
2: 345 \\
2: 357 \\
2: 518 \\
2: 797 \\
2: 812 \\
4: 477 \\
6: 530 \\
8: 572 \\
8: 203\end{array}$ & \begin{tabular}{l} 
OH \\
\hdashline $1: 616$ \\
$1: 640$ \\
$1: 647$ \\
$1: 782$ \\
$2: 333$ \\
$2: 414$ \\
$2: 612$ \\
$3: 083$ \\
$3: 010$ \\
$0: 010$ \\
$0: 0$ \\
$0: 0$ \\
0.0
\end{tabular} \\
\hline $\begin{array}{l}Y F A R \\
1970 \\
1971 \\
1977 \\
1973 \\
1977 \\
1975 \\
1977 \\
1977 \\
1978 \\
1979 \\
1980 \\
1981 \\
1982\end{array}$ & \begin{tabular}{l}
$0 k$ \\
\hdashline $0: 0$ \\
$0: 0$ \\
$0: 0$ \\
$0: 0$ \\
$0: 0$ \\
$0: 0$ \\
$0: 00$ \\
$0: 0$ \\
$0: 0$ \\
$0: 0$ \\
$0: 0$ \\
$0: 0$ \\
$0: 0$
\end{tabular} & \begin{tabular}{l}
$0 k$ \\
\hdashline $0: 0$ \\
00 \\
0 \\
0 \\
0 \\
0 \\
2 \\
0 \\
$0: 055$ \\
$0: 0$ \\
$0: 0$ \\
$0: 0$ \\
$0: 0$ \\
$0: 0$ \\
$0: 0$ \\
$0: 0$
\end{tabular} & $\begin{array}{l}P A \\
1.645 \\
1.670 \\
1: 676 \\
1.816 \\
2: 387 \\
2.535 \\
0.07 \\
0: 0 \\
0: 0 \\
0: 0 \\
0.0 \\
8: 239 \\
8: 113\end{array}$ & \begin{tabular}{l}
$R I$ \\
\hdashline $0: 0$ \\
$0: 0$ \\
$0: 0$ \\
$0: 0$ \\
$0: 0$ \\
$0: 0$ \\
$0: 0$ \\
$0: 0$ \\
$0: 0$ \\
$0: 0$ \\
$0: 0$ \\
$0: 0$ \\
$0: 0$
\end{tabular} & $\begin{array}{l}s c \\
0.0 \\
000 \\
0: 0 \\
0: 0 \\
0: 0 \\
0: 0 \\
0: 0 \\
0: 0 \\
0: 0 \\
0: 0 \\
0: 0 \\
0: 0 \\
0: 0 \\
0: 0\end{array}$ & \begin{tabular}{c} 
so \\
\hdashline $0: 0$ \\
$0: 0$ \\
$0: 0$ \\
$0: 0$ \\
$0: 0$ \\
$0: 0$ \\
0 \\
0 \\
0 \\
0 \\
0 \\
0 \\
0 \\
0 \\
0 \\
0 \\
0 \\
0 \\
0 \\
0
\end{tabular} & \begin{tabular}{l}
$T N$ \\
\hdashline $0: 0$ \\
$0: 0$ \\
$0: 0$ \\
$0: 0$ \\
$0: 0$ \\
$0: 0$ \\
$0: 0$ \\
$0: 0$ \\
$0: 0$ \\
$0: 0$ \\
$0: 0$ \\
$0: 0$ \\
$0: 0$ \\
0.0
\end{tabular} & \begin{tabular}{l}
$T x$ \\
\hdashline $0: 0$ \\
$0: 0$ \\
$0: 0$ \\
$0: 0$ \\
$0: 0$ \\
$0: 0$ \\
$0: 0$ \\
$0: 0$ \\
$0: 0$ \\
$0: 0$ \\
$0: 0$ \\
$0: 0$ \\
$0: 0$
\end{tabular} & 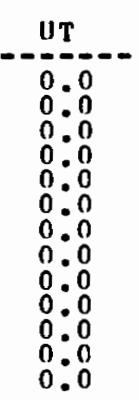 \\
\hline $\begin{array}{l}\text { YEAR } \\
197- \\
1970 \\
1971 \\
1972 \\
1973 \\
1974 \\
1975 \\
1976 \\
1977 \\
1978 \\
1979 \\
1980 \\
1981 \\
1982\end{array}$ & \begin{tabular}{l}
$V A$ \\
\hdashline $0: 0$ \\
00 \\
$0: 0$ \\
$0: 0$ \\
$0: 0$ \\
$0: 0$ \\
$0: 0$ \\
$0: 0$ \\
$0: 0$ \\
$0: 0$ \\
$0: 0$ \\
$0: 0$ \\
$0: 0$ \\
0
\end{tabular} & $\begin{array}{l}V T \\
-1.640 \\
1.664 \\
1.673 \\
1.810 \\
2.421 \\
2.550 \\
2.535 \\
3: 207 \\
0.007 \\
6.611 \\
6.842 \\
8.220 \\
7.944\end{array}$ & $\begin{array}{l}\text { WA } \\
1.747 \\
1: 782 \\
1: 797 \\
19939 \\
2: 626 \\
2.654 \\
2: 600 \\
2: 770 \\
2: 959 \\
4: 968 \\
0: 0 \\
0.01 \\
0.0\end{array}$ & $\begin{array}{l}W 1 \\
1.548 \\
1: 570 \\
1: 575 \\
10998 \\
10902 \\
2: 453 \\
20572 \\
2: 674 \\
0: 0 \\
0: 0 \\
0: 0 \\
0: 0 \\
0.0\end{array}$ & 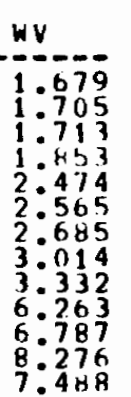 & 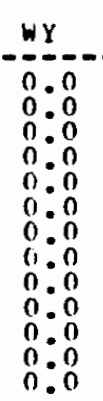 & \begin{tabular}{l} 
US \\
\hdashline 1.394 \\
1.414 \\
1.421 \\
1.535 \\
2.366 \\
$2: 389$ \\
2.508 \\
$2: 756$ \\
2.937 \\
$4: 265$ \\
6.063 \\
7.874 \\
7.743
\end{tabular} & & \\
\hline
\end{tabular}

Source: Pacific Northwest Laboratory 
TABLE 3.24. Residual Fuel Prices: Commercial Sector $\left(\$ / m i l l i o n\right.$ Btu) ${ }^{(a)}$
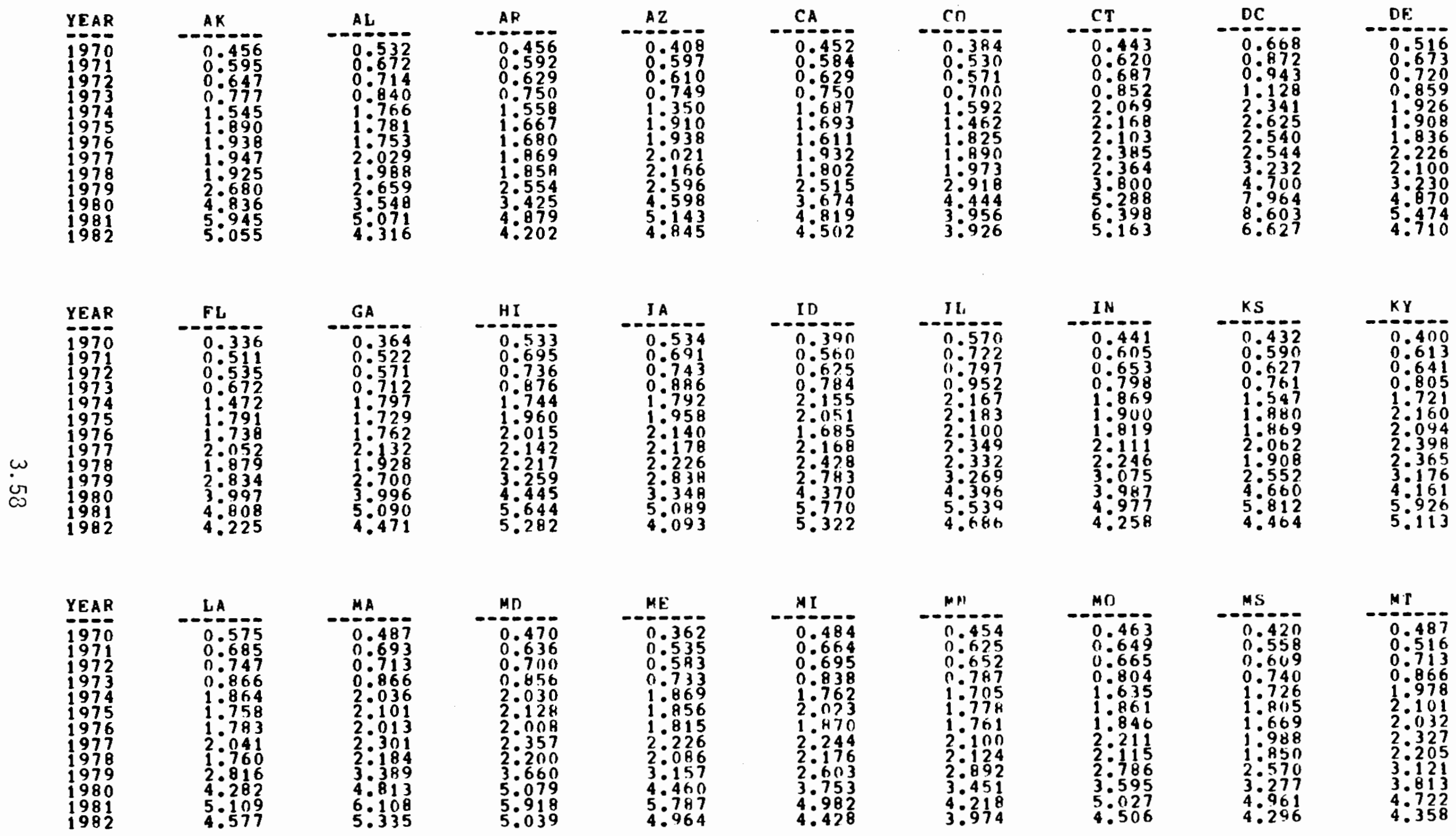

(a) Data for 1982 are provisional estimates and will be updated when data from Annual Survey of Manufactures become available. 
TABLE 3.24 (cont). Residual Fuel Prices: Commercial Sector $\left(\$ /\right.$ million Btu) ${ }^{(a)}$

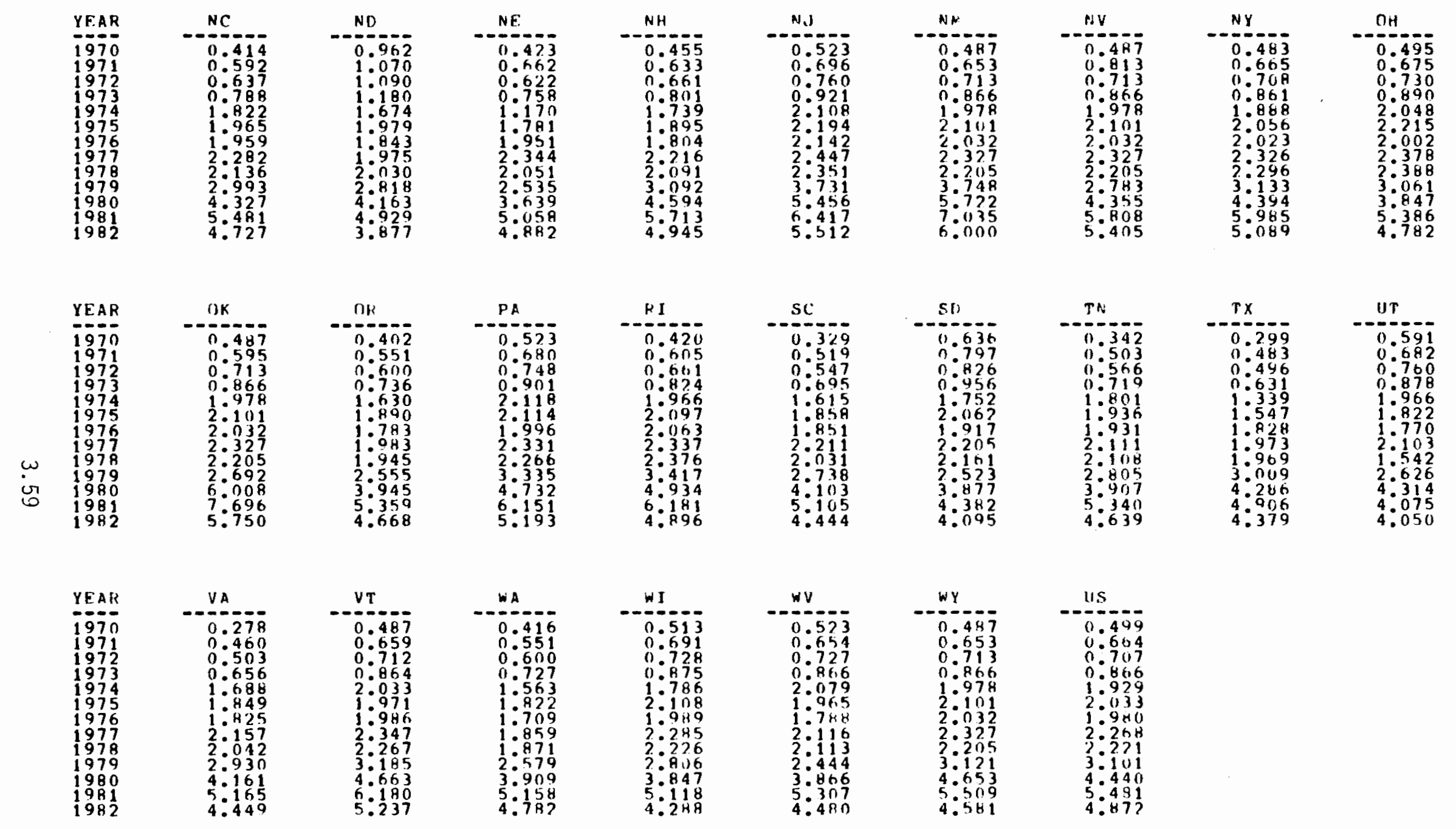

Source: Pacific Northwest Laboratory 
TABLE 3.25. Residual Fuel Prices: Industrial Sector $\left(\$ / m i 11\right.$ ion Btu) ${ }^{(a)}$

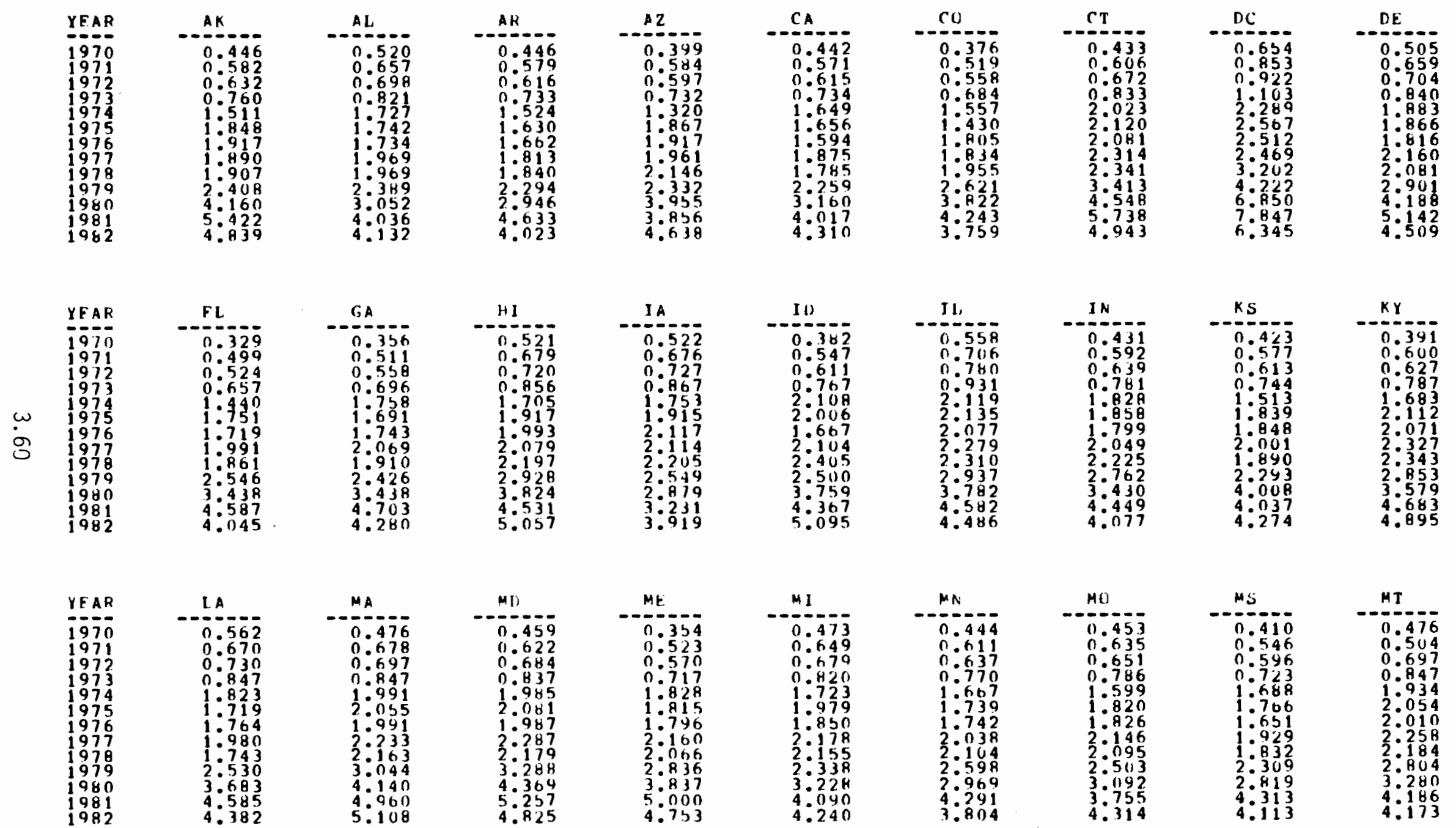

(a) Data for 1982 are provisional estimates and will be updated when data from Annual Survey of Manufactures become available. 
TABLE 3.25 (cont). Residual Fuel Prices: Industrial Sector ( $\$ / \mathrm{milli}$ ion Btu)

\begin{tabular}{|c|c|c|c|c|c|c|c|c|c|}
\hline $\begin{array}{l}\text { YEAR } \\
1970 \\
1971 \\
1971 \\
1977 \\
1974 \\
1975 \\
1976 \\
1977 \\
1978 \\
1979 \\
1980 \\
1981 \\
1982\end{array}$ & $\begin{array}{l}N C \\
-N C=- \\
0.405 \\
0: 579 \\
0: 623 \\
0: 771 \\
1: 782 \\
1: 921 \\
1: 937 \\
2: 214 \\
2: 116 \\
2: 688 \\
3: 722 \\
4: 813 \\
4: 526\end{array}$ & $\begin{array}{l}N D \\
-0.941 \\
1: 947 \\
1: 066 \\
1: 154 \\
1: 697 \\
1: 936 \\
1: 823 \\
10917 \\
20011 \\
20531 \\
3: 5881 \\
3: 909 \\
3.712\end{array}$ & $\begin{array}{l}N E \\
0.713 \\
0: 647 \\
0: 608 \\
0: 742 \\
1: 144 \\
1: 742 \\
1: 929 \\
2: 275 \\
2: 031 \\
2: 277 \\
3: 130 \\
5: 676 \\
4: 674\end{array}$ & $\begin{array}{l}N H \\
-N H \\
-0.445 \\
0: 619 \\
0: 646 \\
0: 783 \\
1: 700 \\
1: 853 \\
1: 785 \\
27151 \\
2: 071 \\
2: 778 \\
3: 951 \\
5: 101 \\
4: 735\end{array}$ & 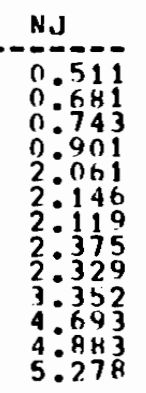 & $\begin{array}{l}\text { NH } \\
-4 .-57 \\
0.476 \\
0.638 \\
0.699 \\
0.847 \\
1.934 \\
2.054 \\
2.0110 \\
2.058 \\
2.184 \\
3.367 \\
4.922 \\
6.416 \\
5.744\end{array}$ & \begin{tabular}{l} 
NV \\
\hdashline 0.476 \\
00795 \\
$0: 697$ \\
0.847 \\
1.934 \\
$2: 054$ \\
2.010 \\
$2: 258$ \\
$2: 184$ \\
$2: 500$ \\
3.746 \\
$4: 820$ \\
$5: 175$
\end{tabular} & 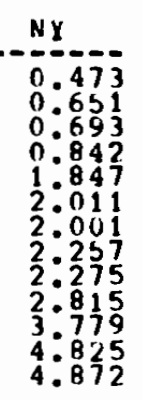 & \begin{tabular}{c}
$n H$ \\
\hdashline 0.484 \\
$0: 660$ \\
$0: 714$ \\
$0: 870$ \\
$2: 003$ \\
$2: 166$ \\
$1: 980$ \\
$2: 308$ \\
$2: 365$ \\
$2: 749$ \\
3.309 \\
$4: 568$ \\
4.579
\end{tabular} \\
\hline $\begin{array}{l}\text { YEAR } \\
1977 \\
1971 \\
1977 \\
1973 \\
1974 \\
1975 \\
1976 \\
1977 \\
1978 \\
1979 \\
1981 \\
1982\end{array}$ & 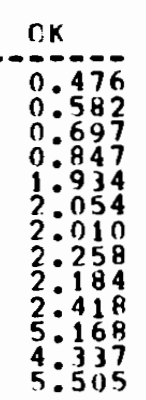 & $\begin{array}{r}0 H \\
-0.394 \\
0: 539 \\
0: 587 \\
0: 720 \\
1: 594 \\
1: 848 \\
1: 764 \\
1: 925 \\
1: 926 \\
2: 295 \\
3.393 \\
3.863 \\
4: 470\end{array}$ & $\begin{array}{l}P A \\
-0.511 \\
0.665 \\
0: 731 \\
0: 841 \\
2: 071 \\
2: 068 \\
1: 974 \\
2: 262 \\
2: 244 \\
2: 996 \\
4: 071 \\
4: 971 \\
4: 971\end{array}$ & $\begin{array}{l}R 1 \\
-0.911 \\
0: 592 \\
0: 646 \\
0: 806 \\
1: 923 \\
2: 050 \\
2: 041 \\
2: 0208 \\
2: 354 \\
3: 070 \\
4: 244 \\
4: 966 \\
4: 687\end{array}$ & \begin{tabular}{l} 
Sc \\
\hdashline 0.321 \\
$0: 507$ \\
$0: 535$ \\
$0: 580$ \\
$1: 579$ \\
$1: 816$ \\
$1: 831$ \\
$2: 146$ \\
2.012 \\
$2: 459$ \\
$3: 529$ \\
4.591 \\
$4: 255$
\end{tabular} & \begin{tabular}{l} 
SD \\
\hdashline 0.622 \\
$0: 779$ \\
$0: 808$ \\
$0: 935$ \\
$1: 713$ \\
$2: 017$ \\
$1: 896$ \\
$2: 139$ \\
$2: 141$ \\
$2: 767$ \\
$3: 335$ \\
$3: 997$ \\
$3: 920$
\end{tabular} & \begin{tabular}{l}
$T N$ \\
\hdashline 0.334 \\
$0: 492$ \\
$0: 554$ \\
$0: 703$ \\
$1: 761$ \\
$1: 893$ \\
$1: 910$ \\
$2: 049$ \\
20088 \\
$2: 519$ \\
$33: 360$ \\
$4: 309$ \\
$4: 441$
\end{tabular} & \begin{tabular}{l}
$T x$ \\
\hdashline 0.292 \\
$0: 472$ \\
$0: 465$ \\
$0: 617$ \\
$1: 309$ \\
$1: 513$ \\
$1: 809$ \\
$1: 915$ \\
$1: 950$ \\
$2: 703$ \\
3.607 \\
39945 \\
$4: 192$
\end{tabular} & \begin{tabular}{c}
$U T$ \\
\hdashline 0.578 \\
$0: 667$ \\
$0: 743$ \\
$0: 858$ \\
$1: 923$ \\
$1: 782$ \\
$1: 751$ \\
$2: 041$ \\
$1: 527$ \\
$2: 359$ \\
3.711 \\
$4: 777$ \\
$3: 877$
\end{tabular} \\
\hline $\begin{array}{l}\text { YEAR } \\
1970 \\
1977 \\
1977 \\
1977 \\
1974 \\
1975 \\
1976 \\
1977 \\
1977 \\
1979 \\
1980 \\
1981 \\
1982\end{array}$ & 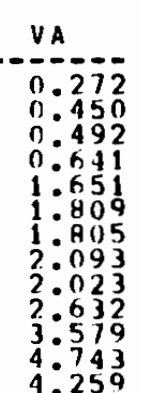 & $\begin{array}{l}V T \\
-10.476 \\
0: 644 \\
0: 696 \\
0: 945 \\
1: 988 \\
1: 928 \\
1: 964 \\
2: 278 \\
2: 246 \\
2: 861 \\
4: 011 \\
4: 957 \\
5.013\end{array}$ & $\begin{array}{l}\text { WA } \\
0.4177 \\
0: 539 \\
0: 587 \\
0: 711 \\
1: 529 \\
1: 782 \\
10991 \\
1: 804 \\
10953 \\
20316 \\
3: 363 \\
4: 046 \\
4.579\end{array}$ & 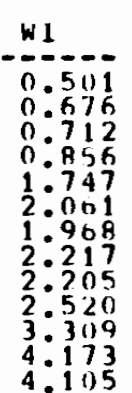 & $\begin{array}{l}W \mathrm{~V} \\
-0.512 \\
0.639 \\
0.711 \\
0.847 \\
2.033 \\
1.921 \\
1.769 \\
2.053 \\
2.093 \\
2.196 \\
3.326 \\
4.682 \\
4.289\end{array}$ & \begin{tabular}{l}
$W y$ \\
\hdashline 0.476 \\
0.639 \\
0.697 \\
$0: 847$ \\
1.934 \\
20054 \\
20010 \\
20258 \\
2.184 \\
2.804 \\
40002 \\
5.024 \\
4.386
\end{tabular} & \begin{tabular}{l} 
US \\
\hdashline 0.459 \\
0.608 \\
0.656 \\
0.746 \\
1.819 \\
$1: 902$ \\
1.878 \\
2.127 \\
2.048 \\
2.771 \\
$3: 720$ \\
4.522 \\
4.5114
\end{tabular} & & \\
\hline
\end{tabular}

Source: Pacific Northwest Laboratory 
TABLE 3.26. Residual Fuel Prices: Transportation Sector $\left(\$ / m i 11\right.$ ion Btu) ${ }^{(a)}$

\begin{tabular}{|c|c|c|c|c|c|c|c|c|c|}
\hline $\begin{array}{l}\text { YFAR } \\
19.7 \\
1971 \\
1971 \\
1972 \\
1974 \\
1975 \\
1976 \\
1977 \\
1978 \\
1979 \\
1981 \\
1981 \\
1982\end{array}$ & \begin{tabular}{l}
$A K$ \\
\hdashline$A$ \\
0.369 \\
$0: 482$ \\
$0: 524$ \\
$0: 630$ \\
$1: 251$ \\
$1: 529$ \\
$1: 621$ \\
$1: 586$ \\
$1: 494$ \\
2019 \\
$3: 730$ \\
$4: 730$ \\
0.07
\end{tabular} & 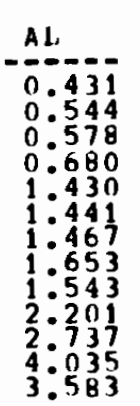 & 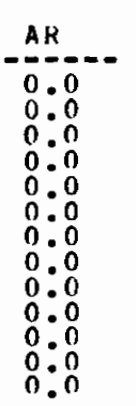 & 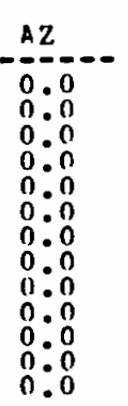 & 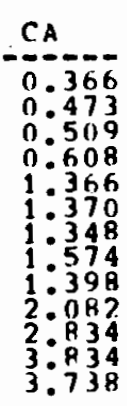 & 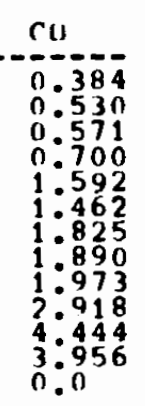 & 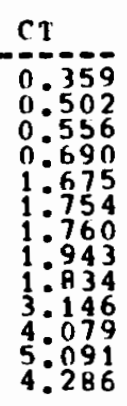 & 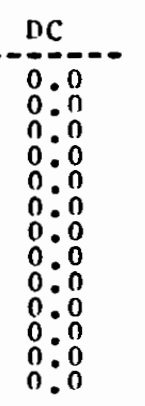 & \begin{tabular}{l} 
DE \\
\hdashline 0.418 \\
$0: 545$ \\
$0: 583$ \\
$0: 696$ \\
$1: 559$ \\
$1: 544$ \\
$1: 537$ \\
$1: 813$ \\
$1: 630$ \\
20674 \\
20756 \\
$4: 355$ \\
$3: 910$
\end{tabular} \\
\hline $\begin{array}{l}\text { YEAR } \\
1971 \\
1971 \\
1971 \\
1972 \\
1973 \\
1974 \\
1975 \\
1976 \\
1977 \\
1978 \\
1979 \\
1981 \\
1982\end{array}$ & \begin{tabular}{l} 
FL \\
\hdashline $0: 272$ \\
$0: 414$ \\
$0: 434$ \\
$0: 544$ \\
$1: 192$ \\
$1: 449$ \\
$1: 455$ \\
$1: 672$ \\
$1: 458$ \\
$2: 346$ \\
$3: 083$ \\
$3: 826$ \\
$3: 508$
\end{tabular} & 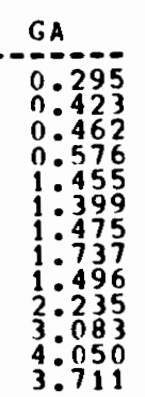 & 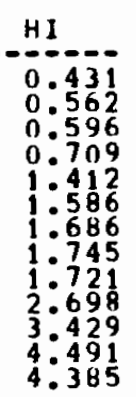 & 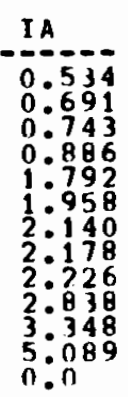 & $\begin{array}{rl}10 & 0 \\
-10 & 0 \\
0 & 0 \\
0 & 0 \\
0 & 0 \\
0 & 0 \\
0 & 0 \\
0 & 0 \\
0 & 0 \\
0 & 0 \\
0 & 0 \\
0 & 0 \\
0 & 0 \\
0 & 0 \\
0 & 0 \\
0 & 0\end{array}$ & 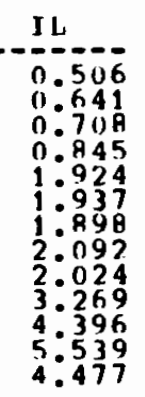 & 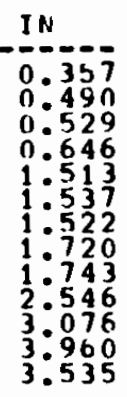 & 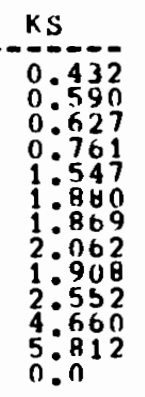 & 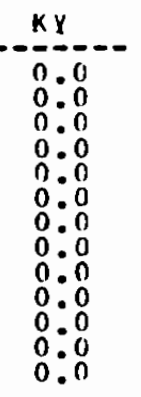 \\
\hline $\begin{array}{l}\text { YEAR } \\
19-70 \\
1971 \\
1971 \\
1973 \\
1973 \\
1974 \\
1975 \\
1977 \\
1978 \\
1979 \\
1980 \\
1981 \\
1982\end{array}$ & $\begin{array}{l}\mathrm{LA} \\
-1.765 \\
0: 465 \\
0: 555 \\
0: 605 \\
0: 701 \\
1: 509 \\
1: 422 \\
1: 492 \\
1: 662 \\
1: 366 \\
2: 332 \\
3: 303 \\
4: 065 \\
3.799\end{array}$ & \begin{tabular}{l}
$M A$ \\
\hdashline 0.394 \\
0.561 \\
0.577 \\
0.701 \\
10649 \\
1.700 \\
10685 \\
10875 \\
10695 \\
20805 \\
3.712 \\
4.860 \\
4.429
\end{tabular} & 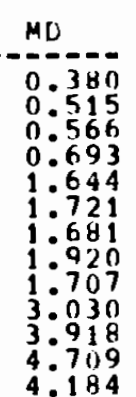 & 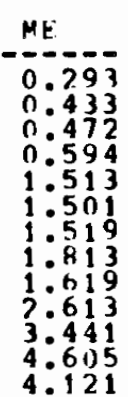 & 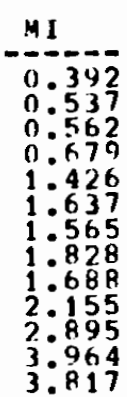 & 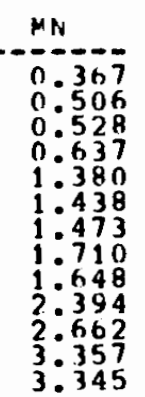 & 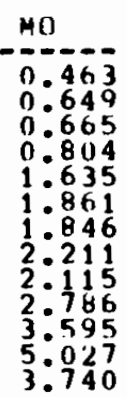 & 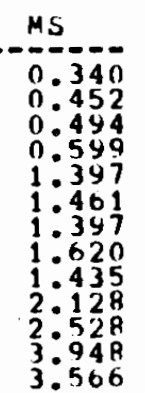 & 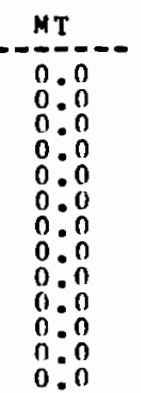 \\
\hline
\end{tabular}

(a) Data for 1982 are provisional estimates and will be updated when data from Annual Survey of Manufactures become available. 
TABLE 3.26 (cont). Residual Fuel Prices: Transportation Sector $(\$ / m i l 1$ ion Btu) $(a)$
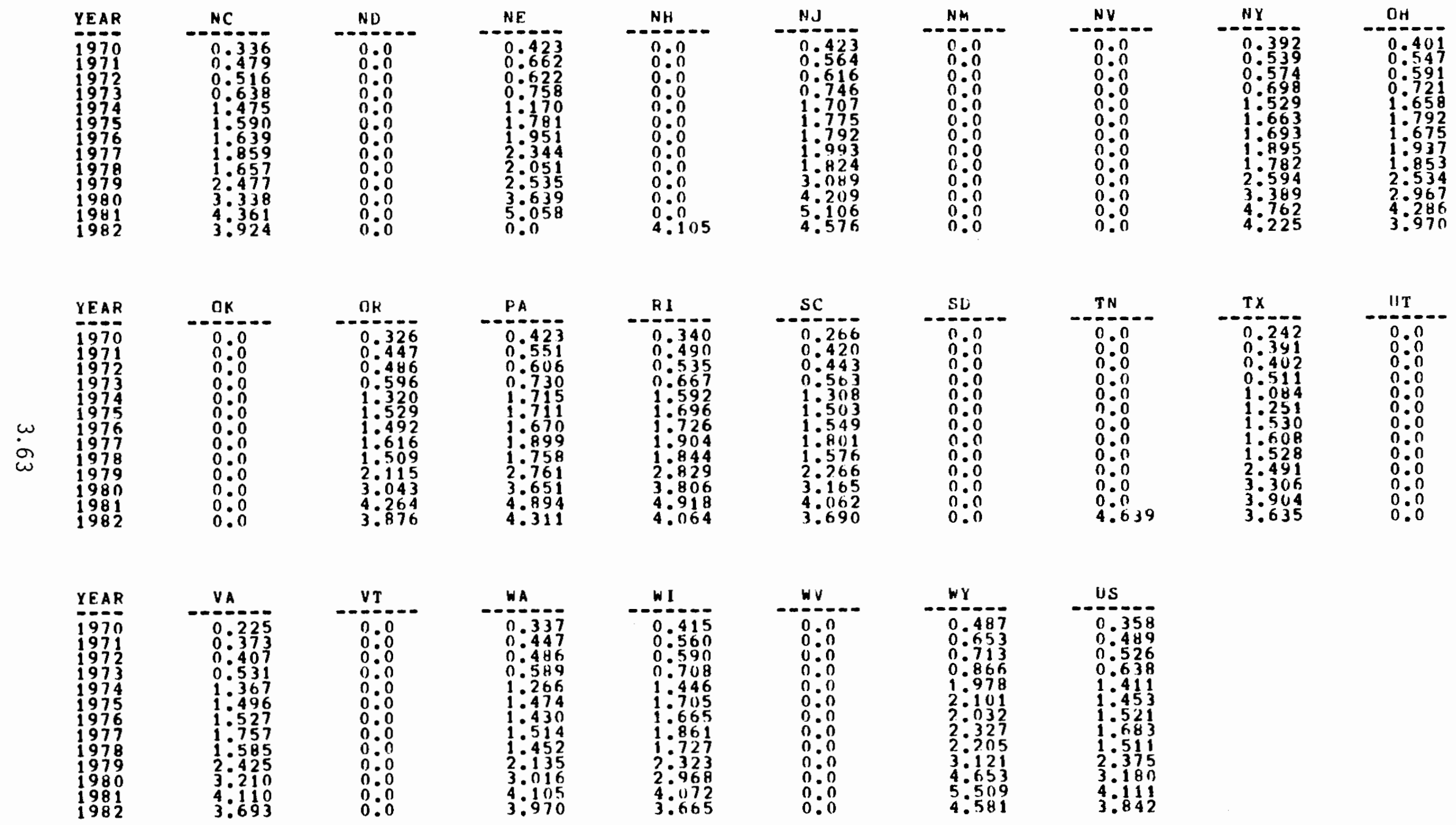

Source: Pacific Northwest Laboratory 
TABLE 3.27. Residual Fuel Prices: Electric Utility Sector (\$/million Btu)
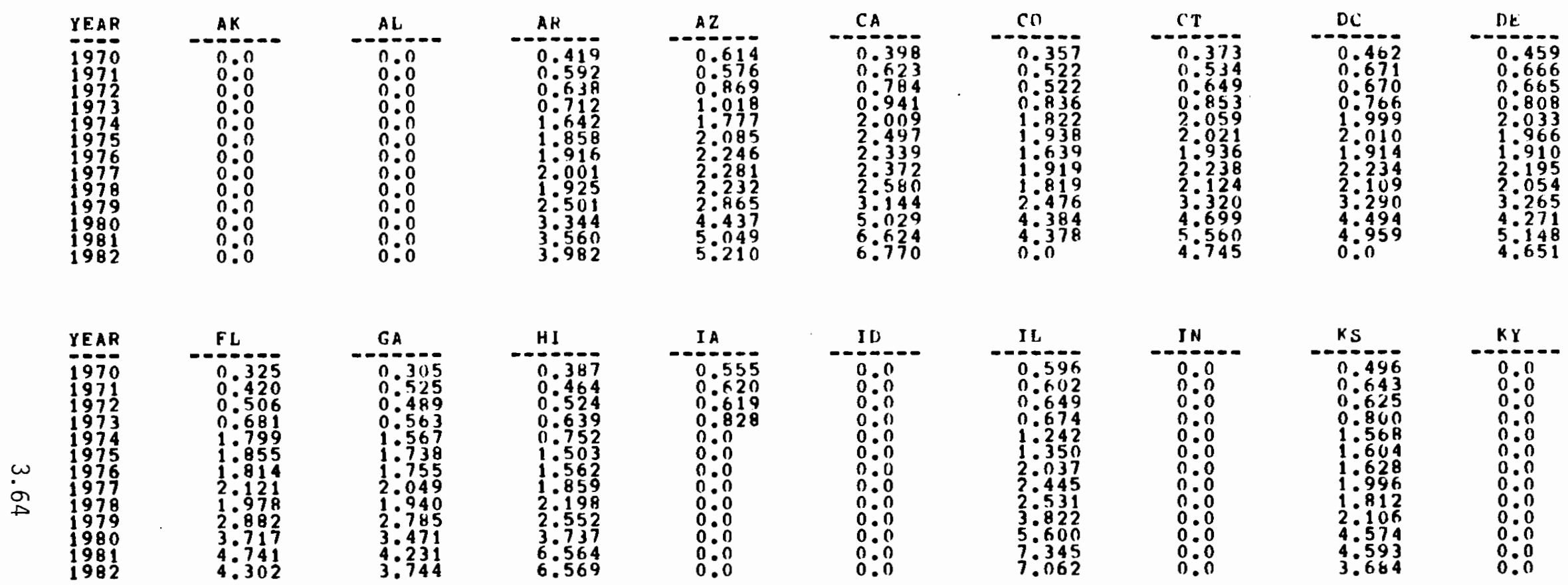

\begin{tabular}{ll} 
YEAR & LA \\
\hline 1970 & 0.494 \\
1971 & 0.515 \\
1973 & 0.693 \\
1973 & 0.738 \\
1974 & 1.656 \\
1975 & 1.730 \\
1976 & 1.732 \\
1979 & 1.891 \\
1978 & 1.820 \\
1979 & 2.700 \\
1980 & 3.576 \\
1981 & 4.362 \\
1982 & 3.907
\end{tabular}
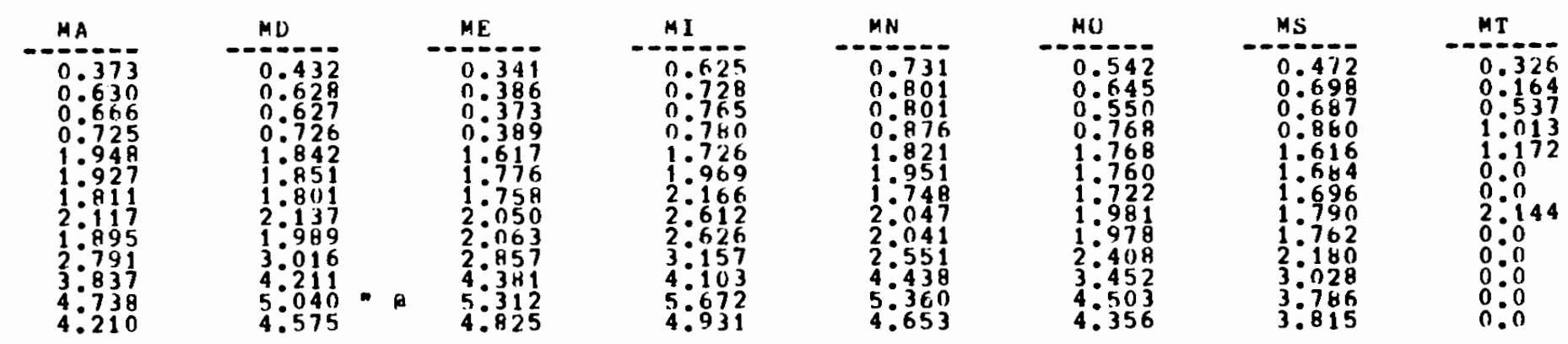
TABLE 3.27 (cont). Residual Fuel Prices: Electric Utility Sector ( $\$ /$ million Btu)
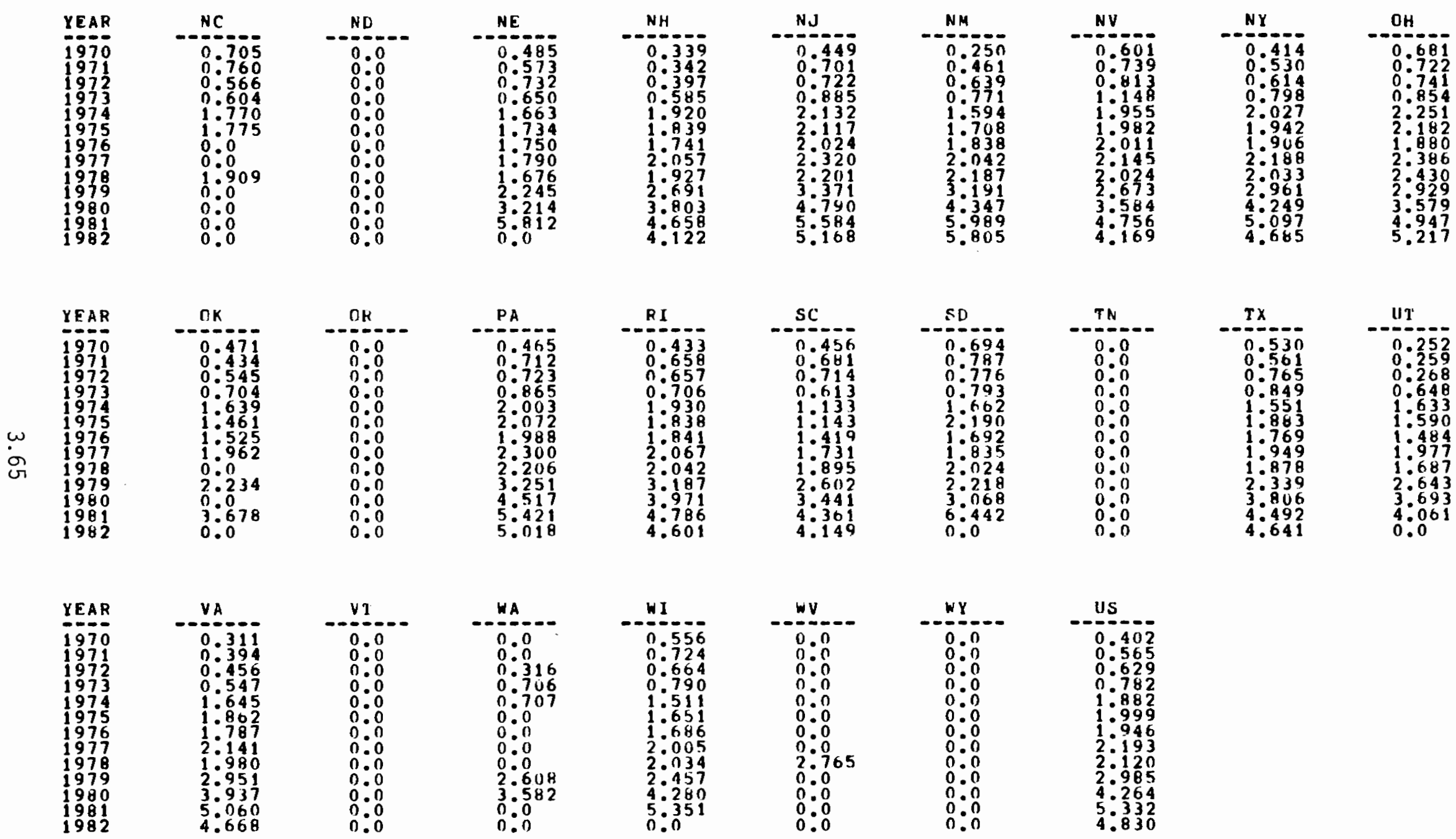

$\vee 1$
$0: 0$
$0: 0$
$0: 0$
$0: 0$
$0: 0$
$0: 0$
$0: 0$
$0: 0$
$0: 0$
$0: 0$
000
$0: 0$
0.0
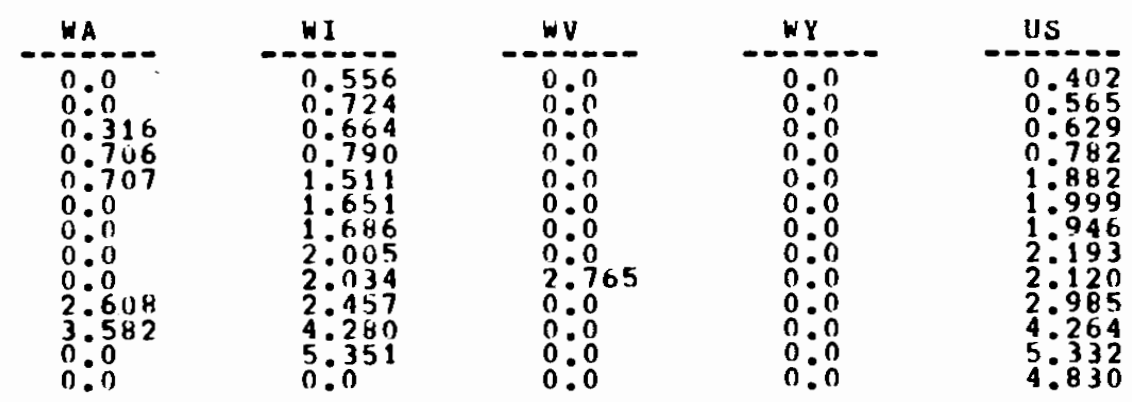

Source: Pacific Northwest Laboratory 
TABLE 3.28. Liquefied Petroleum Gas Prices: Residential Sector $(\$ / m i l l i o n$ Btu)
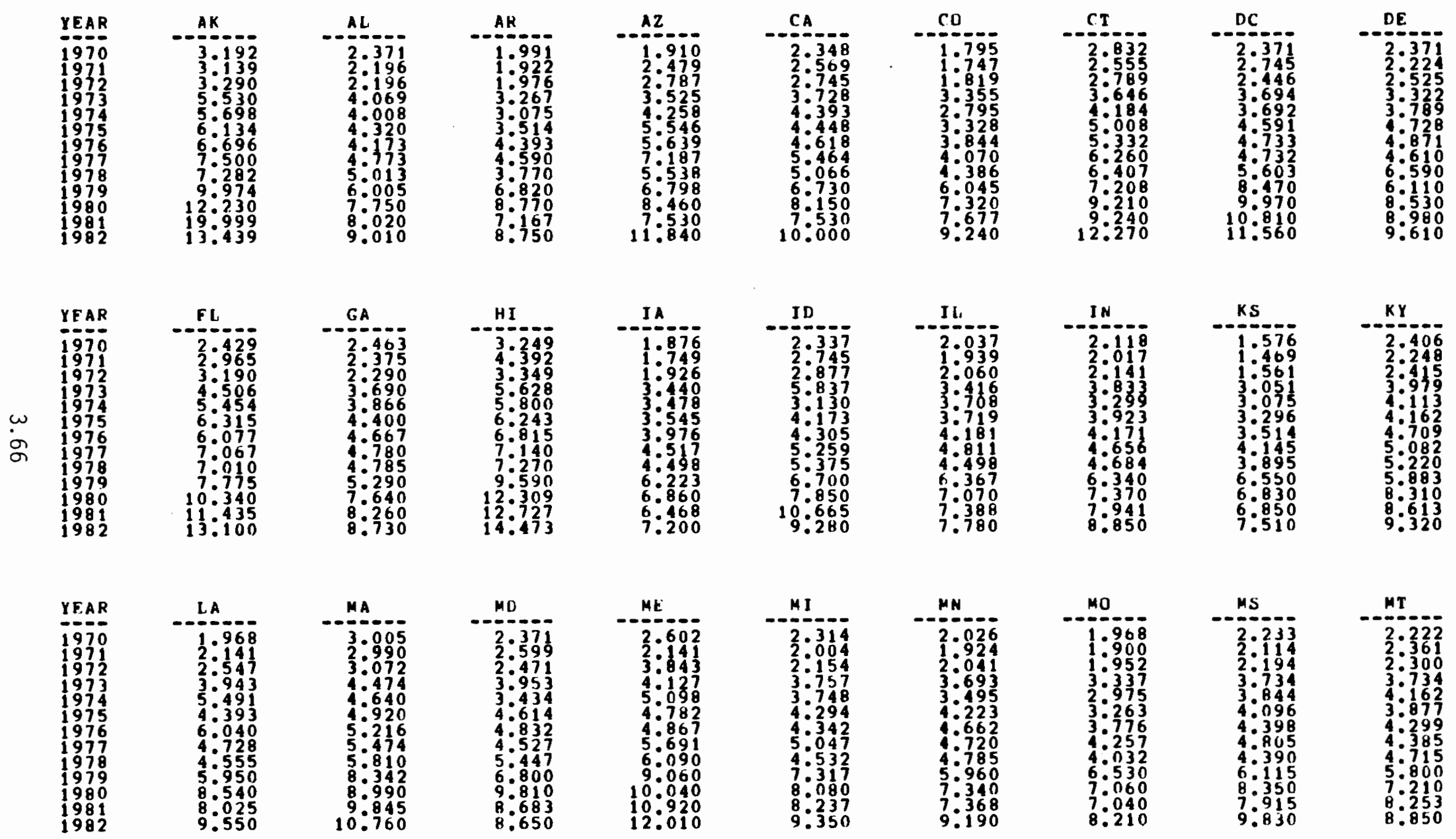
TABLE 3.28 (cont). Liquefied Petroleum Gas Prices: Residential Sector (\$/million Btu)

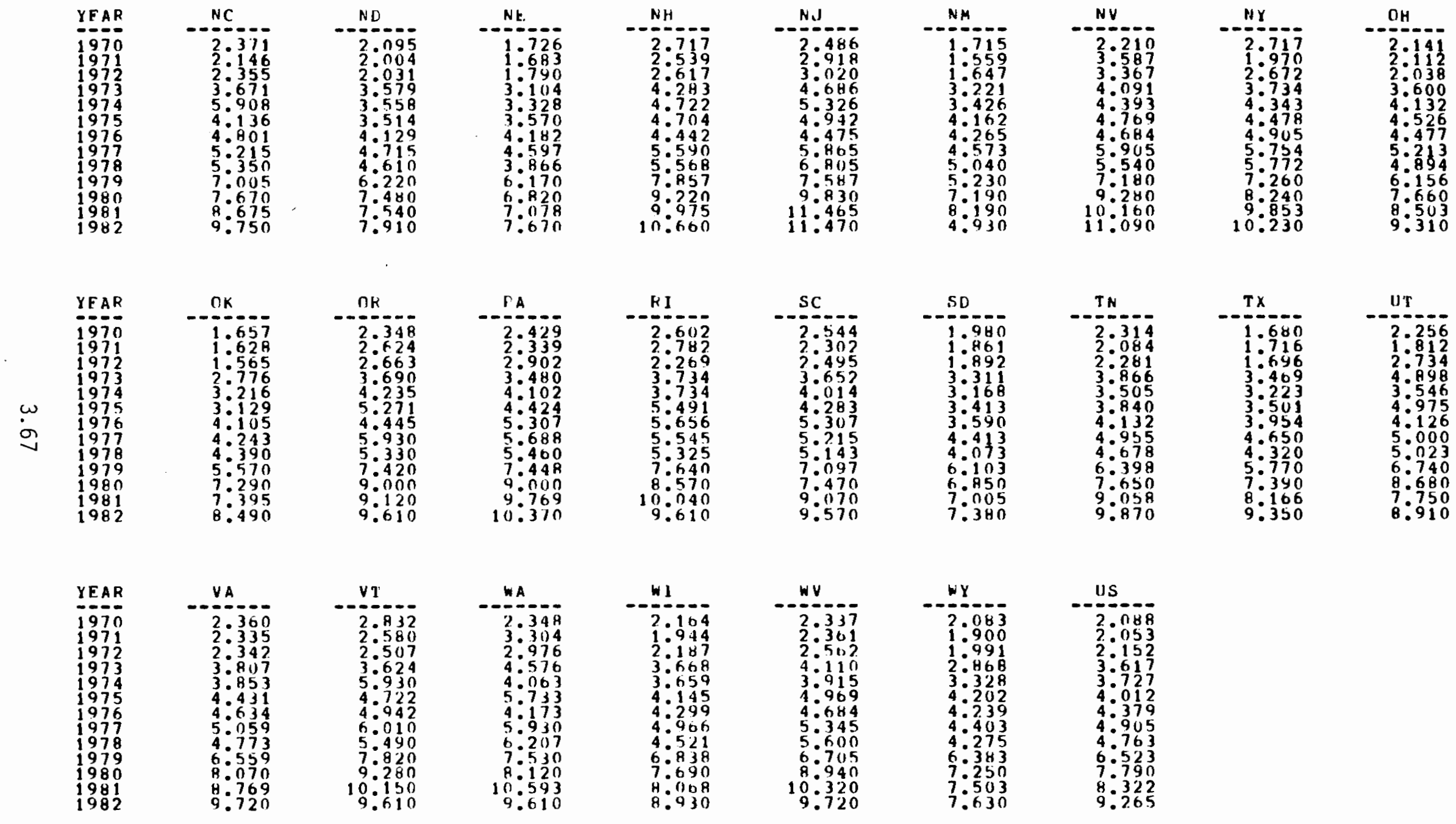

Source: Pacific Northwest Laboratory 
TABLE 3.29. Liquefied Petroleum Gas Priçes: Industrial Sector $(\$ / m i l l i o n B t u)^{(a)}$

\begin{tabular}{|c|c|c|c|c|c|c|c|c|c|}
\hline $\begin{array}{l}\text { YFAR } \\
1970 \\
1971 \\
1971 \\
1972 \\
1973 \\
1974 \\
1975 \\
1976 \\
1977 \\
1978 \\
1979 \\
1980 \\
1981 \\
1982\end{array}$ & \begin{tabular}{l}
$A K$ \\
$A K$ \\
\hdashline $1: 320$ \\
$1: 373$ \\
$1: 378$ \\
$1: 472$ \\
$2: 536$ \\
$2: 746$ \\
33034 \\
33462 \\
33620 \\
$3: 950$ \\
$3: 958$ \\
55464 \\
$5: 586$
\end{tabular} & 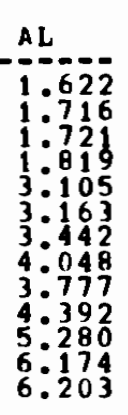 & 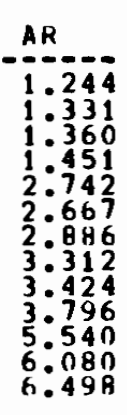 & 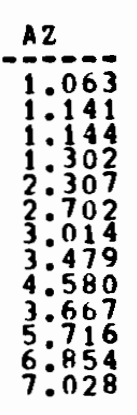 & 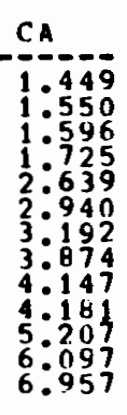 & 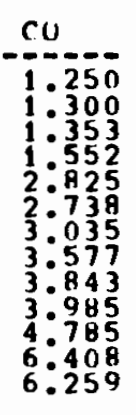 & 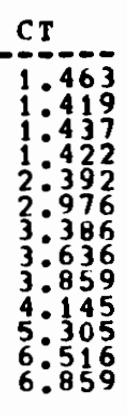 & 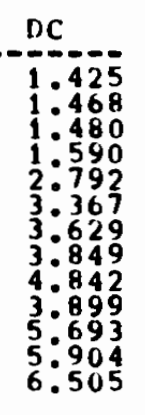 & 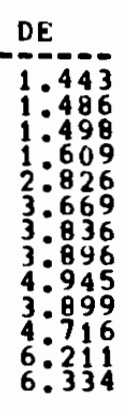 \\
\hline $\begin{array}{l}\text { YFAR } \\
1970 \\
1971 \\
1972 \\
1973 \\
1974 \\
1975 \\
1976 \\
1977 \\
1978 \\
1979 \\
1980 \\
1981 \\
1982\end{array}$ & 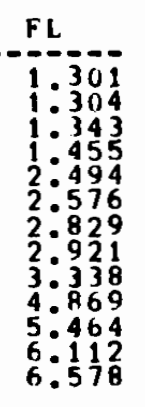 & 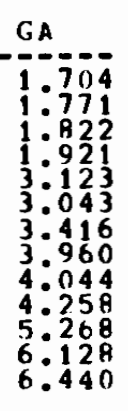 & 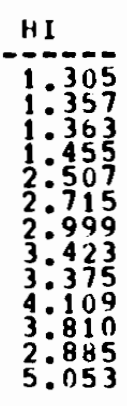 & 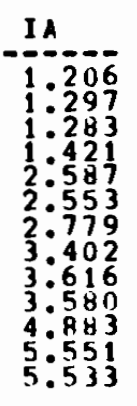 & 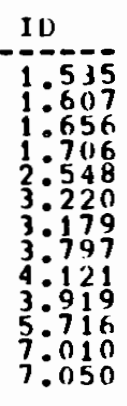 & 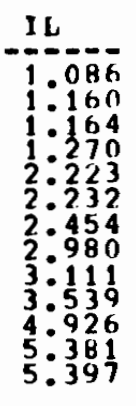 & 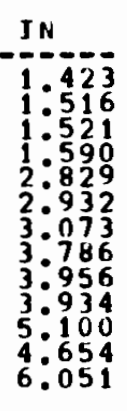 & 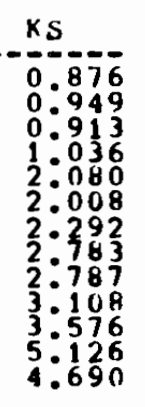 & 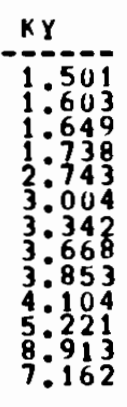 \\
\hline $\begin{array}{l}\text { YFAR } \\
1970 \\
1970 \\
1971 \\
1972 \\
1973 \\
1974 \\
1975 \\
1976 \\
1977 \\
1978 \\
1979 \\
1980 \\
1981 \\
1982\end{array}$ & 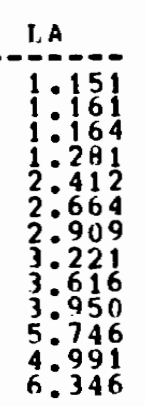 & 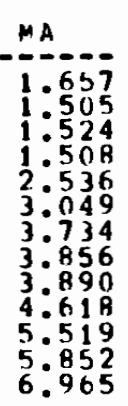 & 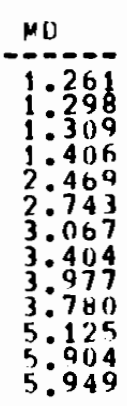 & 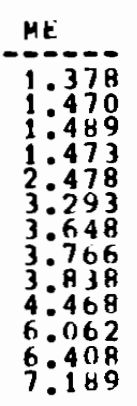 & 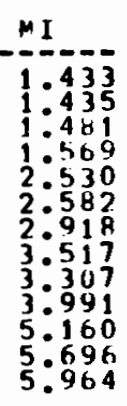 & 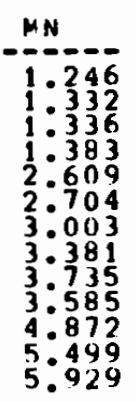 & 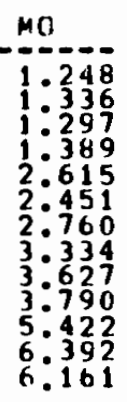 & 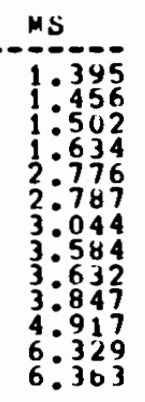 & \begin{tabular}{c}
$M T$ \\
\hdashline $1: 386$ \\
$1: 446$ \\
$1: 443$ \\
$1: 590$ \\
$2: 460$ \\
$2: 574$ \\
$2: 915$ \\
$32: 223$ \\
$2: 064$ \\
$4: 109$ \\
$5: 846$ \\
$6: 854$ \\
$6: 214$
\end{tabular} \\
\hline
\end{tabular}

(a) Data for 1982 are provisional estimates and will be updated when data from Annual Survey of Manufactures become available. 
TABLE 3.29 (cont). Liquefied Petroleum Gas Prices: Industrial Sector (\$/million Btu) ${ }^{(a)}$

\begin{tabular}{|c|c|c|c|c|c|c|c|c|c|}
\hline $\begin{array}{l}\text { YEAR } \\
1970 \\
1970 \\
1971 \\
1972 \\
1973 \\
1974 \\
1975 \\
1976 \\
1977 \\
1978 \\
1979 \\
1980 \\
1981 \\
1982\end{array}$ & 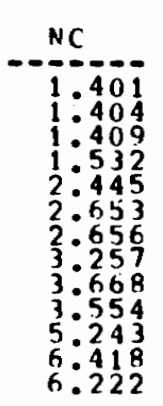 & \begin{tabular}{l}
$N L$ \\
$-N L$ \\
\hdashline $1: 259$ \\
$1: 334$ \\
$1: 339$ \\
$1: 384$ \\
$2: 412$ \\
$2: 512$ \\
$2: 545$ \\
2877 \\
$2: 094$ \\
$2: 483$ \\
$4: 191$ \\
$5: 231$ \\
$5: 686$ \\
$5: 573$
\end{tabular} & 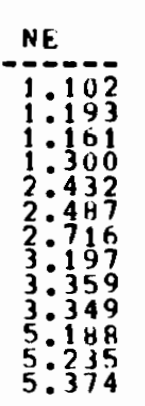 & 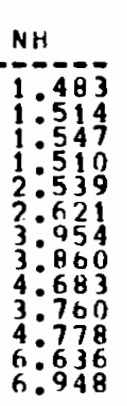 & 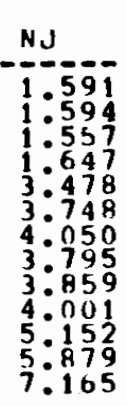 & 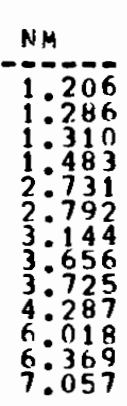 & 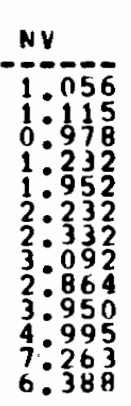 & 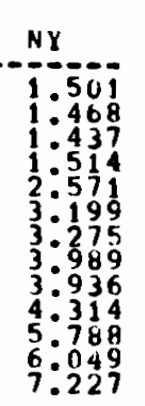 & \begin{tabular}{l} 
OH \\
\hdashline $1: 494$ \\
$1: 524$ \\
$1: 529$ \\
$1: 601$ \\
$2: 837$ \\
$3: 038$ \\
$3: 232$ \\
$3: 799$ \\
$3: 766$ \\
$4: 345$ \\
$5: 341$ \\
$5: 904$ \\
$6: 756$
\end{tabular} \\
\hline $\begin{array}{l}\text { YEAR } \\
1970 \\
1971 \\
1972 \\
1973 \\
1974 \\
1975 \\
1976 \\
1977 \\
1978 \\
1979 \\
1980 \\
1981 \\
1982\end{array}$ & 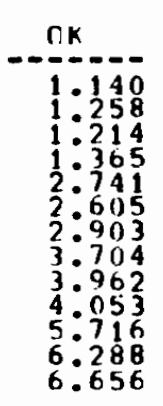 & 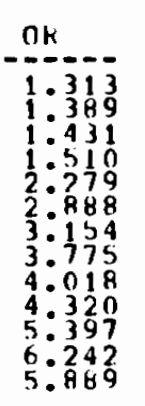 & 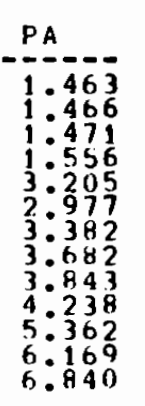 & 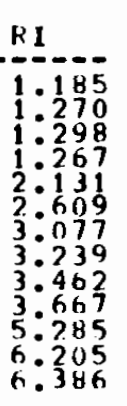 & 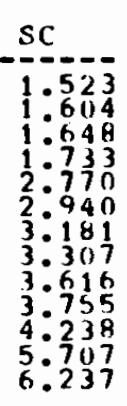 & 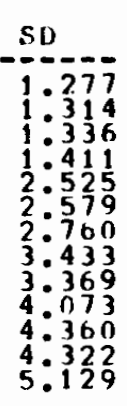 & 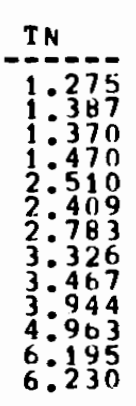 & 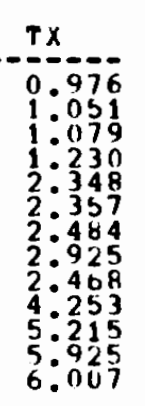 & \begin{tabular}{r}
$U \mathrm{~T}$ \\
\hdashline $1: 351$ \\
$1: 393$ \\
$1: 254$ \\
$1: 395$ \\
$2: 109$ \\
$2: 586$ \\
$2: 913$ \\
$3: 478$ \\
$3: 488$ \\
$4: 386$ \\
$4: 360$ \\
$4: 789$ \\
$5: 901$
\end{tabular} \\
\hline $\begin{array}{l}Y F A R \\
Y 9 A 7 \\
1970 \\
1971 \\
1972 \\
1973 \\
1974 \\
1975 \\
1976 \\
1977 \\
1978 \\
1979 \\
1979 \\
1981 \\
1988\end{array}$ & 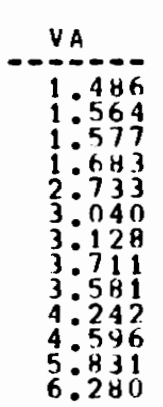 & 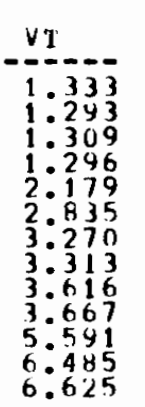 & 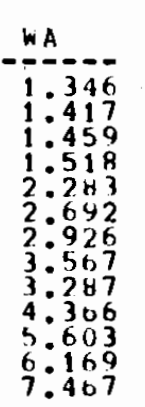 & 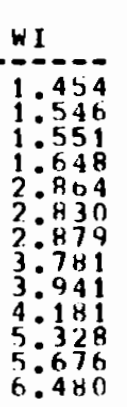 & 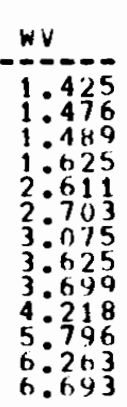 & 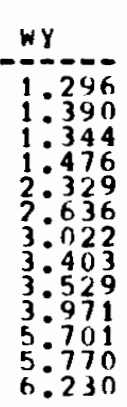 & 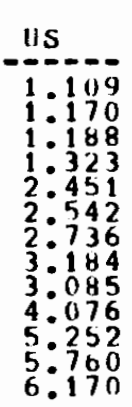 & & \\
\hline
\end{tabular}

Source: Pacific Northwest Laboratory 

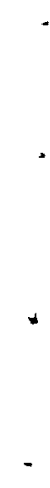


\subsection{STEPS UPDATE DOCUMENTATION}

This chapter documents the changes in sources or estimation procedures where they differ from the procedures described in State Energy Price System, Volume 1 (original documentation), as necessitated by the updates to include price data for 1981 and 1982. Corrections are also included for errors in the original documentation detected in the course of these updates. Any references to page numbers, equation numbers, or tables contained in this chapter refer directly to the original documentation unless otherwise noted. Each of the ten fuels covered by the STEPS data base is discussed separately below.

\subsection{ELECTRICITY}

The basic methodology used to calculate electricity prices for the residential, commercial, industrial, and transportation sectors remains unchanged for the 1981 and 1982 updates. Physical prices are calculated from sales and revenues data taken from the Edison Electric Institute's Statistical Year Book of the Electric Utility Industry, and Btu prices are calculated with a conversion factor of 3,412 Btu per kilowatt-hour. The only imputation done is to assign Maryland prices to the District of Columbia. Note that the original documentation should be amended to reflect the changing table numbers in the Statistical Year Book for both revenues and sales; these table numbers are not always $36 \mathrm{~S}$ and $22 \mathrm{~A}$, respectively, as reported. Table 4.1 shows the actual table numbers used for the 1960 to 1982 period. Note that while the numbers change, the titles, "Revenues: Total Electric Utility Industry" and "Energy Sales: Total Electric Utility Industry," do not.

\subsection{NATURAL GAS}

Natural gas prices for the STEPS series are taken from the EIA's Natural Gas Annual for the residential, commercial, industrial, and transportation sectors, and from the EIA's Cost and Quality of Fuels for Electric Utility Plants, for the electric utility sector. Btu conversion factors for all the sectors except the electric utility sector are also taken from Natural Gas Annual, and 
TABLE 4.1. Revenues and Sales Data Table Numbers in the EEI Statistical Year Book, 1960-1982

\begin{tabular}{|c|c|c|}
\hline Year & $\begin{array}{l}\text { Revenues Data } \\
\text { Table Number }\end{array}$ & $\begin{array}{c}\text { Sales Data } \\
\text { Table Number } \\
\end{array}$ \\
\hline 1960 & 34 & 20 \\
\hline 1961 & 35 & 22 \\
\hline $1962-1970$ & $36 S$ & 22 \\
\hline $1971-1978$ & $36 \mathrm{~s}$ & 225 \\
\hline 1979 & 36 & 22 \\
\hline 1980 & 59 & 41 \\
\hline $1981-1982$ & $59 A$ & $41 \mathrm{~A}$ \\
\hline
\end{tabular}

Source: Pacific Northwest Laboratory

are shown in Table A.2 of Appendix A. The procedures used to calculate natural gas prices for the 1981 and 1982 updates vary only in the way commercial sector physical prices are calculated for 1982. Because value and sales data are not available for the category "other consumers," the commercial sector prices are equal to commercial revenues divided by commercial sales. Equation 10.1 below follows Equation 10 in the original documentation for 1982:

$$
\text { NGCMPPXX }=\text { NCRXX } \div \text { NCCXX }
$$

where:

$$
\begin{aligned}
\text { NGCMPPXX = } & \text { final commercial sector natural gas price for State } X X \\
& \text { or the U.S. }(\$ / 1,000 \text { cubic feet }) \\
\text { NCRXX = } & \text { commercial revenues for State } X X \text { or the U.S. }(\$ 1000) \\
\text { NCCXX = } & \text { commercial volume of natural gas sold in State } X X \text { or } \\
& \text { the U.S. }
\end{aligned}
$$

\subsection{METALLURGICAL COAL}

The procedures for calculating metallurgical coal prices for the industrial sector vary in two minor respects from the original STEPS documentation. First, there is a change in the data source used for prices for the 1981 and 
1982 updates. The data source used to generate the metallurgical coal price series for 1970 to 1980, Coke and Coal Chemicals, ceased publication after the 1980 issue. Prices for 1981 and 1982 were taken from another EIA coal publication, Quarterly Coal Report. Price data given in Coke and Coal Chemicals were collected on Form EIA-5A, "Coke and Coal Chemical Materials, Annual," while the data in Quarterly Coal Report is collected on Form EIA-5, "Coke Plant Report Quarterly." The former publication collected the estimated market value of coals carbonized, while the latter collects the value of coal purchased at coke plants. Data used for the STEPS updates are the year-to-date prices (annual) from the fourth quarters of 1981 and 1982; they are contained in the table entitled "Coal Receipts at Coke Plants." Although data sources change, there is no change in computation or state coverage. The conversion factor of 26.0 million Btu per ton is used for both 1981 and 1982 .

The second modification to the original metallurgical coal documentation is to clarify the state grouping changes from year to year. The price assignments for states with metallurgical coal prices are given in Table 4.2. States whose price assignments changed slightly are: Maryland, Michigan, Minnesota, New Jersey, New York, Wisconsin, Virginia, and West Virginia. No imputations are done for states that do not have prices reported in either Coke and Coal Chemicals (1970-1980) or Quarterly Coal Report (1981-1982).

\subsection{STEAM COAL}

Steam coal prices are computed for the residential, industrial, and electric utility sectors in the STEPS data base. Residential steam coal prices are estimated using regression equations based on Gas Househeating Survey data for 1971-1978. Steam coal industrial sector prices are based on data from form EIA-3, and electric utility sector prices are from Cost and Quality. Conversion factors for all three sectors are given in Table A.1. No procedural changes are needed to do the 1981 or 1982 updates for any of the three sectors, but corrections to the basic documentation and one correction to the data tapes themselves should be made for both the residential and industrial sectors. These corrections and additions are discussed below. 
TABLE 4.2. Metallurgical Coal State Price Assignments, 1970-1982

\begin{tabular}{|c|c|c|}
\hline State & Year & Price Assigned \\
\hline AL & $1970-1982$ & Al abama \\
\hline$C A$ & $1970-1982$ & California, Colorado, Utah \\
\hline $\mathrm{CO}$ & $1970-1982$ & California, Colorado, Utah \\
\hline IL & $1970-1982$ & Illinois \\
\hline IN & $1970-1982$ & Indiana \\
\hline KY & $1970-1982$ & Kentucky, Missouri, Tennessee, Texas \\
\hline MD & $\begin{array}{l}1970-1971 \\
1972-1982\end{array}$ & $\begin{array}{l}\text { Maryl and, New Jersey, New York } \\
\text { Maryl and, Wiscons in }\end{array}$ \\
\hline MI & $\begin{array}{l}1970-1978 \\
1979 \\
1980-1982\end{array}$ & $\begin{array}{l}\text { Michigan } \\
\text { Michigan, Minnesota, Wiscons in } \\
\text { Michigan, Wisconsin }\end{array}$ \\
\hline MN & $1970-1978$ & Minnesota, Wiscons in \\
\hline & 1979 & Michigan, Minnesota, Wisconsin \\
\hline MO & $1970-1982$ & Kentucky, Missouri, Tennessee, Texas \\
\hline NJ & $1970-1971$ & Maryland, New Jersey, New York \\
\hline NY & $\begin{array}{l}1970-1971 \\
1972-1982\end{array}$ & $\begin{array}{l}\text { Maryland, New Jersey, New York } \\
\text { Maryland, New York }\end{array}$ \\
\hline $\mathrm{OH}$ & $1970-1982$ & Oh io \\
\hline PA & $1970-1982$ & Pennsylvania \\
\hline TN & $1970-1982$ & Kentucky, Missouri, Tennessee, Texas \\
\hline TX & $1970-1982$ & Kentucky, Missouri, Tennessee, Texas \\
\hline UT & $1970-1982$ & California, Colorado, Utah \\
\hline VA & $1978-1982$ & Virginia, West Virginia \\
\hline WI & $\begin{array}{l}1970-1978 \\
1979 \\
1980-1982\end{array}$ & $\begin{array}{l}\text { Minnesota, Wiscons in } \\
\text { Michigan, Minnesota, Wiscons in } \\
\text { Michigan, Wiscons in }\end{array}$ \\
\hline WV & $\begin{array}{l}1970-1977 \\
1978-1982\end{array}$ & $\begin{array}{l}\text { West Virginia } \\
\text { Virginia, West Virginia }\end{array}$ \\
\hline US & $1970-1982$ & US \\
\hline
\end{tabular}

Source: Pacific Northwest Laboratory 


\subsubsection{Residential Sector}

There are two basic changes to the residential sector steam coal documentation. First, the values for the variable $c_{x x}$ found in Equation 29 of the original documentation are incorrectly reported in Table 8 and in Table 4.3 of the 1981 update documentation. Table 4.3 below is the correct version of this table. In addition to this correction, the 1981 and 1982 updates require the imputation of values for the variable SPXX of Equation 29 and the variable

TABLE 4.3. Estimated Values of cxX for Residential
Sector Steam Coal Prices (Equation 29)

\begin{tabular}{cc} 
State XX & \multicolumn{1}{c}{$c \times$} \\
AL & Reference State \\
CT & 0.0684 \\
DC & 0.0864 \\
DE & 0.4781 \\
GA & -0.0955 \\
IA & 0.6765 \\
IL & 0.1581 \\
IN & 0.5077 \\
KS & -0.1559 \\
KY & 0.1920 \\
MA & 0.0684 \\
MD & 0.0864 \\
MI & 0.6519 \\
MN & 1.5792 \\
MO & -0.0996 \\
NC & 0.1145 \\
ND & 0.8183 \\
NE & 0.4427 \\
NH & 0.0684 \\
NJ & 0.2563 \\
NY & 0.4761 \\
OH & 0.4530 \\
PA & 0.3589 \\
RI & 0.0684 \\
SC & 0.3516 \\
TN & 0.0239 \\
VA & 0.5047 \\
VT & 0.0684 \\
WI & 1.0446 \\
WV & 1.0446 \\
& \\
\hline
\end{tabular}

Source: Pacific Northwest Laboratory 
ASPXX of Equation 30 for states missing Cost and Quality prices for those two years. To impute these missing price inputs, price assignments are used from nearby states as listed in Table 4.4. For the state of Wyoming in 1982, however, simple use of price assignments from nearby states results in prices that are too high, since Wyoming has unusually low steam coal prices even compared to nearby states. To correct for this, the average price for the states of Colorado, Nebraska, and Utah is calculated both for 1981 and 1982. The ratio of the Wyoming price for 1981 to the 1981 average price for the three nearby states and is then used to estimate the 1982 Wyoming price from the 1982 average price of the three nearby states:

$$
W Y_{1982}=\frac{W Y_{1981}}{A V G_{1981}} \cdot A V G_{1982}
$$

\subsubsection{Industrial Sector}

Corrections for the steam coal industrial sector price series include changes for the calculation of Alaska prices and a clarification of

\section{TABLE 4.4. Price Assignments for States Missing Cost and Quality Prices, 1981 and 1982 (Equations 29 and 30)}

$\begin{array}{lcccc}\text { Variable } & \begin{array}{c}\text { Missing } \\ \text { State }\end{array} \text { SPXX } & \text { ND } & \text { MN } & \text { Price Assignment } \\ \text { SPXX } & \text { CT } & -- & \text { MN } \\ \text { SPXX } & \text { DC } & -- & \text { MA } \\ \text { SPXX } & \text { RI } & -- & \text { MD } \\ \text { ASPXX } & \text { MT } & \text { WY } & \text { MA } \\ \text { ASPXX } & \text { SD } & \text { Average of MN, NE } & \text { CO } \\ \text { ASPXX } & \text { CO } & \text { Average of UT, WY, NE, KS } & \text { Average of MN, NE } \\ \text { ASPXX } & \text { WA } & \text { UT } & -- \\ \text { ASPXX } & \text { ID } & -- & \text { CA } \\ \text { ASPXX } & \text { WY } & -- & \text { Adj. average of CO, NV of CO, NV, UT }\end{array}$


Equation 34. In the original STEPS data base, Btu prices for 1978 to 1980 were erroneously included in the data tapes; the series of prices for Alaska actually ended after 1977. The physical price series for Alaska omitted the 1977 price, ending the series erroneously after 1976. The 1977 physical price has been added to the data base and the 1978 to 1980 Btu prices have been deleted; these changes can be seen in Tables 2.12 and 3.12 of this report. No changes to the original documentation are necessary (see page 77, Step iii(a)).

To clarify Equation 34 on page 76, brackets should be inserted as follows:

$$
\operatorname{SCINPPXX}=\frac{1}{2 N}\left[\sum_{i=1}^{N} \operatorname{PCCR}_{i}+\left(N \cdot \operatorname{PCCR}_{w}\right)\right]
$$

\subsection{DISTILLATE FUEL}

STEPS distillate fuel prices cover the residential, commercial, industrial, and electric utility sectors. No changes in documentation are needed for the electric utility sector update. The industrial sector updates require correction only to update the equations used to estimate prices for 1982 from the Annual Survey of Manufactures. Missing regional prices in both 1981 and 1982 necessitate some procedural changes for the residential sector. The equations to convert physical prices to Btu prices for 1982 are restated so the conversion is made from price per barrel to price per million Btu for all sectors except the electric utility sector. This change allows the use of conversion factors in their published forms. The conversion factors used for the 1981 and 1982 updates and given in Table A.1. Detailed explanation of the corrections for the residential and industrial sectors is presented below.

\subsubsection{Residential Sector}

The distillate residential sector price series is based on state and DOE Region data from Prices and Margins of No. 2 Distillate Fuel $0 i 1$ and Platt's 0il Price Handbook and 0ilmanac. Monthly average heating-degree-day data by state are used in the calculations as weights. Since 1980, D0E Region price 
data have been missing from Prices and Margins for some regions and months. In 1981, DOE Region 9 prices were available for only two months and in 1982 they were missing, along with DOE Region 6 prices, entirely. DOE Regions 7 and 8 were missing prices for some months in 1982 as well.

In the original STEPS documentation, monthly regional prices were weighted into an annual price which was used to adjust state-level prices assigned from Platt's. Procedures to calculate the annual Region 9 price for 1981 are changed as described immediately below. The changes can be inserted into the original documentation on page 81, after Step $i j$ (b), 1978-1980:

1981 1. Calculate the relationship between the U.S. annual heating oil price and the U.S. March and May heating prices:

$$
\mathrm{RDOE}_{9, \mathrm{~m}}=\mathrm{PDOE}_{U S} \div P D O E_{U S, m}
$$

where:

$$
\begin{aligned}
& \begin{aligned}
\text { RDOE }_{9, m}= & \text { ratio of the annual average U.S. price to the } \\
& \text { U.S. price in month } \mathrm{m}
\end{aligned} \\
& \text { POOE } E_{\text {US }}=\text { annual average distillate U.S. price for } 1981 \\
& \text { (\$/gallon) }
\end{aligned}
$$

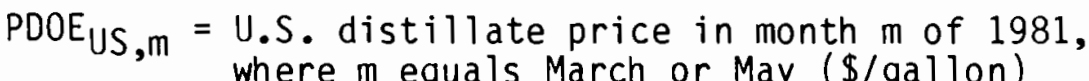

2. Impute the annual Region 9 price:

$$
\mathrm{PDOE}_{9}=\sum_{m=1}^{2}\left[\left(\mathrm{RDOE}_{\mathrm{g}, \mathrm{m}} \cdot \mathrm{PDOE}_{9, \mathrm{~m}}\right) \div 2\right]
$$

where:

$$
\begin{aligned}
\text { PDOE }_{9}= & \begin{array}{r}
\text { annual Region } 9 \text { distillate price for } 1981 \\
(\$ / \text { gallon })
\end{array} \\
\text { PDOE }_{9, m}= & \text { Region } 9 \text { price for month } m \text { of } 1981 \text { ( } \$ / \text { gallon) }
\end{aligned}
$$

3. Complete imputation, as contained in STEPS documentation, with Step ii (c). 
The missing monthly prices for DOE Regions 7 and 8 in 1982 are imputed by averaging the prices in adjacent months and assigning them to the missing months. For example, if the July price is missing, the average of the June and August price is assigned to July. Procedures are then completed as documented, starting with Step $i j$ on page 80 . Since there are no monthly prices for DOE Regions 9 and 6 in 1982, the adjustment factors ( $A_{j}$ in Equation 40) are based on the adjustment factors for previous time periods. The DOE Region 6 adjustment factor is equal to the adjustment factor of the West South Central Census Region for the 1975-1977 period, while the DOE Region 9 adjustment factor is equal to the average adjustment factor for DOE Region 9 from 1978 to 1981.

The equation used to convert prices per gallon into prices per million Btu for the residential sector is given in the original documentation as Equation 49. This equation was used in an altered form in order to use the conversion factor as published in EIA's Annual Energy Review (million Btu per barrel). In the original documentation this conversion factor was translated into million Btu per gallon before it was used to calculate Btu prices from physical prices. The revised equation used in the 1982 update is as follows:

$$
\text { DFRSBPXX }=(\text { DFRSPPXX } \cdot 42) \div 5.825
$$

\subsubsection{Commercial Sector}

Commercial sector distillate prices are based on commercial sector markup data from Foster's and Associates and the STEPS residential distillate price series. The only change made in computational procedures for this sector was to restate Equation 58 of the original documentation similar to Equation 49.1 in the residential sector. The corrected equation was used for the 1982 update and allows for calculation of Btu prices using the published per barrel conversion factor:

$$
\text { DFCMBPXX }=(\text { DFCMPPXX } \cdot 42) \div 5.825
$$




\subsubsection{Industrial Sector}

The distillate industrial sector price series is based on data from the Annual Survey of Manufactures (ASM) or the Census of Manufactures (CM). When the original STEPS data base was completed with prices through 1980, the ASM prices for 1980 were not published. Consequently, prices for 1980 had to be estimated from ASM prices for 1974 to 1979. The lag in availability of ASM and CM data has continued through the 1982 update, which results in a two-part procedure to update industrial sector distillate prices. Estimated data for 1980 and 1981 are replaced with actual ASM data as they become available. Secondly, prices are estimated for years when data are not yet available. States missing actual ASM prices when they are published are assigned the estimated prices.

Actual ASM/CM data are converted into prices using Equation 59. This equation is incorrectly marked in the original documentation as applying only to 1971 data; actually, this equation also applies to ASM/CM data for 1974 to 1981. For the 1981 update, 1981 prices are imputed using an equation similar to Equation 62 on page 93. For the 1982 update (estimating 1982 prices and inserting actual 1981 ASM prices) the equation remains the same except the fraction in front of the summation sign becomes " $1 / 8$ " and the " 80 " above the summation sign becomes " $81 . "$

$$
\text { RAVGXX }=\frac{1}{7} \sum_{t=74}^{80}\left(\text { DFINPPXX }_{t} \div \text { DFPLXX }_{t}\right)
$$

where:

$$
\begin{aligned}
\text { RAVGXX }= & \begin{array}{l}
\text { average ratio of STEPS industrial sector distillate } \\
\text { prices to Platt's prices for State } X X
\end{array} \\
\text { DFINPPXX } & =\begin{array}{l}
\text { STEPS industrial sector distillate price for State } X X \\
\text { in year } t \text { (\$/gallon) }
\end{array} \\
\text { DFPLXX } & =\frac{P \text { latt's distillate price assigned to State } X X \text { in year }}{t(\$ / \text { gallon) }}
\end{aligned}
$$


Calculation of prices continues as recorded in the original documentation with Equation 63.

As with the residential and commercial sectors, the conversion equation used to calculate Btu prices from the physical price series is altered for the 1982 update to allow use of the published per barrel conversion factor. The altered equation corresponds to Equation 65 in the original documentation:

$$
\text { DF INBPXX }=(\text { DF INPPXX } \cdot 42) \div 5.825
$$

\subsection{MOTOR GASOLINE}

The motor gasoline prices in the STEPS series are based on prices for leaded and unleaded gasoline taken from the Consumer Price Index and Platt's. The leaded and unleaded gasoline prices are weighed into the final STEPS prices using leaded and unleaded consumption volume weights. For 1970 to 1981 these consumption weights are taken from the EIA-79 data tapes. For the 1982 prices, these weights are taken from a new source, Form EIA-25, "Prime Supplier Monthly Report." This change affects Equation 75 on page 103, by redefining the variable $\operatorname{COXX} x_{c, m}$ as the EIA-25 product volume weight for produce $c$, in month $m$.

The gasoline conversion equation is restated for the 1982 update similar to changes for the distillate series. Equation 84 from the original documentation is altered as follows:

$$
\text { MGTRBPXX }=(\text { MGTRPPXX } \cdot 42) \div 5.253
$$

\subsection{DIESEL FUEL}

The STEPS diesel price series cover the transportation sector only and are based on physical unit diesel prices from the Department of Agriculture's Agricultural Prices. The 1981 and 1982 updates follow the computational procedures as presented in the original STEPS documentation, except for a change in the conversion equation (Equation 86) for the 1982 update. The 
change in Equation 86 allows the direct use of the per barrel conversion factor published in the Annual Energy Review. The revised equation is as follows:

$$
\text { DLTRBPXX }=(\text { DLTRPPXX } \cdot 42) \div 5.825
$$

\subsection{KEROSENE AND JET FUEL}

The kerosene and jet fuel data base covers the residential, industrial, transportation, and electric utility sectors. For the industrial and electric utility sectors, the computation procedures for the 1981 and 1982 updates are as reported in the original STEPS documentation, except that the 1982 conversion equations have been altered similar to the equations for distillate, motor gasoline, and diesel. In addition to the change in the conversion equation, the transportation and residential sectors require other corrections to the original documentation. These changes are explained by sector below.

\subsubsection{Residential Sector}

In the process of completing the 1982 update, errors were noted in the residential sector kerosene series that required recalculation of both physical and Btu prices for all years in the series (1970-1982). The data sources reported in the original documentation are correct, with the 1981 and 1982 updates using Producer Prices and Price Indexes as given. The section on computational procedures found on pages 110 to 114 of the original documentation should be omitted, and the following section should be used for documentation in its place.

\section{Computational Procedures.}

1970-1976 i. In order to volume weight the Agricultural Prices quarterly prices, monthly quantity weights are calculated by assuming the "other distillate oil" consumption data by PAD Districts from Retail Sales is kerosene, and averaging the data, which covers months from November 1978 to March 1981: 


$$
\mathrm{KSRQ}_{k, m}=\frac{\sum_{t=78}^{81} \mathrm{RSQ}_{k, t, m}}{N} \cdot 42
$$

where:

$$
\begin{aligned}
\mathrm{KSRQ}_{k, m}= & \text { average consumption of kerosene in PAD } \\
& \text { District } k \text { in month } \mathrm{m}(1,000 \text { gallons }) \\
\mathrm{RSQ}_{k, \mathrm{t}, \mathrm{m}=} & \text { sales of other distillate oil in } \mathrm{PAD} \\
& \text { District } k, \text { in month } \mathrm{m} \text { of year } \mathrm{t}(1,000 \\
& \text { barrels }) \\
\mathrm{N}= & \text { number of years of non zero data for month } \mathrm{m}
\end{aligned}
$$

ii. Calculate each month's average share of annual sales (expressed as a fraction) for PAD Districts, then assign the PAD District shares to states within each respective district:

$$
\operatorname{KSAVXX}_{k, m}=\frac{\mathrm{KSRQ}_{k, m}}{\sum_{m=1}^{12} \mathrm{KSRQ}_{k, m}}
$$

where:

$$
\begin{aligned}
\operatorname{KSAVXX}_{k, m}= & \text { average fraction of kerosene consumption in } \\
& \text { State } X X \text { of PAD District } k \text {, in month } m
\end{aligned}
$$

iii. Annual physical prices are calculated as the volume-weighted average of Agricultural Prices quarterly data. The monthly weights developed in Equation 88 are converted to quarterly weights by summing the fractions for months within a particular quarter.

$$
\operatorname{KSRSPPXX}=\sum_{q=1}^{4}\left[\operatorname{KSAPXX} q \cdot \sum_{m=1}^{3} \operatorname{KSAVXX}_{q, m}\right]
$$

where:

$$
\begin{aligned}
\text { KSRSPPXX }= & \text { final residential sector kerosene price for } \\
& \text { State } X X(\$ / \text { gallon })
\end{aligned}
$$




$$
\begin{aligned}
& \begin{aligned}
\operatorname{KSAPXX}_{\mathrm{q}}= & \begin{array}{l}
\text { kerosene price from Agricultural Prices for } \\
\text { State } X X \text { (\$/gallon })
\end{array}
\end{aligned} \\
& \begin{aligned}
& \mathrm{KSAVXX}_{\mathrm{q}, \mathrm{m}}= \text { average fraction of kerosene consumption in } \\
& \text { State } X X \text { in month } m \text { of quarter } q(a)
\end{aligned}
\end{aligned}
$$

iv. The missing 1972 quarterly prices for Nevada (KSAPNV) are imputed by calculating the average ratio between the quarterly Nevada prices and the quarterly Agricultural Prices U.S. prices for 1971 and 1973, and applying the derived ratio to the Agricultural Prices U.S. price for 1972. These quarterly Nevada prices are then inserted into Equation 89 for the variable $\operatorname{KSAPXX}_{\mathrm{q}}$.

$$
\operatorname{KSAPNV}_{72, q}=\operatorname{KSAPUS}_{72, q} \cdot\left[\frac{1}{8} \sum_{q=1}^{8} \operatorname{KSAPNV}_{t, q} \text { KSAPUS }_{t, q}\right]
$$

where:

$$
\begin{aligned}
\text { KSAPNV }_{72, q}= & \begin{array}{l}
\text { estimated kerosene price for Nevada in } \\
\text { quarter q of } 1972(\$ / \text { gallon })
\end{array} \\
\text { KSAPUS } 72, q= & \begin{array}{l}
\text { U.S. kerosene price for quarter } q \text { of } 1972 \\
(\$ / \text { gallon })
\end{array} \\
\text { KSAPNV }_{t, q}= & \begin{array}{l}
\text { kerosene price for Nevada for quarter } q \text { of } \\
\text { year } t(\$ / \text { gallon) }
\end{array} \\
\text { KSAPUS }_{t, q}= & \begin{array}{l}
\text { U.S. kerosene price for quarter } q \text { of year } t \\
(\$ / \text { gallon) }
\end{array} \\
t= & 1971 \text { or } 1973
\end{aligned}
$$

v. Calculate U.S. physical prices using the state physical prices and the SEDS residential kerosene consumption volumes:

(a) $\mathrm{KSAVXX}_{\mathrm{g}, \mathrm{m}}$ is the same as $\mathrm{KSAVXX}_{k}, \mathrm{~m}$; the difference in notation is to preservéclarity in Equations 89 and 88 , respectively. 


$$
\text { KSRSPPUS }=\frac{\sum_{X X=1}^{51}(\text { KSRSPPXX } \cdot K S R V X X)}{\sum_{x X=1}^{51} K S R V X X}
$$

where:

$$
\begin{aligned}
\text { KSRSPPUS }= & \text { final residential sector kerosene price for } \\
& \text { the U.S. (\$/gallon) } \\
\text { KSRVXX = } & \text { residential kerosene consumption volume from } \\
& \text { SEDS for State XX (1,000 barrels) }
\end{aligned}
$$

vi. Calculate Btu prices for states and the U.S. using the completed physical unit price series and the conversion factor:

$$
\text { KSRSPBXX }=\text { KSRSPPXX } \div 0.135
$$

where:

$$
\begin{aligned}
\text { KSRSBPXX }= & \text { final residential kerosene price for State } X X \\
& \text { or the U.S. }(\$ / \text { million Btu })
\end{aligned}
$$

$1977-1982$

Because Agricultural Prices price data are not available after 1976, monthly Census Region Producer Prices and Price Indexes (PPI) prices and price indexes are used as the bas is for the price series from 1977 to 1982. To maintain some consistency in the series, the PPI prices are multiplied by an adjustment factor that accounts for the relationship between PPI and Agricultural Prices prices for quarters where the two series overlap. In the following description of the procedures, the adjustment factor is derived first. The PPI prices for 19771982 are then estimated, and lastly, the final kerosene statelevel physical unit and Btu prices for state and the U.S. are calculated.

i. Calculation of the adjustment factor. PPI and Agricultural Prices kerosene prices overlap from the third quarter of 1975 through the fourth quarter of 1976 (or 1975.3 to 1976.4). The 
Agricultural Prices quarterly prices for these quarters are taken directly from the source documents, but quarterly PPI prices need to be calculated.

a. First, monthly PPI prices are calculated from the July 1975 base prices for Census Regions and price indexes:

$$
\operatorname{KSPPI}_{j, q, m}=\left(\operatorname{KSIDX}_{j, m} \div 100\right) \cdot \mathrm{KSBASE}_{j}
$$

where:

$$
\begin{aligned}
\text { KSPPI }_{j, q, m}= & \frac{P P I}{i n m o n t h ~} m \text { of quarter } q(\$ / g a l l o n) \\
\text { KSIDX }_{j, m}= & \begin{array}{l}
\text { kerosene price index for Census } \\
\text { Region } j \text { in month } m
\end{array} \\
\text { KSBASE }_{j, m}= & \begin{array}{l}
\text { kerosene base price for Census } \\
\text { Region } j \text { in July } 1975 \text { ( } \$ / \text { gallon) }
\end{array} \\
m= & \begin{array}{l}
\text { months from July } 1975 \text { through December } \\
1976
\end{array}
\end{aligned}
$$

b. Assign the Census Region PPI prices equally and identically to each state within the respective Census Region:

$$
\operatorname{KSPPIXX}_{q, m}=\operatorname{KSPPI}_{j, q, m}
$$

c. Calculate volume-weighted quarterly PPI prices for states using the monthly volume weights developed from Retail sales and Inventories of Fuel 0 il sales data for "other distillate oil" (Equation 88):

$$
\operatorname{KSPPIXx_{q}}=\frac{\sum_{m=1}^{3}\left(K S P P I X x_{q, m} \cdot K S A V X x_{q, m}\right)}{\sum_{m=1}^{3} K S A V X x_{q, m}}
$$


where:

$$
\text { KSPPIXX } X_{q}=\frac{P P I}{\text { quarter } q}
$$

d. Calculate the adjustment factor, which relates the quarterly Agricultural Prices prices $\left(K_{S A P X X_{q}}\right)$ to the PPI prices $\left(\right.$ KSPPIXX $\left.X_{q}\right)$ :

$$
\operatorname{KSADJXX}=\frac{1}{6} \sum_{q=1}^{6}\left(\operatorname{KSAPXX} X_{q} \div \operatorname{KSPPIXX} x_{q}\right)
$$

ii. Calculation of monthly PPI prices, 1977-1982.

a. Monthly regional PPI prices are calculated using the July 1975 base prices for Census Regions and monthly price indexes for 1977-1982:

$$
\mathrm{KSPPI}_{j, m}=\left(\mathrm{KSIDX}_{j, m} \div 100\right) \cdot \mathrm{KSBASE}_{j}
$$

where:

$$
\begin{aligned}
\text { KSPPI }_{j, m}= & \frac{\text { PPI kerosene price for Census Region } j \text { in }}{\text { month } m(\$ / g a l l o n)} \\
\text { KSIDX }_{j, m}= & \begin{array}{l}
\text { kerosene price index for Census Region } j \\
\text { in month } m
\end{array} \\
\text { KSBASE }_{j}= & \begin{array}{l}
\text { kerosene base price for Census Region } j \\
\text { in July } 1975 \text { (\$/gallon) }
\end{array} \\
m= & \text { all months in the years } 1977-1982
\end{aligned}
$$

b. Assign the Census Region monthly prices equally and identically to each state within the respective region:

$$
\operatorname{KSPPIXX}_{m}=\operatorname{KSPPI}_{j, m}
$$

where:

$$
\text { KSPPIXX } X_{m}=\underset{\text { assigned }}{\text { in month }} \frac{\text { PP }}{m(\$ / \text { gallon })} \text { price for State } X X
$$


iii. Calculation of annual physical and Btu prices, 1977-1982.

a. Calculate annual physical prices from the monthly PPI statelevel prices, using the volume weights used in Equation 89:

$$
\text { KSPPIXX }=\frac{\sum_{m=1}^{12}\left(K S P P I X X_{m} \cdot K S A V X x_{m}\right)}{\sum_{m=1}^{12} K S A V V X_{m}}
$$

where:

$$
\begin{aligned}
\text { KSPPIXX }= & \underset{\text { annual kerosene price for State } X X}{(\$ / g a l l o n)} \\
\text { KSAVXX }_{m}= & \begin{array}{l}
\text { average fraction of kerosene consumption } \\
\text { in State } X X \text { in month } m(a)
\end{array}
\end{aligned}
$$

b. Calculate physical prices for states using the adjustment factor (KSADJXX) and the annual PPI prices:

$$
\text { KSRSPPXX }=K S A D J X X \cdot K S P P I X X
$$

c. Calculate physical prices for the U.S. using the state physical prices and the SEDS residential consumption volumes (by computer):

$$
\text { KSRSPPUS }=\frac{\sum_{X X=1}^{51}(K S R S P P X X X \cdot K S R V X X)}{\sum_{X X=1}^{51} \operatorname{KSRVXX}}
$$

(a) KSAVXX and KSAVXX, $m$ are equal; the different notation is for clarity with in individual equations. 
d. Calculate Btu prices for states and the U.S. using the completed physical price series and the conversion factors: $\underline{1977-1981 .}$

$$
\text { KSRSBPXX }=\text { KSRSPPXX } \div 0.135
$$

$\underline{1982 .}$

$$
\text { KSRSBPXX }=(\text { KSRSPPXX } \cdot 42) \div 5.670
$$

where:

$$
\begin{aligned}
\text { KSRSBPXX }= & \text { final residential sector kerosene price } \\
& \text { for State } X X \text { or the U.S. }(\$ / \text { million Btu })
\end{aligned}
$$

\subsubsection{Industrial Sector}

The only change to the industrial sector documentation is the alteration to the conversion equation (Equation 105) for the 1982 update. The revised equation below uses the published conversion factor of 5.670 million Btu per barrel:

$$
\text { KSINBPXX }=(\text { KSINPPXX } \cdot 42) \div 5.670
$$

\subsubsection{Transportation Sector}

The transportation sector for the kerosene/jet fuel price series contains prices based on jet fuel prices from Producer Prices and Price Indexes. In addition to a change in the conversion equation (Equation 112) similar to that just done for the industrial sector above, a step was omitted from the original STEPS documentation. The prices contained in the STEPS data base, however, were correctly calculated, so this correction is only needed in the documentation itself. On page 118, after Step $i$ for 1976-1980 (now 1976-1982), the following Step iii should be inserted:

iii. Calculate physical U.S. prices using the state prices and jet fuel consumption volume weights from SEDS: 


$$
\text { JFTRPPUS }=\frac{\sum_{X X=1}^{51} \operatorname{(JFTRPPXX} \cdot \text { JFTVXX) }}{\sum_{x X=1}^{51} J F T V X X}
$$

where:

$$
\begin{aligned}
\text { JFTRPPUS = } & \text { final transportation sector jet fuel price } \\
& \text { for the U.S. (\$/gallon) } \\
\text { JFTVXX = } & \text { transportation sector jet fue } 1 \text { consumption } \\
& \text { volume from SEDS for state XX (1,000 barrels) }
\end{aligned}
$$

The jet fuel transportation sector conversion equation should be altered as follows in order to use the per barrel conversion factor published in Annual Energy Review:

$$
\text { JFTRBPXX }=(\text { JFTRPPXX }-42) \div 5.670
$$

\subsubsection{Electric Utility Sector}

The only correction to this sector in the STEPS data base is to alter the conversion equation for the 1982 update. This change is analogous to changes in the conversion factors for the other sectors in the kerosene/jet fuel series.

$$
\text { KSUTBPXX }=(\text { KSUTPPXX } \cdot 42) \div 5.670
$$

\subsection{RESIDUAL FUEL}

The STEPS residual fuel price series cover the commercial, industrial, transportation and electric utility sectors. No changes to the original documentation are needed for the commercial or electric utility sectors as a result of the 1981 or 1982 updates. The commercial sector prices are based on the industrial residual STEPS series, while the electric utility sector prices are based on data from Cost and Quality. For the industrial sector, the equations used to estimate prices based on available ASM data are updated below. No actual changes in procedure are needed for the transportation sector, but 
states were reassigned to the three groupings in some cases. No changes to conversion equations are needed for the residual fuel series since per barrel conversion factors have always been used.

\subsubsection{Industrial Sector}

The residual fuel industrial sector price series is based on data from the Annual Survey of Manufactures or the Census of Manufactures. When the original STEPS data base was completed with prices through 1980, the ASM prices for 1980 were not yet published. Consequently, prices for 1980 had to be estimated from ASM prices for 1974 to 1979. The lag in availability of ASM and CM data has continued through the 1982 update, which results in a two-part procedure to update industrial sector residual fuel prices. Estimated data for 1980 (1981 for the 1982 update) are replaced with actual ASM data as they become available. Second, prices are estimated for years when data are not yet available. States missing actual ASM prices when they are published are assigned the estimated prices.

Actual ASM/CM data are converted into prices using Equation 122. For the 1981 update, 1981 prices were imputed using an equation similar to Equation 124 on page 128. For the 1982 update (estimating 1982 prices and inserting actual 1981 ASM prices) the equation remains the same except the fraction in front of the summation sign becomes " $1 / 8$ " and the " 80 " above the summation sign becomes "81."

$$
\text { RAVGXX }=\frac{1}{7} \sum_{t=74}^{80}\left(\operatorname{RFINPPXX}_{t} \div \operatorname{RFPLXX}_{t}\right)
$$

where:

$$
\begin{aligned}
& \text { RAVGXX = average ratio of STEPS industrial sector residual } \\
& \text { prices to Platt's prices for State } X X \\
& \text { RFINPPXX } X_{t}=\text { final industrial sector residual price (STEPS) for } \\
& \text { State } X X \text { in year } t \text { (\$/gallon) } \\
& \text { RFPLXX } X_{t}=\frac{\text { Platt's }}{\text { (\$/gallon) }} \text { residual price assigned to State } X X \text { in year } t
\end{aligned}
$$

Calculation of prices continues as recorded in the original documentation with Equation 125. 


\subsubsection{Transportation Sector}

To calculate residual fuel transportation sector prices, states are segregated into groups depending on the major transportation use of residual fuel in each state. Group A states use all or most of their residual fuel for vessel bunkering purposes. Group B states use all or most of their residual fuel for railroads, and Group $C$ states use residual fuel for both railroads and vessel bunkering. A fourth group includes states with no reported residual use in the transportation sector. The 1982 update used a revised list of group assignments in comparison to the original assignments found on page 136. Table 4.5 gives the new listing.

TABLE 4.5. Residual Fuel Transportation Use List for 1982 Update

\begin{tabular}{|c|c|c|c|}
\hline Group A & Group B & Group C & No Use \\
\hline $\begin{array}{l}\text { Alabama } \\
\text { California } \\
\text { Connecticut } \\
\text { Delaware } \\
\text { Florida } \\
\text { Georgia } \\
\text { Hawaii. } \\
\text { Indiana } \\
\text { Louisiana } \\
\text { Massachusetts } \\
\text { Maryland } \\
\text { Maine } \\
\text { Missouri } \\
\text { North Carolina } \\
\text { New Hampshire } \\
\text { New Jersey } \\
\text { New York } \\
\text { Ohio } \\
\text { Oregon } \\
\text { Pennsylvania } \\
\text { Rhode Island } \\
\text { South Carolina } \\
\text { Texas } \\
\text { Virginia } \\
\text { Washington }\end{array}$ & $\begin{array}{l}\text { Tennessee } \\
\text { Wyoming }\end{array}$ & $\begin{array}{l}\text { Illinois } \\
\text { Michigan } \\
\text { Minnesota } \\
\text { Wisconsin }\end{array}$ & $\begin{array}{l}\text { Alaska } \\
\text { Arkansas } \\
\text { Arizona } \\
\text { Colorado } \\
\text { District of Columbia } \\
\text { Iowa } \\
\text { Idaho } \\
\text { Kansas } \\
\text { Kentucky } \\
\text { Montana } \\
\text { North Dakota } \\
\text { Nebraska } \\
\text { New Mexico } \\
\text { Nevada } \\
\text { Oklahoma } \\
\text { South Dakota } \\
\text { Utah } \\
\text { Vermont } \\
\text { West Virginia }\end{array}$ \\
\hline
\end{tabular}

Source: Pacific Northwest Laboratory 


\subsection{LIQUEF IED PETROLEUM GAS}

The liquefied petroleum gas (LPG) series for the STEPS data base covers the residential and industrial sectors. The conversion equations for both sectors are changed similarly to other petroleum products for the 1982 update. In addition, the equation used for the industrial sector updates for 1981 and 1982 is updated.

\subsubsection{Residential Sector}

The residential sector LPG series involved no changes to the original documentation except for the change to the conversion equation (Equation 148) to allow for the calculation of 1982 physical prices using the conversion factor published in Annual Energy Review. The revised equation is as follows:

$$
\text { LGRSPPXX }=(\operatorname{LGRSBPXX} \cdot 3.643) \div 42
$$

\subsubsection{Industrial Sector}

The LPG industrial sector price series is based on data from the Annual Survey of Manufacturers or the Census of Manufacturers. When the original STEPS data base was completed with prices through 1980, the ASM prices for 1980 were not yet published. Consequently, prices for 1980 had to be estimated from ASM prices for 1978 to 1979. The lag in availability of $\underline{\text { ASM }}$ and $\underline{\text { CM data has }}$ continued through the 1982 update, which results in a two-part procedure to update industrial sector LPG prices. Estimated data for 1980 (1981 for the 1982 update) are replaced with actual ASM data as they become available. Second, prices are estimated for years when data are not yet available. States missing actual ASM prices when they are published are assigned the estimated prices.

Actual $\mathrm{ASM} / \mathrm{CM}$ data are converted into prices using Equation 149. For the 1981 update, the prices were imputed using an equation similar to Equation 150 on page 143. For the 1982 update inserting actual 1981 ASM prices and estimating 1982 prices the equation remains the smae except the fraction in front of the summation sign becomes " $1 / 4$ " and the " 80 " above the summation sign becomes " $81 . "$ 


$$
\text { RAVGXX }=\frac{1}{3} \sum_{t=78}^{80}\left(\operatorname{LGINPPXX}_{t} \div \operatorname{LGAPXX}_{t}\right)
$$

where:

$$
\begin{aligned}
& \text { RAVGXX = average ratio of STEPS industrial sector LPG prices to } \\
& \text { LGINPPXX } X_{t}=\text { final industrial sector LPG price (STEPS) for State } X X \\
& \text { in year } t \text { (\$/gallon) }
\end{aligned}
$$

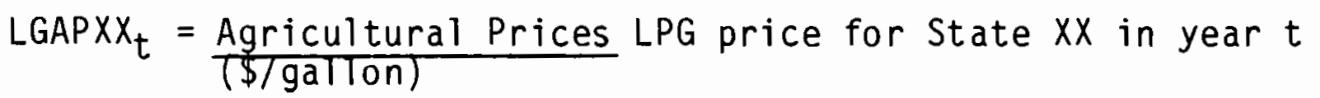

Calculation of prices continues with Equation 152.

The conversion equation (Equation 153) for the LPG industrial sector is altered as follows for the 1982 update:

$$
\text { LGINBPXX }=(\operatorname{LGINPPXX} \cdot 42) \div 3.643
$$




\section{REFERENCES}

American Gas Association. 1983. Gas Househeating Survey: 1982. Arlington, Virginia.

American Gas Association. 1982. Gas Househeating Survey: 1981. Arlington, Virginia.

Bureau of Labor Statistics, U.S. Department of Labor. 1983. Consumer Prices: Energy. Washington, D.C.

Bureau of Labor Statistics, U.S. Department of Labor. 1982. Consumer Prices: Energy. Washington, D.C.

Bureau of Labor Statistics, U.S. Department of Labor. 1983. Producer Prices and Price Indexes. Washington, D.C.

Bureau of Labor Statistics, U.S. Department of Labor. 1982. Producer Prices and Price Indexes. Washington, D.C.

Bureau of the Census, U.S. Department of Commerce. 1982. 1980 Annual Survey of Manufactures: Fuels and Electric Energy Consumed. Washington, D.C.

Bureau of the Census, U.S. Department of Commerce. 1983. 1982 Census of Manufactures: Fuels and Electric Energy Consumed. MC82-5-4 (Part 2), Washington, D.C.

Edison Electric Institute. 1983. Statistical Year Book of the Electric Utility Industry. Washington, D. C.

Edison Electric Institute. 1982. Statistical Year Book of the Electric Utility Industry. Washington, D.C.

Energy Information Administration, U.S. Department of Energy. 1983. Cost and Quality of Fuels for Electric Utility Plants. DOE/EIA-0191(82), Washington, D.C.

Energy Information Administration, U.S. Department of Energy. 1982. Cost and Quality of Fuels for Electric Utility Plants. DOE/EIA-0191(81), Washington, D.C.

Energy Information Administration, U.S. Department of Energy. 1982. Monthly Energy Review. DOE/EIA-0035, Washington, D.C.

Energy Information Administration, U.S. Department of Energy. 1983. Monthly Energy Review. D0E/EIA-00335, Washington, D.C.

Energy Information Administration, U.S. Department of Energy. 1982. Monthly Petroleum Product Price Report. DOE/EIA-0032(82/12), Washington, D.C. 
Energy Information Administration, U.S. Department of Energy. 1981. Monthly Petroleum Product Price Report. D0E.EIA-0032(81/12), Washington, $D . \bar{C}$.

Energy Information Administration, U.S. Department of Energy. 1983. Natural Gas Annua 1, 1982. DOE/E IA-0131(82), Washington, D.C.

Energy Information Administration, U.S. Department of Energy.. 1982. Natural Gas Annual, 1981. D0E/EIA-0131(81), Washington, D.C.

Energy Information Administration, U.S. Department of Energy. 1982. Prices and Margins of No. 2 Distillate Fuel 0il. DOE/E IA-0013, Washington, D.C.

Energy Information Administration, U.S. Department of Energy. 1981. Prices and Margins of No. 2 Distillate Fuel 0il. DOE/EIA-0013, Washington, D.C.

Energy Information Administration, U.S. Department of Energy. 1983. "Prime Supplier Monthly Report." EIA Form 25, Washington, D.C.

Energy Information Administration, U.S. Department of Energy. 1983. Quarterly Coal Report. DOE/EIA-0121(82/4Q), Washington, D.C.

Energy Information Administration, U.S. Department of Energy. 1982. Quarterly Coal Report. DOE/EIA-0121(81/4Q), Washington, D.C.

Fang, J. M., K. L. Imhoff and L. J. Hood. 1983. State Energy Price System: 1981 Update. PNL-4802, Pacific Northwest Laboratory, Richland, Washington.

McGraw-Hil1, Inc. 1983. Platt's 0il Price Handbook and 0ilmanac. 59th edition, New York, New York.

McGraw-Hill, Inc. 1982. Platt's 0il Price Handbook and 0ilmanac. 58th edition, New York, New York.

Pacific Northwest Laboratory. 1982. State Energy Price System. DOE/NBB-0029, Volumes 1 and 2, published by Energy Information Administration, Washington, D.C.

U.S. Department of Agriculture, Crop Reporting Board. 1983. Agricultural Prices. PR 1-3(83), Washington, D.C.

U.S. Department of Agriculture, Crop Reporting Board. 1982. Agricultural Prices. $\operatorname{Pr~1-3(82),~Washington,~D.C.~}$

U.S. Department of Transportation. 1983. Highway Statistics 1982. Washington, D.C.

U.S. Department of Transportation. 1982. Highway Statistics 1981. Washington, D.C. 
APPENDIX A

CONVERSION FACTORS 


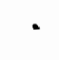

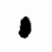

. 
TABLE A.1. Btu Conversion Factors for 1981 and 1982

\begin{tabular}{|c|c|c|c|}
\hline \multirow[b]{2}{*}{ Fuel } & \multirow[b]{2}{*}{ Sector } & \multicolumn{2}{|c|}{ Conversion Factor } \\
\hline & & 1981 & 1982 \\
\hline 1. Electricity & Al1 1 & $3412 \mathrm{Btu} / \mathrm{kWh}$ & $3412 \mathrm{Btu} / \mathrm{kWh}$ \\
\hline 2. Natural Gas & $\begin{array}{l}\text { Residential } \\
\text { Commercial } \\
\text { Industrial } \\
\text { Transportation } \\
\text { Electric Utility }\end{array}$ & $\begin{array}{l}\text { See Table A.2 } \\
\text { See Table A.2 } \\
\text { See Table A.2 } \\
\text { See Table A.2 } \\
\text { Embedded in data(a) }\end{array}$ & $\begin{array}{l}\text { See Table A.2 } \\
\text { See Table A.2 } \\
\text { See Table A.2 } \\
\text { See Table A.2 } \\
\text { Embedded in data (a) }\end{array}$ \\
\hline $\begin{array}{l}\text { 3. Metallurgical } \\
\text { Coal }\end{array}$ & Industrial & $26.0 \mathrm{Million} \mathrm{Btu} /$ ton & 26.0 Million Btu/ton \\
\hline 4. Steam Coal & $\begin{array}{l}\text { Residential } \\
\text { Industrial } \\
\text { Electric Utility }\end{array}$ & $\begin{array}{l}25.97 \mathrm{MMBtu} / \text { ton } \\
25.97 \mathrm{MMBtu} / \mathrm{ton} \\
\text { Embedded in data }(\mathrm{a})\end{array}$ & $\begin{array}{l}24.96 \mathrm{MMBtu} / \mathrm{ton} \\
24.96 \mathrm{MMBtu} / \mathrm{ton} \\
\text { Embedded in data }(\mathrm{a})\end{array}$ \\
\hline 5. Distillate Fuel & $\begin{array}{l}\text { Residential } \\
\text { Commercial } \\
\text { Industrial } \\
\text { Electric Utility }\end{array}$ & $\begin{array}{l}0.13869 \mathrm{MMBtu} / \mathrm{gallion} \\
0.13869 \mathrm{MMBtu} / \mathrm{gall} \text { ion } \\
0.13869 \mathrm{MMBtu} / \mathrm{gal} \text { lon } \\
\text { Embedded in datala }\end{array}$ & $\begin{array}{r}5.825 \mathrm{MMBtu} / \text { barrel } \\
5.825 \mathrm{MMBtu} / \text { barrel } \\
5.825 \mathrm{MMBtu} / \text { barre] } \\
\text { Embedded in data }(\mathrm{a})\end{array}$ \\
\hline 6. Motor Gasoline & Transportation & $0.12507 \mathrm{MMBtu} / \mathrm{gal}$ lon & $5.253 \mathrm{MMBtu} / \mathrm{barrel}$ \\
\hline 7. Diesel Fuel & Transportation & $0.14 \mathrm{MMBtu} / \mathrm{gal}$ lon & $5.825 \mathrm{MMBtu} /$ barrel \\
\hline $\begin{array}{l}\text { 8. Kerosene/Jet } \\
\text { Fuel }\end{array}$ & All & $0.135 \mathrm{MMBtu} / \mathrm{gal} 1 \mathrm{on}$ & $5.670 \mathrm{MMBtu} / \mathrm{barre}$ \\
\hline 9. Residual Fuel & $\begin{array}{l}\text { Commercial } \\
\text { Industrial } \\
\text { Transportation } \\
\text { Electric Utility }\end{array}$ & $\begin{array}{r}\text { 6.287 MMBtu/barrel } \\
6.287 \mathrm{MMBtu} / \text { barrel } \\
6.287 \mathrm{MMBtu} / \mathrm{barrel} \\
\text { Embedded in data(a) }\end{array}$ & $\begin{array}{r}6.287 \mathrm{MMBtu} / \text { barrel } \\
6.287 \mathrm{MMBtu} / \text { barrel } \\
6.287 \mathrm{MMBtu} / \text { barrel } \\
\text { Embedded in data(a) }\end{array}$ \\
\hline 10. $L P G$ & $\begin{array}{l}\text { Residential } \\
\text { Industrial }\end{array}$ & $\begin{array}{c}10.917 \mathrm{gallons} / \mathrm{MMBtu} \\
0.0875 \mathrm{MMBtu} / \mathrm{gallon}\end{array}$ & $\begin{array}{l}\text { 3.836 MMBtu/barrel } \\
\text { 3.643 MMBtu/barrel }\end{array}$ \\
\hline
\end{tabular}

\footnotetext{
(a) Cost and Quality publishes both physical and Btu prices so no conversion
} 
TABLE A.2. Natural Gas Conversion Factors by State, 1981 and 1982 (Btu per cubic foot)

\begin{tabular}{|c|c|c|c|c|c|}
\hline State & 1981 & 1982 & State & 1981 & 1982 \\
\hline$A K$ & 1004 & 1000 & MT & 1011 & 1011 \\
\hline$A L$ & 1036 & 1052 & NC & 1012 & 1033 \\
\hline$A R$ & 1001 & 1002 & ND & 1042 & 1026 \\
\hline$A Z$ & 1053 & 1054 & NE & 978 & 981 \\
\hline$C A$ & 1048 & 1049 & $\mathrm{NH}$ & 1022 & 1020 \\
\hline $\mathrm{CO}$ & 994 & 1000 & $\mathrm{NJ}$ & 1034 & 1031 \\
\hline CT & 1025 & 1027 & NM & 1047 & 1045 \\
\hline$D C$ & 1014 & 1017 & NV & 1076 & 1070 \\
\hline $\mathrm{DE}$ & 1035 & 1033 & NY & 1020 & 1023 \\
\hline $\mathrm{FL}$ & 1059 & 1044 & $\mathrm{OH}$ & 1023 & 1029 \\
\hline GA & 1027 & 1030 & OK & 1035 & 1023 \\
\hline HI & 959 & 989 & OR & 1044 & 1044 \\
\hline IA & 1003 & 1008 & PA & 1022 & 1028 \\
\hline ID & 1070 & 1072 & RI & 1022 & 1036 \\
\hline IL & 1020 & 1022 & SC & 1023 & 1030 \\
\hline IN & 993 & 1016 & SD & 1002 & 999 \\
\hline$K S$ & 987 & 999 & TN & 1016 & 1024 \\
\hline$K Y$ & 1014 & 1014 & $T X$ & 1032 & 1032 \\
\hline LA & 1037 & 1047 & UT & 1073 & 939 \\
\hline MA & 1016 & 1024 & VA & 1024 & 1027 \\
\hline MD & 1014 & 1018 & $V T$ & 993 & 993 \\
\hline ME & 1025 & 1025 & WA & 1050 & 1053 \\
\hline MI & 1017 & 1022 & WI & 1009 & 1012 \\
\hline MN & 995 & 1005 & WV & 1040 & 1047 \\
\hline MO & 1015 & 1018 & WY & 1059 & 1002 \\
\hline MS & 1025 & 1028 & US & 1027 & 1025 \\
\hline
\end{tabular}

Source: Energy Information Administration, Natural Gas Annual 


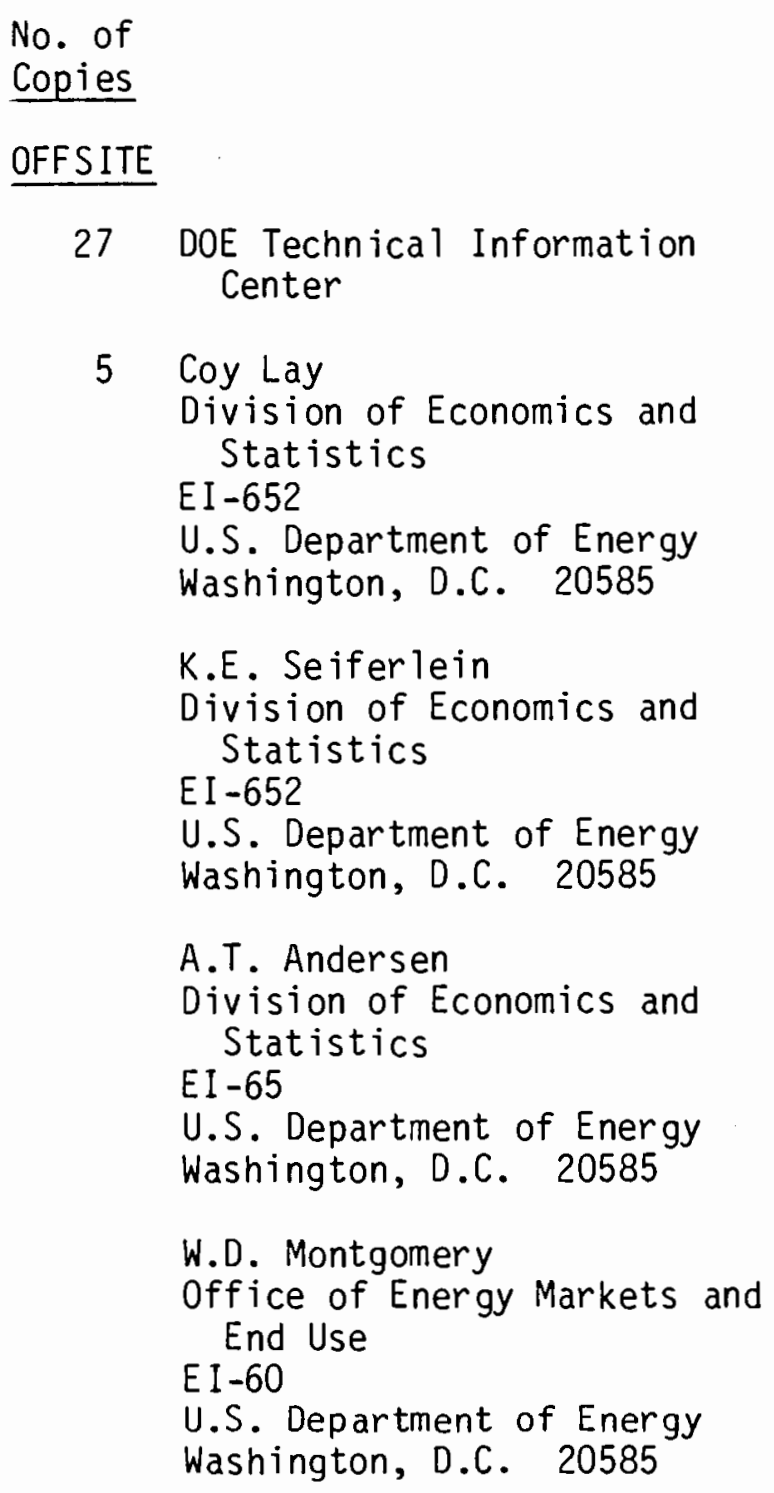

No. of

Copies

J.L. Peterson

Office of $0 i 1$ and Gas

E I -40

U.S. Department of Energy

Washington, D.C. 20585

J.C. Geidl

Office of Coal, Nuclear,

Electric and Alternate Fuels EI -50

U.S. Department of Energy Washington, D.C. 20585

D.R. Hale Quality Assurance Division EI -74

U.S. Department of Enregy Washington, D.C. 20585

ONSITE

DOE Richland Operations

$$
\text { H.E. Ransom }
$$

23 Pacific Northwest Laboratory

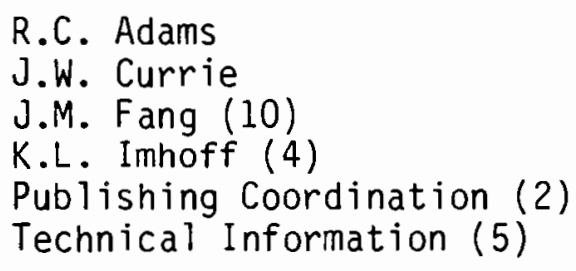




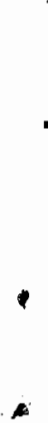

